\title{
ASSESSMENT OF VITRIFIED CBN WHEELS FOR PRECISION GRINDING
}

\section{RUI CAI}

A thesis submitted in partial fulfilment of the requirements of Liverpool John Moores University for the degree of Doctor of Philosophy

May, 2002 


\section{Abstract}

The aim of the investigation was to achieve a method of assessment of vitrified CBN wheels for precision grinding based on investigation of relationships between the changing features of grinding wheels and grinding performance.

Feasible measurement and observation techniques for the changing topographical features of vitrified $\mathrm{CBN}$ wheels in grinding were investigated. Comparisons included measurement resolution, measuring depth, ease of application, and data analysis and interpretation. The optical interferometer was found to be good for measurement of vitrified CBN wheel topography, and a microscope was found to be helpful for understanding the measurement results. In addition, replication techniques were compared and analyzed. A newly developed replica material with high resolution ( 0.1 microns) and easy operation, Microset 101 , was employed as a negative replica material in the experimental work.

A set of parameters was defined which describe the wheel characteristics including active cutting edge density, cutting edge dullness, effective porosity volume ratio and wheel scratch hardness. A parametric study was made of the effects of wheel characteristics on $\mathrm{CBN}$ grinding performance including grinding force, grinding temperature, wheel life and workpiece accuracy using elevated wheel speed with different workpiece materials.

An experimental study was carried out with grinding wheels having different grain sizes, pore volumes, and wheel hardness values for grinding different workpiece materials. Experimental results are discussed in relation to the theoretical understanding of the process. The effects of varying wheel structure, wheel speed and workpiece material are discussed in relation to grinding power, workpiece surface roughness, size error and wheel topography. 
The mechanics of grinding wheel wear and loading are reviewed. Grinding wheel wear and loading trends were predicted. Suggestions are made for the avoidance of grinding wheel wear and loading. 


\section{Acknowledgements}

I would like to express my sincere gratitude to Professor W. Brian Rowe, my Director of Studies, for his invaluable guidance and encouragement throughout the research and his creative suggestions in preparing this thesis.

Thanks are expressed to my second supervisor, Dr. X. Chen, for his assistance in my initial work. Thanks are also due to my third supervisor Professor Ben Mills, particular for his support in providing information concerning new products and suggestions for the experimental system.

I would like to thank Mr. Stephen Ebbrell who cooperated in the EPSRC supported project, for his help and cooperation thoughout my study. I would also like to thank Dr. Michael Morgan for his suggestions for this thesis.

Thanks are due to Mr. Paul Wright and Mr. Peter Moran for technical support and advice in organizing the experimental facilities, Mr. Thomas Scargill, for his hard work and patience throughout the whole series of experiments, Mr. Michael Noord for his practical help in many aspects.

Thanks are due to the Advanced Manufacturing Technology Research Laboratory of Liverpool John Moores University for funding the research.

Acknowledgements are due to EPSRC for grant support and also to the collaborating partners, Castrol, Dittel, Jones \& Shipman, Rolls-Royce, Timken Europe (Research) and Wendt-Boart for financial support, advice and practical assistance.

Finally, I wish to thank my parents and my husband for their encouragement and patience over the last few years. 


\section{Nomenclature}

\begin{tabular}{|c|c|}
\hline Symbol & Meaning \\
\hline$a_{c}$ & depth of cut \\
\hline A & wear flat area in unit area of wheel surface \\
\hline $\mathrm{A}_{\text {an }}$ & $\begin{array}{l}\text { wear flat area per unit surface area on the wheel after } \\
\text { wheel } n \text { revolutions }\end{array}$ \\
\hline$A_{w}$ & wear flat area on one cutting edge \\
\hline $\mathrm{C}_{\mathrm{a}}$ & active cutting edge density \\
\hline $\mathrm{C}_{\mathrm{an}}$ & $\begin{array}{l}\text { active cutting edge density on the wheel after wheel } n \\
\text { revolutions }\end{array}$ \\
\hline $\mathrm{C}_{\text {stat }}$ & static cutting edge density \\
\hline $\mathrm{C}_{\mathrm{dyn}}$ & dynamic cutting edge density \\
\hline $\mathrm{C}_{\mathrm{ga}}$ & grain area density \\
\hline $\mathrm{C}_{\mathrm{gv}}$ & grain volumetric density \\
\hline $\mathrm{C}_{\mathrm{pa}}$ & pore area density \\
\hline $\mathrm{C}_{\mathrm{pv}}$ & pore volumetric density \\
\hline $\mathrm{C}_{\mathrm{ba}}$ & $\begin{array}{l}\text { grain density removed by bond fracture and grain } \\
\text { fracture per unit scratch length }\end{array}$ \\
\hline $\mathrm{C}_{\mathrm{bf}}$ & $\begin{array}{l}\text { grain density of the grains removed by bond fracture per } \\
\text { unit scratch length }\end{array}$ \\
\hline $\mathrm{C}_{\mathrm{gf}}$ & $\begin{array}{l}\text { grain density of the grains removed by grain fracture } \\
\text { per unit scratch length }\end{array}$ \\
\hline$d_{c}$ & equivalent wheel diameter \\
\hline$d_{s}$ & wheel diameter \\
\hline
\end{tabular}


$d_{b a}$

$d_{p}$

$d_{g}$

f

$\mathrm{F}_{\text {gst }}$

$f_{c n}$

$f_{c t}$

$h_{\max }$

$\mathrm{h}_{\mathrm{cu}}$

$\mathrm{H}_{\mathrm{p}}$

$\mathrm{H}_{\mathrm{ss}}$

$\mathrm{H}_{\mathrm{sb}}$,

$\mathrm{H}_{\mathrm{s}}$

K

$\mathrm{k}_{\mathrm{a}}$

L

m

n

$\mathrm{p}$

$\mathrm{P}_{\mathrm{f}}$

$\mathrm{P}_{\mathrm{r}}$ mean diameter ratio of the grains removed

pore size

grain size

overall grinding force ratio

average tangential force on the grains

normal load on one cutting edge

tangential force on one cutting edge

maximum uncut chip thickness

mean uncut chip thickness

penetration hardness

wheel scratch hardness

theoretical wheel bond hardness

indentation hardness of abrasive

attritious or adhesive wear rate

volume ratio of adhesive particles which adhered to

cutting edges to the whole adhesive particles

grain spacing

number of bond bridges around each grain

active cutting edges on active grain

pressure between cutting edge and workpiece

probability of grain fracture wear and of bond fracture

wear when contact occurs between active grains and the

workpiece

probability of a new active cutting edge appearing after

grain and bond fracture 
pore loading probability between two cutting edges ratio of mean chip width to mean uncut chip thickness initial cutting edge radius

cutting edge radius

sliding distance for contact with the cutting edge temperature

workpiece speed

wheel peripheral speed

attritious wear volume on one cutting edge

adhesive wear volume from workpiece under one

cutting edge

percentage volume of bond

percentage volume of grains

percentage volume of pores

effective porosity volume ratio of the wheel

volume of wheel

radial distance

cutting edge dullness

infeed angle

contact time between cutting edge and workpiece in one

wheel revolution

friction coefficient

interfacial energy which depends on the grain and workpiece pair

surface energy of the workpiece material

surface energy of the grain material 
Abstract I

Acknowledgments III

Nomenclature IV

Contents

List of Figures $\quad$ XI

List of Tables $\quad X X$

Chapter 1 Introduction 1

1.1. Benefits of vitrified CBN wheels 1

1.2. Limitations in the use of CBN grinding wheels 2

1.3. The need for the research 3

1.4. Aim and objectives 5

1.5. Scope of the investigation 5

PART 1 MEASUREMENT AND CHARACTERIZATION OF VITRIFIED CBN GRINDING WHEEL TOPOGRAPHY

Chapter 2 Measurement of vitrified CBN grinding wheel topography 8

2.1. Review of techniques for examination of wheel topography 8

2.2. Principles for selection of measurement techniques 12

2.3. Experimental equipment and procedure 13

2.3.1. Experimental equipment 13

$\begin{array}{ll}\text { 2.3.2. Experimental procedure } & 16\end{array}$

$\begin{array}{ll}\text { 2.4. Experimental results } & 17\end{array}$

$\begin{array}{ll}\text { 2.5. Discussion } & 19\end{array}$

2.5. Conclusions 21

Chapter 3 Evaluation of vitrified CBN grinding wheel topography 22

3.1. Review of evaluation parameters 22

3.2. Selection of evaluation parameters 25

3.2.1. Definition of active cutting edge density 25

3.2.2. Definition of cutting edge dullness 26

3.2.3. Definition of effective porosity volume ratio 26 
3.3.1. The measurement and observation instruments and analysis software

3.3.2. The micromachining rig and dynamometer 28

$\begin{array}{ll}\text { 3.3.3. Grinding wheels } & 30\end{array}$

3.4. Experimental procedure 30

3.5. Experimental results $\quad 30$

3.5.1. Active cutting depth 30

3.5.2. Active cutting edge density 31

3.5.3. Cutting edge dullness $\quad 32$

3.5.4. Effective porosity volume ratio 32

3.5.5. Wheel scratch hardness $\quad 32$

3.6. Discussion of experimental results 33

3.6.1. Active cutting depth 33

3.6.2. Wheel evaluation parameters $\quad 35$

$\begin{array}{ll}3.7 . \text { Conclusions } & 36\end{array}$

\section{PART 2 RELATIONSHIPS BETWEEN WHEEL STRUCTURE AND GRINDING PERFORMANCE}

Chapter 4 Literature review $\quad 40$

4.1. Structure of vitrified CBN wheels $\quad 40$

4.2. Grinding force and specific energy 42

4.3. Grinding temperature $\quad 47$

4.4. Grinding wheel wear $\quad 50$

4.5. Grinding wheel loading $\quad 53$

Chapter 5 Theory $\quad 57$

5.1. A model for a vitrified CBN wheel structure 57

5.2. Effect of vitrified $\mathrm{CBN}$ wheel structure on active cutting edge density 59

5.3. Effect of vitrified CBN wheel structure on porosity volume ratio 62 
5.4. Effect of vitrified CBN wheel structure on wheel scratch hardness 63

5.5. Prediction of grinding wheel wear $\quad 74$

$\begin{array}{ll}\text { 5.6. Prediction of grinding wheel loading } & 79\end{array}$

\section{Chapter 6 Experimental investigation of vitrified CBN wheel}

$$
\text { performance }
$$

6.1. The experimental system $\quad 84$

6.1.1. The grinding machines $\quad 84$

6.1.2. The grinding wheels 85

6.1.3. The workpiece materials $\quad 87$

6.1.4. The coolant delivery system $\quad 89$

6.1.5. The dressing conditions $\quad 89$

6.1.6. Power measurement 90

6.1.7. Workpiece measurement 90

6.1.8. The application and measurement of replicas for wheel topography 90

6.2. The grinding conditions 91

6.2.1. The grinding conditions for internal grinding 91

6.2.2. The grinding conditions for external grinding 94

6.3. Experimental procedure $\quad 97$

6.4. Experimental results for internal grinding 98

6.4.1. M2 tool steel 98

6.4.2. Inconel 718 aerospace alloy $\quad 102$

6.5. Experimental results for external grinding on the Suprema machine 103

$\begin{array}{ll}\text { 6.5.1. AISI52100 bearing steel } & 104\end{array}$

6.5.2. Inconel 718 aerospace alloy $\quad 110$

$\begin{array}{ll}\text { 6.6. Discussion of experimental results } & 114\end{array}$

6.6.1. Porosity volume ratio 114

$\begin{array}{ll}\text { 6.6.2. Wheel speed } & 115\end{array}$ 
7.1. Prediction of wheel wear and loading

7.1.1. Calibration of parameters for prediction of wheel wear

7.1.2. Calibration of parameters for prediction of wheel loading

7.2. Prediction values of wheel wear and loading

7.3. Discussion

7.3.1. Effect of wheel structure

7.3.2. Effect of wheel speed

7.4. Suggestions for optimization of grinding wheels

A 1. Matlab program for calculating grain area density along the radial depth of wheel (whelstruc11.m)

A 2. Matlab program for calculating porosity volume ratio (porosity2.m) 149

A 3. Matlab program for prediction of grinding wheel wear (wheelwear3.m)

A 4. Matlab program for prediction of grinding wheel loading (wheelloading2.m) 


\section{List of Figures}

Fig. 2.1 Basic principle of an optical interferometer [11]

Fig. 2.2 Basic principle of laser triangulation [12]

Fig. 2.3 A resolution and range of different measuring techniques

Fig. 2.4 Talysurf (3D) instrument

Fig. 2.5 Wyko RST plus instrument

Fig. 2.6 Uniscan OSP 100 instrument

Fig. 2.7 Olympus BH2-UMA optical microscope with a mounted Olympus SC 35 camera

Fig. 2.8 The replication rig for lead replicas

Fig. 2.9 Calibration of the elastic coefficient of the spring

Fig. 2.10 The replication rig for Microset 101 replicas

Fig. 2.11 Comparison of wheel surface roughness measured by three measurement methods

Fig. 2.12 (a) 2D image measured by Talysurf on the wheel surface

Fig. 2.12 (b) 3D image measured by Talysurf on the wheel surface

Fig. 2.12 (c) 2D image measured by Wyko RST on the wheel surface

Fig. 2.12 (d) 3D image measured by Wyko RST on the wheel surface

Fig. 2.12 (e) 2D image measured by Uniscan OSP 100 on the wheel surface

Fig. 2.12 (f) 3D image measured by Uniscan OSP 100 on the wheel surface

Fig. 2.13 (a) Measurements from the same part of a wheel by optical interferometry with Magnification of $41.3 \times$

Fig. 2.13 (b) Measurements from the same part of a wheel by optical interferometry with Magnification of $10.3 \times$

Fig. 2.14 (a) Microscope photograph of stylus before measuring a CBN wheel area of $1 \mathrm{~mm}^{2}$ 
Fig. 2.14 (b) Microscope photograph of stylus after measuring a CBN wheel area of $1 \mathrm{~mm}^{2} 5$ times

Fig. 2.15 Microscope photograph of wheel surface after grinding (Wheel: B91-150V1)

Fig. 2.16 SEM photograph lead replica after making a replication of wheel

Fig. 2.17 SEM photograph of a Microset 101 replica which melted during coating

Fig. 2.18 (a) Interferometry measurement of Microset replica

Fig. 2.18 (b) Interferometry measurement of wheel

Fig. 3.1 Cutting edge dullness

Fig. 3.2 Illustration of infeed angle $\varepsilon$ of material flow direction relative to a cutting point on the wheel periphery and wheel rotation angle $\theta^{\prime}$

Fig. 3.3 Volume of pores

Fig. 3.4 Scheme of transfering measurement results to Matlab software to calculate cutting edge density

Fig. 3.5 Function to calculate pore volume and peak volume between a certain depth

Fig. 3.6 Measurement of grinding wheel hardness by the scratch method

Fig. 3.7 Microcutting system

Fig. 3.8 (a) Schematic view of the two-component dynamometer in the microcutting system

Fig. 3.8 (b) Scheme of corresponding beam with applied forces and bending moment

Fig. 3.8 (c) The bridge connection for measuring $P_{z}$ and $P_{y}$ forces

Fig. $3.9 \quad$ Force calibration for microcutting

Fig. 3.10 (a) Surface grinding process

Fig. 3.10 (b) Uncut chip thickness, $h_{c u}$ and maximum uncut chip thickness, $h_{\max }$

Fig. 3.10 (c) Uncut chip width, $b_{c u}$ 
Fig. 3.11(a) Active cutting edge density of the newly dressed wheel, worn wheel and loaded wheel

Fig. 3.11(b) Cutting edge dullness of the newly dressed wheel, worn wheel and loaded wheel

Fig. 3.11(c) Porosity volume ratio of the newly dressed wheel, worn wheel and loaded wheel

Fig. 3.12 Results of wheel scratch hardness tests

Fig. 4.1 Representative face structure of vitrified-bond wheels

Fig. 4.2 Basic three-component diagram for abrasive tools [39]

Fig. 5.1 Assumed wheel structure

Fig. 5.2 Grain area density with radial depth into wheel

Fig. 5.3 Grain spacing

Fig. 5.4 (a) Distribution of grain spacings at radial depth of wheel $z=0.5 . d g$ with different pore sizes dp

Fig. 5.4 (b) Distribution of grain spacings at radial depth of wheel $z=0.5 . \mathrm{dg}$ with different grain sizes $\mathrm{dg}$

Fig. 5.5 (a) Effect of wheel concentration and depth of wheel surface on porosity volume ratio of vitrified $\mathrm{CBN}$ wheel structure

Fig. 5.5 (b) Effect of percentage of pore volume and pore size on porosity volume ratio of vitrified $\mathrm{CBN}$ wheel structure

Fig. 5.6 Grains are assumed to be arrayed within cone envelopes

Fig. 5.7 (a) Force analysis when 3 grains are above the active grain

Fig. 5.7 (b) Force analysis when 4 grains are above the active grain

Fig. 5.7 (c) Force analysis when 5 grains are above the active grain

Fig. 5.8 The reduction of bond force with the increasing of half vertex angle of the cone envelope

Fig. 5 .9 Relationship between grain tangential force and scratch force

Fig. 5.10 The basic steps in prediction of wheel wear 
Fig. $5.11 \quad$ Attritious wear of cutting edge

Fig. 5.12 The basic steps in prediction of wheel wear and wheel loading

Fig. 6.1 Jones \& Shipman 1300X internal grinding machine

Fig. 6.2 Installation of workpiece for internal grinding

Fig. 6.3 Jones \& Shipman Suprema machine

Fig. 6.4 Internal grinding wheels

Fig. 6.5 (a) SEM photograph of HP- B64 wheel

Fig. 6.5 (b) SEM photograph of MP- B91 wheel

Fig. 6.5 (c) SEM photograph of HP-B91 wheel

Fig. 6.6 A wheel for external grinding (B91-150VR)

Fig. 6.7 Workpieces for external grinding

Fig. 6.8 Coolant delivery system for $\mathrm{CBN}$ internal grinding

Fig. 6.9 Internal grinding dressing system

Fig. 6.10 The dressing disc for external grinding

Fig. 6.11 The power measurement system

Fig. 6.12 Calibration results of power analyzer D4355

Fig. 6.13 (a) Maximum power when grinding M2 at a wheel speed of $45 \mathrm{~m} / \mathrm{s}$

Fig. 6.13 (b) Specific energy when grinding M2 at a wheel speed of $45 \mathrm{~m} / \mathrm{s}$

Fig. 6.13 (c) Workpiece size error when grinding M2 at a wheel speed of $45 \mathrm{~m} / \mathrm{s}$

Fig. 6.13 (d) Workpiece roundness when grinding M2 at a wheel speed of 45 $\mathrm{m} / \mathrm{s}$

Fig. 6.13 (e) Workpiece Ra surface roughness when grinding M2 at a wheel speed of $45 \mathrm{~m} / \mathrm{s}$

Fig. 6.13 (f) Workpiece $R z$ surface roughness when grinding $M 2$ at a wheel speed of $45 \mathrm{~m} / \mathrm{s}$

Fig. $6.13(\mathrm{~g}) \quad$ Workpiece Rt surface roughness when grinding M2 at a wheel speed of $45 \mathrm{~m} / \mathrm{s}$ 
Fig. $6.13(\mathrm{~h})$ Active cutting edge density when grinding M2 at a wheel speed of $45 \mathrm{~m} / \mathrm{s}$

Fig. 6.13 (i) Cutting edge dullness when grinding M2 at a wheel speed of 45 $\mathrm{m} / \mathrm{s}$

Fig. 6.14 (a) Maximum power when grinding M2 at a wheel speed of $55-60 \mathrm{~m} / \mathrm{s}$

Fig. 6.14 (b) Specific energy when grinding M2 at a wheel speed of $55-60 \mathrm{~m} / \mathrm{s}$

Fig. 6.14 (c) Workpiece size error when grinding M2 at a wheel speed of 55-60 $\mathrm{m} / \mathrm{s}$

Fig. 6.14 (d) Workpiece roundness when grinding M2 at a wheel speed of 55-60 $\mathrm{m} / \mathrm{s}$

Fig. 6.14 (e) Workpiece Ra surface roughness when grinding M2 at a wheel speed of $55-60 \mathrm{~m} / \mathrm{s}$

Fig. 6.14 (f) Active cutting edge density when grinding M2 at a wheel speed of $55-60 \mathrm{~m} / \mathrm{s}$

Fig. 6.14 (g) Cutting edge dullness when grinding M2 at a wheel speed of 55-60 $\mathrm{m} / \mathrm{s}$

Fig. 6.15 (a) Maximum power when grinding M2 at a wheel speed of $110 \mathrm{~m} / \mathrm{s}$

Fig. 6.15 (b) Specific energy when grinding M2 at a wheel speed of $110 \mathrm{~m} / \mathrm{s}$

Fig. 6.15 (c) Workpiece size error when grinding M2 at a wheel speed of 110 $\mathrm{m} / \mathrm{s}$

Fig. 6.15 (d) Workpiece roundness when grinding M2 at a wheel speed of 110 $\mathrm{m} / \mathrm{s}$

Fig. 6.15 (e) Workpiece Ra surface roughness when grinding M2 at a wheel speed of $110 \mathrm{~m} / \mathrm{s}$

Fig. 6.15 (f) Active cutting edge density when grinding M2 at a wheel speed of $110 \mathrm{~m} / \mathrm{s}$

Fig. $6.15(\mathrm{~g})$ Cutting edge dullness when grinding M2 at a wheel speed of 110 $\mathrm{m} / \mathrm{s}$

Fig. 6.16 (a) Maximum power when grinding Inconel 718 at a wheel speed of $50-60 \mathrm{~m} / \mathrm{s}$ 
Fig. 6.16 (b) Specific energy when grinding Inconel 718 at a wheel speed of 50$60 \mathrm{~m} / \mathrm{s}$

Fig. 6.16 (c) Workpiece size error when grinding Inconel 718 at a wheel speed of $50-60 \mathrm{~m} / \mathrm{s}$

Fig. 6.16 (d) Workpiece roundness when grinding Inconel 718 at a wheel speed of $50-60 \mathrm{~m} / \mathrm{s}$

Fig. 6.16 (e) Workpiece Ra surface roughness when grinding Inconel 718 at a wheel speed of $50-60 \mathrm{~m} / \mathrm{s}$

Fig. 6.16 (f) Active cutting edge density when grinding Inconel 718 at a wheel speed of $50-60 \mathrm{~m} / \mathrm{s}$

Fig. $6.16(\mathrm{~g})$ Cutting edge dullness when grinding Inconel 718 at a wheel speed of $50-60 \mathrm{~m} / \mathrm{s}$

Fig. 6.16 (h) Effective wheel porosity volume ratio when grinding Inconel 718 at a wheel speed of $50-60 \mathrm{~m} / \mathrm{s}$

Fig. 6.17 (a) Direct effects charts of process parameters on the mean specific energy for AISI 52100 on Suprema

Fig. 6.17 (b) Direct effects charts of process parameters on the mean surface roughness for AISI 52100 on Suprema

Fig. 6.17 (c) Direct effects charts of process parameters on the mean roundness for AISI 52100 on Suprema

Fig. 6.17 (d) Direct effects charts of process parameters on the mean size holding for AISI 52100 on Suprema

Fig. 6.17 (e) Direct effects charts of process parameters on the mean G-ratio for AISI 52100 on Suprema

Fig. 6.18(a) Specific energy of Confirmation Trial 1 for improved workpiece quality grinding AISI 52100 on the J\&S Suprema

Fig. 6.18(b) Surface roughness of Confirmation Trial 1 for improved workpiece quality grinding AISI 52100 on the J\&S Suprema

Fig. 6.18(c) Roundness of Confirmation Trial 1 for improved workpiece quality grinding AISI 52100 on the J\&S Suprema

Fig. 6.18(d) Size holding of Confirmation Trial 1 for improved workpiece quality grinding AISI 52100 on the J\&S Suprema 
Fig. 6.19(a) Specific energy of Confirmation Trial 2 for improved workpiece quality grinding AISI 52100 on the J\&S Suprema

Fig. 6.19(b) Surface roughness of Confirmation Trial 2 for improved workpiece quality grinding AISI 52100 on the J\&S Suprema

Fig. 6.19(c) Roundness of Confirmation Trial 2 for improved workpiece quality grinding AISI 52100 on the J\&S Suprema

Fig. 6.19(d) Size holding of Confirmation Trial 2 for improved workpiece quality grinding AISI 52100 on the J\&S Suprema

Fig. 6.20 (a) Direct effects charts of process parameters on the mean specific energy for Inconel 718 on Suprema

Fig. 6.20 (b) Direct effects charts of process parameters on the mean surface roughness for Inconel 718 on Suprema

Fig. 6.20 (c) Direct effects charts of process parameters on the mean roundness for Inconel 718 on Suprema

Fig. 6.20 (d) Direct effects charts of process parameters on the mean size holding for Inconel 718 on Suprema

Fig. 6.20 (e) Direct effects charts of process parameters on the mean G-ratio for Inconel 718 on Suprema

Fig. 6.21(a) Specific energy of wheel life trial for grinding Inconel 718 on the J\&S Suprema

Fig. 6.21(b) Surface roughness wheel life trial for grinding Inconel 718 on the J\&S Suprema

Fig. 6.21(c) Roundness of wheel life trial for grinding Inconel 718 on the J\&S Suprema

Fig. 6.21(d) Size holding of wheel life trial for grinding Inconel 718 on the J\&S Suprema

Fig. 6.22 (a) Adhesive loaded grains when grinding Inconel 718

Fig. 6.22 (b) Compact mass loading when grinding Inconel 718

Fig. 6.22 (c) Grains pulled off when grinding Inconel 718

Fig. 6.23 Changes in material hardness with temperature. 
Fig. 6.24 Proposed trend of adhesive loading with increase of wheel speed for a particular set of grinding conditions and a particular grinding distance

Fig. 7.1(a) The effect of grain size on cutting edge density in prediction of wheel wear

Fig. 7.1(b) The effect of grain size on wear flat radius in prediction of wheel wear

Fig. 7.1(c) The effect of grain size on workpiece Ra surface roughness in prediction of wheel wear

Fig. 7.2(a) The effect of percentage of pore volume on cutting edge density in prediction of wheel wear

Fig. 7.2(b) The effect of percentage of pore volume on wear flat radius in prediction of wheel wear

Fig. 7.2(c) The effect percentage of pore volume on workpiece Ra surface roughness in prediction of wheel wear

Fig. 7.3(a) The effect of wheel speed on cutting edge density in prediction of wheel wear

Fig. 7.3(b) The effect of wheel speed on wear flat radius in prediction of wheel wear

Fig. 7.3(c) The effect wheel speed on workpiece Ra surface roughness in prediction of wheel wear

Fig. 7.4(a) The effect of grain size on cutting edge density in prediction of wheel loading

Fig. 7.4(b) The effect of grain size on wear flat radius in prediction of wheel loading

Fig. 7.4(c) The effect of grain size on workpiece Ra surface roughness in prediction of wheel loading

Fig. 7.4(d) The effect of grain size on adhesive wear coefficient in prediction of wheel loading

Fig. 7.4(e) The effect of grain size on pore loading probability in prediction of wheel loading

Fig. 7.5(a) The effect of percentage of pore volume on cutting edge density in prediction of wheel loading 
Fig. 7.5(b) The effect of percentage of pore volume on wear flat radius in prediction of wheel loading

Fig. 7.5(c) The effect of percentage of pore volume on workpiece Ra surface roughness in prediction of wheel loading

Fig. 7.5(d) The effect of percentage of pore volume on adhesive wear coefficient in prediction of wheel loading

Fig. 7.5(e) The effect of percentage of pore volume on pore loading probability in prediction of wheel loading

Fig. 7.6(a) The effect of wheel speed on cutting edge density in prediction of wheel loading

Fig. 7.6(b) The effect of wheel speed on wear flat radius in prediction of wheel loading

Fig. 7.6(c) The effect of wheel speed on workpiece Ra surface roughness in prediction of wheel loading

Fig. 7.6(d) The effect of wheel speed on adhesive wear coefficient in prediction of wheel loading

Fig. 7.6(e) The effect of wheel speed on pore loading probability in prediction of wheel loading

Fig. 7.6(f) The adhesive wear coefficient at wheel speed of $90 \mathrm{~m} / \mathrm{s}$ in prediction of wheel loading

Fig. 7.6(g) The pore loading probability at wheel speed of $90 \mathrm{~m} / \mathrm{s}$ in prediction of wheel loading 


\section{List of Tables}

Table 2.1 Comparison of measurement techniques

Table 3.1 Relationship between active cutting edge density and radial depth of wheel

Table 3.2 Experimental results of wheel scratch hardness

Table 5.1 Relationship between half vertex angle of the cone, $\theta$, pore size $\mathrm{dp}$, and grain size dg

Table 5.2 Relationship between pore volume $\mathrm{Vp}$ and average half vertex angle of the cone $\theta$

Table 6.1 Composition of the workpiece materials

Table 6.2 Mechanical and thermal properties of the workpiece materials

Table 6.3 Experimental arrangement for AISI52100 on the Suprema machine

Table 6.4 Two levels for each of the process parameters for AISI 52100

Table 6.5 Experimental arrangement for Inconel 718 on the Suprema machine

Table 6.6 Two levels for each of the process parameters for Inconel 718

Table 6.7 Value of $\mathrm{t}_{\mathrm{obt}}$ for performance indicator

Table 6.8 Results for Confirmation Trial 1 - AISI 52100 on the Suprema machine

Table 6.9 Results for Confirmation Trial 2 - AISI 52100 on the Suprema machine

Table 6.10 Calculated grinding temperatures when grinding Inconel 718

Table 7.1 Relationship between initial active cutting edge density and percent of pore volume

Table 7.2 Tangential cutting force with different maximum uncut chip thickness when grinding M2 workpiece material at a wheel speed of $45 \mathrm{~m} / \mathrm{s}$

Table 7.3 Tangential cutting force with different maximum uncut chip thickness when grinding M2 workpiece material at a wheel speed of $60 \mathrm{~m} / \mathrm{s}$ 
Table 7.4 Tangential cutting force with different maximum uncut chip thickness when grinding M2 workpiece material at a wheel speed of $110 \mathrm{~m} / \mathrm{s}$

Table 7.5 Parameter value in Equation 7.9 at a wheel speed of $45 \mathrm{~m} / \mathrm{s}$

Table 7.6 Parameter value in Equation 7.9 at a wheel speed of $60 \mathrm{~m} / \mathrm{s}$

Table 7.7 Parameter value in Equation 7.9 at a wheel speed of $110 \mathrm{~m} / \mathrm{s}$

Table 7.8 Calibration of workpiece surface for M2

Table 7.9 Tangential cutting force with different maximum uncut chip thickness when grinding Inconel 718 workpiece material at a wheel speed of $50 \mathrm{~m} / \mathrm{s}$

Table 7.10 Tangential cutting force with different maximum uncut chip thickness when grinding Inconel 718 workpiece material at a wheel speed of $60 \mathrm{~m} / \mathrm{s}$

Table 7.11 Parameter value in Equation 7.10

Table 7.12 Calibration of workpiece surface for Inconel 718 


\section{Chapter 1 Introduction}

\subsection{Benefits of vitrified CBN wheels}

Cubic Boron Nitride (CBN) is a commercially available superabrasive that is synthesized at high temperature and high pressure. CBN is the second hardest material in the world, it is 2 to 3 times harder than aluminium oxide abrasive, and it is chemically inert. The properties of CBN are exploited in grinding a range of materials including hard-to-grind ferrous alloys as well as nickel and cobalt-based alloys. The hardness and wear characteristics of CBN allow low grinding forces, low grinding temperatures, high cutting efficiency and consistent part quality to be maintained for a long period before redressing is required. CBN wheels have been successfully used in a variety of precision grinding applications including internal, external, cam-contour, centreless, surface, double-disc, creep-feed, endmill feature, form, fluting, slot/groove, thread and jig grinding operations [1]. Improvements in quality and productivity were reported from its application.

Vitrified bond CBN wheels offer potential advantages compared to conventional dressable wheels due to the greater wear resistance and thermal stability of CBN [1]. Vitrified bond CBN wheels offer an advantage over plated CBN wheels in that vitrified wheels can be redressed. The fact that vitrified $C B N$ wheels can be redressed also improves the economics of CBN grinding compared with single layer wheels since the life of the wheel is extended each time the wheel is redressed. The pores in vitrified bond wheels play an important role in assisting the flow of grinding fluid into the grinding contact zone. The pores also ease the problem of achieving effective removal of the chips from the wheel surface [1].

The benefits claimed when using vitrified CBN under ideal conditions included improved size and form holding, more consistent surface texture, virtual elimination of thermal damage, improved grinding wheel redress life, higher machine utilization, reduced lead time and improved productivity [2]. It is also claimed that vitrified CBN not only offers increased productivity, and improved workpiece quality in the right applications but also offers reduced grinding costs. 


\subsection{Limitations in the use of $\mathrm{CBN}$ grinding wheels}

The successful application of CBN wheels is not as straightforward as with conventional abrasives [3]. Success requires both an understanding of the grinding process fundamentals and a commitment of resources to make CBN work. The grinding system when using conventional abrasives is to some extent forgiving in that system faults or weaknesses can generally be overcome by redressing the grinding wheel with a diamond tool after every part or several times per part. Continual dressing aims to restore the correct grinding wheel form and a sharp cutting surface.

CBN wheels, are orders of magnitude more expensive than conventional wheels. The justification for using CBN therefore relies on achieving improved quality and accuracy, reducing costs by extending redress life and increasing removal rates. Increased productivity must more than offset the increased costs of the wheels, the dressing and conditioning. However, too frequent redressing and large dressing depth removes expensive abrasive from the wheel and hence tends to negate the cost benefits of increased productivity. Also, large dressing depth may detract from the cutting ability of the wheel by closing up the wheel surface [4]. The high wear resistance of $\mathrm{CBN}$ makes grinding performance very sensitive to bond structure and initial wheel preparation.

While, some companies have apparently experienced substantial gains in accuracy and productivity employing vitrified $\mathrm{CBN}$, many have encountered difficulties in conditioning vitrified $\mathrm{CBN}$ wheels and have reverted to conventional abrasives. A CIRP international round robin experiment [3] showed that even knowledgeable users had difficulty maintaining $3 \mathrm{~mm}^{3} / \mathrm{mm} . \mathrm{s}$ removal rate using vitrified $\mathrm{CBN}$ to finish-grind precision bearing rings. The problems were related to the difficulties of wheel conditioning. Combined with high initial cost, the difficulties of implementation have limited the application of $C B N$ wheels and led to a failure to realize the full benefits of $C B N$ grinding. 


\subsection{The need for the research}

Research into the implementation of vitrified CBN has mainly concentrated on achieving high removal rates up to $30 \mathrm{~mm}^{3} / \mathrm{mm} . \mathrm{s}$ for grinding free cutting materials [5]. Less attention has been paid into the benefits for accuracy, surface roughness and surface integrity when grinding high precision parts. In research by Klocke, rubber bonded $\mathrm{SiC}$ conditioning sticks were required to achieve high removal rates and a substantial volume of grinding production was wasted in the process. Westkämper [6] reported problems due to insufficient pores in plated CBN. Onchi [7] proposed vitrified CBN wheels with giant pores to overcome the problem of wheel loading in super-finishing.

The problems of implementation can be very significant. If a CBN wheel does not cut efficiently, the temperature is increased, a hardened workpiece may be softened, the wheel surface may load with workpiece material and workpiece surface texture rapidly deteriorates. Under these conditions, normal forces may rapidly increase and may lead to vibration and even to catastrophic failure. In consequence, none of the benefits of $\mathrm{CBN}$ may be realized and grinding performance may be far worse than with conventional abrasives.

Initial experimental investigations within the AMT Research Laboratory (AMTTREL) led to the conclusion that while harder grains require stronger bonds, this strength is often achieved at the cost of reduced grinding efficiency. Fine surface texture requirements may be achievable through the application of small grit sizes and higher wheel speeds. Increased bond strength tends to be associated with small pore size consequent on small grit size and increased bond volume. This has the unfortunate consequence that the chip removal space is reduced. Reduced chip removal space encourages wheel loading.

This problem is widely tackled by undertaking conditioning processes to erode the bond and create larger chip spaces. Conditioning processes are wasteful on several accounts. Conditioning takes up production time, it wears the expensive CBN grains causing wheel bluntness and introduces errors into the wheel profile. 
Initially when grinding after conditioning, it may be necessary to operate at reduced removal rates. The economics of production with $\mathrm{CBN}$ require that dressing be greatly reduced compared with conventional abrasives.

To some extent, the problem can be overcome by a special dressing technique known as 'touch dressing' where the depths dressed from the wheel are very small. Contact with the wheel is detected using acoustic emission sensing and contact analysis techniques. Work already accomplished at AMTREL on CBN grinding has demonstrated the potential of acoustic emission techniques for touch dressing using fluid coupling [8]. This technique using monitoring frequencies up to $1 \mathrm{MHz}$ was found to be reliable to detect $0.1 \mu \mathrm{m}$ cuts.

Wheel redress life of vitrified CBN wheels is limited in some cases by wheel loading rather than by grinding wheel wear. Whilst this is less of a problem for conventional grinding wheels that are redressed frequently, it undermines the main advantage of using an expensive $\mathrm{CBN}$ wheel to achieve a long redress life with stable grinding conditions. In order to achieve cost effective CBN grinding it is necessary to investigate the causes of wheel loading, its prevention by the selection of optimal grinding and dressing parameters, the application of wheel cleaning techniques and the optimal application of fluid, the engineering of stronger bonds and the application of more porous wheel structures. A potentially fruitful way forward is to employ wheels with artificially increased pore size. It is found that if an optimum wheel-workpiece combination can be found, wheel redress life can be improved by a factor of ten [2]. This has potential for enormous improvements in productivity.

In order to optimise wheel structures, it is necessary to investigate the performance and characterization of $\mathrm{CBN}$ wheels in different grinding stages. This calls for efficient and convenient methods to observe, measure and characterize the changing topographical features of the wheel surface including grit size, shape, spacing, density in the surface and loading proportion. In addition, the mechanisms of grinding wheel loading and wheel wear are not well 
understood nor how the grinding parameters, workpiece material, and wheel specification affect these processes. It is therefore necessary to know how to assess a grinding wheel to optimise wheel selection and process operation to achieve better grinding performance.

\subsection{Aim and objectives}

The aim of the research was to determine methods for assessment of vitrified CBN grinding wheels and their performance in precision grinding. Major objectives of the research were to achieve,

(i) Measurement and observation techniques for the changing topographical features of vitrified CBN wheels in grinding;

(ii) A set of parameters which describe the wheel characteristics including grit size, shape, spacing, density, grit wear, and loading proportion;

(iii) A parametric study of the effects of wheel characteristics on CBN grinding performance including the effects on grinding force, grinding temperature, wheel life, workpiece accuracy at low and elevated wheel speeds with different workpiece materials;

(iv) An approach to assessment and optimisation of wheel structure of vitrified $\mathrm{CBN}$ wheels for precision grinding.

\subsection{Scope of the investigation}

The research programme was divided into three main parts:

(i) A comparison of measurement and observation techniques for the changing topographical features of vitrified CBN wheels in grinding.

(ii) An investigation of the description of grinding wheel characteristics.

(iii) An investigation of the effect of grinding wheel structure on grinding performance leading to proposals for a strategy for evaluation and optimisation of vitrified CBN wheels for precision grinding.

The first part of the research on measurement and observation techniques for CBN grinding wheel topography includes a comparison of measurement resolutions, measuring depths, ease of application, and data analysis and 
interpretation for different techniques. Optical interferometry was found to be a good method for measuring vitrified CBN wheel topography, and microscopy was found to be useful for understanding the measurement results. In addition, replication techniques were compared and analysed. A recently developed replica material with high resolution ( 0.1 microns) and easy operation, Microset 101, was employed as a negative replica material in the experimental work. Working parameters were defined for the characterization of the grinding wheels used for the subsequent investigations of grinding performance.

In the second part, an experimental study was undertaken with grinding wheels of different grain sizes, pore volumes, and wheel hardnesses to grind different workpiece materials. Experimental results are discussed in relation to the theoretical understanding of the process. The effects of varying wheel structure and workpiece material are discussed in relation to grinding power, size error and associated changes in wheel topography.

In the third part of the research, the mechanics of grinding wheel wear and loading are examined. A method for prediction of grinding wheel wear and loading is proposed. Suggestions are made for avoidance of grinding wheel wear and loading. A strategy is proposed for evaluation and optimisation of vitrified $\mathrm{CBN}$ wheels for precision grinding. 
PART 1. MEASUREMENT AND CHARACTERIZATION OF CBN GRINDING WHEEL TOPOGRAPHY 


\section{Chapter 2 Measurement of vitrified CBN grinding wheel topography}

\subsection{Review of techniques for examination of wheel topography}

A grinding wheel is composed of abrasive grains, bond material and pores. The grains provide the hard edges to cut the workpiece material being ground. The bond material holds the abrasive grains in position and spaces the grains apart. The empty pores provide clearance space for workpiece swarf and also space for coolant to be conveyed into the grinding zone. The three phases form a threedimensional matrix. The spatial distribution of abrasive grains over the wheel surface and the morphology of the grains in the contact surface of the grinding wheel comprise the grinding wheel topography. The wheel topography has a strong effect on grinding performance. The topography together with other elements of the abrasive system determines grinding forces, power consumption, temperatures, workpiece precision and wheel redress life.

A detailed knowledge of the topography of a grinding wheel reveals the results of wheel preparation. Topographical changes during grinding can help to explain the effects of grinding parameters on grinding performance. A knowledge of topographical changes can also help to improve control of the grinding process in general. For the advancement of grinding technology it is therefore helpful to establish procedures for measurement and monitoring of wheel topography in grinding. Numerous techniques have been employed for measurement including stylus methods, optical methods, imprint methods, scratch methods and SEM methods. Each technique has advantages and limitations, according to such factors as resolution, measuring depth, ease of application, data analysis and interpretation. The results obtained vary from method to method and instrument to instrument.

Surface replication is used to measure grinding wheel topography when a wheel is too large to put on the measurement table of a particular instrument, or when the wheel needs to be measured in-situ to avoid continual removal and replacement. 
A replica of the surface is prepared and is then measured. With the development of new replication materials it is possible to achieve high resolution, rapid curing and ease of preparation.

Four main kinds of measurement and observation technique are often used for measuring wheels. These and replication technique are reviewed below.

\section{(i) Stylus-methods}

Stylus methods have been employed since 1927 at least [9]. Stylus methods are widely used for measuring surface roughness because of the ease of use and low cost. A stylus instrument incorporates a contact stylus and a transducer. The transducer is most often inductive or possibly an optical interferometer. The transducer is used to measure the displacements of the stylus as it is moved across the surface. The accuracy of the instrument particularly for optical transducers depends on the atmospheric pressure and temperature. Accuracy of a stylus instrument depends on stylus size and shape, on the measurement speed and on the stylus load. The stylus size and shape are usually characterized by the radius and cone angle of the tip. Errors in stylus size and shape and other sources of error affect the accuracy of measurement [9]. For grinding wheel measurement, the radial penetration of the stylus into the wheel is limited to about $70 \mu \mathrm{m}$, or less, as pointed out by Malkin [4]. This depth is greater than the typical radial cutting depth of the wheel but possibly smaller than the depth affected by dressing.

3D-stylus measurement provides a more complete description of a wheel surface than traditional 2D-stylus measurement. In 2D cases, only a part of the 3D surface topographical information is addressed. 2D measurement does not allow 3D functional features of the bearing surface such as peaks, pores and cracks, to be clearly identified even where the measurement is carefully controlled. 


\section{(ii) Optical interferometry}

Optical methods make it possible to achieve quantitative characterization of surface topography without making physical contact. There are several optical techniques. Interferometry is illustrated schematically in Fig. 2.1[11].

An optical interferometer works on the principle of interference of two beams of light where at least one beam is reflected off the surface to be measured. The two most widely used techniques are phase shifting interferometry and scanning differential interferometry [10]. The resolution of the instrument for resolution less than one wavelength depends on the wavelength of the light used and on the accuracy of interpolation. By interpolation, resolution can be achieved within the nanometer range.

The main limitation of interferometry is the need for surfaces with reflectivity greater than $15 \%$ [11]. The maximum vertical range is limited to a level approaching the wavelength of the incident beam and slope changes are sometimes difficult to measure with interferometry. A further feature of interferometry is that a high degree of environmental vibration isolation is required when operating the instrument if useful data are to be realized.

\section{(iii) Laser triangulation}

The principle of measurement by laser triangulation is shown in Fig. 2.2 [12]. The sensor employs a combination of a laser diode to create a structured light pattern and a CCD camera to capture the image of the laser light reflected from the measured surface. Typically, the laser and CCD camera are mounted in a housing in which the $\mathrm{CCD}$ camera is fixed at a triangulation angle of $18^{\circ}$ from the laser source. Lenses form the light into a thin laser plane. When a feature intersects the laser plane, a bright red laser line appears on its surface. Within the camera field of view, the detected red line represents the contour of the part to be measured. With the aid of image analysis, the detected contours can be turned into measurements [12]. Laser triangulation measurement can be applied either offline or for in-situ measurement on a grinding machine. Brinksmeier [13] developed a triangulation laser reflection system for in-process $2 \mathrm{D}$ grinding wheel 
characterization. The system provided information for characterization of grinding wheel form deviations, waviness and roughness.

Results obtained with optical methods do not always correlate well with those obtained with stylus methods [14,15]. This is due in part to the fact that stylus methods are contact methods and optical methods are non-contact. It is also due in part to the limitation of light diffraction. Whitehouse [16] concluded that the two methods are complementary rather than competitive since different types of information are provided.

\section{(iv) Microscopy}

Microscopy involves the use of an optical microscope or a scanning electron microscope (SEM) to observe the topography of the wheel surface. Fine detail of the surface can be seen clearly using a large magnification. When viewing normal to the wheel surface, flattened areas on the grain tips reflect light and appear shiny against a dark background. The number of distinct wear-flat areas per unit area on the wheel surface may be counted and is sometimes interpreted as the cuttingedge density.

Microscopy is not primarily a depth measurement method. It is difficult to relate the topographical features of a grinding wheel to the radial depth of the wheel using an optical microscope or a SEM. A stereoscopic microscope utilizing a built-in vertical illuminator or a link to a computer partially overcomes this problem. SEM provides stereographic photographs and can also provide contour plots with high magnification and large depth of field, allowing cutting edge density to be observed at various depths of penetration. The limitation of these two methods is the requirement to employ removable segments or sections of a wheel small enough to fit into the observation table or the SEM chamber. A microscope may be mounted directly on the grinding machine, thereby making it unnecessary to remove the wheel for observation or to make replicas of the wheel surface. Yet another problem encountered is that replicas tend to melt when being coated in preparation for measurement by SEM. 


\section{(v) Other methods}

Other techniques, such as the scratch method, acoustic emission, thermocouple, and razor blade techniques have been employed in an attempt to measure and observe the features of grinding wheel topography. A review of techniques was carried out by a CIRP co-operative research [17].

\section{(vi) Replication techniques}

Replication is not a measurement technique in itself. Replication may be applied to overcome difficulties of making direct measurements on large wheels or of monitoring a wheel in-situ when it is undesirable to remove and replace the wheel without having to redress the wheel surface. This situation applies, for example, when it is desired to monitor wheel changes within a redress cycle.

A replica is prepared using a setting compound or soft metal. The replica material is poured or pressed onto the area of interest of the wheel. When cured, this provides a solid surface that ideally should be identical with the area of interest. The replicas can be measured and observed by stylus, optical and microscope techniques. Investigations by Eckert [18] on a water-based plastic system and investigations by George [19], and James and Thum [20] on epoxy-resin systems have shown that both types have the ability to replicate surface structure and have relatively low shrinkage effect.

\subsection{Principles for selection of measurement techniques}

In the choice of the 'best' instrument to adopt for a specific application, knowledge of resolution and range is fundamental. Limiting factors are the vertical range and resolution of the system, the horizontal range and resolution, the horizontal datum, the probe size and geometry. Another consideration is the effect of the colour and texture of CBN wheels and replica surfaces for different measurements. For example, CBN wheels usually have hard abrasives and dark coloured bonds. Replica materials may be of different colours too. 
In choosing a replica material, resolution of the replica, ease of application and whether there is damage to the wheel when a replica is applied on the surface are all essential questions.

\subsection{Experimental equipment and procedure}

\subsubsection{Experimental equipment}

In Fig. 2.3, the measurement domains are plotted for the above techniques. In the figure, the two axes represented the resolution and range. The resolution is indicated by the boundary nearest the origin of the axes and the range by the boundary farthest from the origin. From Fig. 2.3, it can be seen that stylus and laser triangulation instruments have a large working area. An optical interferometer has the finest vertical resolution but a more limited vertical and horizontal range.

Three particular instruments for measuring CBN wheel and replica topography were used. These were a stylus instrument, the FormTalysurf 120 (3D), an optical interferometer, the Wyko RST plus and a laser triangulation instrument, the Uniscan OSP 100.

The Form Talysurf 120 (3D) has a $120 \mathrm{~mm}$ traverse unit in which an inductive transducer is employed. 120,000 data points are gathered during a single measurement traverse, thus allowing analysis of data without loss of detail. Surface texture could be measured over a wide gauging range, which, with the appropriate software package, also allowed the measurement of form deviations from simple radius and angular relationships or from complex mathematical forms.

The Wyko RST Plus is a research grade instrument for measuring surface topography and surface roughness. Quantitative results are based on calibrated optics and a scanner with closed loop feedback to maintain accuracy. The equipment is capable of vertical scanning interferometry for larger scale vertical measurements and phase shifting interferometry for smooth surfaces where the instrument can resolve to less than 1 angstrom unit. 
The RTS Plus interferometer takes data by combining the path of light reflected off the test part with the path of light reflected off an internal reference surface. When the two paths combine, the light waves interfere to produce a pattern of dark and light bands called fringes. Fringes are obtained quickly with the RTS Plus system. In phase shifting interferometry (PSI) mode, a precise mechanical translation system precisely alters the optical path length of the test and reference beams in a series of shifts. Each optical path change causes a lateral shift in the fringe pattern. A detector inside the interferometer, producing a series of interferograms, periodically records the shifted fringes. Computerized calculations combine the interferograms to determine the surface height profile. In vertical scanning interferometry (VSI) mode, the surface is profiled by scanning vertically downward so that each point on the surface produces an interference signal. The software program calculates and displays the analysis output, from which further analyses can be performed.

The Uniscan OSP 100 is a line-scanning product for surface profiling and measurement. Utilizing laser displacement sensors the OSP 100 measures surface topography in the ranges of tens of millimetres to sub-microns, over an area up to $100 \mathrm{~mm} \times 100 \mathrm{~mm}$. Line profiles can be scanned at up to 256,000 data points per line and $3 \mathrm{D}$ surface profiles at many giga samples per surface area map.

The above three instruments are compared in Table 2.1.

From Table 2.1, it can be seen that the Wyko RST plus offers a smaller measurement area for $3 \mathrm{D}$ analysis either of the wheel or of replicas but with more accuracy. The Talysurf (3D) provides a larger area for 3D measurement either of the wheel or of replicas with a slightly worse accuracy. The Uniscan OSP 100 provides the largest area for 3D measurement either of the wheel or of replicas with the worst accuracy. A large area is useful to obtain representative data for statistical purposes. 
Measurement errors vary according to the measurement technique. A measurement error includes not only an instrument error, but also an image processing error. In order to analyse the peripheral surface of a grinding wheel, the peripheral curved shape needs to be fitted and regressed to a flat surface by the software. Inadequate data at the measurement border and inconsistency of the local form of the wheel surface introduces fitting errors. In addition, the raw data from measurement usually includes some bad data when interferometry is applied. Bad data means the surface cannot be measured at that point due to a limitation of darkness, slope or some other reason. The measurement software provides processed data for such points usually by interpolation. This process introduces interpolation errors. Both fitting and interpolation errors depend on the uniformity of the wheel surface.

Table 2.1: Comparison of measurement techniques

\begin{tabular}{|l|l|l|l|}
\hline Principle & Stylus & Interferometry & $\begin{array}{l}\text { Laser } \\
\text { triangulation }\end{array}$ \\
\hline Instrument & $\begin{array}{l}\text { Talysurf (3D) } \\
\text { (See Fig. 2.4) }\end{array}$ & $\begin{array}{l}\text { Wyko RST plus } \\
\text { (See Fig. 2.5) }\end{array}$ & $\begin{array}{l}\text { Uniscan OSP 100 } \\
\text { (see Fig. 2.6) }\end{array}$ \\
\hline $\begin{array}{l}\text { Measurement } \\
\text { area (Typical } \\
\text { value) }\end{array}$ & $120 \mathrm{~mm} \times 50 \mathrm{~mm}$ & $\begin{array}{l}8 \mathrm{~mm} \times 6 \mathrm{~mm} \\
\text { (depends on } \\
\text { variable } \\
\text { objectives) }\end{array}$ & $\begin{array}{l}100 \mathrm{~mm} \mathrm{x} \mathrm{100} \\
\mathrm{mm}\end{array}$ \\
\hline $\begin{array}{l}\text { Measurement } \\
\text { depth }\end{array}$ & $1.5 \mathrm{~mm}$ & $0.1 \mathrm{~nm}-500 \mu \mathrm{m}$ & $35 \mathrm{~mm}$ \\
\hline $\begin{array}{l}\text { Measurement } \\
\text { accuracy }\end{array}$ & $0.03 \mu \mathrm{m}$ & $0.01 \mu \mathrm{m}$ & $0.5 \mu \mathrm{m}$ \\
\hline
\end{tabular}

For the Talysurf (3D), the measurement error of the mean square surface roughness $R_{q}$ is less than $7 \%$ if the grinding wheel is measured. For the Wyko RST, the measurement error $\mathrm{R}_{\mathrm{q}}$ is less than $5 \%$ and for the Uniscan OSP 100 , the measurement error $R_{q}$ is less than $15 \%$ if the wheel is properly measured. 
The Olympus BH2-UMA metallurgical microscope shown in Fig. 2.7 was employed to observe wheel and replica surfaces. This microscope has objective magnifications of $5 \times, 10 \times, 20 \times, 50 \times, 100 x$. Photographs were taken with a mounted Olympus SC 35 camera.

Different replication materials were prepared including Thixoflex and Contrast $T$ (Addition curing silicone-based impression materials).

Soft lead was also tested as a replication material. The lead was prepared in the form of a cylinder with a surface roughness of $0.02 \pm 0.03$ microns $\mathrm{Ra}$. The replication rig is shown in Fig. 2.8. The force was applied through a spring, which was compressed by a screw thread. The spring stiffness was calibrated as shown in Fig. 2.9.

Another replication material tested was a relatively new material called Microset 101. The material consists of a two-part synthetic rubber compound. Microset 101 was developed for high resolution and easy operation. The replicas have a resolution of 0.1 microns and exhibit high contrast characteristics. The compounds are supplied in cartridges, which are dispensed using hand-operated dispensers. The cartridges contain both a polymer and a curing agent, which are automatically mixed in a disposable static-mixing nozzle during application to the surface. The replication rig is shown in Fig. 2.10.

\subsubsection{Experimental procedure}

The experimental procedure included the following steps:

(i) The same area on the grinding wheel surface was measured ten times using the Wyko RST and the Uniscan OSP 100 and measured 5 times using the Talysurf (3D). The same area was also observed using the microscope. The measured values were compared and analysed.

(ii) $\mathrm{CBN}$ wheels of different structures and colours were measured using the three measurement instruments. The results were compared and analysed.

(iii) Different replication techniques and materials were tried including Thixoflex and Contrast $\mathrm{T}$ (Addition curing silicone-based impression 
materials), soft lead metal and Microset 101. The replication results were compared and analysed.

\subsection{Experimental results}

(i) The surface roughness values measured by the three different measurement methods are listed in Fig. 2.11. From this figure it can be seen that the $\mathrm{Rq}$ surface roughness measured by the Uniscan OSP 100 gave the highest values, the roughness is more than double the roughness measured using the Wyko RST and three times the value using the Talysurf (3D). The Talysurf (3D) gave the lowest Rq surface roughness.

Fig. 2.12 shows measurements obtained from the same part of a wheel using the Talysurf (3D), the Wyko RST and the Uniscan OSP 100, and the blocking areas in Fig. 2.12 (a) and (e) are the area in Fig. 2.12 (c). In a colour diagram, when assuming high point are grains, the grains and wear flats can be distinguished using both the Talysurf (3D) and the Wyko RST according to grain shape and size, but cannot be distinguished using the Uniscan OSP 100 because the high points are too big to be a grain according to grain size. Fig. 2.13 shows measurements from the same part of a wheel by Wyko RST with different magnifications of 41.3:1 and 10.3:1. It can be seen that high magnification gives a clear image of the top of the grain.

Fig. 2.14 shows microscopic photographs of the Talysurf stylus before and after measuring a CBN wheel area 5 times over an area of $1 \mathrm{~mm}^{2}$. There is no obvious wear on the top of stylus.

Fig. 2.15 shows a photograph of the same area of the wheel surface observed under the microscope. The wear flat areas on the cutting edges shown as bright areas can be seen clearly. The density of the active cutting edges, which have a wear flat, can be counted from a photograph. 
(ii) Different structures and colours of the measured surface have the strongest effect on use of the Wyko RST due to its limit of measurement depth and the requirement for reflection of light from the surface. Because of the poorer reflectivity of the bond material and because of the depth of the pores it is more difficult to make measurements of these aspects. Better measurements can be obtained for fine-grain grinding wheels than for coarse wheels. This is because coarse wheels have deeper pores. Only a small area of measurement is possible due to the limitation of the objective lens. As with interferometry, laser triangulation requires environmental vibration isolation if useful data are to be realized when operating the instrument. Laser scanning speed, the nature of the measurement samples, and the texture of the objects to be measured all affect the level of detail obtainable. It is difficult to obtain accurate measurement results when measurement samples are too dark or too bright.

(iii) Different replication techniques and materials were tried including Thixoflex and Contrast $\mathrm{T}$ (Addition-curing silicone-based impression materials). Due to the complicated and time-consuming operation, which made it difficult to obtain satisfactory results, they were all excluded.

Soft lead is easy to prepare and is practically unaffected by the coolant. Lead was successful as a material, although there were some disadvantages. The force applied was about $170 \mathrm{~N}$ in order to get a clear replica for an internal wheel with diameter about $13 \mathrm{~mm}$. The force applied on the lead to achieve the surface replication can cause occasional breakage of a fragile wheel bond bridge and also a small amount of lead may sometimes be left on the surface of the wheel (see Fig.2.16). While this probably does not seriously affect the measurement accuracy or the behaviour of the grinding wheel, it was considered to be a disadvantage in using lead replicas.

Microset 101 replica material has high strength and flexibility that enables easy removal from re-entrant geometry without damage and shrinkage. 
Unfortunately, Microset 101 replica cannot be measured by the stylus method due to its softness. The measured surface was dragged to one side. Also there was a melting problem when Microset 101 was used in SEM (see Fig. 2.17). No problem was experienced using optical interferometry and laser triangulation even though the Microset 101 is dark in colour. A problem was experienced initially due to residual coolant on the surface of the grinding wheel, which affected the curing of the Microset 101. Running the wheel for a period of time to remove the coolant and cleaning the wheel using high-pressure air was necessary before taking replicas. Microset 101 replica material has a high resolution. With proper operation, a clear negative replica can be obtained especially for the topmost layer of abrasive grains as shown in Fig. 2.18. In most cases, it was found that the rubber did not penetrate to the full depth of the pores in the wheels. The Rt surface roughness of the replica was about $60 \%-90 \%$ of the values for the wheel depending on the surface roughness of the wheel. The error using Microset replica was estimated to be less than $20 \%$ for the fine-grain wheels.

\subsection{Discussion}

Although there was no obvious wear on the top of the stylus after measuring a CBN wheel, the stylus method is not recommended for further measurement of hard abrasive wheels such as CBN and diamond. The similar hardness of the CBN abrasive and the stylus, which is usually made of diamond, causes a stylus to wear. This is expensive since the stylus will need continual replacement and leads to measurement inaccuracy. Soft replicas are not suitable for stylus measurement either, because the stylus penetrates the surface leading to inaccurate results. In order to overcome this problem, a harder replica material is required and may be achieved by making a positive replica on the surface of a soft negative replica.

High reflectivity of abrasive grains on the surface of a grinding wheel makes it feasible to use interferometry. Detailed information can be obtained concerning the abrasive grains and even details on a particular grain in the wheel surface. 
This is because coarse wheels have deeper pores and because of the limitation in the vertical range which can be achieved. Only a small area of measurement is possible due to the limitation of the objective lens. Nowadays, a larger measurement area is possible if special software is employed to connect together data from neighbouring small areas. Replicas of a dark colour can be measured by using an appropriate filter.

Laser triangulation tends to allow a larger measurement area and deeper measurement depth than interferometry but yields reduced accuracy. Laser triangulation is useful for obtaining statistical results for fine grinding wheels and also for coarse wheels and replicas. However, the topographical detail is not as clear as with interferometry.

Although microscopy is not ideal for depth measurement, microscopy provides direct observation of the wheel and helps to understand the nature of the wheel topography. Microscopy is convenient for counting cutting edges whereas depth information is more conveniently obtained using stylus or optical instruments based on measurement of bearing area at different depths. Each method has advantages and disadvantages depending on the information required. Depth measurement using stylus or optical methods allows the number of grains to be related to the radial depth below the wheel surface. This is straightforward, if it is assumed that any high point is a cutting edge of the grain. The high points can be counted by software in the depth measurement method. Sometimes, however, a high point may not mean a cutting edge, but may be caused by swarf, by dust or by a loose particle of grain or bond. It is impossible to distinguish between grains, bond, swarf or dust using the depth measuring method. When looking through a microscope, some points of the grain tips come into sharp focus. Since the depth of the focus is very shallow, all the grain tips can be considered to come into focus within the same vertical focal plane. The number of grains on a surface area of the wheel can be observed by eye and analysed directly. 
Microset 101 was the best replica material tried. Using a microscope, a replica provides a clear picture of the wear flats. But, in preparation of the specimens for SEM, melting problems were sometimes encountered when coating the replica.

\subsection{Conclusions}

(i) Stylus instruments are not recommended for measuring a CBN wheel surface due to the hardness of CBN and stylus wear. Soft replicas are not recommended for use with stylus instruments due to wear of the replicas.

(ii) The interferometer (E.g. Wyko RST plus) offers the best method for 3D measurement of a small area both of a wheel or of a replica. Fine surface detail can be detected such as wear flats and adhesive loading of grains.

(iii) The laser triangular measurement (E.g. Uniscan OSP 100) provides a method for 3D measurement of a large area both of a wheel or of a replica but with reduced accuracy compared with interferometry.

(iv) Microscopy provides direct observation of the wheel and helps to provide understanding of the nature of the wheel topography.

(v) Interferometry, laser triangular and microscopy can all be employed to measure and observe wheel structure depending on the particular requirements.

(vi) A replica material developed for high resolution and easy operation (Microset 101 with a resolution of 0.1 microns) was selected as the main method to be used in experiments. 


\section{Chapter 3 Evaluation of vitrified CBN grinding wheel topography}

\subsection{Review of evaluation parameters}

It is well known that wheel surface topography has a critical influence on grinding performance. Wheel topography is partly determined by initial wheel structure, and partly by wheel dressing. As far as superabrasive wheels are concerned, changes in topography of the wheel surface during a grinding process do not appear to be as significant as for conventional wheels. This is because superabrasives are much tougher and more wear resistant than conventional abrasives. It has been asserted that once the topography of a superabrasive wheel is identified, its grinding process behaviour can be predicted with greater reliability [21]. However, parameters are required to allow the grinding wheel structure to be characterised and related to wheel performance in grinding. The characterisation must be possible from data produced by measurements of the wheel surface. Clearly, more comprehensive descriptive parameters of the grinding wheel topography allow grinding performance to be more accurately explained and the effects of changes in wheel specification to be predicted.

Wheel characterisation requires topographical parameters and internal structure parameters such as wheel hardness and strength.

There are a large number of parameters that currently exist for characterising 2D and 3D topographies [22-24]. Stout [25] presented sixteen 3D topographic parameters including amplitude parameters, spatial parameters, hybrid parameters and function parameters for characterising bearing and fluid retention properties. This list illustrates the problem of determining evaluation parameters for $\mathrm{CBN}$ wheels. Given the large number of parameters that currently exist, parameters selected should relate closely to the grinding process itself.

The cutting edges at the wheel surface play an important role in grinding especially cutting edge size, shape and density. 
Cutting edge size and shape determines the degree of wheel sharpness. Sharpness of the grinding wheel depends not only on the geometry of the wheel but also on the physical process. Sometimes in the literature [25], a sharp wheel is taken to mean that a high metal removal rate can be achieved, and at other times [26] refers to the condition where a good surface integrity can be obtained. The time constant $\tau$ of the grinding system is a parameter which has been used to indicate relative wheel sharpness. Time constant represents the combined effect of wheel sharpness and system stiffness on grinding behaviour. The force ratio $F_{t} / F_{n}$ and the specific energy $e_{c}$ are also used to indicate wheel sharpness.

In grinding, a sharp wheel implies sharp cutting edges on the wheel surface. This means the apex angles of cutting edges illustrated schematically in Figure 3.1 are small. King [27] et al presented a truncated amplitude density function as a basis for a wear model. A model of this type is widely used to evaluate wheel wear in grinding. Malkin [28] related wear flat area with grinding forces and specific energy. The slope of the cutting edges is also widely used in grinding.

König [29] pointed out that not all static cutting edges within a particular area come into contact with the workpiece in grinding. Shaw [30] also pointed out that even edges at the same level do not necessarily all cut, since it is possible that a preceding edge may have already removed the material in the path of a succeeding edge. Verkerk et al [17] proposed that two parameters be used, $\mathrm{C}_{\text {stat, }}$, the total number of static cutting edges per unit area and $\mathrm{C}_{\mathrm{dyn}}$, the number of cutting edges per unit area when dynamics are included in the assessment. From a basic kinematic analysis, it appears that the dynamic cutting edge density for a given wheel topography should depend uniquely on the quantity $\left(v_{w} / v_{s}\right) /\left(a_{e} / d_{e}\right)^{1 / 2}[4]$, which in turn can be shown to be directly related to the infeed angle $\varepsilon$ rather than the radial distance z. Fig. 3.2 illustrates the parameters $\varepsilon$ and $\theta^{\prime}$ in a diagram. The density of dynamic cutting edges in the grinding zone can be related to the static value by [4], 


$$
C_{d y n}=C_{s t a t}\left(\frac{v_{w}}{v_{s}}\right)^{c}\left(\theta^{\prime}\right)^{c}
$$

where, $v_{w}$ is workpiece speed,

$\mathrm{v}_{\mathrm{s}}$ is wheel speed

$\theta^{\prime}$ is wheel rotation angle during contact length

$\mathrm{c}$ is a constant for a particular wheel and dressing condition. Values of c range from about 0.4 to 0.8 .

It can be seen that $\mathrm{C}_{\mathrm{dyn}}$ is smaller than $\mathrm{C}_{\text {stat }}$ for the same grinding conditions.

Another factor, which affects cutting edge density, is wheel-workpiece deflection. Rowe and Qi [31] found that real contact length is much larger than the theoretical geometric value. The difference was explained by factors including elastic deformation of the wheel and workpiece, roundness errors of the wheel and workpiece, surface roughness of the workpiece, and unevenly distributed cutting edges. Shaw [32] concluded that the total local elastic deformation for an individual grit is typically $1-6 \mu m$ for a vitrified wheel of conventional structure as used in fine grinding.

Porosity is another important feature of grinding wheel structure, which relates to coolant supply, cutting edge distribution and wheel hardness. Wheel porosity has not been as widely researched as wheel cutting edges. Usually, the ratio of the volume of the pores to the volume of the whole wheel is employed to describe wheel porosity. This parameter is termed the porosity volume ratio.

Wheel hardness, is an elusive characteristic of a grinding wheel because a wheel is not a homogeneous structure. Wheel hardness or grade depends on the strength of the bond holding the abrasive grains together. For a particular abrasive and bond material, a harder wheel contains more bond and less porosity. Therefore, harder wheels are stronger, and the abrasive grits are more firmly held by the bond. Methods for testing the wheel hardness usually involve measurement of the forces required to remove wheel material with a particular shape of tool. Peklenik [33] used a triangular tool to scratch a 
groove in a wheel with the depth of penetration set equal to the nominal grit dimension. Peklenik proposed that despite the somewhat greater complexity of a dynamic method of testing grinding wheel hardness using a dynamic technique, it correlated quite well with the static method. Measurement of the elastic modulus has also been employed to characterise wheel hardness since hardness can be assumed to correlate with elastic modulus [34]. Relatively simple methods have been developed for determining the elastic modulus based on measuring the natural frequency of a grinding wheel excited by impact [35]. It was decided, however, to use the Peklenik method of scratch testing for reasons of simplicity and in the absence of any other convenient method.

\subsection{Selection of wheel evaluation parameters}

Four basic parameters were selected for comparative wheel evaluation. These four parameters were used to monitor the changing cutting surface of the grinding wheels as grinding progressed. The parameters chosen were,

Active cutting edge density [17] $\left(\mathrm{C}_{\mathrm{a}}\right)$,

Cutting edge dullness $(\gamma)$,

Effective porosity volume ratio $\left(\mathrm{V}_{\mathrm{pw}}\right)$, and

Wheel scratch hardness $\left(\mathrm{H}_{\mathrm{ss}}\right)$.

This set of parameters obviously reflects important features of grinding wheels, which change as the grinding process is performed. Monitoring these parameters can contribute to the analysis of grinding performance, optimisation of grinding wheel structures and optimisation of grinding process conditions.

\subsubsection{Definition of active cutting edge density $\left(C_{a}\right)$}

Active cutting edge density $\left(C_{a}\right)$ is the number of active cutting edges per unit area of the wheel surface. Active cutting edge density can be determined by counting the cutting edges above the active radial depth of the wheel. 


\subsubsection{Definition of cutting edge dullness $(\gamma)$}

Cutting edge dullness $(\gamma)$ is defined here as the ratio of the effective cutting edge radius $r_{0}$ to the grain radius $r_{g}$ as illustrated in Fig. 3.1. i.e.

$\gamma=\frac{r_{0}}{r_{g}}$

\subsubsection{Definition of effective porosity volume ratio $\left(V_{p w}\right)$}

Effective porosity volume ratio $\left(\mathrm{V}_{\mathrm{pw}}\right)$ is the ratio of the effective volume of the pores to the volume of the wheel on the wheel surface as shown in Fig. 3.3. That is.

$V_{p w}=\frac{\left.V_{p}\right|_{z \geq z_{i}}}{\left.V_{w}\right|_{z \geq z_{i}}}$

where, $V_{p}$ is the volume of the pores

$\mathrm{V}_{\mathrm{w}}$ is the volume of wheel, which includes pores, abrasive and bond $\mathrm{z}$ is radial depth into the wheel

$z_{i}$ is the active radial depth into the wheel below which the cutting edges are active

The term 'effective' is associated with the porosity volume ratio because the porosity volume ratio at the surface may be different to the porosity volume ratio within the body of the abrasive. The value at the surface below active radial depth $z_{i}$ is significant for grinding performance considering wheel deflection.

\subsubsection{Wheel scratch hardness $\left(H_{s s}\right)$}

Wheel scratch hardness is defined here as the ratio of average tangential grain force to the mean diameter of the grains removed when scratching a wheel surface with a depth equal to the average grain size using a particular size of cutting tool. i.e.

$H_{s s}=\frac{F_{g s t}}{d_{b a}}$

where, $d_{b a}$ is the mean diameter of the grains removed. 
$\mathrm{F}_{\mathrm{gst}}$ is the average tangential force on the grains.

The wheel scratch hardness not only reflects the bond strength, but also relates to the grain strength.

\subsection{Experimental equipment}

3.3.1. The measurement and observation instruments and analysis software

Measurement of the wheel surface topography is necessary in order to obtain the active cutting edge density, the cutting edge dullness and the effective porosity volume ratio,. The measurement equipment employed was the Wyko RST plus and the Olympus BH2-UMA microscope as described in Section 2.4.1.

Blunt [36] proposed that for the general measurements of the grains, the optimum sample spacing $\mathrm{SS}_{\text {opt }}$ required to measure the grains should fulfil the relationship $3 \leq \frac{d_{8}}{S S_{\text {opt }}} \leq 4$. Normally, a $10 \times$ objective lens with measurement sampling of $1.65 \mu \mathrm{m}$ was used when measuring with the Wyko RST. The measurement sampling requirement was satisfied with $S S_{o p t}<\frac{d_{g}}{4}$, which enables values of cutting edge density to be obtained rather than grain density.

After measurement of wheel topography, the measurement datum was put into an image processing software, Matlab Image Processing for the analysis of wheel topography. 3D analysis allowed measurement results to be presented in $2 \mathrm{D}$ working slice by slice from the top surface of a wheel as shown in Fig.3.4 (c) and (d). The 2D image was transformed into a binary image and the bearing area shown in white represents cutting edges at the top of the wheel. The density of the cutting edges above a particular radial depth of a particular slice was determined by counting the unconnected bearing areas by software. Also, the wear flat area can be calculated. Active cutting edge density was obtained by counting the wear flats from microscope photographs as shown in Fig. 3.4 (e). 
Comparing the active cutting edge density observed through the microscope, with active cutting edge density and wear flat area determined by Matlab Image Processing software, the difference was less than $10 \%$.

The Wyko software itself provides a function to calculate pore volume and peak volume between particular depths as shown in Fig 3.5. The wheel volume is taken as the whole volume including pore, grain and bond volume. Thus, the wheel effective porosity volume ratio can be determined once active cutting depth is decided.

\subsubsection{The micromachining rig and dynamometer}

Fig. 3.6 illustrates schematically the experimental technique for measuring wheel scratch hardness. Tests were carried out on the micro-machining rig to measure wheel scratch hardness. (See Figures 3.7 and 3.8) The assembly comprised of a co-ordinate table, a workpiece holder with adjustable cutting speeds and a tool holder. The workpiece holder has variable speeds of rotation in the range from 0.01 to $1 \mathrm{rev} / \mathrm{min}$. The scratch depth was adjusted by means of a worm and wheel gear system and a micrometer screw with an accuracy of $0.003 \mathrm{~mm}$.

A two-component dynamometer was used. A schematic illustration of the dynamometer is shown in Fig. 3.8(a). Fig. 3.8(b) shows the corresponding beam with the applied forces and bending moments. Fig. 3.8(c) shows the strain-gauge bridge connections for measuring the $\mathrm{P}_{\mathrm{y}}$ and $\mathrm{P}_{\mathrm{z}}$ components of cutting force.

According to the loading diagram the beam is subject to the $P_{z}$ and the $P_{y}$ components of the cutting force. Eight strain gauges were mounted on the body of the elastic element as shown in Fig. 3.8(a) and are indicated by corresponding numbers from 1 to 8 . Strains at each of the indicated points depend on their location and the values of applied forces and bending moments. The strain gauges mounted in a Wheatstone bridge, allows 
independent measure of $P_{z}$ and $P_{y}$, so that crosstalk between $P_{z}$ and $P_{y}$ components is absent. The output signal for each of the circuits reflects only the action of the corresponding $\mathrm{P}_{\mathrm{z}}$ or $\mathrm{P}_{\mathrm{y}}$ component.

Previous calibration [37] confirmed good characteristics of the dynamometer, including linearity, and repeatability. The main characteristics of the dynamometer were:

Measurement range: $\quad 0$ to $500 \mathrm{~N}$ (Principal Force)

Stiffness $\left(J_{D}\right)$ and sensitivity $\left(S_{D}\right)$ for the principal cutting force were $30 \mathrm{~N} / \mu \mathrm{m}$ and $0.38 \mu$ strain $/ \mathrm{N}$ respectively.

Only the principal force $P_{z}$ was used in evaluation of wheel scratch hardness. So, only $\mathrm{P}_{\mathrm{z}}$ was calibrated. A photograph of the calibration set-up is shown in Fig. 3.9. Sequential loading of the dynamometer was carried out separately in the directions of the principal $P_{z}$ forces.

The wheel was scratched at a cutting speed of $1 \mathrm{rev} / \mathrm{min}$ and the depth of cut was set equal to the average grain size. The infeed for scratching the wheel was set equal to the average grain size. The actual scratch depth changed around the wheel due to wheel roundness errors and wheel misalignment errors. The groove depth was measured using the Wyko RST Plus after scratching. The experimental results were accepted with scratch depth error estimated at less than $10 \% .10$ grooves were measured for each wheel and the repeatability of the scratch force was more than $80 \%$.

The removed grains were collected on a piece of paper laid under the wheel. The grains removed and the grain fragments were collected from the paper onto the surface of a piece of bluetack using light pressure and observed under microscope. The shiny grains and grain fragments were separated from bond material under the microscope. The grain and grain fragment size was evaluated from the calibrated length scale in the eyepiece. The error was estimated to be less than $10 \% .40$ removed grains and grain fragments were evaluated for each test. 


\subsubsection{Grinding wheels}

In order to explore the relationship between wheel structure and wheel evaluation parameters, two grinding wheels with different wheel structures were measured, observed and analyzed as follows:

Wheel MP-B91: A medium-porosity vitrified CBN wheel of conventional structure, specified as B91-150V1 having a diameter of $13.4 \mathrm{~mm}$.

Wheel HP-B64: A high-porosity vitrified CBN wheel specified as B64-150VX having a diameter of $13.6 \mathrm{~mm}$. For this wheel the grain size was made smaller than the grain size of the wheel of conventional structure in an attempt to maintain a similar workpiece roughness for the two wheels.

\subsection{Experimental procedure}

The experimental procedure was as follows:

(i) Six different areas of each wheel with wear flats were measured using the Wyko RST plus. The results were analyzed using the Matlab image processing software and the static cutting edges evaluated at a particular radial depth into the wheel. The wheels were also examined under a microscope. The number of active cutting edges in the same area, were counted by observation of the wear flats. Results from the two methods were compared allowing the active cutting depth to be estimated using the image processing analysis.

(ii) Active cutting edge density, cutting edge dullness and effective porosity volume ratio were evaluated for the wheels when new, halfworn and worn.

(iii) Wheel scratch hardness was evaluated.

\subsection{Experimental results}

\subsubsection{Active cutting depth $\left(\mathrm{z}_{\mathrm{i}}\right)$}

The cutting edge density resulting from the two methods of depth measurement and direct observation were compared. The results are presented in Table 3.1 (Given at the end of the chapter). 
The topmost cutting edges in a wheel surface are the ones most likely to come into active contact with the workpiece in the grinding process. These might be expected to be the cutting edges, which lie at a depth less than the theoretical maximum uncut chip thickness $h_{\max }$ as shown in Fig.3.10. Fig.3.10 illustrates schematically, (a) the grinding process, (b) the uncut chip thickness and the maximum uncut chip thickness and (c) the uncut chip width. Usually, the maximum uncut chip thickness $h_{\max }$ is assumed to be twice the mean uncut chip thickness $h_{c u}$, that is

$$
h_{\max }=2 . h_{c u}
$$

Backer, Marshall and Shaw [38] proposed that,

$$
h_{\max }=\sqrt{\left[\frac{4}{C_{a} r} \frac{v_{w}}{v_{s}} \sqrt{\frac{a_{e}}{d_{s}}}\right]}
$$

where, $v_{w}$ is the workpiece speed

$\mathrm{V}_{\mathbf{s}}$ is the wheel peripheral speed

$a_{c}$ is the depth of cut

$\mathrm{d}_{\mathrm{s}}$ is the wheel diameter

$\mathrm{C}_{\mathrm{a}}$ is the number of active cutting edges per unit area of the wheel periphery

$r$ is the ratio of mean chip width to mean uncut chip thickness (approximately 15 in fine grinding [32])

From Table 3.1, it can be seen that when the wheel was measured statically, the radial depth into the grinding wheel above which the cutting edges were considered to be active was 8 - 44 times greater than the values estimated for the maximum uncut chip thickness $h_{\max }$. This is discussed in Section 3.6.

The average active cutting depth for the medium-porosity wheel MP-B91 was $11 \mu \mathrm{m}$ and was $20 \mu \mathrm{m}$ for the high-porosity wheel HP-B64. These values were used for evaluation of the active cutting edge density, the cutting edge dullness and the effective porosity volume ratio.

\subsubsection{Active cutting edge density}


Fig. 3.11(a) shows the active cutting edge density for a newly dressed wheel, a half-worn wheel and a worn wheel. It can be seen that there were more active cutting edges for the newly dressed wheel and less cutting edges for the worn wheel. For the conventional structure MP-B91 wheel, the active cutting edge density was more than the active cutting edge density of the high-porosity wheel, HP-B64 wheel. This was as expected.

\subsubsection{Cutting edge dullness}

Cutting edge dullness depends on the degree of wheel wear and the degree of wheel loading. The wheel cutting edge dullness increased for the half-worn wheel and even more for the worn wheel as shown in Fig. 3.11(b). This was as expected due to wheel wear and loading which tend to increase as grinding progresses. For the wheel of conventional structure, MP-B91, the cutting edge dullness was smaller than the cutting edge dullness for the high-porosity HPB64 wheel. This might be expected because the greater number of active cutting edges for the MP-B91 wheel reduces the average volume of material removed per active cutting edge. This would be expected to reduce the rate of wear. However, if the removal rates were increased to the extent that grains started to suffer significant fractures, it might be expected that the highporosity wheel would be sharpened more rapidly by the grinding process and become sharper than the wheel of conventional structure.

\subsubsection{Effective porosity volume ratio}

As mentioned in Section 3.2, the volume of the pores and the volume of the wheel were calculated below the active cutting depth of wheel, $z_{\mathrm{i}}$. As shown in Fig. 3.11(c), effective porosity volume ratio does not change much from the newly dressed wheel to the worn wheel. For the wheel of conventional structure, MP-B91, the effective wheel porosity volume ratio was about $10 \%$ less than for the high-porosity HP-B64 wheel.

\subsubsection{Wheel scratch hardness $\left(H_{s s}\right)$}

Fig.3.12 shows the results obtained for wheel scratch hardness. It can be seen that the scratch forces were different for the two wheels. Also, the distribution 
of broken and dislodged grain sizes were different. For the high porosity wheel, HP-B64, the scratch force was smaller and more whole grains were dislodged, while for the wheel of conventional structure, MP-B91, the scratch force was larger and more grains were broken.

Table. 3.2 Experimental results for wheel scratch hardness

\begin{tabular}{|c|c|c|}
\hline Wheel & MP-B91 & HP-B64 \\
\hline $\mathrm{F}_{\mathrm{gsc}}$ & $20.0 \mathrm{~N}$ & $9.8 \mathrm{~N}$ \\
\hline $\mathrm{d}_{\mathrm{ba}}$ & $43.3 \mathrm{~mm} / 91 \mathrm{~mm}$ & $48.5 \mathrm{~mm} / 64 \mathrm{~mm}$ \\
\hline $\mathrm{H}_{\mathrm{ss}}=\mathrm{F}_{\mathrm{gsc}} / \mathrm{d}_{\mathrm{ba}}$ & $42.0 \mathrm{~N}$ & $12.9 \mathrm{~N}$ \\
\hline
\end{tabular}

Table 3.2 shows the experimental results for wheel scratch hardness. It can be seen that the HP-B64 wheel had a lower scratch hardness than the MP-B91 wheel as would be expected. It indicates that high porosity reduced wheel hardness although it should help coolant enter the grinding zone and help to prevent wheel loading. This prediction was confirmed in later grinding performance trials.

\subsection{Discussion of experimental results}

\subsubsection{Active cutting depth}

Active cutting depth measurements appear, at first sight, to be surprisingly high, since it might have been expected that the depth above which grains are active should be similar in magnitude to the theoretical maximum uncut chip thickness.

This major discrepancy can be explained as follows.

(i) The real value of the maximum uncut chip thickness is much higher than the theoretical values given by Equations 3.5 and 3.6 due to a combination of factors including roundness errors of the wheel and workpiece, surface roughness of the workpiece, and unevenly distributed cutting edges etc. 
Under the above grinding conditions, the average roundness of the wheel and workpiece were $5 \mu \mathrm{m}$ and $7 \mu \mathrm{m}$. Thus, the maximum real depth of cut may be estimated to increase to a maximum of $12 \mu \mathrm{m}$. The average $R_{t}$ surface roughness of the workpiece was about $1.2 \mu \mathrm{m}$. Therefore, the maximum real depth of cut is increased by another $1.2 \mu \mathrm{m}$ to $13.2 \mu \mathrm{m}$.

In addition, the cutting edge density represented by $C_{a}$ is not even. This increases the maximum uncut chip thickness. For example, the minimum value of $C_{a}$ is $26 / \mathrm{mm}^{2}$ for the MP-B91 wheel and $7 / \mathrm{mm}^{2}$ for the HP-B64. Under the particular grinding conditions, when maximum real depth of cut and minimum cutting edge density are introduced into Equation 3.6, it was estimated that $h_{\max }$ for the MP-B91 wheel was 0.9 $\mu \mathrm{m}$ and for the HP-B64 wheel was $1.7 \mu \mathrm{m}$.

(ii) Wheel-workpiece contact deflection: Under load, individual grits are pushed away from the surface and the shape of the arc of contact is changed in a similar way to the contact between a car tyre and the road. These elastic deflections can have a significant influence on the effective value of $C_{a}$. There is ample opportunity for the number of cutting edges to increase due to local deflections of the wheel and of the grains. Comparing the grinding force in these two trials with the values given by Shaw [32], the total local elastic deformation of individual grits would be 5-6 $\mu \mathrm{m}$ for the MP-B91 wheel. The whole wheel deflection for the HP-B64 wheel is twice as large as for the MPB91 wheel at a normal force of about $15 \mathrm{~N}$. It is assumed that the total local elastic deformation of individual grits for the HP-B64 wheel is also twice as large as for the MP-B91 wheel. So, the total local elastic deformation of individual grits for the HP-B64 wheel was estimated to be $10-12 \mu \mathrm{m}$.

(iii) Sometimes, the high point on a cutting edge, could be swarf, dust, adhesive loading, or a loose particle of grain or bond. In this case, the 
active cutting depth measured in the static wheel will be larger than the theoretical maximum uncut chip thickness.

For the high-porosity wheel, HP-B64, the active cutting depth was $20 \mu \mathrm{m}$. This depth includes the total local elastic deformation of approximately $10-12 \mu \mathrm{m}$. For the medium-porosity wheel, MP-B91, the active cutting depth was $11 \mu \mathrm{m}$ with a total local elastic deformation of approximately $5-6 \mu \mathrm{m}$. For these two wheels, the active cutting depth was about 12 times larger than the theoretical maximum uncut chip thickness. The deflection for the HP-B64 wheel was estimated to be twice as large as for the MP-B91 wheel at a normal force of $15 \mathrm{~N}$. The active cutting depth for the HP-B64 wheel was correspondingly found to be twice that for the MP-B91 wheel.

It was therefore concluded that the active layer of a grinding wheel surface is many times larger than would be expected from Equation 3.6. Also, it may be concluded that the active cutting depth for the same grinding conditions is related to wheel deflection.

In order to determine the active cutting edge density by measuring either a static grinding wheel or a replica, it is first necessary to determine the active cutting depth of the grinding wheel. This is the depth for which the cutting edges can be shown to be active for a particular wheel and workpiece and for particular grinding process conditions.

\subsubsection{Wheel evaluation parameters}

Based on Equation 3.1, $C_{\text {dyn }}$ is smaller than $C_{\text {stat }}$. But, experimental work in internal grinding showed that the effect of wheel deformation on the active cutting depth is stronger than the effect of penetration angle of the cutting edges.

The four parameters, active cutting edge density, cutting edge dullness, effective porosity volume ratio and wheel scratch hardness can describe basic wheel characteristics. For example, the high-porosity wheel has a lower active cutting edge density, a larger cutting edge dullness, a higher effective porosity 
volume ratio and a lower wheel scratch hardness compared to the mediumporosity wheel of conventional structure. Also, these four parameters reflect the different grinding behaviour experienced at each stage of grinding. For instance, there are more active cutting edges on a newly dressed wheel and the cutting edge dullness becomes larger when a wheel is worn.

\subsection{Conclusions}

(i) The four parameters, active cutting edge density, cutting edge dullness, effective porosity volume ratio and wheel scratch hardness reflect basic wheel characteristics.

(ii) There are many more active cutting edges in grinding than expected based on chip thickness formulae mainly due to wheel deflection in grinding.

(iii) The active depth of the grinding wheel for which the cutting edges are active needs to be determined for each grinding system and measurement technique. 


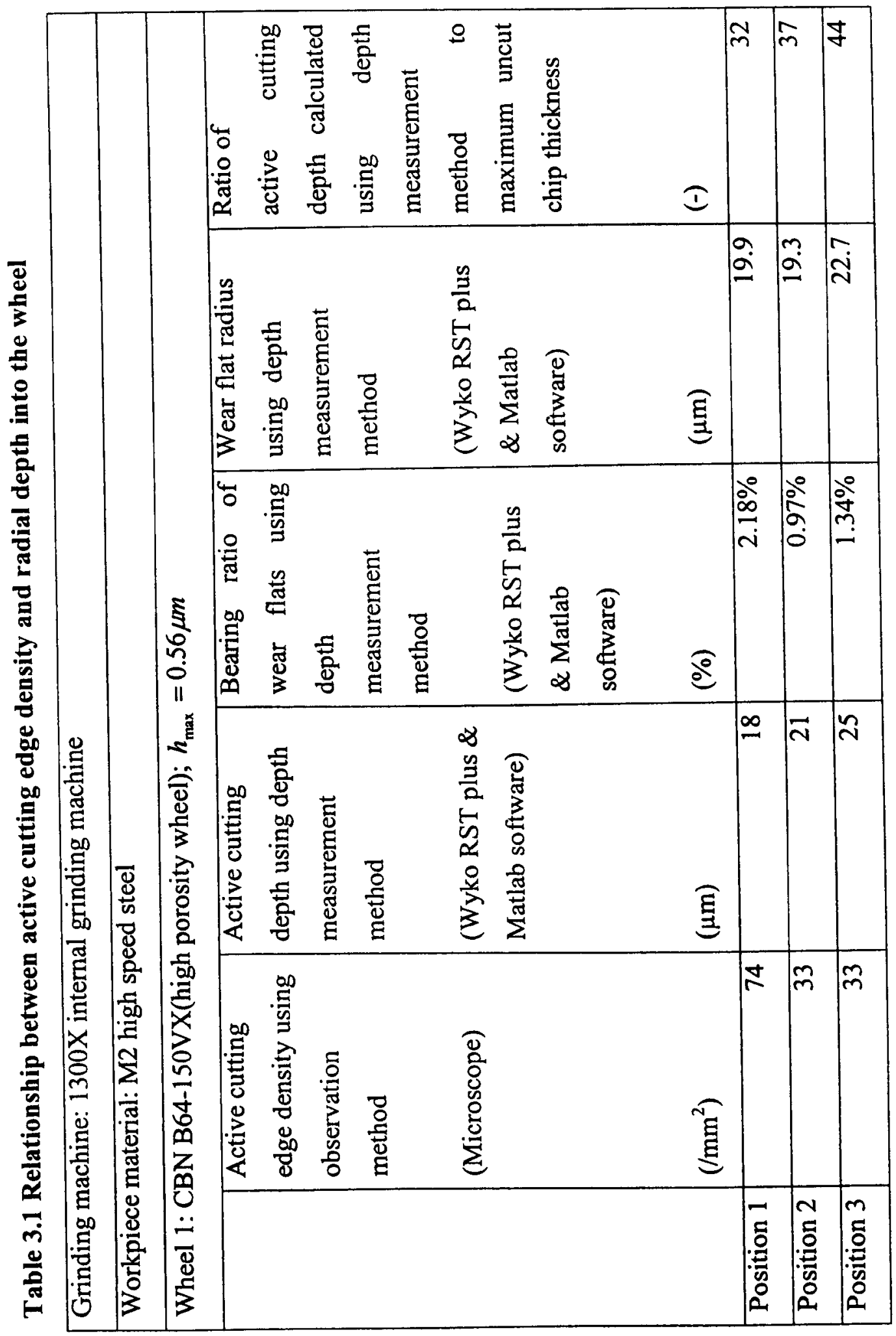




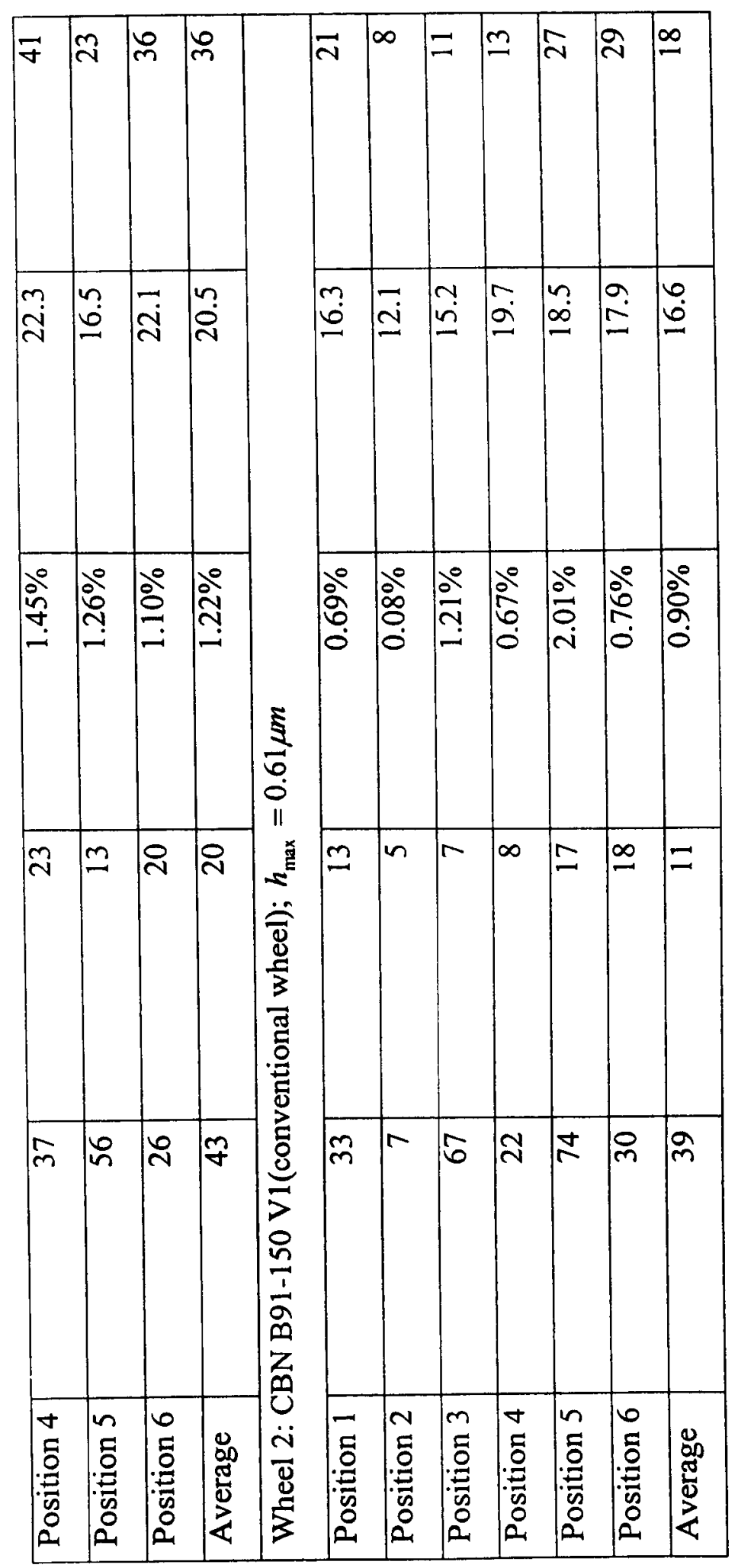


PART 2. RELATIONSHIPS BETWEEN WHEEL STRUCTURE AND GRINDING PERFORMANCE. 


\section{Chapter 4 Literature review}

\subsection{The structure of vitrified CBN wheels}

A vitrified CBN wheel is composed of an abrasive phase, a bond phase that holds the abrasive grains in a complex three-dimensional matrix, and a distribution of pores, which provides clearance for workpiece swarf, and the supply of coolant to the working surface. Wheel structure plays an important role in wheel wear and wheel loading.

The volumetric composition and distribution of grains, bond and pores can be expressed in the following form.

$V=V_{g}+V_{b}+V_{p}$

where,

$V$ is the volume of the wheel,

$V_{g}$ is the volume of the grains,

$V_{b}$ is the volume of the bond,

$V_{p}$ is the volume of the pores.

If the individual volumes are expressed as a percentage of the total volume of the abrasive body,

$V_{g}+V_{b}+V_{p}=100 \%$

The variations resulting from this volumetric relationship are shown in Fig. 4.1. Taking the sample specification of $V_{g}, V_{b}$, and $V_{p}$, shown in Fig. 4.1(a), it can be seen that the hardness of the grinding tool can be increased by raising the volume of bond by the amount $V_{x}$, as shown in Fig. 4.1(b). If, as is the case in this example, the volume of grain is kept constant, the volume of pores must be decreased by the same amount, $V_{x}$. In Fig. 4.1(c), the amount of abrasive has been increased at the expense of porosity, resulting in increased tool hardness since the amount of bond is the same and it will, therefore, form stronger bond bridges. At the same time, the force determining grit particle pullout is reduced, as the particle-to-particle distance is smaller, which also leads to a harder grinding 
action of the wheel. Fig. 4.1(d) shows an increased proportion of abrasive and a decreased proportion of bond. Although the bond bridges in this case are much smaller, paradoxically, the hardness does not decrease. This is due to the effect of the decreased moment on the abrasive grain, as previously mentioned. It can be seen in Fig. 4.1(e) that there is an increase of $V_{g}+V_{b}$, at the expense of $V_{p}$. The result of this relationship is a dramatic increase in the effective hardness, whilst, at the same time, the advantages of high porosity are lost.

There are limits between which the volumetric proportions can vary and these are, of course, determined by the strength requirements, grinding requirements and manufacturing conditions under which the grinding tools are produced. The manufacturing conditions include the compaction pressure, the particle size of the bond material used and the reaction of the bond during the firing process. Fig. 4.2 shows a three-component diagram in accordance with Equation 4.1[39].

The wheel hardness depends on porosity to some extent, regardless of the proportions of abrasive and bond. Selection of appropriate hardness of a grinding wheel is of paramount importance for the achievement of optimum performance for a particular workpiece material and a particular application. It is commonly found that the lower porosity of the wheel leads to the wheel behaving as a harder wheel in grinding.

Although high porosity decreases wheel hardness, decreases wheel stiffness and increases workpiece surface roughness, high porosity benefits clearance of workpiece swarf from the surface of the wheel and improves supply of coolant to the contact surface [5]. It was found, as described later, that increased porosity tends to increase wheel life with difficult-to-grind workpiece materials but not with easy-to-grind materials.

Onchi [7] designed a fine CBN stone with a porous structure for superfinishing which gave improved performance in terms of stable stock removal rate, low rate of wheel wear and fine finish. Di llio and Paoletti [40] found that a decrease in cutting ability of grinding wheels is mainly caused by clogging of the active 
surface due to chip adhesion rather than flattening of the grits caused by the abrasion of the hard reinforcements. Grinding wheels with conventional abrasives and open structure were found to be less prone to clogging and to lead to lower forces and lower surface roughness when grinding $\mathrm{SiC}$-aluminium composites. It may be concluded that the optimum wheel structure depends on the requirements of the particular workpiece material and of the particular grinding operation.

Several wheel surface models are described in the literature. Chen [41] investigated the effects of different grinding cycles by simulation of the grinding process. The grains were represented as spheres. Koshy et al [42] simulated a 3D diamond grinding wheel to study the effects of grinding parameters on workpiece surface roughness. No account was taken of the effect of wheel structure on active cutting edge density, wheel porosity, wheel hardness or wheel sharpness.

\subsection{Grinding force and specific energy}

Grinding is a process where numerous grains of a wheel pass though the workpiece surface. Uncut chip thickness and length are often used as geometric and kinematic parameters in the study of the grinding process. Such theoretical studies date back to the earliest research on the process $[43,44]$.

In practice, there are three phases in the grinding process including rubbing, ploughing and cutting, as proposed by Hahn [45]. When a grain engages with the workpiece in the rubbing stage, the grain slides on the workpiece surface with elastic deformation of the system and negligible plastic deformation. Ploughing occurs as the grain initially plastically deforms the workpiece and even after chip formation begins, ploughing may still exist [46-52]. A chip is formed when the workpiece material can no longer withstand the tearing stress. For efficient metal removal, cutting can be considered to be the most desirable deformation process. In rubbing and ploughing, the energy is wasted in deformation and friction without contributing to metal removal.

A fundamental parameter derived from the power and machining conditions is the specific energy, defined as the energy per unit volume of material removed. i.e. 
$e_{c}=\frac{P}{Q_{w}}=\frac{P}{v_{w} d_{w} v_{f} b}$

where, $\mathrm{P}$ is the grinding power and

$\mathrm{Q}_{\mathrm{w}}$ is the volumetric removal rate from the workpiece.

The total specific grinding energy can be considered to consist of chip formation, ploughing, and sliding components:

$e_{c}=e_{c h}+e_{p l}+e_{s l}$

The specific chip formation energy is actually expended by material removal and is the minimum grinding energy required. The sliding energy accounts quantitatively for the influence of wheel grade and dressing conditions on grinding forces. With harder-grade wheels and finer dressing, the wear flat area is larger and so the sliding forces are proportionally larger [4].

In grinding, it has been said that typically, about $35 \%$ of the total energy is consumed by the cutting phase [53,54]. The specific energy is much larger than in other metal-cutting operations. Also, larger specific energies were found when uncut chip thickness was reduced, as proposed by Backer, Marshall and Shaw in describing the 'size effect' $[38,55]$. The size effect is that small chip size tends to increase specific energy. It was proposed by Backer, Marshall and Shaw that small uncut chip thickness reduced the defects in the metal to be removed and allowed the workpiece material to achieve its theoretical strength.

However, Von Turkovich [56], Nakayama and Tamura [57] cast doubt on Backers' description, because their research showed that the shear strength would not be larger as a result of the decrease of uncut chip thickness. Graham and Baul [58] and also Kannapan and Malkin [59] suggested that the size effect in grinding could be attributed to the occurrence of a relatively greater proportion of sliding and ploughing energy when the uncut chip thickness is decreased. Rowe [60] gave a sliced bread analogy. The thinner the loaf is sliced, the more energy is required to slice the whole loaf. In part, this is because a greater surface area is created. However, Rowe points out that the increase in specific energy is the 
combination of several physical effects and that for a complete explanation it is important to take account of the geometry of the active part of the cutting edges of the grains. Shaw [61] states that, in general, specific energy varies exponentially with uncut chip thickness.

Merchant [62] formulated a classic model of orthogonal chip formation. According to this model, chip formation occurs by an intense shearing process in an extremely thin shear plane followed by friction as the chip slides over the tool rake face. Typically, shearing at the shear plane is predicted to account for about $75 \%$ of the total chip formation energy, and chip tool friction the remaining $25 \%$. Although many other secondary effects have been observed over the years, this same model is still considered to present a reasonably accurate description of orthogonal chip formation. Unfortunately, grinding is far removed from an orthogonal chip formation process and the Merchant model is unrealistic for prediction of grinding energies.

One of the difficulties encountered in grinding is determining how a given set of grinding wheel, dressing conditions and workpiece material properties will affect the forces and power generated. Lindsay [63-65] presented a 'system characteristic chart'. For a specific set of conditions, the grinding results were fitted by straight-line equations. These equations can be used as a basis for controlling the process. The primary disadvantage of this method is that tests were run on a narrow set of grinding conditions so that separate charts must be made to cover all possible combinations for a given workpiece.

Peters et al $[66,67]$ defined equivalent chip thickness $h_{\mathrm{eq}}$ as

$$
h_{e q}=\frac{a_{e} \cdot v_{w}}{v_{s}}
$$

where, $a_{e}$ is depth of cut

$\mathrm{v}_{\mathrm{w}}$ is workpiece speed and

$\mathrm{v}_{\mathrm{s}}$ is wheel speed 
The relationship between normal and tangential forces per unit width in grinding, $F_{n}^{\prime}$ and $F_{\mathrm{t}}{ }^{\prime}$, and $h_{e q}$ can be represented by,

$$
\begin{aligned}
& F_{n}{ }^{\prime}=C_{1} \cdot h_{e q}{ }^{f} \\
& F_{t}{ }^{\prime}=C_{2} \cdot h_{e q}{ }^{f}
\end{aligned}
$$

Where, $C_{1}$ and $C_{2}$ are the intercepts of the graphs on a log-log scale

$f$ is the slope of the graphs on a log-log scale and can be considered to reflect the grindability of the material

Malkin [28] related wear flat area and grinding force. It was suggested that almost all sliding energy is generated at the interface between the wear flat of the grain and the workpiece. Grinding force can be separated into two kinds of forces. One is associated with cutting, and another is associated with sliding, so that

$$
\begin{aligned}
& F_{t}=F_{t c}+F_{t s l} \\
& F_{n}=F_{n c}+F_{n s l}
\end{aligned}
$$

where, $F_{t c}$, and $F_{n c}$ are the tangential and normal forces for cutting, and $F_{t s l}$ and $F_{\text {nsl }}$ are those for sliding.

It was assumed that the cutting force components are unaffected by the size of the wear flats while tangential and normal sliding forces are both linearly related to the wear flat area. The friction coefficient $\mu$ and the average contact pressure $\bar{p}$ between the flats and the workpiece were assumed to be constants. Equations 4.8 and 4.9 become

$$
\begin{aligned}
& F_{n}=F_{n c}+\bar{p} A \\
& F_{t}=F_{t c}+\mu \bar{p} A
\end{aligned}
$$

where, $A$ is the wear flat area per unit area of the wheel surface.

Although the inclusion of wear flat area in the analysis makes it easier to evaluate grinding force, the prediction is still particular to a set of wheel, workpiece, dressing and grinding conditions. 
Another force model was proposed by Torrance and Badger [68] based on a threedimensional abrasive model due to Xie and Williams [69-71]. The relationships were as follows.

$$
\begin{aligned}
& K=\frac{0.003 \times 3 \sqrt{3} \tan ^{3} \alpha}{f k l^{0.5}} \sqrt{\frac{H_{b}}{H_{s}}} \\
& \mu=(2 / \pi)^{0.5} \frac{\tan \alpha}{l^{0.25}}\left(1-\frac{f}{3 \sqrt{3}}\left[1+\frac{\pi}{4 \tan ^{2} \alpha}\right]^{0.5}\right) \\
& F_{n}{ }^{\prime}=\frac{h_{e q}}{K} . \\
& F_{t}{ }^{\prime}=\mu F_{n}{ }^{\prime}
\end{aligned}
$$

where,

$\mathrm{K}$ is the specific wear rate (1/MPa)

$\mu$ is the ratio of tangential force to normal force

$\alpha$ is the attack angle or slope of the cutting asperity

$\mathrm{H}_{b}$ is the bulk hardness of the workpiece

$\mathrm{H}_{\mathrm{s}}$ is the surface hardness of the workpiece

$l$ is a dimensionless distance between adjacent tracks for transition from ploughing to cutting

$f$ is equal to $\tau / k$, the ratio of the shear strength of the lubricant film to the shear strength of the workpiece

$k$ is the shear flow stress of the workpiece

This model takes into account the distribution of cutting slopes based on surface profiles taken from the grinding wheels. It also takes account of the ratio of workpiece hardness at the surface to bulk workpiece hardness. This model provides a more comprehensive prediction of how grinding forces are affected although some coefficients are difficult to determine from the grinding process. 


\subsection{Grinding temperature}

The temperatures generated during grinding are a direct consequence of the energy input to the process. Virtually all of this energy is converted to heat which is concentrated within the grinding zone. The high temperatures produced can cause various types of thermal damage to the workpiece such as burning, phase transformations, softening (tempering) of the surface layer with possible rehardening, residual tensile stresses, cracks and reduced fatigue strength $[72,73]$. High temperatures also cause workpiece size inaccuracy.

The analysis of moving heat sources was provided by Carslaw and Jaeger[74] in 1942. The moving band source model is widely used as a basis for modelling shallow cut grinding processes. Notable early studies of grinding temperature were carried out by Outwater and Shaw [75], Hahn [76], Des Ruisseaux and Zerkle [77], Maris and Snoeys [78].

Lavine, Malkin and Jen analysed the heat transfer into grains of the grinding wheel assuming the grains to be conical in shape using one-dimensional simplifications to represent two-dimensional heat conduction [79]. Later Rowe and Black [80] showed that the simplified cone assumption was inaccurate and particularly so for the large negative rake angles applicable in grinding. The plane grain assumption of Hahn [75] is an accurate model for three dimensional heat conduction into a plane surface and has the advantage of yielding a very simple expression. Jen and Lavine [81] used the cone assumption despite the complicated expression involving the unknown cone angle to compare workpiece temperature rises in up-grinding and down-grinding, and to explore the effect of the location of heat generation.

Rowe and Pettit [82] modelled the energy partitioning to all four heat sinks which are workpiece, wheel, coolant and chips. Upper and lower bound estimates of the energy to the workpiece provided confidence limits on the temperature calculation. In later work by Rowe [83-85], it was shown that it was important to combine the effect of the four heat sinks but also that a simpler and more accurate 
result is achieved if the wheel-workpiece contact is modelled as a set of discrete contacts as proposed by Hahn in 1962.

In Rowes' later work, the maximum value of the workpiece background temperature was calculated from the following equations [83-85].

$$
\begin{gathered}
T_{\max }=\left.\frac{q_{t}-h_{c h} \cdot T_{m p}}{\frac{h_{w}}{R_{w s}}+h_{f}}\right|_{T_{\max } \leq T_{b}} \\
T_{\max }=\left.\frac{q_{t}-h_{c h} \cdot T_{m p}}{\frac{h_{w}}{R_{w s}}}\right|_{T_{\max }>T_{b}}
\end{gathered}
$$

where,

$R_{\mathrm{ws}}$ is the workpiece-wheel partition ratio explained below

$\mathrm{q}_{\mathrm{t}}$ is the total grinding energy expressed as a heat flux (i.e. power/contact area)

$r_{0}$ is the effective radius of the grain contact area

$\mathrm{h}_{\mathrm{w}}$ is the workpiece convection factor

$h_{c h}$ is the maximum chip convection factor based on melting of the chips

$T_{b}$ is the boiling temperature of the coolant

$\mathrm{T}_{\mathrm{mp}}$ is the melting temperature of the workpiece material

The derivation of the main terms in the equations is indicated below.

The surface temperature of a grain subject to a steady state heat flux as shown by Hahn is,

$$
\theta_{g}=\frac{q_{g} \cdot r_{0}}{K_{g}}
$$

where,

$r_{0}$ is the radius of the contact area of the grain.

$\mathrm{q}_{\mathrm{g}}$ is the steady state heat flux entering the grain at a grain contact

$\mathrm{K}_{\mathrm{g}}$ is the thermal conductivity of the grains 
The temperature of the workpiece at a grain contact is found by application of Jaegers sliding source equation. Equating the grain contact temperature with the workpiece contact temperature at a grain contact leads to the partition of energy between the grains and the workpiece. This partition is defined in terms of Rws.

$$
R_{w s}=\frac{q_{w}}{q_{w}+q_{s}}=\left[1+\frac{0.97 x K_{g}}{\beta_{w} \cdot \sqrt{r_{0} \cdot v_{s}}}\right]^{-1}
$$

where

$\mathrm{q}_{\mathrm{w}}$ is the heat flux entering the workpiece and $\mathrm{q}_{\mathrm{s}}$ is the heat flux entering the grains and is therefore the same as the heat entering the wheel.

$\beta_{\mathrm{w}}$ is the workpiece thermal property i.e $\sqrt{K_{w} \cdot \rho_{w} \cdot c_{w}}$

$\mathrm{K}_{\mathrm{w}}$ is the thermal conductivity of the workpiece

$\rho_{\mathrm{w}}$ is the density of the workpiece

$c_{w}$ is the specific heat capacity of the workpiece.

$R_{w s}$ is defined as the energy going into the workpiece expressed as a proportion of the energy going into both the workpiece and the grains.

The derivation of the term $R_{w s}$ is made clearer if the energy terms are made explicit to a single grain contact as in the following equation.

$$
R_{w s}=\frac{q_{w g}}{q_{w g}+q_{g}}=\frac{\frac{q_{w g}}{q_{g}}}{\frac{q_{w g}}{q_{g}}+1}
$$

where,

$\mathrm{q}_{\mathrm{wg}}$ is the rate of heat per unit area (i.e. the heat flux) entering the workpiece at a grain contact and

$\mathrm{qg}_{\mathrm{g}}$ is the heat flux entering the same grain. 
Coolant convection factors are empirically based. Typical values were assumed to be

$$
\begin{aligned}
& h_{\text {fwater }}=6700(\mathrm{~W} / \mathrm{m} \cdot \mathrm{K}) \text { - Water based } \quad \mathrm{T}_{\mathrm{b}}=120^{\circ} \mathrm{C} \\
& h_{f . o i l}=2700(W / m \cdot K)-\text { Neat oils } \quad T_{b}=300^{\circ} \mathrm{C}
\end{aligned}
$$

Later work by Rowe and Jin [86] showed these values for coolant convection could be orders of magnitude greater.

\subsection{Grinding wheel wear}

As grinding proceeds, a wheel wears and either becomes sharper or dulled. In fine grinding, a wheel of conventional structure often becomes dull rather than sharp. As the cutting edges of the grains become blunt, the grinding force and grinding temperature increase and workpiece accuracy deteriorates. At some point, the wheel needs to be dressed. Dressing is a term, which implies restoration of a true form and sharp cutting edges. The duration from commencement of grinding to the point when re-dressing is required is termed the wheel redress life. Wheel redress life is an important feature of wheel performance, which strongly influences the economic efficiency of grinding.

Basically, there are three ways in which grinding wheel performance deteriorates: $[28,87,88]$

(i) Gradual or rapid wear of the grains which reduces the size of the wheel and may cause the wheel to become dull;

(ii) Loading of workpiece material into the surface of the wheel and

(iii) Loss of wheel shape.

Active cutting edge density, cutting edge dullness, and effective wheel porosity ratio change during the grinding process. This is indicated by the wear and 
loading of the grinding wheel. The wear and loading of a grinding wheel in grinding is the combined effect of the wear and loading of individual grains in the wheel.

When the wheel is rotated at grinding speed and applied to the workpiece, the abrasive grains cut the material and remove small chips. Under the action of the forces imposed during grinding, flats are worn on the abrasive cutting points. This causes an increase in friction, workpiece heating and magnitude of the forces imposed on the wheel. An increase in the magnitude of grinding force increasingly causes the abrasive to fracture, exposing new cutting edges, or fractures the bond bridges to expose fresh abrasive grains. If the fracture wear allows the wheel to remain sharp, the grinding wheel is said to be self-sharpening. If the wheel is not self-sharpening, the wheel has to be dressed more frequently.

Grinding wheel wear is a complex process. Generally, three main mechanisms of wheel wear are attrition, grain fracture and bond fracture [88-93].

(i) Attritious wear involves slow dulling of the abrasive grains and the growth of wear flats.

(ii) Grain fracture refers to removal of abrasive fragments from the grain when the internal stress exceeds the fracture strength.

(iii) Bond fracture occurs when the force on a grain causes the stresses within the bond to exceed the fracture stress of the bond. This results in abrasive grains being dislodged from the bond.

In grinding, hard particles in the workpiece material physically displace abrasive material from the surface of the grains. The generic scientific term for this type of action is abrasive wear. Abrasive wear is a complex process involving a range of physical and chemical interactions. The abrasive grains are subject to high temperatures and pressures in grinding. In consequence, oxidation wear, chemical wear, diffusion wear, fracture wear by thermal stress and plastic deformation wear all play a part. The sharpness of a wheel is reduced by attritious wear of the grains and by adhesion of workpiece material. 
Slow attritious wear is produced by rubbing contact between the workpiece and the grains involving various physical and chemical reactions within the grinding zone in the regions of plastic deformation of the workpiece material. Although the consumption of the wheel caused by attritious wear is relatively small, grinding quality and productivity are affected. Attritious wear is often the most important form of wear since it controls the grinding forces and hence also the rate of bond fracture. Where possible, the abrasive should be considerably harder than the material being ground and should not demonstrate chemical affinity for the workpiece material.

It is known that grain fracture wear consists principally of the detachment of fragments from the sliding surface of the grains. It was found that crystallographic orientation plays a role. A thermal analysis of grinding indicated that the thermal gradient along the grain can be as high as $4 \times 10^{5} \mathrm{~K} / \mathrm{mm}[76,94]$. Under such extreme conditions, it is evident that thermal stress can cause grain fracture. The occurrence of these temperature gradients during grinding followed by rapid cooling during the free path around the wheel periphery causes thermal fatigue. It is apparent that the fracture wear of the grains during grinding is a complex process, which has not yet been fully explained. Because of the random distribution and orientation of grains in a grinding wheel, statistical prediction of grain fracture is possible for a particular set of grinding conditions.

Grain fracture and bond fracture in grinding depend on the grinding conditions, grain friability and the wheel hardness [95]. More friable grains are more susceptible to grain fracture prior to bond fracture. With increasing wheel hardness, there is relatively more grain fracture and less bond fracture, because of the greater bond strength allied to the greater probability of the abrasive grain undergoing fracture prior to being finally dislodged [4]. Grain fracture and bond fracture is an important factor in restricting the size of wear flat areas initially dressed onto the grains and their subsequent growth by attrition. In the absence of self-sharpening, abrasive grains become dull, thereby causing large grinding forces and possible thermal damage to the workpiece. 
Ye and Pearce [96] found wheel profile wear was strongly influenced by the total distance travelled by an individual grit in contact with the workpiece and by the workpiece feed rate.

It is commonly accepted $[89,97-99]$ the wheel wear process tends to follow three stages, an initial rapid wear stage, a slower steady stage and a final rapid wear stage where the wheel surface rapidly deteriorates and quality becomes poor.

The initial stage is characterized by intensive wheel wear and the shape of the grinding force characteristic is strongly dependent on the dressing operation. There are many cracks in the wheel surface after dressing, which cause grains to fracture or dislodge from the wheel surface when the wheel engages with the workpiece. In the second stage, wheel wear is mainly due to a combination of attritious wear and grain fracture. Grinding wheel wear is slower and steady grinding behaviour is experienced. The steady stage is desirable for predictable control of the grinding process. The first and second stages represent the useful redress life of the grinding wheel.

In the final stage, either grinding forces become excessive or vibration develops and the wheel has to be redressed. Wheel wear in this stage is due to bond post rupture and whole grains are dislodged from the wheel. This leads to rapid deterioration in surface texture, workpiece form and size tolerance. If the surface roughness, roundness and size errors of the workpiece fall outside the tolerance limits, the wheel has to be redressed.

\subsection{Grinding wheel loading}

Wheel loading is a term used to describe the undesirable condition where workpiece material attaches itself to the wheel or becomes embedded in the pores [100]. Under wheel loading conditions, workpiece material accumulates on the wheel surface, filling the surface between the grains and causing metal-to-metal contact with the workpiece. This is particularly noticeable with increasing wear flats on the wheel. When loaded, the wheel does not cut efficiently, the 
temperature increases, the workpiece softens, roughness deteriorates, and normal forces may rapidly increase leading to vibration and even to catastrophic failure. Even with slight loading the surface roughness increases and deep scratches become evident on the surface of the workpiece [100].

The most common form of wear according to Rabinowicz is adhesive wear [101]. If two surfaces are brought together and then separated, either normally or tangentially, attractive forces act in such a way as to attempt to pull material from one surface onto the other. It has been argued that whenever material is removed from its original surface in this way, an adhesive wear fragment is created [101]. Whether the fragment adheres to one or the other depends on the strength of the interfacial bond depending on the nature of the friction couple. At the atomic scale, Sarkar [102] described this as atom plucking. This means as an atom is displaced, it attempts to return to its original position. This does not occur each time, and it is equally probable that as an atom is dislodged from its equilibrium position, it may adhere to another atom in the opposite surface instead of returning to its original position.

Various investigators have tried to explain the mechanism of the loading phenomenon. Komanduri [103] explained metal build-up on abrasive grits in terms of a mechanism in which the work material initially oxidises and solid phase reaction between the metal oxide and grit material gives a graded interface between the build-up layer and grit material. Chemical interaction between abrasive and workpiece material has also been suggested [104-107]. Shaw and Yang [108] suggested that loading of a wheel surface might be due to pressure welding of grinding chips to abrasive grits. Nakayama [109] concluded there are mainly three factors, which lead to adhesive loading in grinding.

(i) Workpiece material affinity to the grains of the wheel

(ii) An interfacial layer between the workpiece and grain

(iii) Load and temperature

Yossifon and Rubenstein [110-112] suggested that wheel loading occurs due to adhesion of individual grinding chips that first adhere locally to the grain tips and 
then to each other to form a compact mass that, after going though its life cycle, becomes detached from the wheel.

Archard presented an adhesive wear law [113]. According to Archards' Law the adhesive wear in grinding is,

$V_{a d}=\frac{c F_{c n^{x}}}{H_{w}}$

where,

$V_{a d}$ is the adhesive wear volume from the workpiece under a cutting edge $c$ is a dimensionless constant dependent on the materials in contact and their exact degree of cleanliness

$F_{c n}$ is the normal load on the workpiece under a cutting edge

$H_{w}$ is the surface hardness of the workpiece

$x$ is the sliding distance

It was proposed [102] that, as in other types of adhesive contacts in grinding, some adhered wear particles will stay adhered and thus be in a position to grow by further accretion of material. Others will detach in loose form and thus limit the growth of loading. The formation of loose particles is often a result of chemical changes in the adherent fragments.

Estimation of the diameter of adherent wear particles is obtained by noting that such particles are typically a factor of 3 times smaller than loose particles [101]:

$d_{a d h}<\frac{1}{3}\left(60,000 \frac{W_{a b}}{H_{p}}\right)=20,000 \frac{W_{a b}}{H_{p}}$

where,

$\mathrm{H}_{\mathrm{p}}$ is the penetration hardness of adhering material

$\mathrm{W}_{\mathrm{ab}}$ is the work of adhesion of the contacting materials, which is equal to the energy that must be applied to separate unit area of the interface between two materials a and $b$. To do this, it is necessary to create two 
surfaces of energy $\gamma_{w}$, and $\gamma_{g}$, respectively, which destroys an interface that has interfacial energy of amount $\gamma_{a b}$. i.e.

$W_{a b}=\gamma_{a}+\gamma_{b}-\gamma_{a b}$

Then,

$\frac{W a b}{H_{p}}=\frac{\gamma_{a}}{H_{p}}+\frac{\gamma_{b}}{H_{p}}-\frac{\gamma_{a b}}{H_{p}}$

In summary, the literature review has shown that the explanation of grinding behaviour requires a complex set of physical processes to be modelled. The next chapter deals with this problem in greater detail. 


\section{Chapter 5 Theory}

\subsection{A model for a vitrified CBN wheel structure}

The previous review showed the qualitative relationship between wheel structure and grinding performance. A quantitative analysis was performed to achieve a better understanding about how wheel structure affects grinding performance.

For the purposes of analysis and calculation, the 3D wheel structure was modelled as follows. Pores and grains in the wheel were assumed to be spheres with diameter $d_{p}$ of the pores and diameter $d_{g}$ of the grains. The grains were assumed to be randomly distributed in the wheel and to surround the pores as shown in Fig. 5.1.

Definition of the terms used in the analysis is given below:

'On the wheel surface' is to be understood as meaning that the radial depth into the wheel is equal to or less than the pore size, i.e. $z \leq d_{p}$, as in Fig. 5.1. 'Within the wheel' is to be understood as meaning that the radial depth into the wheel is more than the pore size, i.e. $z>d_{p}$, as in Fig 5.1.

$\mathrm{C}_{\mathrm{ga}}$ is the grain area density defined as the number of grains per unit area at a particular radial depth within the wheel.

$\mathrm{C}_{\text {gas }}$ is the grain area density defined as the number of grains per unit area at a particular radial depth on the wheel surface.

$\mathrm{C}_{\mathrm{gv}}$ is the grain volumetric density defined as the number of grains per unit volume.

$\mathrm{C}_{\mathrm{pa}}$ is the pore area density defined as the number of pores per unit area at a particular radial depth within the wheel.

$\mathrm{C}_{\text {pas }}$ is the pore area density defined as the number of pores per unit area at a particular radial depth on the wheel surface.

$\mathrm{C}_{\mathrm{pv}}$ is the pore volumetric density defined as the number of pores per unit volume. 
$\mathrm{L}_{\text {gamin }}$ is the minimum grain area spacing at a particular radial depth within the wheel

$\mathrm{L}_{\text {gamax }}$ is the maximum grain area spacing at a particular radial depth within the wheel

$\mathrm{L}_{\text {gasmin }}$ is the minimum grain area spacing at a particular radial depth on the wheel surface

$\mathrm{L}_{\text {gasmax }}$ is the maximum grain area spacing at a particular radial depth on the wheel surface

According to the grain concentration and grain size, the percentage grain volume $\mathrm{V}_{\mathrm{g}}$ and grain volumetric density within the wheel $\mathrm{C}_{\mathrm{gv}}$ can be evaluated as follows,

$$
V_{g}=\text { Conc. } \frac{0.24}{100}
$$

where, Conc is the wheel concentration number which indicates the amount of abrasive contained in the wheel. The concentration number is based upon a proportional scale with a value of 100 corresponding to an abrasive content of 4.4 carats $/ \mathrm{cm}^{3}$. For CBN wheels, 100 concentration is $24 \%$ grit by volume.

So, $C_{g v}=\frac{V_{g}}{V_{1 g}}=\frac{\text { Conc. } \frac{0.24}{100}}{\frac{4}{3} \pi\left(\frac{d_{g}}{2}\right)^{3}}$

where, $V_{1 \mathrm{~g}}$ is average grain volume for each grain.

A software program based on this model of the wheel structure was established to calculate the variation of grain area density with increasing radial depth into the wheel. This program is given in Appendix A1.1. When the grain volumetric density and pore volumetric density are determined, it is found that the grain area density varies linearly with radial depth below the surface of the wheel down to a depth equal to the grain size $d_{g}$. Beyond this depth, the grain area density remains approximately constant due to the even distribution of grains in this range, as shown in Fig. 5.2. The slope of the linearly increasing region is the grain volumetric density, i.e,

$$
C_{g a}=C_{g v} \cdot d_{g} \quad \text { for } \quad z>d_{g}
$$


$C_{g a s}=C_{g v} \cdot z$ for $z \leq d_{g}$

Where, $\mathrm{z}$ is radial depth into the wheel.

The pore distribution follows a similar relationship to the grain distribution, i.e.

$C_{p a}=C_{p v} \cdot d_{p} \quad$ for $\quad z>d_{p}$

$C_{p a s}=C_{p v} . z \quad$ for $\quad z \leq d_{p}$

According to Equations 5.5 and 5.6, at a wheel radial depth equal to the pore size $d_{p}$, the maximum pore area density on the wheel surface is $C_{p a}$. Thus, from measurements and analysis of the wheel surface as described in Chapter 3 , pore size $d_{p}$ and pore area density within the wheel $C_{p a}$ can be evaluated (the $C_{p a s}$ reaches maximum at wheel radial depth of $d_{p}$, and at this time $C_{p v}$ equals $\mathrm{C}_{\mathrm{pa}}$ can be known according to Equation 5.5 and 5.6). Pore volumetric density can then be achieved by application of Equation 5.5. Thus, a quantitative description of the wheel structure can be established.

\subsection{Effect of vitrified CBN wheel structure on active cutting edge density}

Initially, grains are considered rather than cutting edges in order to simplify the analysis. From Equations 5.1-5.4, it can be shown that,

$C_{\text {gas }}=\frac{\text { Conc. } \frac{0.24}{100}}{\frac{4}{3} \pi\left(\frac{d_{g}}{2}\right)^{3}} \cdot z \quad$ for $z \leq d_{g}$

A smaller grain size increases the grain area density. However, a conclusion from this equation is that grain area density on the wheel surface has no relationship with pore characteristics including pore size, pore volume, pore area density and pore volumetric density once the percentage volume of pore is fixed. In Chapter 3, it was shown that not all grains cut the workpiece even if they are at the same radial depth. It depends on grinding kinematics. Active grain area density meaning the density of grains actively involved in cutting the workpiece is less than static grain area density. It is found that active grain area density is affected by pore size and grain size unlike grain area density, 
which is unaffected by pore size as explained previously. The reason that active grain area density is affected is explained as follows.

The minimum and maximum grain area spacing $\mathrm{L}_{\text {gamin }}, \mathrm{L}_{\text {gamax }}$ in a particular area may be evaluated as follows:

Fig. 5.3 shows the grain area spacing is measured within a 'bearing area' at a radial depth $\mathrm{z}$ into the wheel. Within the wheel, the minimum grain spacing approaches zero because one grain may lie almost on top of another. Also the pores are surounded by grains, the maximum grain spacing is $2 r_{g}+d_{p}$ i.e. $d_{g}+d_{p}$

The minimum grain area spacing on the wheel surface $L_{\text {gasmin }}$ is $\sqrt{d_{g}{ }^{2}-z^{2}}$ which may be deduced from Fig.5.3. The maximum grain area spacing on the wheel surface $L_{\text {gasmax }}$, is affected by the maximum grain area spacing $L_{\text {gamax }}$ within the wheel. If it is assumed there is a proportional relationship, the maximum grain area spacing $\mathrm{L}_{\text {gasmax }}$ on the wheel surface is $k .\left(d_{g}+d_{p}\right)$ where $\mathrm{k}$ is a coefficient, which has a relationship with the radial depth into the wheel $z$. When $z$ approaches the value of the grain size $d_{g}, k$ approaches the value 1 . When $\mathrm{z}$ approaches zero, $\mathrm{k}$ approaches the value $1 /\left(d_{g}+d_{p}\right)$, i.e. the maximum grain area spacing is $1 \mathrm{~mm}$ in fine wheels although in realistic it could be larger than $1 \mathrm{~mm}$.

It can be seen that, the minimum grain area spacing on the wheel surface has relationships with grain size and radial depth into the wheel, the maximum grain area spacing has relationships with grain size, pore size and radial depth into the wheel.

Assuming that the distribution of grain area spacings $\mathrm{L}_{\mathrm{ga}}$ is normal on the wheel surface,

$P_{N}\left(L_{g a}\right)=\frac{1}{\sqrt{2 \pi} \sigma} e^{-\frac{\left(L_{g a}-\mu\right)^{2}}{2 \sigma^{2}}}$ 
Where, the mean grain spacing is $\mu=\sqrt{\frac{1}{C_{\text {gas }}}}$ at the particular depth into the wheel and $\sigma$ is the standard deviation of the grain area spacings.

From Equation 5.3 it can be shown that,

$$
\mu=\sqrt{\frac{1}{C_{g a s}}}=\sqrt{\frac{1}{C_{g \nu \cdot z}}} \quad \text { for } \quad z \leq d_{p}
$$

$\mu$ is a constant at a particular depth into the wheel for a given grain volumetric density.

Based on the normal distribution, it is assumed that all (more than $99.7 \%$ ) are within 3 standard deviations of the mean. It is therefore assumed that the standard deviation is given approximately by

$\sigma=\frac{L_{g a \max }-L_{g a \min }}{6}$

From Equations 5.8-5.10, a distribution of grain area spacings on the surface of a wheel is shown in Fig. 5.4. The values used in this example were $\mathrm{C}_{\mathrm{gas}}=20 / \mathrm{mm}^{2}, z=0.5 . \mathrm{g}_{\mathrm{g}}$ and $\mathrm{k}=2.5$. From the figure, it can be seen that the bigger the pore size, the wider is the distribution of grain area spacings for a particular grain size. The bigger the grain size, the wider is the distribution of grain area spacings for a particular pore size.

As mentioned in Chapter 3, in grinding, some active grains remove material in front of other grains thus rendering them inactive. For the same grinding conditions, there is less chance for grains to become active when the grain area spacing is small. So, there are fewer active grains in a wheel with a larger standard deviation in the grain distribution although the grain area density is the same. Thus, the larger the pore size and the larger the grain size, the wider will be the distribution of grain area spacings and the fewer the active grains left. Thus, the mean active grain spacing will become larger. 
In conclusion, increasing or reducing pore size and grain size affects active grain spacing. Grain area density and grain volumetric density, do not change with pore size and pore volumetric density. Active grain density, however, decreases with increasing pore size and grain size, while, mean active grain area spacing increases. Assuming there are the same numbers of cutting edges as grains, the above conclusion also applies to active cutting edge density and spacing.

\subsection{Effect of vitrified CBN wheel structure on porosity volume ratio}

In Chapter 3, effective porosity volume ratio $\mathrm{V}_{\mathrm{pw}}$ was defined as the ratio of the effective volume of the pores to the volume of the wheel on the wheel surface. In order to simplify the analysis, the total volume of the pores on the wheel surface was assumed to be the effective pore volume.

When the radial depth into the wheel $\mathrm{z} \leq \mathrm{d}_{\mathrm{g}}$, the porosity volume ratio can be expressed as

$$
\left.V_{p w}\right|_{z \leq d_{g}}=1-\left.V g\right|_{z \leq d_{g}}-\left.V_{b}\right|_{z \leq d_{g}}
$$

Within the radial depth into the wheel $z=d_{g}$, there are $C_{\text {gasmax }}$ grains per unit area. It is assumed that the bond volume around each grain within the wheel $\mathrm{V}_{\mathrm{bg}}$ is

$V_{b g}=\frac{1-V_{g}-V_{p}}{C_{g v}}$

It is assumed that on the wheel surface, the average bond volume around each grain is half that within the wheel. Equation 5.11 can then be expressed as,

$$
\begin{aligned}
& \left.V_{p w}\right|_{z \leq d_{g}}=1-\left.V g\right|_{z \leq d_{g}}-\frac{1}{2} \cdot C_{g a s \max } \cdot V_{b g} \\
& =1-\left.V g\right|_{z \leq d_{g}}-\frac{1}{2} \cdot C_{g a s \max } \cdot \frac{1-V_{g}-V_{p}}{C_{g v}}
\end{aligned}
$$

Because $\mathrm{C}_{\text {gasmax }}=\mathrm{C}_{\mathrm{gv}}$, Equation 5.13 can be expressed as, 


$$
\begin{aligned}
& V_{\left.p w\right|_{z} \leq d_{g}}=1-\left.V g\right|_{z \leq d_{g}}-\frac{1}{2} \cdot C_{g a s \max } \cdot V_{b g} \\
& =\frac{1}{2}-\left.V g\right|_{z \leq d_{g}}+\frac{1}{2}\left(V_{g}+V_{p}\right)
\end{aligned}
$$

where, $\mathrm{n}$ is positive integrals.

When the radial depth of the wheel $z \leq n . d_{g}$, the porosity volume ratio is

$$
V_{\left.p w\right|_{z \leq n . d}}=\frac{1}{2}-\left.V g\right|_{z \leq n . d_{g}}+\frac{1}{2}\left(V_{g}+V_{p}\right)
$$

A software program based on this model of the wheel structure was established to calculate wheel porosity ratio on the wheel. This program is given in Appendix A1.2. Fig. 5.5 shows the effect of wheel structure on porosity volume ratio. As shown in Fig. 5.5(a), for a larger concentration, the porosity volume ratio is smaller for a particular percentage of pore volume (in this figure, the percentage of pore volume is $30 \%$ ). Pore size affects the depth of the wheel surface, the larger the pore size, the greater is the depth of the wheel surface. With a deeper wheel surface, the porosity volume ratio is smaller for a particular pore volume and wheel concentration. The effect of depth of wheel surface on porosity volume ratio is less than that of percentage of pore volume. Fig. 5.5(b) shows that when the pore size increases from $d_{g}$ to 1.26. $\mathrm{d}_{\mathrm{g}}$, the depth of the wheel surface increases to $1.26 . \mathrm{d}_{\mathrm{g}}$ and the percentage of pore volume increases from $30 \%$ to $60 \%$. The porosity volume ratio increases above 0.1 .

In conclusion, a larger concentration reduces the porosity volume ratio for a particular percentage of pore volume. The effect of depth of the wheel surface on porosity volume ratio is less than the effect of percentage of pore volume on porosity volume ratio.

\subsection{Effect of vitrified CBN wheel structure on wheel scratch hardness}

In Chapter 3, wheel scratch hardness was defined as the ratio of average tangential grain force to the mean diameter of the grains removed when scratching the wheel surface with a depth equal to the mean grain size using a particular sized cutting tool, i.e. 


$$
H_{s s}=\frac{F_{g s t}}{d_{b a}}
$$

Based on the definition of wheel scratch hardness in Equation 5.16, wheel bond hardness $\mathrm{H}_{\mathrm{sb}}$ and grain fracture hardness $\mathrm{H}_{\mathrm{sa}}$ are defined as

$$
\begin{gathered}
H_{s b}=\frac{F_{g s t}}{\left(\frac{\int_{i=1}^{C_{b}} d_{b i}}{C_{b a}}\right)} \\
H_{s a}=\frac{F_{g s t}}{\left(\frac{\int_{j=1} d_{a j}}{C_{b a}}\right)}
\end{gathered}
$$

where,

$\mathrm{C}_{\mathrm{ba}}$ is the grain density in the scratch direction of grains removed by bond fracture and grain fracture per unit scratch length;

$\mathrm{C}_{\mathrm{b}}$ is the grain density in the scratch direction of grains removed by bond fracture per unit scratch length;

$\mathrm{C}_{\mathrm{a}}$ is the grain density in the scratch direction of grains removed by grain fracture per unit scratch length;

$\mathrm{d}_{\mathrm{b}}$ is the diameter of the grains removed by bond fracture. $\mathrm{d}_{\mathrm{a}}$ is the diameter of the grains removed by grain fracture.

So,

$$
H_{s s}=\frac{F_{g s t}}{d_{b a}}=\frac{F_{g s t}}{\frac{\int_{i=1}^{C_{b}} d_{b i}+\int_{j=1}^{C_{a}} d_{a j}}{C_{b a}}}=\frac{F_{g s t}}{\frac{\int_{i=1}^{C_{b}} d_{b i}}{C_{b a}}+\frac{\int_{j=1}^{C_{a}} d_{a j}}{C_{b a}}}
$$

So. wheel scratch hardness can be split into two parts, wheel bond hardness $\mathrm{H}_{\mathrm{sb}}$ and grain fracture hardness $\mathrm{H}_{\mathrm{sa}}$ as follows, 


$$
H_{s s}=\frac{1}{\frac{1}{H_{s b}}+\frac{1}{H_{s a}}}
$$

$\mathrm{H}_{\mathrm{sa}}$ reflects the inherent nature of the abrasive, it does not change if the type of abrasive remains the same.

Two factors affect wheel bond hardness in terms of wheel structure. One is the bond volume between the grains at each bond bridge, i.e. $\frac{V_{b}}{m \cdot C_{g v}}$, where, $m$ is the number of bond bridges around each grain. The other factor is the force applied to the bond bridge for a particular grinding force. A criterion to evaluate wheel bond hardness was defined as the ratio of bond bridge volume to bond bridge force on the grain when a unit grinding force is applied to the grain, i.e.

$$
H_{s b^{\prime}}=\frac{V_{g b}}{m \cdot F_{b}}
$$

where, $H_{s b}$ ' is propotional to theoretical wheel bond hardness.

In order to calculate the bond force for each grain, grains were assumed to be arrayed within a conical space as shown in Fig. 5.6. Every grain on the wheel surface is assumed to be located at the top of a cone. The cone density is defined as the number of cones per unit area at a particular depth on the surface of the wheel. In order to make the cone density consistent with the grain area density in the wheel surface, a new cone is assumed to appear at each depth interval of $\mathrm{d}_{\mathrm{g}} / \mathrm{C}_{\mathrm{ga}}$. The grain area density in the wheel surface at a radial depth equivalent to the grain size $d_{g}$ reaches its maximum value. At a radial depth equal to the pore size $d_{p}$, the bearing area ratio of grain and the bearing area ratio of the bond summed together are assumed to be $100 \%$. This means the bearing area ratio for the pores is assumed to be equal to $0 \%$ at this depth. Also, pore size is assumed to be larger than the grain size. Thus, different pore sizes and grain sizes correspond to different values of cone angle, $\theta$ as shown in Table. 5.1. 
Table: 5.1. Relationship between half vertex angle of the cone, $\theta$, pore size $d_{p}$, and grain size $d_{g}$

\begin{tabular}{|c|c|c|c|c|}
\hline \multicolumn{5}{|c|}{$\mathrm{V}_{\mathrm{g}}=30 \%, \mathrm{~V}_{\mathrm{b}}=10 \%, \mathrm{~V}_{\mathrm{p}}=60 \%$} \\
\hline \multicolumn{5}{|c|}{ Grain size $\mathrm{d}_{\mathrm{g}}: 64 \mu \mathrm{m}$} \\
\hline $\begin{array}{l}\text { Pore size } \\
d_{p}(\mu \mathrm{m})\end{array}$ & 81 & 90 & 110 & 130 \\
\hline $\mathrm{C}_{\mathrm{pv}}\left(/ \mathrm{mm}^{3}\right)$ & 2188 & 1273 & 861 & 522 \\
\hline $\mathrm{C}_{\mathrm{gv}} / \mathrm{C}_{\mathrm{pv}}$ & 1 & 1.72 & 2.54 & 4.19 \\
\hline$\theta\left(^{\circ}\right)$ & 42.5 & 45.0 & 43.0 & 44.0 \\
\hline \multicolumn{5}{|c|}{ Grain size $d_{g}: 91 \mu \mathrm{m}$} \\
\hline $\begin{array}{l}\text { Pore size } \\
d_{p}(\mu \mathrm{m})\end{array}$ & 118 & 130 & 150 & \\
\hline $\mathrm{C}_{\mathrm{pv}}\left(/ \mathrm{mm}^{3}\right)$ & 760 & 522 & 340 & \\
\hline $\mathrm{C}_{\mathrm{gv}} / \mathrm{C}_{\mathrm{pv}}$ & 1 & 1.46 & 2.24 & \\
\hline$\theta\left(^{\circ}\right)$ & 42.0 & 42.5 & 42.5 & \\
\hline \multicolumn{5}{|c|}{ Grain size $\mathrm{d}_{\mathrm{g}}: 126 \mu \mathrm{m}$} \\
\hline $\begin{array}{l}\text { Pore size } \\
d_{p}(\mu \mathrm{m})\end{array}$ & 159 & & & \\
\hline $\mathrm{C}_{\mathrm{pv}}\left(/ \mathrm{mm}^{3}\right)$ & 287 & & & \\
\hline $\mathrm{C}_{\mathrm{gv}} / \mathrm{C}_{\mathrm{pv}}$ & 1 & & & \\
\hline$\theta\left(^{\circ}\right)$ & 42.5 & & & \\
\hline \multicolumn{5}{|c|}{ Grain size $d_{g}: 151(\mu \mathrm{m})$} \\
\hline $\begin{array}{l}\text { Pore size } \\
d_{p}(\mu \mathrm{m})\end{array}$ & 194 & & & \\
\hline $\mathrm{C}_{\mathrm{pv}}\left(/ \mathrm{mm}^{3}\right)$ & 166 & & & \\
\hline $\mathrm{C}_{\mathrm{gv}} / \mathrm{C}_{\mathrm{pv}}$ & 1 & & & \\
\hline$\theta\left(^{\circ}\right)$ & 42.0 & & & \\
\hline
\end{tabular}


Table 5.1 Continued

\begin{tabular}{|c|c|c|c|c|c|c|c|c|}
\hline \multicolumn{9}{|c|}{$\mathrm{V}_{\mathrm{g}}=30 \%, \mathrm{~V}_{\mathrm{b}}=50 \%, \mathrm{~V}_{\mathrm{p}}=20 \%$} \\
\hline \multicolumn{9}{|c|}{ Grain size $d_{g}: 64(\mu \mathrm{m})$} \\
\hline $\begin{array}{l}\text { pore size } \\
d_{p}(\mu \mathrm{m})\end{array}$ & 58 & \multicolumn{2}{|l|}{70} & 90 & \multicolumn{2}{|c|}{110} & 130 & 150 \\
\hline $\mathrm{C}_{\mathrm{pv}}\left(/ \mathrm{mm}^{3}\right)$ & 2188 & \multicolumn{2}{|c|}{1111} & 525 & \multicolumn{2}{|c|}{287} & 173 & 113 \\
\hline $\mathrm{C}_{\mathrm{gv}} / \mathrm{C}_{\mathrm{pv}}$ & 1 & \multicolumn{2}{|l|}{1.97} & 4.17 & \multicolumn{2}{|c|}{7.62} & 12.72 & 19.36 \\
\hline$\theta\left(^{\circ}\right)$ & 56.0 & \multicolumn{2}{|c|}{57.5} & 57.5 & \multicolumn{2}{|c|}{57.5} & 57.5 & 57.5 \\
\hline \multicolumn{9}{|c|}{ Grain size $d_{g}: 91 \mu \mathrm{m}$} \\
\hline $\begin{array}{l}\text { pore size } \\
d_{p}(\mu \mathrm{m})\end{array}$ & 82 & \multicolumn{2}{|l|}{90} & \multicolumn{2}{|l|}{110} & \multicolumn{2}{|l|}{130} & 150 \\
\hline $\mathrm{C}_{\mathrm{pv}}\left(/ \mathrm{mm}^{3}\right)$ & 760 & \multicolumn{2}{|l|}{525} & \multicolumn{2}{|l|}{287} & \multicolumn{2}{|l|}{172} & 113 \\
\hline $\mathrm{C}_{\mathrm{gv}} / \mathrm{C}_{\mathrm{pv}}$ & 1 & 1.45 & & 2.65 & & 4.42 & & 6.73 \\
\hline$\theta\left(^{\circ}\right)$ & 57.5 & $\overline{57.5}$ & & 57.5 & & 57.5 & & 57.5 \\
\hline Grain size $d_{\varepsilon}$ & $126 \mu \mathrm{m}$ & & & & & & & \\
\hline $\begin{array}{l}\text { pore size } \\
d_{p}(\mu \mathrm{m})\end{array}$ & 112 & & 130 & & 150 & & & \\
\hline $\mathrm{C}_{\mathrm{pv}}\left(/ \mathrm{mm}^{3}\right)$ & 287 & & 172 & & 113 & & & \\
\hline $\mathrm{C}_{\mathrm{gv}} / \mathrm{C}_{\mathrm{pv}}$ & 1 & & 1.67 & & 2.54 & & & \\
\hline$\theta\left({ }^{\circ}\right)$ & 57.5 & & 57.0 & & 57.0 & & & \\
\hline Grain size d & $\mathrm{g}: 151 \mu \mathrm{m}$ & & & & & & & \\
\hline $\begin{array}{l}\text { pore size } \\
d_{p}(\mu \mathrm{m})\end{array}$ & 135 & & 150 & & & & & \\
\hline $\mathrm{C}_{\mathrm{pv}}\left(/ \mathrm{mm}^{3}\right)$ & 166 & & 113 & & & & & \\
\hline $\mathrm{C}_{\mathrm{gv}} / \mathrm{C}_{\mathrm{pv}}$ & 1 & & 1.47 & & & & & \\
\hline$\theta\left(^{\circ}\right)$ & 57.0 & & 57.0 & & & & & \\
\hline
\end{tabular}


Table 5.1 Continued.

\begin{tabular}{|c|c|c|c|c|c|c|c|c|c|c|}
\hline \multicolumn{11}{|c|}{$V_{g}=35 \%, V_{b}=50 \%, V_{p}=15 \%$} \\
\hline \multicolumn{11}{|c|}{ Grain size $\mathrm{d}_{\mathrm{g}}: 64 \mu \mathrm{m}$} \\
\hline $\begin{array}{l}\text { pore size } \\
d_{p}(\mu \mathrm{m})\end{array}$ & \multicolumn{2}{|l|}{50} & 70 & 90 & \multicolumn{3}{|c|}{110} & \multicolumn{2}{|c|}{130} & 150 \\
\hline $\mathrm{C}_{\mathrm{pv}}\left(/ \mathrm{mm}^{3}\right)$ & \multicolumn{2}{|c|}{2555} & 836 & 393 & \multicolumn{3}{|c|}{215} & \multicolumn{2}{|c|}{130} & 85 \\
\hline $\mathrm{C}_{\mathrm{gv}} / \mathrm{C}_{\mathrm{pv}}$ & \multicolumn{2}{|l|}{1} & 3.06 & 6.50 & \multicolumn{3}{|c|}{11.88} & \multicolumn{2}{|c|}{19.65} & 30.06 \\
\hline$\theta\left(^{\circ}\right)$ & \multicolumn{2}{|c|}{60.0} & 61.0 & 61.0 & \multicolumn{3}{|c|}{61.0} & \multicolumn{2}{|c|}{61.5} & 61.5 \\
\hline \multicolumn{11}{|c|}{ Grain size $d_{g}: 151 \mu \mathrm{m}$} \\
\hline $\begin{array}{l}\text { pore size } \\
\mathrm{d}_{\mathrm{p}}(\mu \mathrm{m})\end{array}$ & \multicolumn{3}{|c|}{116} & 130 & \multicolumn{3}{|c|}{150} & & & \\
\hline $\mathrm{C}_{\mathrm{pv}}\left(/ \mathrm{mm}^{3}\right)$ & \multicolumn{3}{|c|}{194} & 130 & \multicolumn{3}{|c|}{85} & & & \\
\hline $\mathrm{C}_{\mathrm{gv}} / \mathrm{C}_{\mathrm{pv}}$ & \multicolumn{3}{|c|}{1} & 1.49 & \multicolumn{3}{|c|}{2.28} & & & \\
\hline$\theta\left(^{\circ}\right)$ & & & & $61.5^{\circ}$ & & $1.5^{\circ}$ & & & & \\
\hline$V_{g}=50 \%, V$ & & $V_{p}=15$ & & & & & & & & \\
\hline Grain size & $64 \mu$ & & & & & & & & & \\
\hline pore size $d_{p}$ & & 44 & & 70 & 90 & & 110 & & 130 & 150 \\
\hline $\mathrm{C}_{\mathrm{pv}}\left(/ \mathrm{mm}^{3}\right)$ & & 3645 & & 836 & 393 & & 215 & & 130 & 85 \\
\hline $\mathrm{C}_{\mathrm{gv}} / \mathrm{C}_{\mathrm{pv}}$ & & 1 & & 4.36 & 9.2 & & 16.95 & & 28.04 & 42.88 \\
\hline$\theta\left(^{\circ}\right)$ & & 60.0 & & 61.0 & 61. & & 61.0 & & 61.0 & 61.5 \\
\hline
\end{tabular}

From this table we can see that the half vertex angle of the cone $\theta$ stays the same with fixed pore volume $V_{p}$. These two parameters have a relationship as follows.

Table 5.2 Relationship between pore volume $V_{p}$ and average half vertex angle of the cone $\theta$

\begin{tabular}{|l|l|l|l|}
\hline Pore volume $\mathrm{V}_{\mathrm{p}}$ & $15 \%$ & $20 \%$ & $60 \%$ \\
\hline Average half vertex angle of the cone $\theta$ & $61.1^{\circ}$ & $57.4^{\circ}$ & $42.9^{\circ}$ \\
\hline
\end{tabular}


By curve fitting the values in the table, the following relationship was identified.

$\theta=0.1 . V_{p}^{2}-1.1 V_{p}+75.9$ degrees for $60 \% \geq V_{p} \geq 15 \%$

From Table 5.1 it can be seen that the half vertex angle of the cone $\theta$ has no relationship with grain size $d_{g}$, or grain volume $V_{g}$, but the minimum pore size $d_{p}$ does depend on grain size $d_{g}$. Pores are separated by grains so two pores cannot be adjacent. The pore volume density should therefore be less than the grain volume density.

The next step is to estimate the bond force for a given grinding force. With reference to Fig. 5.7, 3, 4 and 5 grains above the active grain are considered. The force on a bond bridge can be calculated assuming the tangential grinding force on a grain is $1 \mathrm{~N}$, and the normal force is $2 \mathrm{~N}$ (a grinding force coefficient of 0.5 ).

The half-cone angle is defined as the half-apex angle of the cone. When the half-cone angle is between $30^{\circ}$ and $45^{\circ}$, there are 3 grains above the active grain. When the half-cone angle is between $45^{\circ}$ and $58^{\circ}$, there are 4 grains above the active grain. When the half-cone angle is greater than $58^{\circ}$, there are 5 grains above the active grain.

(i) $30^{\circ}-40^{\circ}$ half-cone angle. When the half-cone angle lies in the range $30^{\circ}$ $45^{\circ}$, there are 3 grains above the active grain as shown on Fig. 5.7(a). The grain normal force $\mathrm{F}_{\mathrm{gn}}$, and grain tangential force $\mathrm{F}_{\mathrm{gt}}$ are applied on the active grain. Also, there are three bond forces applied on the grain. Each bond force can be divided into forces in the directions $x, y, z$ as shown in Fig. 5.7(a).

Based on the balance of forces and moments along each of the $x, y, z$ axes, 6 equations result as follows. 
Direct forces in $\mathrm{x}, \mathrm{y}$ and $\mathrm{z}$ directions:

$$
\begin{aligned}
& F_{b 1 x}+F_{b 2 x}+F_{b 3 x}+F_{g t}=0 \\
& F_{b 1 y}+F_{b 2 y}+F_{b 3 y}=0 \\
& F_{b 1 z}+F_{b 2 z}+F_{b 3 z}+F_{g n}=0
\end{aligned}
$$

Moments about the $\mathrm{x}$ axis

$$
\begin{aligned}
& \left(F_{b 1 y}+F_{b 3 y}\right) \cdot r_{g} \cdot \sqrt{\left(\sin \alpha \cdot \sin 30^{\circ}\right)^{2}+\cos ^{2} \alpha}-\left(F_{b 1 z}+F_{b 3 z}\right) \cdot r_{g} \cdot \sin \alpha \cdot \cos 30^{\circ} \\
& +F_{b 2 y} \cdot r_{g} \cdot \cos \alpha=0
\end{aligned}
$$

Moments about the y axis

$\left(F_{b 1 x}+F_{b 2 x}+F_{b 3 x}\right) \cdot r_{g} \cdot \cos \alpha-\left(F_{b 1 z}+F_{b 3 z}\right) \cdot r_{g} \cdot \sin \alpha \cdot \sin 30^{\circ}+F_{b 2 z} \cdot r_{g} \cdot \sin \alpha$ $+F_{g t} \cdot r_{g}=0$

Moment about the $\mathrm{z}$ axis

$\left(F_{b 1 x}-F_{b 3 x}\right) \cdot r_{g} \cdot \sin \alpha \cdot \cos 30^{\circ}+\left(F_{b 1 y}+F_{b 3 y}\right) \cdot r_{g} \cdot \sin \alpha \cdot \sin 30^{\circ}$

$-F_{b 2} y \cdot r_{g} \cdot \sin \alpha=0$

Because of symmetry of the forces on Bond 1 and Bond 3, the bond forces can be found from,

$$
\begin{aligned}
& F_{b 1 x}=F_{b 3 x} \\
& F_{b 1 y}=-F_{b 3 y}, F_{b 2 y}=0 \\
& F_{b 2 z}=-\frac{F_{g t} \cdot(\cos \alpha+1)+F_{g n} \sin \alpha \cdot \sin 30^{\circ}}{\sin \alpha\left(1+\sin 30^{\circ}\right)} \\
& F_{b 1 z}=F_{b 3 z}=-\frac{F_{g n}+F_{b 2 z}}{2}
\end{aligned}
$$

In order to simplify the analysis, it was assumed that,

$$
\begin{aligned}
& F_{b 1 x}=F_{b 2 x}=F_{b 3 x}=-\frac{F_{g t}}{3} \\
& F_{b 1 y}=-F_{b 3 y}=0
\end{aligned}
$$


(ii) $45^{\circ}-58^{\circ}$ half-cone angle. If the half cone angle lies in the range $45^{\circ}-58^{\circ}$, there are 4 grains above the top grain as shown in Fig. 5.7(b). Grain normal force $\mathrm{F}_{\mathrm{gn}}$, and grain tangential force $\mathrm{F}_{\mathrm{gt}}$ are applied on the top grain. Also, there are four bond forces applied on the top grain. The bond forces in three directions $\mathrm{x}, \mathrm{y}, \mathrm{z}$ are as shown in Fig. 5.7(b).

Based on balance of forces and moments for each of the $x, y, z$ axes.

$$
\begin{aligned}
& F_{b 1 x}+F_{b 2 x}+F_{b 3 x}+F_{b 4 x}+F_{g t}=0 \\
& F_{b 1 y}+F_{b 2 y}+F_{b 3 y}+F_{b 4 y}=0 \\
& F_{b 1 z}+F_{b 2 z}+F_{b 3 z}+F_{b 4 z}+F_{g n}=0 \\
& \left(F_{b 1 y}+F_{b 2 y}+F_{b 3 y}+F_{b 4 y}\right) \cdot r_{g} \cdot \cos \alpha- \\
& \left(F_{b 2 z}+F_{b 3 z}-F_{b 1 z}-F_{b 4 y}\right) \cdot r_{g} \cdot \sin \alpha \cdot \sin 45^{\circ}=0 \\
& -\left(F_{b 1 x}+F_{b 2 x}+F_{b 3 x}+F_{b 4 x}\right) \cdot r_{g} \cdot \cos \alpha+ \\
& \left(F_{b 1 z}+F_{b 2 z}-F_{b 3 z}-F_{b 4 z}\right) \cdot r_{g} \cdot \sin \alpha \cdot \cos 45^{\circ}+F_{g t} \cdot r_{g}=0 \\
& \left(F_{b 1 x}+F_{b 4 x}-F_{b 2 x}-F_{b 3 x}\right) \cdot r_{g} \cdot \sin \alpha \cdot \sin 45^{\circ}+ \\
& \left(-F_{b 1 y}-F_{b 2 y}+F_{b 3 y}+F_{b 1 y}\right) \cdot r_{g} \cdot \sin \alpha \cdot \cos 45^{\circ}=0
\end{aligned}
$$

Because of symmetry of the forces on Bond 1 and Bond 2, Bond 3 and Bond 4 , the bond force can be calculated as follows,

$$
\begin{aligned}
& F_{b 1 x}=F_{b 2 x}, F_{b 3 x}=F_{b 4 x}, F_{b 2 x}+F_{b 3 x}=-\frac{F_{g^{\prime}}}{2} \\
& F_{b 1 y}=-F_{b 2 y}, F_{b 3 y}=-F_{b 4 y} \\
& F_{b 1 z}=F_{b 2 z}=\frac{1}{2}\left(\frac{-F_{g t} \cdot(\cos \alpha+1)}{\left.2 \sin \alpha \cos 45^{\circ}\right)}-\frac{F_{g n}}{2}\right. \\
& F_{b 3 z}=F_{b 4 z}=-\frac{F_{g n}}{2}-F_{b 2 z}
\end{aligned}
$$

In order to simplify the analysis, it is assumed that, 
$F_{b 1 x}=F_{b 2 x}=F_{b 3 x}=F_{b 4 x}=-\frac{F_{g t}}{4}$

$F_{b 1 y}=F_{b 2 y}=F_{b 3 y}=F_{b 4 y}=0$

(iii) Greater than $58^{\circ}$ half-cone angle. If the half-cone angle is larger than $58^{\circ}$, there are 5 grains above the top grain as shown in Fig. 5.7(c). Grain normal force $F_{g n}$, and grain tangential force $F_{g t}$ are applied on the top grain. Also, there are four bond forces applied on the top grain. The bond forces in three directions $x, y, z$ are as shown in Fig. 5.7(c).

Based on balance of forces and moments for each of the $x, y, z$ axes.

$$
\begin{aligned}
& F_{b 1 x}+F_{b 2 x}+F_{b 3 x}+F_{b 4 x}+F_{b 5 x}+F_{g t}=0 \\
& F_{b 1 y}+F_{b 2 y}+F_{b 3 y}+F_{b 4 y}+F_{b 5 y}=0 \\
& F_{b 1 z}+F_{b 2 z}+F_{b 3 z}+F_{b 4 z}+F_{b 5 z}+F_{g n}=0
\end{aligned}
$$

$-\left(F_{b 1 y}+F_{b 2 y}+F_{b 3 y}+F_{b 4 y}+F_{b 5 y}\right) \cdot r_{g} \cdot \cos \alpha+\left(F_{b 2 z}-F_{b 5 z}\right) \cdot r_{g} \cdot \sin \alpha \cdot \sin 72^{\circ}$ $+\left(F_{b 3 z}-F_{b 4 z}\right) \cdot r_{g} \cdot \sin \alpha \cdot \sin 36^{\circ}=0$

$\left(F_{b 1 x}+F_{b 2 x}+F_{b 3 x}+F_{b 4 x}+F_{b 5 x}\right) \cdot r_{g} \cdot \cos \alpha-F_{b 1 z} \sin \alpha \cdot \sin 36^{\circ}$ $-\left(F_{b 2 z}+F_{b 5 z}\right) \cdot r_{g} \cdot \sin \alpha \cdot \cos 72^{\circ}+\left(F_{b 3 z}+F_{b 4 z}\right) \cdot r_{g} \cdot \sin \alpha \cdot \cos 36^{\circ}-F_{g t} \cdot r_{g}=0$

$\left(-F_{b 2 x}+F_{b 5 x}\right) \cdot r_{g} \cdot \sin \alpha \cdot \sin 72^{\circ}+\left(-F_{b 3 x}+F_{b 4 x}\right) \cdot r_{g} \cdot \sin \alpha \cdot \sin 36^{\circ}+$ $F_{b 1} \cdot r_{g} \cdot \sin \alpha \cdot+\left(F_{b 2 y}+F_{b 5 y}\right) \cdot r_{g} \cdot \sin \alpha \cdot \cos 72^{\circ}$ $-\left(F_{b 3} y+F_{b 4}\right) \cdot r_{g} \cdot \sin \alpha \cdot \cos 36^{\circ}=0$

Because of symmetry of the forces on Bond 2 and Bond 5, Bond 3 and Bond 4 , the bond force can be calculated as follows,

$$
\begin{aligned}
& F_{b 2 z}=F_{b 3 z}=F_{b 4 z}=F_{b 5 z}=\frac{1}{2}\left(\frac{-F_{g t} \cdot(\cos \alpha+1)-F_{g n} \cdot \sin \alpha}{\sin \alpha \cos 72^{\circ}-2 \cdot \sin \alpha-\sin \alpha \cos 36^{\circ}}\right) \\
& F_{b 1 z}=-F_{g n}-4 . F_{b 2 z}
\end{aligned}
$$


In order to simplify the analysis, it is assumed that,

$$
\begin{aligned}
& F_{b 1 x}=F_{b 2 x}=F_{b 3 x}=F_{b 4 x}=F_{b 5 x}=-\frac{F_{g t}}{5} \\
& F_{b 1 y}=F_{b 2 y}=F_{b 3 y}=F_{b 4 y}=F_{b 5 y}=0
\end{aligned}
$$

Fig. 5.8 shows the reduction of bond force $F_{b}$ in 3,4 or 5 grains on the top grain with the increasing half cone angle obtained from the above equations. The larger bond force in these three cases can be curve-fitted to yield the following equation.

$$
F_{b}=0.4 \times 10^{-2} \cdot \theta^{2}-0.8 \times 10^{-1} \theta+5.1(N)
$$

Combining Equations 5.57 and 5.22, the following single empirical equation is obtained

$$
F_{b}=0.2 . \times 10^{-1} V_{p}+1.5(N)
$$

Both $\mathrm{H}_{\mathrm{sb}}$ and $\mathrm{H}_{\mathrm{sb}}$ ' express the bond hardness. $\mathrm{H}_{\mathrm{sb}}$ ' is based on assumptions concerning a theoretical wheel structure, while, $\mathrm{H}_{\mathrm{sb}}$ is a definition based on experiments. They may be related as follows,

$$
H_{s b}=k \cdot H_{s b}{ }^{\prime}
$$

where, $\mathrm{k}$ is a coefficient.

From Equation 5.20,

$$
H_{s s}=\frac{1}{\frac{1}{H_{s b}}+\frac{1}{H_{s a}}}=\frac{1}{\frac{1}{k \cdot H_{s b^{\prime}}}+\frac{1}{H_{s a}}}
$$

Combining Equations 5.58 and 5.20,

$$
\begin{aligned}
& H_{s b^{\prime}}=\frac{V_{g b}}{m \cdot\left(0.2 \cdot \times 10^{-1} V_{p}+1.5\right)} \\
& =\frac{V_{b}}{m \cdot C_{g v} \cdot\left(0.2 \cdot \times 10^{-1} \cdot V_{p}+1.5\right)} \\
& =V_{b} \cdot \frac{4}{3} \pi\left(\frac{d_{g}}{2}\right)^{3} \cdot \frac{1}{V_{g} \cdot m \cdot\left(0.2 \cdot \times 10^{-1} \cdot V_{p}+1.5\right)}
\end{aligned}
$$


Based on the experimental results presented in Table $3.2, \mathrm{H}_{\text {as }}$ and $\mathrm{k}$ have the following values,

$$
\begin{aligned}
& H_{s a}=85.3(N) \\
& k=1.7 \times 10^{6}\left(\mathrm{~N}^{2} / \mathrm{mm}^{3}\right)
\end{aligned}
$$

From which,

$$
H_{s S}=\frac{1}{\frac{1}{85.3}+\frac{1}{H_{s b}}}
$$

where,

$$
H_{s b}=1.7 \times 10^{6} \cdot V_{b} \cdot \frac{4}{3} \pi\left(\frac{d_{g}}{2}\right)^{3} \cdot \frac{1}{V_{g} \cdot m \cdot\left(0.2 \times 10^{-1} \cdot V_{p}+1.5\right)}
$$

This expression for vitrified CBN wheel scratch hardness is general for all wheels.

\subsection{Prediction of grinding wheel wear}

As discussed in Section 4.2, grinding force, specific energy, workpiece surface roughness, wheel wear and wheel loading are directly affected by wheel structure. In order to grind workpieces accurately and efficiently, it is necessary to determine an appropriate wheel structure. The selection of an optimum wheel structure depends on the workpiece accuracy requirements and acceptable energy dissipation. In this section, prediction of wheel wear, loading and workpiece accuracy are made for different wheel structures. The simulation of grinding behaviour provides a basis for further investigation of the wheel structure. The simulation is based on the mechanics of wheel wear and wheel loading as reviewed in the chapter on previous work and on the theoretical assumptions outlined in the previous sections.

As discussed previously, there are three main mechanisms of wheel wear: attrition, grain fracture and bond fracture. The prediction of wheel wear includes the three mechanisms. 
In this prediction, it was assumed that, there are ' $n$ ' active cutting edges on each active grain depending on the dressing process. The value of $n$ used in the prediction was 1.2 , that is 2 cutting edges on every fifth grain based on the measurements described in Chapter 3. The overall shape of a grain was assumed to be a sphere, while the cutting edge was assumed to be a cone as described in Chapter 3.

The attritious wear volume of the cutting edge was assumed to follow a relationship of the form [101],

$$
V=\frac{K \cdot f_{c n} \cdot S}{H_{S}}=\frac{K \cdot \bar{p} \cdot A_{\mathcal{W}} \cdot S}{H_{S}}
$$

where, $\mathrm{V}$ is the wear volume under attritious wear conditions

$\mathrm{K}$ is the attritious wear rate of the cutting edge

$\mathrm{S}$ is the sliding distance for contact with the cutting edge

$\mathrm{H}_{\mathrm{s}}$ is the indentation hardness of abrasive

$f_{c n}$ is the normal load on one cutting edge.

$\bar{p}$ is the average contact pressure between wear flat and workpiece

$A_{w}$ is the wear flat area on one cutting edge

The probability of grain fracture wear and of bond fracture wear when contact occurs between active grains and the workpiece is dependent on the wheel scratch hardness. According to the definition of wheel scratch hardness given by Equation 3.5 ,

$$
d_{b a}=\frac{F_{g s t}}{H_{s s}}
$$

where $d_{b a}$ is the mean diameter of the grain particles removed. $d_{b a}$ takes into account the combined occurrence of bond fractures and grain fractures for a particular grain tangential force when the wheel scratch hardness $\mathrm{H}_{\mathbf{s s}}$ has previously been determined or is given. It can be seen from this equation that there is a greater proportion of bond fractures when $\mathrm{d}_{\mathrm{ba}}$ is larger and a greater proportion of grain fractures when $d_{b a}$ is smaller. It was assumed to be a measure of the probability of bond and grain fracture and was used in the wheel wear prediction. 
Values of $H_{s s}$ were obtained from the experiments described in Chapter 3. The hardness may be related to a situation where a force $F_{g s t}$ is applied towards the centre of a grain, while the tangential force $f_{c t}$, is the tangential force applied at the cutting edge of the grain as shown in Fig.5.9. Taking moments about the cutting edge of the grain,

$$
F_{g s t}=\frac{1.2 \times 2 . d_{x}}{d_{g}} \cdot f_{C t}=c 1 \cdot f_{\mathcal{C} t}
$$

where, $\mathrm{c} 1$ is a coefficient.

$d_{x}$ is perpadicular distance to the force $f_{c t}$.

When grains are in contact with the workpiece, bond and grain fractures are a stochastic process. It was assumed that the probability of grain fracture wear and of bond fracture wear when contact occurs between active grains and the workpiece $\mathrm{P}_{\mathrm{f}}$ is,

$P_{f}=A \cdot \tau^{c 2 \cdot \frac{1}{d b a}}=A \cdot \tau^{c 2 \cdot \frac{H_{S S}}{c 1 \cdot f_{c t}}}=A \cdot \tau^{c \cdot \frac{H}{S S}_{f_{c t}}} \quad(\tau<1 \mathrm{~s})$

where, $\mathrm{A}, \mathrm{c1}, \mathrm{c} 2$, and $\mathrm{c}$ are coefficients and

$\tau$ is the contact time between cutting the edge and the workpiece.

As discussed in Chapter 3, some inactive cutting edges become active during the course of grinding as preceding edges become dislodged due to bond fracture. Also, grain fracture produces new cutting edges. It was assumed that the probability of new active cutting edges appearing after each grain and bond fracture is $\mathrm{Pr}$. The value of $\mathrm{Pr}$ was calibrated from the experimental results presented in Chapter 7 .

Prediction of wheel wear was carried out to corroborate the relationships between wheel structure determined by grinding experiments. The prediction concentrated on the behaviour of the active cutting edge density per unit area in grinding. The active cutting edge density and grinding force both change with wheel wear in grinding after each revolution of the grinding wheel corresponding to each grain contact with the workpiece. The active cutting edge density can be estimated according to the probability of grain fracture 
wear and bond fracture wear and the probability of new active cutting edges appearing by grain and bond fracture after each revolution of the wheel. According to the relationship between workpiece surface roughness and cutting edge spacing $\mathrm{L}$ [4], the workpiece surface roughness $\mathrm{Ra}$ was obtained by assuming,

$$
\begin{aligned}
& R a=a 1 .\left(\frac{v_{w} \cdot L}{v_{s} \cdot d_{s}^{1 / 2}}\right)^{0.8}+a 2 \\
& \text { where, } L=\sqrt{\frac{1}{C_{a}}}
\end{aligned}
$$

and a1 and a2 are empirical constants.

From the specifications of the grinding wheel, workpiece, dressing conditions and grinding conditions, the prediction was designed to give values of active cutting edge density, cutting edge wear flat radius and workpiece surface roughness. The basic steps in the prediction are shown in Fig. 5.10.

For a specified wheel structure, the wheel scratch hardness $H_{s s}$ could be determined using Equation 5.62. Initial active cutting edge density $C_{a 0}$ and mean cutting edge radius $r^{\prime}{ }_{a} 0$ were determined experimentally based on wheel structure and dressing conditions.

At the beginning of one grinding wheel revolution, which includes one contact of a particular cutting edge and workpiece, the mean wear flat area of a cutting edge, $A^{\prime}{ }_{a 0}$ is

$$
A_{a 0}^{\prime}=\pi \cdot r^{\prime} a 0^{2}
$$

Only attritious wear is considered at first. After a grinding time $t$, the volume of attritious wear $V_{a t}$ from a cutting edge based on the geometry of a cone as shown in Fig 5.11 is

$$
V_{a t}=\frac{1}{3} \pi \cdot \operatorname{ctg} \gamma \cdot\left(r^{\prime} a^{3}-r^{\prime} a 0^{3}\right)
$$

where, $r^{\prime}{ }_{a}$ is the mean cutting edge radius after time $t$ when only attritious wear is considered. 
The cutting edge sliding distance is

$S=n_{s} \cdot t \cdot l_{c}$

where, $n_{s}$ is the rotary speed of the wheel.

Combining Equation 5.64, the mean cutting edge wear flat radius based on the geometry of a cone is given by

$r_{a}=\left(a t+r_{a 0}^{\prime}\right)^{1 / 3}$

The wear flat area is

$$
\begin{aligned}
& A^{\prime}{ }_{a}=\pi\left(a t+r^{\prime}{ }_{a 0}^{3}\right)^{2 / 3} \\
& \text { where, } a=\frac{K \cdot F_{c n} \cdot n_{s} \cdot \sqrt{a_{e} \cdot d_{e}}}{H_{s} \cdot \frac{1}{3} \pi \cdot \operatorname{ctg} \gamma}
\end{aligned}
$$

$A^{\prime}{ }_{a}$ is the mean wear flat area on the cutting edge.

Then, grain fracture and bond fracture were considered according to Equation 5.67. So, the cutting edge density was calculated after $n$ wheel revolutions, by taking into account the probability of grain fracture and of bond fracture and the probability of new cutting edges appearing,

$$
C_{a n}=C_{a 0} .\left(1-P_{f 1}+P_{r 1}\right) \cdot\left(1-P_{f 2}+P_{r 2}\right) \ldots\left(1-P_{f n}+P_{r n}\right)
$$

where, the suffix 1 to $n$ is the number of revolutions a particular cutting edge passes through the contact length.

Then, the wear flat area per unit surface area after $n$ revolutions $A_{a n}$ is,

$$
\begin{aligned}
& A_{a n}=\left(C_{a 0}-C_{f 1}-C_{f 2}-\ldots-C_{f n}\right) \cdot\left(\pi \cdot r_{a n}^{\prime}{ }^{2}\right) \\
& +C_{r 1} \cdot\left(\pi \cdot r_{a(n-1)}{ }^{2}\right)+C_{r 2} \cdot\left(\pi \cdot r_{a(n-2)^{\prime}}^{2}\right)+\ldots+C_{r n} \cdot\left(\pi \cdot r_{a 0}^{\prime}\right)
\end{aligned}
$$

where, $\mathrm{C}_{\boldsymbol{f}}$ is the cutting edge density taking account of cutting edge fracture and dislodgement. $C_{r}$ is the new value of cutting edge density. The suffix 1 to $\mathrm{n}$ is the number of times the cutting edges pass through the contact length. 
Then, the mean cutting edge radius $r_{a n}$ considering all three kinds of wheel wear after $\mathrm{n}$ wheel revolutions was,

$$
r_{a n}=\sqrt{\frac{A_{a n}}{\pi C_{a n}}}
$$

This radius was used as the initial mean cutting edge radius for the next wheel revolution.

So, wheel wear flat area and cutting edge density which change after each wheel revolution lead to the change in grinding forces $F_{\mathrm{cn}}$ and $\mathrm{F}_{\mathrm{ct}}$ according to Section 4.2. If workpiece surface roughness is less than the specified requirement, another cycle begins until workpiece surface roughness exceeds the specification.

The cutting edge density and cutting edge radius can be determined for each wheel revolution. The workpiece surface roughness can be determined based on Equation 5.68.

\subsection{Prediction of grinding wheel loading}

Two forms of wheel loading were observed in experiments as discussed further in the next chapter. One form of loading is where workpiece material adheres to the cutting surface of the grinding wheel grains. This case will be referred to as 'adhesive loading'. Another form of loading is where the chips block the pores on the surface of the wheel and is termed 'pore loading'.

Adhesive loading on the top and side surfaces of the cutting edges follows Archards' adhesive wear law, reviewed previously in Chapter 4. While pore loading was found to occur at the same time as wheel adhesive loading and based on experimental observation, it appears the two forms of loading affect each other. This aspect is described further in the next chapter.

The relationship between wheel adhesive loading and pore loading can be explained theoretically as follows. With an increase of wheel wear flats in grinding, the workpiece background temperature increases, and the workpiece material softens. This promotes wheel adhesive loading based on Archards' 
adhesive wear law. Also, workpiece ductility increases and the probability is increased that long filament chips will be formed rather than short fracture chips. It is also proposed that long chips are more likely to become lodged in the pores rather than be washed out by coolant as is more likely with short chips. Long filament chips were found to enter the pores causing pore loading. This was confirmed from examination of the wheels used in grinding experiments. When pore loading occurs with long filament chips, there is normally more severe surface adhesive loading on the grain as well. Conversely, pore loading is also likely to increase the probability of adhesive loading between grains causing a large adhesive block. So, pore loading is expected to have a proportional relationship with adhesive loading and both effects will interact with each other.

In this prediction, wheel loading including both adhesive loading and pore loading take place together with wheel wear at the same time. Adhesive loading on the top and side surfaces of the cutting edges follows Archards' adhesive wear law, discussed previously in this chapter.

$$
V_{a d}=\frac{c \cdot f_{c n} \cdot S}{H w}=\frac{c \cdot \bar{p} \cdot A_{w} \cdot S}{H w}
$$

where,

$V_{a d}$ is the adhesive wear volume from the workpiece under a cutting edge

$c$ is a dimensionless constant dependent on the materials in contact and their exact degree of cleanliness

$f_{c n}$ is the normal load on the workpiece under a cutting edge

$H_{w}$ is the surface hardness of the workpiece

$S$ is the sliding distance

The coefficient $k_{a}$, reflects the volume ratio of particles adhered to the cutting edges $\mathrm{V}^{\prime}$ ad to the total number of adhesive wear particles $\mathrm{V}_{\text {ad. }}$.

$$
V^{\prime}{ }_{a d}=k_{a} \frac{c \cdot \bar{p} \cdot A_{w} \cdot S}{H w}
$$


Adhesive loading volume can be represented by a coefficient $\mathrm{B}_{\mathrm{a}}$, which reflects the degree of adhesive loading.

$$
B_{a}=k \cdot V^{\prime} a d
$$

The probability of pore loading between two cutting edges is assumed to follow a relationship of the form,

$$
P_{b}=\frac{B_{b} \cdot B_{a}}{V_{p} \cdot L}
$$

where,

$$
\begin{aligned}
& B_{b} \text { is a coefficient; } \\
& B_{a} \text { is a coefficient for adhesive loading and } \\
& L \text { is the cutting edge spacing }
\end{aligned}
$$

Based on the previous discussion, the following assumptions were made,

(i) For a particular grinding condition, workpiece surface hardness remains stable.

(ii) Adhesive loading takes place over $100 \%$ on the wear flat area. This is considered reasonable based on obervations where adhesive loading has taken place.

The prediction process is similar to the grinding wheel wear simulation. Fig. 5.12 shows the steps. From the specifications of the grinding wheel, workpiece, dressing conditions and grinding conditions, predictions were made of active cutting edge density, cutting edge radius, grinding wheel loading and workpiece surface roughness.

After one grinding wheel revolution, which includes one contact of a particular cutting edge and workpiece, wheel attritious wear may be calculated from Equations 5.69-5.74. The wheel adhesive loading was calculated using Equation 5.79 in which $\mathrm{ka}$ was determined as described in Chapter 7. The probability of pore loading between two cutting edges was determined using Equation 5.81. 
So, the cutting edge density was calculated after $\mathrm{n}$ wheel revolutions, taking account of the probability of grain fracture and of bond fracture, probability of new cutting edges appearing, wheel pore loading which has relationships with wheel adhesive loading is

$$
C_{a n}=C_{a 0} \cdot\left(1-P_{f 1}+P_{r 1}-P_{b 1}\right) \cdot\left(1-P_{f 2}+P_{r 2}-P_{b 2}\right) \ldots\left(1-P_{f n}+P_{r n}-P_{b n}\right)
$$

where, the suffix 1 to $\mathrm{n}$ is the number of times the cutting edges pass through the contact length.

Then, the wear flat area per unit surface area after $\mathrm{n}$ revolutions and the mean cutting edge radius follow Equations 5.76 and 5.77.

The wheel wear flat area and cutting edge density is changed after each wheel revolution leading to the changed grinding forces $F_{c n}$ and $F_{c t}$ according to Section 4.2. The changed grinding force affects wheel wear and loading inversely. If workpiece surface roughness is less than the specified values, another cycle begins until workpiece surface roughness exceeds the specification.

The cutting edge density, cutting edge radius, wheel adhesive loading coefficient, pore loading probability and workpiece surface roughness including the effects of all three mechanisms were determined after succeeding wheel revolutions.

In summary, relationships have been presented for the effects of wheel structure on the nature and distribution of the cutting edges. Models have been presented which allow the wheel-wear and wheel-loading processes to be predicted based on coefficients derived from grinding experiments. Predictions from these models will be correlated with the variations in grinding performance measured in the experimental investigation. 


\section{Chapter 6 Experimental investigation of vitrified CBN wheel performance}

From the review of previous work and the theoretical analysis, it was proposed that a more porous grinding wheel would allow cooler grinding, reduce the tendency for a grinding wheel to load and maintain a sharper grinding wheel surface but with lower wheel scratch hardness. Since the objective was to investigate the achievement of low surface roughness with long wheel redress life, it was decided to perform grinding experiments with grinding wheels having two basically different types of structure and porosity. The performance indicators used to assess the performance of the process were:

- Specific energy

- Surface roughness, $\mathrm{Ra}$ and $\mathrm{Rz}$

- Roundness

- Size holding

- G-Ratio

The upper limit for surface roughness level for internal grinding was set at 0.25 $\mu \mathrm{m} \mathrm{Ra}$ and $2 \mu \mathrm{m} \mathrm{Rz}$ for external grinding.

The main purpose of internal grinding trials was to investigate changes in wheel topography with wear. The purpose of external grinding trials was to further validate research findings over an extended range of grinding parameters using the capability of a specially-designed high-speed CBN grinding machine. In the internal grinding trials, replicas were employed to monitor wheel topography and were taken frequently during the redress cycle while in external grinding trials, replicas were taken only at the beginning and end of each trial. This was because of the requirement to monitor size holding without disturbing the thermal equilibrium of the machine. In external grinding, the Students t-test was employed to test for statistical significance between different sets of results.

Experiments were performed to compare the performance of a high-porosity grinding wheel with a medium-porosity grinding wheel. Experiments were 
performed with easy-to-grind materials and with a difficult-to-grind material to determine whether the conclusions reached might be valid for both situations. Grinding wheel speed was also varied because surface roughness should be reduced at high speed according to the kinematic principles discussed in Chapter 4.

\subsection{The experimental system}

\subsubsection{The grinding machines}

The experimental system was designed to investigate the relationships between the changing features of grinding wheel topography and grinding performance. Internal grinding experiments were undertaken on a Jones \& Shipman $1300 \mathrm{X}$ universal grinding machine with a PC $2000 \mathrm{CNC}$ interface as shown in Fig. 6.1. The minimum programmable increment of the wheelhead was $0.001 \mathrm{~mm}$. The maximum wheel rotary speed was $75,000 \mathrm{rev} / \mathrm{min}$. The maximum output power was $6 \mathrm{~kW}$. The workpiece was held within a collet, which was then held in a three-jaw chuck as shown in Fig. 6.2. This arrangement distributed the pressure evenly around the workpiece and the deformation of the workpiece caused by the three-jaw chuck was reduced. This machine was employed because with small grinding wheels it was relatively straightforward to obtain different CBN grinding wheels engineered for the research.

Later parametric experiments were performed on the Suprema external cylindrical grinding machine. The external grinding machine was tested as part of a collaborative project to include an investigation of machine aspects in the optimisation of precision CBN grinding. The results obtained in external grinding were obtained jointly with the input of the engineer responsible for investigating that machine. The Suprema research machine employed for this purpose was a unique prototype developed by Jones and Shipman especially for precision highspeed CBN grinding research. The Jones \& Shipman Suprema machine shown in Fig. 6.3 was designed to grind small cylindrical parts such as diesel injector nozzles using relatively small grinding wheels. The wheelhead incorporated hydrostatic bearings capable of delivering wheelspeeds up to $150 \mathrm{~m} / \mathrm{s}$. The wheel mounting was designed to allow the interchange of grinding wheels with 
minimum wheel dressing. The dressing unit was a high-speed unit capable of $15000 \mathrm{rev} / \mathrm{min}$, which enabled a wide range of speed ratios to be programmed. The workpiece centre mounted in the workhead rotates with the drive plate. A dead centre was employed in the tailstock. Five diameters were ground consecutively starting at a diameter nearest the workhead and working towards the final diameter nearest the tailstock. The grinding cycle used was a plunge cycle.

\subsubsection{The grinding wheels}

(i) Internal grinding wheels. Wheels of different structures and porosity were tested. The width of the wheels was $25 \mathrm{~mm}$. The diameter of the wheels in the two main series of experiments varied from $16 \pm 0.001 \mathrm{~mm}$ to $13 \pm 0.001 \mathrm{~mm}$ for lower peripheral wheelspeeds and from $31 \pm 0.001 \mathrm{~mm}$ to $27 \pm 0.001 \mathrm{~mm}$ for higher wheelspeeds. These ranges resulted due to wheel wear as the trials proceeded. Fig. 6.4 shows these two series of wheels.

The specifications of the wheels used for the easy-to-grind materials are listed below:

Series 1: Wheel diameters, $16 \pm 0.001 \mathrm{~mm}$ to $13 \pm 0.001 \mathrm{~mm}$

$\begin{array}{lll}\text { MP-B91: } & \text { Medium-porosity wheel } & : B 91-150 \mathrm{~V} 1 \\ & \text { Wheel scratch hardness }: \mathrm{H}_{\mathrm{ss}}: 42.0 \mathrm{~N} \\ \text { HP-B64: } & \text { High-porosity wheel } & : \mathrm{B} 64-150 \mathrm{VX} \\ & \text { Wheel scratch hardness }: \mathrm{H}_{\mathrm{ss}} & : 12.9 \mathrm{~N}\end{array}$

Series 2: Wheel diameters, $31 \pm 0.001 \mathrm{~mm}$ to $27 \pm 0.001 \mathrm{~mm}$

HP-B64: High-porosity wheel : B64-150 VR

Wheel scratch hardness : $H_{s s}: 15.3 \mathrm{~N}$

The high-porosity small-grain B64 CBN wheels had large pores and were referenced by the notation HP-B64. The performance of the high-porosity wheel was compared with the performance of a medium-porosity B91 CBN wheel with larger grains and smaller pores, referenced by the notation MP-B91. A smaller 
grain size was selected for the high-porosity wheel to assist the achievement of low surface roughness while cutting with sharp cutting edges. Fig. 6.5 shows SEM photographs comparing the HP-B64 wheel with the MP-B91 wheel. The average volume of a pore in the HP-B64 wheel was about 4 times larger than in the MP-B91 wheel. The effective porosity volume ratio on the HP-B64 wheel surface was 0.45 , and on the MP-B91 wheel was 0.35 .

Further observations indicated that greater porosity with finer grains caused the grains to agglomerate together, resulting in fewer active cutting edges and larger cutting edge spacing as mentioned in Chapter 5 . The number of active cutting edges measured after dressing for the HP-B64 wheel was found to be only two third that for the MP-B91 wheel.

The specifications of the wheels for grinding the difficult-to-grind material Inconel 718 are listed below. Both the medium-porosity MP-B91 wheel, and the high-porosity HP-B91wheel were manufactured with the same grain size. The only difference was the porosity. The wheel diameters ranged from $16 \pm 0.001$ $\mathrm{mm}$ to $13 \pm 0.001 \mathrm{~mm}$.

$\begin{array}{cl}\text { MP-B91: Medium-porosity wheel } & : \text { B91-150V1 } \\ \text { Wheel scratch hardness: } \mathrm{H}_{s s} & : 42.0 \mathrm{~N} \\ \text { HP-B91: High-porosity wheel } & : B 91-150 \mathrm{VR} \\ \text { Wheel scratch hardness: } \mathrm{H}_{\mathrm{ss}} & : 36.3 \mathrm{~N}\end{array}$

Fig. 6.5 also shows SEM photographs comparing the high-porosity HP-B91 wheel with the medium-porosity MP-B91 wheel. The average volume of a pore was about 3 times larger in the high-porosity HP-B91 wheel than in the mediumporosity MP-B91 wheel. The porosity volume ratio on the surface of the highporosity HP-B91 wheel was close to 0.4 compared with 0.35 on the mediumporosity MP-B91 wheel. 
(ii) External grinding wheels

Two vitrified CBN wheels were tested on the Suprema machine. One wheel was for AISI 52100 and another was for Inconel 718. The width of the wheels was 15 $\mathrm{mm}$. The diameter of the wheels was $247 \pm 0.1 \mathrm{~mm}$.

The specifications of the wheels are listed below:

MP-B91(E) for AISI 52100: Medium-porosity wheel $\quad$ : B91-150VR

Wheel scratch hardness $H_{s s}: 39.3 \mathrm{~N}$

HP-B151(E) for Inconel 718: High-porosity wheel $\quad$ : B151-150VR

Wheel scratch hardness $\mathrm{H}_{\mathrm{ss}}: 55.0 \mathrm{~N}$

Fig. 6.6 shows the B91-150VR wheel and the B151-150VR wheel were the same size. The effective porosity volume ratio on the surface of the high-porosity HPB151 wheel was measured and found to be 0.50 and for the medium-porosity MPB91 wheel was 0.35 . The grain size of the high-porosity wheel used for external grinding of Inconel 718, was increased compared with the high-porosity wheel for internal grinding in order to maintain surface roughness within the target range of $2 \mu \mathrm{m}$ Rz.

\subsubsection{The workpiece materials}

\section{(i) Materials for internal grinding.}

An M2 tool steel with hardness of HRC 60-62 was selected as an easy-to-grind material. Inconel 718 with hardness of HRC $42-43$ was selected as a difficult-togrind material. The compositions are listed in Table 6.1. The thermal properties of the materials are listed in Table 6.2.

The diameter of the M2 workpieces varied between $53 \pm 0.001 \mathrm{~mm}$ and $49 \pm 0.001 \mathrm{~mm}$, and the Inconel 718 workpieces varied between $40 \pm 0.001 \mathrm{~mm}$ and $38 \pm 0.001 \mathrm{~mm}$ depending on the number of times the specimens were ground. The width of the workpieces was $20 \mathrm{~mm}$. 
(ii) Materials for external grinding.

Two materials were tested in external grinding. One was a bearing steel AISI 52100 with hardness of HRC 57-58. The other material was Inconel 718. Fig.6.7 shows the workpiece dimensions. Five diameters were ground on each workpiece.

Table.6.1 Composition of the workpiece materials

Material Composition

M2 $\quad 5 \%$ Mo $\quad 6 \% \mathrm{~W} \quad 2 \% \mathrm{~V}$

Inconel $718 \quad 54 \% \mathrm{Ni} \quad 19 \% \mathrm{Cr} \quad 5 \% \mathrm{Nb} \quad 3 \% \mathrm{Mo}$

AISI $52100 \quad 1 \% \mathrm{C} \quad 1 \% \mathrm{Cr}$

Table.6.2 Mechanical and thermal properties of the workpiece materials

$\begin{array}{llllll}\text { Material } & \mathrm{Hv} & \mathrm{E} & \kappa & \rho & \mathrm{c} \\ & \mathrm{kg} / \mathrm{mm}^{2} & \mathrm{GPa} & \mathrm{W} / \mathrm{mK} & \mathrm{kg} / \mathrm{mm}^{3} & \mathrm{~J} / \mathrm{kgK} \\ \text { M2 } & 810 & 270 & 23.5 & 7860 & 515 \\ \text { Inconel 718 } & 420 & 208 & 11.4 & 8220 & 424 \\ \text { AISI 52100 } & 650 & 221 & 34.3 & 7815 & 506\end{array}$

\subsubsection{The coolant delivery system}

(i) Coolant for internal grinding

The coolant used for dressing and grinding was an emulsion, HYSOL-X. HYSOL-X is a semi-synthetic cutting and grinding fluid that was employed with a dilution ratio of 10:1 in water. In order to reduce wheel loading, the pressure of the coolant delivery system was increased to 4 bar. The coolant delivery is illustrated schematically in Fig.6.8. The system included a 4 bar low volume coolant supply for wheel cleaning and a lower pressure high volume coolant delivery for lubricating and cooling the grinding process. By using this arrangement for coolant delivery, the stability of the grinding wheel performance was improved [116]. 


\section{(ii) Coolant for external grinding}

The coolant used for external grinding was also HYSOL-X with a dilution ratio of 10:1 in water. A flat pipe nozzle was employed. The pump pressure for coolant delivery was 30 bar with a flowrate of $33 \mathrm{l} / \mathrm{min}$ for Inconel 718 and $36 \mathrm{l} / \mathrm{min}$ for AISI 52100, which keeps the same as on another external machine for the same workpiece materials.

\subsubsection{The dressing conditions}

\section{(i) Internal grinding}

A rotary cup diamond-dressing tool was used for touch dressing with a $50 \mathrm{~mm}$ diameter rotary cup. Fig. 6.9 shows the dressing system for internal grinding. The diamond rim used was either $3 \mathrm{~mm}$ or $1 \mathrm{~mm}$ wide. The two different sizes were used in order to confirm that the dressing condition was optimised for the highporosity wheels. No obvious difference between the wheel topography achieved was found after dressing with these two different widths. Further grinding trials confirmed that 10 passes should be taken across the wheel at an increment of 1 micron to achieve a stable topography. The dressing lead was optimised based on previous research depending on the grain size. The value of dressing lead selected for the tests was slightly smaller than the grain size based on the previous work by Chen [114].

\section{(ii) External grinding}

A diamond disc-dressing tool was used for touch dressing with a $150 \mathrm{~mm}$ diameter and a width of $2 \mathrm{~mm}$, as shown in Fig. 6.10. Values of the dressing parameters and changes made during experiments are listed in Section 6.2.

\subsubsection{Power measurement}

The procedure for making power measurements was the same for internal grinding and for external grinding. The spindle power level was monitored by fitting a power transducer (shunt) in the power supply lines to the grinding wheel motor as illustrated schematically in Fig. 6.11. The shunt translated voltage into current, and power was calculated by multiplying voltage and current. A power analyser Norma D4355 was used to measure the power value. Grinding power 
was assumed to be the difference between grinding wheel spindle power when grinding and when running under the no-load condition but with the coolant switched on and the grinding wheel in proximity with the workpiece. The power analyser Norma D4355 was calibrated using a power function meter B1081 which was calibrated by Siemens, the manufacturer. A power range of $700 \mathrm{~W}$ to $1300 \mathrm{~W}$ for the calibration was achieved by adjusting wheel speed. The results of the calibration are shown in Fig. 6.12. The power analyser Norma D4355 was found to have good linearity and to give good agreement with the function meter readings within an accuracy of $\pm 3 \%$.

A Tektronix 027400 digital storage oscilloscope was used as a visual monitor. The oscilloscope was capable of storing 10,000 points on each channel and a zoom function was used to analyse the detail. The oscilloscope allowed up to 5,000 waveforms per second to be acquired for examination of rapidly changing signals or intermittent signal irregularities. An inbuilt floppy disk was used to store, and recall waveforms and to transfer hardcopies to a PC.

\subsubsection{Workpiece measurement}

Workpiece size measurements were carried out using a micrometer gauge of 1 micron resolution. Workpiece surface texture was measured on a Taylor-Hobson Form Talysurf 120 (later upgraded for 3D measurement) after each batch of workpieces was ground. For each workpiece, three measurements were performed at different positions around the cylindrical surface and an average value was used. Workpiece roundness was measured on a Taylor-Hobson Talyrond 210. The measurement accuracies of the Talysurf and of the Talyrond were better than \pm 0.03 microns. Workpiece measurements followed the same procedure for both internal and external grinding operations.

6.1.8 Measurement of wheel topography including replicas. Overall wheel wear was measured by the razor-blade method. The grinding wheel profile is checked by grinding a razor blade. The wheel wear corresponds to the depth of the groove reproduced on the razor-blade profile. The razor-blade profiles were 
measured on the Talysurf. The accuracy of measurement was judged to be better than 1 micron.

A device was designed to take topographical replicas of the wheel surface as shown in Fig. 2.10. The replica material employed was Microset 101 RT Thixo, described in Chapter 2. Replicas were taken after dressing the wheel and after grinding a number of workpieces in order to monitor changes in wheel topography. Before taking replicas, the wheel was run for a few minutes in order to clean the surface of the wheel of coolant as well as of solvent liquid. An air gun was used to blow away the dust and broken grains attached to the wheel. After preparation, the replica material was poured into a small container, which was then placed in contact with the wheel surface. After setting, the replicas were removed from the jig and observed through the microscope. The replicas were measured using the Wyko RST Plus and Uniscan OSP 100 as described in Chapter 2.

\subsection{Grinding conditions}

\subsubsection{The grinding conditions for internal grinding}

Two groups of internal grinding experiments were carried out to test the effects of wheel structure. One group was for the M2 workpiece material. The other was for Inconel 718. For each group, two different wheel structures and wheel speeds were used (The medium-porosity medium-grain CBN wheel denoted MP-B91 and the high-porosity fine-grain CBN wheel denoted HP-B64)

The basic grinding conditions are listed below:

Internal Grinding Machine:

Grinding mode:

Grinding wheel traverse speed $v_{t r}$ :

Grinding wheel traverse length $l_{\text {tr: }}$ :

Workpiece rotary speed:

Stock removal:
$1300 \mathrm{X}$

Plunge with oscillation

$506 \mathrm{~mm} / \mathrm{min}$

$3 \mathrm{~mm}$

$30 \mathrm{rev} / \mathrm{min}$

$0.3 \mathrm{~mm}$ on diameter 
Roughing infeed a1:

Finishing infeed a2:

Number of spark-out passes:

Coolant:

Dressing method:

Dressing tool:

Diameter of rotary cup dresser:
$6 \mu \mathrm{m}$ per traverse for $270 \mu \mathrm{m}$ stock removal on diameter

$2 \mu \mathrm{m}$ per traverse for $30 \mu \mathrm{m}$ stock removal on diameter

75

Emulsion HYSOL - X 10\%

Touch dressing

Rotary cup dresser

$50 \mathrm{~mm}$

The grinding wheel speeds were approximate since as the wheel wears the speed is reduced for a particular rotational speed. The maximum variation from the nominal speed was less than $5 \%$.

\section{Group 1: M2 workpiece material}

(i) Grinding wheel speed of $45 \mathrm{~m} / \mathrm{s}$

Grinding wheel rotary speed $n_{\mathbf{s}}$ :

$60,000 \mathrm{rev} / \mathrm{min}$

Width of diamond rim:

$3 \mathrm{~mm}$

Dressing parameters:

Dressing depth $a_{d}$ :

Dresser rotating speed $\mathrm{n}_{\mathrm{d}}$ :

Dressing lead $\mathrm{f}_{\mathrm{d}}$ for the MP-B91 wheel

Dressing lead $\mathrm{f}_{\mathrm{d}}$ for the HP-B64 wheel
$1 \mu \mathrm{m}$

$2800 \mathrm{rev} / \mathrm{min}$

$0.083 \mathrm{~mm}$

$0.058 \mathrm{~mm}$

\section{(ii) Grinding wheel speed of $55-60 \mathrm{~m} / \mathrm{s}$}

The grinding conditions were the same as the previously stated standard conditions, except that the wheel speed was increased from $60,000 \mathrm{rev} / \mathrm{min}$ to $75,000 \mathrm{rev} / \mathrm{min}$ and the dressing conditions were adjusted to provide the same dressing lead for both wheels.

Wheel rotational speed

$75,000 \mathrm{rev} / \mathrm{min}$

Dressing parameters:

Dressing depth $a_{d}$ : 
Dresser rotating speed $\mathrm{n}_{\mathrm{d}}$ :

Dressing lead $\mathrm{f}_{\mathrm{d}}$ for the MP-B91 wheel: $\quad 0.067 \mathrm{~mm}$

Dressing lead $\mathrm{f}_{\mathrm{d}}$ for the HP-B64 wheel: $\quad 0.067 \mathrm{~mm}$

\section{(iii) Grinding wheel speed of $110 \mathrm{~m} / \mathrm{s}$}

The wheel speed of $110 \mathrm{~m} / \mathrm{s}$ was obtained by employing a larger wheel diameter of $30 \mathrm{~mm}$. Except where stated below, the test conditions remained the same as the previously listed standard conditions. Only a high-porosity HP-B64 wheel was tested for improvement of surface roughness with increased wheel speed.

The specifications of the wheels are as listed previously:

Grinding wheel rotary speed $\mathrm{n}_{\mathbf{s}}$ :

$70,000 \mathrm{rev} / \mathrm{min}$

Dressing lead $\mathrm{f}_{\mathrm{d}}$ for the HP-B64 wheel:

$0.058 \mathrm{~mm}$

\section{Group 2: Inconel 718 workpiece material}

\section{(i) Grinding wheel speed of $50 \mathrm{~m} / \mathrm{s}$}

The particular grinding conditions are listed below. Other conditions are the same as the previously listed standard conditions. The wheels used were the MP-B91 wheel and the HP-B91 wheel.

Workpiece material:

Grinding wheel rotary speed $\mathrm{n}_{\mathbf{s}}$ :

Grinding wheel traverse speed $\mathbf{v}_{\mathbb{t}}$ :

Dressing parameters:

Dresser rotational speed $n_{d}$ :

Dressing lead $\mathrm{f}_{\mathrm{d}}$ :

(ii) Grinding wheel speed of $60 \mathrm{~m} / \mathrm{s}$

Workpiece material:

Grinding wheel rotary speed $n_{\mathbf{s}}$ :

Grinding wheel traverse speed $v_{\mathbf{t r}}$ :
Inconel 718

$60,000 \mathrm{rev} / \mathrm{min}$

$506 \mathrm{~mm} / \mathrm{min}$

$46 \mathrm{rev} / \mathrm{s}$

$0.083 \mathrm{~mm}$

Dressing parameters:

Inconel 718

$75,000 \mathrm{rev} / \mathrm{min}$

$506 \mathrm{~mm} / \mathrm{min}$ 

Dresser rotational speed $\mathrm{n}_{\mathrm{d}}$ :
$46 \mathrm{rev} / \mathrm{s}$
Dressing lead $\mathrm{f}_{\mathrm{d}}$ :
$0.083 \mathrm{~mm}$

\subsubsection{Grinding conditions for external grinding}

Two groups of external grinding experiments were carried out on the Suprema machine to test the effects of high wheelspeed. These tests were carried out collaboratively, as mentioned previously using the MP-B91 and HP-B151 wheels.

Table 6.3 Experimental arrangement for AISI 52100 on the Suprema machine

$\begin{array}{lllllllll}\text { Parameter A } & \text { B } & \text { C } & \text { D } & \text { E } & \text { F } & \text { G } & \text { H }\end{array}$

Test

$\begin{array}{lllllllll}1 & 1 & 1 & 1 & 1 & 1 & 1 & 1 & 1 \\ 3 & 1 & 1 & 1 & 2 & 2 & 2 & 2 & 1 \\ 4 & 1 & 2 & 2 & 1 & 1 & 2 & 2 & 1 \\ 5 & 1 & 2 & 2 & 2 & 2 & 1 & 1 & 1 \\ 6 & 2 & 1 & 2 & 1 & 2 & 1 & 2 & 1 \\ 7 & 2 & 1 & 2 & 2 & 1 & 2 & 1 & 1 \\ 8 & 2 & 2 & 1 & 1 & 2 & 2 & 1 & 1 \\ 9 & 2 & 2 & 1 & 2 & 1 & 1 & 2 & 1 \\ 10 & 2 & 2 & 2 & 2 & 2 & 2 & 2 & 2 \\ 11 & 2 & 2 & 2 & 1 & 1 & 1 & 1 & 2 \\ 12 & 2 & 1 & 1 & 2 & 2 & 1 & 1 & 2 \\ 13 & 2 & 1 & 1 & 1 & 1 & 2 & 2 & 2 \\ 14 & 1 & 2 & 1 & 2 & 1 & 2 & 1 & 2 \\ 15 & 1 & 2 & 1 & 1 & 2 & 1 & 2 & 2 \\ 16 & 1 & 1 & 2 & 2 & 1 & 1 & 2 & 2 \\ 1 & 1 & 1 & 2 & 1 & 2 & 2 & 1 & 2\end{array}$

\section{Group 1: AISI 52100 workpiece material}

To determine the effect of each of the parameters tested a standard $L_{16} 2^{8}$ orthogonal array was used. This provides the most economical testing plan for 
evaluating the direct effects of eight parameters. According to Taguchi [117] the plan may be checked for reliability by running confirmation trials based on the results collected. If the results from the confirmation trials agree with the expected results, then the experimental plan may be considered to be reliable. Table 6.3 shows the parameter array used in the experimental plan.

In accordance with the experimental design sixteen trials were carried out with the process parameters set at either of two levels as indicated in the table.

The two levels set for each of the process parameters are listed below:

Table 6.4 Two levels for each of the process parameters for AISI 52100

\section{$\underline{\text { Parameter }}$}

A. Dressing direction

B. Dressing overlap

C. Dressing increment

D. Number of dressing passes

E. Wheel speed

F. Workpiece speed

G. Fine feed / Dwell

H. Dresser speed.

$\begin{array}{lc} & \text { Level } \\ 1 & 2 \\ \text { down } & \text { up } \\ 2 & 10 \\ 2 \mu \mathrm{m} & 10 \mu \mathrm{m} \\ 2 & 10 \\ 60 \mathrm{~m} / \mathrm{s} & 120 \mathrm{~m} / \mathrm{s} \\ 36 \mathrm{~m} / \mathrm{min} & 54 \mathrm{~m} / \mathrm{min} \\ 10 \mu \mathrm{m} @ 1 \mu \mathrm{m} / \mathrm{s} & 10 \mathrm{~s} \\ 42 \mathrm{~m} / \mathrm{s} & 72 \mathrm{~m} / \mathrm{s}\end{array}$

For all trials, the specific volumetric removal rate, $Q^{\prime} \mathrm{w}=10 \mathrm{~mm}^{3} / \mathrm{mm} \mathrm{s}$ was kept constant.

Two confirmation trials were carried out for each wheel with the parameters set to give improved surface roughness and roundness.

\section{Group 2: Inconel 718 workpiece material}

A standard $\mathrm{L}_{8}{ }^{7}$ orthogonal array was followed for the experimental plan. Table 6.4 shows the experimental array: 
Table 6.5 Experimental arrangement for Inconel 718 on the Suprema machine

\begin{tabular}{cccccccc}
\multicolumn{1}{c}{ Pest } & Aarameter & B & C & D & E & F & G \\
1 & & & & & & & \\
2 & 1 & 1 & 1 & 1 & 1 & 1 & 1 \\
3 & 1 & 1 & 1 & 2 & 2 & 2 & 2 \\
4 & 1 & 2 & 2 & 1 & 1 & 2 & 2 \\
5 & 1 & 2 & 2 & 2 & 2 & 1 & 1 \\
6 & 2 & 1 & 2 & 1 & 2 & 1 & 2 \\
7 & 2 & 1 & 2 & 2 & 1 & 2 & 1 \\
8 & 2 & 2 & 1 & 1 & 2 & 2 & 1 \\
& 2 & 2 & 1 & 2 & 1 & 1 & 2
\end{tabular}

In accordance with the experimental design eight trials were carried out with the process parameters set as indicated in the table above.

The process parameters selected for investigation were tested at two levels as listed below:

Table 6.6 Process parameter levels for Inconel 718

Parameter

$\begin{array}{lll}\text { A - Dressing direction } & 1 & 2 \\ \text { B - Dressing overlap } & 2 & \text { up } \\ \text { C - Dressing increment } & 2 \mu \mathrm{m} & 10 \\ \text { D - No. of dressing passes } & 2 & 10 \mu \mathrm{m} \\ \text { E - Wheel speed } & 60 \mathrm{~m} / \mathrm{s} & 10 \\ \text { F - Workpiece speed } & 36 \mathrm{~m} / \mathrm{min} & 120 \mathrm{~m} / \mathrm{s} \\ \text { G - Dwell } & 2 \mathrm{~s} & 120 \mathrm{~m} / \mathrm{min} \\ \end{array}$


For all Inconel 718 trials, the specific volumetric removal rate $Q^{\prime}$ w was $2 \mathrm{~mm}^{3} / \mathrm{mm}$ s. The stock removal off each diameter ground was $200 \mu \mathrm{m}$.

Two confirmation trials were carried out for the wheel, with parameters set either to give improved surface roughness and roundness or to give reduced specific energy for high removal-rate.

The following parameters were used to determine wheel life, which were chosen from the earlier grinding results as discussed in the next section.

Parameter

Dressing direction

Dressing overlap

Dressing increment

No. of dressing passes

Wheel speed

Workpiece speed

Dwell

Dresser rotational speed

Specific volumetric removal rate

\section{Suprema}

up-dressing

$2 \mathrm{x}$

$2 \mu \mathrm{m}$

10

$120 \mathrm{~m} / \mathrm{s}$

$36 \mathrm{~m} / \mathrm{min}$

$2 \mathrm{~s}$

$42 \mathrm{~m} / \mathrm{s}$

$2 \mathrm{~mm}^{3} / \mathrm{mm}$.s

\subsection{Experimental procedure}

(i) Internal grinding

The experimental procedure was as follows.

i. The workpieces were ground to the same size and the internal diameters were measured before grinding. Each workpiece was numbered.

ii. The power measurement system was set up.

iii. The grinding wheel was dressed and the wheel diameter was measured.

iv. A wheel surface replica was taken before grinding.

v. A workpiece was set in position. Coolant delivery was turned on. The no-load power was measured.

vi. The internal surface of the workpiece was ground. The power was monitored and the power signal was recorded. The grinding conditions 
were as given according to different trials. The specific energy was evaluated.

vii. Wheel surface replicas were taken after pre-determined intervals of grinding. The grinding tests were ceased after workpiece surface roughness reached the maximum level corresponding to the set target.

viii. A razor blade was ground across a step in the wheel to monitor wheel wear.

ix. The wheel was removed after grinding and observed under the microscope.

x. The workpiece diameters, roughness and roundness were measured after the batch of workpieces was ground.

xi. The replicas were measured and analysed.

\section{(ii) External grinding}

The procedure was slightly different for the Suprema machine. The wheel size was not measured before and after trials since very little wear occurs with touch dressing. Replicas were only taken before and after trials to avoid disturbance to the system temperatures and size-holding during the trials.

\subsection{Experimental results for internal grinding}

\subsubsection{M2 tool steel}

\section{(i) Grinding wheel speed of $45 \mathrm{~m} / \mathrm{s}$}

Grinding performance of the high-porosity fine-grain HP-B64 wheel and the medium-porosity medium-grain MP-B91 wheel was compared in relation to wheel redress life, specific energy, surface roughness, and size error. The grinding results are shown in Fig.6.13 (a)-(i). With the high-porosity fine-grain HP-B64 wheel, the power and specific energy were lower than for the medium-porosity medium-grain MP-B91 wheel. The workpiece surface roughness was higher and the size errors were lower. For the same specific material removal, the MPB91wheel gave the better grinding performance with a G-ratio of 5000, an average surface roughness of 0.15 microns $\mathrm{Ra}$ and a wheel redress life of 4100 $\mathrm{mm}^{3} / \mathrm{mm}$ before the acceptable roughness level of 0.25 microns $\mathrm{Ra}$ was exceeded. The HP-B64 wheel yielded a G-ratio of 3000 , an average surface roughness of 
0.18 microns $\mathrm{Ra}$ and a wheel redress life of $1650 \mathrm{~mm}^{3} / \mathrm{mm}$ before the acceptable roughness level was exceeded.

The measured values of active cutting edge density are shown in Fig.6.13(h). The active cutting edge density for the MP-B91 wheel was higher than for the HPB64 wheel. Initially, this was considered to be the opposite of expectation. However, the studies of topography described in Chapter 3 revealed the occurrence of grain agglomeration with the fine grain wheel as the cause of reduced active cutting edge density. The active cutting edge density decreased in both trials. The rate of decrease was quite rapid at the beginning of each trial. The rates of decrease were nearly the same for both wheels.

The cutting edge dullness, shown in Fig.6.13 (i), was lower for the MP-B91 wheel indicating that the wheel was sharper than the HP-B64 wheel. The cutting edge dullness increased in both trials indicating increasing wear flat area. The rate of increase in cutting edge dullness of the HP-B64 wheel was 3 times higher than for the MP-B91 wheel. The effective porosity volume ratio for both wheels remained reasonably stable. For the MP-B91 wheel, the effective porosity volume ratio was $35 \%$ while for the HP-B64 wheel, the effective porosity volume ratio was $45 \%$.

The explanation proposed for the differences in performance between these two wheels is based on the evidence revealed in the examination of the wheel topography. As mentioned previously, it was found that the finer grains of the HP-B64 wheel were agglomerated together in groups. This had the effect of reducing the active grain density. The reduced active grain density, meant that a smaller number of cutting edges were involved in the removal process. The consequences of reduced active grain density are increased removal per active cutting edge leading to reduced specific energy and power due to the size effect. Even though the cutting edge dullness was greater for the HP-B64 wheel, the size effect appears to have been predominant in the effect on specific energy, which was lower. As a further consequence of the increased removal performed by each active cutting edge, the cutting edges would be expected to be subject to an 
increased rate of wear. This explains why the wear flat area increased more rapidly and explains the increased cutting edge dullness.

Unfortunately, perhaps, the cutting edge dullness parameter is based only on the wear flat area and the grain size. As the wear flat area increases, it is assumed that the grain becomes dull. In practice, the effective slopes of the sides of the grains are also important. It was realized, towards the end of this research that, as the wear flat area increases, the effective slopes of the sides of the grains may be increased. This would suggest that there is possibly, a need for two parameters to describe grain geometry. Arguments such as these are summarized in the discussion section in an attempt to generalize the results.

\section{(ii) Grinding wheel speed of $55-60 \mathrm{~m} / \mathrm{s}$}

The grinding results for $55-60 \mathrm{~m} / \mathrm{s}$ are shown in Fig.6.14 (a)-(g). For the highporosity fine-grain HP-B64 wheel, the power and specific energy were lower than for the MP-B91 wheel, the workpiece surface roughness and the size error were lower as well. For the same specific material removal, the MP-B91 wheel gave a G-ratio of 2500 , an average surface roughness of 0.22 microns $\mathrm{Ra}$ and a wheel redress life of $500 \mathrm{~mm}^{3} / \mathrm{mm}$ before the acceptable roughness was exceeded. The HP-B64 wheel gave a better grinding performance with a G-ratio of 4300, an average surface roughness of 0.17 microns $\mathrm{Ra}$ and a wheel redress life exceeding $1200 \mathrm{~mm}^{3} / \mathrm{mm}$ before the acceptable roughness was exceeded.

The change of wheel topography was nearly the same as at the wheel speed of 45 $\mathrm{m} / \mathrm{s}$. The active cutting edge density for the MP-B91 wheel was still higher and the cutting edge dullness was lower. The rate of increase of the cutting edge dullness of the HP-B64 wheel was 1.5 times higher than for the MP-B91 wheel. The effective porosity volume ratio of both wheels remained stable.

The same arguments for the effects of topography could be given as for the lower speed of $45 \mathrm{~m} / \mathrm{s}$. The high-porosity wheel would be expected to reduce the specific and energy power compared with the medium-porosity wheel as previously. This aspect was therefore as expected. 
It was not expected however, that the specific energy and power would be lower at the higher wheel speed than at the lower speed or that the workpiece roughness would be increased when using the MP-B91 wheel at the same removal rate. These results are contrary to usual findings unless the removal rate is increased at least in proportion to the wheelspeed or greater. Neither was it expected that the MP-B91 wheel would suffer a reduced G-ratio and reduced redress life compared with the lower wheelspeed and also compared with the HP-B64 wheel. For some reason, the wheel-wear rates were increased which is consistent with reduced specific energies and increased workpiece roughness at the higher wheelspeed.

These results suggest that some uncontrolled factor played a part in the tests. For example, it is known that increased vibrations can reduce specific energy and increase workpiece roughness, so that the possibility of increased vibration at the increased spindle speed of $75000 \mathrm{rev} / \mathrm{min}$ compared with the lower spindle speed of $60,000 \mathrm{rev} / \mathrm{min}$ must be considered a possibility. However, machine vibrations would be expected to affect the performance of the B64 wheel as much as the B91 wheel. It therefore seems unlikely that increased machine vibration provides the whole answer. Another possibility is that differences in the particular dressing operations in some way affected the vibrations during the grinding process. In the absence of scientific evidence for the levels of vibration during dressing, a complete explanation of the results could not be provided.

Further tests were carried out at the much higher wheel speed of $110 \mathrm{~m} / \mathrm{s}$ using larger wheels at a spindle speed of $70,000 \mathrm{rev} / \mathrm{min}$ in order to clarify the significance of wheel topography and kinematic effects.

\section{(iii) Grinding wheel speed of $110 \mathrm{~m} / \mathrm{s}$}

The grinding results for a high-porosity HP-B64 wheel at $110 \mathrm{~m} / \mathrm{s}$ are shown in Fig.6.15. It can be seen that the specific energy is about twice that at the wheel speed of $45-60 \mathrm{~m} / \mathrm{s}$. The workpiece average surface roughness was 0.15 microns $\mathrm{Ra}$, which, as expected, was better than at a wheel speed of $45-60 \mathrm{~m} / \mathrm{s}$. The larger wheel, grinding at a higher wheel surface speed reduces the material removed per 
cutting edge by a factor of approximately 4 times. This reduces the wear per cutting edge and reduces workpiece roughness. The G-ratio was 3800 after a specific volume of material removed of $1700 \mathrm{~mm}^{3} / \mathrm{mm}$. This volume of material removed corresponds to the length of the test and was not the same as the previously defined wheel redress life. The size error was up to $85 \mu \mathrm{m}$, which is related to machine thermal drift and was not greatly different from the lower wheelspeed. The active cutting edge density remained much more stable than at a wheel speed of $45 \mathrm{~m} / \mathrm{s}$. At the lower speed there was a steady reduction in the active cutting edge density. The cutting edge dullness increased much more slowly with the larger wheel and with the higher wheel surface speed. These results reflect the expected benefits of a larger wheel diameter and higher surface speeds of the wheel.

The results for $110 \mathrm{~m} / \mathrm{s}$ compared with the results for $45 \mathrm{~m} / \mathrm{s}$ are so much in line with expectations from the previous topographic, kinematic and physical considerations, that it would tend to support the conclusion that the results for 55 $60 \mathrm{~m} / \mathrm{s}$ are anomalous probably due to dressing and process vibrations.

\subsubsection{Inconel 718 aerospace alloy}

The grinding results are shown in Fig.6.16. At a wheel speed of $50 \mathrm{~m} / \mathrm{s}$, the conventional MP-B91 wheel failed to grind Inconel 718 satisfactorily, after a specific material removal of $60 \mathrm{~mm}^{3} / \mathrm{mm}$ due to heavy loading. In contrast, for the same specific material removal, the high-porosity fine grain HP-B91 wheel gave a good grinding performance for a difficult-to-grind material with a G-ratio of 2000 , an average surface roughness of 0.18 microns $\mathrm{Ra}$ and a wheel redress life of $500 \mathrm{~mm}^{3} / \mathrm{mm}$ before the acceptable roughness was exceeded.

The grinding performance of the high-porosity medium-grain HP-B91 wheel was even better when the wheel speed was increased from $50 \mathrm{~m} / \mathrm{s}$ to $60 \mathrm{~m} / \mathrm{s}$. The Gratio increased to 4000 , workpiece roughness decreased to 0.13 microns $\mathrm{Ra}$ and wheel redress life was increased to $1400 \mathrm{~mm}^{3} / \mathrm{mm}$. The results are illustrated in Figure Fig. 6.16(a)-(h), which demonstrates the improvements for precision 
grinding using the high-porosity medium-grain HP-B91 wheel at a high grinding speed.

In grinding, the active cutting edge density decreased in both trials and more quickly at the beginning. The active cutting edge density for the wheel speed of $50 \mathrm{~m} / \mathrm{s}$ was about 5 per $\mathrm{mm}^{2}$ less than for the $60 \mathrm{~m} / \mathrm{s}$ wheelspeed. The rate of decrease then became nearly the same. The cutting edge dullness increased quickly at both speeds. The effective porosity volume ratio of both wheels decreased and for $50 \mathrm{~m} / \mathrm{s}$ the value was approximately halved by the end of the trial.

These results tend to confirm the proposal that high-porosity wheels with higher wheelspeeds are an advantage for a difficult-to-grind material. There were fewer problems with loading than with a medium-porosity wheel and reducing the volume of material removed per cutting edge allowed redress life to be increased and workpiece roughness to be reduced.

\subsection{Experimental results for external grinding on the Suprema machine}

The purpose of the trials was to confirm whether improved grinding results could be achieved using increased wheelspeeds and wheels selected according to the previous findings in relation to the type of workpiece material and the type of structure. The trials also provided an opportunity to validate the relationships between grinding parameters, grinding performance and wheel topography that would be expected for an easy-to-grind material and a difficult-to-grind material.

Students' t-test was employed to check if a significant difference exists between the parameter levels and their effect on the performance indicators. The equation for Students t-test is given as:

$$
t_{o b t}=\frac{x_{1}-x_{2}}{\sqrt{\frac{s_{1}^{2}}{n_{1}}+\frac{s_{2}^{2}}{n_{2}}}}
$$

Where: $t_{o b l}=$ the value of $t$ determined for the sample $x=$ the mean of the sample 
$s^{2}=$ the variance of the sample

$n=$ the sample size

The subscripts $1 \& 2$ denote the parameter level

$t_{c r i t}=$ the value of $\mathrm{t}$ determined from tables. The significance level was 0.05 , the degrees of freedom were greater than 120 , therefore, $t_{\text {crit }}=1.96$.

The test was two-tailed.

If a null hypothesis is taken then $\mathrm{H}_{0}: x_{1}=x_{2}$

Thus, if $t_{o b t}>t_{c r i t}$ the hypothesis is rejected and a significant difference between means does exist.

\subsubsection{AISI 52100 bearing steel}

The wheel used for this material was the medium-porosity medium-grain MP-B91 wheel. The effective porosity volume ratio of the wheel was 0.35 . The active cutting edge density was approximately 40 per $\mathrm{mm}^{2}$ depending on different dressing conditions and grinding conditions.

Initial 2- level trials at a moderately high removal-rate for precision grinding of $10 \mathrm{~mm} / \mathrm{mm}$.s showed the strength of particular grinding parameters. The following results were determined, as listed in Table 6.7 in which the parameters from $A$ to $H$ have the same meanings as in Table 6.4. More detail can be seen in Figure 6.17:

Table 6.7 Value of $t_{o b t}$ for performance indicator

$\underline{\text { Parameter }}$

Value of $t_{o b t}$ for Performance Indicators

Specific Energy Ra Roundness Size Holding

A

$-130$

8

13

14

B

$-45$

4

2

5

C

57

$-8$

$-7$

$-20$

D

$-13$

$-3$

1.3

$-29$

E

$-115$

49

23

46

F

$-38$

$-8$

$-3$

1.6

G

62

8

17

$-6$

$\mathrm{H}$

$-153$

$-22$

$-8$

$-31$ 
As can be seen, values for $t_{o b t}$ are higher than $t_{c r i t}(=1.96)$ in all but two instances. This shows that the parameter levels tested were of the correct magnitude to determine the direction in which they influence the performance indicators. The values in the table are also a good indication as to how strongly a parameter affects a particular performance indicator. The greater is $t_{o b t}$ the greater is the influence of that parameter on the performance indicator relative to the other parameters.

\section{Direct-effect charts:}

The direct-effects charts are shown in Fig.6.17. It can be seen that when wheel speed increased from $60 \mathrm{~m} / \mathrm{s}$ to $120 \mathrm{~m} / \mathrm{s}$, the specific energy' increased from 15.2 $\mathrm{J} / \mathrm{mm} 3$ to $22.0 \mathrm{~J} / \mathrm{mm} 3$; the workpiece surface roughness decreased from 0.66 $\mu \mathrm{mRa}$ to $0.20 \mu \mathrm{mRa}$; the workpiece roundness decreases from $1.25 \mu \mathrm{m}$ to 0.72 $\mu \mathrm{m}$; the size holding decreased from $30 \mu \mathrm{m}$ to $5 \mu \mathrm{m}$ and the G-ratio increased from 850 to 2480 . The results showed the benefits of high-speed grinding particularly in terms of workpiece quality and high G-ratio. Although high wheelspeed increased specific energy, workpiece roughness was reduced, size holding was improved, G-ratio was increased and wheel wear was reduced.

It can be seen that high-speed grinding not only gives benefits with a highporosity wheel as demonstrated from the internal grinding trials, but also gives benefits with a medium-porosity wheel as demonstrated from this trial.

Of the eight parameters, four had a stronger effect on grinding performance than the other four. The strongest effects were found due to variations in dressing direction, dresser speed, wheel speed and dwell period. It was found that, detailed interactions between process parameters and grinding performance could be correlated with the topographical parameters for the wheel. The active cutting edge density was $38 / \mathrm{mm}^{2}$ for up-dressing and $25 / \mathrm{mm}^{2}$ for down-dressing. A greater active cutting edge density, caused higher specific energy due to the size effect and lower surface roughness. A higher dresser speed gave an active cutting 
edge density of $23 / \mathrm{mm}^{2}$ and a cutting edge dullness of 0.13 . A lower dresser speed gave an active cutting edge density of $40 / \mathrm{mm}^{2}$ and a cutting edge dullness of 0.09 . This could be due to a greater number of bond fractures resulting from the higher dressing speed. Higher wheel speed allowed wheel sharpness to be maintained with cutting dullness of 0.08 compared with a cutting edge dullness of 0.12 for the lower wheel speed. This means higher wheel speed reduced wheel wear. A very fine stock removal of $10 \mu \mathrm{m}$ from diameter at $1 \mu \mathrm{m} / \mathrm{s}$ for $5 \mathrm{~s}$ was substituted for a true dwell for the one set of trials. This short 'dwell' gave an active cutting edge density of $29 / \mathrm{mm}^{2}$ and a cutting edge dullness of 0.12 . For a true 10 s dwell without the fine infeed, the active cutting edge density was $37 / \mathrm{mm}^{2}$ and the cutting edge dullness was 0.10 . This implies that a longer dwell without infeed, reduces the number of fractures of the wheel compared with a very fine infeed for a short duration. This was also demonstrated for the confirmation trials as follows.

\section{Confirmation trials:}

On the basis of the initial trials, two confirmation trials were carried out. Confirmation Trial 1 was based on values of parameters selected for high quality and high G-ratio. Confirmation Trial 2 was based on values of parameters selected for high removal rate and productivity. From the initial trial, it appeared that the duration of the dwell had an effect on the wheel condition. The dwell was therefore maintained at $10 \mathrm{~s}$ for the high quality objective but reduced to $2 \mathrm{~s}$ for reduced cycle-time in Confirmation Trial 2. For Confirmation Trial 1, specific volumetric removal rate was maintained constant at $Q^{\prime}{ }_{w}=10 \mathrm{~mm}^{3} / \mathrm{mm} . \mathrm{s}$ but increased to $20 \mathrm{~mm}^{3} / \mathrm{mm}$.s for Confirmation Trial 2. Thus, from the previous direct-effects charts shown in Fig.6.17, the following parameters were selected for the confirmation trials: 


\section{Confirmation Trial 1}

For workpiece quality and high G-ratio:

Up-dressing

Dressing overlap, 10x

Dressing increment, $2 \mu \mathrm{m}$

Dressing passes, 2

High wheelspeed, $120 \mathrm{~m} / \mathrm{s}$

Workspeed, $36 \mathrm{~m} / \mathrm{min}$

Dwell, $10 \mathrm{~s}$

Low dresser speed, $42 \mathrm{~m} / \mathrm{s}$

Specific removal-rate, $10 \mathrm{~mm}^{3} / \mathrm{mm}$.s

\section{Confirmation Trial 2}

For high removal rate:

Down-dressing

Dressing overlap , $2 \mathrm{x}$

Dressing increment, $10 \mu \mathrm{m}$

Dressing passes, 2

High wheelspeed, $120 \mathrm{~m} / \mathrm{s}$

Workspeed, $36 \mathrm{~m} / \mathrm{min}$

Dwell, 2 s

Low dresser speed, $42 \mathrm{~m} / \mathrm{s}$

Specific removal-rate, $20 \mathrm{~mm}^{3} / \mathrm{mm}$.s

For the trial aimed at workpiece quality and high G-ratio, the following results were achieved:

Table 6.8 Results for Confirmation Trial 1 - AISI 52100 on the Suprema machine

\begin{tabular}{l|ccccc} 
& Specific Energy & $\mathrm{Ra}$ & $\mathrm{Rz}$ & Roundness & Size Holding \\
\hline Mean & $26.5 \mathrm{~J} / \mathrm{mm}^{3}$ & $0.14 \mu \mathrm{m}$ & $1.0 \mu \mathrm{m}$ & $0.5 \mu \mathrm{m}$ & $-0.5 \mu \mathrm{m}$ \\
Median & 25.5 & 0.14 & 0.95 & 0.5 & -1 \\
Mode & 25.3 & 0.13 & 0.92 & 0.5 & -3 \\
Maximum & 49 & 0.18 & 1.4 & 0.65 & 10 \\
Minimum & 21.5 & 0.1 & 0.6 & 0.3 & -6 \\
Range & 27.5 & 0.08 & 0.8 & 0.35 & 16 \\
Variance & 11.3 & 0.0003 & 0.02 & 0.008 & 14 \\
Standard & & & & & \\
Deviation & 3.4 & 0.017 & 0.14 & 0.09 & 3.7
\end{tabular}

G-ratio was 5,700 measured after a fixed volume of material removed, at $V_{w}{ }^{\prime}=76,140 \mathrm{~mm}^{3} / \mathrm{mm}$.

The results for Confirmation Trial 1 are detailed in Figure 6.18(a)-(d). The maximum workpiece roughness for this trial was 1.4 microns $\mathrm{Rz}$, which is below 
the set target value of 2 microns Rz. Roughness tended to increase during the trial and specific energy reduced. These results are consistent with a stable wheel in which the active cutting edge density tended to reduce over the trial. This was confirmed from the measurements at the beginning and end of the trial.

Roundness varied about a mean value of 0.5 microns with no strong pattern regarding which of the five diameters was being ground on each workpiece. The size holding for each diameter showed the same trend. The size-holding trend did not follow the thermal drift trend of the machine or of the coolant, both of which were found to remain reasonably constant throughout the trials.

For Confirmation Trial 2, removal-rate was increased to $20 \mathrm{~mm}^{3} / \mathrm{mm} . \mathrm{s}$, and the dwell was reduced to $2 \mathrm{~s}$. This change represents a substantial reduction in cycle time. The following results were achieved:

Table 6.9 Results for Confirmation Trial 2 - AISI 52100 on the Suprema machine

\begin{tabular}{l|lllcc} 
& Specific Energy & $\mathrm{Ra}$ & $\mathrm{Rz}$ & Roundness & Size Holding \\
\hline Mean & $16 \mathrm{~J} / \mathrm{mm}^{3}$ & $0.5 \mu \mathrm{m}$ & $3.5 \mu \mathrm{m}$ & $1.0 \mu \mathrm{m}$ & $3.2 \mu \mathrm{m}$ \\
Median & 16.3 & 0.5 & 3.4 & 0.95 & 2 \\
Mode & 16.5 & 0.6 & 3.4 & 0.8 & - \\
Maximum & 20.5 & 0.7 & 4.4 & 2.7 & 15 \\
Minimum & 11.4 & 0.4 & 2.9 & 0.6 & -5 \\
Range & 9.1 & 0.3 & 1.5 & 2.1 & 20 \\
Variance & 2 & 0.004 & 0.11 & 0.1 & 27.5 \\
Standard & & & & & \\
Deviation & 1.4 & 0.06 & 0.34 & 0.32 & 5.24
\end{tabular}

G-ratio was 720 measured for a fixed volume of material removed, at $V_{w}{ }^{\prime}=76,140 \mathrm{~mm}^{3} / \mathrm{mm}$ and not at the end of the redress life. Thus, although the removal-rate was doubled and the cycle time more than halved, these results were at a cost of greatly reduced G-ratio and increased workpiece roughness. Fig 6.19 
(a)-(d) shows the progressive changes in specific energy, surface roughness, roundness and size holding as the trial proceeded.

From Confirmation Trial 2, it can be seen that the specific energy was very low and surface roughness quite high. This would be expected at high removal-rate, according to the size effect. It can also be confirmed that the active cutting edge density was lower for Confirmation Trial 2 than for Confirmation Trial 1. In Confirmation Trial 1, the active cutting edge density changed from 42 to $30 / \mathrm{mm}^{2}$ from the beginning of the trial to the end of the trial. While in Confirmation Trial 2 , the active cutting edge density changed from 34 to $22 / \mathrm{mm}^{2}$ from the beginning of the trial to the end of the trial. This would further enhance the size effect.

From the graphs of the performance indicators, it can be deduced that the wheel surface was changing throughout the course of the trial. Lower workpiece roughness was found in the middle of the trial where the workpiece size holding was more stable and increased again towards the end of the trial. This can be explained because in the first half of the trial, the active cutting edge density tended to increase. This is consistent with an initial steady progression of grain fracture wear followed by an increased rate of bond fracture wear later in the trial with a reduced active cutting edge density. At the end of the trial, it therefore appears, that the wheel was approaching the end of its' redress life.

In Confirmation Trial 2, G-ratio was 720, which is low compared to the value of 5700 obtained in Confirmation Trial 1 for high quality. This is because high removal rates leads to high forces on the grains and more self-sharpening. This was confirmed by the change of cutting edge dullness. In Confirmation Trial 1 , the cutting edge dullness increased from 0.05 to 0.14 while in Confirmation Trial 2 , the cutting edge dullness only increased 0.02 from 0.06 . This means there are more grains pulled out by bond fracture at the end of Confirmation Trial 2 while in Confirmation Trial 1, wheel wear is mainly attritious.

In summary, for the easy-to-grind bearing steel, no wheel loading was experienced in any of these trials. It was therefore concluded that the process was 
controlled by a steady wear mechanism with a tendency towards self-sharpening of the wheel, particularly at the high removal rate, rather than by a mechanism of wheel loading. For these conditions, the previous analysis and tests suggested that there would be no benefit in using a high-porosity grinding wheel. No further wheels were therefore tested for the easy-to grind material and these results are considered to provide strong support for the conclusion that high-porosity wheels offer no advantage for easy-to-grind materials.

\subsubsection{Inconel 718 aerospace alloy}

Previous analysis and test results suggested that for the difficult-to-grind material, wheel redress life tends to be limited by wheel loading. It was therefore decided to use a high-porosity and relatively large-grain CBN wheel for grinding the difficult-to-grind material. This combination assists effective coolant delivery into the wheel contact area and ease of chip removal with the long thin chips expected with a difficult-to-grind material.

The wheel used in this trial was the high-porosity large grain B151 wheel. The average effective porosity volume ratio of the wheel was 0.5 . The average active cutting edge density was about $20 / \mathrm{mm}^{2}$ depending on dressing conditions and grinding conditions. It was expected that with long thin chips there would be more loading. Pore loading is also expected to increase with grain wear flat adhesive loading. The wheel design and the grinding conditions were designed to make the chip volume small in relation to the pore volume.

\section{Direct-effect charts:}

A complete set of direct-effects charts for grinding Inconel 718 with the HP-B151 wheel may be found in Fig. 6.20. The tests were conducted at a removal-rate of 2 $\mathrm{mm}^{3} / \mathrm{mm}$.s which although much lower than used for the easy-to-grind material was considered to be a sensible removal-rate for a difficult-to-grind material. Reducing the removal-rate, reduces the chip thickness and is expected to reduce the problem of wheel loading. The extent of wheel loading observed depended on the dressing and grinding conditions employed. It was found that there was a tendency for some loading to be observed at the commencement of a test, but the 
loading problem reduced as the test progressed. Qualitatively, it was judged that the high-porosity wheel at high speed gave better performance compared with either the medium-porosity or high-porosity wheels in previous tests at low speed. Further evidence on wheel loading is presented in the discussion of the results.

From this figure, it can be seen that high wheelspeed was an advantage for workpiece quality, for G-ratio and for removal-rate but was a disadvantage for specific energy. This is consistent with the previous findings for easy-to-grind materials.

\section{Confirmation trials:}

As in previous trials for easy-to-grind materials, parameters were selected for confirmation trials. The trials were designed to demonstrate two contrasting situations for the difficult-to-grind material. The grinding conditions and results are presented below:

\section{Confirmation Trial 1}

For workpiece quality and high

\section{G-ratio:}

Up-dressing

Dressing overlap, $2 \mathrm{x}$

Dressing increment, $2 \mu \mathrm{m}$

Dressing passes, 10

High wheelspeed, $120 \mathrm{~m} / \mathrm{s}$

Workspeed, $36 \mathrm{~m} / \mathrm{min}$

Dwell, $10 \mathrm{~s}$

Dresser rotational speed, $42 \mathrm{~m} / \mathrm{s}$

$\mathrm{Q}_{\mathrm{w}}{ }_{\mathrm{w}}=2 \mathrm{~mm}^{3} / \mathrm{mm} . \mathrm{s}$

$\mathrm{V}_{\mathrm{w}}=4,100 \mathrm{~mm}^{3}$

$\mathrm{V}_{\mathrm{w}}^{\prime}=342 \mathrm{~mm}^{3} / \mathrm{mm}$

\section{Confirmation Trial 2}

For high removal rate:

Down-dressing

Dressing overlap, $2 \mathrm{x}$

Dressing increment, $10 \mu \mathrm{m}$

Dressing passes, 2

High wheelspeed, $120 \mathrm{~m} / \mathrm{s}$

Workspeed, $36 \mathrm{~m} / \mathrm{min}$

Dwell, $10 \mathrm{~s}$

Dresser rotational speed, $42 \mathrm{~m} / \mathrm{s}$

Q' ${ }^{\prime}=2 \mathrm{~mm}^{3} / \mathrm{mm}$.s

$\mathrm{V}_{\mathrm{w}}=4,100 \mathrm{~mm}^{3}$

$V_{w}^{\prime}=342 \mathrm{~mm}^{3} / \mathrm{mm}$

Results for these trials are listed below: 


\section{Confirmation Trial 1}

Mean specific energy

Mean Ra

Mean Rz

Mean roundness

Mean size holding

G-ratio (At $V_{w}=4100 \mathrm{~mm}^{3}$ )
$122 \mathrm{~J} / \mathrm{mm}^{3}$

$0.18 \mu \mathrm{m}$

$1.18 \mu \mathrm{m}$

$0.5 \mu \mathrm{m}$

$4.5 \mu \mathrm{m}$

85
Confirmation Trial 2

$73 \mathrm{~J} / \mathrm{mm}^{3}$

$0.45 \mu \mathrm{m}$

$3.0 \mu \mathrm{m}$

$0.6 \mu \mathrm{m}$

$2 \mu \mathrm{m}$

35

Confirmation Trial 1 was aimed at high quality and high G-ratio, Confirmation Trial 2 was aimed at conditions suitable for low specific energy and high-removal rate. For Confirmation Trial 2, the removal rate was maintained constant at 2 $\mathrm{mm}^{3} / \mathrm{mm}$.s as in Confirmation Trial 1 , but the dressing increment was increased from 2 microns in Confirmation Trial 1 to 10 microns in Confirmation Trial 2. This uses up fives times as much of the wheel but has the effect of reducing any residual loading. It also places more stress on the grains which become more susceptible to grain fracture [114]. Heavy dressing cuts tend to close up the wheel, requiring the abrasive bond to be eroded away to restore the open structure of the wheel. This was not expected to be a problem with a high-porosity wheel.

It can be seen that the conditions selected for high removal rate, have the effect of reducing specific energy but increasing roughness. The conditions selected for high removal rate also have the effect of reducing G-ratio. These results can be explained in terms of the effect of the dressing conditions on wheel topography. It was found that the cutting edge density after dressing in Confirmation Trial 1 was $25 / \mathrm{mm}^{2}$ while in Confirmation Trial 2 , the cutting edge density was only $16 / \mathrm{mm}^{2}$. Larger cutting edge density decreases workpiece surface roughness as previously discussed. It was also found that the cutting edge dullness showed similar results as in grinding AISI 52100. That is, there was more self-sharpening in Confirmation Trial 2 than in Confirmation Trial 1.

\section{Wheel life trial:}

A further trial was conducted to establish performance over a whole wheel redress life cycle. The grinding conditions employed were different from Confirmation 
Trial 1 in that the dwell was reduced from the previous value of $10 \mathrm{~s}$ to $2 \mathrm{~s}$ in order to increase productivity and reduce wear.

The results from the wheel life trial were as summarized below. Fig. 6.21 shows how the results varied during the trial.

$\begin{array}{ll}\text { Mean specific energy } & 120 \mathrm{~J} / \mathrm{mm}^{3} \\ \text { Mean Ra } & 0.26 \mu \mathrm{m} \\ \text { Mean Rz } & 1.6 \mu \mathrm{m} \\ \text { Mean roundness } & 0.7 \mu \mathrm{m} \\ \text { Mean size holding } & 2 \mu \mathrm{m} \\ \text { G-ratio } & 710 \\ V_{w}=10,530 \mathrm{~mm}^{3} & \\ V_{w}^{\prime}=878 \mathrm{~mm}^{3} / \mathrm{mm} & \end{array}$

It can be seen that after an initial decrease, specific energy remained constant. This shows that a stable grinding wheel topography had been achieved. It can be seen that the specific energy was unchanged from Confirmation Trial 1. This is mainly because the dressing conditions and the removal-rate were unchanged and the wheel topography was similar to the previous case in Confirmation Trial 1. The mean surface roughness was higher than in Confirmation Trial 1, which is considered to be mainly due to the shorter dwell. The G-ratio at a value of 710 was greatly increased compared to the value of 85 in Confirmation Trial 1. This is mainly because in a short test the G-ratio is strongly affected by the initial rapid wheel wear whereas in this wheel life trial where a stable wheel condition was achieved the G-ratio was much greater. The specific volume of material removed was $878 \mathrm{~mm}^{3} / \mathrm{mm}$.

These results can be explained in terms of wheel topography. It was found that the cutting edge density at the beginning of the trial was $26 / \mathrm{mm}^{2}$ while at the end it was $13 / \mathrm{mm}^{2}$. It was also found that the cutting edge dullness changed from 0.04 to 0.13 . That means cutting edge wear flat area increased with grinding. 
Large benefits were found from using high-speed vitrified CBN grinding with a high-porosity wheel for Inconel 718 on the Suprema machine as clearly shown by the results for the wheel life trial. Grinding Inconel 718 workpieces with a medium porosity wheel either aluminium oxide or with vitrified CBN at a wheel speed of $45 \mathrm{~m} / \mathrm{s}$ resulted in relatively poor grinding performance as reported previously [115].

\subsection{Discussion of experimental results}

From the experimental results, it can be seen that workpiece material, wheel speed and wheel porosity can have a strong effect on grinding performance and hence on the grinding wheel requirements.

\subsubsection{Porosity volume ratio}

For the relatively easy-to-grind M2 tool steel, wheel loading was not found to be a severe problem. For such a material, it was found that a high-porosity wheel did not show advantages under conditions where wheel wear was of more significance than wheel loading. This is because under these conditions, a highporosity wheel increases workpiece roughness and wheel wear-rate leading to shorter wheel life and lower G-ratio. At higher speeds of $110 \mathrm{~m} / \mathrm{s}$, workpiece surface roughness ground by the high-porosity wheel was improved at the expense of higher specific energy. When grinding M2, slower attritious wheel wear was evidenced at the beginning of grinding. As the wheel approached the end of the redress life, bond wear increased and active cutting edge density decreased more quickly. Wheel porosity volume ratio reduced by $14 \%$ in grinding. The medium-porosity wheel also showed advantages of an increased wheelspeed of $120 \mathrm{~m} / \mathrm{s}$ in external grinding when grinding AISI 52100 bearing steel in a situation where whee! loading was not a problem.

As discussed in Chapter 5, high porosity combined with fine grains creates a greater tendency for the grains to agglomerate together and reduce the active cutting edge density. Reduced active cutting edge density leads to larger uncut chip thickness, increased workpiece surface roughness and reduced specific 
energy. Also, high porosity leads to low wheel scratch hardness, which accelerates wheel bond wear and shortens the wheel life.

High porosity helps avoid wheel loading. When grinding M2 steel in internal grinding, the ratio of the mean volume of chips removed by each grain to the mean volume of each pore is only about $2 \%$ for the HP-B64 wheel and $7 \%$ for the MP-B91 wheel. It is considered that there was adequate space for chips to be accommodated in both wheels for the removal rate required in the experiments. The redress life of the wheels was limited more by wheel wear than by wheel loading.

For a difficult-to-grind workpiece material such as Inconel 718, wheel loading was a problem. For such a material, a high-porosity wheel was much more effective than a medium-porosity wheel. A high-porosity wheel was less prone to experience wheel loading and gave longer wheel life and higher G-ratio. Wheel adhesive loading was observed to occur on some of the grain tips at the beginning of grinding. Then local adhered material on the grain tips formed a compact mass and pulled off. Fig. 6.22 shows the three stages. These observations confirmed the observation of Yossifon and Rubenstein [110-112]. With small pores, adhered material is more likely to bridge across from one grain to another. Under these circumstances, the adhered material is less easily detached. A high-porosity wheel is therefore expected to be less prone to loading as was found in practice.

Higher wheelspeed helped to improve grinding performance even with the difficult-to-grind material. The results obtained support the conclusion that a high-porosity wheel is an advantage under conditions where there would otherwise be a loading problem as when grinding difficult-to-grind materials. It also helps to reduce loading using larger grain wheels, and small chip thickness.

\subsubsection{Wheel speed}

The wheelspeed results can be explained by further consideration of the analysis of adhesive wear and loading wear. 
Combining equations $4.12,4.13,4.14,4.15$ and 4.23 , it may be argued that, the adhesive loading on the top of cutting edge is:

$$
V_{s}=c k_{a} \cdot x \cdot \frac{1}{C} \frac{v_{w}}{v_{s}} \frac{f l^{0.5}}{0.003 * 27 * \tan ^{3} \alpha} \sqrt{\frac{H_{b}}{H_{s}}} \sqrt{\frac{a_{e}}{d_{e}}}
$$

where,

$$
\begin{aligned}
& c \text { is a coefficient, } \\
& k_{a} \text { is the ratio of adhesive loading volume to adhesive wear volume. }
\end{aligned}
$$

From this equation, it can be seen that, if the ratio of workpiece bulk hardness to surface hardness does not change, the adhesive loading decreases with increasing wheelspeed (the effect of $f$ can be neglected if the change is small compared to that of $\mathbf{v}_{\mathbf{s}}$ ).

However, the ratio of workpiece bulk hardness to surface hardness does change with grinding temperature, which is a likely consequence of the change in wheel speed.

Table 6.10 shows the grinding temperature evaluated according to the Rowe model [82-86]. It can be seen that grinding temperature increases with speed. Also, grain surface temperatures are all calculated to exceed $1000^{\circ} \mathrm{C}$ in these trials.

Table 6.10 Calculated grinding temperatures when grinding Inconel 718

\begin{tabular}{|l|l|l|}
\hline \multicolumn{3}{|c|}{ Internal grinding (J\&S 1300X). Workpiece: Inconel 718 } \\
\hline Wheelspeed (m/s) & $\begin{array}{l}\text { Mean workpiece back } \\
\text { ground temperature }\left({ }^{\circ} \mathrm{C}\right)\end{array}$ & $\begin{array}{l}\text { Mean grain surface } \\
\text { temperature }\left({ }^{\circ} \mathrm{C}\right)\end{array}$ \\
\hline $50 \mathrm{~m} / \mathrm{s}$ & 1269 & 1925 \\
\hline $60 \mathrm{~m} / \mathrm{s}$ & 1333 & 2000 \\
\hline External grinding (J\&S Suprema); workpiece: Inconel 718 \\
\hline $60 \mathrm{~m} / \mathrm{s}$ & 1147 & 1900 \\
\hline $120 \mathrm{~m} / \mathrm{s}$ & 1576 & 2595 \\
\hline
\end{tabular}


Most steels begin softening at temperatures in the region of $500^{\circ} \mathrm{C}$. Below 500 ${ }^{\circ} \mathrm{C}$, the change in deformation stress is relatively small although the cutting conditions are changed [109]. At low temperatures, the effects on workpiece material work-hardening, strain-rate hardening and high-temperature softening tend to balance out, and the basic characteristics of the material do not change very rapidly. This is called Stage 1 in Fig. 6.23, which shows the changes of the material hardness with temperature. The characteristics of the material change more rapidly above a transition temperature depending on the particular material. In Stage 2, the deformation-stress changes more greatly with temperature and with strain-rate. The transition temperature for $\mathrm{M} 2$ steel is approximately $550^{\circ} \mathrm{C}$ and for Inconel 718 it is approximately $750^{\circ} \mathrm{C}$. In Stage 3, the characteristics of the material become more stable again.

It is clear that temperature plays a very important role in affecting material characteristics. The background temperature is likely to be below $500^{\circ} \mathrm{C}$ when grinding steels with a sharp grinding wheel. However, the background temperature is more likely to be above $750^{\circ} \mathrm{C}$ when grinding super-alloys such as Inconel 718. If the background temperature rises above the transition temperature, material softening occurs with increased risk of wheel loading.

In Stage 1, the material is below the softening temperature; the ratio of workpiece bulk hardness to surface hardness does not change much, although the grinding temperature increases with an increase in wheel speed and adhesive loading decreases. When the material begins to soften, i.e. in Stage 2 , the ratio of workpiece bulk hardness to surface hardness increases with increased temperature. At this stage, the surface hardness of the material reduces rapidly while the bulk hardness remains almost unchanged with surface background temperature. If the increase of $\sqrt{H_{b} / H_{s}}$ exceeds the effect of increase of $\mathrm{v}_{\mathrm{s}}$, the adhesive loading will increase. If temperature is further increased with wheel speed, i.e. in Stage 3, the workpiece material approaches the melting point and the ratio of workpiece bulk hardness to surface hardness returns to a stable condition. It is therefore proposed that adhesive loading will decrease again. Fig. 6.24 shows 
the proposed trend of adhesive loading with increase of wheelspeed for a particular set of grinding conditions and a particular grinding distance. The above analysis offers one explanation based on the theoretical analysis of why higher speed has advantages for a difficult-to-grind material such as Inconel 718. 


\section{Chapter 7 Theoretical results for wheel wear and loading}

\subsection{Prediction of wheel wear and loading}

\subsubsection{Calibration of parameters for prediction of wheel wear}

Wheel adhesive loading was not considered in the wheel wear model. From the experiments discussed in Chapter 6, no obvious wheel adhesive loading was found when grinding M2 workpiece material. So, the calibration of parameters for the grinding wheel wear prediction was performed by fitting the experimental results obtained in internal grinding for the M2 workpiece material. Based on this technique, further results can be predicted for internal grinding with easy-to-grind workpiece materials in cases where there is no obvious wheel adhesive loading. This technique can be used to explore the effect of different wheel structure and wheelspeed on grinding performance.

The calibration of parameters for prediction of wheel wear is listed below:

(i) Based on the experimental measurements of the wheel surface according to Chapter 6, the initial average active cutting edge radius after dressing, was set equal to $r_{a 0}=2.5 \mu \mathrm{m}$.

(ii) Based on measurements of the wheel surface (Method described in Chapter 3), the initial values of average active cutting edge density after optimised dressing of different wheels are listed in Table 7.1. 
Table 7.1 Relationship between initial active cutting edge density and percent of pore volume

\begin{tabular}{|c|c|c|c|}
\hline Wheel & $\begin{array}{c}\text { initial active cutting } \\
\text { edge density Ca }\end{array}$ & $\begin{array}{c}\text { Maximum } \\
\text { grain density } \\
\text { on wheel } \\
\text { surface } \\
\text { (Equation 5.3) }\end{array}$ & $\begin{array}{c}\text { Percentage of } \\
\text { pore volume } \\
\mathrm{Vp}\end{array}$ \\
\hline B64-150VX & 40 & 174 & 0.4 \\
\hline B91-150V1 & 60 & 86 & 0.2 \\
\hline B91-150 VR & 40 & 86 & 0.3 \\
\hline
\end{tabular}

The initial average active cutting edge density values were curve-fitted to give the following equation.

$$
C a=m \cdot \frac{V g \cdot d g}{\frac{4}{3} \pi \cdot\left(\frac{d g}{2}\right)^{3}} \cdot(-2 \cdot 4 \cdot V p+1.1) \quad \text { for } \quad 0.2 \leq V p \leq 0.4
$$

where, $m$ is the ratio of the cutting edge density to the grain density. The value, $\mathrm{m}=1.2$ is used here according to Chapter 3 .

(iii) Force calculation: The total tangential grinding force was obtained from the grinding power,

$F_{t}=\frac{P}{v_{s}}$

It was assumed that the lowest tangential grinding force is not affected by incomplete conditioning. About $35 \%$ of the total energy was assumed to be related to cutting $[79,81]$

Thus,

$$
F_{t c}=\frac{0.35 . P}{v_{s}}
$$

Based on Shaw's assumption [61], the tangential cutting force was expressed as

$$
F_{t c}=\frac{a}{h_{\max }}
$$

where, $a$ is a coefficient. 
Based on the grinding trials for the M2 workpiece material at a wheelspeed of 45 $\mathrm{m} / \mathrm{s}$, values of tangential cutting force with varying maximum uncut chip thickness are shown in Table 7.2. The coefficient a in Equation 7.4 was evaluated.

Table 7.2: Tangential cutting force and maximum uncut chip thickness when grinding M2 workpiece material at a wheel speed of $45 \mathrm{~m} / \mathrm{s}$

\begin{tabular}{|c|c|c|c|}
\hline Wheel & $\mathrm{h}_{\max }\left(10^{-5} \mathrm{~mm}\right)$ & $\mathrm{F}_{\mathrm{tc}}(\mathrm{N})$ & $\mathrm{a}$ \\
\hline B64-150 VX & 2.0 & 2.8 & $5.6 \times 10^{-5}$ \\
\hline B91-150V1 & 1.5 & 3.8 & $5.7 \times 10^{-5}$ \\
\hline
\end{tabular}

A mean value of a in Equation 7.3, gives

$$
F_{t c}=\frac{5.7 \times 10^{-5}}{h_{\max }}
$$

Based on the grinding trials, for the M2 workpiece material at a wheelspeed of 60 $\mathrm{m} / \mathrm{s}$, the tangential cutting force with varying maximum uncut chip thickness is shown in Table 7.3. The constant a in Equation 7.3 was evaluated.

Table 7.3: Tangential cutting force and maximum uncut chip thickness when grinding M2 workpiece material at a wheel speed of $60 \mathrm{~m} / \mathrm{s}$

\begin{tabular}{|c|c|c|c|}
\hline Wheel & $\mathrm{h}_{\max }\left(10^{-5} \mathrm{~mm}\right)$ & $\mathrm{F}_{\mathrm{tc}}(\mathrm{N})$ & $\mathrm{a}$ \\
\hline $\mathrm{B} 64-150 \mathrm{VX}$ & 1.8 & 3.4 & $6.1 \times 10^{-5}$ \\
\hline B91-150V1 & 1.3 & 3.7 & $4.8 \times 10^{-5}$ \\
\hline
\end{tabular}

Substituting a into Equation 7.3,

$$
F_{t c}=\frac{5.5 \times 10^{-5}}{h_{\max }}
$$

Based on the grinding trials, for the M2 workpiece material at a wheelspeed of $110 \mathrm{~m} / \mathrm{s}$, the tangential cutting force with varying maximum uncut chip thickness is shown in Table 7.4. The constant a in Equation 7.3 was evaluated. 
Table 7.4: Tangential cutting force and maximum uncut chip thickness when grinding M2 workpiece material at a wheel speed of $110 \mathrm{~m} / \mathrm{s}$

\begin{tabular}{|c|c|c|c|}
\hline Wheel & $\mathrm{h}_{\max }\left(10^{-5} \mathrm{~mm}\right)$ & $\mathrm{F}_{\mathrm{tc}}(\mathrm{N})$ & $\mathrm{a}$ \\
\hline $\mathrm{B} 64-150 \mathrm{VX}$ & 1.3 & 3.7 & $4.8 \times 10^{-5}$ \\
\hline
\end{tabular}

Substituting a into Equation 7.3 using a mean value of a,

$$
F_{t c}=\frac{4.8 \times 10^{-5}}{h_{\max }}
$$

The coefficient a was obtained by curve-fitting,

$$
a=\left(-1.4 \times 10^{-2} \cdot v_{s}+6.3\right) * 10^{-5} \quad 45 \leq v_{s} \leq 110
$$

From Equation 4.10 and 4.11 , the average contact pressure $\bar{p}$ between the wear flats and the workpiece was obtained as follows,

$$
\bar{p}=\frac{\frac{d F_{t}}{d A}-\frac{d F_{t c}}{d A}}{\mu b\left(d_{e} a_{e}\right)^{1 / 2}}
$$

Re-arranging,

$$
\bar{p}=\frac{\frac{d F_{t}}{d V_{w}}-\frac{d F_{t c}}{d V_{w}}}{\left(\mu b\left(d_{e} a_{e}\right)^{1 / 2}\right) \frac{d A}{d V_{w}}}
$$

where, $\mathrm{V}_{\mathrm{w}}$ is the specific volume of material removed.

Tables 7.5, 7.6, 7.7 show the calculated values of parameters based on grinding trials. 
Table 7.5: Parameter value in Equation 7.9 at a wheelspeed of $45 \mathrm{~m} / \mathrm{s}$ Wheel: B91-150 V1:

\begin{tabular}{|c|c|c|c|c|}
\hline $\begin{array}{c}\frac{d F_{1}}{d V_{w}} \\
\mathrm{~N} /\left(\mathrm{mm}^{3} / \mathrm{mm}\right)\end{array}$ & $\begin{array}{c}\frac{d F_{\kappa}}{d V_{w}} \\
\mathrm{~N} /\left(\mathrm{mm}^{3} / \mathrm{mm}\right)\end{array}$ & $\begin{array}{c}\frac{d A}{d V_{w}} \\
1 /\left(\mathrm{mm}^{3} / \mathrm{mm}\right)\end{array}$ & $\mu$ & $\begin{array}{c}\bar{p} \\
\mathrm{~N} / \mathrm{mm}^{2}\end{array}$ \\
\hline 0.0029 & -0.00027 & $3.6 \times 10^{-6}$ & 0.5 & 147 \\
\hline
\end{tabular}

Wheel: B64-150 VX:

\begin{tabular}{|c|c|c|c|c|}
\hline $\begin{array}{c}\frac{d F_{1}}{d V_{w}} \\
\mathrm{~N} /\left(\mathrm{mm}^{3} / \mathrm{mm}\right)\end{array}$ & $\begin{array}{c}\frac{d F_{w}}{d V_{w}} \\
\mathrm{~N} /\left(\mathrm{mm}^{3} / \mathrm{mm}\right)\end{array}$ & $\begin{array}{c}\frac{d A}{d V_{w}} \\
1 /\left(\mathrm{mm}^{3} / \mathrm{mm}\right)\end{array}$ & $\mu$ & $\begin{array}{c}\bar{p} \\
\mathrm{~N} / \mathrm{mm}^{2}\end{array}$ \\
\hline 0.0030 & -0.00033 & $6.4 \times 10^{-6}$ & 0.5 & 87 \\
\hline
\end{tabular}

The mean value $\bar{p}=117 \mathrm{~N} / \mathrm{mm}^{2}$ was used in the grinding wheel wear prediction.

Table 7.6: Parameter value in Equation 7.9 at a wheelspeed of $60 \mathrm{~m} / \mathrm{s}$ Wheel: B91-150 V1:

\begin{tabular}{|c|c|c|c|c|}
\hline $\begin{array}{c}d F_{i} \\
d V_{*} \\
\mathrm{~N} /\left(\mathrm{mm}^{3} / \mathrm{mm}\right)\end{array}$ & $\begin{array}{c}\frac{d F_{*}}{d V_{w}} \\
\mathrm{~N} /\left(\mathrm{mm}^{3} / \mathrm{mm}\right)\end{array}$ & $\begin{array}{c}\frac{d A}{d V_{w}} \\
1 /\left(\mathrm{mm}^{3} / \mathrm{mm}\right)\end{array}$ & $\mu$ & $\begin{array}{c}\bar{p} \\
\mathrm{~N} / \mathrm{mm}^{2}\end{array}$ \\
\hline 0.0018 & -0.00043 & $10.0 \times 10^{-6}$ & 0.5 & 43.3 \\
\hline
\end{tabular}

Wheel: B64-150 VX:

\begin{tabular}{|c|c|c|c|c|}
\hline $\begin{array}{c}d F_{i} \\
d V_{w} \\
\mathrm{~N} /\left(\mathrm{mm}^{3} / \mathrm{mm}\right)\end{array}$ & $\begin{array}{c}\frac{d F_{\kappa}}{d V_{w}} \\
\mathrm{~N} /\left(\mathrm{mm}^{3} / \mathrm{mm}\right)\end{array}$ & $\begin{array}{c}\frac{d A}{d V_{w}} \\
1 /\left(\mathrm{mm}^{3} / \mathrm{mm}\right)\end{array}$ & $\mu$ & $\begin{array}{c}\bar{p} \\
\mathrm{~N} / \mathrm{mm}^{2}\end{array}$ \\
\hline 0.0018 & -0.00073 & $7.3 \times 10^{-6}$ & 0.5 & 60.0 \\
\hline
\end{tabular}

The mean value $\bar{p}=52 \mathrm{~N} / \mathrm{mm}^{2}$ was used in the grinding wheel wear prediction. 
Table 7.7: Parameter value in Equation 7.9 at a wheelspeed of $110 \mathrm{~m} / \mathrm{s}$ Wheel: B64-150 VX:

\begin{tabular}{|c|c|c|c|c|}
\hline $\begin{array}{c}\frac{d F_{i}}{d V_{w}} \\
\mathrm{~N} /\left(\mathrm{mm}^{3} / \mathrm{mm}\right)\end{array}$ & $\begin{array}{c}\frac{d F_{c}}{d V_{w}} \\
\mathrm{~N} /\left(\mathrm{mm}^{3} / \mathrm{mm}\right)\end{array}$ & $\begin{array}{c}\frac{d A}{d V_{w}} \\
1 /\left(\mathrm{mm}^{3} / \mathrm{mm}\right)\end{array}$ & $\mu$ & $\begin{array}{c}\bar{p} \\
\mathrm{~N} / \mathrm{mm}^{2}\end{array}$ \\
\hline 0.00025 & -0.00034 & $1.5 \times 10^{-6}$ & 0.5 & 68 \\
\hline
\end{tabular}

The mean value $\bar{p}=68 \mathrm{~N} / \mathrm{mm}^{2}$ was used in the grinding wheel wear prediction.

The contact pressure obtained by curve-fitting was,

$$
\bar{p}=-0.3 v_{s}+101.0 \quad 45 \leq v_{s} \leq 110
$$

(iv) Workpiece surface roughness calibration:

The empirical constants a 1 and a2 in Equation 5.68 were calibrated using the values given below:

Table 7.8: Calibration of workpiece surface for $M 2$

\begin{tabular}{|c|c|c|c|c|}
\hline Wheel & B64-150 VX & B91-150 V1 & B64-150 VX & B91-150 V1 \\
\hline $\begin{array}{c}\text { Wheel diameter } \\
(\mathrm{mm})\end{array}$ & 15.0 & 14.8 & 15.4 & 13.7 \\
\hline $\begin{array}{c}\text { Wheel speed } \\
(\mathrm{m} / \mathrm{s})\end{array}$ & 45 & 45 & 60 & 55 \\
\hline $\begin{array}{c}\text { Workpiece speed } \\
\mathrm{v}_{\mathrm{w}}(\mathrm{m} / \mathrm{s})\end{array}$ & 5 & 5 & 5 & 5 \\
\hline $\begin{array}{c}\text { cutting edge } \\
\text { spacing L (mm) } \\
\text { Workpiece } \mathrm{R}_{\mathrm{a}} \\
\text { surface } \\
\text { roughness }(\mu \mathrm{m})\end{array}$ & 0.18 & 0.15 & 0.17 & 0.15 \\
\hline a1 $\left(\mu \mathrm{m}{ }^{0.6}\right)$ & 0.18 & 0.15 & 0.17 & 0.22 \\
\hline a2 $(\mu \mathrm{m})$ & & \multicolumn{3}{|c|}{646.0} \\
\hline
\end{tabular}


So, values of workpiece surface roughness were obtained from,

$R a=646.0 \times\left(\frac{v_{w} \cdot L}{v_{s} \cdot d_{s}^{1 / 2}}\right)^{0.8}-0.2$

(v) Parameters adjusted to obtain a fit between theory and experiment include the following:

The grinding wheel attritious wear rate: $K=2 \times 10^{-7}$;

Coefficients in probability of bond and grain fracture: $\mathrm{A}=1.0 \times 10^{-2} ; \mathrm{c}=5.0 \times 10^{-5}$

The probability of new active cutting edge appearing after bond and grain fracture: $\operatorname{Pr}=0.1$.

\subsubsection{Calibration of parameters for prediction of wheel loading}

Not only wheel wear but also wheel adhesive loading was considered in the wheel loading model. From the experiments described in Chapter 6, it was found that there was an adhesive loading problem when grinding Inconel 718 workpiece material. The calibration of the parameters for the grinding wheel loading prediction was based on the internal grinding results for the Inconel 718 workpiece material. The parameters were obtained by curve-fitting the experimental results. Using the model, further internal grinding results can be predicted for workpiece materials, which tend to cause wheel adhesive loading. The effect of varying wheel structure and wheelspeed on grinding performance can be predicted.

(i) Based on the experimental measurements of the wheel surface according to Chapter 6, the initial average active cutting edge radius after dressing, was set equal to $r_{a 0}=2.5 \mu \mathrm{m}$.

(ii) Force calculation: The tangential grinding force and tangential cutting force were obtained from the grinding power and from Equations 7.1 and 7.2 .

Based on the grinding trials, for the Inconel 718 workpiece material at a wheel speed of $50 \mathrm{~m} / \mathrm{s}$, the tangential cutting force with the different values of maximum uncut chip thickness is shown in Table 7.9. The constant a in Equation 7.4 was 
calculated from the measured lowest force which it was assumed was unaffected by incomplete conditioning. The theoretical maximum uncut chip thickness in this grinding condition is also given.

Table 7.9: Tangential cutting force and maximum uncut chip thickness when grinding Inconel 718 workpiece material at a wheel speed of $50 \mathrm{~m} / \mathrm{s}$

\begin{tabular}{|l|l|l|l|}
\hline Wheel & $\mathrm{h}_{\max }\left(10^{-5} \mathrm{~mm}\right)$ & $\mathrm{F}_{\mathrm{tc}}(\mathrm{N})$ & $\mathrm{a}$ \\
\hline B91-150VR & 1.5 & 6.8 & $10.1 \times 10^{-5}$ \\
\hline
\end{tabular}

Substituting a into Equation 7.4,

$$
F_{t c}=\frac{10.1 \times 10^{-5}}{h_{\max }}
$$

Based on the grinding trials, for the Inconel 718 workpiece material at a wheel speed of $60 \mathrm{~m} / \mathrm{s}$, the tangential cutting force with maximum uncut chip thickness is shown in Table 7.10. The constant a in Equation 7.4 was calculated from this table.

Table 7.10: Tangential cutting force and maximum uncut chip thickness when grinding Inconel 718 workpiece material at a wheel speed of $60 \mathrm{~m} / \mathrm{s}$

\begin{tabular}{|l|l|l|l|}
\hline Wheel & $h_{\max }\left(10^{-5} \mathrm{~mm}\right)$ & $F_{\mathrm{tc}}(\mathrm{N})$ & $\mathrm{a}$ \\
\hline B91-150V1 & 1.3 & 6.0 & $7.8 \times 10^{-5}$ \\
\hline
\end{tabular}

Substituting a into Equation 7.4,

$$
F_{t c}=\frac{7.8 \times 10^{-5}}{h_{\max }}
$$

The coefficient a determined by curve-fitting was,

$$
a=\left(-2.3 \times 10^{-1} \cdot v_{s}+21.6\right) * 10^{-5} \quad 50 \leq v_{S} \leq 60
$$


Table 7.11 shows the parameters evaluated from the Inconel 718 internal grinding trials in order to calculate the average contact force between the workpiece and the cutting edges using Equation 7.10.

\section{Table 7.11: Parameter value in Equation 7.10}

Wheel: B91-150 VR: Wheel speed: $50 \mathrm{~m} / \mathrm{s}$

\begin{tabular}{|l|l|l|l|l|}
\hline $\begin{array}{l}d F_{\ell} \\
d V_{w}\end{array}$ & $\frac{d F_{t c}}{d V_{w}}$ & $\frac{d A}{d V_{w}}$ & $\mu$ & $\bar{p}$ \\
$\mathrm{~N} /\left(\mathrm{mm}^{3} / \mathrm{mm}\right)$ & $\mathrm{N} /\left(\mathrm{mm}^{3} / \mathrm{mm}\right)$ & $1 /\left(\mathrm{mm}^{3} / \mathrm{mm}\right)$ & & $\mathrm{N} / \mathrm{mm}^{2}$ \\
\hline-0.0093 & -0.0016 & $3.0 \times 10^{-6}$ & 0.5 & 442 \\
\hline
\end{tabular}

Wheel: B91-150 VR: Wheel speed: $60 \mathrm{~m} / \mathrm{s}$

\begin{tabular}{|l|l|l|l|l|}
\hline $\begin{array}{l}d F_{t} \\
d V_{w} \\
\mathrm{~N} /\left(\mathrm{mm}^{3} / \mathrm{mm}\right)\end{array}$ & $\begin{array}{l}\frac{d F_{t c}}{d V_{w}} \\
\mathrm{~N} /\left(\mathrm{mm}^{3} / \mathrm{mm}\right)\end{array}$ & $\begin{array}{l}\frac{d A}{d V_{w}} \\
1 /\left(\mathrm{mm}^{3} / \mathrm{mm}\right)\end{array}$ & & $\bar{p}$ \\
\hline-0.012 & -0.00074 & $6.1 \times 10^{-6}$ & 0.5 & $\mathrm{~N} / \mathrm{mm}^{2}$ \\
\hline
\end{tabular}

The contact pressure was fitted as

$$
\bar{p}=-12.4 . v_{s}+1062.0 \quad 50 \leq v_{s} \leq 60
$$

(iii) As mentioned in Chapter 5, the coefficient $\mathrm{k}_{\mathrm{a}}$ is used to reflect the volume ratio of particles adhered to the cutting edges to the total number of adhesive wear particles. It was assumed that the size of adhesive particles on the wear flats were normally distributed on an average wear flat diameter of 20 microns. That is, the minimum diameter of adherent particle tends to 0 and the maximum diameter is 20 microns. The ratio of the volume of loose particles to the total volume of adhesive particles will be 0.3 if the size of adherent particles is 3 times smaller than loose particles [101]. When cutting edges with adhered particles on the wear flat, contact the workpiece again, the adhered particles may accumulate on the cutting edges and may attach to the workpiece.

(iv) Workpiece surface roughness calibration: 
Table 7.12: Calibration of workpiece surface for Inconel 718

\begin{tabular}{|c|c|c|}
\hline Wheel & B91-150 VR & B91-150 VR \\
\hline Wheel diameter $(\mathrm{mm})$ & 15.8 & 14.9 \\
\hline Wheel speed $(\mathrm{m} / \mathrm{s})$ & 50 & 60 \\
\hline Workpiece speed $\mathrm{v}_{\mathrm{w}}(\mathrm{m} / \mathrm{s})$ & 4 & 4 \\
\hline cutting edge spacing $\mathrm{L}(\mathrm{mm})$ & 0.22 & 0.19 \\
\hline Workpiece $\mathrm{R}_{\mathrm{a}}$ surface roughness $(\mu \mathrm{m})$ & 0.18 & 0.13 \\
\hline $\mathrm{a} 1\left(\mu \mathrm{m}^{0.6}\right)$ & \multicolumn{2}{|c|}{16.7} \\
\hline $\mathrm{a} 2(\mu \mathrm{m})$ & \multicolumn{3}{|c|}{$3.7 \times 10^{-2}$} \\
\hline
\end{tabular}

So, values of workpiece surface roughness were obtained from,

$R a=16.7 \times\left(\frac{v_{w} \cdot L}{v_{s} \cdot d_{s}^{1 / 2}}\right)^{0.8}-0.037$

(v) Other parameters were adjusted to make the predicted values match the measured values. The parameters adjusted in this way to suit the experimental trials included: $c=40000 ; k=0.7 ; B_{b}=1$.

\subsection{Predicted values of grinding wheel wear and loading}

The grinding wheel wear predictions are shown in Fig. 7.1 to 7.3. It can be seen that when the percentage of grain volume and pore volume is fixed, a smaller the grain size, causes a greater initial active cutting edge density and wheel wear is reduced. The wear flat is smaller and workpiece surface roughness is reduced. When the wheel specification is fixed, a larger percentage of pore volume, and reduces the initial active cutting edge density. The wear flat is larger and the workpiece surface roughness is greater. When the wheel specification is fixed, a higher wheelspeed, reduces the wheel wear, reduces the wear flat and reduces workpiece surface roughness.

The predictions of grinding-wheel loading are shown in Fig. 7.4 to 7.6. The predictions of grinding performance including both wheel wear and loading are similar to the predictions including wheel wear only. In this prediction, higher 
wheelspeed improves grinding performance. At a wheelspeed of $90 \mathrm{~m} / \mathrm{s}$, the adhesive wear coefficient and probability of pore loading increase a little and then decrease during the course of grinding.

\subsection{Discussion}

In the model of wheel wear and loading, wheel structure, grinding force and grinding conditions are all considered. The coefficients employed in the simulation were adjusted by reference to experimental results in which different wheel structures and wheelspeeds were employed. The model of wheel wear is suitable for a grinding situation in which adhesive loading can be ignored. While the model of wheel loading is suitable for a grinding situation in which adhesive loading and pore loading need to be considered. From these models, more grinding performance results can be predicted by inputting different wheel structure parameters and different wheelspeeds. The results are subject to the initial assumptions remaining valid. In practice, other factors may come into play such as the onset of loading in a case where loading had not previously been significant.

\subsubsection{Effect of wheel structure}

The wheel structure refers to the grain size, percentage of abrasive volume, percentage of pore volume and wheel hardness. The previous theoretical analysis in Chapter 5 was based on the assumption that the cutting edge density of the grains is a constant when the abrasive volume and pore volume are fixed. In this case, grain size cannot affect cutting edge density if the number of cutting edges on each grain is assumed to be a constant. As a result, the smaller the grain size, the higher is the abrasive volume and the lower is the pore volume leading to increased cutting edge density. The increased number of cutting edges leads to higher specific energy due to the 'size effect'. In this case, wheel wear and loading are higher after the same material volume is removed. The increased number of cutting edges also leads to lower surface roughness. The lower is the surface roughness of the workpiece, the longer it should be possible to maintain the surface roughness of the workpiece within the specified limit. The wheel life 
should also be increased. The power and the grinding temperature are also increased, which may cause thermal problems and wheel loading.

Wheel scratch hardness is another factor, which affects wheel wear and loading. It has less effect on grinding performance compared to that of grinding force in this model.

\subsubsection{Effect of wheel speed}

Based on Equation 3.3, higher wheelspeed leads to a smaller uncut chip thickness. Due to the smaller uncut chip thickness, the grinding force on each cutting edge is smaller. So, in this prediction, at higher wheelspeed, wheel wear is lower and surface roughness of the workpiece is reduced. In this case, it should be possible to maintain the surface roughness of the workpiece longer within the specified limit. The wheel life will be increased.

In wheel loading, the grinding performance is greatly improved by using high wheelspeed. The main reason is the lower grinding force and lower rate of attritious wear of the wheel.

\subsection{Suggestions for optimisation of grinding wheels}

There are four factors which affect the use of CBN grinding wheels in precision grinding: the material to be ground which includes the properties of wheel hardness and adhesive character; the surface roughness required; the coolant delivery and wheelspeed.

When grinding materials which load, high-porosity wheels are suggested for increased chip space and coolant delivery. High porosity weakens the wheel and makes it easier for loaded grains to be pulled out. When grinding an easy-to-grind material, conventional porosity wheels are suggested for lower workpiece roughness and increased redress life.

In order to achieve low roughness and good surface integrity, sharp wheels with smaller grain size, are preferable. However, not all wheels of small grain size 
produce low surface roughness, grain agglomeration and wheel porosity are other factors, which affect active cutting edge density and hence surface roughness.

Coolant delivery is important for the ability to lubricate, conduct heat and carry swarf from the grinding surface. Decreasing wheel porosity is possible when using effective coolant delivery.

Based on the previous theoretical and experimental work, it has been found that higher wheelspeed helps to improve grinding performance. High wheelspeed brings higher grinding temperature and requires a sharper wheel to guarantee surface integrity. High porosity is helpful because it is good for coolant delivery.

In order to design a suitable wheel for a particular grinding condition, it is important to decide which parameter plays the most significant role. 


\section{Chapter 8 Discussion and Conclusions}

\subsection{Discussion}

Feasible measurement and observation techniques for the changing topographical features of vitrified CBN wheels in grinding were investigated. This comparison includes measurement resolution, measuring depth, ease of application, and data analysis and interpretation. Stylus instrument was not recommended for measuring a CBN wheel surface due to the hardness of CBN and stylus wear. Soft replicas were not recommended for use with stylus instruments due to inaccuracy of measurement. Optical interferometer was found to be a good method to measure vitrified $\mathrm{CBN}$ wheel topography. The laser triangular measurement provided a method for 3D measurement of a large area both of a wheel or of a replica but with reduced accuracy compared with interferometry. Microscope is helpful to understand the measurement results. In addition, replication technique has been compared and analyzed. A new developed replica material with high resolution ( 0.1 microns) and easy operation, Microset 101, had been employed as negative replica material in the experimental work.

A set of parameters which describe the wheel characteristics including active cutting edge density, cutting edge dullness, effective porosity volume ratio and wheel scratch hardness were defined. The four parameters reflect basic wheel characteristics. There were many more active cutting edges in grinding than expected based on chip thickness formulae mainly due to wheel deflection in grinding. The active depth of the grinding wheel above which cutting edges were active needs to be determined for each grinding system and measurement technique. A parametric study of the effects of wheel characteristics to CBN grinding performance was investigated.

An experimental study was carried out with different grinding wheels which have different grain size, pore volume, and wheel hardness to grind different workpiece materials. Experimental results were discussed in relation to the theoretical understanding of the process. The effects of varying wheel structure, wheel speed and workpiece material were discussed in relation to grinding power, workpiece 
surface roughness, size error and wheel topographical changes. When grinding M2 steel with the high porosity fine grain wheel at wheel speed of $45-60 \mathrm{~m} / \mathrm{s}$, the power, specific energy and size error were lower than for the conventional wheel. The workpiece surface roughness was higher. For the same specific material removal, the conventional structural wheel gave the better grinding performance with higher $\mathrm{G}$ ratio and longer wheel redress life. Because there was not a serious loading problem when grinding M2 steel, and the porosity for these two wheels are all enough for accommodating the chips, the high porosity wheel did not show much advantage. High porosity leads grains to agglomerate together which decreases active cutting edge density. High porosity also weakens the wheel leading to shorter wheel life. Increased wheel speed improves grinding performance for these two kinds of wheels.

When grinding difficult-to-grind material such as Inconel 718 , there were serious loading problems, high porosity increases the space for chip flow and reduces problem of pore loading. Also, high porosity weaken the wheel which helps loaded grains pull out. Lower specific energy and grinding temperature reduces wheel loading further. The benefits of CBN for a high speed grinding with high porosity wheel application as on the Suprema clearly shown by the results for the wheel life trial on the Suprema for Inconel 718. The Inconel 718 workpieces have been ground with aluminum oxide wheel and CBN vitrified wheel at wheel speed of $45 \mathrm{~m} / \mathrm{s}$ with poor grinding performance. In many ways high speed vitrified CBN grinding offered the same benefits for grinding Inconel 718 as for AISI 52100 .

The mechanics of grinding wheel wear and loading was reviewed and discussed in the research. A prediction of grinding wheel wear and loading has been established based on mechanics.

The grinding wheel wear prediction results showed that when the percentage of grain volume and pore volume was fixed, the smaller the grain size, the greater is the initial active cutting edge density, the smaller the wheel scratch hardness, and wheel wear is reduced. The wear flat is smaller and workpiece surface roughness 
is reduced. When the wheel specification is fixed, the larger the percentage of pore volume, the lower is the initial active cutting edge density, the smaller is the wheel scratch hardness. The wear flat is larger and the workpiece surface roughness is greater. When the wheel specification is fixed, the higher the wheel speed, the wheel wear is less, the wear flat is smaller and workpiece surface roughness is better.

The grinding wheel loading predictions indicate that the effects on grinding performance including wheel wear and loading are similar with the effects including wheel wear only. At the commencement of grinding, there is unstable wheel loading. In order to avoid grinding wheel wear and loading, the wheel should be designed to suit the workpiece material.

\subsection{Conclusions}

The grinding performance of a particular wheel-workpiece combination under different grinding conditions can be correlated with four grinding wheel parameters. These are active cutting edge density, cutting edge dullness, effective porosity volume ratio and wheel scratch hardness.

Techniques have been described for the evaluation of the three topographical parameters using optical interferometer, laser triangulation and microscopy. For many situations, it is necessary to employ surface replication techniques.

Grinding performance is reflected in grinding energy, surface roughness, removal rates, and wheel redress life. Investigations of grinding performance have demonstrated that the optimum grinding wheel porosity for easy-to-grind materials is lower than for difficult-to-grind materials.

It was found that high grinding wheel speeds can be advantageous for easy-togrind materials. The advantages of high wheel speeds include reduced workpiece roughness and increased G-ratio. The disadvantage for a particular removal rate is increased energy consumption. 
It was found that the problem with the difficult-to-grind material related to wheel loading. A high-porosity wheel can alleviate the wheel loading problem. These conclusions are supported by an analysis of wheel wear and wheel loading. 


\section{Chapter 9 Recommendations for future work}

The following areas need further investigation:

In this research, all the bond strengths and CBN abrasive properties for the different wheels were considered to be the same. In fact, the bond strength and CBN abrasive properties such as fracture strength are different for different wheels and for different providing companies. Further investigations of relationships between wheel structure and grinding performance are required taking account of differences in bond strength and CBN abrasive properties.

The methodology for predicting wheel wear and loading needs to be further modified to apply more grinding conditions. The grinding force and workpiece material properties were assumed to be unaffected by the changing grinding temperature. Further research is needed for the grinding force and workpiece material properties to take account of the changes in grinding temperature. Also in this research, the ratio of adhesive loading of the wheel to adhesive wear was assumed to be constant. Further improvement considering how this ratio changes with grinding temperature and coolant effect is required. 


\section{References}

[1] R. L. Mahar, Grinding with Superabrasives, Handbook of Modern Grinding Technology, composed by R. I. King and R. S. Hahn, Chapman and Hall. 1986, pp. $98-118$

[2] X. Chen, W. B. Rowe, Precision Grinding with Vitrified CBN, EPSRC Proposal, EPSRC Grant GR/M35161, 1998

[3] H. K. Tönshoff, I. Inasaki, B. Karpuschewski, T. Mandrysch, Grinding Process Achievements and their Consequences on Machine Tools-Challenges \& Opportunities, Annals of the CIRP, Vol. 47/2, 1998, pp. 651-668

[4] S. Malkin, Grinding Technology, Ellis Horwood Ltd. 1989

[5] F. Klocke, W. König, Appropriate Conditioning Strategies to Increase the Performance Capabilities of Vitrified Bond CBN Grinding Wheels, Annals of the CIRP, Vol. 44/2, 1995, pp.305-310

[6] E. Westkämper, H. K. Tönshoff, CBN or CD grinding of profiles, Annals of the CIRP, Vol. 42/1, 1993, pp. 371-374

[7] Y. Onchi, N. Ikawa, Porous fine CBN Stones for High Removal Rate Super Finishing, Annals of the CIRP, Vol. 44/2, 1995, pp.291-294

[8] W. B. Rowe, X. Chen and D. R. Allanson, The Coolant Coupling Method Applied to Touch Dressing in High Frequency Internal Grinding, Proceedings of the Thirty- second International MATADOR Conference, Manchester, 1997, pp. 337-342

[9] P. M. Lonardo, H. Trumpold, L. De Chiffre, Progress in 3D Surface Microtopography Characterisation, Annals of the CIRP, Vol. 45/2, 1996, pp. 589-598

[10] T. C. Bristow, Surface Topography Goes beyond Just RMS Roughness, Laser Focus World, Vol. 28(3), 1992, pp. 149-150

[11] L. Blunt, X. Q. Jiang, Three Dimensional Measurement of the Surface Topography of Ceramic and Metallic Orthopaedic Joint Prostheses, Journal of Materials Science: Materials in Medicine Vol. 11, 2000, pp.235-246 
[12] T. Rzeznik, In-line Laser Measurement in the Assembly Process, Industrial robot, Vol. 24(1), 1997, pp.16-23

[13] E. Brinksmeier and F. Werner, Monitoring of Grinding Wheel Wear, Annals of the CIRP, Vol. 41/2, 1992, pp.373-376

[14] Hillmann, 1990, Surface Profiles Obtained by Means of Optical MethodsAre They True Representation of the Real Surface? Annals of the CIRP, Vol. 39/1, 1990, pp.581-584

[15] De Chiffre, L., Henson, N. H., Investigation of the wheel topography in polishing using Atomic Force Microscopy, Annals of the CIRP, Vol. 45/1, 1996, pp.523-529

[16] D. J. Whitehouse, Comparison between Stylus and Optical Method for Measuring Surfaces, Annals of the CIRP, Vol. 37/1, 1988, pp. 281-285

[17] J. Verkerk et al., Final report concerning CIRP cooperative work on Characterisation of Grinding Wheel Topography, Annals of the CIRP, Vol. 26/1, 1977, pp.385-395

[18] J.D. Eckert, Replica Techniques for the Study of Fractures Surfaces and Topography Study in General, Prakt, Metallogr. Vol. 33, 1996, pp. 369-372

[19] A. F. George, A Comparative Study of Surface Replicas, Wear, Vol. 57, 1979, pp. 51-61

[20] P.J. James, W.Y. Thum, The Replication of Metal Surfaces by Filled Epoxy Resins, Precision Engng, Vol. 4, 1982, pp. 201-204.

[21] I. Inasaki, Grinding Process Simulation Based on the Wheel Topography Measurement, Annals of the CIRP, Vol. 45/1, 1996, pp. 347-350

[22] J. Peters, P. Vanherck, M. Sastrodinoto, 1979, Assessment of Surface Typology Analysis Techniques, Annals of the CIRP 28/2, 1979, pp.539-553

[23] B. Nowicki, Multiparameter representation of surface roughness, Wear, Vol. 102, 1985, pp161-176

[24] D. J. Whitehouse, Handbook of Surface Metrology, Institute of Physics Publishing, Bristol, 1994

[25] K. J. Stout, P. J. Sullivan, W. P. Dong, E. Mainsah, N. Luo, T. Mathia and H. Zahyouani, The Development of Methods for the Characterisation of 
Roughness in Three Dimensions, Commission of the European Communities, 1993

[26] R. S. Hahn, R. Lindsay, The Influence of Process Variables on Material Removal, Surface Integrity, Surface Finish and Vibration in Grinding, Proceedings of 10th Int. MTDR Conference, The Macmillan Press, 1969, pp. $95-117$

[27] T. G. King, D. J. Whitehouse, K. J. Stout, Some Topographic Features of Wear Process-Theory and Experiment, Annals of the CIRP 25/1, 1977, pp. 351-357

[28] S. Malkin and N. H. Cook, the wear of grinding wheels, Part 1-Attritious wear, J. of Eng. For Ind., Trans. ASME, Vol. 93, 1971, pp.1120-1128

[29] W. König, W. Lortz, Properties of Cutting Edges Related to Chip Formation in Grinding, Annals of the CIRP, Vol. 24/1, 1975, pp. 231-236

[30] M. Shaw, R. Komanduri, The Role of Stylus Curvature in Grinding Wheel Surface Characterisation, Annals of the CIRP, Vol. 26/1, 1977, pp. 139-142

[31] W. B. Rowe, M. W. Morgan, H. S. Qi, and H. W. Zheng, The Effect of Deformation on the Contact Area in Grinding, Annals of CIRP 42/1, 1993, pp. 409-412

[32] M. Shaw, Principles of abrasive processing, Oxford science publications, 1996

[33] J. Peklenik, H. Opitz, 1962, Testing of Grinding Wheels, Proceedings of the Ninth International Machine Tool Design and Research Conference, pp.163170

[34] J. Peters, R. Snoey and A. Deceut, Sonic Testing of Grinding Wheels, Proceedings of the Ninth International Machine Tool Design and Research Conference, 1968, pp. 1113-1131

[35] J. N. Brecker, Grading Grinding Wheels by Elastic Modulus, Proceedings, First North American Metalworking Research Conference, 3, 1973, pp. 149164 
[36] L. Blunt, S. Ebdon, The Application of Three-Dimensional Surface Measurement Techniques to Characterising Grinding Wheel Topography, Int. J. Mach. Tools \& Manufact. Vol. 36, 1993, pp. 1207-1266

[37] R. Bradley, The effect of Workpiece composition of Free-Cutting Steels on Machinability Using Coated and Uncoated Gigh Speed Steel Tools, PhD thesis, Liverpool John Moores University, 1997

[38] W. R. Backer, E. R. Marshall, M. C. Shaw, The size effect in Metal Cutting, Trans. ASME, Vol. 74, 1952, pp. 61-72

[39] H. O. Juchem, B. A. Cooley, Vitrified Bond Abrasive Boron Nitride Tools and Their Advantages, De Beers Industrial Diamond Division, 1993

[40] A. Di Ilio, A. Paoletti, A Comparison between Conventional Abrasives and Superabrasives in Grinding of SiC-aluminum Composites, Int. J. of Machine Tools \& Manufacture, Vol. 40(2), Jan 2000, pp173-184

[41] X. Chen, Strategy for the Selection of Grinding Wheel Dressing Conditions, $\mathrm{PhD}$ thesis, Liverpool John Moores University, 1995

[42] P. Koshy, LK. Ives and S. Jahanmir, Simulation of Diamond-ground Surfaces, Int. J. of Machine Tools \& Manufacture, 39(9), Sep 1999, pp. $1451-1470$

[43] J. J. Guest, Grinding Machinery, Edward Arnold, London, 1915

[44] Alden, I. Geo, Operation of Grinding Wheels in Machine Grinding, ASME Trans., Vol. 36, 1914, pp. 451-460

[45] R. S. Hahn, On the Nature of the Grindinf Process, Proc. of 3rd MTDR Conf., 1962, pp. 129-154

[46] E. D. Doyle, S. K. Dean, An Insight into Grinding from a Materials Viewpoint, Annals of the CIRP, 29/2, 1980, pp. 571-576

[47] A. J. Sedricks, T. O. Mulhearn, The Mechanics of Cutting and Rubbing in Simulated Abrasives Processes, Wear, 6, 1963, pp. 457-465

[48] T. O. Mulhearn, T. O. Samuels, The abrasion of Metals: a Model of the Process, Wear, 5, 1962, pp. 478-484

[49] L. E. Samuels, Metallographic Polishing by Mechanical Methods, 3rd edn., Chapter 3, ASM, 1982 
[50] N. Maan, A. Broese Van Groenou, Low Speed Scratch Experiments on Steels, Wear, 42, 1977, pp. 365-373

[51] P. Gilormini, E. Felder, Theoretical and Experimental Study of the Ploughing of a Rigid-Plastic Semi-infinite Body by a Rigid Pyramidal Indentor, Wear, 88(2). 1983, pp. 195-206

[52] A. A. Torrance, A New Approach to the Mechanics of Abrasion, Wear, 67(2), 1981, pp. 233-257

[53] M. D. Demetriou, A. S. Lavine, Thermal Aspects of Grinding: The Case of Upgrinding, ASME, Vol. 122, 2000, pp. 605-611

[54] S. Malkin, R. B. Anderson, Thermal Aspects of Grinding: Part 1-Energy Partition, J. Eng. Ind., Vol. 96, 1974, pp. 1177-1183

[55] E. R. Marshall, M. C. Shaw, Forces in Dry Surface Grinding, Trans. ASME, 74, 1952, pp.61-70

[56] B. F. Von Turkovich, Shear Stress in Metal Cutting, Trans. ASME Journal of Engineering for Industry, 1970, v.92, 151-157.

[57] K. Nakayama, K. Tamura, Size Effect in Metal-Cutting Force, Trans. Of the ASME, Journal of Engineering for Industry, 1968, v. 90, pp. 119-126

[58] D. Graham, R. M. Baul, An Investigation into the Mode of Metal Removal in the Grinding Process, Wear, 1972, v. 19, pp. 301-314

[59] S. Kannappan, S. Malkin, Effects of Grain Size and Operating Parameters on the Mechanics of Grinding, Journal of Engineering for Industry, Aug. 1972, pp. 833-842

[60] W. B. Rowe, X. Chen, Characterization of the Size Effect in Grinding and the Sliced Bread Analogy, Int. J. of Production Research, Vol.35, No. 3, 1997, pp. 887-899

[61] M. C. Shaw, Energy Conversion in Cutting and Grinding, Annals of the CIRP, Vol. 45/1, 1996, pp. 101-104

[62] E. Merchant, Mechanics of the Metal Cutting Process, J. Appl. Phys., 16, 1945, pp. 207-215 
[63] P. R. Lindsay, Dressing and its Effect on Grinding Performance, Technical Paper MP 29-568, American Society of Tool Manufacturing in Engineering, 1969

[64] P. R. Lindsay, The Effect of Wheel Wear Rate on Grinding Performance of Three Wheel Grades. Annals of CIRP, 32/1, 1983, pp.247-249

[65] P. R. Lindsay, The Effect of Contact Time on Forces, Wheelwear Rate and G-Ratio during Internal and External Grinding. Annals. CIRP, 33/1, 1984, pp.193-198

[66] R. Snoeys, J. Peters, The Significance of Chip Thickness in Grinding Annals of CIRP, 23/2, 1974. Pp. 227-237

[67] J. Peters, Contribution of CIRP Research to Industrial Problem in Grinding, Annals of CIRP, 33/1, 1984. pp. 193-197

[68] J. A. Badger, A. A. Torrance, A Comparison of two models to predict grinding Forces from Wheel Surface Topography, Int. J. Mach. Tool. Manu, 40(8), 2000, pp. 1099-1120

[69] J. A. Williams, Y. Xie, The Generation of Wear Surfaces by the Interaction of Parallel grooves. Wear, 155, 1992, pp. 363-379

[70] Y. Xie and J. A. Williams, The Generation of Worn Surfaces by the Repeated Interaction of Parallel grooves. Wear, 194, 1993, pp. 864-872

[71] Y. Xie and J. A. Williams, The Prediction of Friction and Wear when a Soft Surface Slides against a Hard Rough Surface, Wear, 196, 1996, pp. 21-34

[72] R. Snoeys, M. Maris and J. Peters, Thermally Induced Damage in Grinding, Annals of CIRP, 27/2, 1978, pp.571-581

[73] A. A. Torrance, Metallurgical Effects Associated with Grinding, Proceedings of the Twelfth International Machine Tool Design and Research Conference, 1978, pp.637-643

[74] H. Carslaw, J. C. Jaeger, Conduction of Heat in Solids, Oxford University Press, Oxford, 1959

[75] J. O. Outwater, M. C. Shaw, Surface temperatures in grinding, Trans ASME, 74, 1952, pp.73-78 
[76] R. S. Hahn, On the Nature of the Grinding Process, Proc. of the 3rd MTDR Conf., 1962, pp. 129-154

[77] N. R. Des Ruisseaux, R. D. Zerkle, Temperatures in Semi-infinite and Cylindrical Bodies Subject to Moving Heat Sources and Surface Cooling, Journal of Heat Transfer, 92, 1970, pp. 456-464

[78] M. Maris, R. Snoeys, Heat Affected Zone in Grinding Oprations, 14th MTDR Conf. 1973, pp. 504-510

[79] A. S. Lavine, S. Malkin, Thermal Aspects of Grinding with CBN Wheels, Annals of CIRP, 38/1, 1989, pp. 557-560

[80] W. B. Rowe, S. C. E. Black, Experimental Investigation of Heat Transfer in Grinding, Annals of CIRP, 44/1. 1995, pp. 409-412

[81] A. S. Lavine, T. C. Jen, Thermal Aspects of Grinding: Heat Transfer to Workpiece, Wheel and Fluid, Journal of Heat Transfer, Trans. Of ASME, 113, 1991, pp. 296-303

[82] W. B. Rowe, J. A. Pettit, A. Boyle and J. L. Moruzzi, Avoidance of the Thermal Damage in Grinding and Prediction of the Damage threshold, Annals of CIRP, 37/1, 1990, pp. 327-330

[83] W. B. Rowe, S. C. E. Black, B. Mills, M. N. Morgan, H. S. Qi, Grinding Temperatures and Energy Partitioning, Proceedings of the Royal Society, Part A, 453, 1997, pp. 1083-1104

[84] W. B. Rowe, M. N. Morgan, S. C. E. Black, B. Mills, A Simplified Approach to Thernmal Damage in Grinding, Annals of CIRP, Vol. 45/1, 1996, pp. 299-302

[85] W. B. Rowe, Thermal Analysis of High Efficiency Deep Grinding, Int. J. Mach. Tool Manu, 41, 2001, pp. 1-19

[86] W. B. Rowe, T. Jin, Annals of CIRP, Vol. 50/1, 2001, pp. 205-208

[87] H. Yoshikawa and T. Sata, Study on wear of grinding wheels, J. of Eng. For Ind., Trans. ASME, 85, 1963, p.39-46

[88] H. Yoshikawa, Fracture wear grinding wheels, International Research in Production Engineering, 1963, p.209-217 
[89] T. N. Loladze, G.V.Bokuchava, Tribological Aspects of the Grinding Process, ASME, PED, 1985, v. 16, pp.401-407

[90] H. Grisbrook, R.H.Hollier, P.G. Varley, Related Patterns of Grinding Forces, Wheel Wear and Surface Finish, Int. Jnl. Of Prod. Res., 1962, v. 1, 3, pp. $57-74$

[91] W. R. Backer, M. E. Merchant, On the Basic Mechanics of the Grinding Process, ASME Paper No 56-A-43, 1957, pp.1-6

[92] J. Koloc, On the Wear of Grinding Wheels, MicroTechnic, 1959, Vol. 81, pp.13-15

[93] S. K. Bhattacharyya, H. Grisbrook, H. Moran, Analysis of Grit Fracture with Change in Grinding Conditions, Microtechnics, 1968, v.22, pp. 114-116

[94] A. G. Wetton, A Review of Published Fundamental Research of the Grinding of Metals, Research Report No. 38, The Machine Tool Industry Research Association (MTIRA), 1970

[95] S. K. Bhattacharyya, V. L. Moffatt, Characteristics of Micro Wheel Wear in Grinding, Microtechnics, Int. J. Mach. Tool Des. Res., 1976, v. 16, pp. 325334

[96] N. E. Ye, T. R. A. Pearce, Some Observations on Profile Wear in Creep-Feed Grinding, Wear, v. 92(1), 1983, pp.51-66

[97] E. J. Pattinson, A. W. J. Chisholm, The Effect of Dressing Techniques on Grinding Wheel Wear, Proc. Int. Conf. On Manufacturing Technology, A. S. T. M. E., Univ. of Michigan, 1967, pp. 601-616

[98] W. B. Rowe, K. J. Stout, Review of Grinding Process Parameters, Engineers' Digest, 1971, v. 32, 10, pp. 41-48

[99] S. J. Pande, S. N. Halder, G. K. Lal, Evaluation of Grinding Wheel Performance, Wear, v.58, 2, 1975, pp. 237-248

[100] W.B.Rowe, Application and Performance of CBN Wheels for Precision Grinding, Abrasives Magazine, Oct./Nov. 2000, pp.36-41

[101] E. Rabinowicz, Friction and wear of materials, John Wiley \& Sons, Inc. 1995

[102] A. D. Sarkar, Friction and wear, Academic press, 1980 
[103] R. Komanduri, M. C. Shaw, The Mechanisam of Metal Build-up on Aluminum Oxide Abrasive, Annals of CIRP, 25, 1976, pp. 191-196

[104] G. J. Geopfert, J. L.Williams, The Wear of Abrasives in Grinding, Mech. Eng., ASME, Vol. 81, 1969, pp. 69-73

[105] L. Coes, Abrasives, Springer, New York, 1971

[106] W. R. Brown, N. S. Eiss and H. T. McAdams, Chemical Mechanisms Contributing to Wear of Single-Crystal Sapphire on Steel, J. Am. Ceramic Soc. Vol. 47, 1964, pp. 157-162

[107] N. S. Eiss, R. C. Fabiniak, Chemical and Mechanical Mechanisms in Wear of Sapphire on Steel, J. Am. Ceramic Soc. Vol. 49, 1966, pp. 221-226

[108] C. T. Yang, M. Shaw, The Grinding of Titanium Alloys, Trans. ASME., J. Engng Ind. Vol. 77, 1955, pp. 645-660

[109] I. Nakayama, Theory of Metal Cutting (translated in Chinese), press of mechanical industry ,Beijing, 1985

[110] S. Yossifon, C. Rubenstein, The Grinding of Workpiece Materials Exhibility High Adhesion 1. Mechanics, Trans. ASME, J. Engng Ind. 103, 1981, pp. 144-155

[111] S. Yossifon, C. Rubenstein, The Grinding of Workpiece Materials Exhibility High Adhesion 2. Forces, Trans. ASME, J. Engng Ind. 103, 1981, pp. 156-164

[112] S. Yossifon, C. Rubenstein, Wheel Wear When Grinding Workpieces Exhibition High Adhesion, Int. J. Mach. Tool Des. Res. 22, 1982, pp. 159176

[113] J. F. Archard, Contact and rubbing of flat surfaces, J.Appl. Phys., 24, 1953, pp.981-988

[114] X. Chen, Strategy for the Selection of Grinding Wheel Dressing Conditions, $\mathrm{PhD}$ thesis, Liverpool John Moores University, 1995

[115] S. Ebbrel, R. Cai, V. Gviniashvili, Technical Report, Precision Grinding with Vitrified CBN Wheels, Liverpool John Moores University, 2002 
[116] X. Chen, W. B. Rowe, R. Cai, Precision grinding with CBN wheels, Internal Journal of Machine tools and Manufacture, Vol. 42, pp. 585-593, 2002

[117] D. M. Grove \& T. P. Davis, Engineering, Quality and Experimental Design, Longman Science and Technical, 1992 


\section{Appendix}

\section{A 1. Matlab program for calculating grain area density along the radial depth of wheel (whelstruc11.m)}

\& Establishing CBN wheel structure and calculating grain area density along the radial depth of wheel (whelstrucl1.m)

Conc $=150 ;$ wheel concentration

$\mathrm{Vp}=0.3 ; \quad$ spercentage of pore volume $\mathrm{dg}=0.064 ;$ o diameter of grits $\mathrm{dp}=0.120 ;$ \& diameter of pores $\mathrm{Cpv}=\mathrm{Vp} /\left((4 / 3){ }^{\star} \mathrm{pi} *(\mathrm{dp} / 2)^{\wedge} 3\right) ; \quad$ \& number of pores, $1 / \mathrm{mm} 3$ $\mathrm{Cgv}=\left(\mathrm{Conc}^{\star} 0.24 / 100\right) /((4 / 3) * \mathrm{pi} *(\mathrm{dg} / 2) \wedge 3) ;$ o number of grits, $1 / \mathrm{mm} 3$

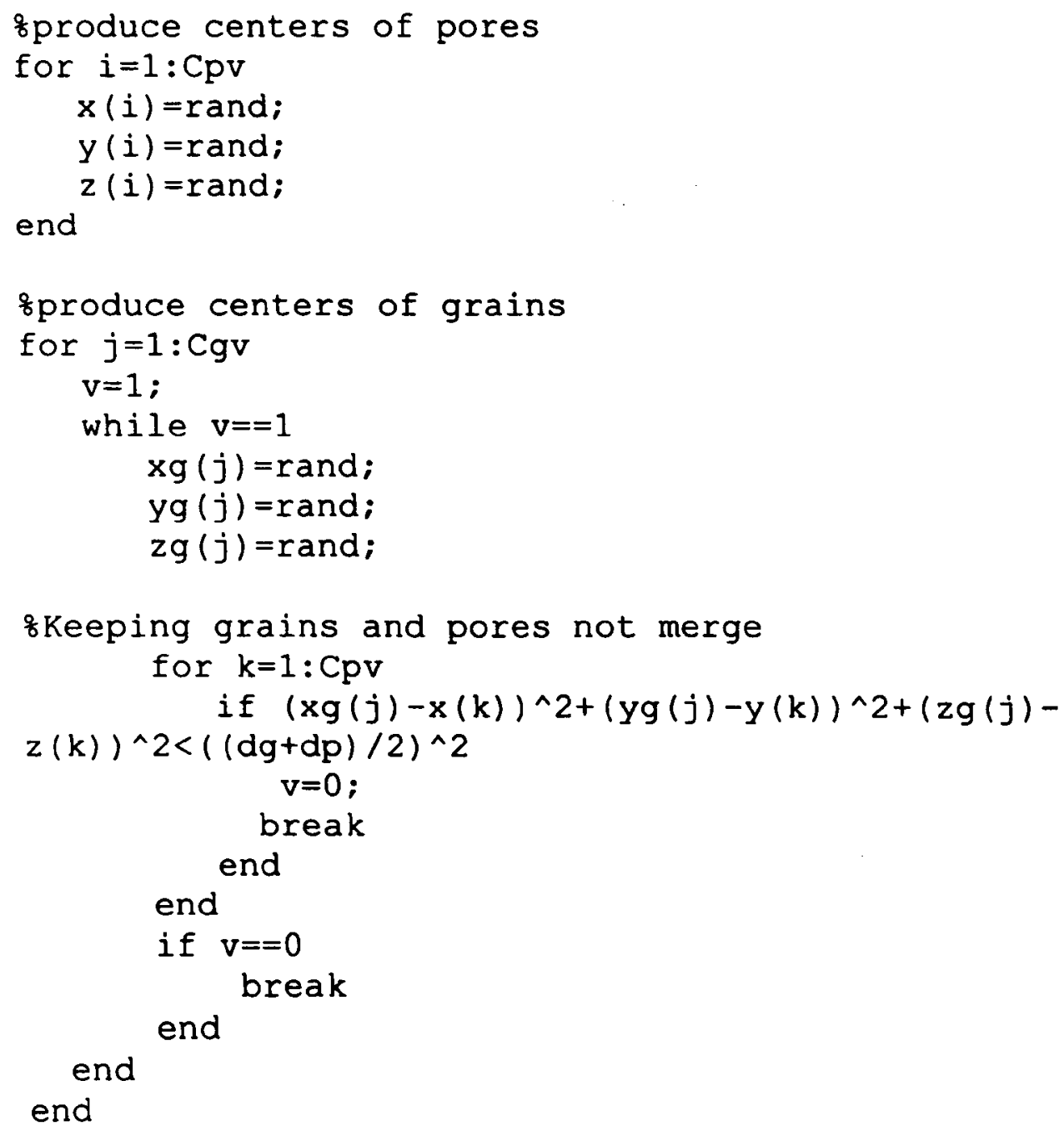

ofcalculating grain area density along the radial depth of wheel

$\mathrm{zz}=0.5$; 8 randomly adopted from 0 to 1 


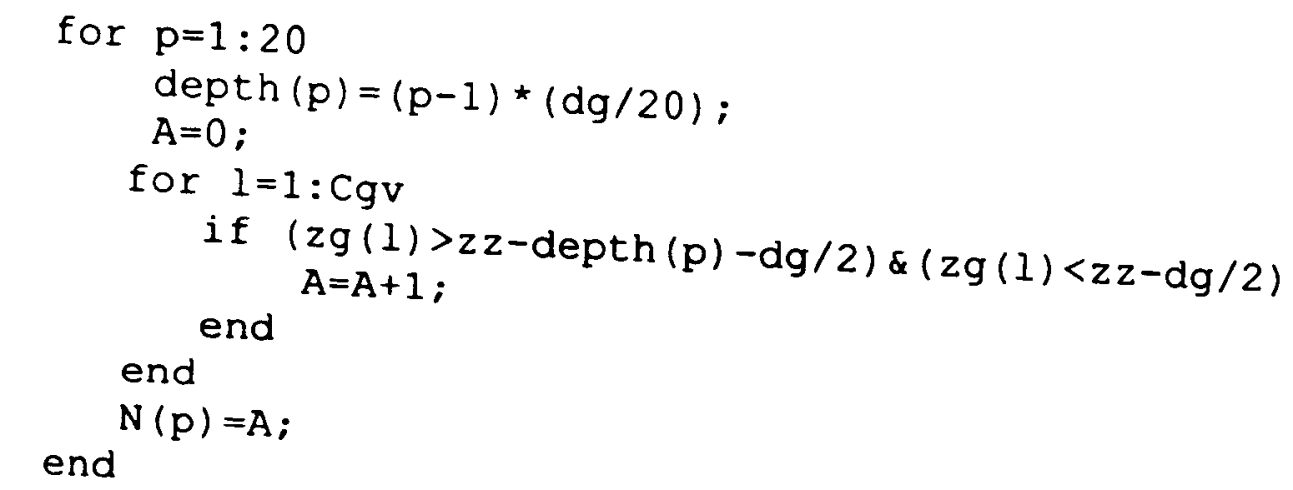

plot (depth, N)

grid 


\section{A 2. Matlab program for calculating porosity volume ratio (porosity2.m)}

\&Calculating porosity volume ratio (porosity $2 . m$ )

format long

Conc $=200$;

of wheel concentration

$\mathrm{Vg}=0.24^{\star}$ Conc $/ 100$;

$\mathrm{Vp}=0.3$;

spercentage of grain volume

$\mathrm{dg}=0.091$ \&percentage of pore volume

$r=d g / 2 ; 8 g r a i n$ radius

Cgv $=\left(\right.$ Conc $\left.^{\star} 0.24 / 100\right) /\left((4 / 3){ }^{*} \mathrm{pi}^{\star}(\mathrm{dg} / 2)^{\wedge} 3\right) ;$; grain volumetric density

Cgamax $=\mathrm{Cgv}^{\star} \mathrm{dg} / 2$; gorain maximum area density $z=1.26^{\star} \mathrm{dg}$;

\&calculating grain volume on the wheel surface $\mathrm{u}=\mathrm{dg} / 4$; ormean

sigma $=\mathrm{dg} / 12$; \&deviation

$\mathrm{a}=0: \mathrm{dg} / 10: \mathrm{dg} / 2$;

$\mathrm{h}=\mathrm{r}-\operatorname{sqrt}\left(\mathrm{r}^{\wedge} 2-\mathrm{a} \cdot{ }^{\wedge} 2\right)$;

$\mathrm{P} 1=\left(\exp \left((-1 / 2) \cdot{ }^{*}((\mathrm{a}-\right.\right.$

u) / sigma ).^(2)))/(( sqrt $(2 * p i)) \star s i g m a)$;

$\mathrm{V} 1=\left((\mathrm{pi} / 3) \cdot{ }^{\star} \mathrm{h} \cdot \wedge^{\wedge} 2 \cdot{ }^{\star}\left(3^{\star} \mathrm{r}-\mathrm{h}\right)\right)+(4 / 6) \star \mathrm{pi} * \mathrm{r}^{\wedge} 3$;

$\mathrm{P} 11=\mathrm{Cgamax} .{ }^{\star} \mathrm{P} 1 .{ }^{*} \mathrm{~V} 1$;

$\mathrm{Vg} 1=\operatorname{trap} z(a, P 11)$

$\mathrm{V} 2=(4 / 3){ }^{\star} \mathrm{pi}{ }^{\star} \mathrm{r}^{\wedge} 3-\left((\mathrm{pi} / 3) .{ }^{\star} \mathrm{h} \cdot{ }^{\wedge} 2 .{ }^{*}\left(3^{\star} \mathrm{r}-\mathrm{h}\right)\right) ;$

$\mathrm{P} 22=$ Cgamax $.{ }^{*} \mathrm{P} 1 .{ }^{*} \mathrm{~V} 2$;

$\mathrm{Vg} 2=\operatorname{trap} z(a, \mathrm{P} 22)$

$\mathrm{Vgdg}=(\mathrm{Vg} 1+\mathrm{Vg} 2) / \mathrm{dg}$;

$V g s=\left(V g d g{ }^{\star} d g+V g^{\star}(z-d g)\right) / z$ sporosity volume ratio of grain on the wheel surface

$\mathrm{Vps}=0.5-\mathrm{Vgs}+0.5^{*}(\mathrm{Vg}+\mathrm{Vp}) \quad$ sporosity volume ratio of pore on the wheel surface 


\section{A 3. Matlab program for wheel wear prediction (wheelwear3.m)}

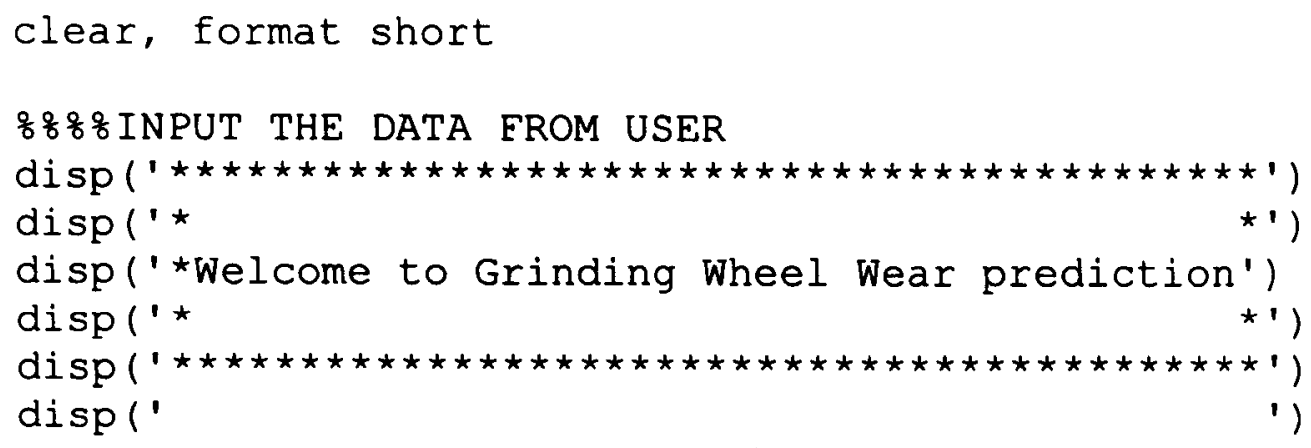

\&: ON WHEEL STRUCTURE-CO

$\mathrm{m}=1.2$; gnumber of cutting

edges on each grain

$\mathrm{C} 0=\mathrm{m}^{\star} 6 \star \mathrm{Vg} /\left(\mathrm{pi} \star \mathrm{dg}^{\wedge} 2\right) \star(-2.4 * \mathrm{Vp}+1.1) ; \frac{\circ}{8}\left(/ \mathrm{mm}^{\wedge} 2\right)$

\% disp('ENTER THE PARAMETER OF GRINDING CONDITION') ds=input ('wheel diameter in (mm):'); $\mathrm{dw}=$ input ('workpiece diameter in $(\mathrm{mm}):$ '); $\mathrm{de}=\mathrm{ds} /(1-\mathrm{ds} / \mathrm{dw}) ; \quad \&(\mathrm{~mm})$, equivalent diameter

bw=input ('workpiece width in (mm): '); ns=input ('wheel revolution speed in (rev/s):'); $\mathrm{vs}=\mathrm{pi}{ }^{*} \mathrm{ds}{ }^{*} \mathrm{~ns} / 1000 ; \quad \%(\mathrm{~m} / \mathrm{s})$, wheel speed $\mathrm{nw}=$ input ('workpiece revolution speed in (rev/s):'); $\mathrm{vw}=\mathrm{pi}{ }^{\star} \mathrm{dw} \mathrm{w}^{\star} \mathrm{nw} / 1000 ; \quad \&(\mathrm{~m} / \mathrm{s})$, work speed ae=input ('wheel cutting depth in $(\mathrm{mm}):$ '); lc=sqrt $\left(a e^{\star} \mathrm{de}\right) ; \quad$ o $(\mathrm{mm})$, contact length 


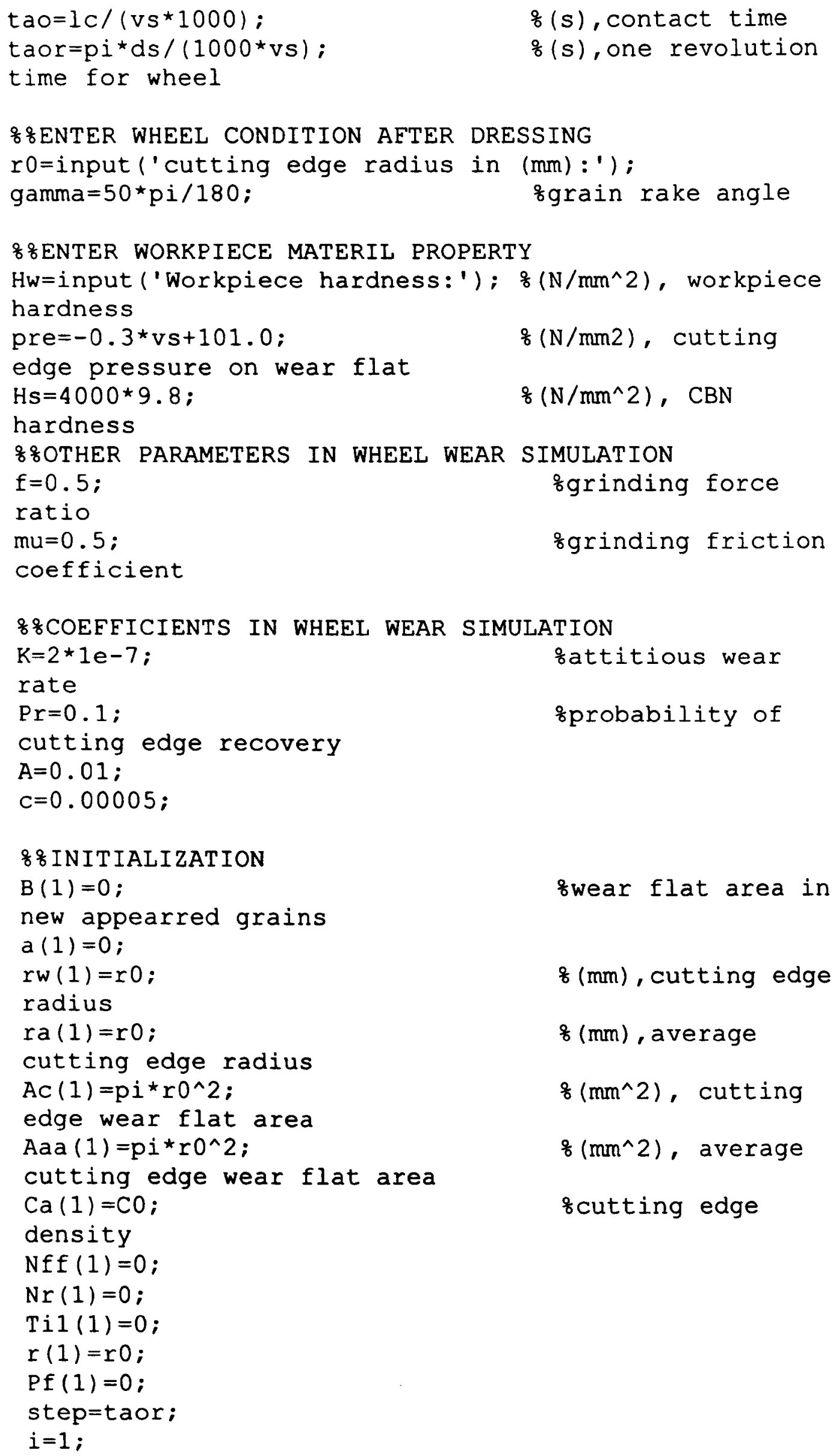


$j=1 ;$

$a a=\left(-1 \cdot 4^{\star} 10^{\wedge}(-2) \star v s+6 \cdot 3\right) \star 10^{\wedge}(-5) ;$

for $t=0:$ step: $(16000 \star 200 *$ step $)$

ogrinding time

$\mathrm{T} i(i)=t$;

$t 1=t *$ tao/taor; ogrinding time ocontact time

$\%$ CALCULATE MAXIMUM UNCUT CHIP THICKNESS-hmax $\operatorname{hmax}(\mathrm{i})=2 * \operatorname{sqrt}(1 / \mathrm{Ca}(\mathrm{i}))$ * (vw/vs) ${ }^{*} \operatorname{sqrt}(\mathrm{ae} / \mathrm{de})$; $\&(\mathrm{~mm})$, maximum uncut chip thickness

$\because \%$ CALCULATE GRINDING FORCE

Fctc (i) $=a a /(h \max (i) * \mathrm{Ca}(i) * 1 c * b w)$;

stangential cutting force on each cutting edge Fcts $(i)=$ mu*pre*pi*ra (i)^2;

otangential sliding force on each cutting edge Fct $(i)=F c t c(i)+F c t s(i)$;

stangential force on each cutting edge $\mathrm{FCn}(\mathrm{i})=\mathrm{Fct}$ (i) /f;

snormal force on each cutting edge

$F t(i)=(\operatorname{Fctc}(i)+\operatorname{Ects}(i)) * C a(i) \star b w * l c$;

Power $(i)=F t(i) * v s$;

\% 8 CALCULATE ATTRITIOUS WEAR

$a(i)=3 * \tan ($ gamma $){ }^{*}{ }^{\star} \mathrm{Fcn}(\mathrm{i}){ }^{*} \mathrm{~ns}{ }^{\star} l \mathrm{c} /\left(\mathrm{p} i{ }^{*} \mathrm{Hs}\right)$;

8 constant change with Aaa(i)

$r w(i+1)=\left(a(i) *(t 1+t a o)+r 0^{\wedge} 3\right)^{\wedge}(1 / 3)$;

ocutting edge radius after attritious wheel wear

$A c(i+1)=p i \star r w(i+1)^{\wedge} 2$;

scutting edge wear flat after attritious wheel wear

\% 8 CALCULATE ABRASIVE WEAR AND BOND WEAR

$\operatorname{Pf}(i)=A \star t a o^{\wedge}\left(C^{\star}(\right.$ Hss $/($ Fct $\left.(i) * m))\right) ; \quad$ opossibility

of abrasive and bond wear

$\mathrm{Nf}(i)=\mathrm{Ca}(i) * \mathrm{Pf}(i)$;

zabrasive and bond wear density

$\mathrm{Nff}(i+1)=\mathrm{Nff}(i)+\mathrm{Nf}(i)$;

8 total abrasive and bond wear density

$\mathrm{N}(\mathrm{i})=\mathrm{Nf}(\mathrm{i}){ }^{*} \mathrm{Pr}$;

fcutting edge recovery density

$\mathrm{Nr}(\mathrm{i}+1)=\mathrm{Nr}(\mathrm{i})+\mathrm{N}(\mathrm{i})$;

stotal cutting edge recovery density

$\mathrm{Ca}(i+1)=\mathrm{C} 0-\mathrm{Nff}(i+1)+\mathrm{Nr}(i+1)$;

of Active cutting edge density

$\%$ CALCULATE WEAR AREA RATIO IN A UNIT AREA

for $g=2: j+1$ 


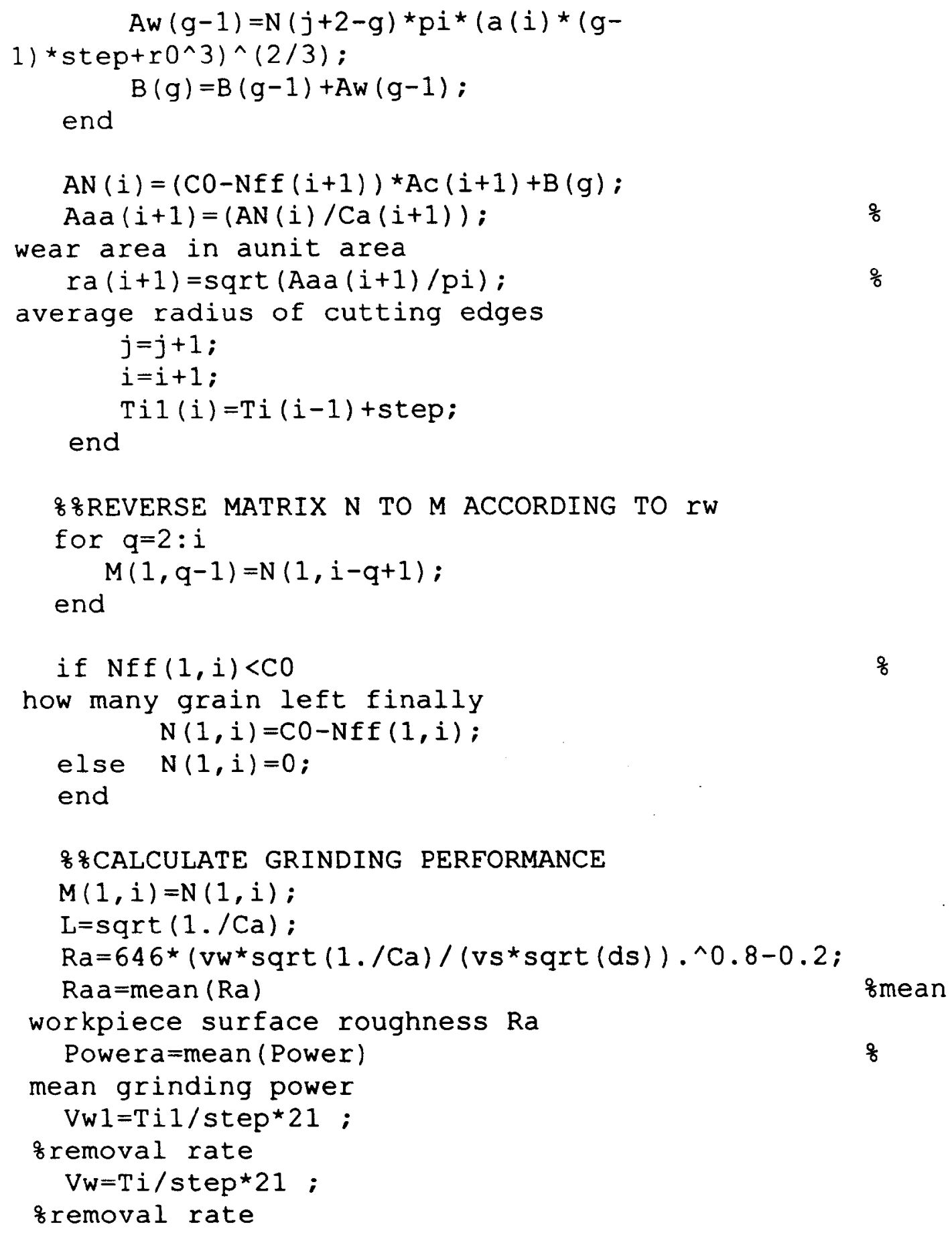

\section{용 PLOTS}

figure, plot(Vw1, Ca) \& cutting edge density grid

$x$ label ('specific volume of material removed (mm^3/mm) ') 
xlabel('specific volume of material removed (mm^3/mm) ')

ylabel('average radius of wear flat (micron)')

figure, plot(Vw1, Ra) \% workpiece surface roughness Ra $x l a b e l$ ('specific volume of material removed $\left.\left(\mathrm{mm}^{\wedge} 3 / \mathrm{mm}\right)^{\prime}\right)$

ylabel('Ra (micron)') 


\title{
A 4. Matlab program for wheel loading prediction (wheelloading2.m)
}

\author{
clear, format short
}

䒠各各各 INPUT THE DATA FROM USER

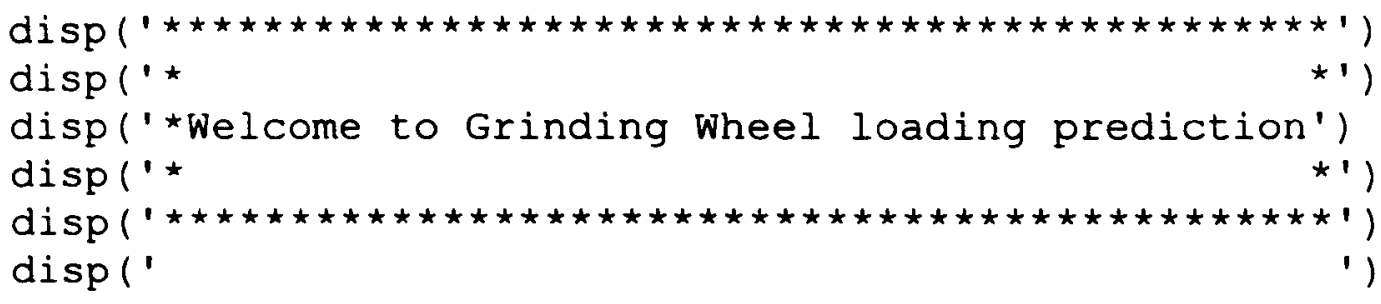

\& $\&$ DETERMINE WHEEL SCRATCH HARDNESS-HSS disp('DETERMINE WHEEL SCRATCH HARDNESS-HSS:')

$\mathrm{Vg}=$ input ('percent of abrasive volume: ');

$V p=i n p u t$ ('percent of pore volume:');

$\mathrm{dg}=$ input ('grain diameter in $(\mathrm{mm}):$ ');

$\mathrm{Vb}=1-\mathrm{Vg}-\mathrm{Vp}$;

$k=1.6794 \star 10^{\wedge} 6$;

$8\left(1 /\left(\mathrm{mm}^{\wedge} 3\right)\right)$, coefficient

Hsa $=85.3162$;

$\%(\mathrm{~N})$, wheel abrasive

hardness

$\mathrm{mm}=6$;

gnumber of bond

bridges abound each grain

$\mathrm{C}=\mathrm{Vg} \star \mathrm{mm} \star(0.0181 * \mathrm{Vp}+1.5094) /(\mathrm{Vb} \star 4 / 3 * \mathrm{pi} *(\mathrm{dg} / 2) *(\mathrm{dg} / 2) \star(\mathrm{d}$ $\mathrm{g} / 2)$ ); zcoefficient

Hss $=1 /(\mathrm{C} / \mathrm{k}+1 / \mathrm{Hsa})$

\% ON WHEEL STRUCTURE-CO

$\mathrm{m}=1$. 2;

edges on each grain

znumber of cutting

$\mathrm{C} 0=\mathrm{m}^{\star} \sigma^{\star} \mathrm{Vg} /\left(\mathrm{pi} \star \mathrm{dg}^{\wedge} 2\right) \star(-2.4 * \mathrm{Vp}+1.1) ; \quad$ o $\left(/ \mathrm{mm}^{\wedge} 2\right)$

\% 8 ENTER THE PARAMETER OF GRINDING CONDITION

disp('ENTER THE PARAMETER OF GRINDING CONDITION')

ds=input ('wheel diameter in (mm): ');

$\mathrm{dw}=$ input ('workpiece diameter in $(\mathrm{mm}):$ ');

$\mathrm{de}=\mathrm{ds} /(1-\mathrm{ds} / \mathrm{dw}) ; \quad \&(\mathrm{~mm})$, equivalent

diameter

bw=input ('workpiece width in (mm): '),

ns=input ('wheel revolution speed in (rev/s): ');

$\mathrm{vs}=\mathrm{pi}{ }^{\star} \mathrm{ds} \mathrm{s}^{\star} \mathrm{ns} / 1000 ; \quad 8(\mathrm{~m} / \mathrm{s})$, wheel speed

$n w=i n p u t$ ('workpiece revolution speed in (rev/s): ');

$\mathrm{vw}=\mathrm{pi} \star \mathrm{dw} \mathrm{ww}^{*} / 1000 ; \quad \frac{8}{8}(\mathrm{~m} / \mathrm{s})$, work speed

ae=input ('wheel cutting depth in $(\mathrm{mm}):$ ');

$l c=\operatorname{sqrt}\left(a e^{\star} \mathrm{de}\right) ; \quad z(\mathrm{~mm})$, contact

length 
tao $=1 \mathrm{c} /\left(\mathrm{vs}^{\star} 1000\right)$;

$\%$ (s), contact time

taor $=\mathrm{pi} * \mathrm{ds} /(1000 * \mathrm{vs})$;

time for wheel $\%(s)$, one revolution

$\%$ ENTER WHEEL CONDITION AFTER DRESSING

rO=input ('cutting edge radius in (mm): ');

gamma $=50 \star \mathrm{pi} / 180$;

ơgrain rake angle

\& \& ENTER WORKPIECE MATERIL PROPERTY

$\mathrm{HW}=$ input ('Workpiece hardness:'); $8\left(\mathrm{~N} / \mathrm{mm}^{\wedge} 2\right)$, workpiece hardness

hardness

pre $=-12.4^{\star} \mathrm{vs}+1062.0$;

edge pressure on wear flat

$\%(\mathrm{~N} / \mathrm{mm} 2)$, cutting

\& 8 OTHER PARAMETERS IN WHEEL WEAR SIMULATION

$\mathrm{f}=0.5$;

\%grinding force

ratio

mu=0.5;

friction coefficient

$\mathrm{K}=2$ * $1 \mathrm{e}-7$;

ogrinding

rate

$\operatorname{Pr}=0.1$;

\%attitious wear

cutting edge recovery

opprobability of

$\%$ COEFFICIENTS IN WHEEL WEAR SIMULATION

$A=0.01$

$\mathrm{C}=0.00005$;

$\%$ \%

$\mathrm{Caa}=40000$;

$\mathrm{Bb}=1 * 10^{\wedge}(0)$;

$\mathrm{k}=0.7$;

\& 8 INITIALIZATION

$\mathrm{ca}(1)=0$;

oadhesion constant

$\mathrm{cb}(1)=0$;

\&adhesion constant

$\mathrm{B}(1)=0$;

flat area in new appearred grains

ofwear

$a(1)=0$;

$r w(1)=r 0$;

$\%(\mathrm{~mm})$, cutting edge radius

$\mathrm{ra}(1)=\mathrm{r} 0$;

$\frac{\circ}{\partial}(\mathrm{mm})$, average cutting edge radius

$\mathrm{Ac}(1)=\mathrm{pi}{ }^{\star} \mathrm{rO}^{\wedge} 2$;

cutting edge wear flat area

Aaa $(1)=\mathrm{pi}^{\star} \mathrm{r} 0^{\wedge} 2$;

$8\left(\mathrm{~mm}^{\wedge} 2\right)$,

average cutting edge wear flat area 


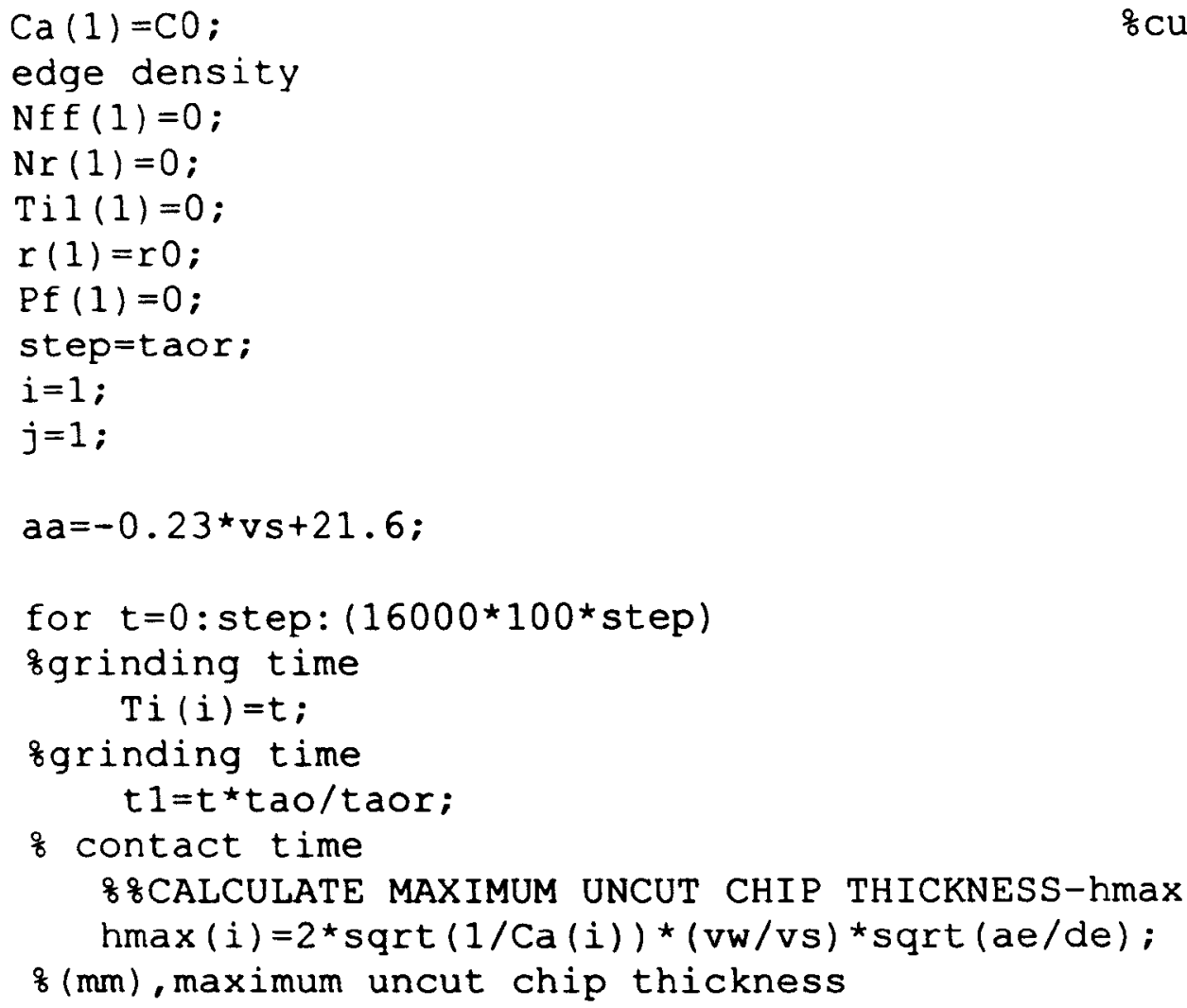

\% 8 CALCULATE ATTRITIOUS WEAR

$a(i)=3 * \tan (g a m m a) * K \star F c n(i) * n s \star l c /(p i * H w)$; sconstant change with Aaa(i)

$$
r w(i+1)=(a(i) *(t 1+t a o)+r 0 \wedge 3) \wedge(1 / 3) \text {; }
$$

scutting edge radius after attritious wheel wear $A c(i+1)=p i \star r w(i+1)^{\wedge} 2$;

scutting edge wear flat after attritious wheel wear

$\%$ CALCULATE ADHESIVE LOADING $\mathrm{Ca}(i+1)=\mathrm{Caa} *(\mathrm{Fcn}(i) \star l \mathrm{c}) / \mathrm{Hw}$; $c a(i+1)=(c a(i) * 0.3+k * c a(i+1)) * 0.3$; 
$\operatorname{Pf}(i)=A^{\star}\left(\text { tao* } 10^{\wedge} 4\right)^{\wedge}\left(c^{\star}(\right.$ Hss $/($ Fct $\left.(i) \star m))\right)$;

spossibility of abrasive and bond wear

$\mathrm{Nf}(i)=\mathrm{Ca}(i) \star \mathrm{Pf}(\mathrm{i})$;

\&abrasive and bond wear density

$\mathrm{Nff}(i+1)=\mathrm{Nff}(i)+\mathrm{Nf}(i)$;

stotal abrasive and bond wear density

$\mathrm{N}(\mathrm{i})=\mathrm{Nf}(\mathrm{i})$ *Pr;

scutting edge recovery density

$\mathrm{Nr}(\mathrm{i}+1)=\mathrm{Nr}(\mathrm{i})+\mathrm{N}(\mathrm{i})$;

ototal cutting edge recovery density

$\mathrm{Pb}(i)=\mathrm{Bb}^{\star} \mathrm{Ca}(i+1) /\left(\mathrm{Vp}^{\star} \operatorname{sqrt}(1 / \mathrm{Ca}(i))\right)$;

spossibility of two grains blocking together

$\mathrm{Nb}(\mathrm{i})=(\mathrm{Ca}(\mathrm{i})) * \mathrm{~Pb}(\mathrm{i})$;

$\mathrm{Ca}(i+1)=\mathrm{C} 0-\mathrm{Nff}(i+1)+\mathrm{Nr}(i+1)-\mathrm{Nb}(i) / 2 ;$

of Active cutting edge density

$\%$ CALCULATE WEAR AREA RATIO IN A UNIT AREA

for $g=2: j+1$

$A w(g-1)=N(j+2-g) \star p i *(a(i) *(g-$

1) *step $\left.+\mathrm{r} 0^{\wedge} 3\right)^{\wedge}(2 / 3)$;

$B(g)=B(g-1)+A w(g-1)$;

end

$A N(i)=(C O-N f f(i+1)) \star A C(i+1)+B(g)$;

Aaa $(i+1)=(\mathrm{AN}(i) / \mathrm{Ca}(i+1))$;

wear area in aunit area

ra $(i+1)=\operatorname{sqrt}($ Aaa $(i+1) / p i)$ :

average radius of cutting edges

$j=j+1$;

$i=i+1 ;$

$\operatorname{Til}(i)=\mathrm{T} i(i-1)+$ step;

end

\%

for $q=2: i$

end

$$
M(1, q-1)=N(1, i-q+1) ;
$$

if $\operatorname{Nff}(1, i)<\mathrm{CO}$

how many grain left finally

$\mathrm{N}(1, \mathrm{i})=\mathrm{CO}-\mathrm{Nff}(1, \mathrm{i})$;

else $N(1, i)=0$;

end

$\%$ CALCULATE GRINDING PERFORMANCE

$M(1, i)=N(1, i)$;

$\mathrm{Ra}=16.7^{\star}\left(\mathrm{Vw}^{\star} \operatorname{sqrt}(1 . / \mathrm{Ca}) /\left(\mathrm{vs}{ }^{\star} \operatorname{sqrt}(\mathrm{ds})\right) . \wedge 0.8-0.037\right.$; 
Raa $=$ mean $(\mathrm{Ra})$

workpiece surface roughness $\mathrm{Ra}$

Powera=mean (Power)

mean grinding power

Vw1 $=\mathrm{Ti1} /$ step ${ }^{\star} 21 ;$

oremoval rate

$\mathrm{Vw}=\mathrm{Ti} /$ step ${ }^{2} 2$;

oremoval rate

$\because$ PLOTS

figure, plot(Vw1, Ca) \& cutting edge density grid

xlabel('specific volume of material removed (mm^3/mm)

')

ylabel('cutting edge density $\left(1 / \mathrm{mm}^{\wedge} 2\right)$ ')

figure,plot(Vw1,ra) $\%$ average wear flat area grid

xlabel('specific volume of material removed (mm^3/mm) ')

ylabel('average radius of wear flat (micron)')

figure, plot(Vw1, Ra) $\frac{8}{8}$ workpiece surface roughness $\mathrm{Ra}$ grid

$x$ label('specific volume of material removed

$\left.\left(\mathrm{mm}^{\wedge} 3 / \mathrm{mm}\right)^{\prime}\right)$

ylabel('Ra (micron)')

figure, plot (Vw1,ca) \& grid

xlabel('specific volume of material removed

$\left.\left(\mathrm{mm}^{\wedge} 3 / \mathrm{mm}\right)^{\prime}\right)$

ylabel('adhesive loading coefficient')

figure, plot $(\mathrm{Vw}, \mathrm{Pb})$ \& pore loading coefficient grid

xlabel('specific volume of material removed $\left.\left(\mathrm{mm}^{\wedge} 3 / \mathrm{mm}\right)^{\prime}\right)$

ylabel (pore loading probability') 


\section{PAGES NOT SCANNED AT THE REQUEST OF THE UNIVERSITY}

SEE ORIGINAL COPY OF THE THESIS FOR THIS MATERIAL 


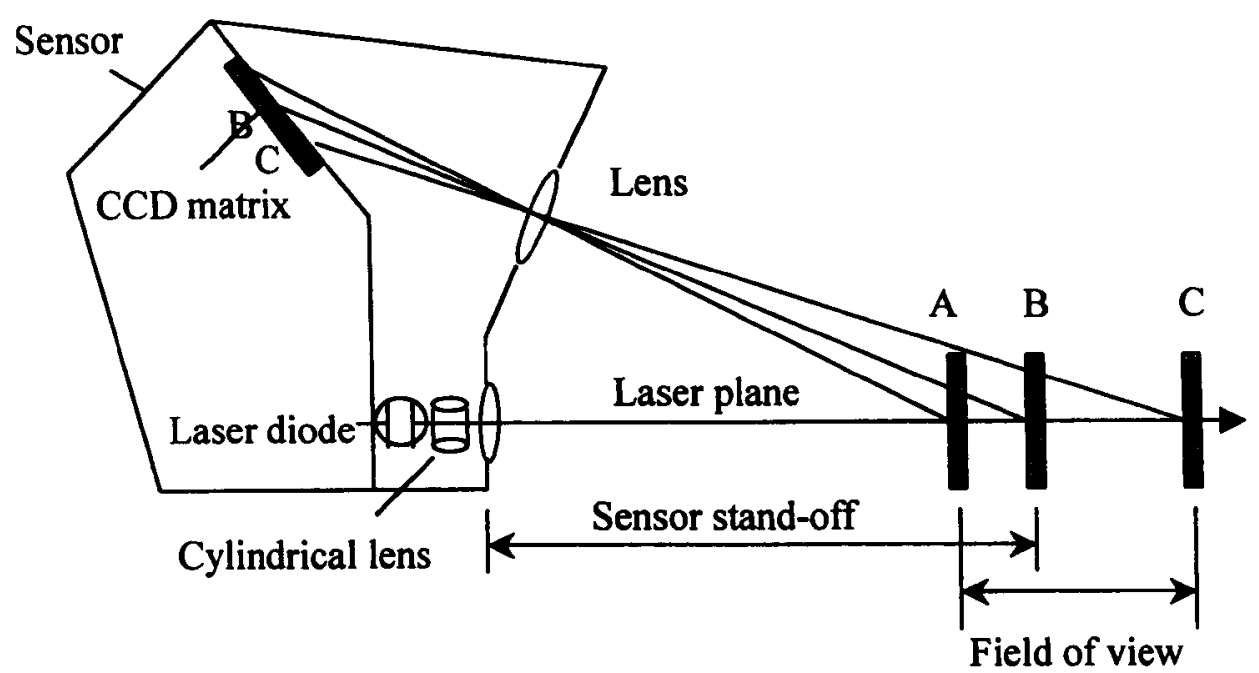

Fig. 2.2 Basic principle of laser triangulation [12]

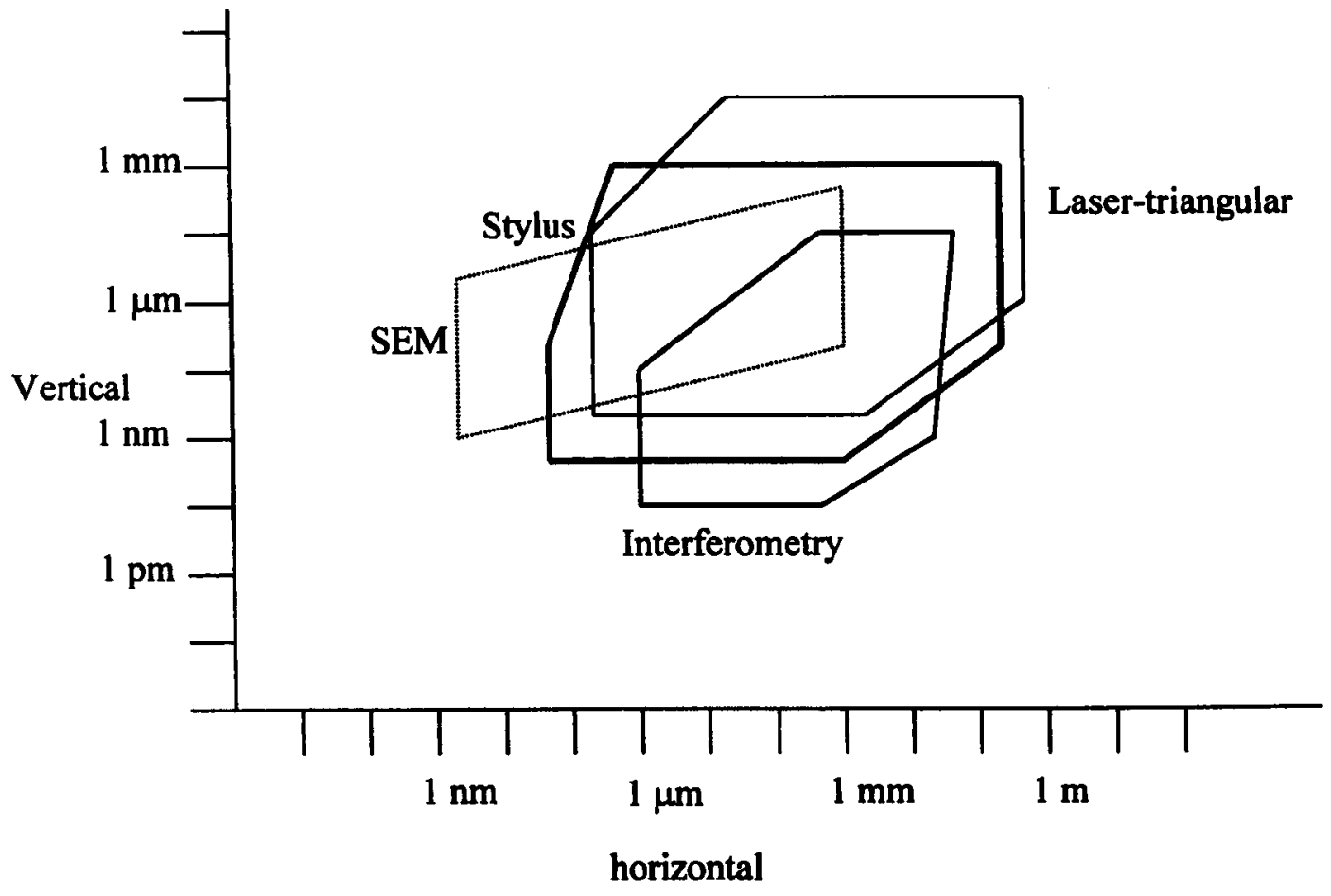

Fig. 2.3 A resolution and range of different measuring techniques 


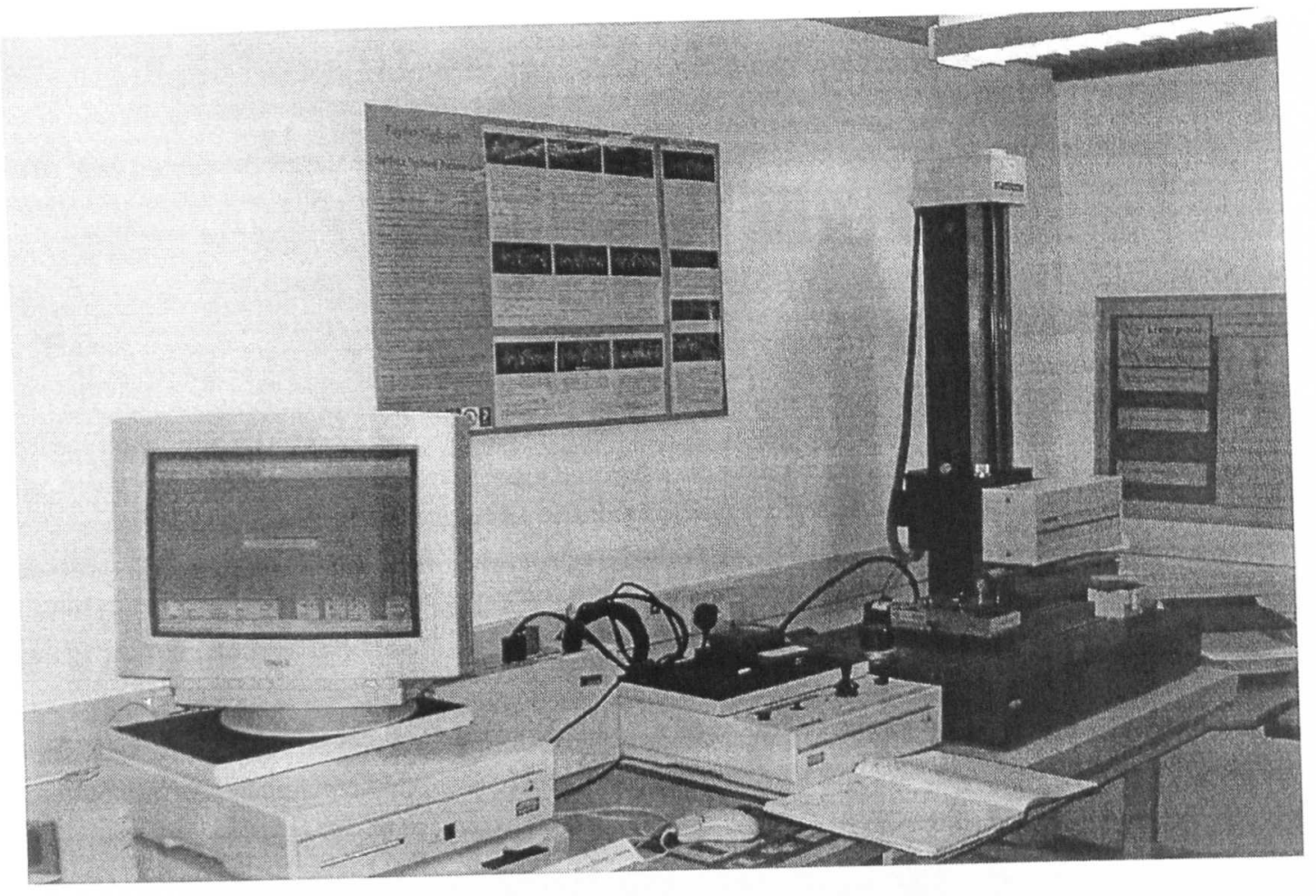

Fig. 2.4 Talysurf instrument (3D)

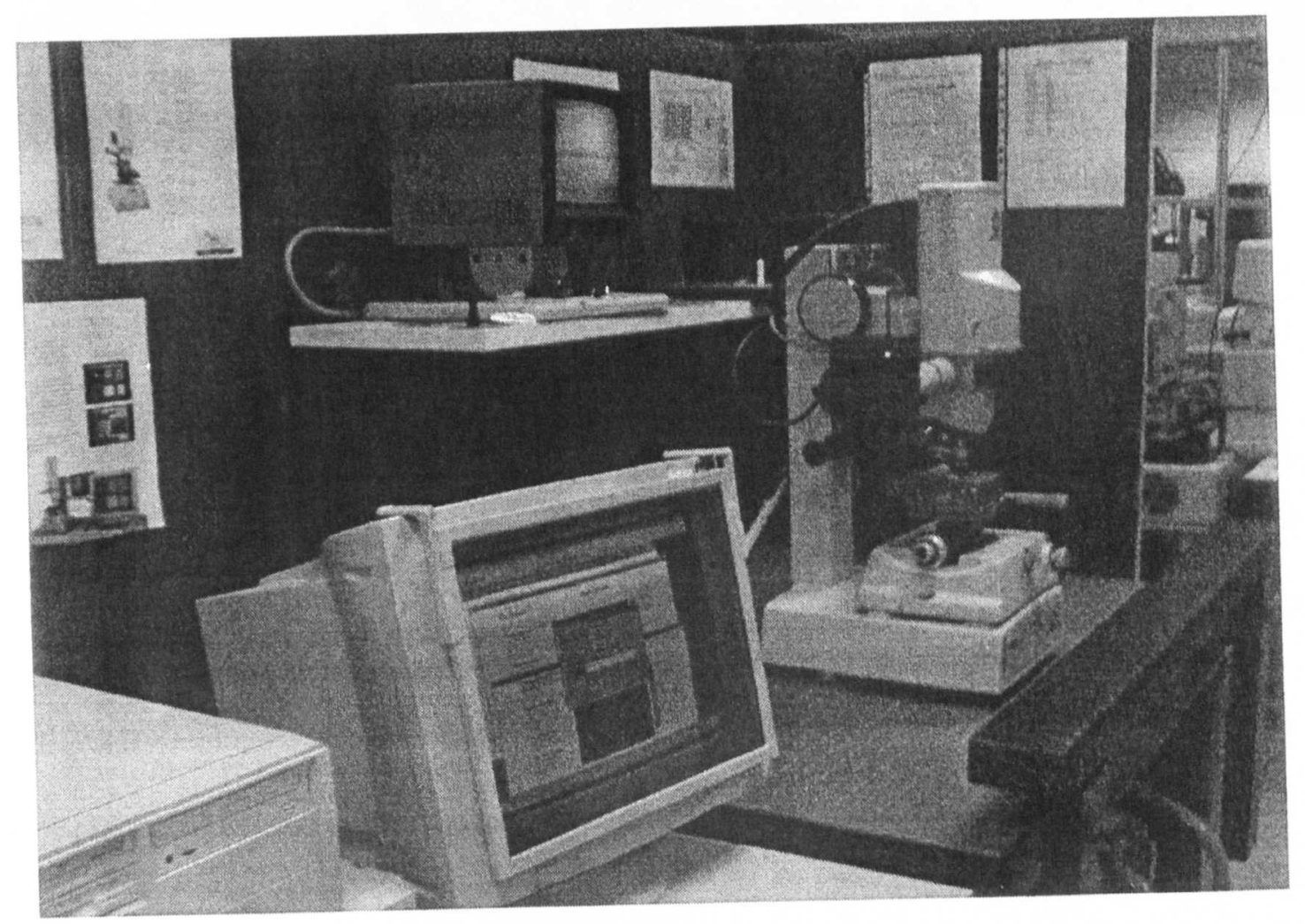

Fig. 2.5 Wyko RST Plus instrument 


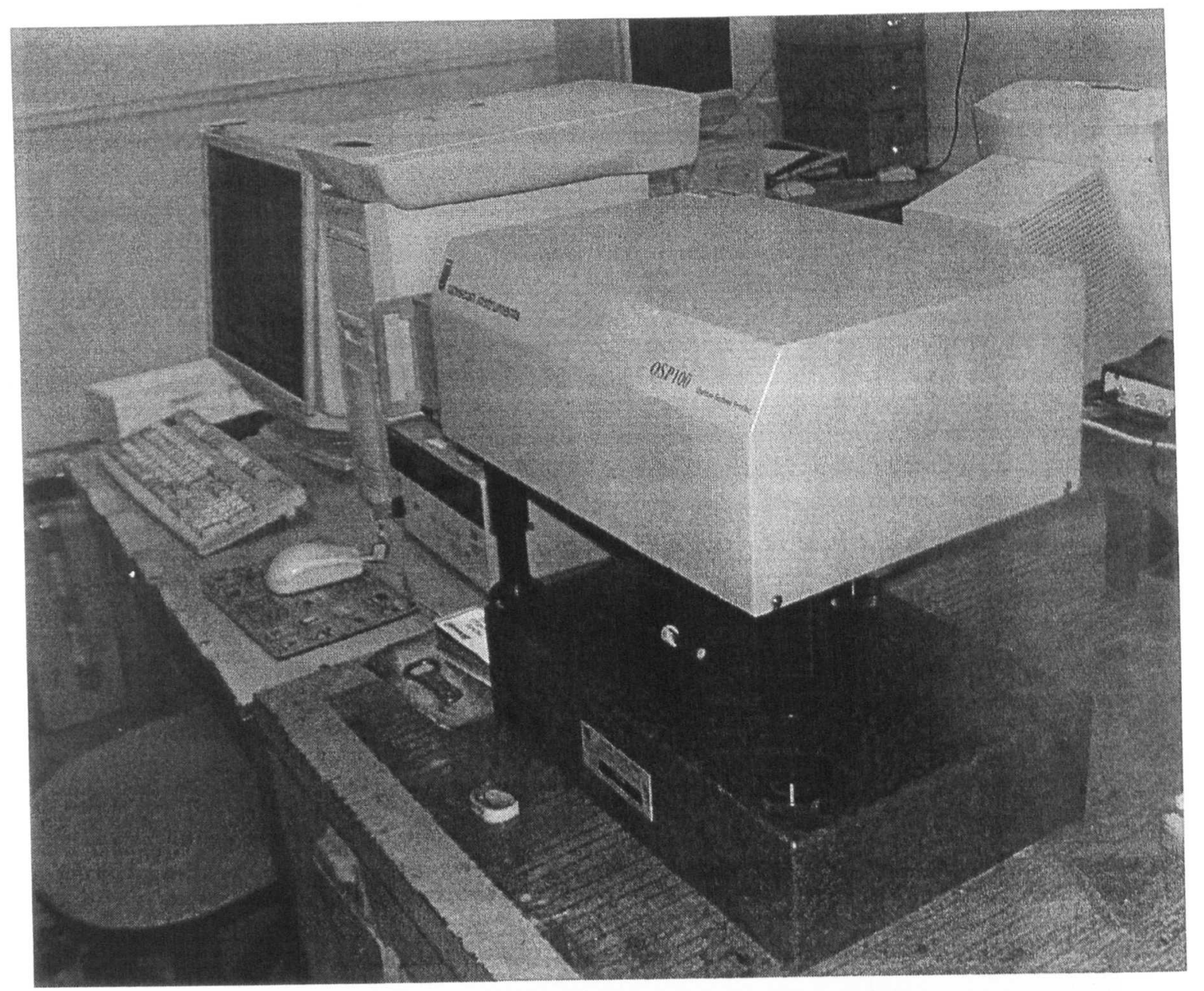

Fig. 2.6 Uniscan OSP 100 instrument 


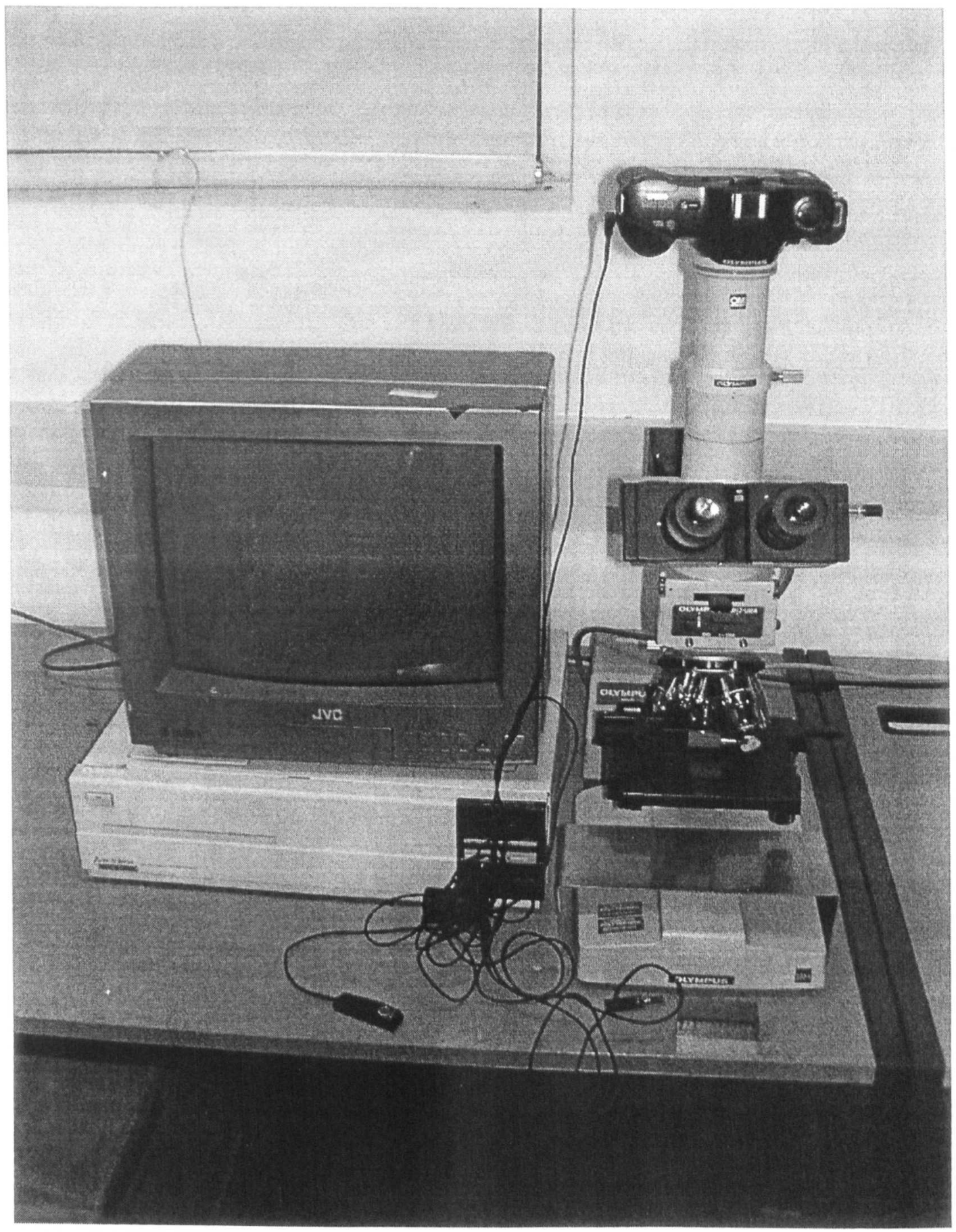

Fig. 2.7 Olympus BH2-UMA optical microscope with a mounted Olympus SC 35 camera 


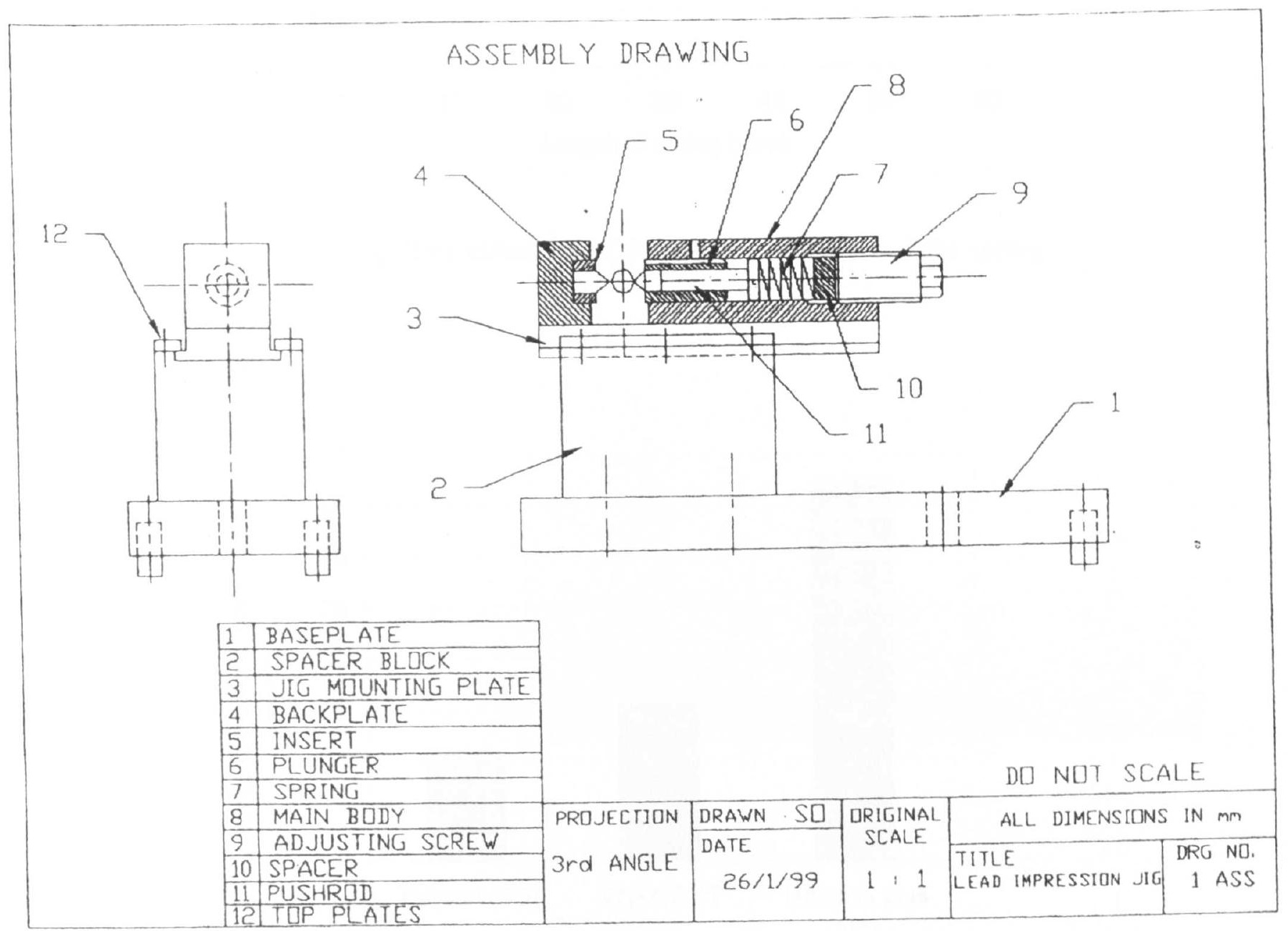

Fig. 2.8 The replication rig for lead replica 


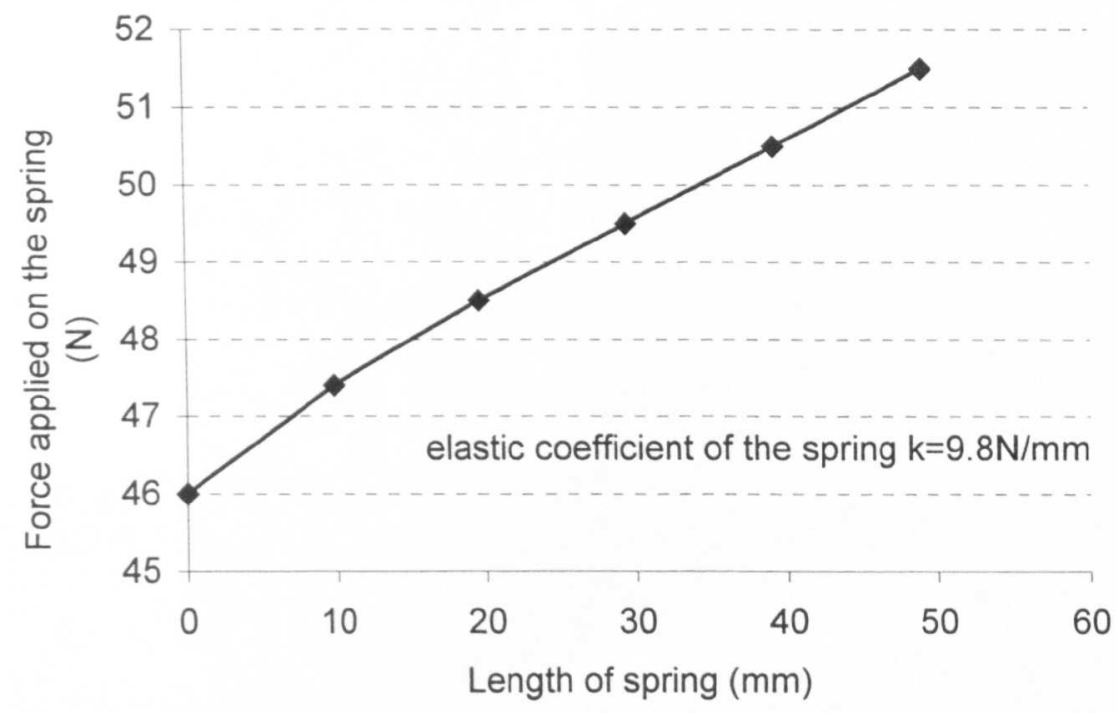

Fig. 2.9 Calibration of the elastic coefficient of the spring

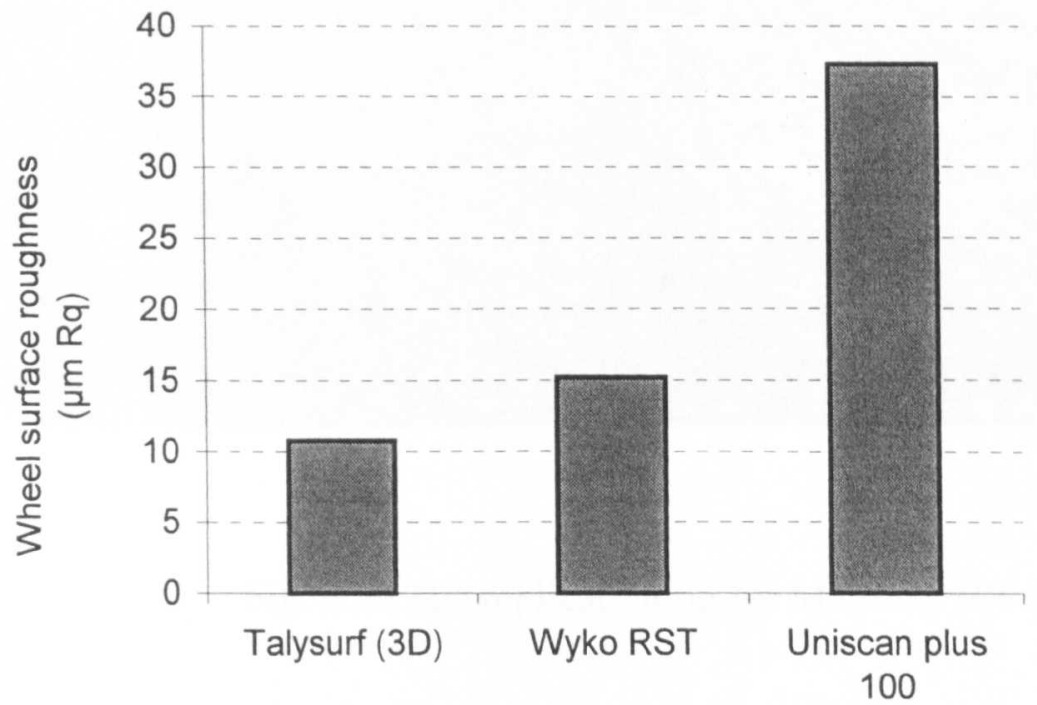

Fig. 2.11 Comparison of wheel surface roughness measured by three measurement methods 


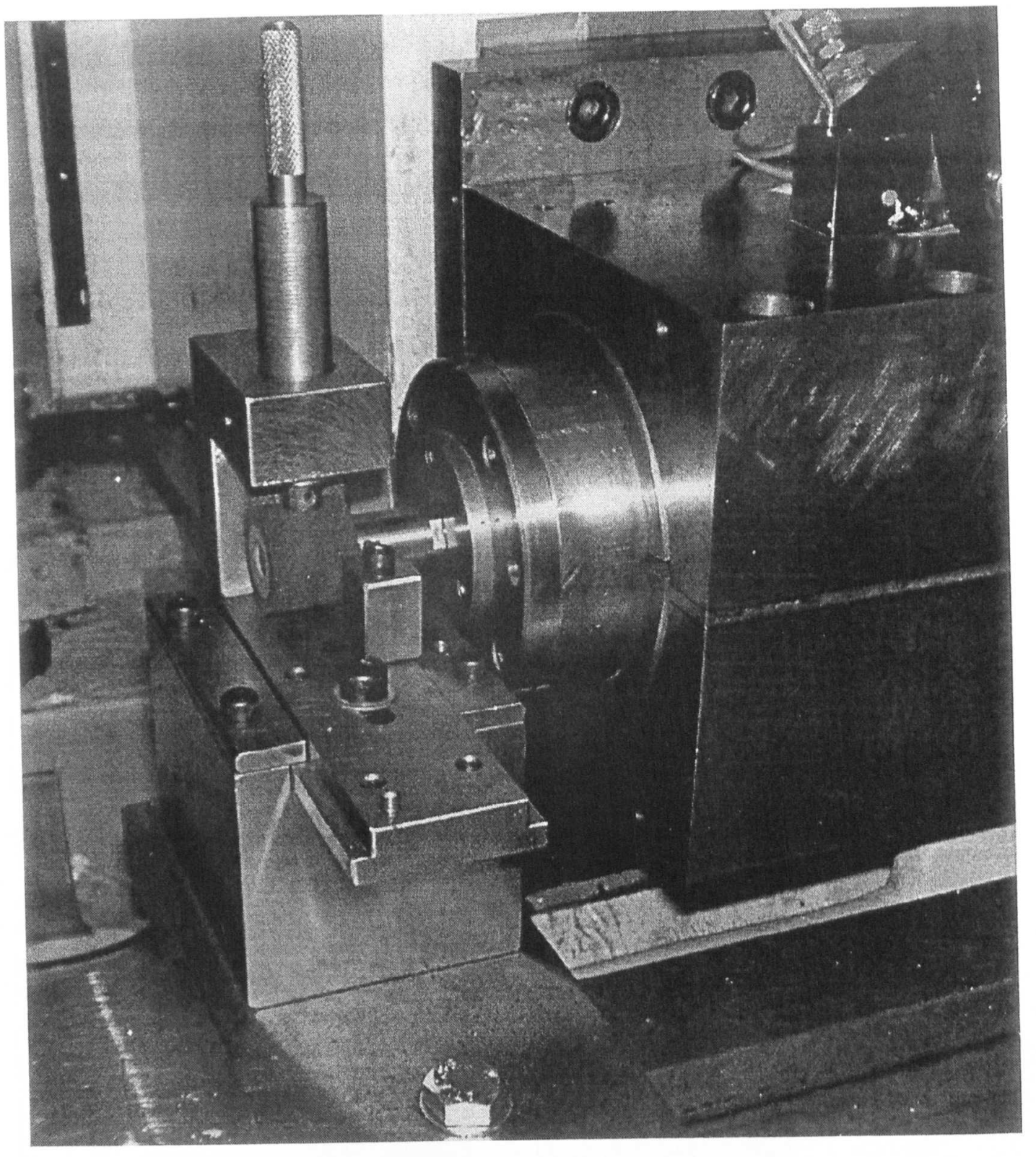

Fig. 2.10 The replication rig for Microset 101 replicas 

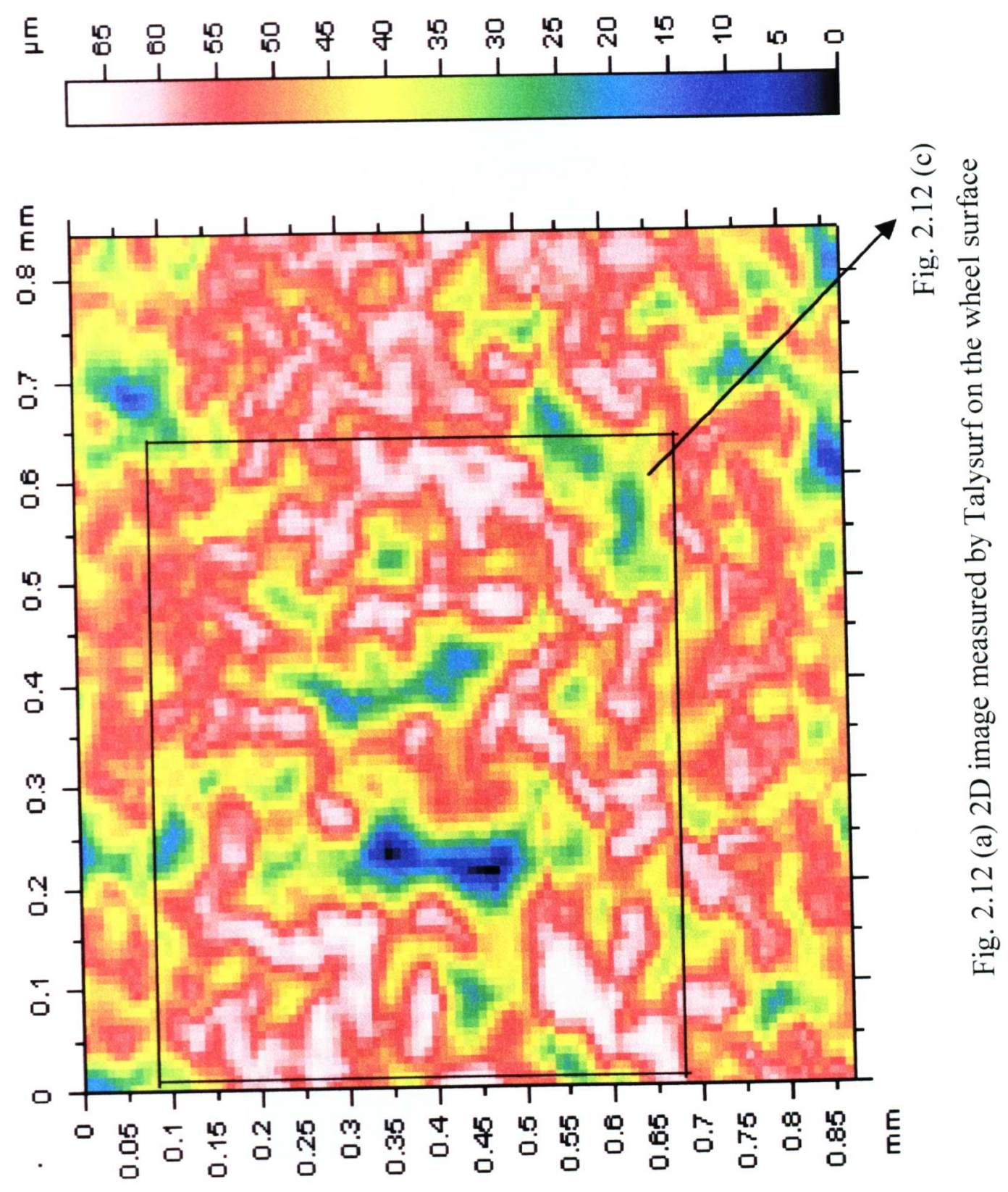

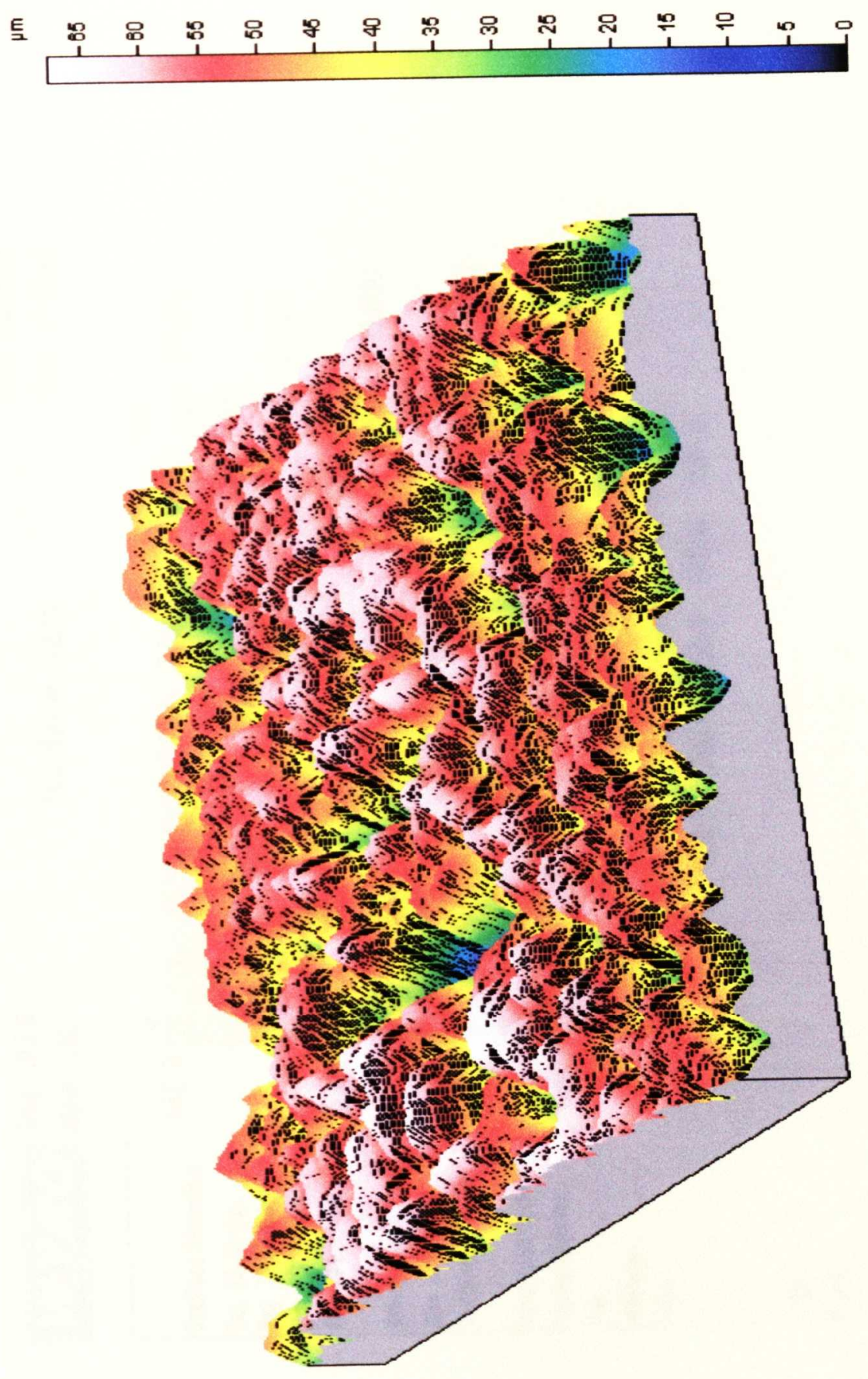

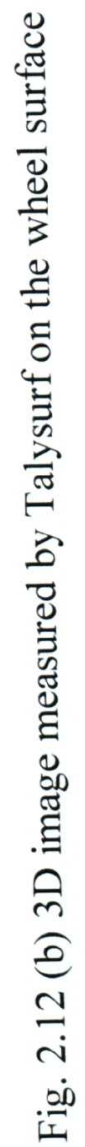


嘉商
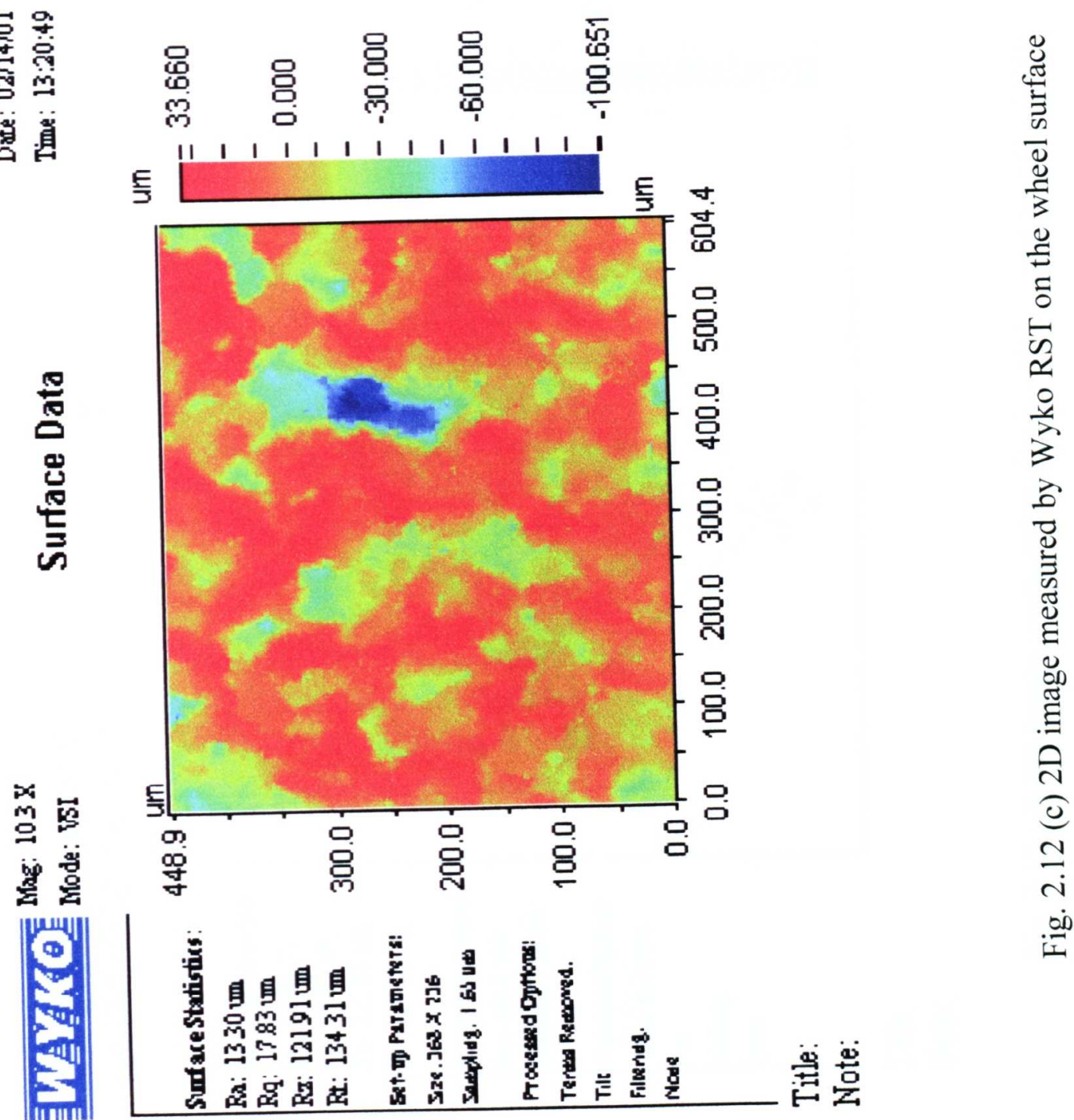

io 


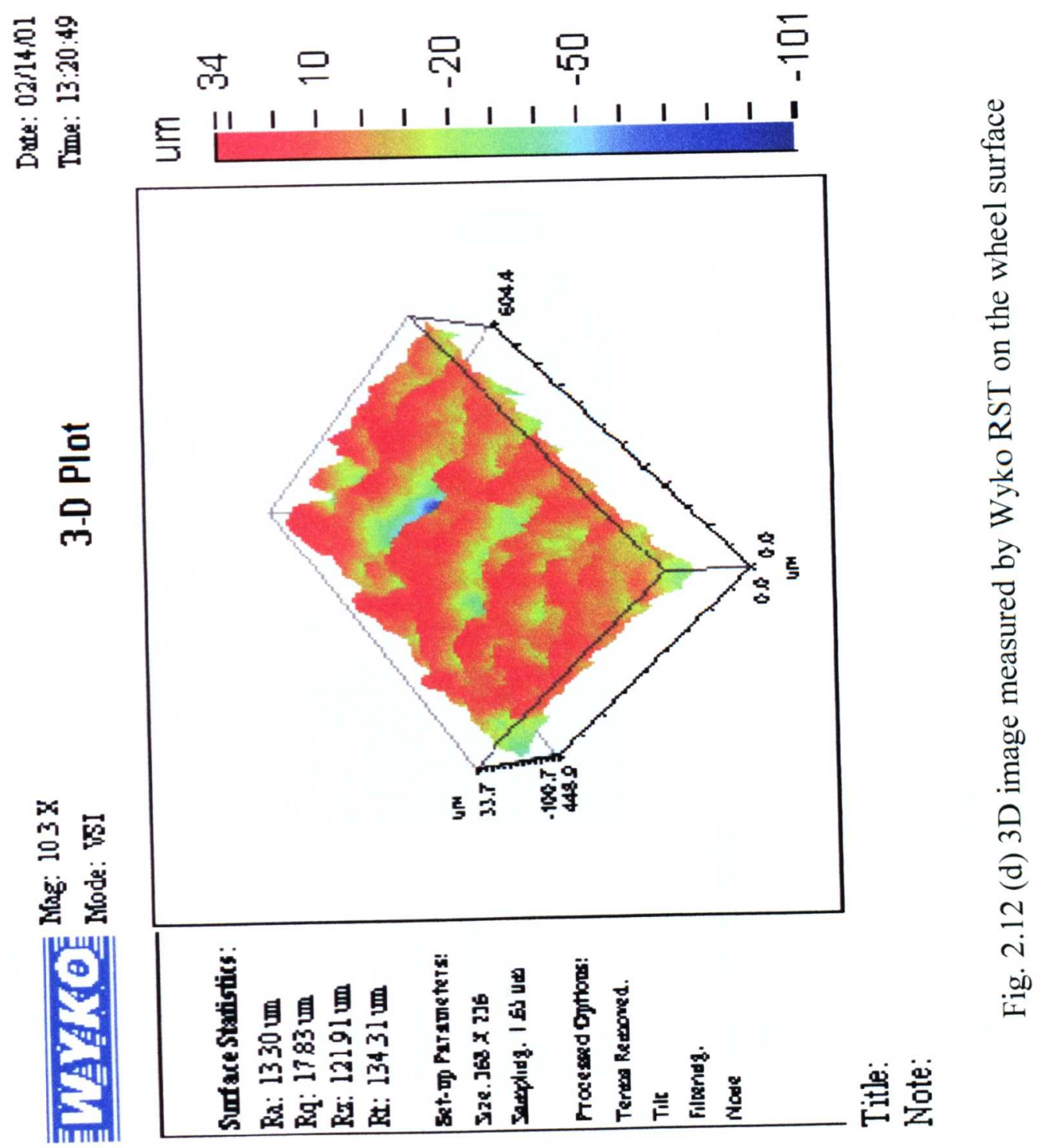




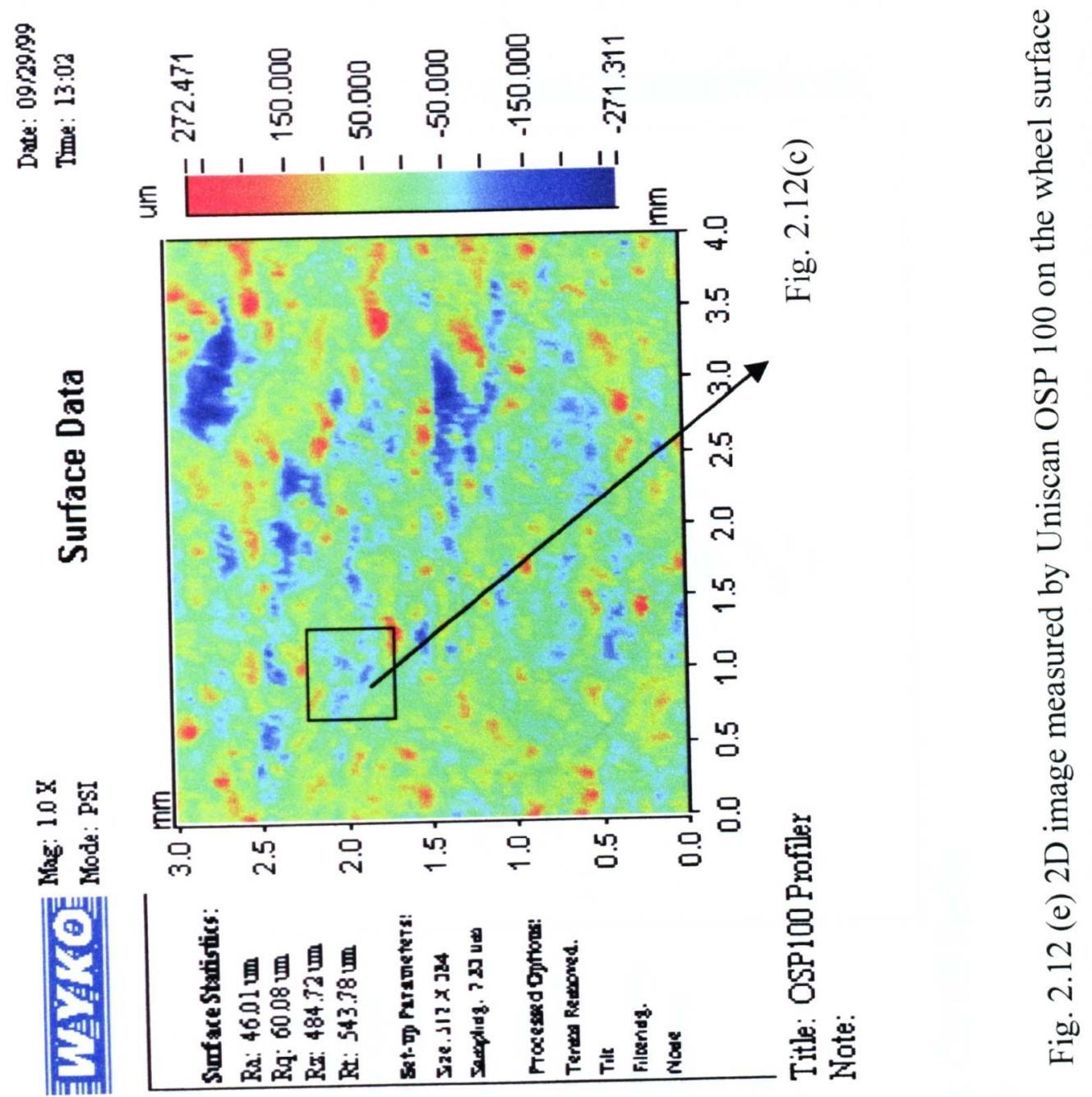




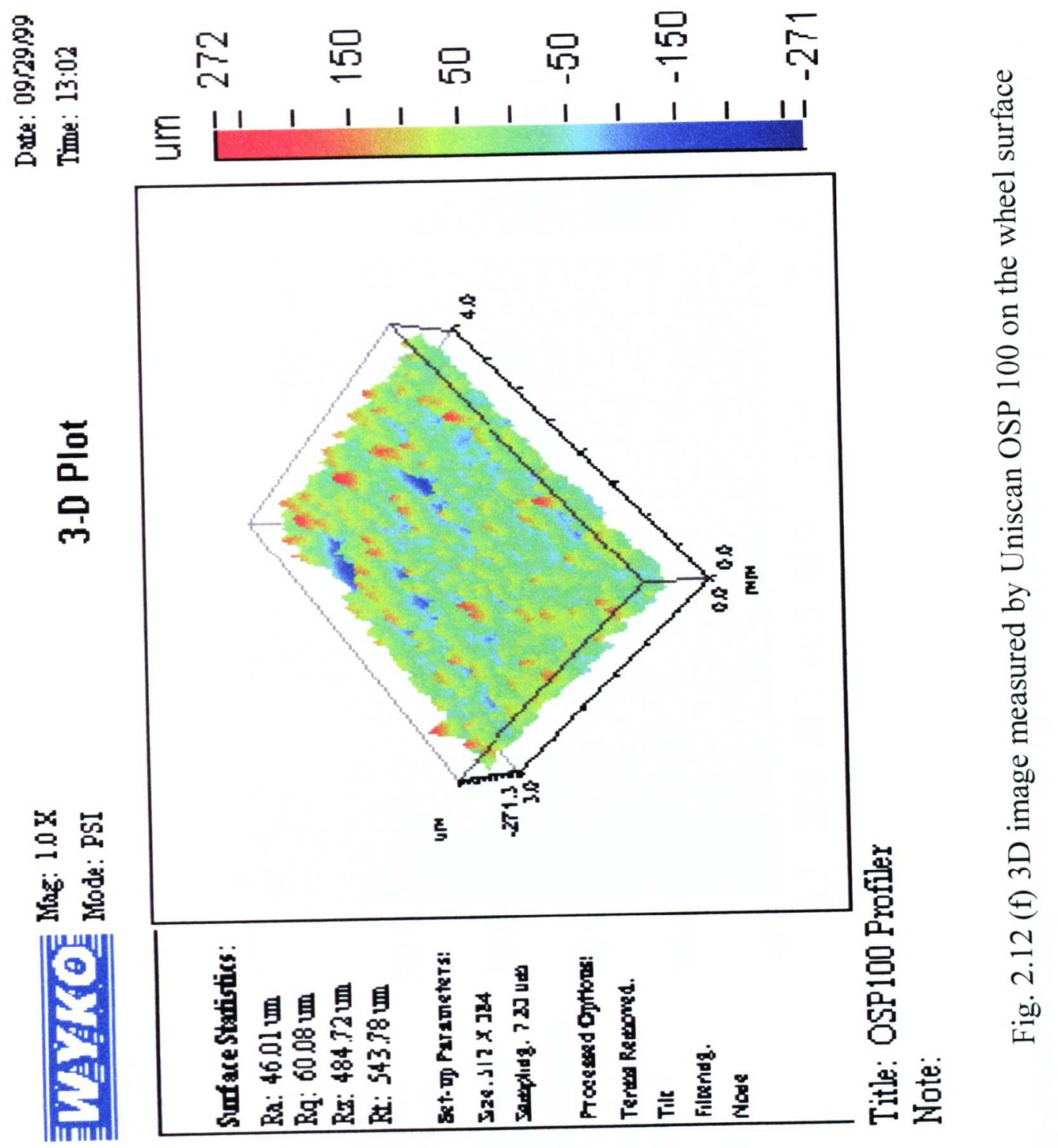




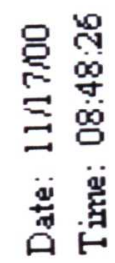

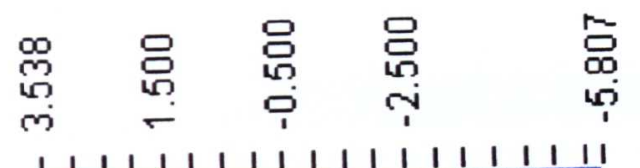

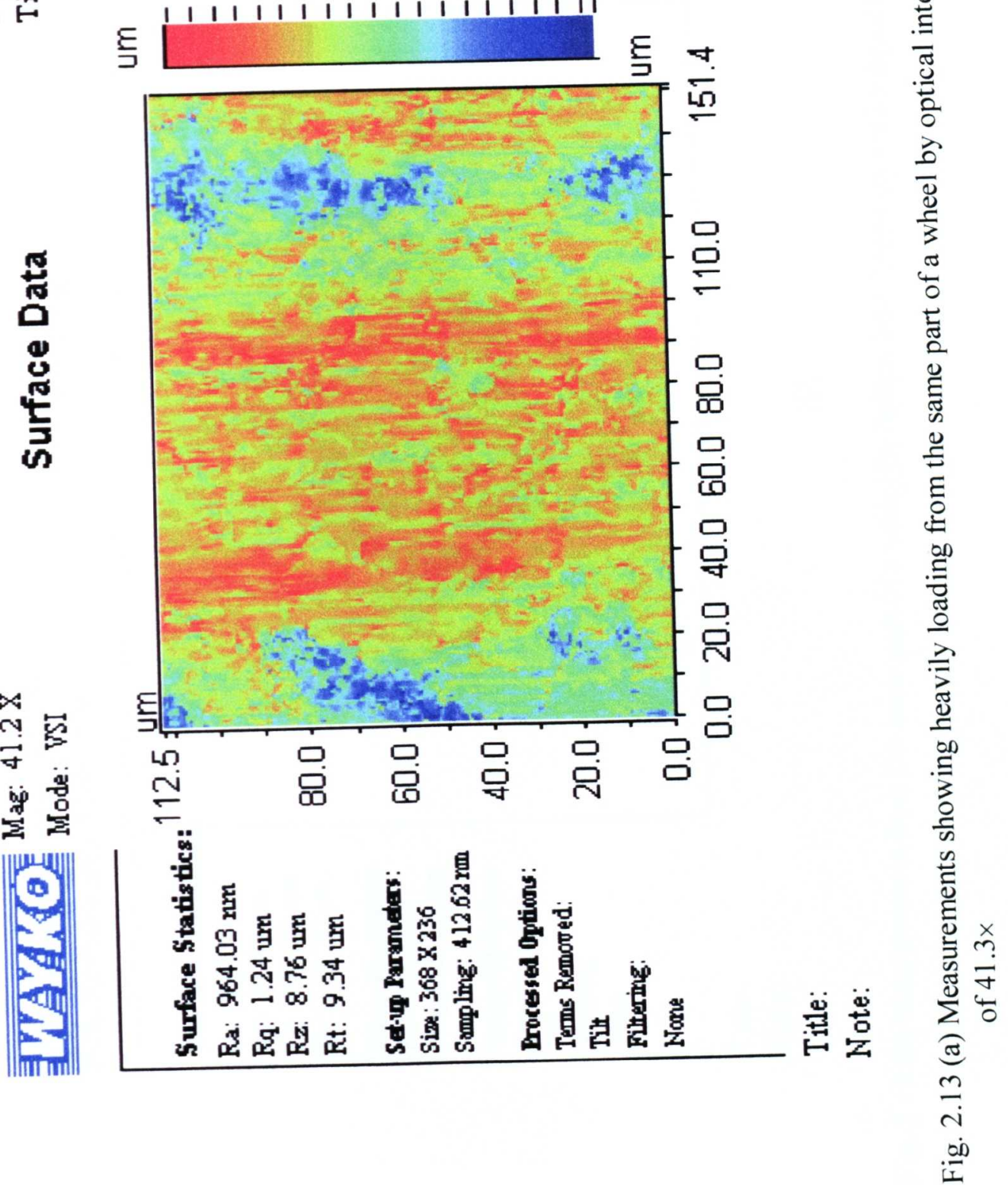




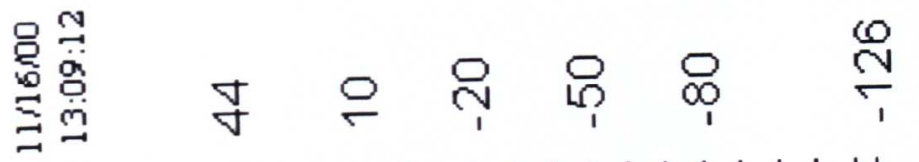

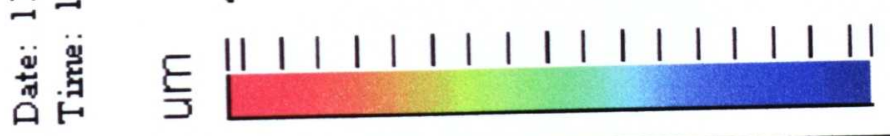

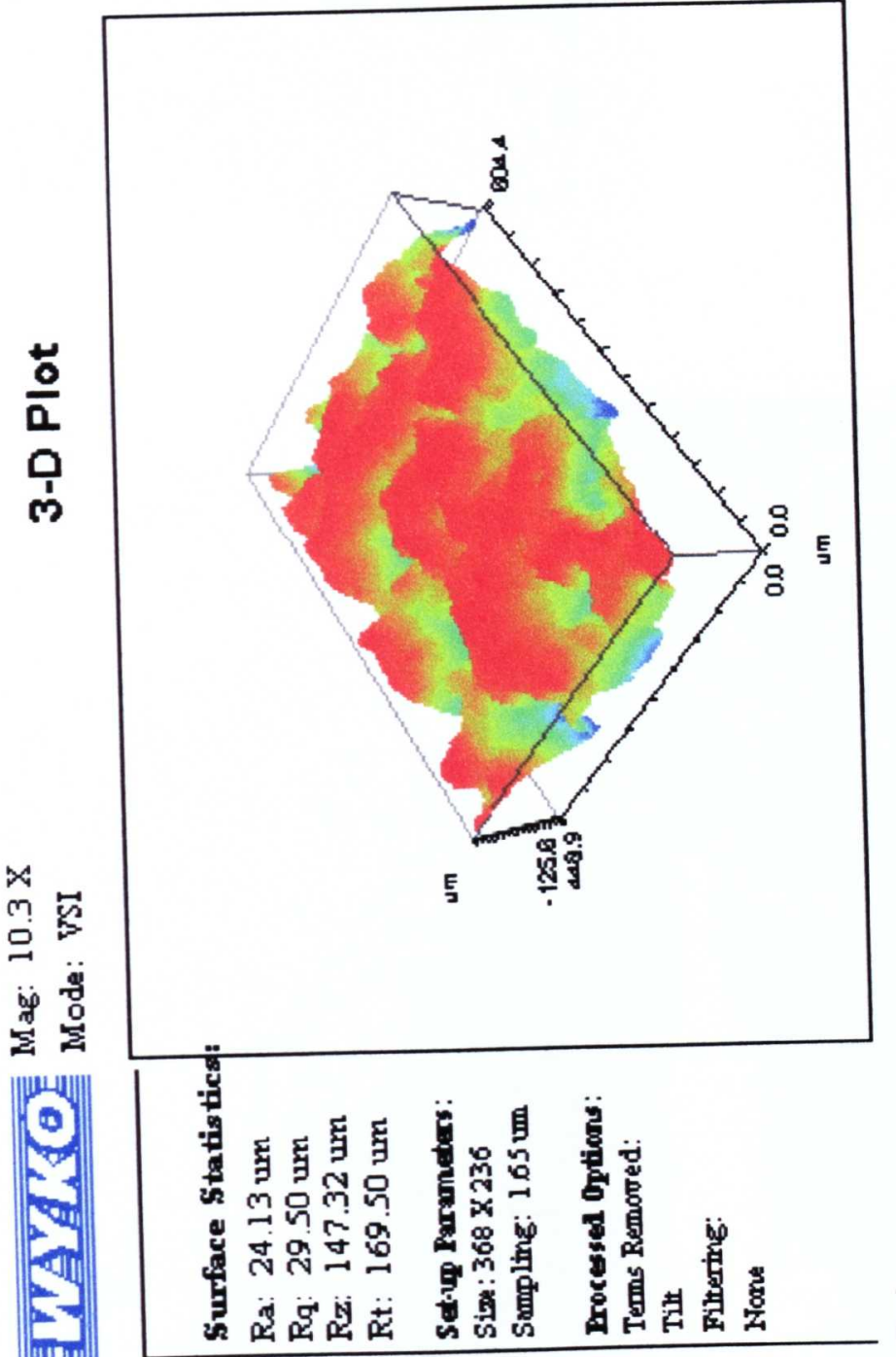

열 흠

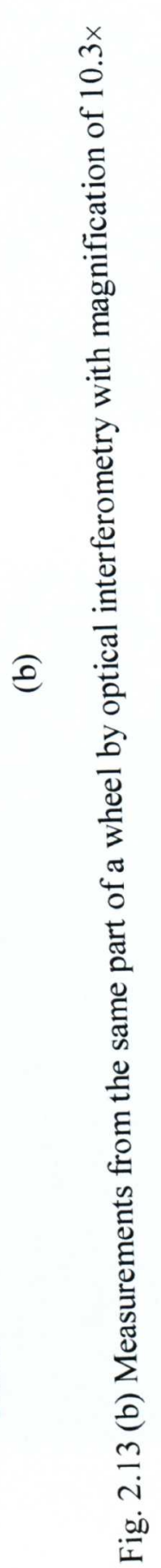




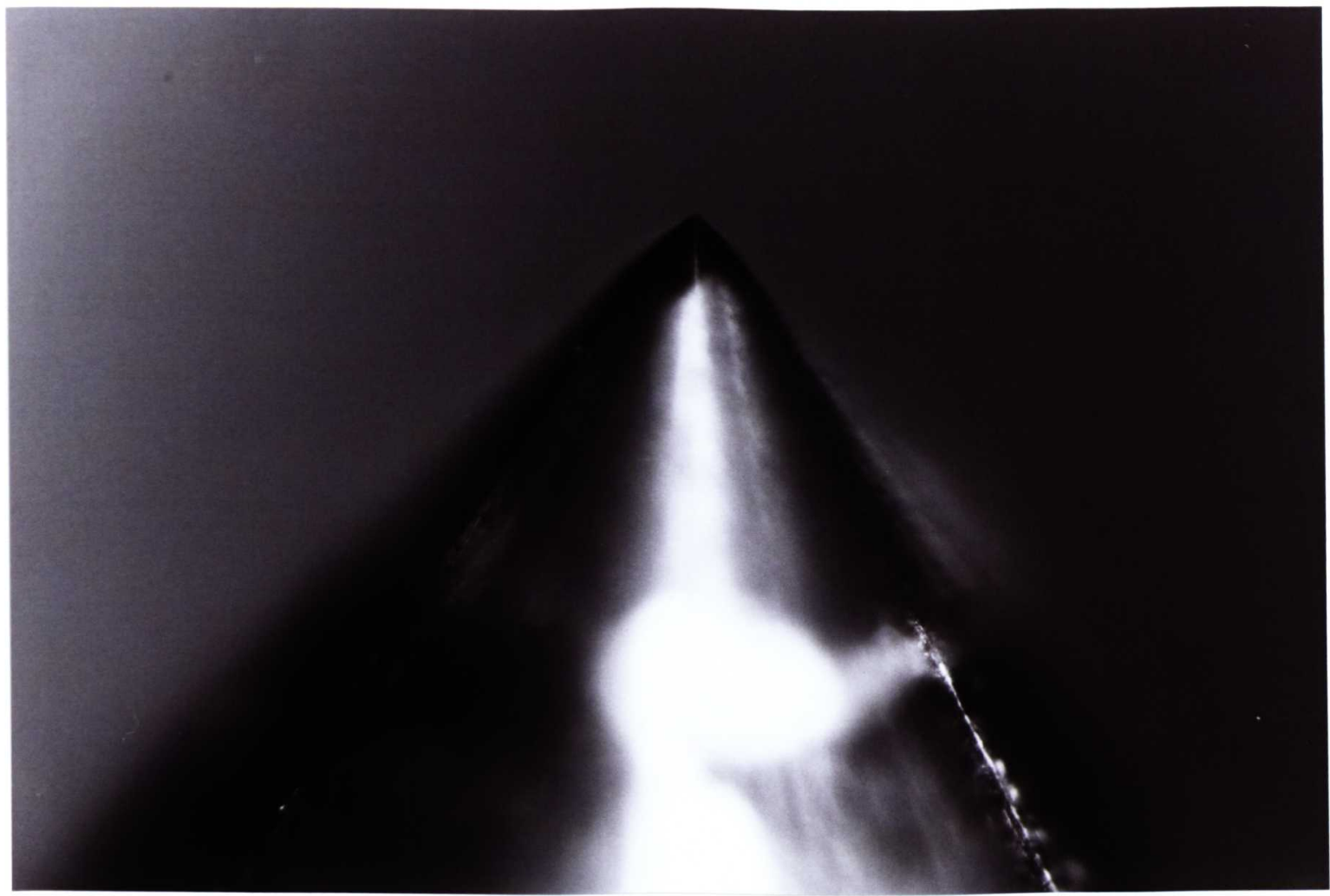

Fig. 2.14 (a) Microscope photograph (20x) of stylus before measuring a CBN wheel area of $1 \mathrm{~mm}^{2}$

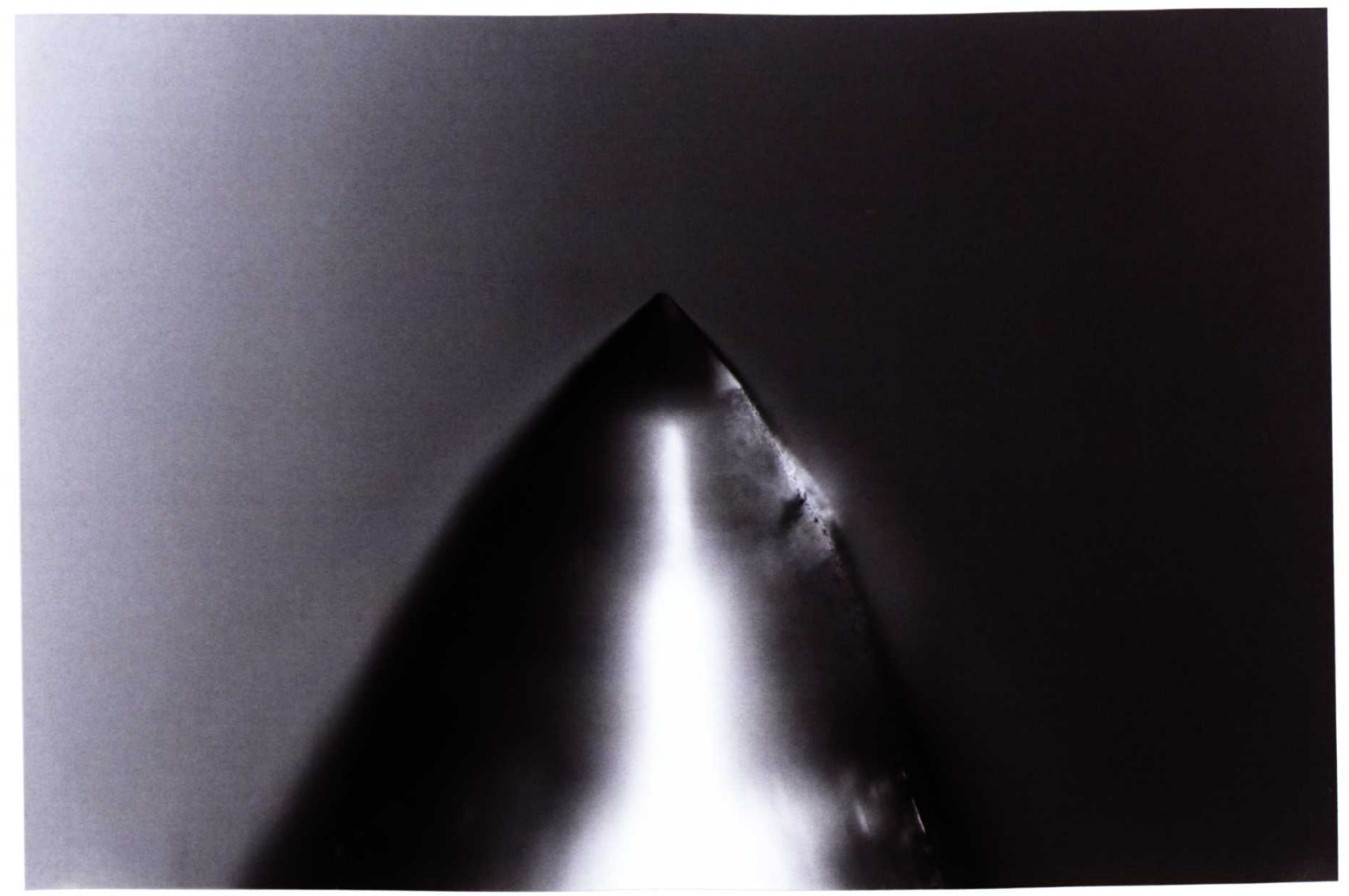

Fig. 2.14 (b) Microscope photograph (20x) of stylus after measuring a CBN wheel area of $1 \mathrm{~mm}^{2} 5$ times 


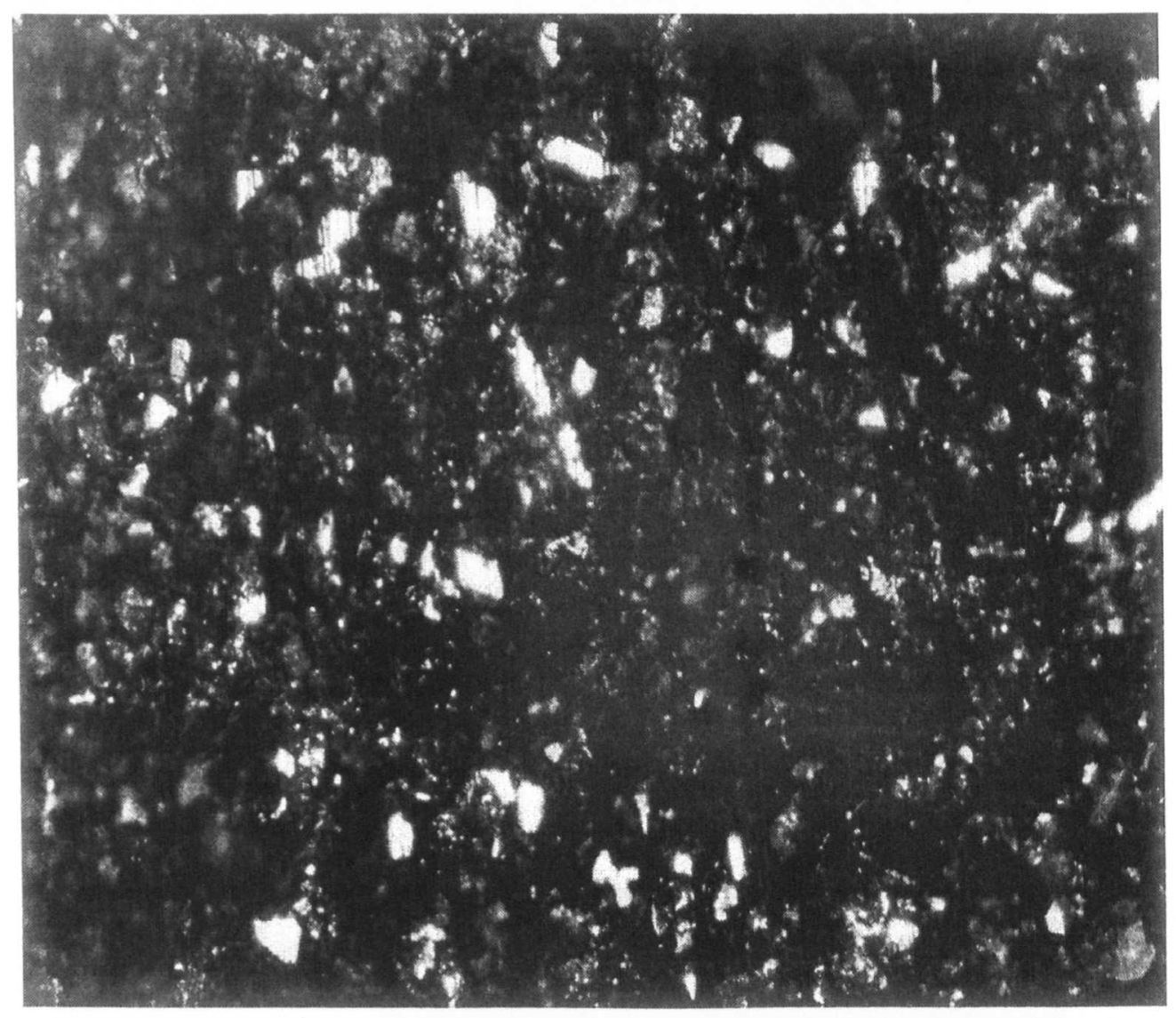

Fig. 2.15 Microscope photograph of wheel surface after grinding (Wheel: B91150V1) 


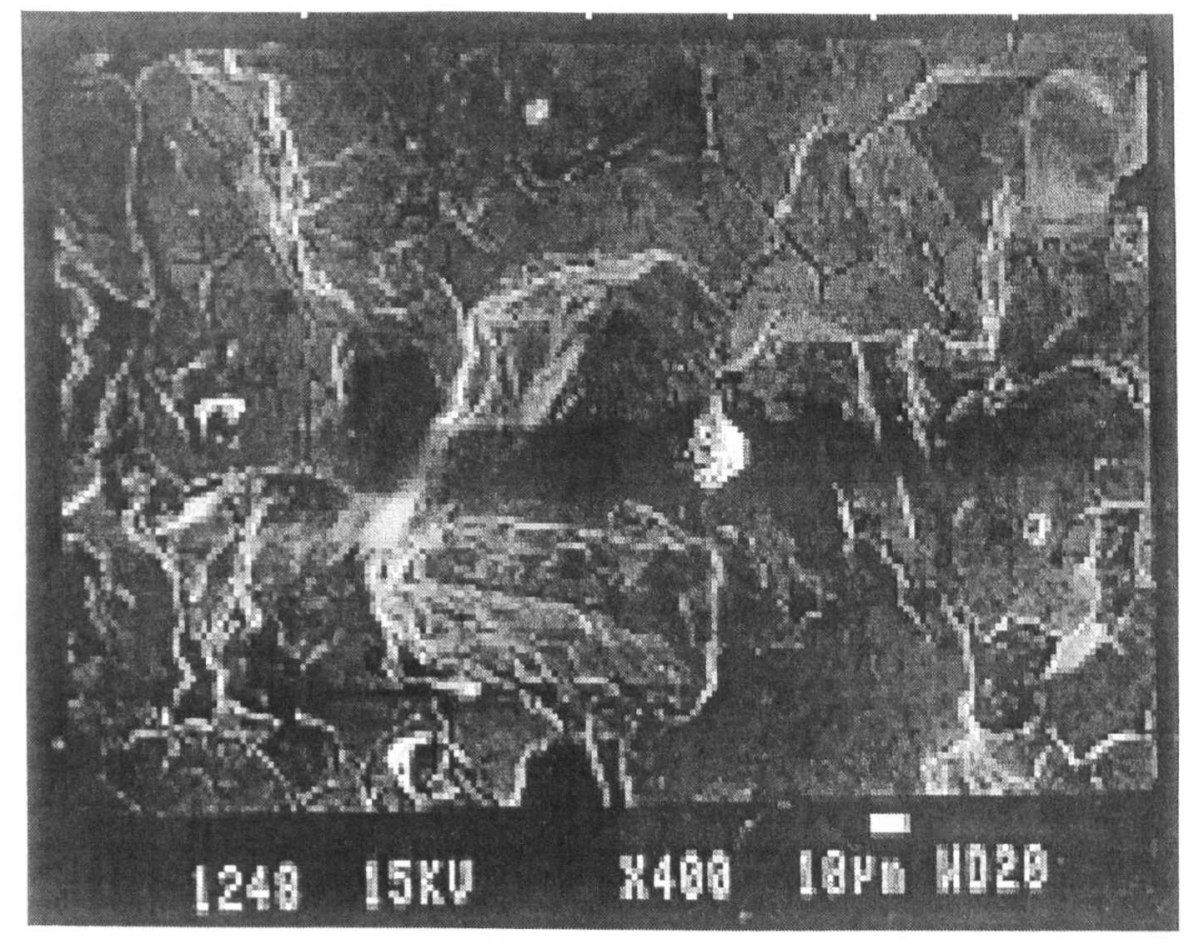

Fig. 2.16 SEM photograph lead replica after making a replication of wheel

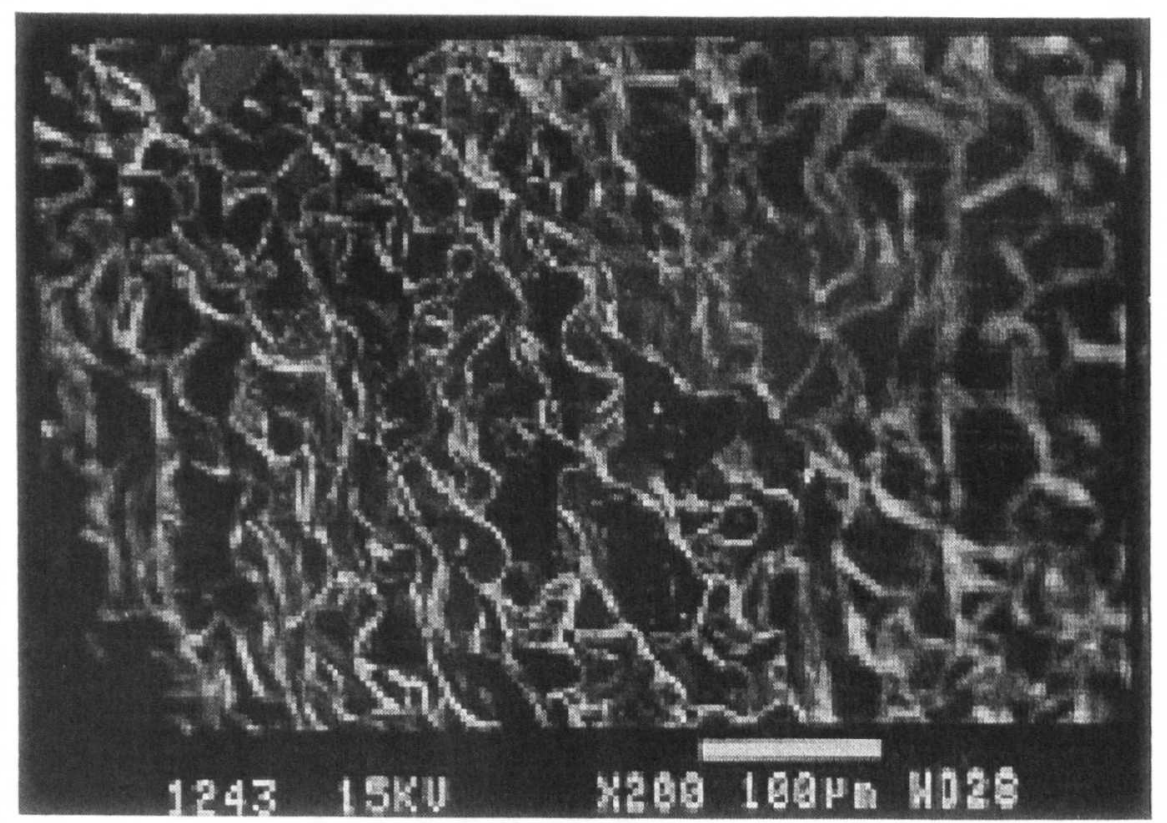

Fig. 2.17 SEM photograph of a Microset 101 replica which melted during coating 


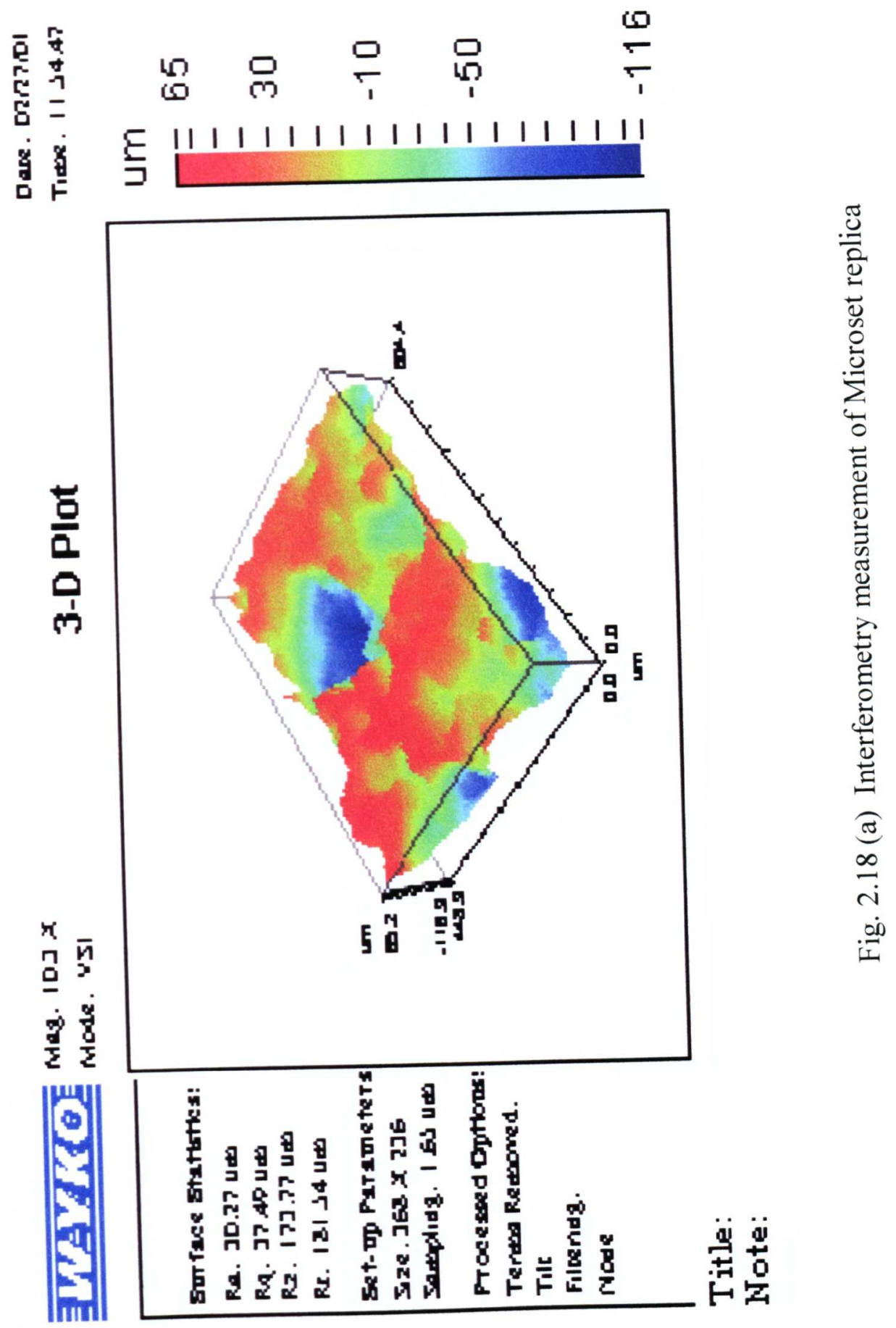



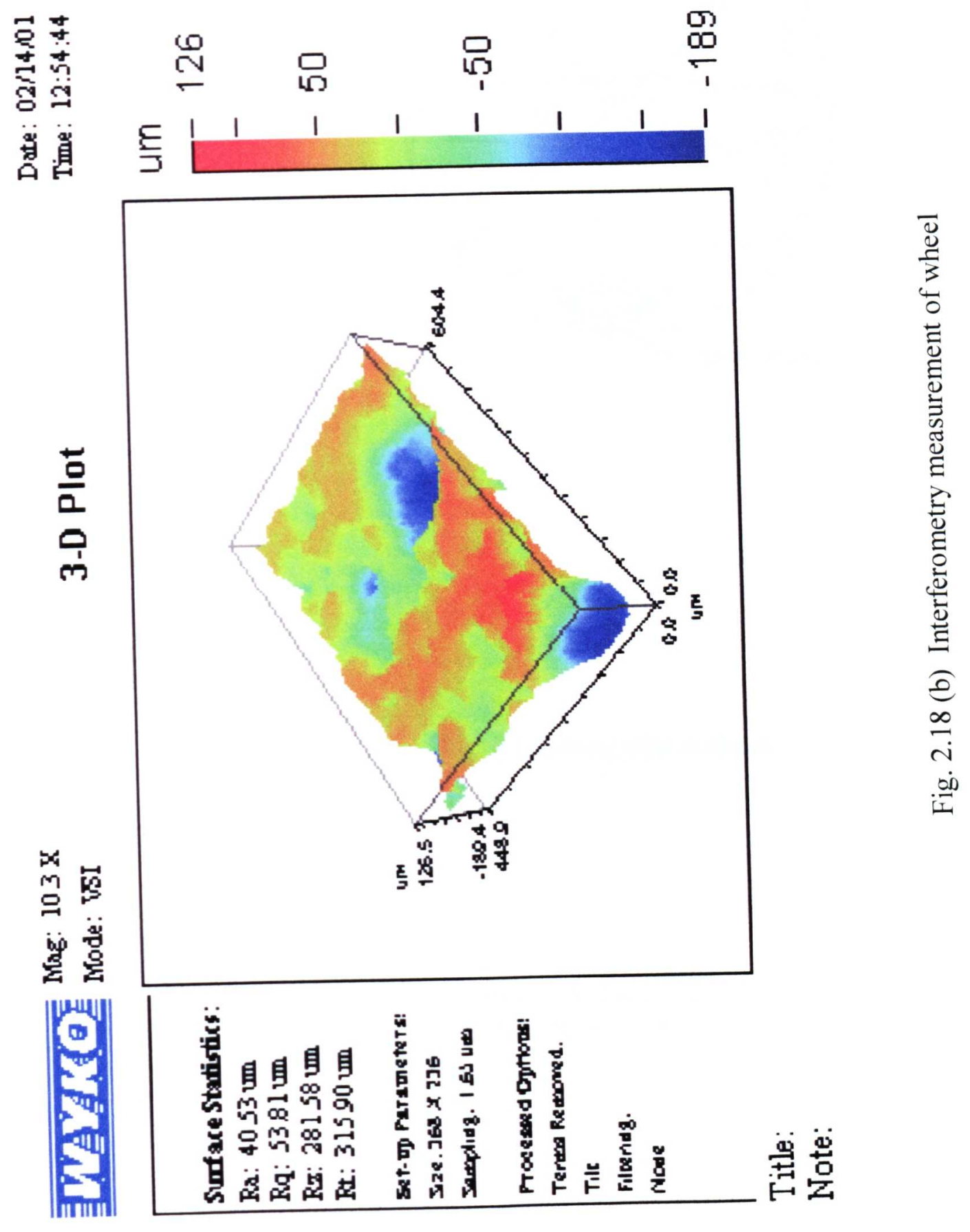


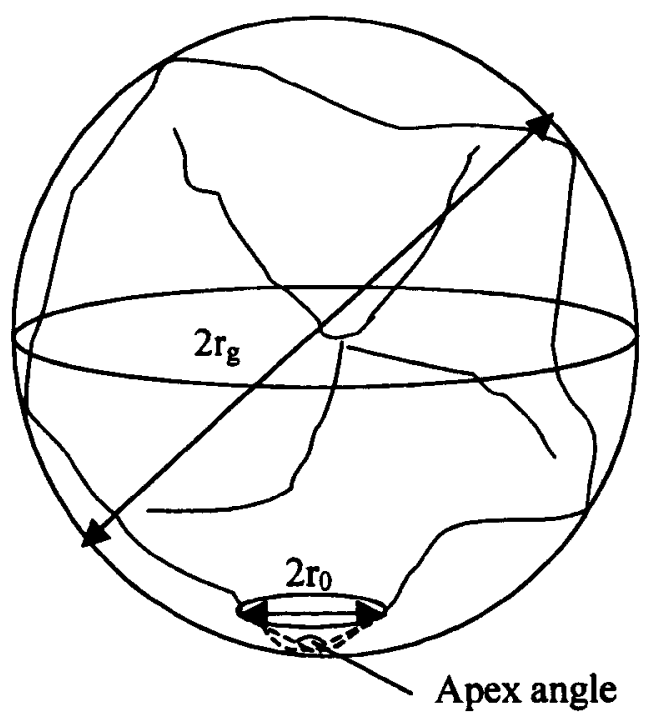

Fig. 3.1 Cutting edge dullness 


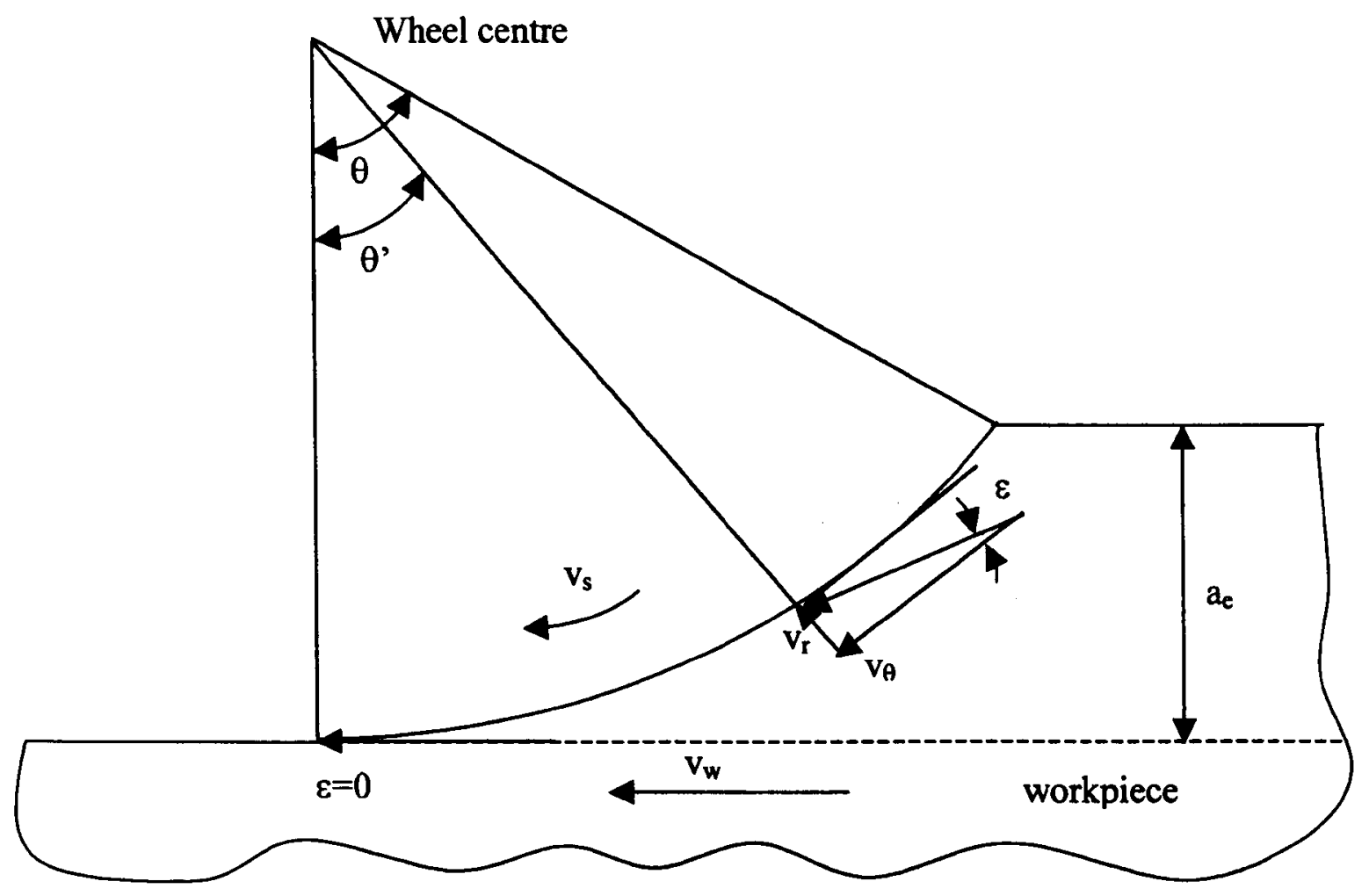

Fig. 3.2 Illustration of infeed angle $\varepsilon$ of material flow direction relative to a cutting point on the wheel periphery and wheel rotation angle $\theta^{\prime}$ 


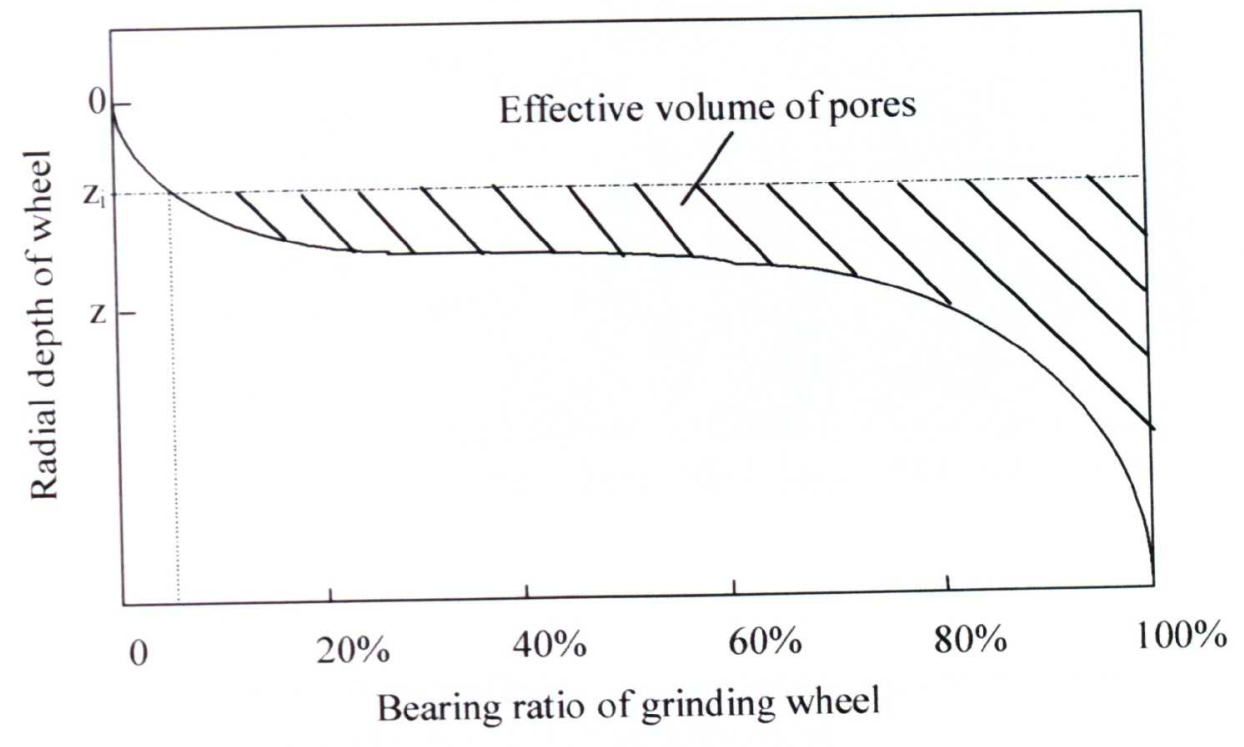

Fig. 3.3 Effective volume of pores
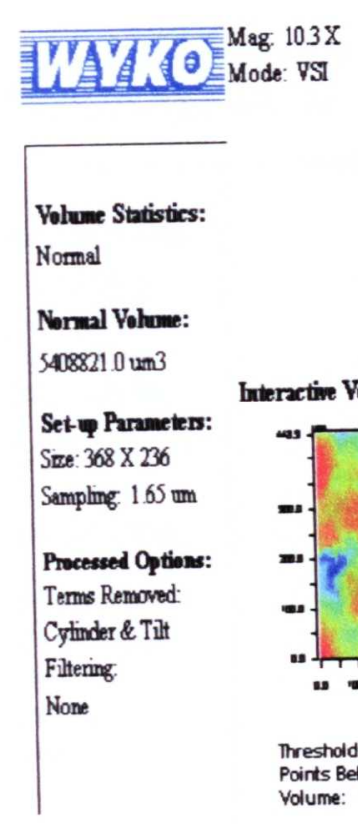

Title.

Note:
Date: $1207 / 01$

Time: $15: 10: 18$

Volume

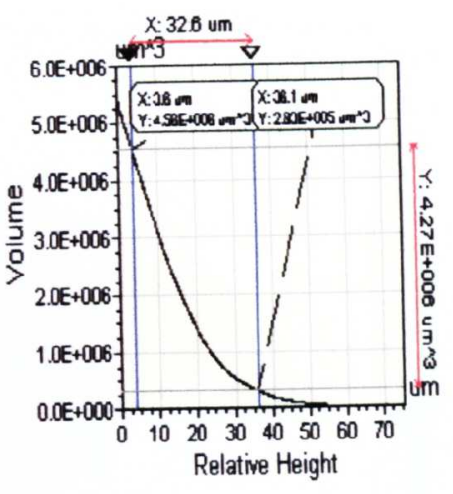

$0.00 \mathrm{~nm} \quad 0.00 \%$ of $P . Y$

Points Below: $100.00 \%$ of Total

Volume: $5.41 \mathrm{e}+006 \mathrm{um} 3 \quad 100.00 \%$ of Total

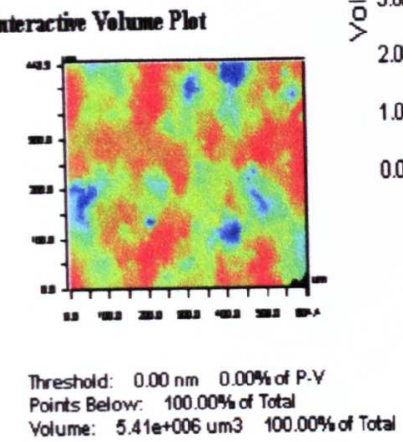

Relative Height

Fig. 3.5 Function to calculate pore volume and peak volume between a certain depth 


\section{MATPOE 1038}

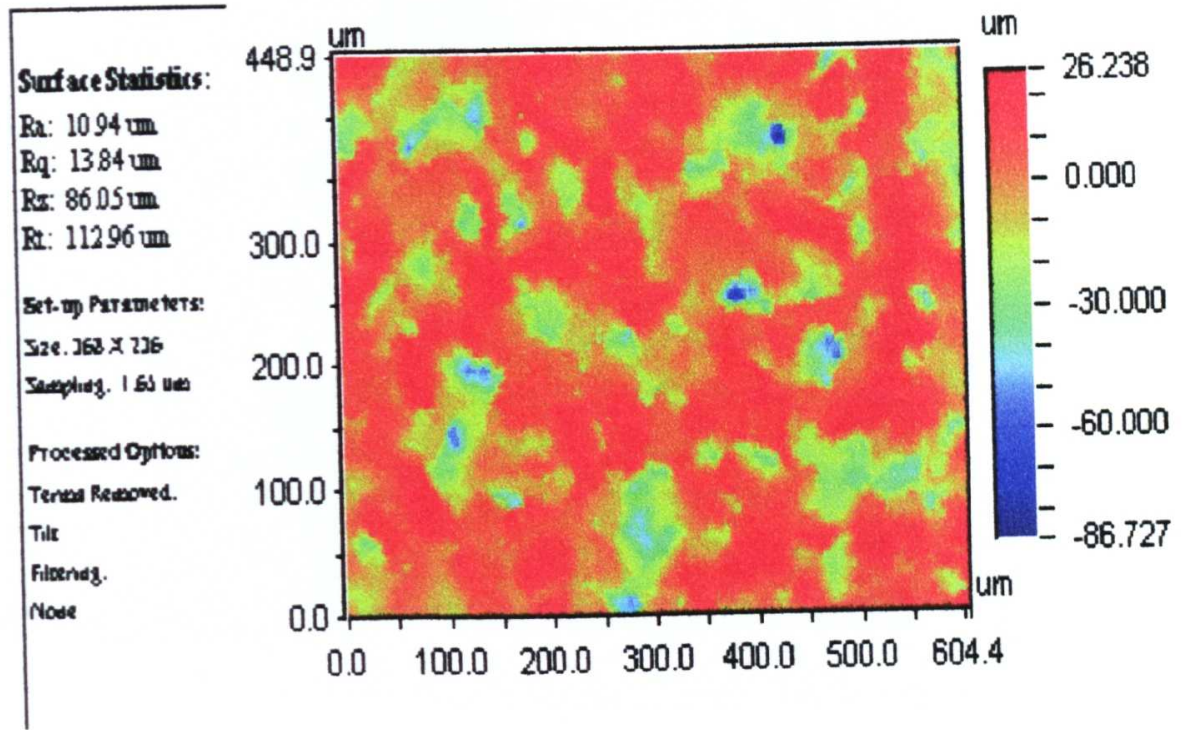

Title:

Note:

Fig. 3.4 (a) Scheme of transferring measurement results to Matlab software to calculate cutting edge density: 2D measurement of CBN wheel surface

\begin{tabular}{|c|c|}
\hline 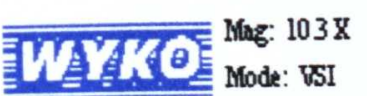 & 3-D Plot \\
\hline
\end{tabular}

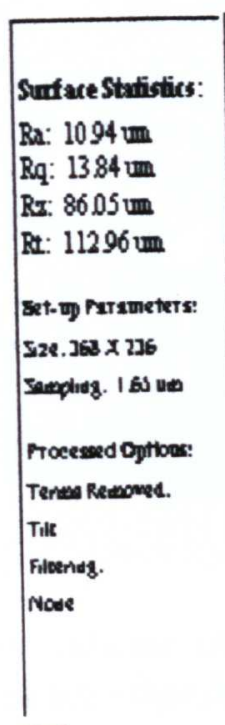

Title:

Note:

\section{Surface Data}




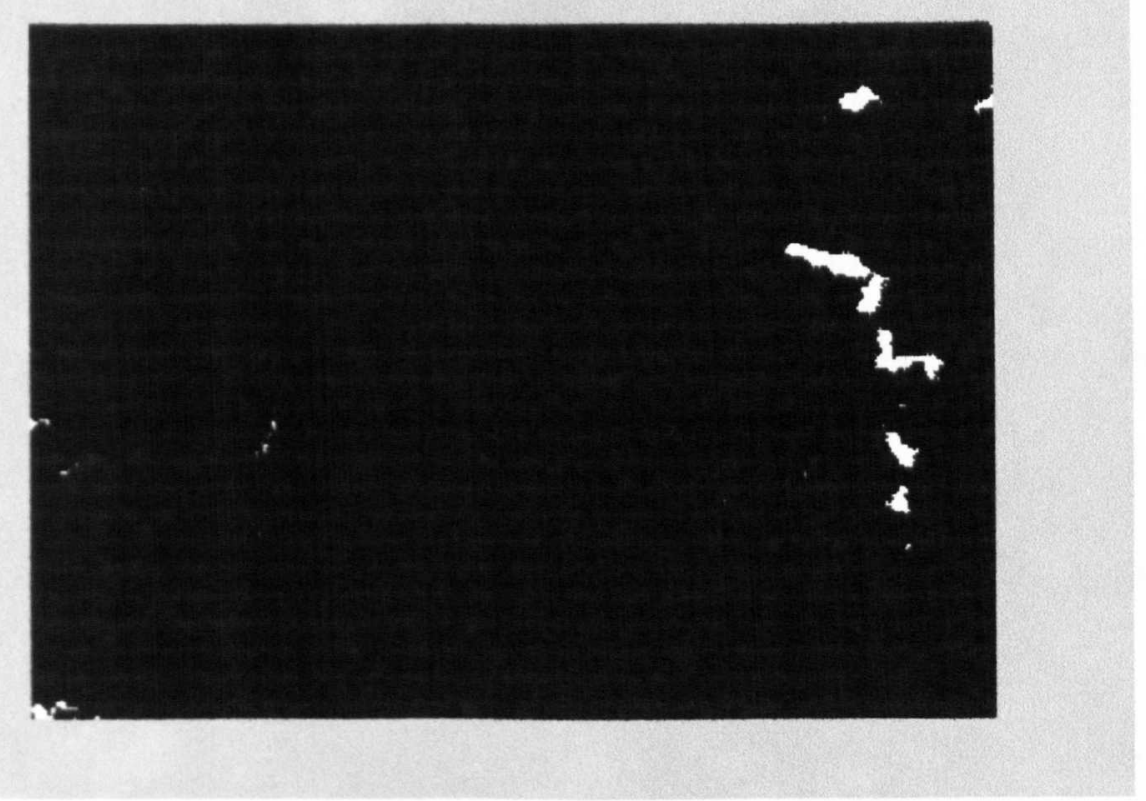

Fig. 3.4 (c) Scheme of transferring measurement results to Matlab software to calculate cutting edge density: $2 \mathrm{D}$ binary image at radial depth of $5 \mu \mathrm{m}$

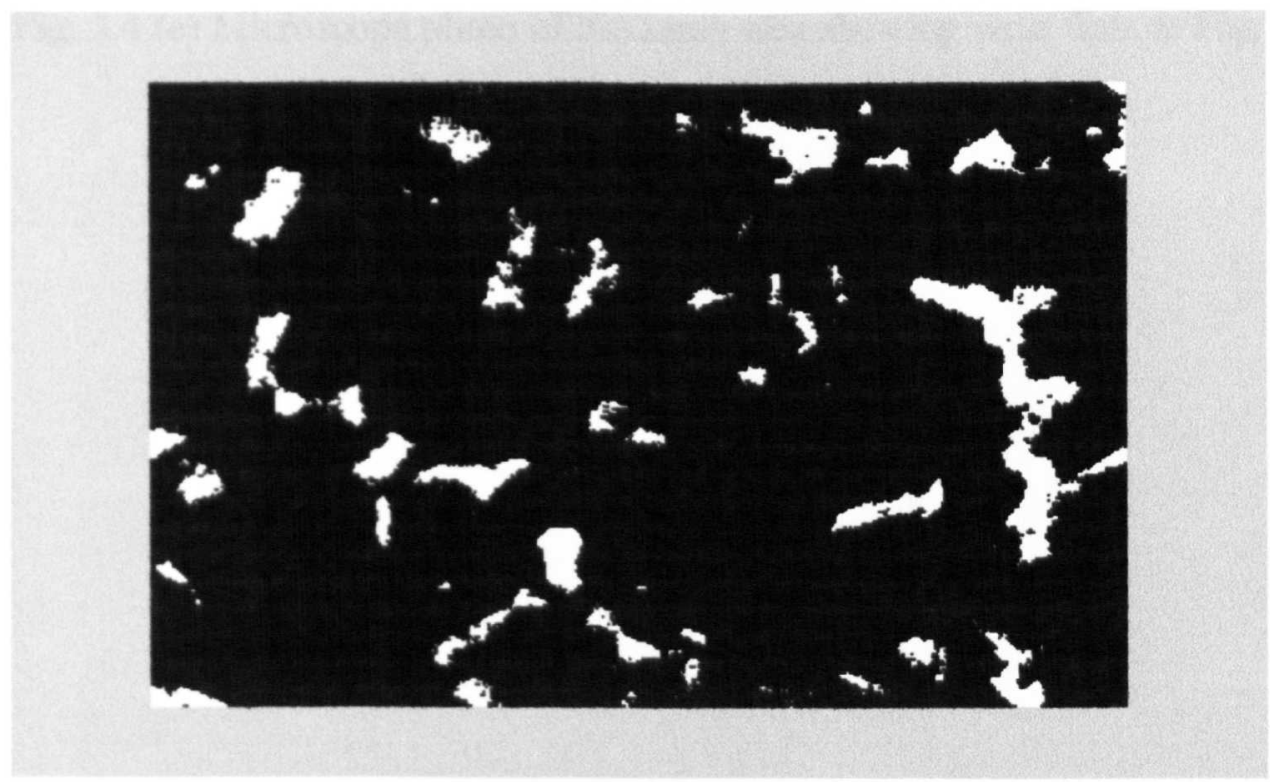

Fig. 3.4 (d) Scheme of transferring measurement results to Matlab software to calculate cutting edge density: $2 \mathrm{D}$ binary image at radial depth of $15 \mu \mathrm{m}$ 


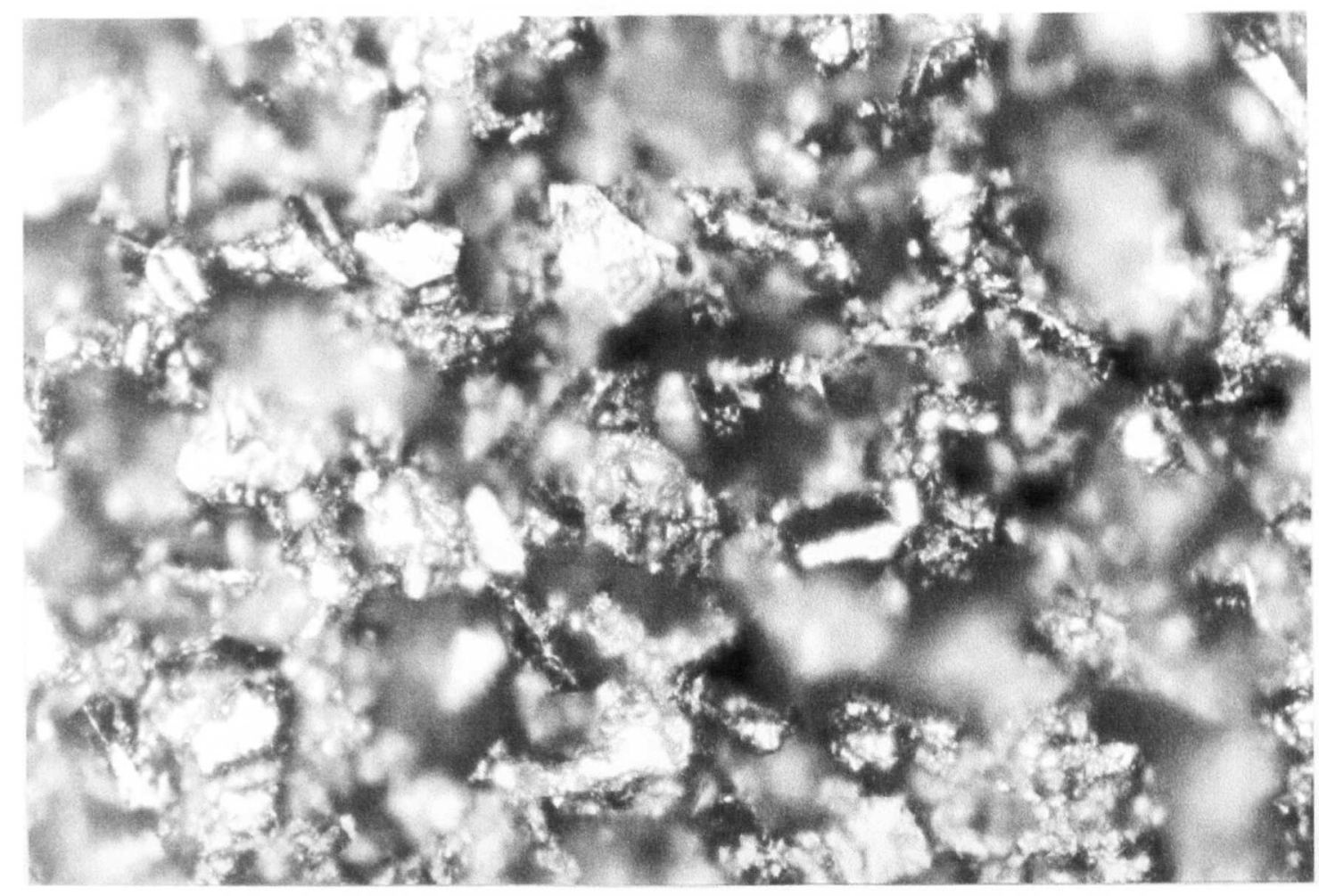

Fig. 3.4 (e) Microscope photo of the same area showing wear flats as Fig. 3.4 (d) 


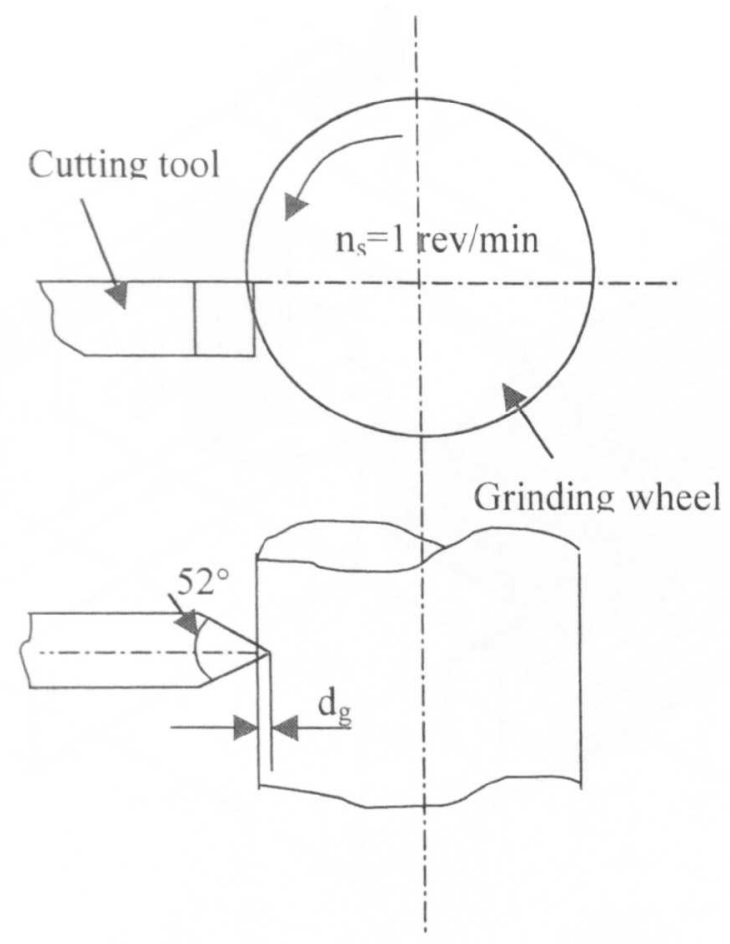

Fig. 3.6 Measurement of grinding wheel hardness by the scratch method

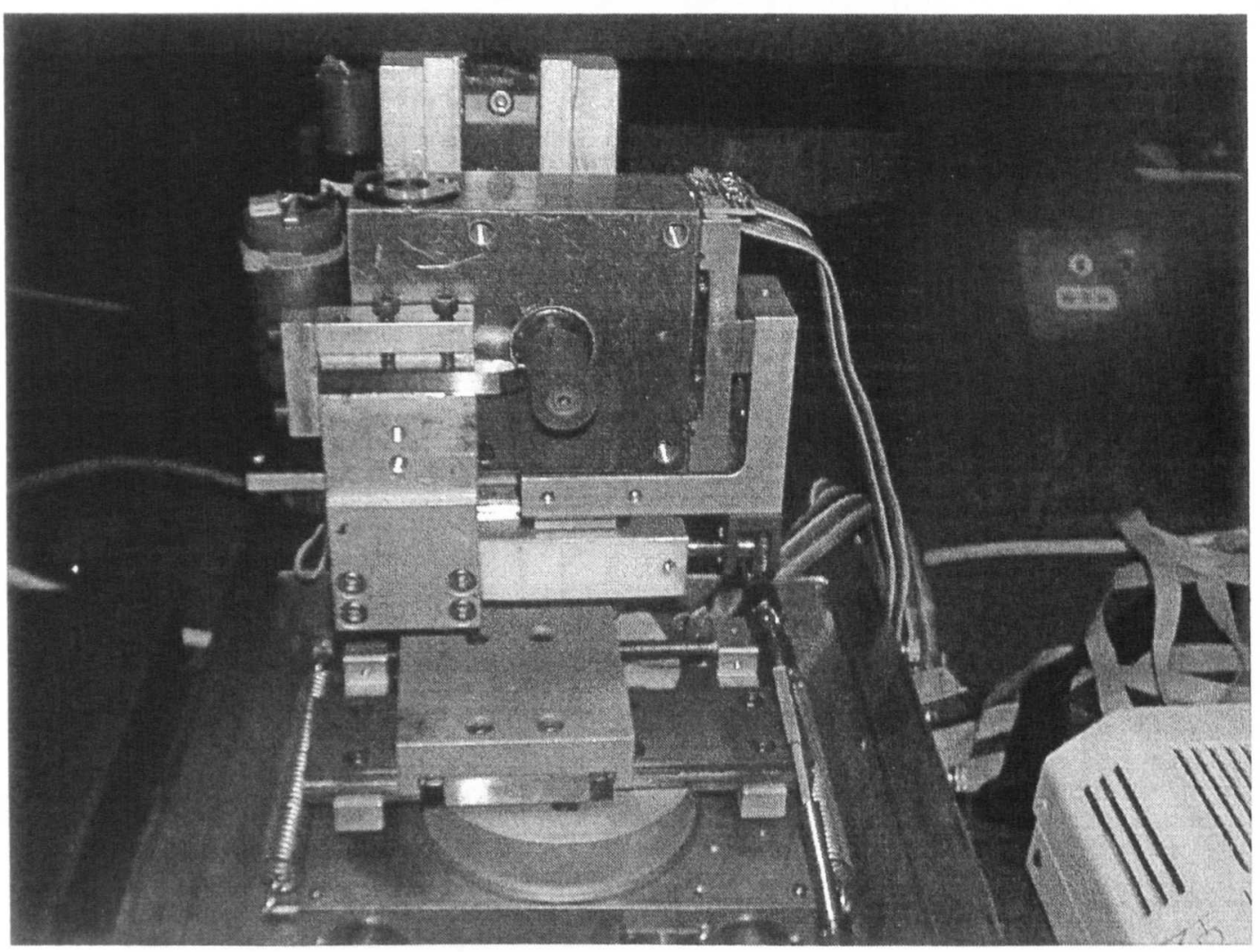

Fig. 3.7 Microcutting system 


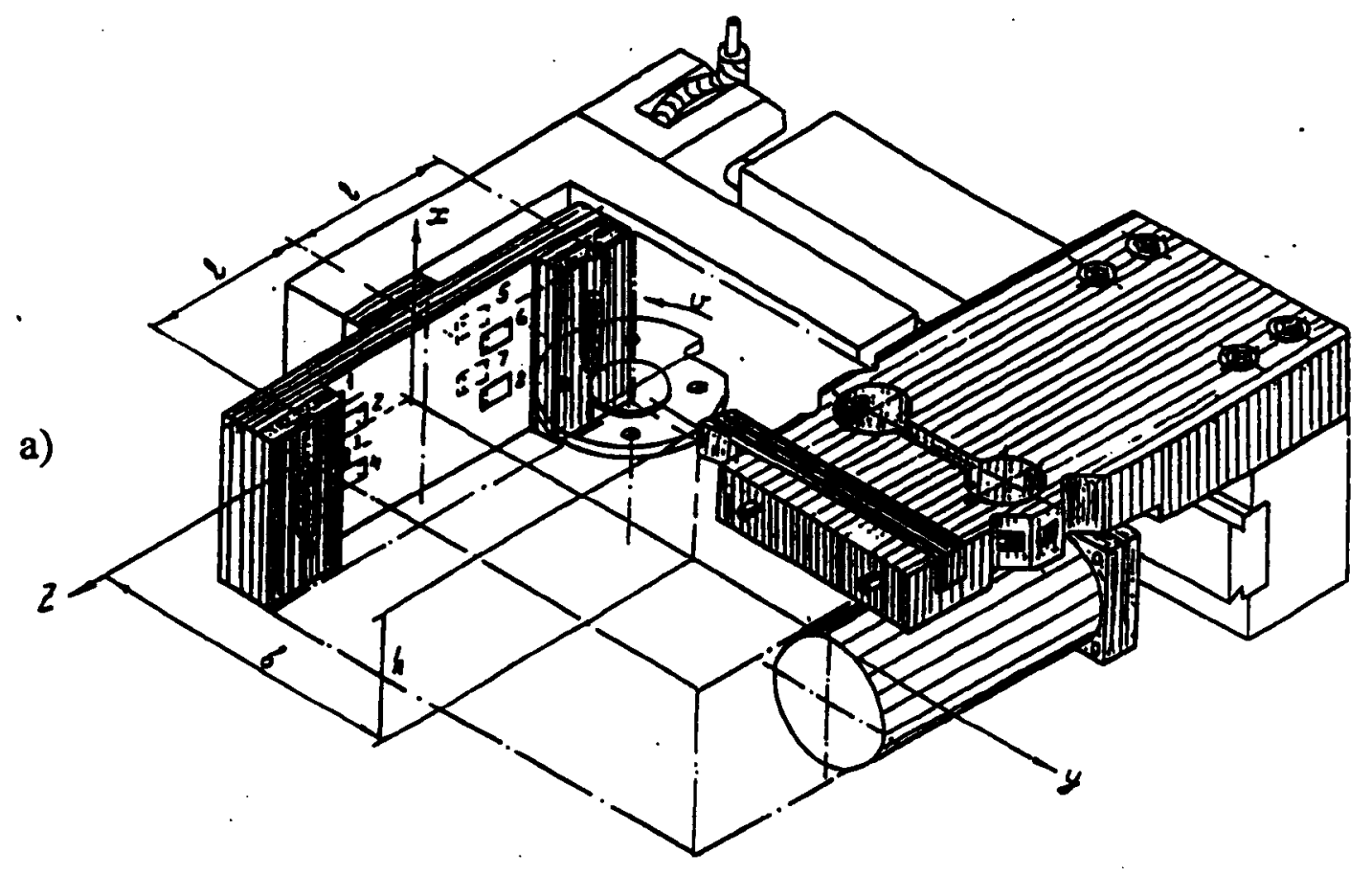

b)

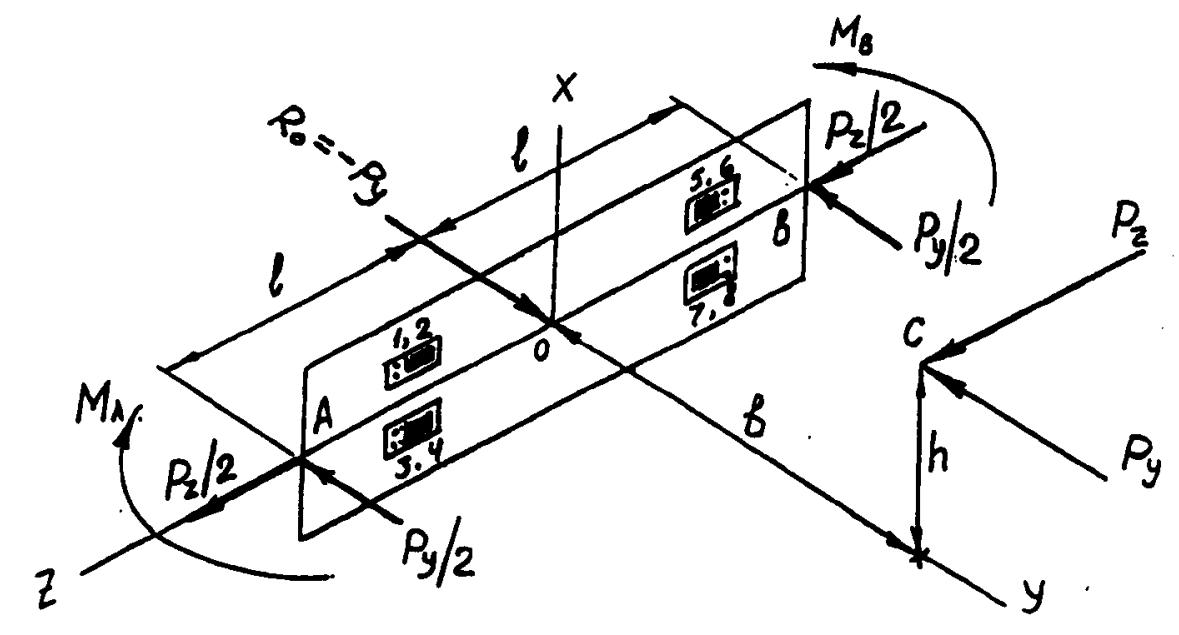

c)

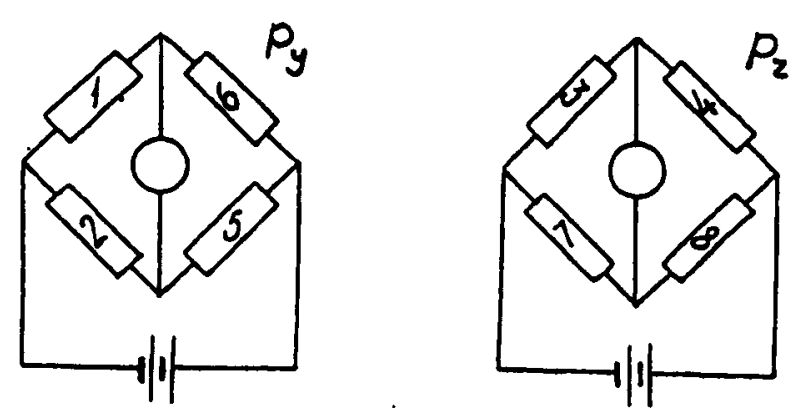

Fig. 3.8 (a) Schematic view of the two-component dynamometer in the microcutting system

(b) Scheme of corresponding beam with applied forces and bending moment

(c) The bridge connection for measuring $\mathrm{P}_{\mathrm{z}}$ and $\mathrm{P}_{\mathrm{y}}$ forces 


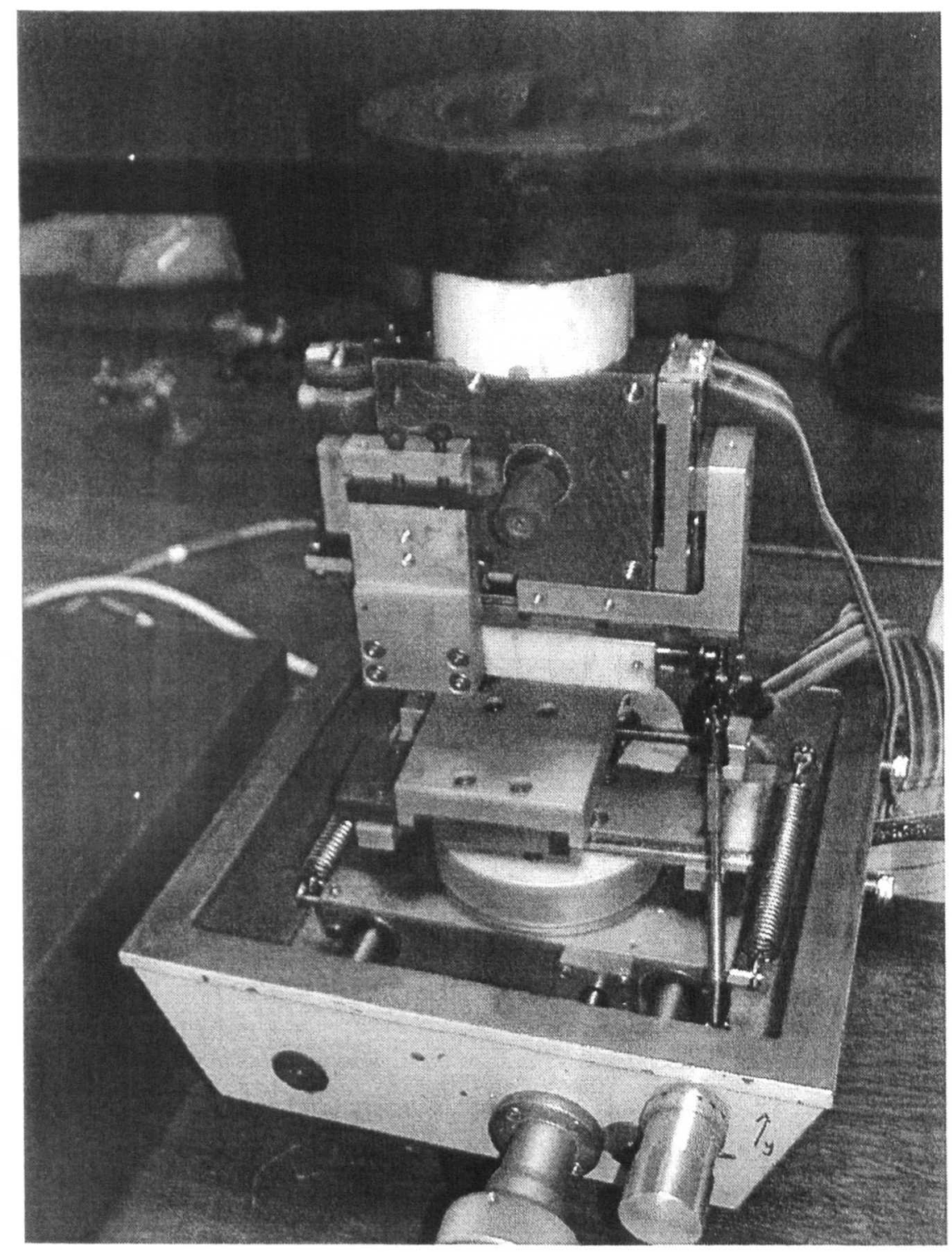

Fig. 3.9 Force calibration for microcutting 


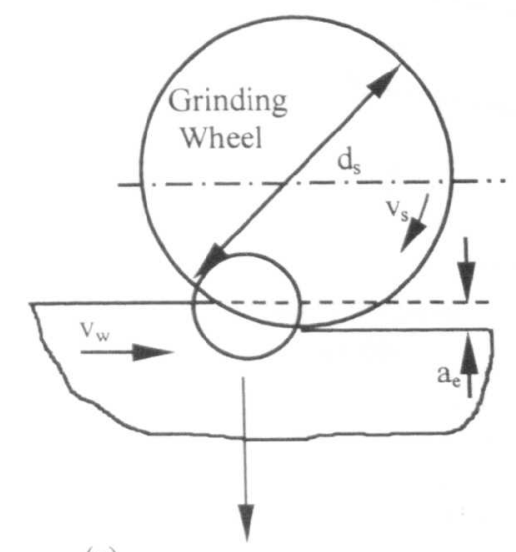

(a)

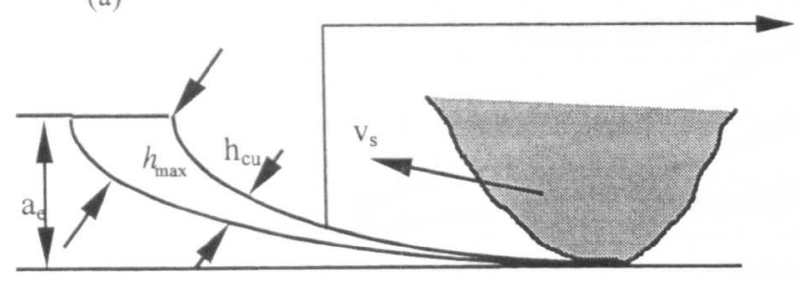

(b)

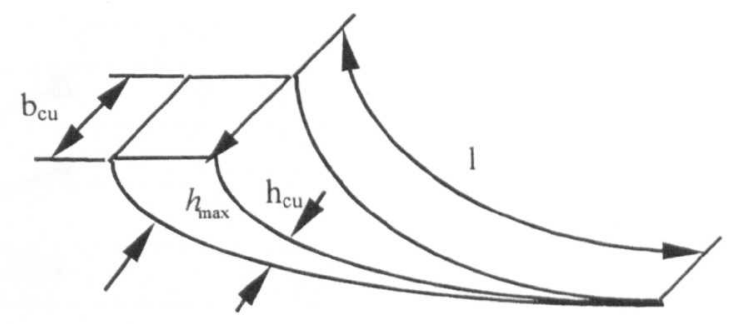

(c)

Fig. 3.10 (a)Surface grinding process

(b)Uncut chip thickness, $\mathrm{h}_{\mathrm{cu}}$ and maximum uncut chip thickness, $\mathrm{h}_{\max }$

(c) Uncut chip width, $b_{\mathrm{cu}}$ 


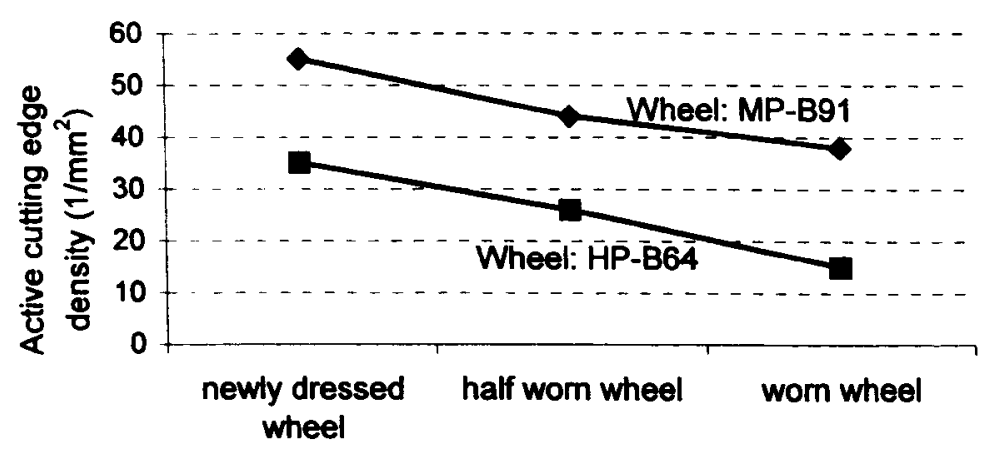

(a)

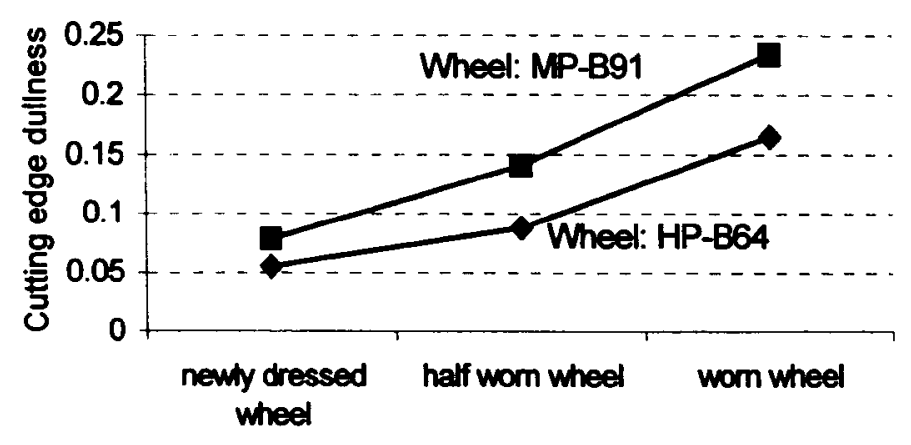

(b)

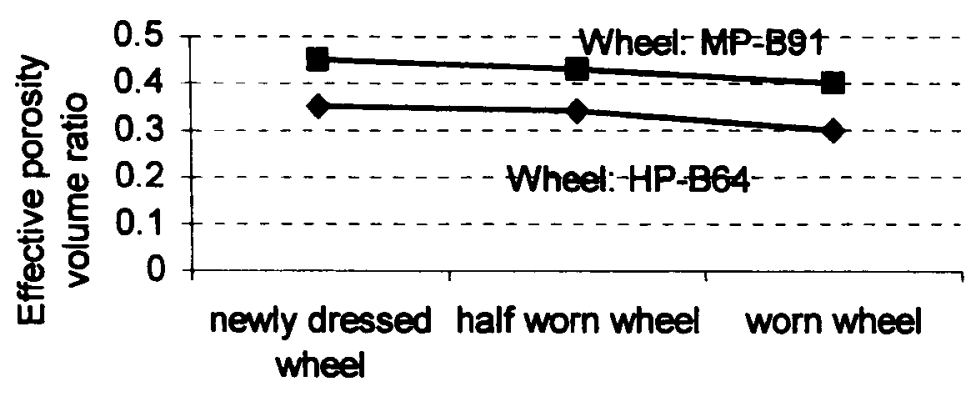

(c)

Fig. 3.11(a) Active cutting edge density of the newly dressed wheel, worn wheel and loaded wheel

(b) Cutting edge dullness of the newly dressed wheel, worn wheel and loaded wheel

(c) Effective porosity volume ratio of the newly dressed wheel, worn wheel and loaded wheel 


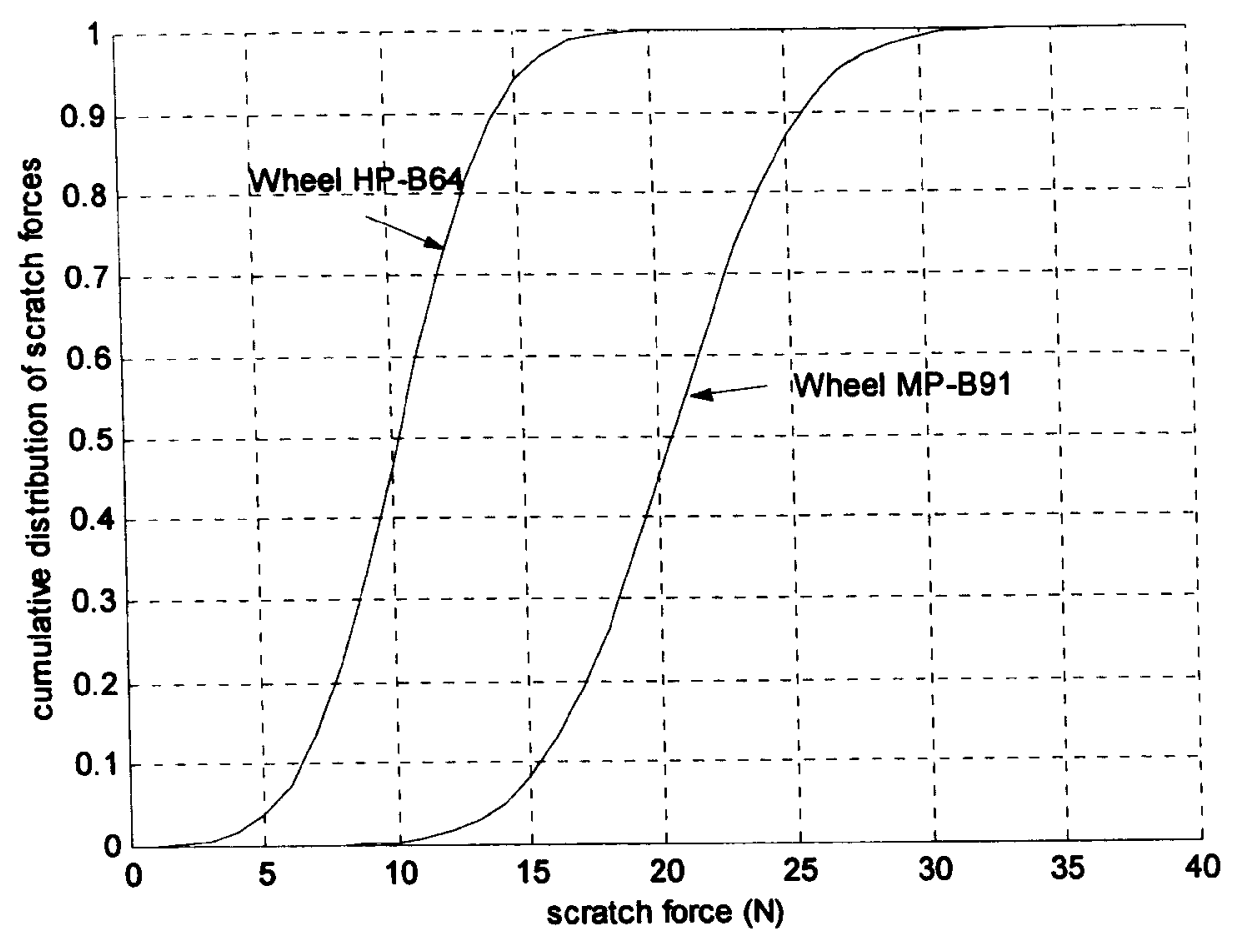

(a) Cumulative distribution of bond forces

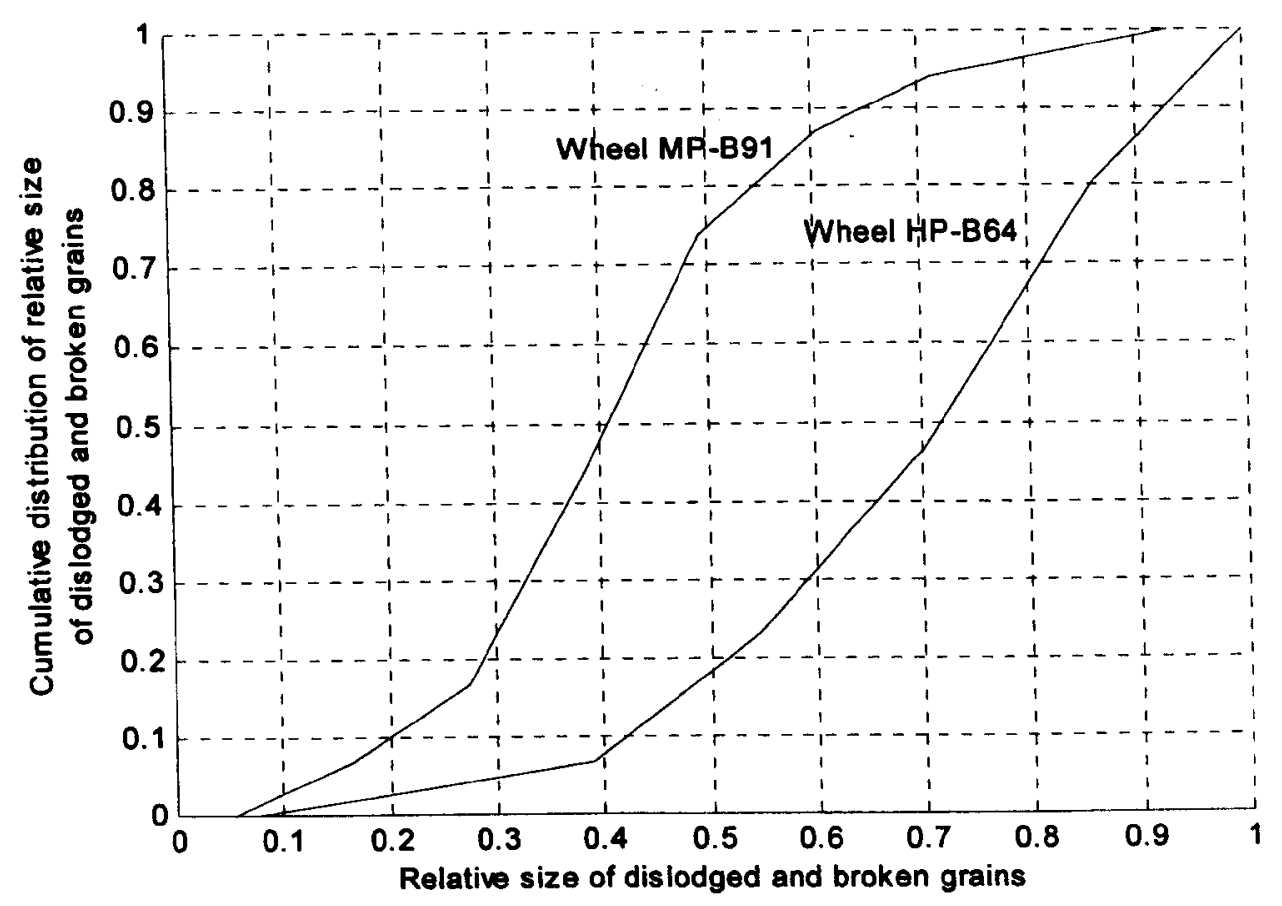

$\left(d_{b a}\right)$

(b) Cumulative distribution of broken and dislodged grain sizes

Fig. 3.12 Results of wheel scratch hardness tests 


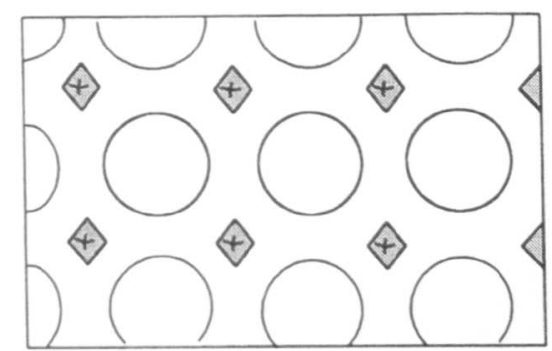

(a)

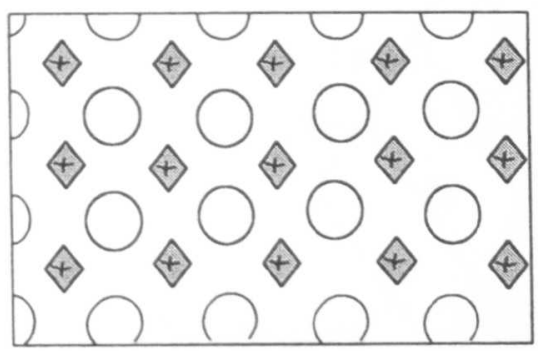

(c)

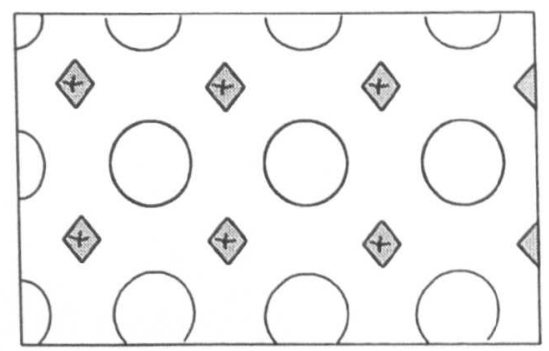

(b)

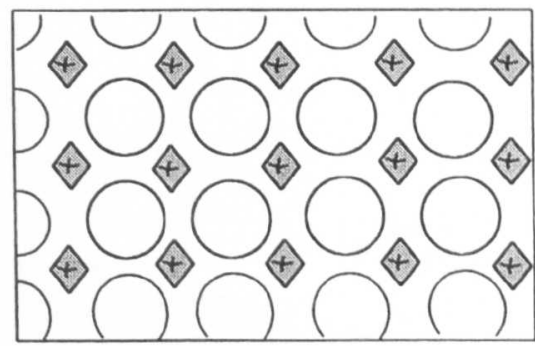

(d)

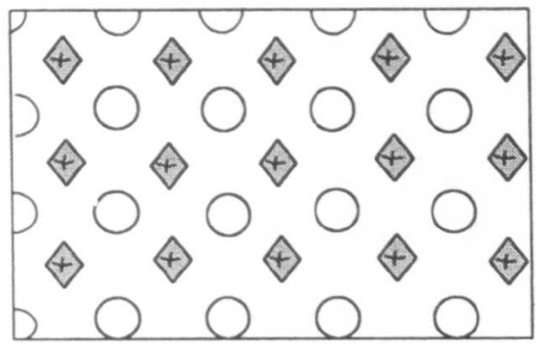

(e)
(a) $\mathrm{V}_{\mathrm{g}}+\mathrm{V}_{\mathrm{b}}+\mathrm{V}_{\mathrm{p}}=100 \%$
(b) $\mathrm{V}_{\mathrm{g}}+\left(\mathrm{V}_{\mathrm{b}}+\mathrm{V}_{\mathrm{x}}\right)+\left(\mathrm{V}_{\mathrm{p}}-\mathrm{V}_{\mathrm{x}}\right)=100 \%$
(c) $\left(\mathrm{V}_{\mathrm{g}}+\mathrm{V}_{\mathrm{x}}\right)+\mathrm{V}_{\mathrm{b}}+\left(\mathrm{V}_{\mathrm{p}}-\mathrm{V}_{\mathrm{x}}\right)=100 \%$
(d) $\left(\mathrm{V}_{\mathrm{g}}+\mathrm{V}_{\mathrm{x}}\right)+\left(\mathrm{V}_{\mathrm{b}}-\mathrm{V}_{\mathrm{x}}\right)+\mathrm{V}_{\mathrm{p}}=100 \%$
(e) $\left(\mathrm{V}_{\mathrm{g}}+\mathrm{V}_{\mathrm{x}}\right)+\left(\mathrm{V}_{\mathrm{b}}-\mathrm{V}_{\mathrm{y}}\right)+\left[\mathrm{V}_{\mathrm{p}}-\left(\mathrm{V}_{\mathrm{x}}+\mathrm{V}_{\mathrm{y}}\right)\right]=100 \%$

Fig. 4.1 Representative phase structure of vitrified-bond wheels 


\section{PAGES NOT SCANNED AT THE REQUEST OF THE UNIVERSITY}

SEE ORIGINAL COPY OF THE THESIS FOR THIS MATERIAL 


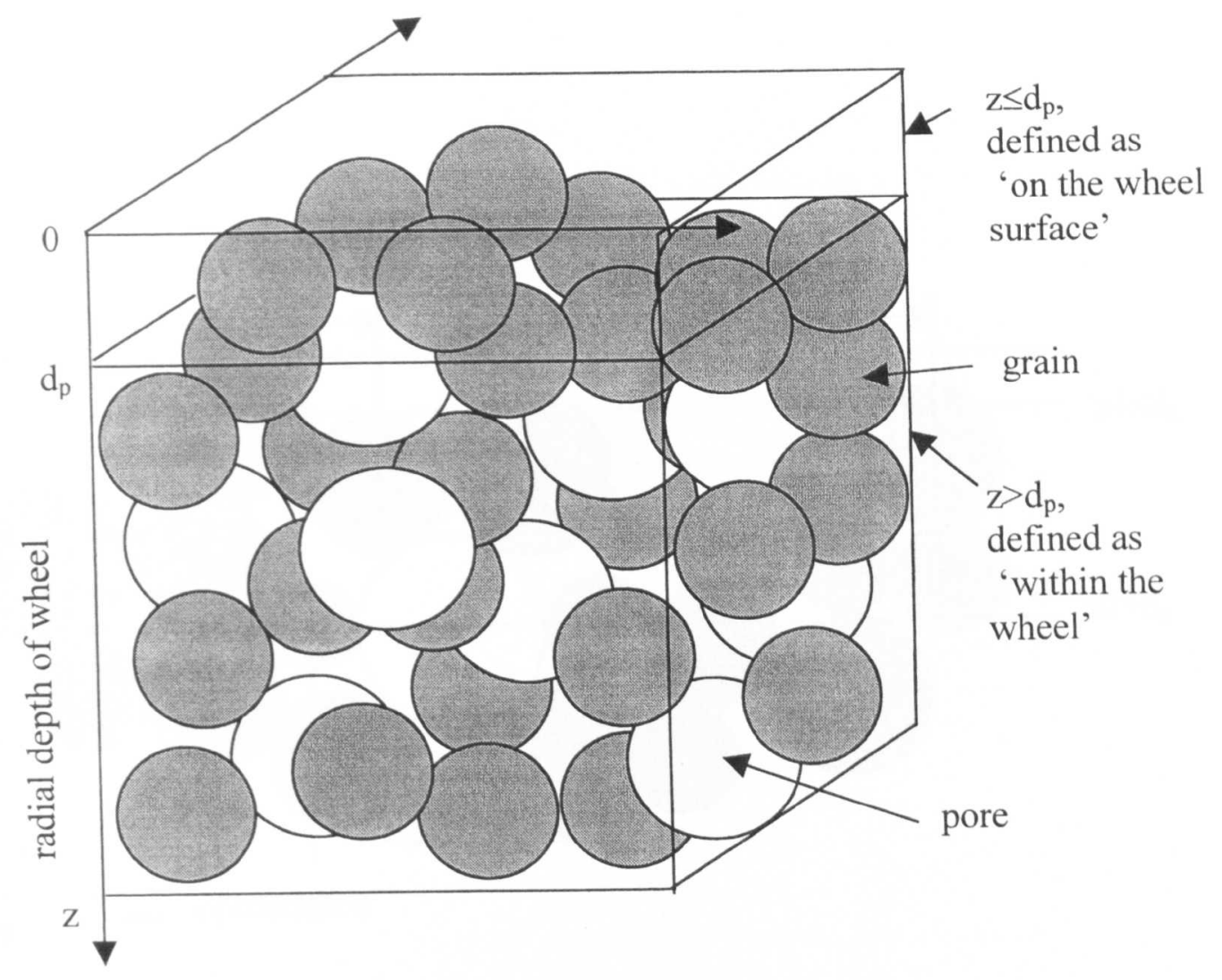

Fig. 5.1 Assumed wheel structure

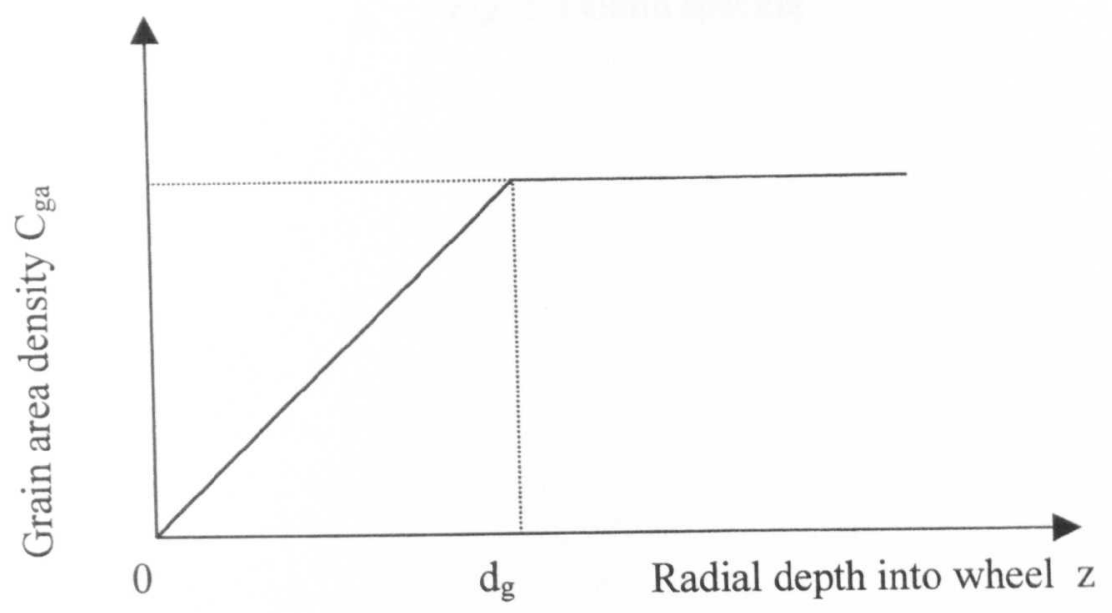

Fig.5.2 Grain area density with radial depth into the wheel 


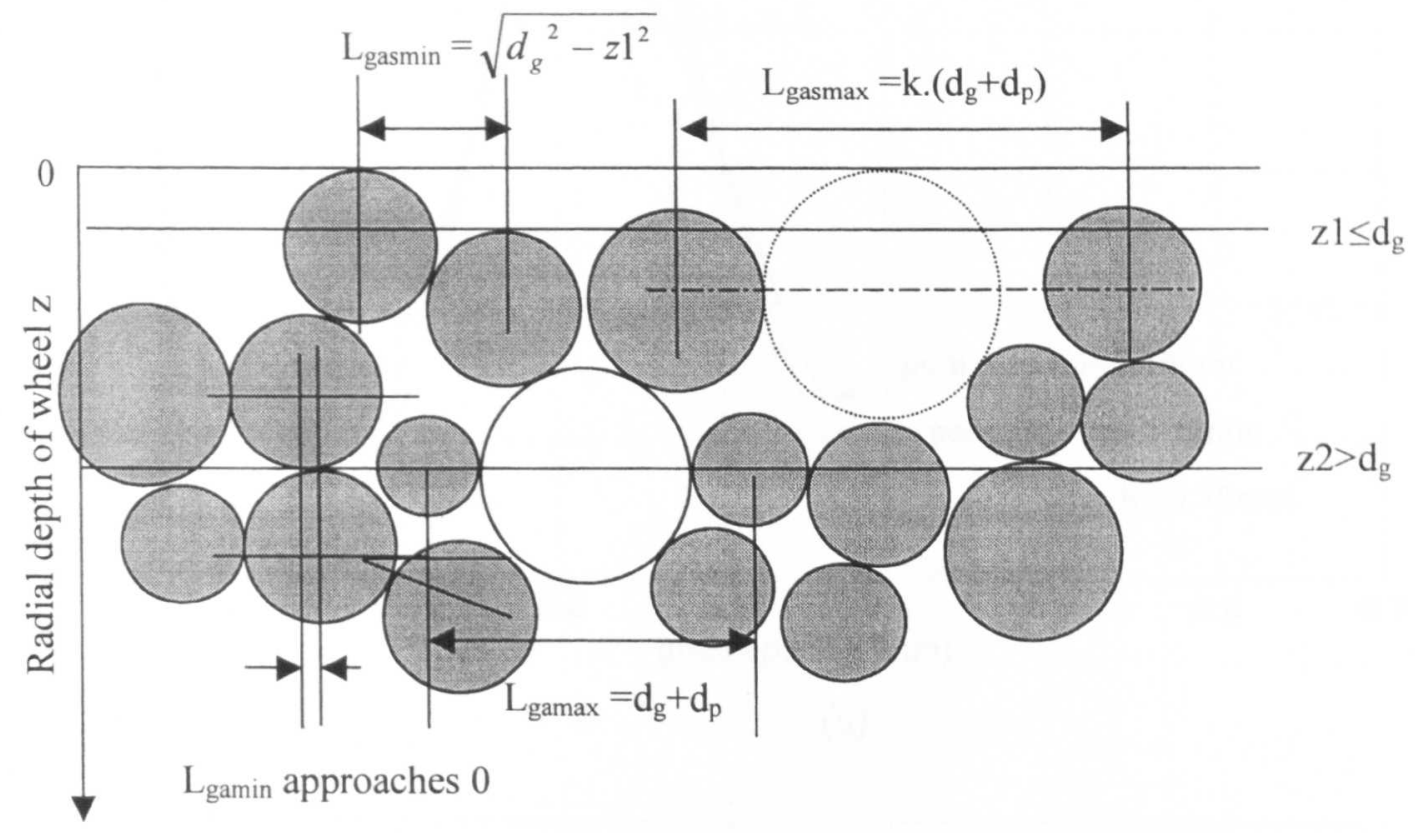

Fig. 5.3 Grain spacing 


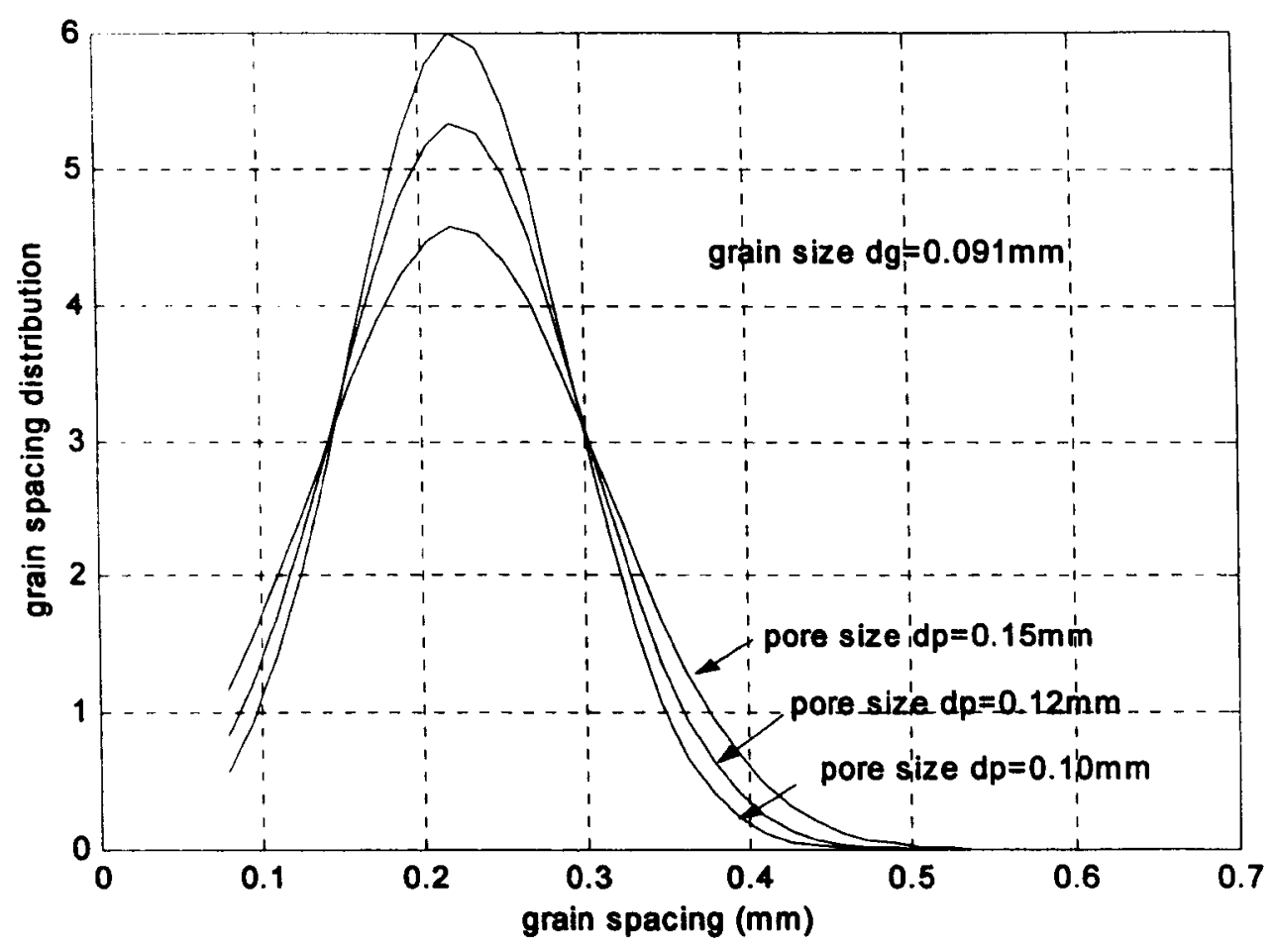

(a)

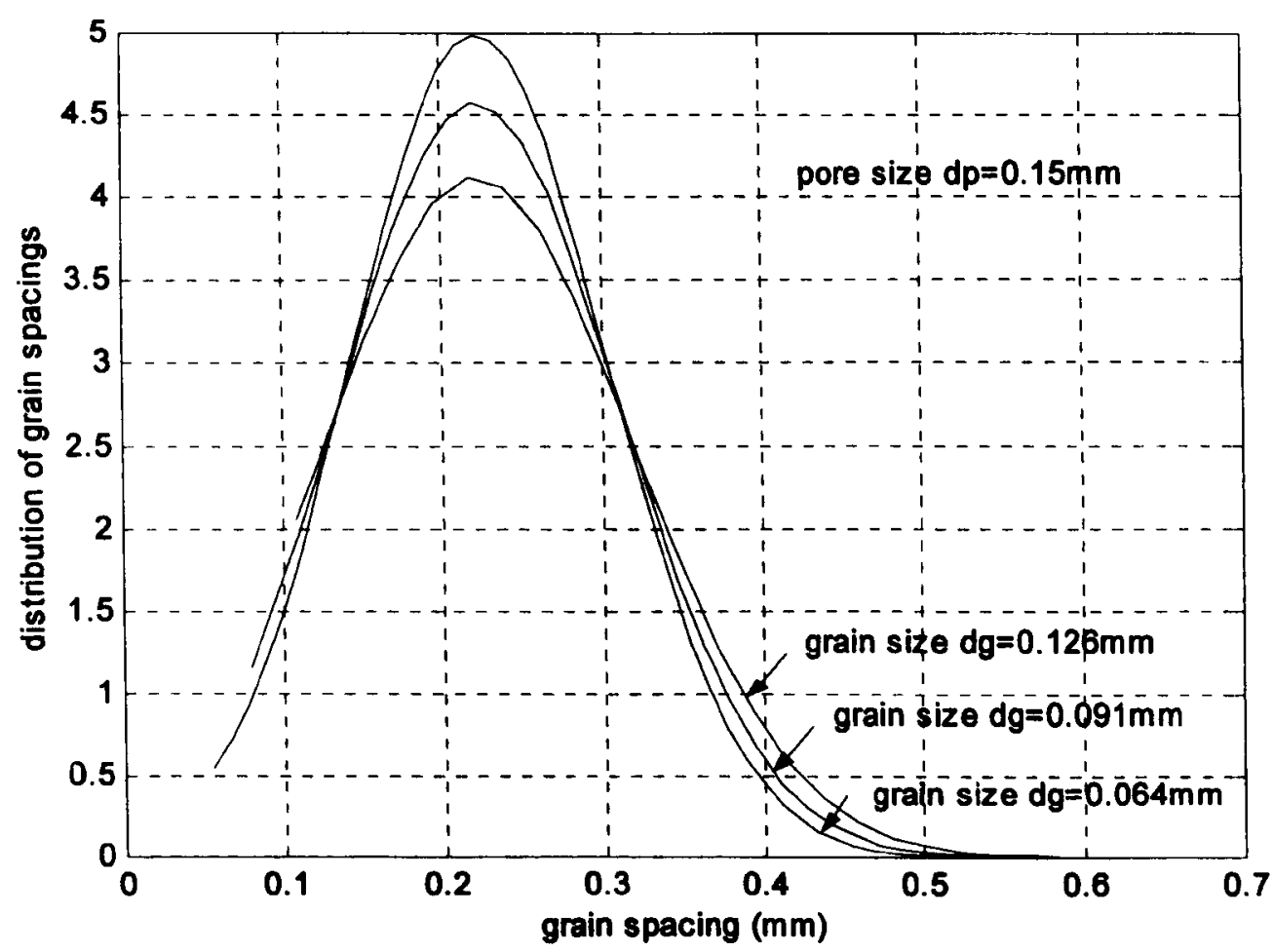

(b)

Fig. 5.4 (a) Distribution of grain spacings at radial depth of wheel $z=0.5 . \mathrm{d}_{\mathrm{g}}$ with different pore sizes $d_{p}$

(b) Distribution of grain spacings at radial depth of wheel $z=0.5 . \mathrm{d}_{\mathrm{g}}$ with different grain sizes $d_{g}$ 


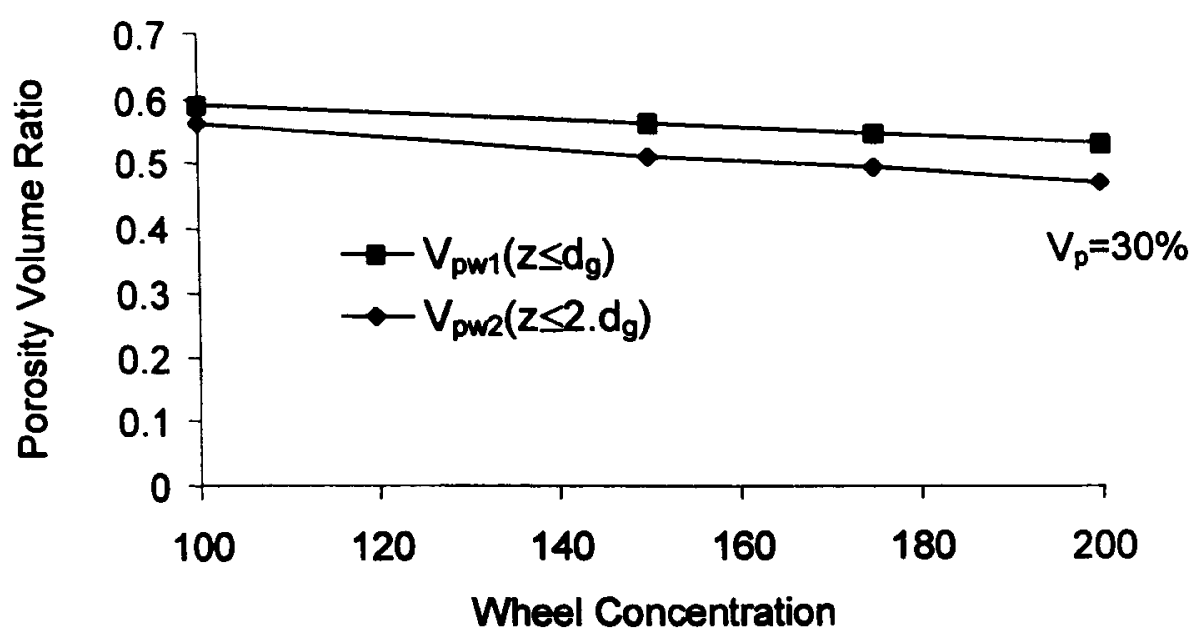

(a)

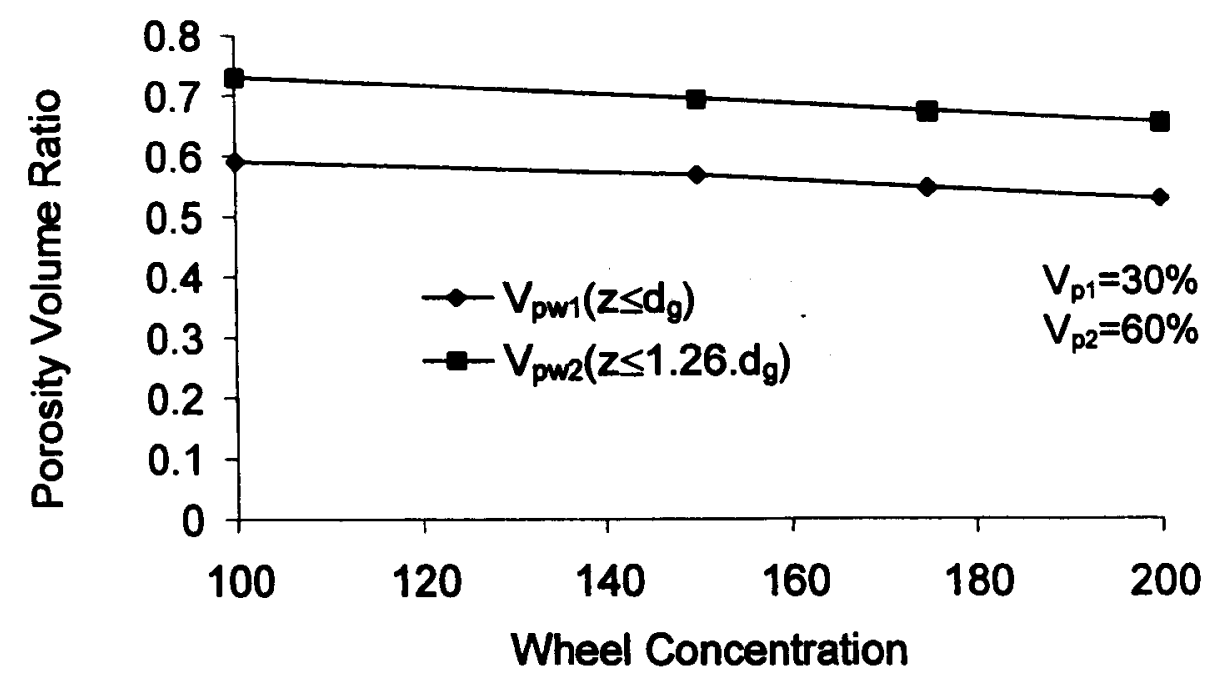

(b)

Fig. 5.5 (a) Effect of wheel concentration and depth of wheel surface on porosity volume ratio of vitrified $\mathrm{CBN}$ wheel structure

(b) Effect of percentage of pore volume and pore size on porosity volume ratio of vitrified $\mathrm{CBN}$ wheel structure 


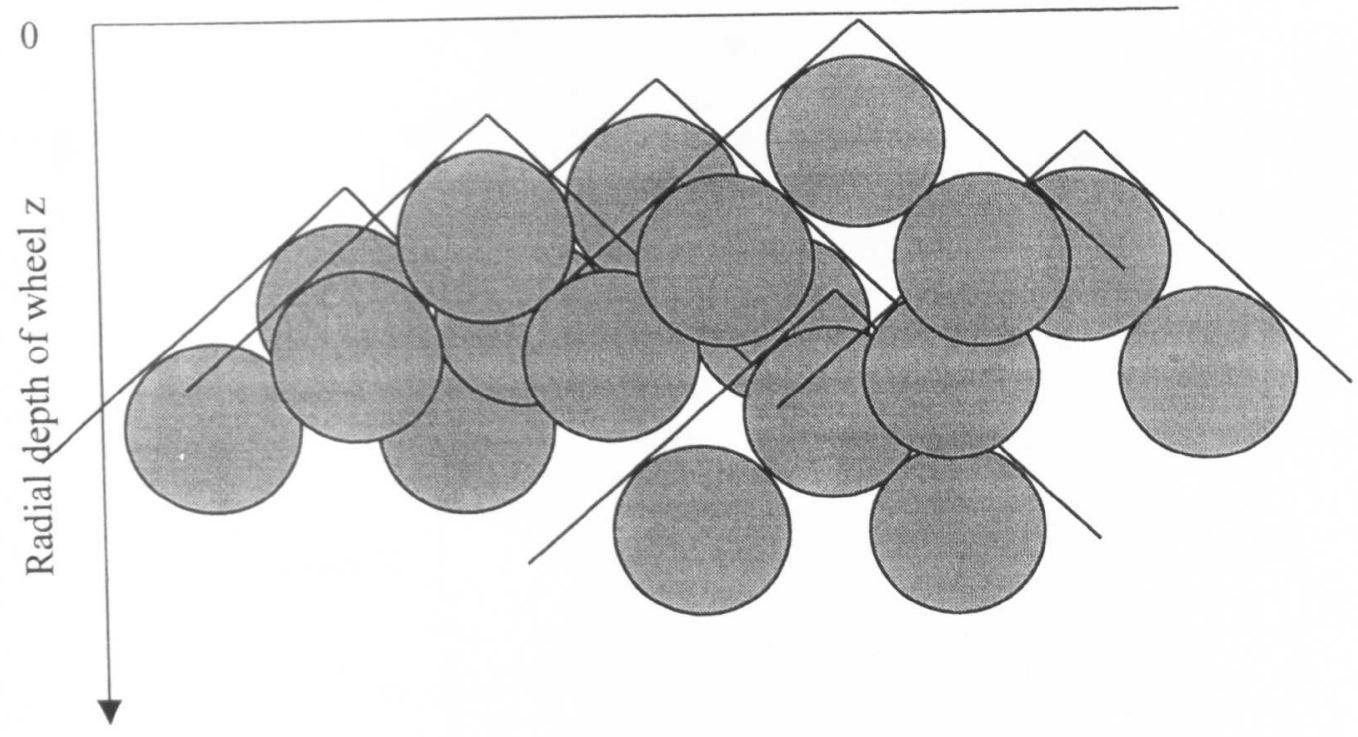

Fig. 5.6 Grains are assumed to be arrayed within cone envelopes 


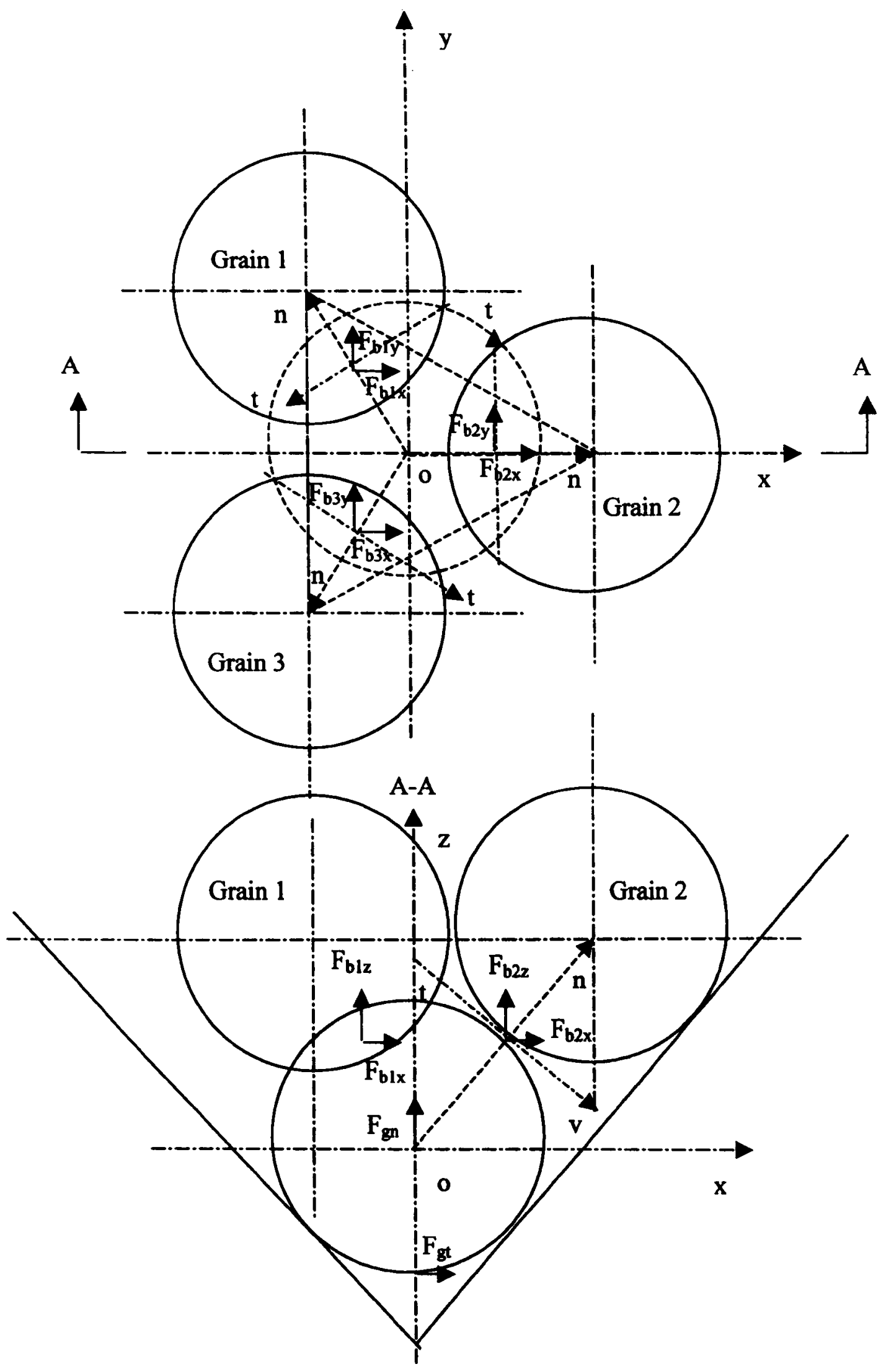

Fig. 5.7(a) Force analysis when 3 grains are above the active grain 


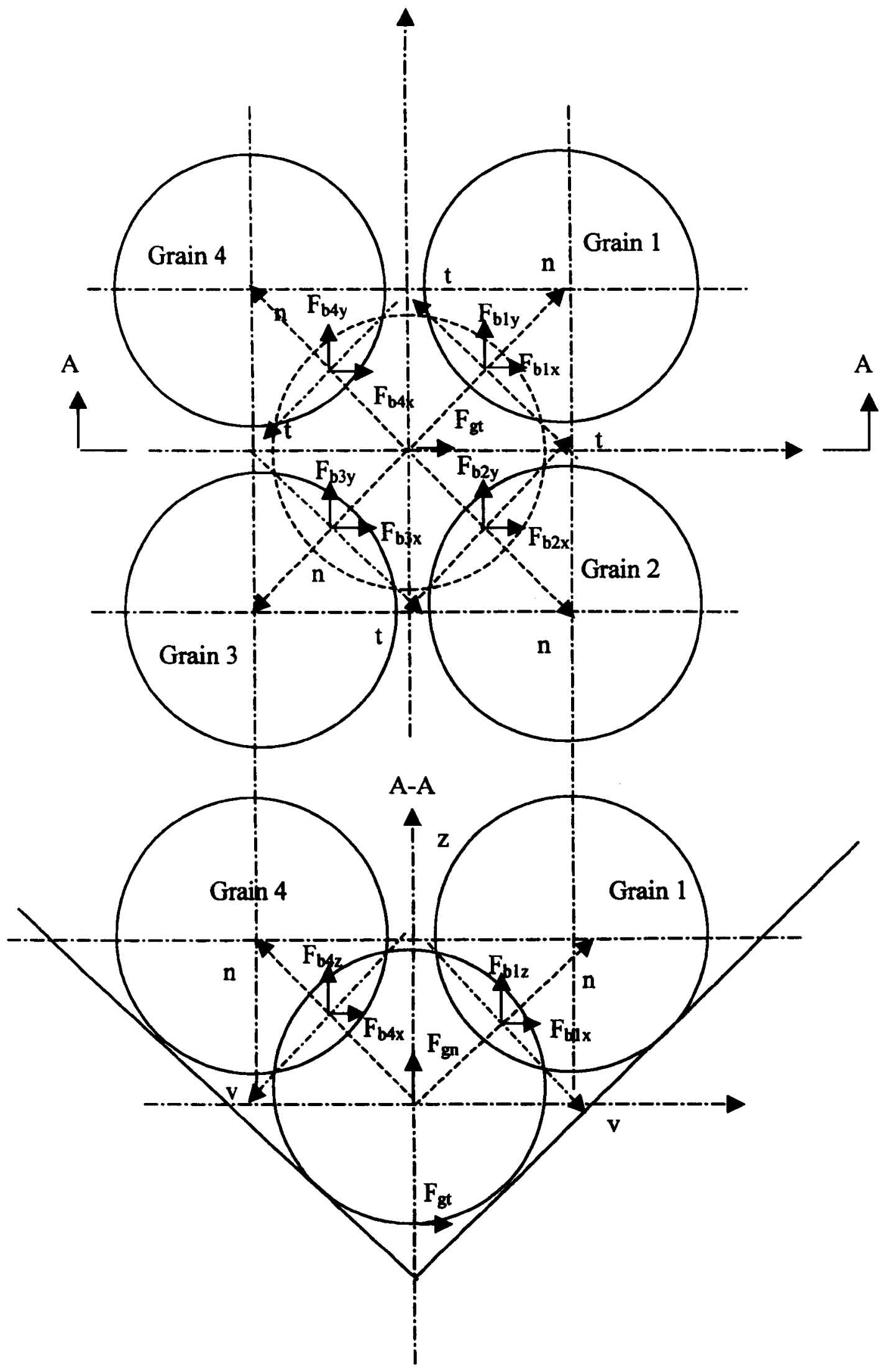

Fig. 5.7(b) Force analysis when 4 grains are above the active grain 


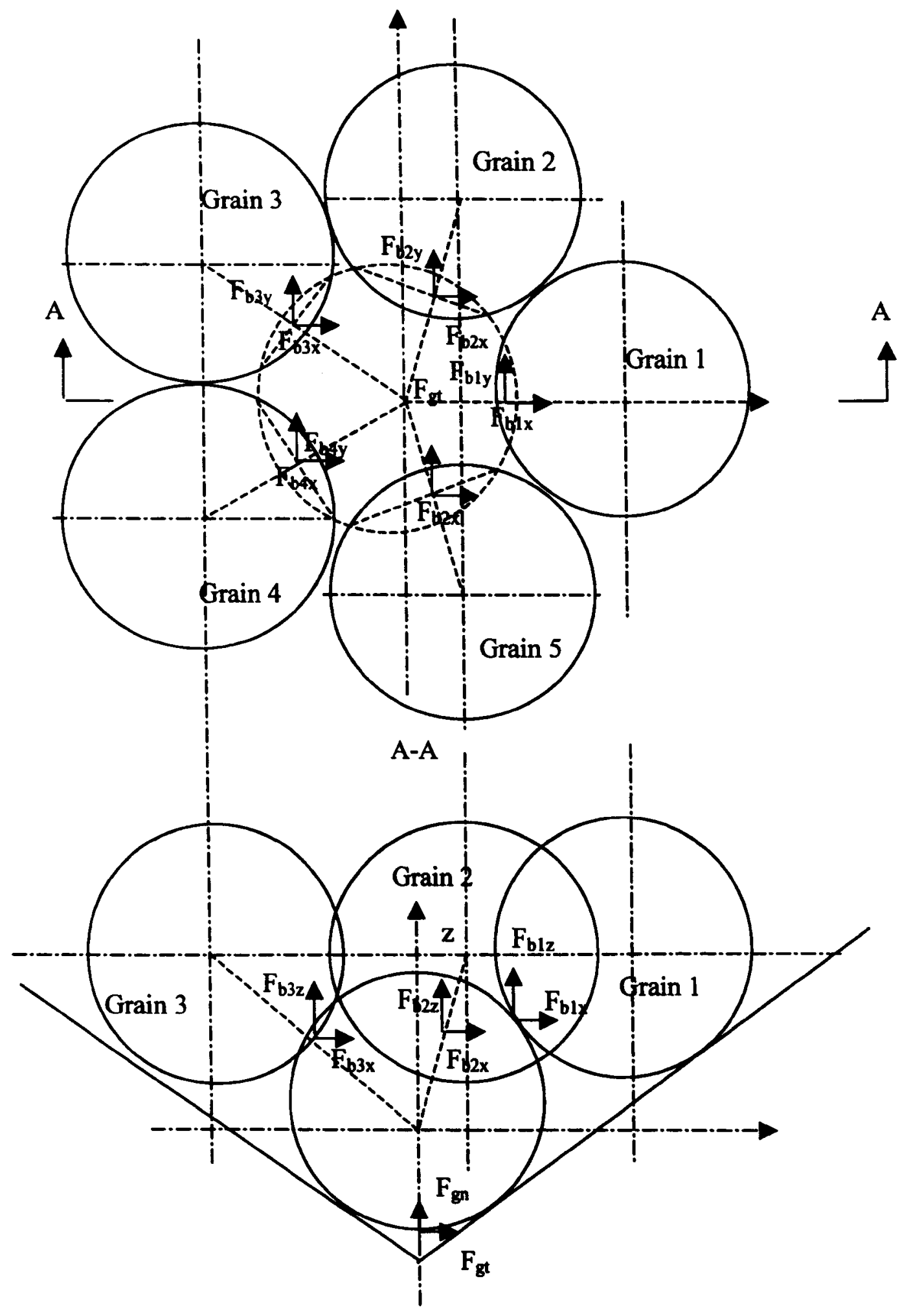

Fig. 5.7 (c) Force analysis when 5 grains are above the active grain 


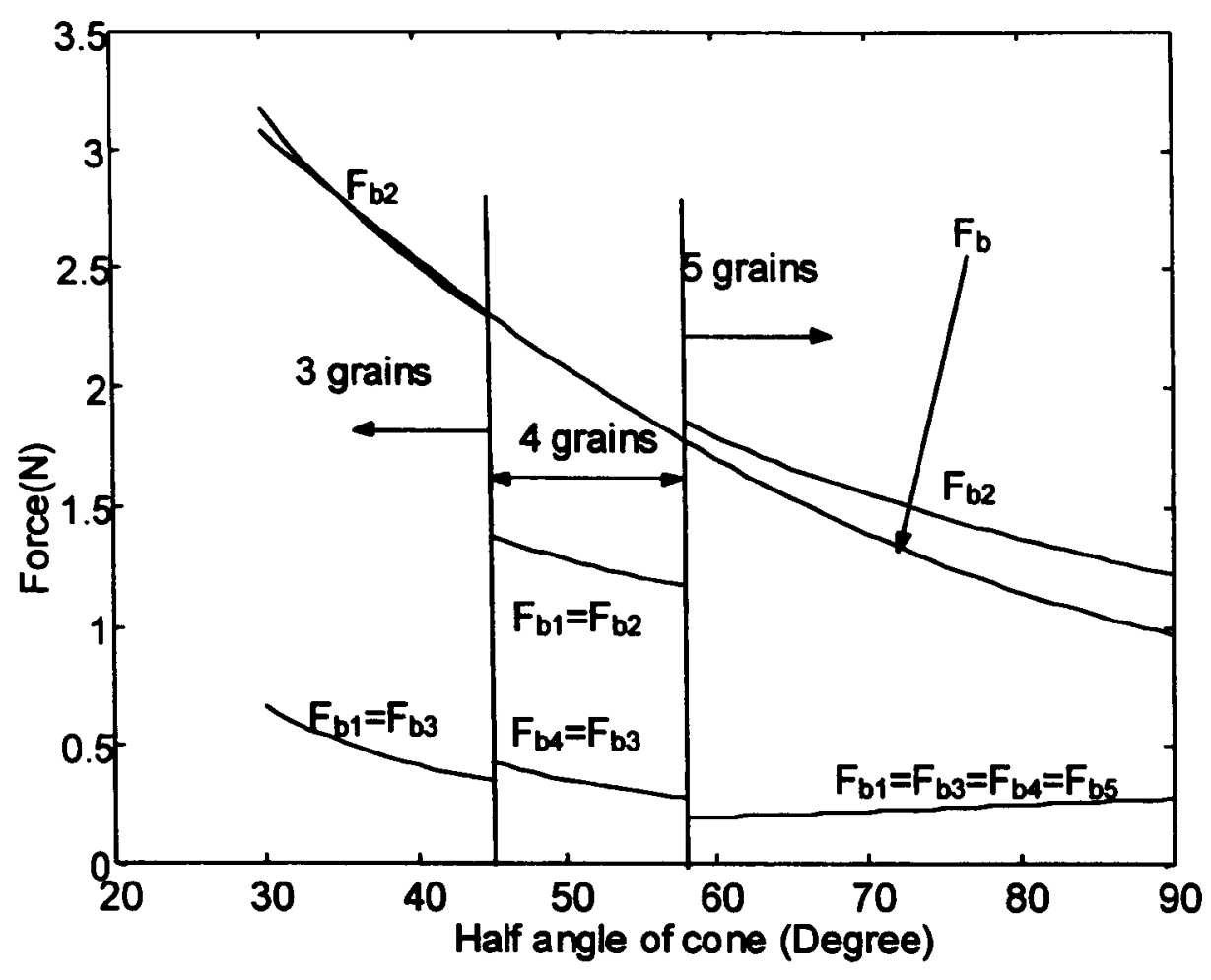

$$
F_{b}=0.4 \times 10^{-2} \theta^{2}-0.8 \times 10^{-1} \theta+5.1(N)
$$

Fig. 5.8 The reduction of bond force with the increasing of half vertex angle of the cone envelope 


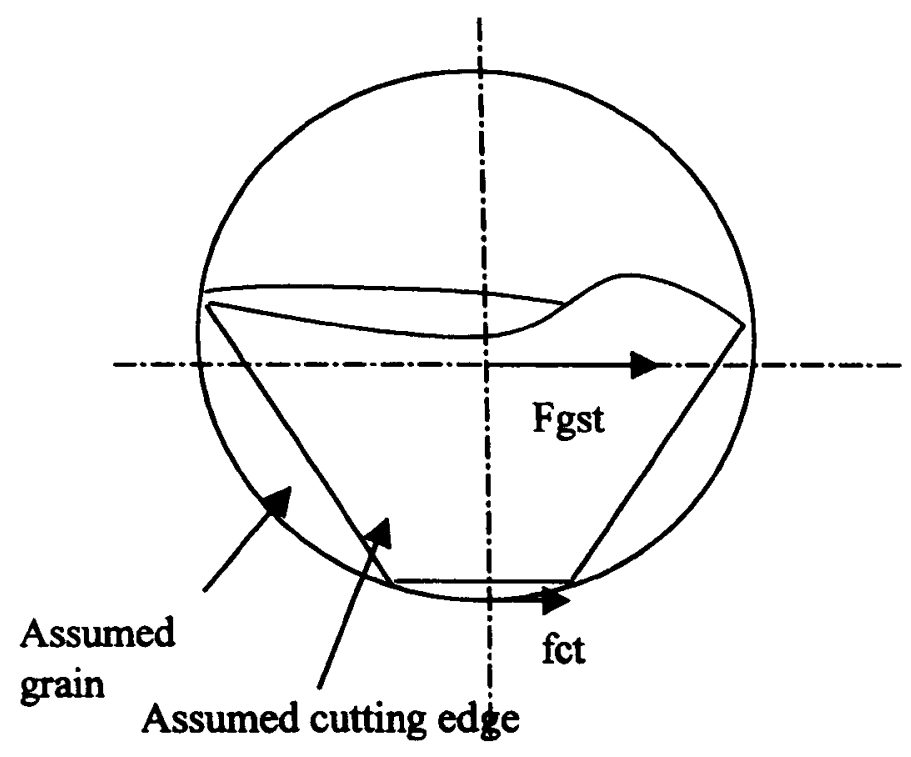

Fig. 5.9 Relationship between grain tangential force and scratch force

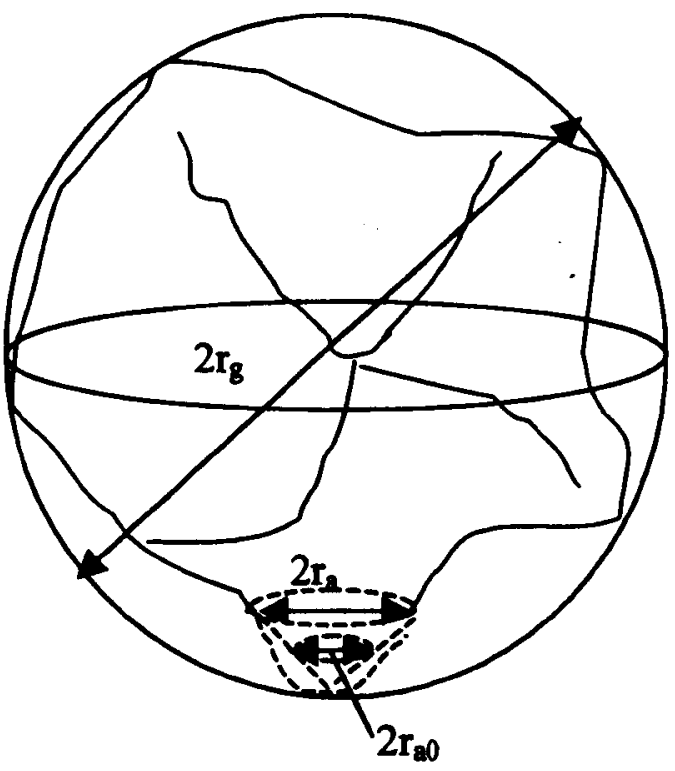

Fig. 5.11 Attritious wear of cutting edge 


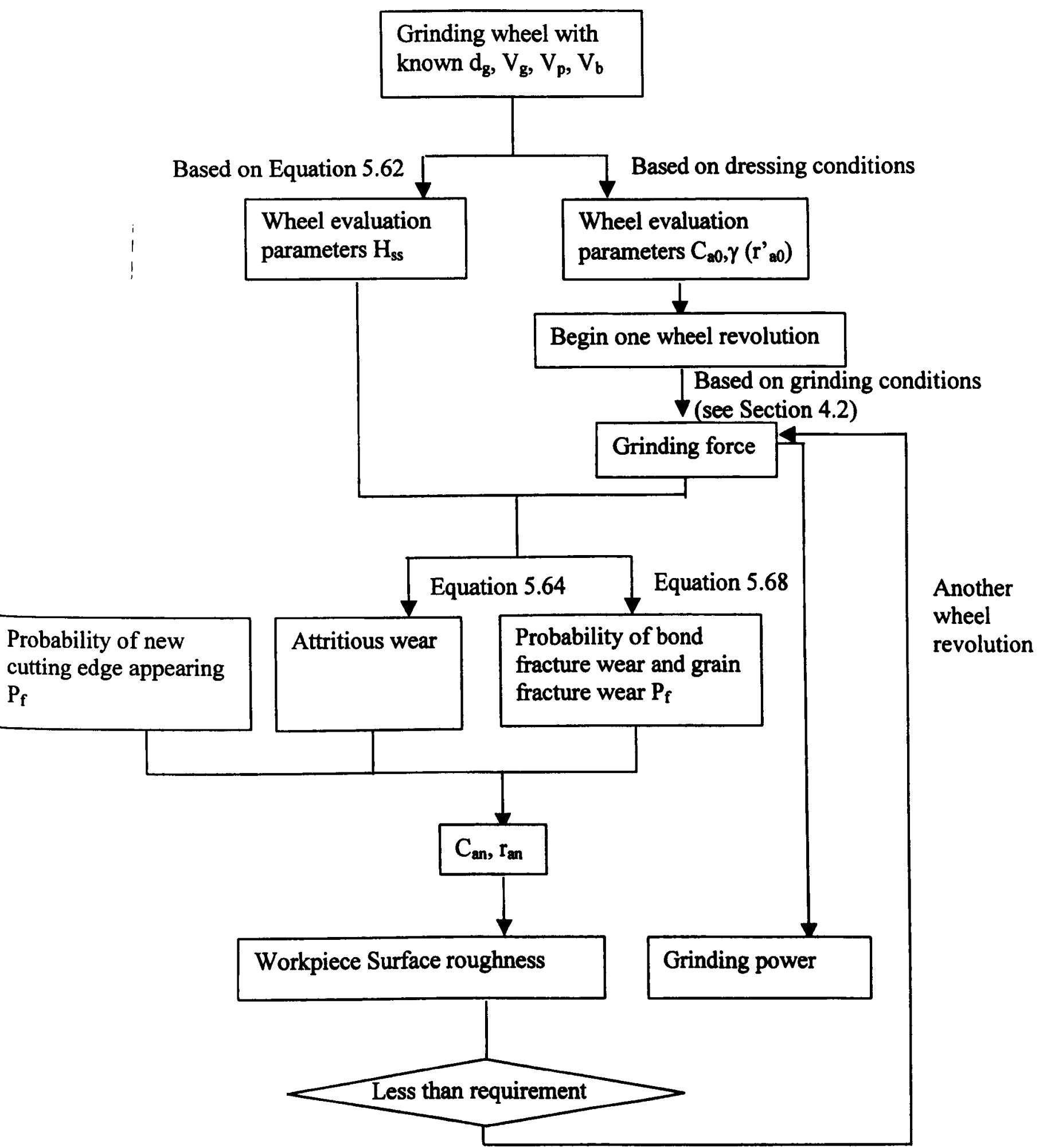

Fig. 5.10 The basic steps in prediction of wheel wear 


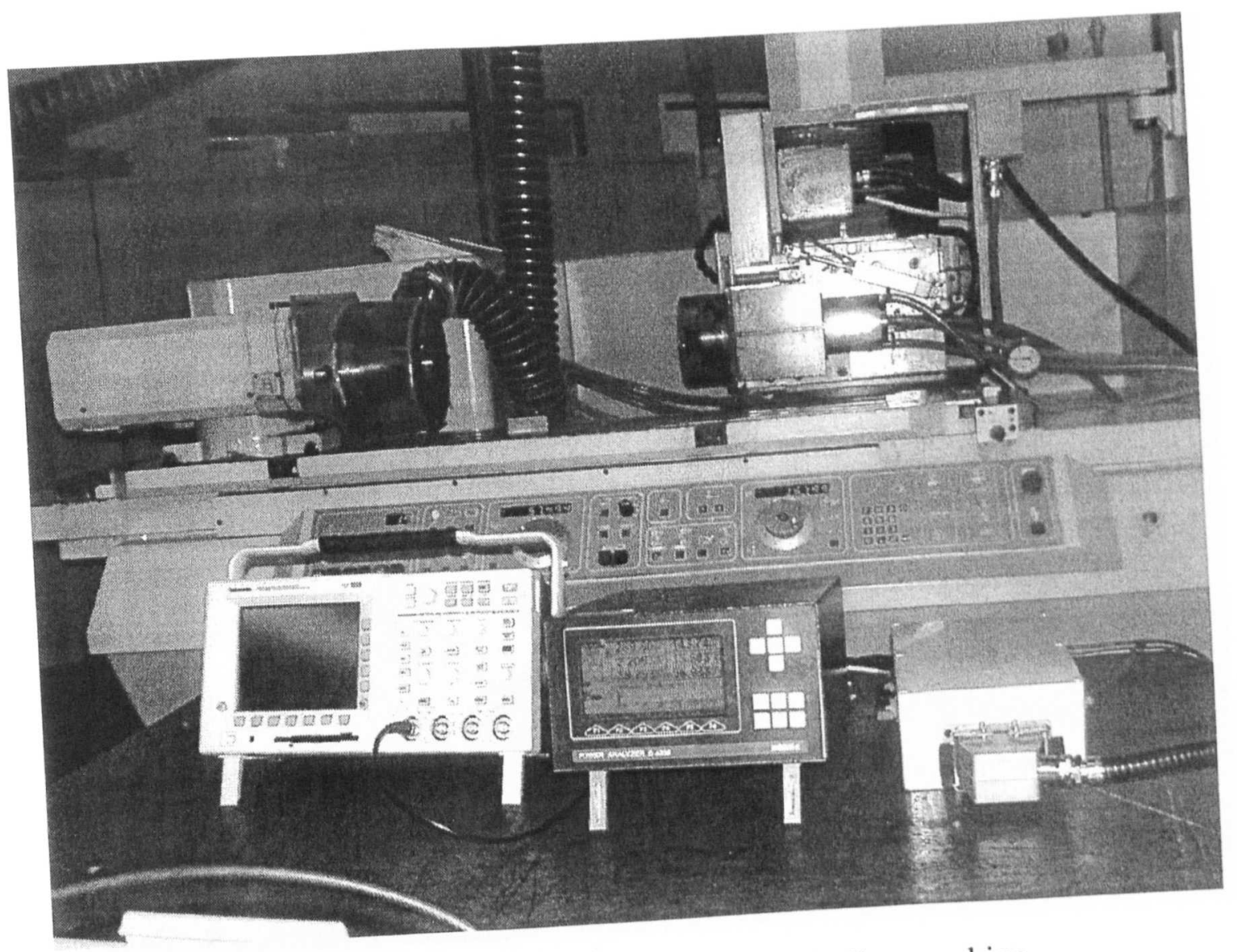

Fig. 6.1 Jones \& Shipman 1300X internal grinding machine

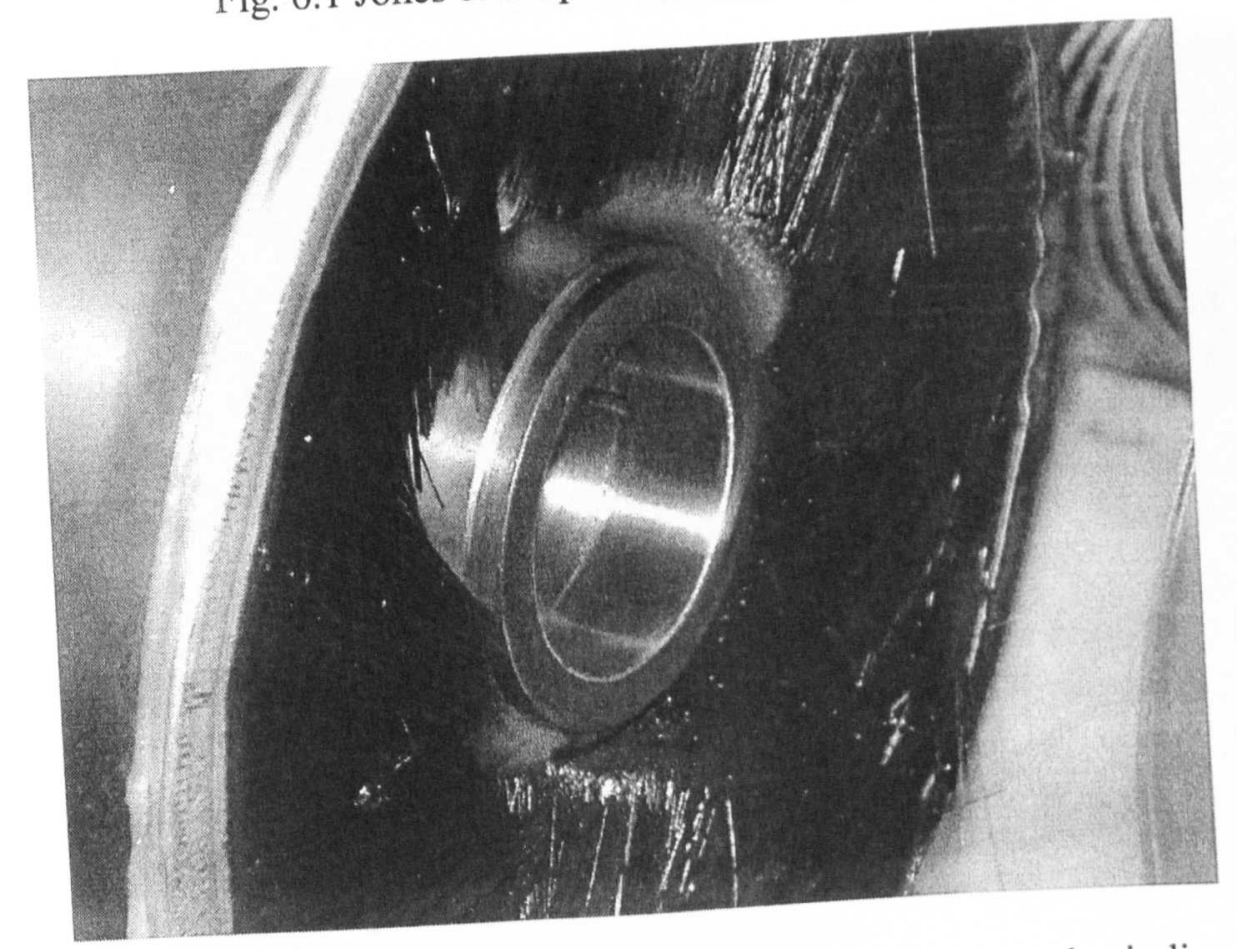

Fig. 6.2 Installation of workpiece for internal grinding 


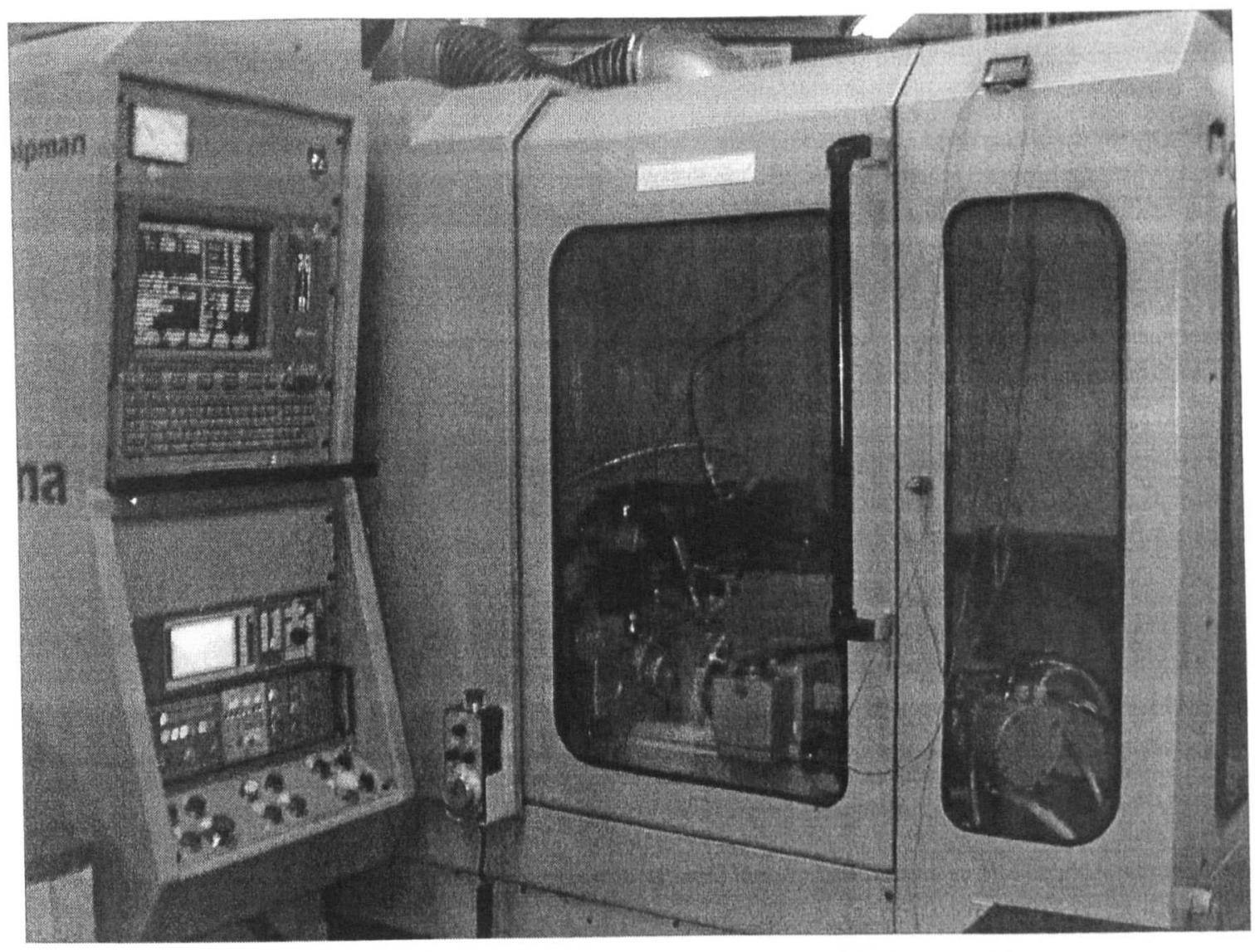

Fig. 6.3 Jones \& Shipman Suprema machine

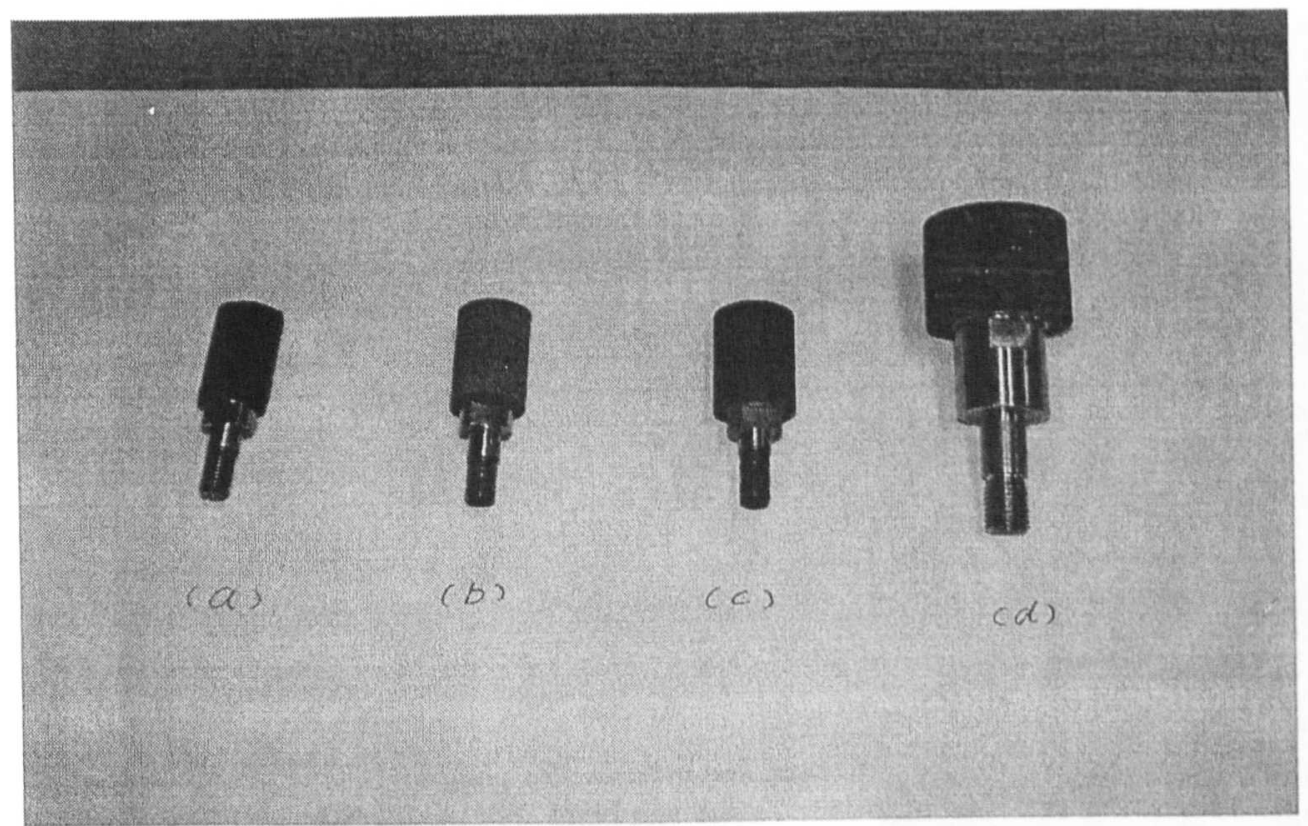

Fig. 6.4 Internal grinding wheels
(a) B91-150V1
(b) B64-150 VR
(c) B91-150 VR (for Inconel 718)
(d) B64-150 VX 


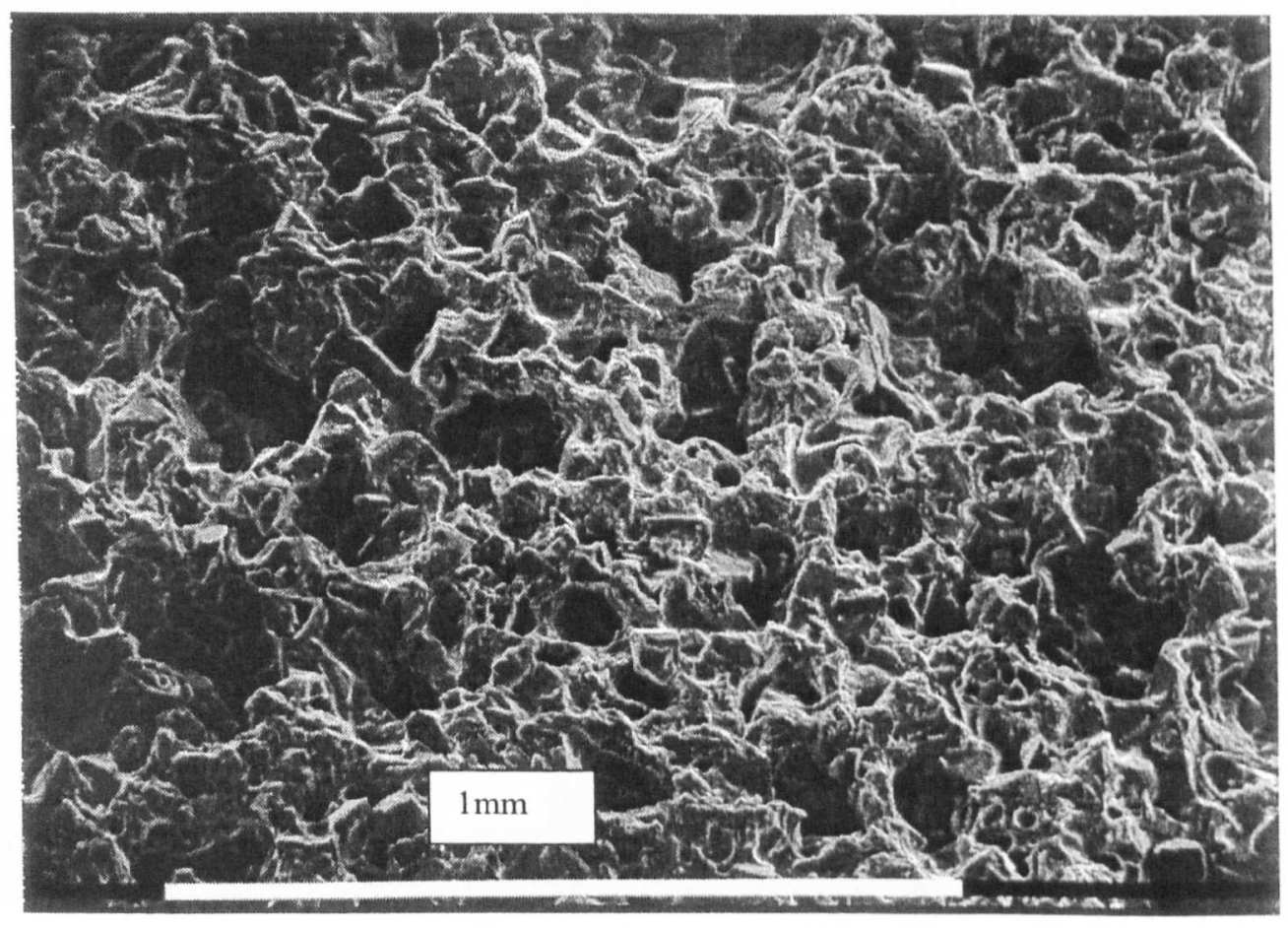

Fig. 6.5 (a) SEM photograph of HP- B64 wheel

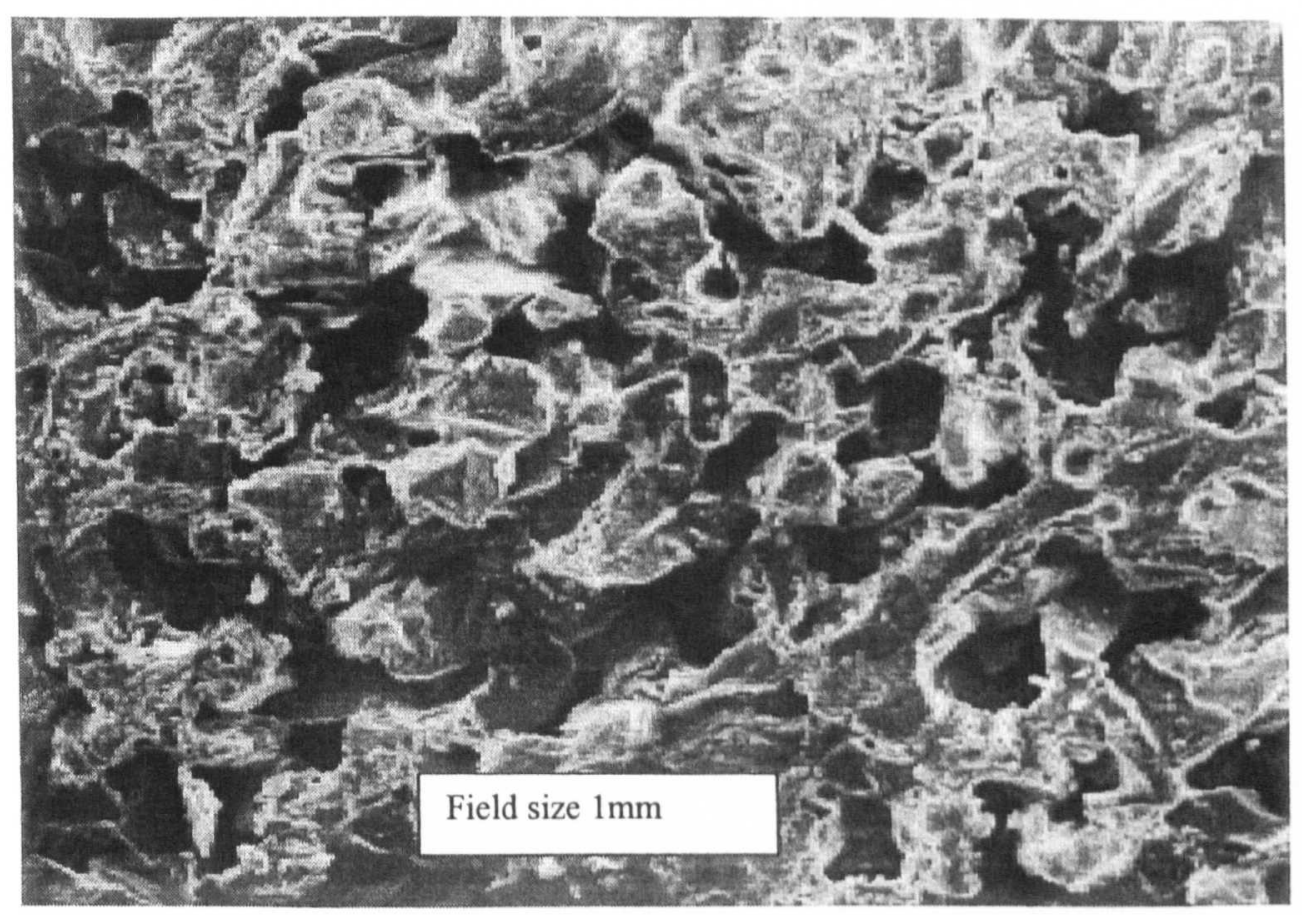

Fig. 6.5 (b) SEM photograph of MP- B91 wheel 


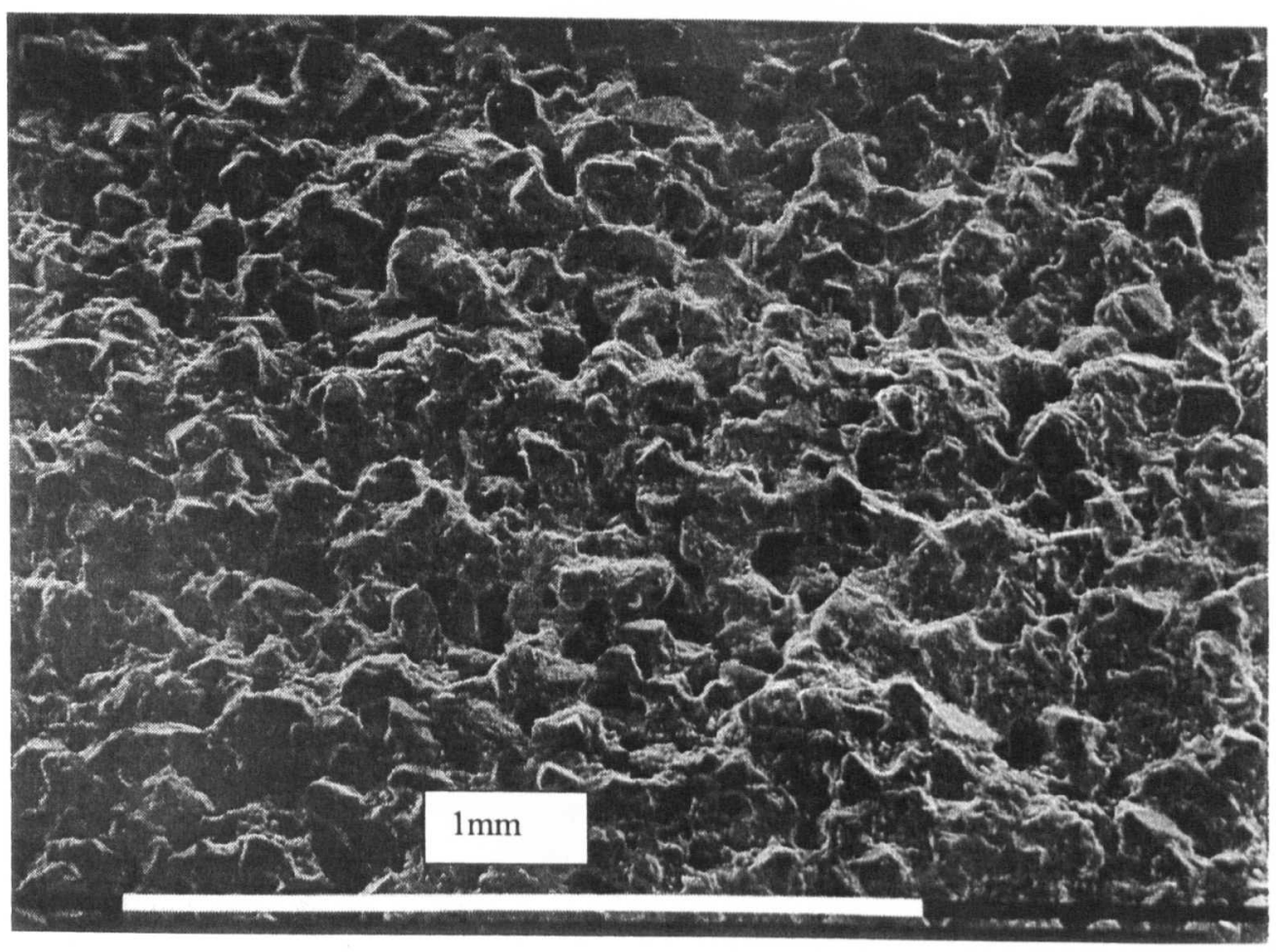

Fig. 6.5 (c) SEM photograph of HP-B91 wheel 


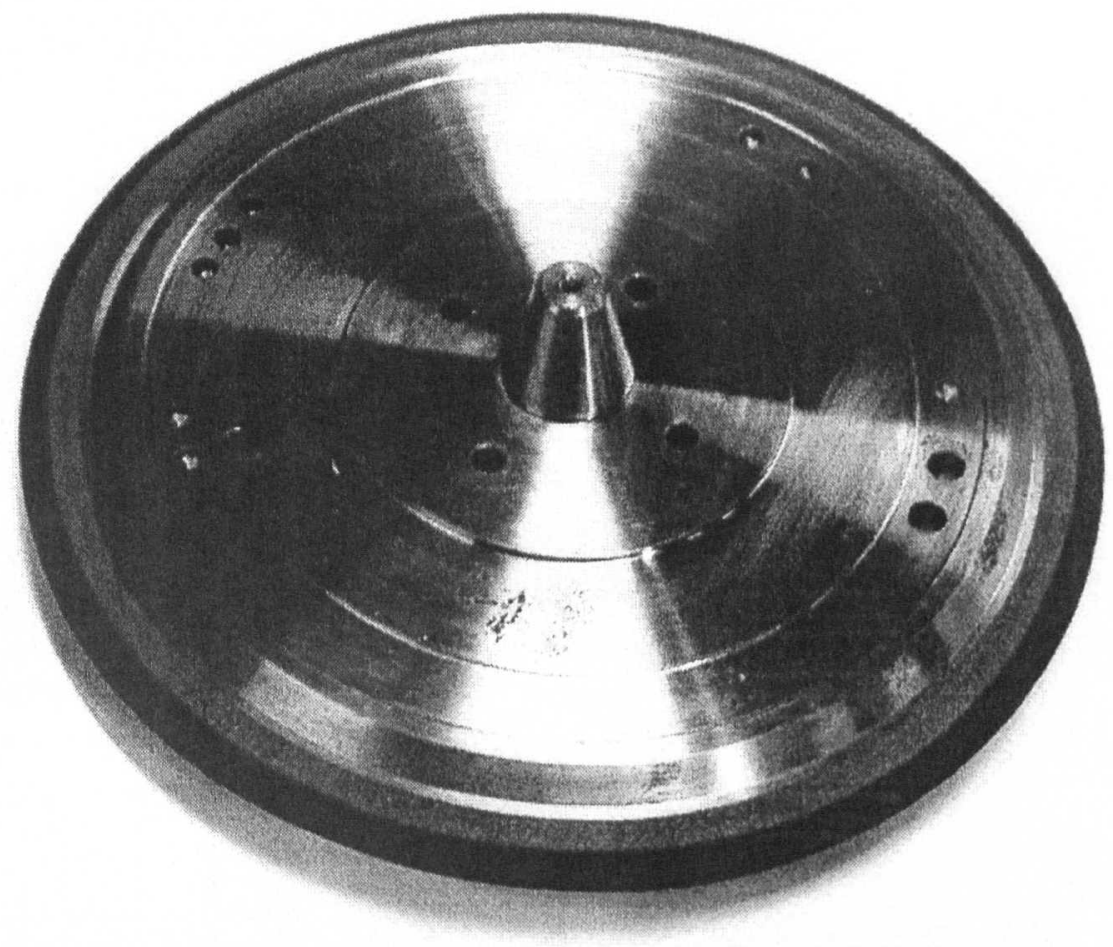

Fig. 6.6 A wheel for external grinding (B91-150VR) 


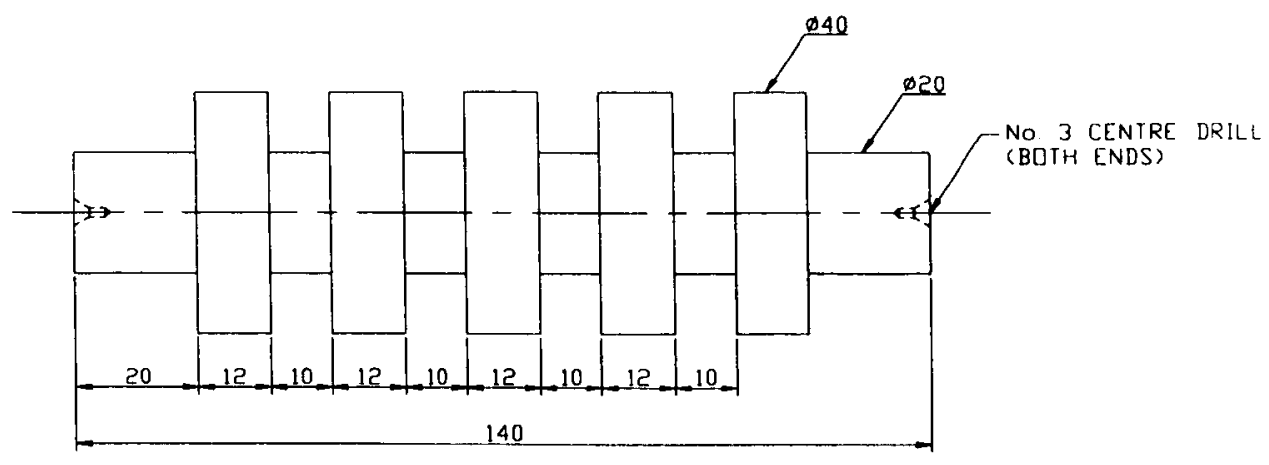

(a) AISI 52100 workpiece

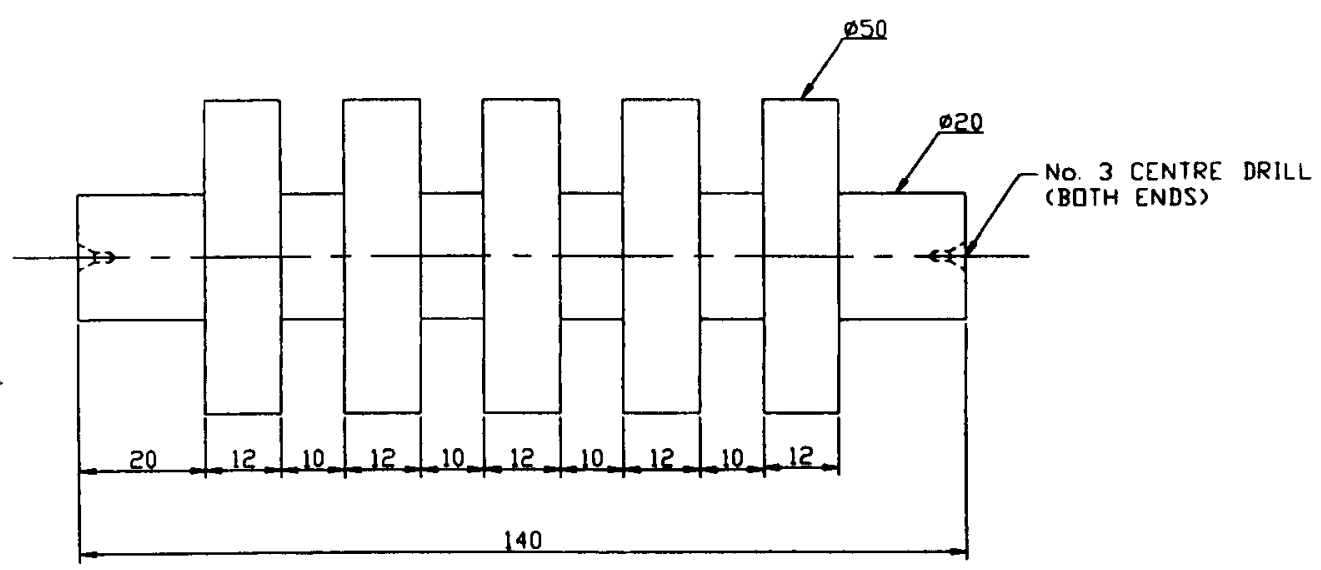

(b) Inconel 718 workpiece

Fig. 6.7 Workpieces for external grinding (all sizes shown in $\mathrm{mm}$ ) 


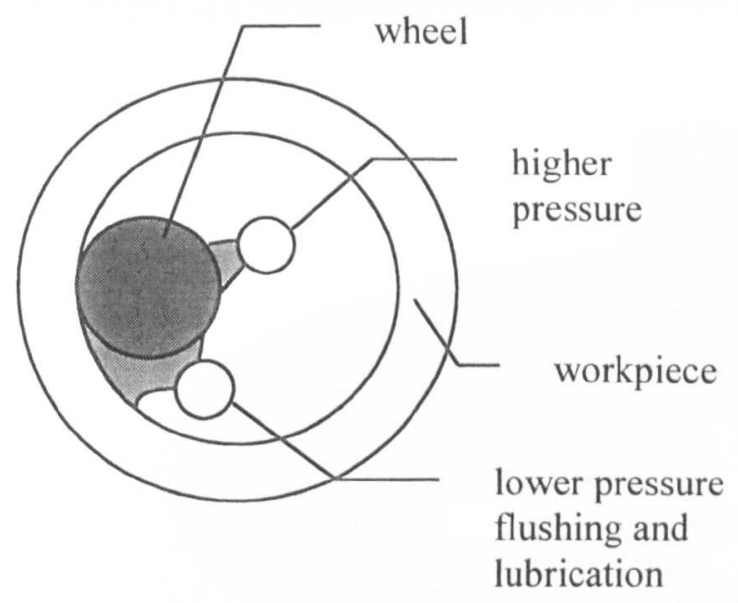

Fig. 6.8 Coolant delivery system for CBN internal grinding

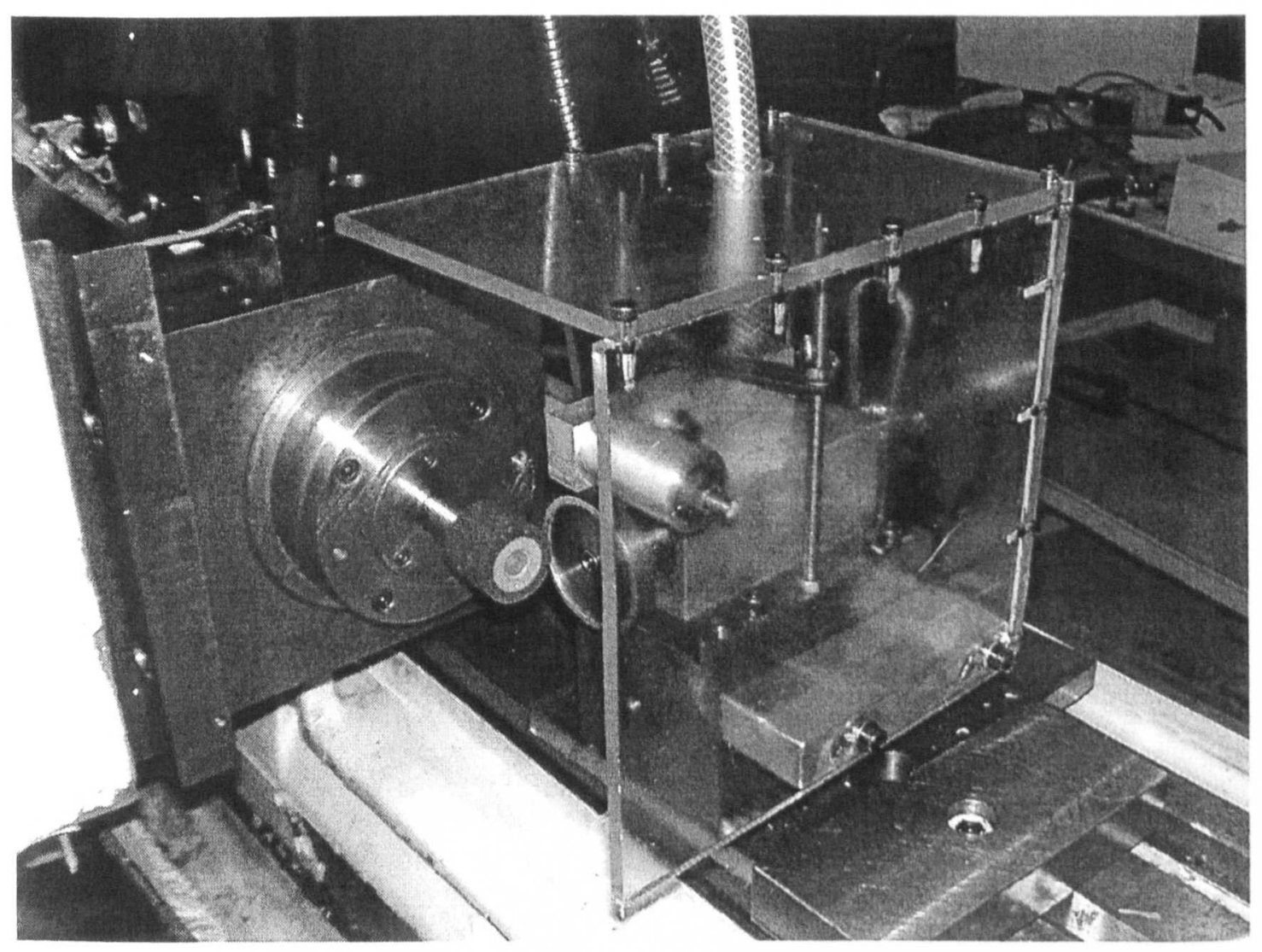

Fig. 6.9 Internal grinding dressing system 


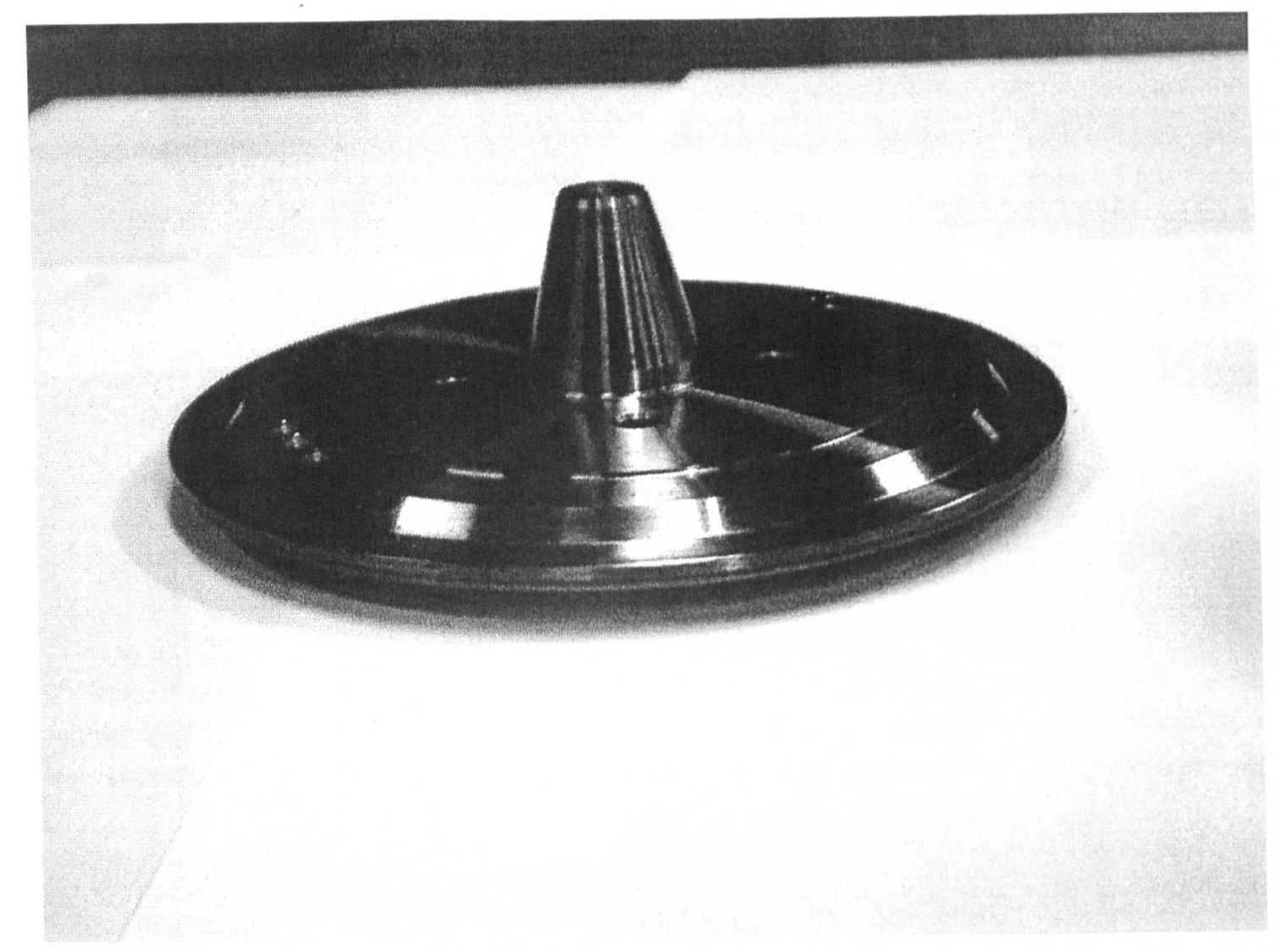

Fig. 6.10 The dressing disc for external grinding 


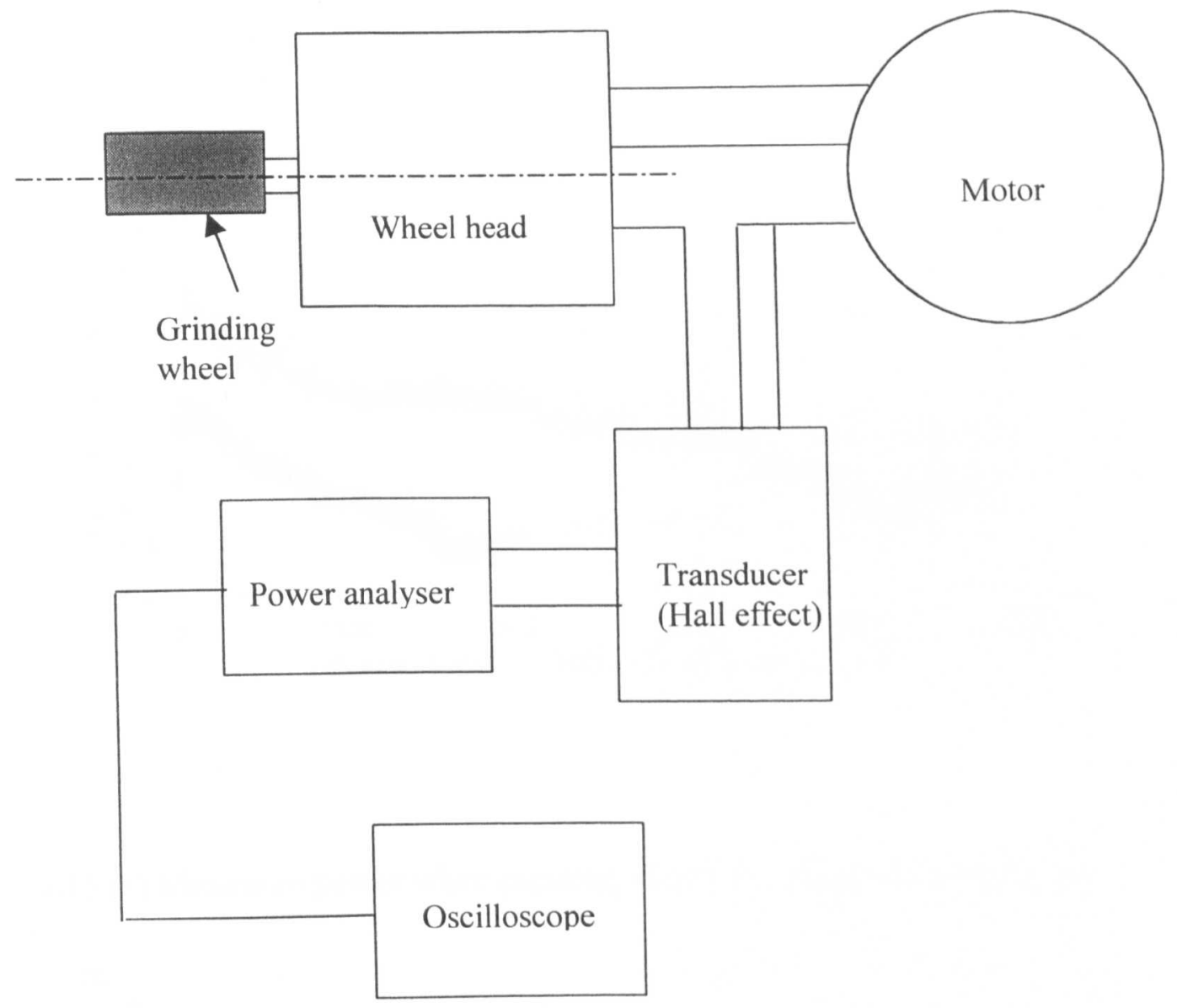

Fig. 6.11 The power measurement system

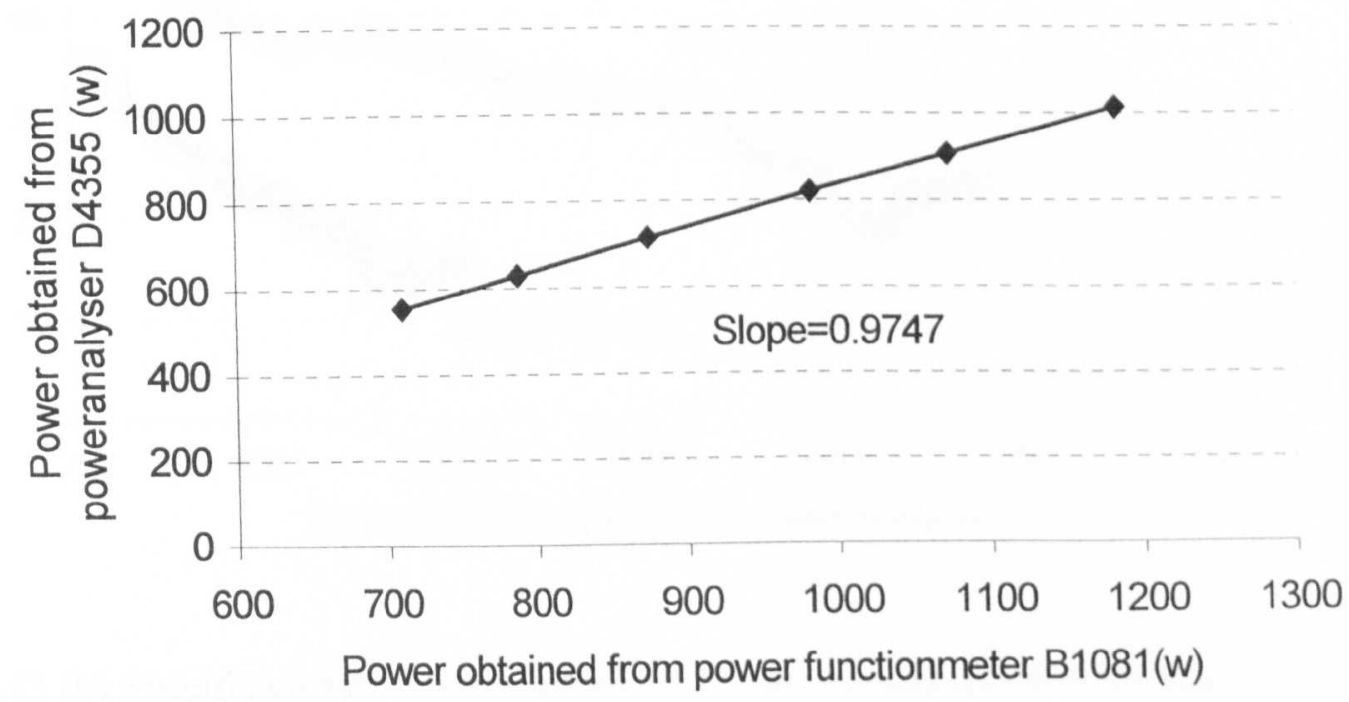

Fig. 6.12 Calibration results of power analyser D4355 


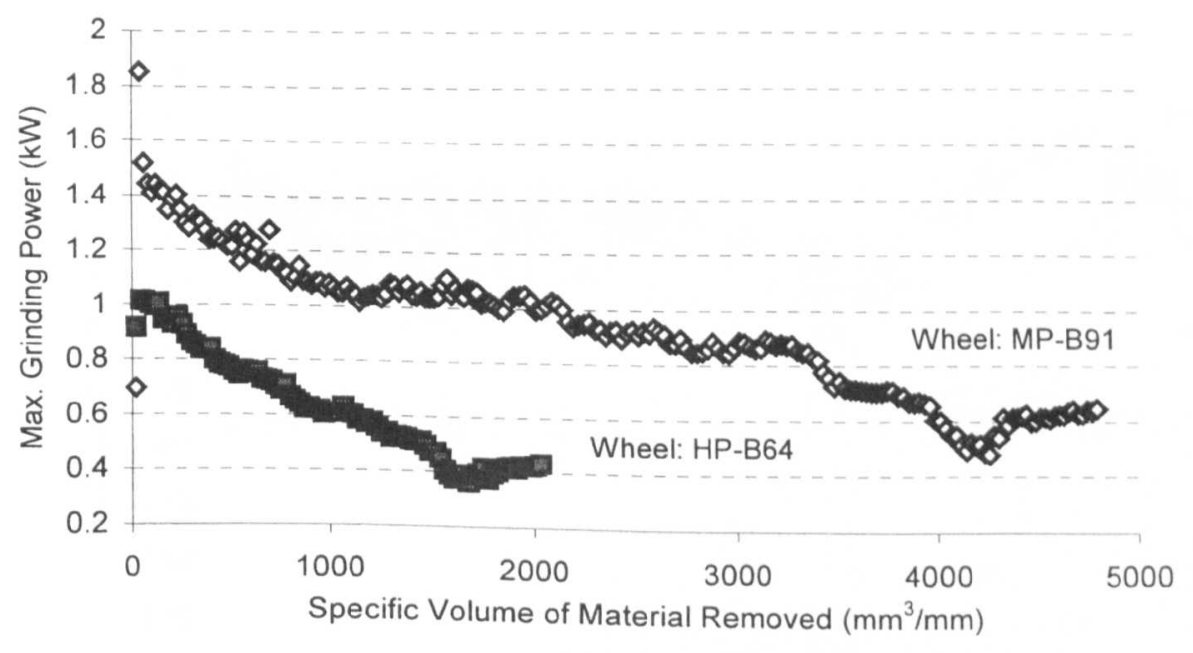

Fig. 6.13 (a) Maximum power when grinding of M2 at a wheel speed of $45 \mathrm{~m} / \mathrm{s}$

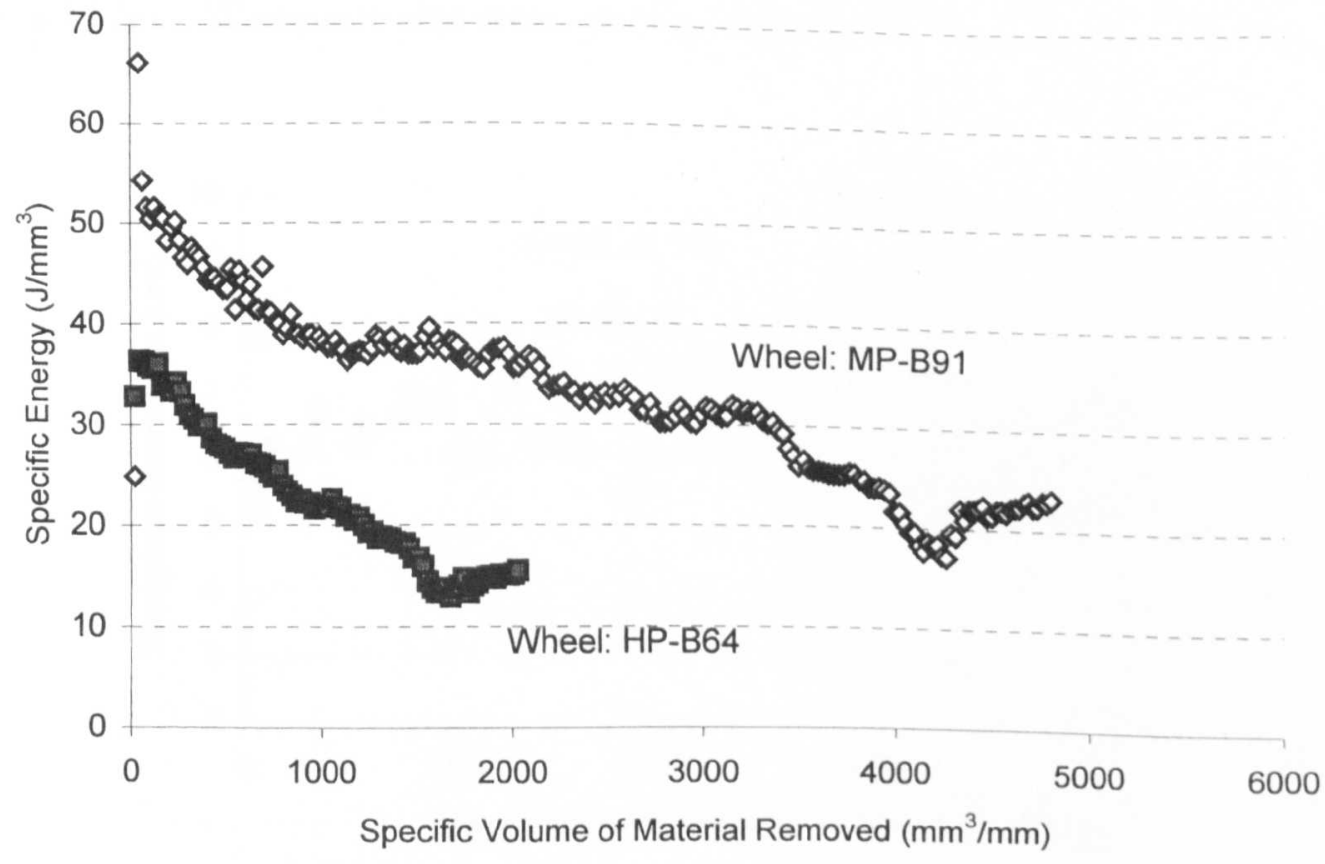

Fig. 6.13 (b) Specific energy when grinding of M2 at a wheel speed of $45 \mathrm{~m} / \mathrm{s}$ 


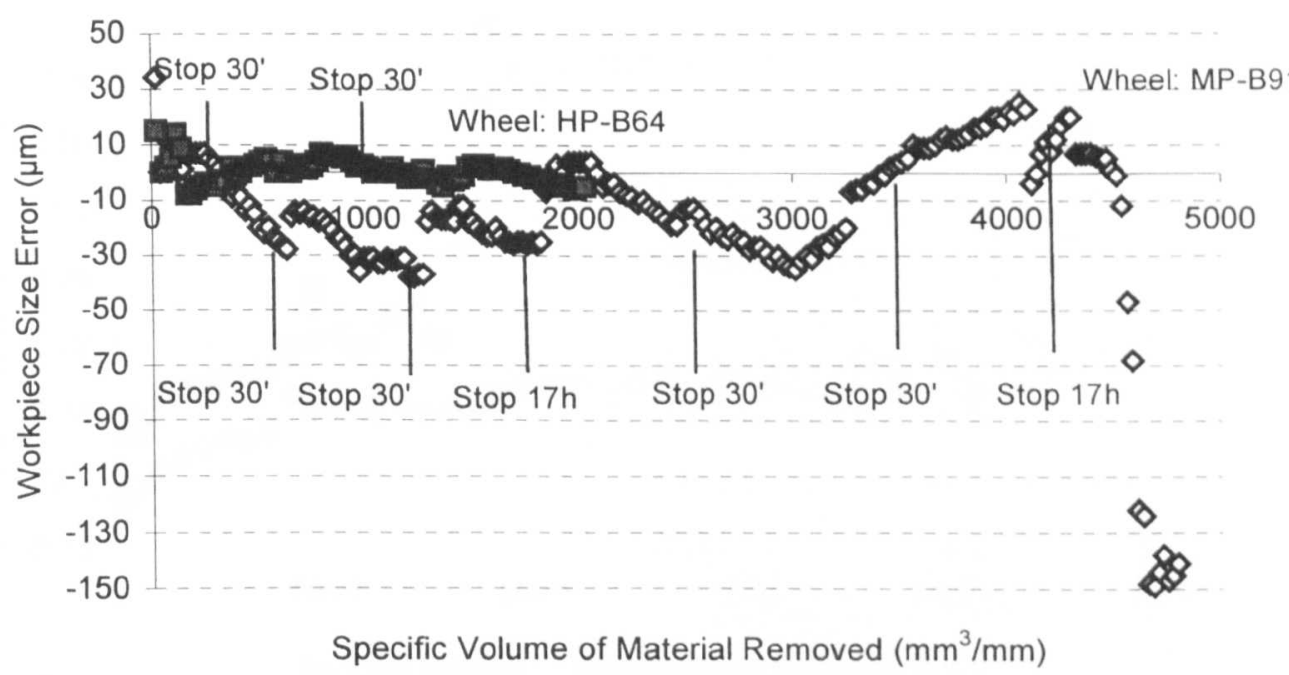

Fig. 6.13 (c) Workpiece size error when grinding M2 at a wheel speed of $45 \mathrm{~m} / \mathrm{s}$

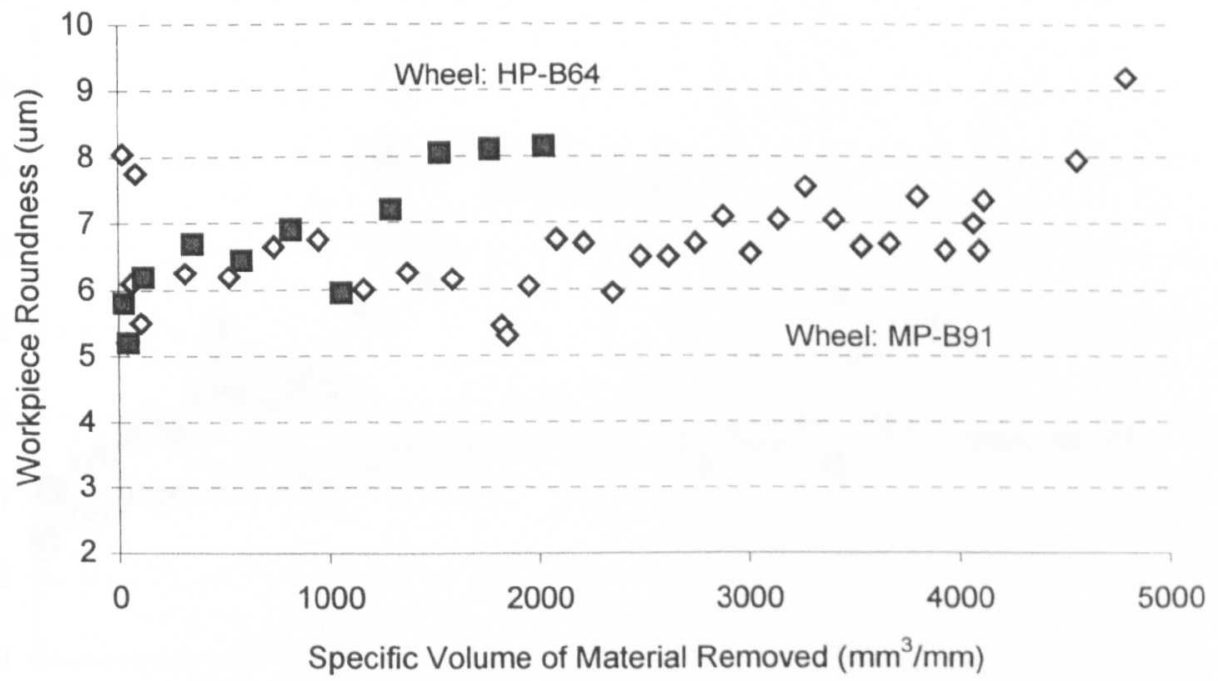

Fig. 6.13 (d) Workpiece roundness when grinding M2 at a wheel speed of 45 $\mathrm{m} / \mathrm{s}$ 


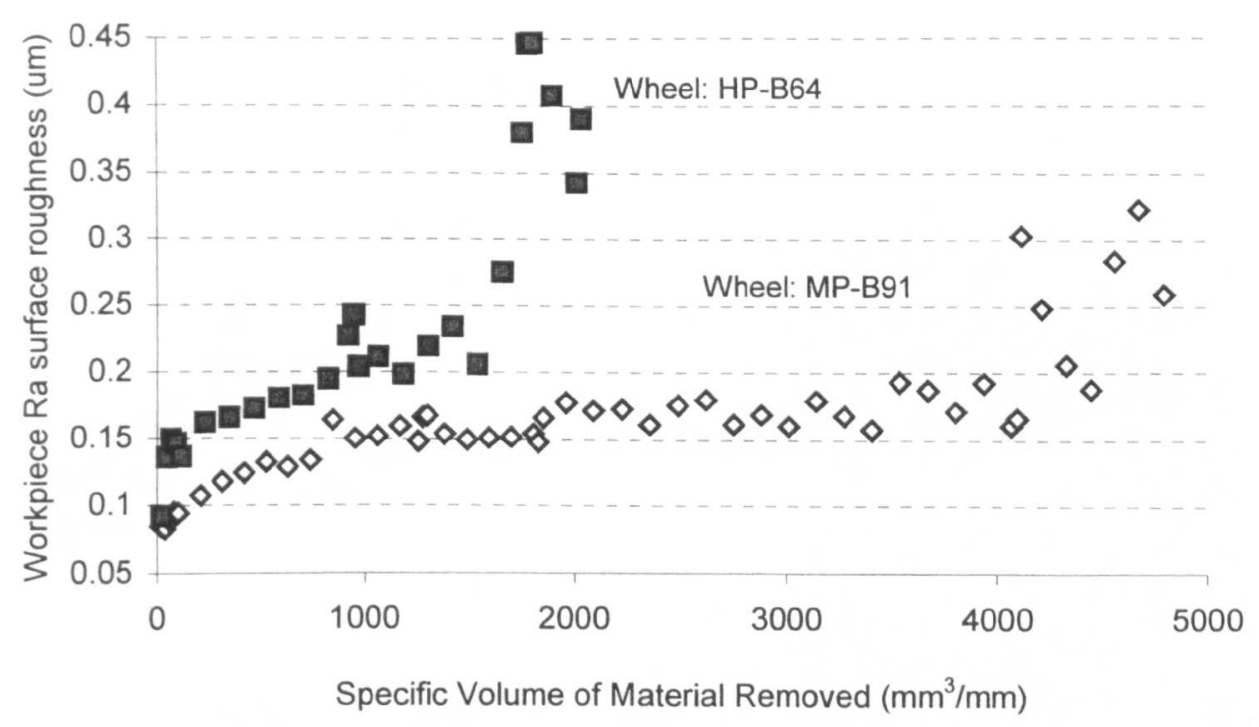

Fig. 6.13 (e) Workpiece Ra surface roughness when grinding M2 at a wheel speed of $45 \mathrm{~m} / \mathrm{s}$

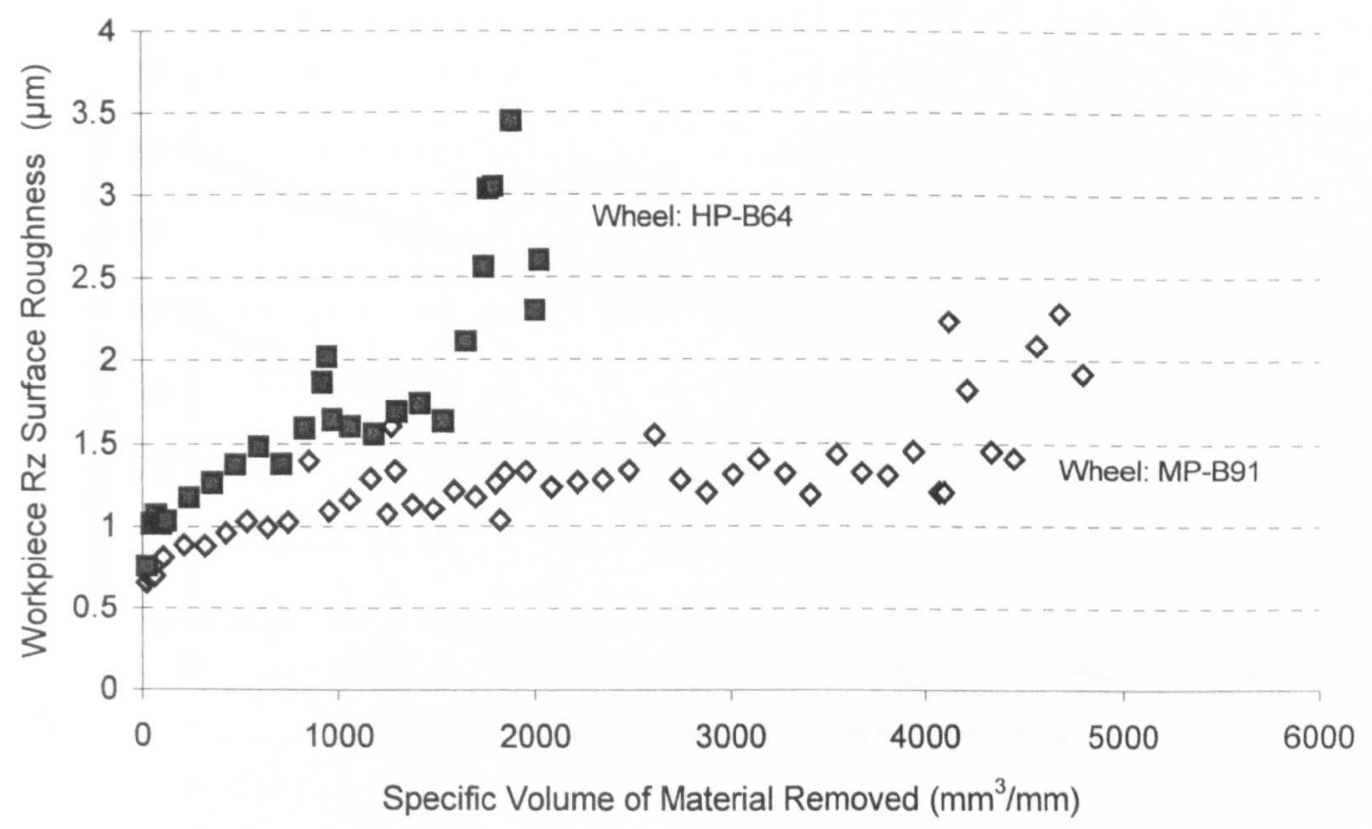

Fig. 6.13 (f) Workpiece Rz surface roughness when grinding M2 at a wheel speed of $45 \mathrm{~m} / \mathrm{s}$ 


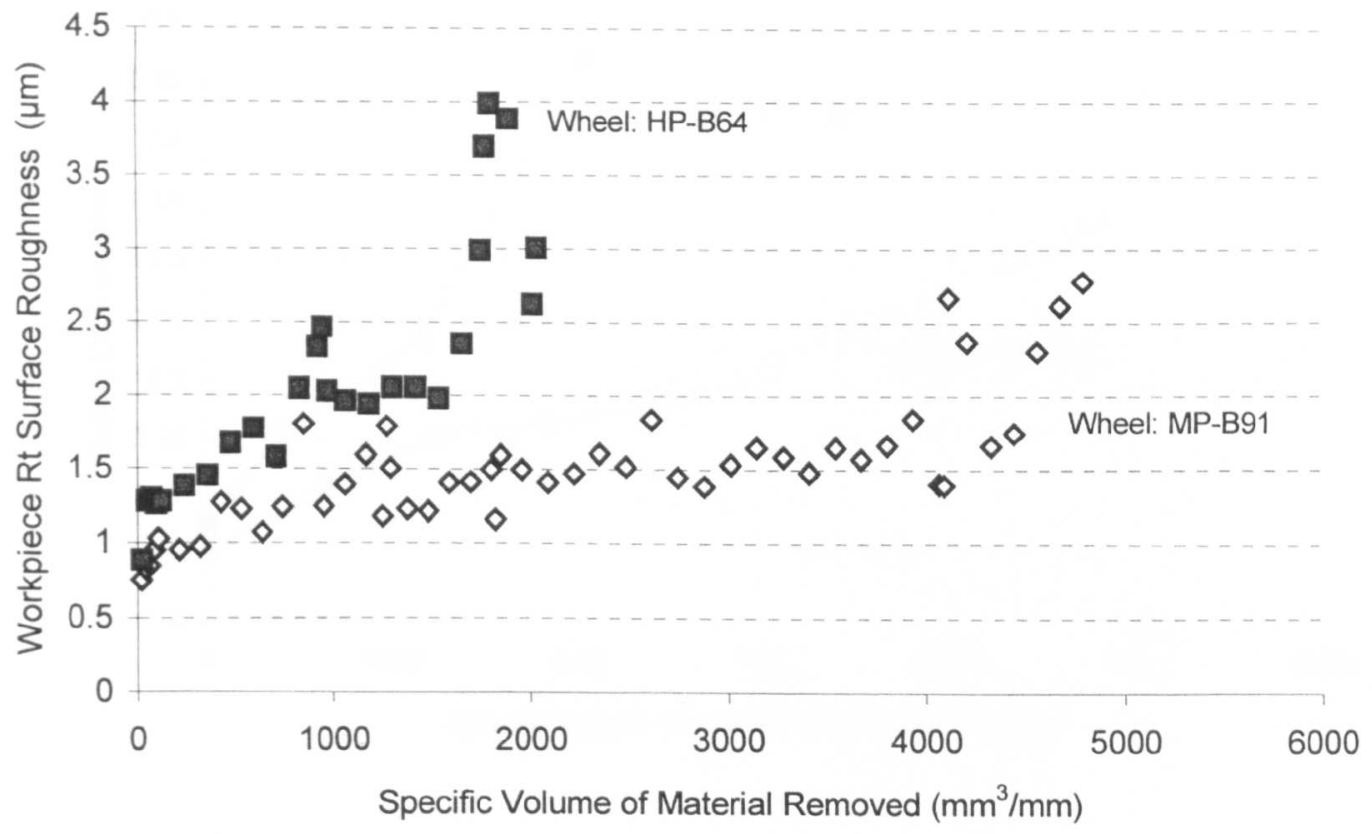

Fig. 6.13 (g) Workpiece Rt surface roughness when grinding M2 at a wheel speed of $45 \mathrm{~m} / \mathrm{s}$

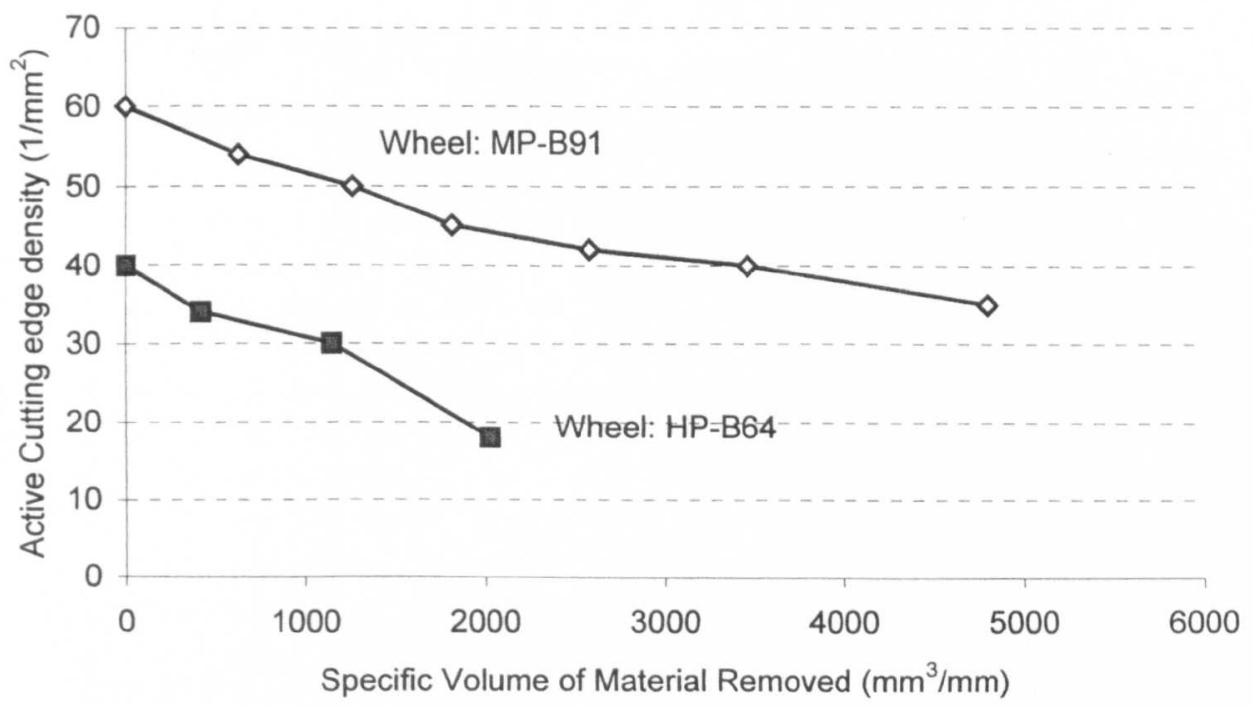

Fig. 6.13 (h) Active cutting edge density when grinding M2 at a wheel speed of $45 \mathrm{~m} / \mathrm{s}$ 


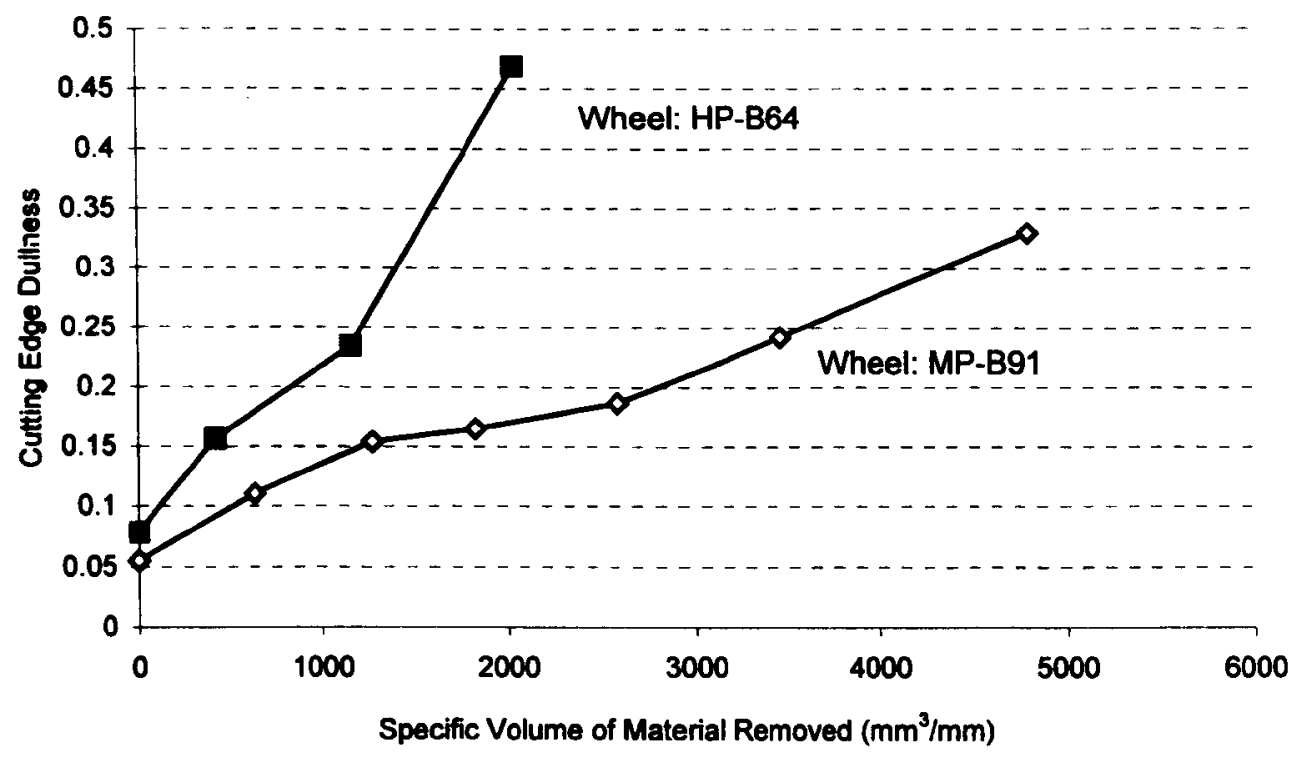

Fig. 6.13 (i) Cutting edge dullness when grinding M2 at a wheel speed of 45 $\mathbf{m} / \mathbf{s}$ 


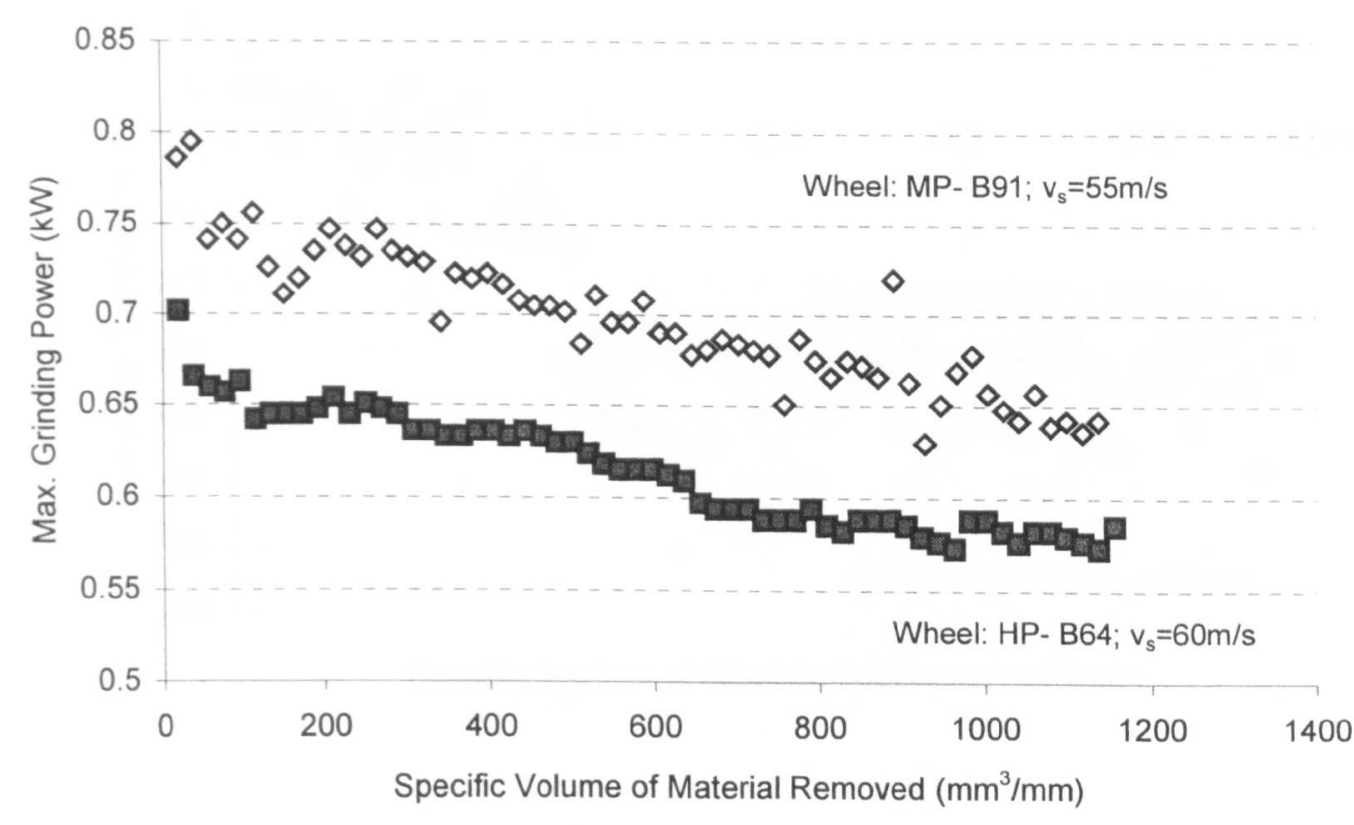

Fig. 6.14 (a) Maximum power when grinding M2 at a wheel speed of $55-60 \mathrm{~m} / \mathrm{s}$

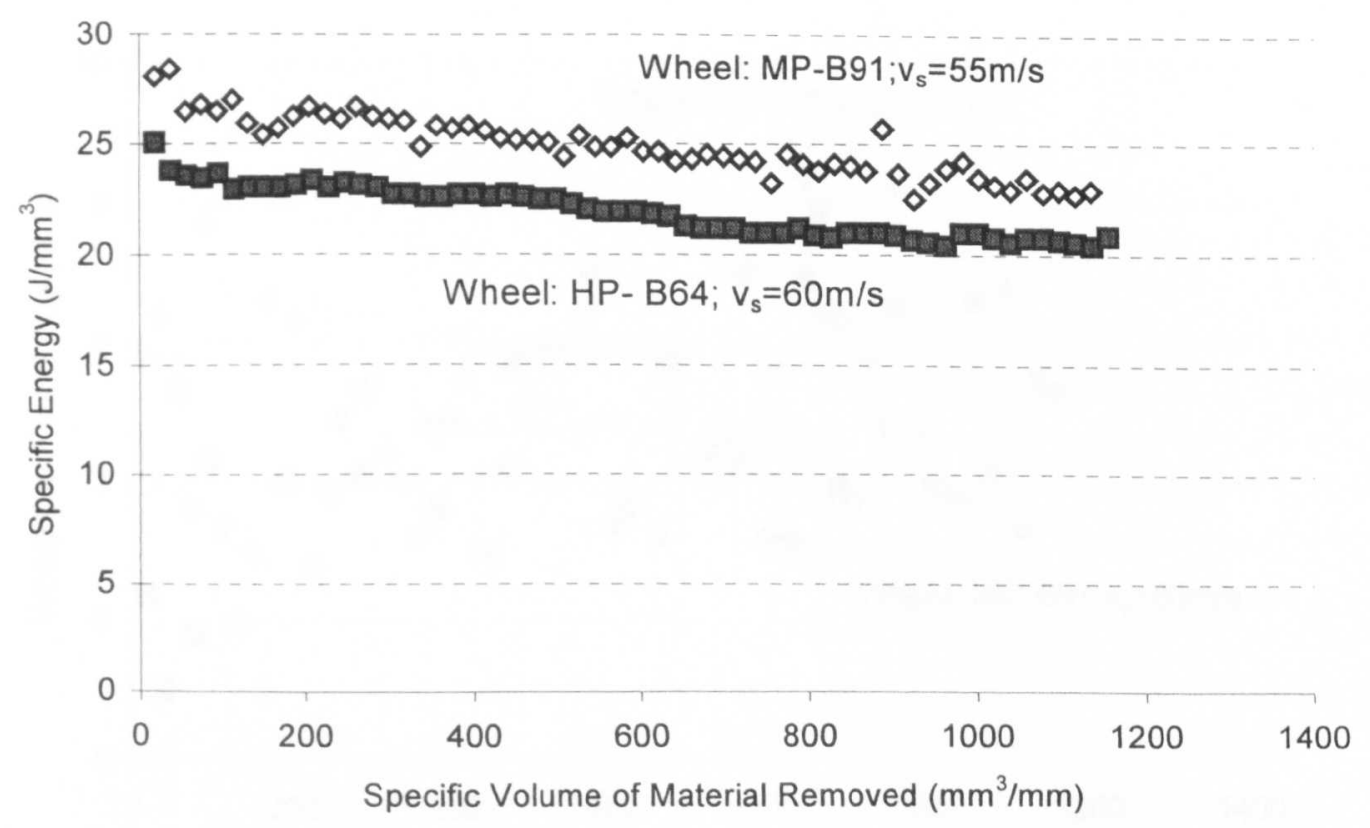

Fig. 6.14 (b) Specific energy when grinding M2 at a wheel speed of $55-60 \mathrm{~m} / \mathrm{s}$ 


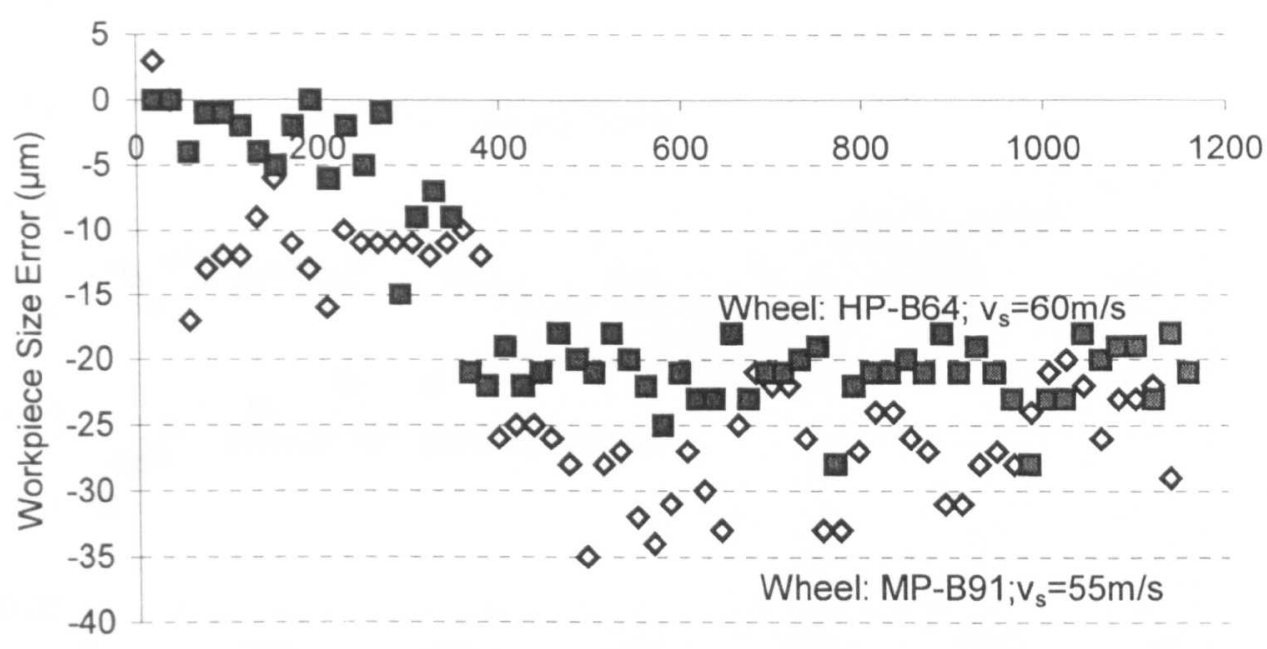

Specific Volume of Material Removed $\left(\mathrm{mm}^{3} / \mathrm{mm}\right)$

Fig. 6.14 (c) Workpiece size error when grinding M2 at a wheel speed of 55-60 $\mathrm{m} / \mathrm{s}$

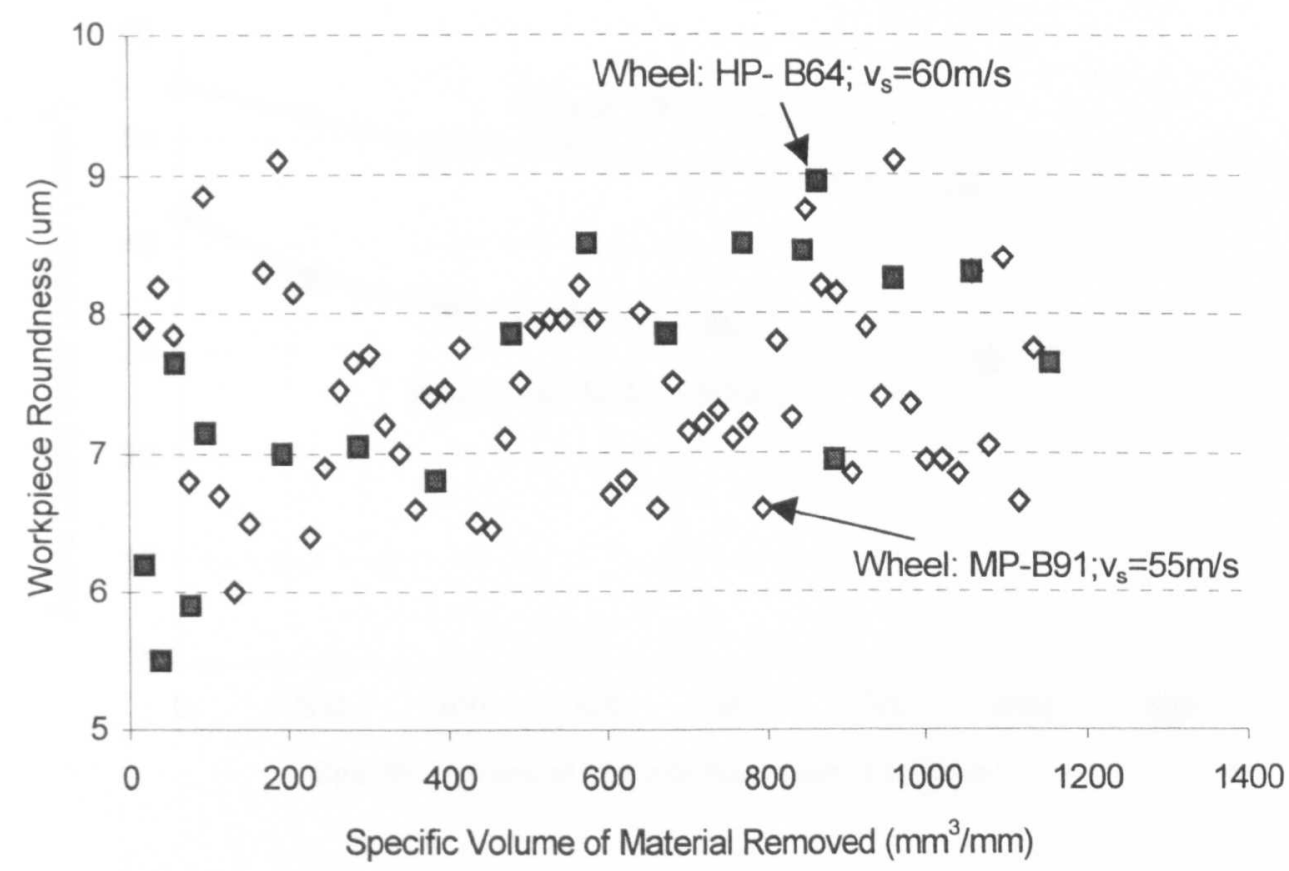

Fig. 6.14 (d) Workpiece roundness when grinding M2 at a wheel speed of 55-60 $\mathrm{m} / \mathrm{s}$ 


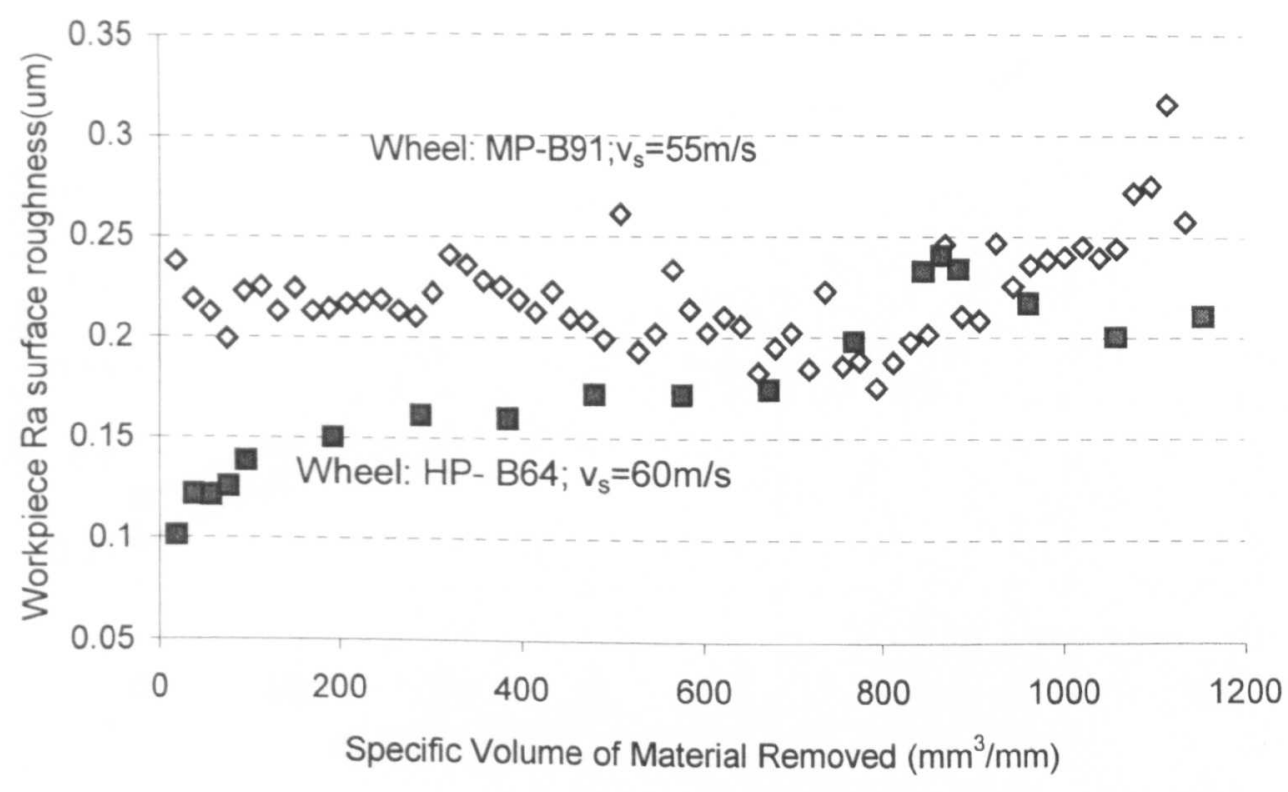

Fig. 6.14 (e) Workpiece Ra surface roughness when grinding M2 at a wheel speed of $55-60 \mathrm{~m} / \mathrm{s}$

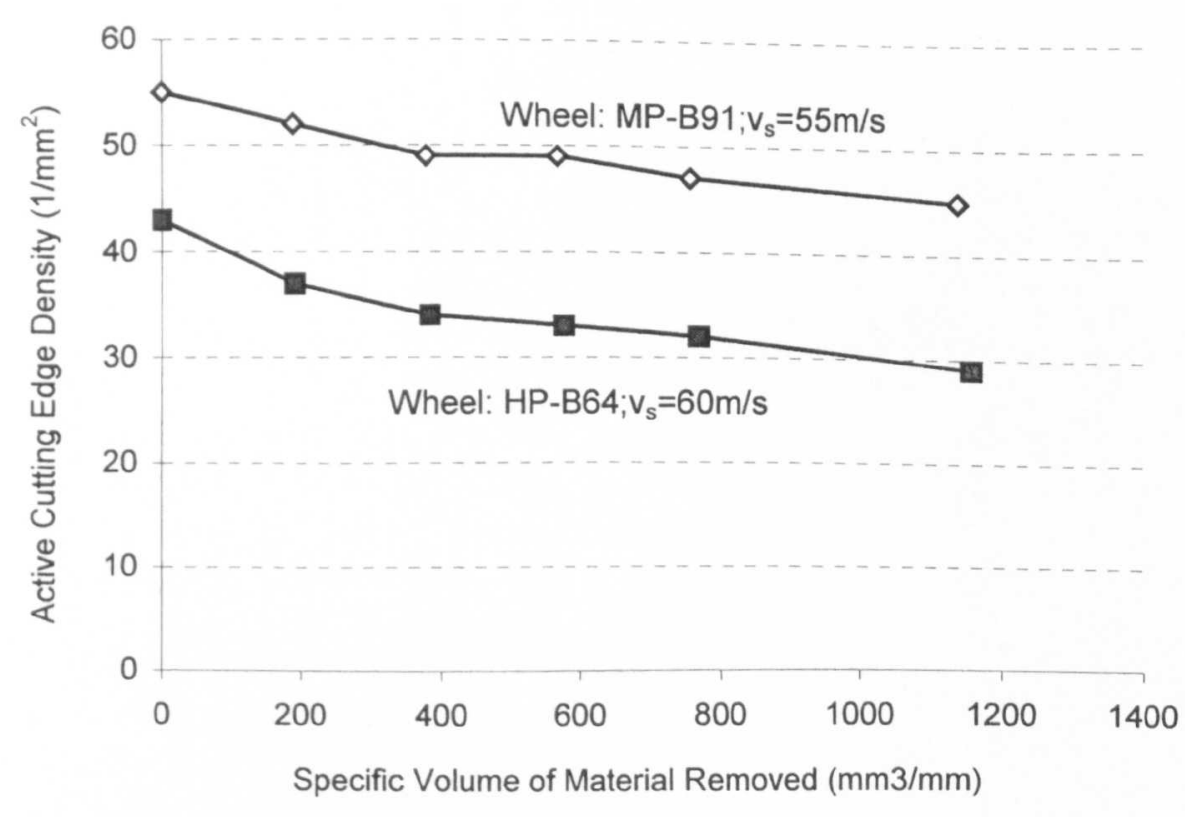

Fig. 6.14 (f) Active cutting edge density when grinding M2 at a wheel speed of $55-60 \mathrm{~m} / \mathrm{s}$ 


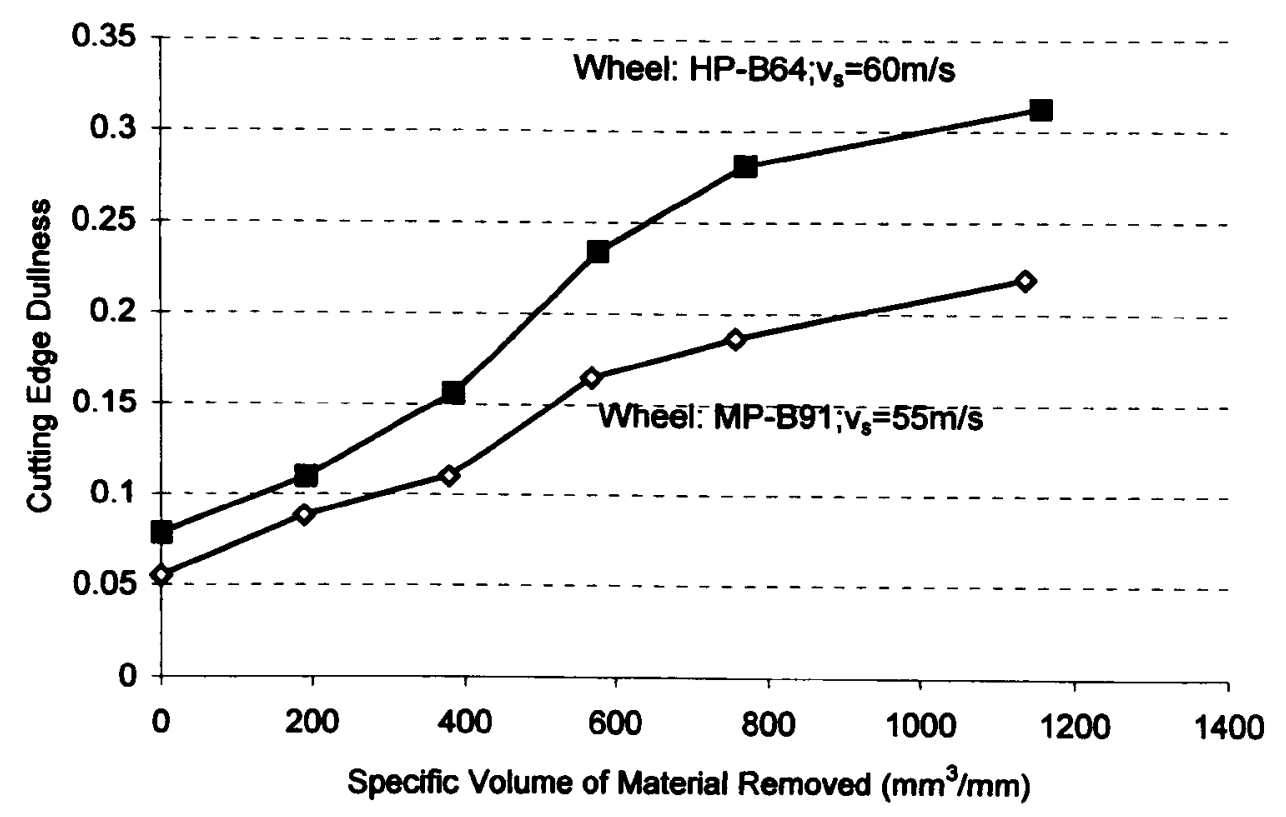

Fig. 6.14 (g) Cutting edge dullness when grinding M2 at a wheel speed of 55-60 $\mathrm{m} / \mathrm{s}$ 


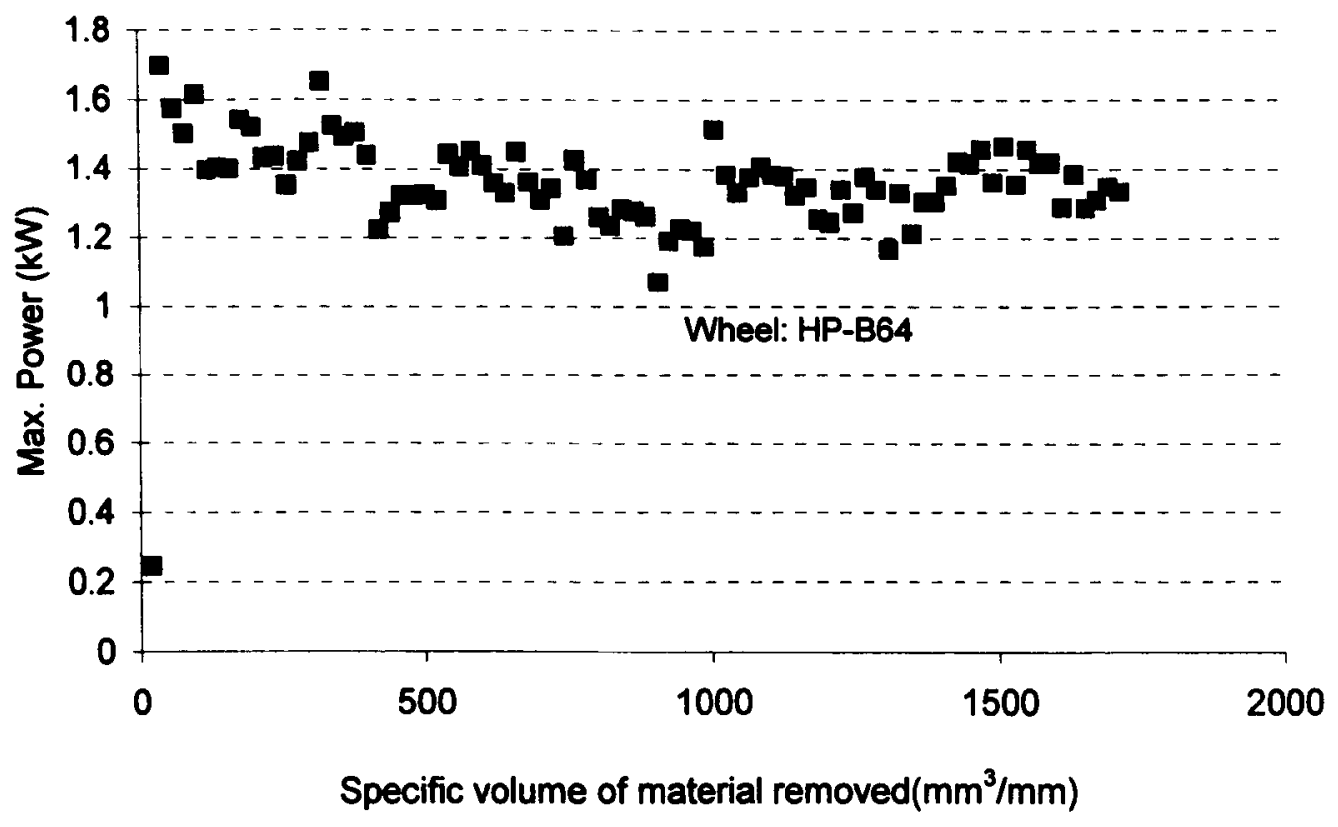

Fig. 6.15 (a) Maximum power when grinding $M 2$ at a wheel speed of $110 \mathrm{~m} / \mathrm{s}$

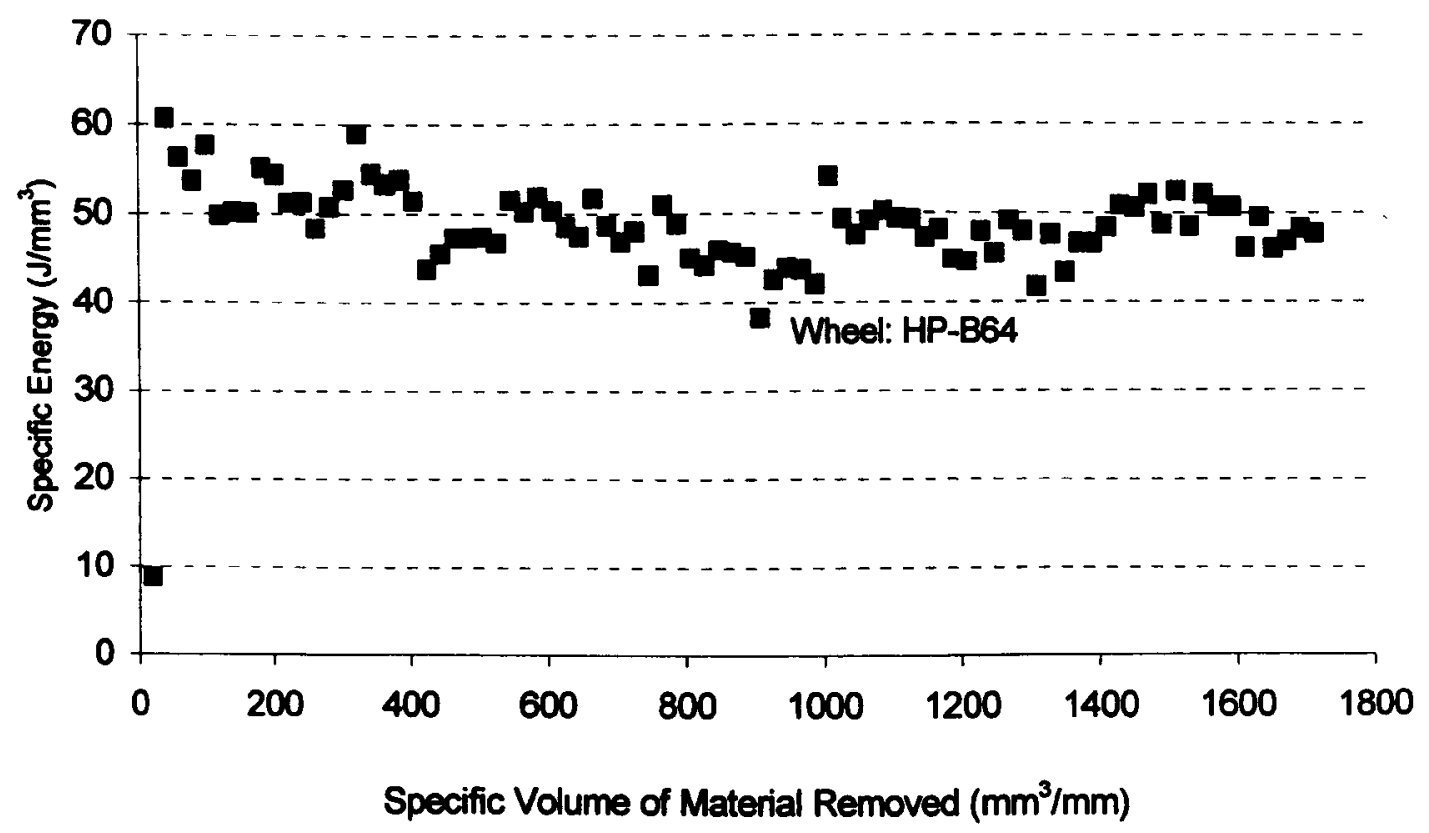

Fig. 6.15 (b) Specific energy when grinding M2 at a wheel speed of $110 \mathrm{~m} / \mathrm{s}$ 


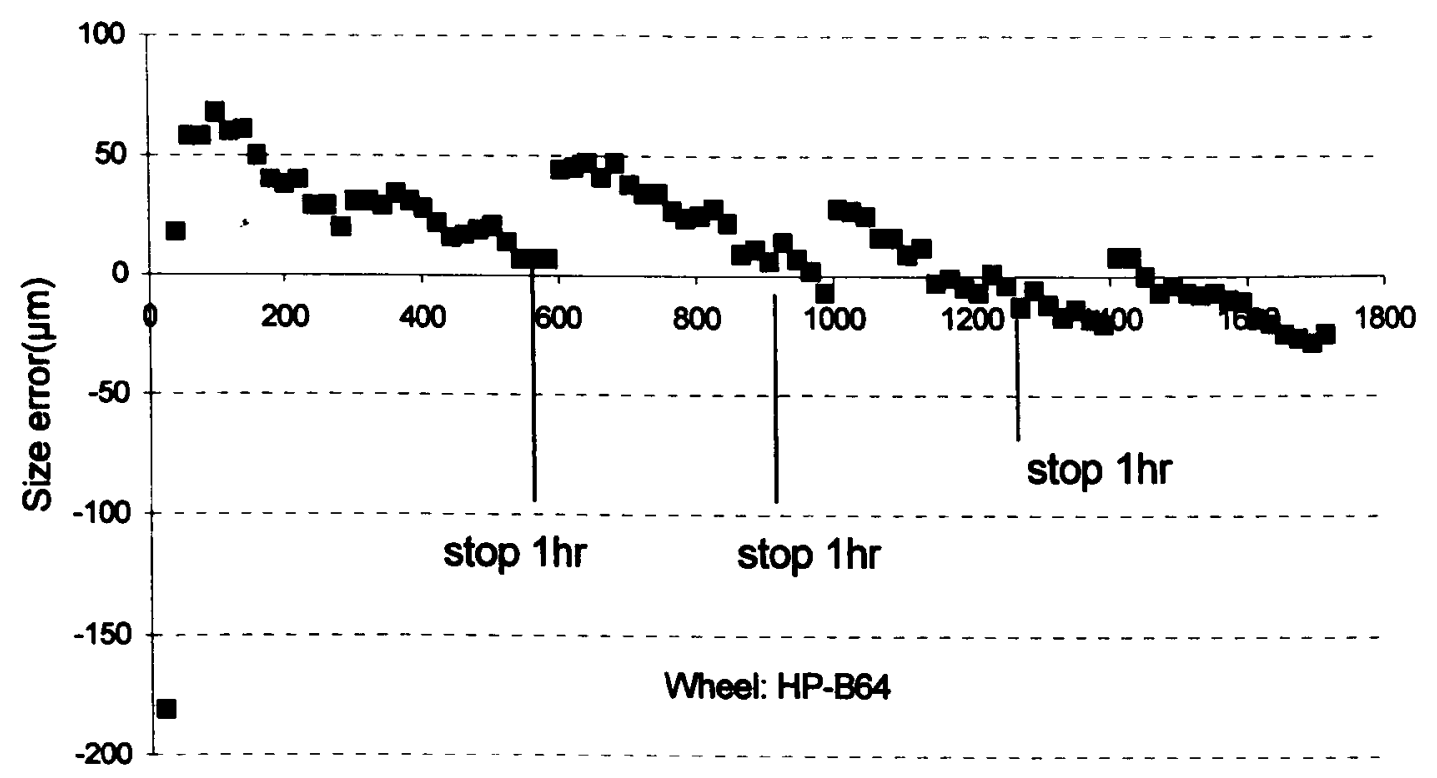

Specific volume of material removed $\left(\mathrm{mm}^{3} / \mathrm{mm}\right)$

Fig. 6.15 (c) Workpiece size error when grinding M2 at a wheel speed of 110 $\mathrm{m} / \mathrm{s}$

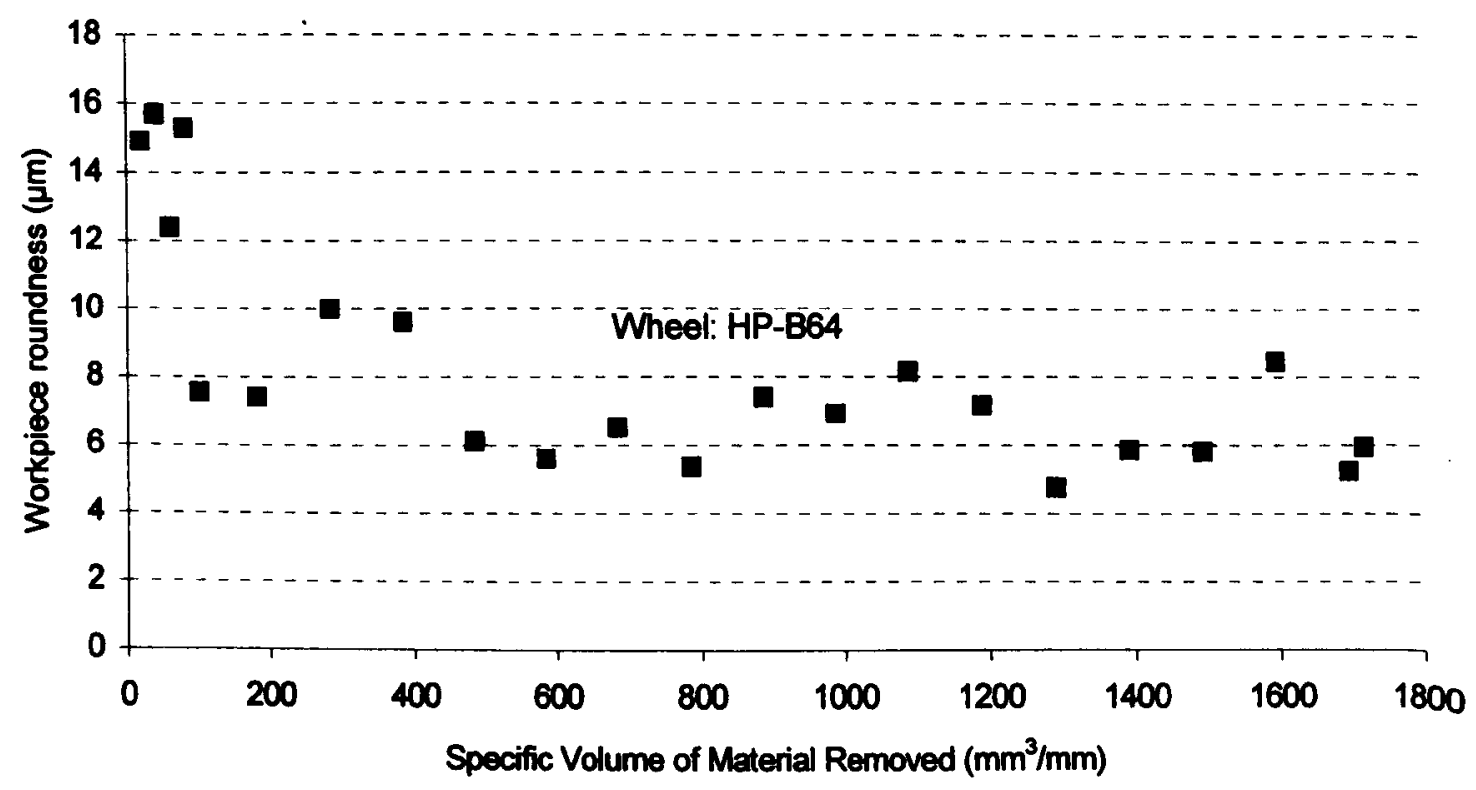

Fig. 6.15 (d) Workpiece roundness when grinding M2 at a wheel speed of 110 $\mathbf{m} / \mathbf{s}$ 


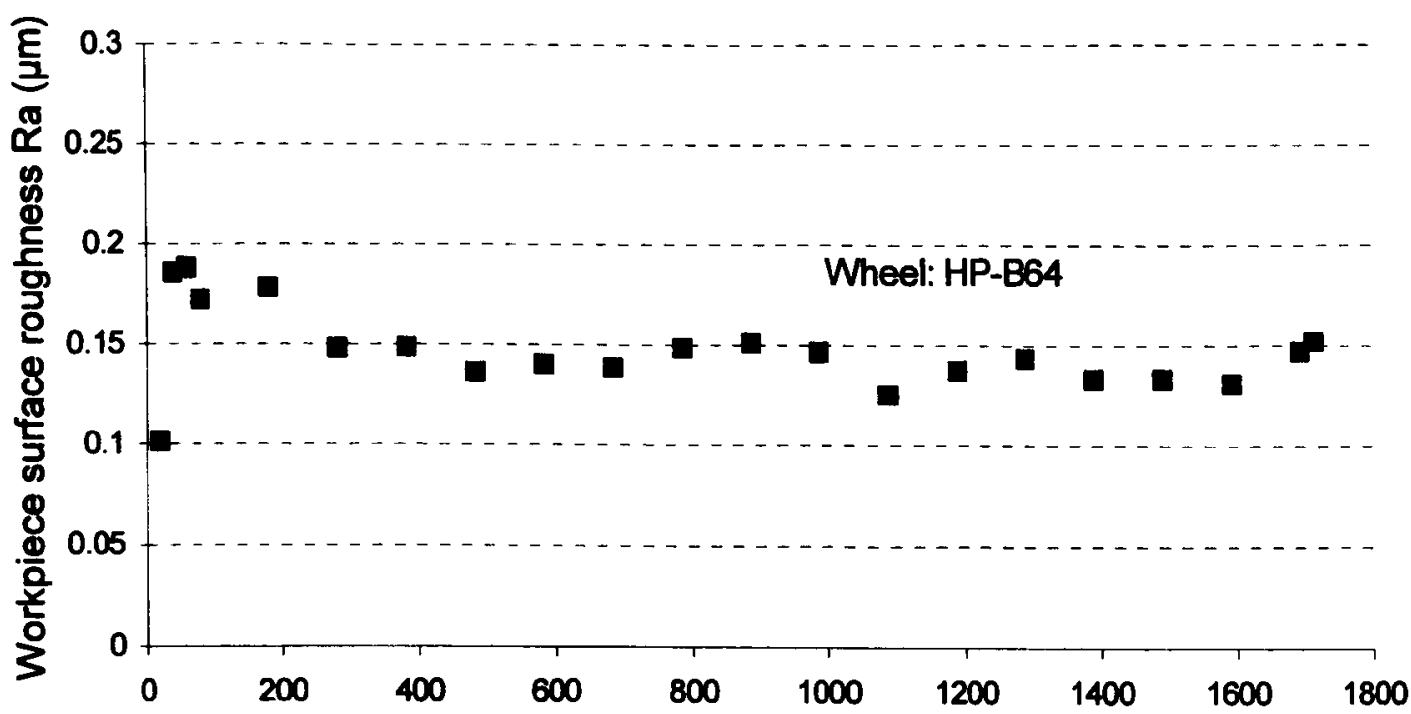

Specific volume of material removed $\left(\mathrm{mm}^{3} / \mathrm{mm}\right)$

Fig. 6.15 (e) Workpiece Ra surface roughness when grinding M2 at a wheel speed of $110 \mathrm{~m} / \mathrm{s}$

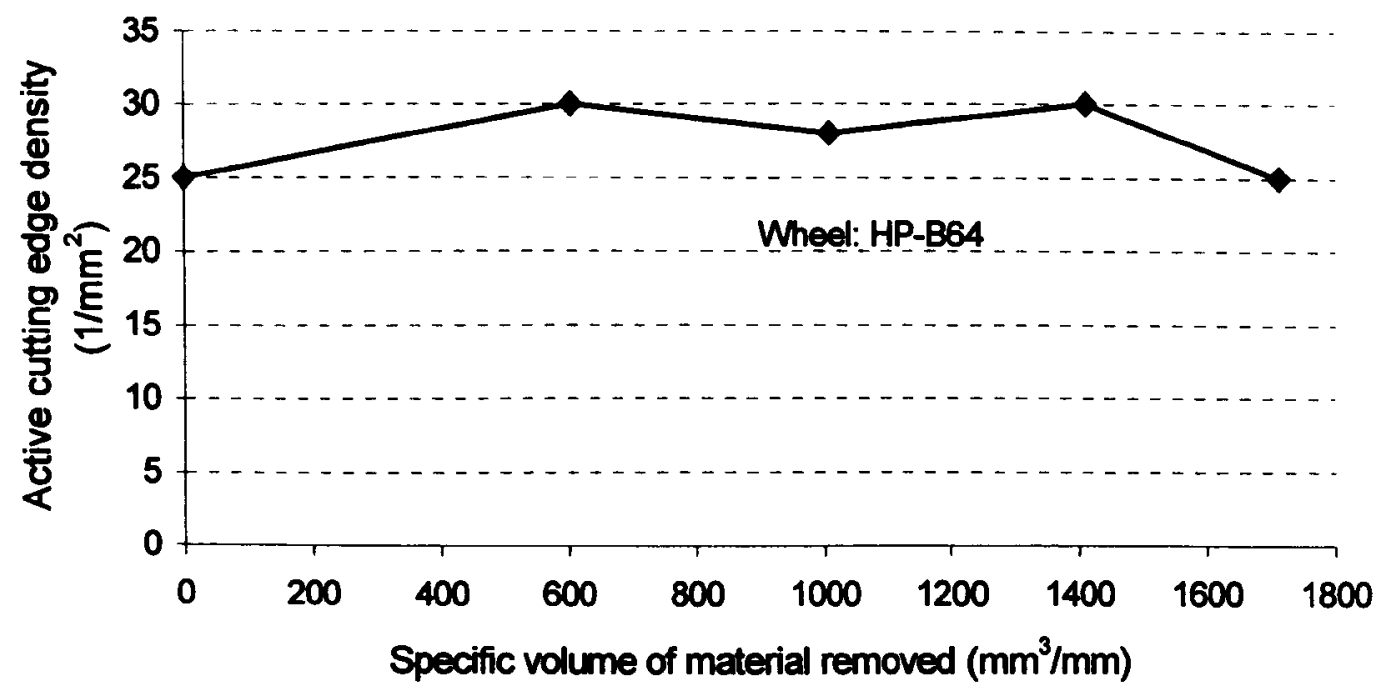

Fig. 6.15 (f) Active cutting edge density when grinding M2 at a wheel speed of $110 \mathrm{~m} / \mathrm{s}$ 


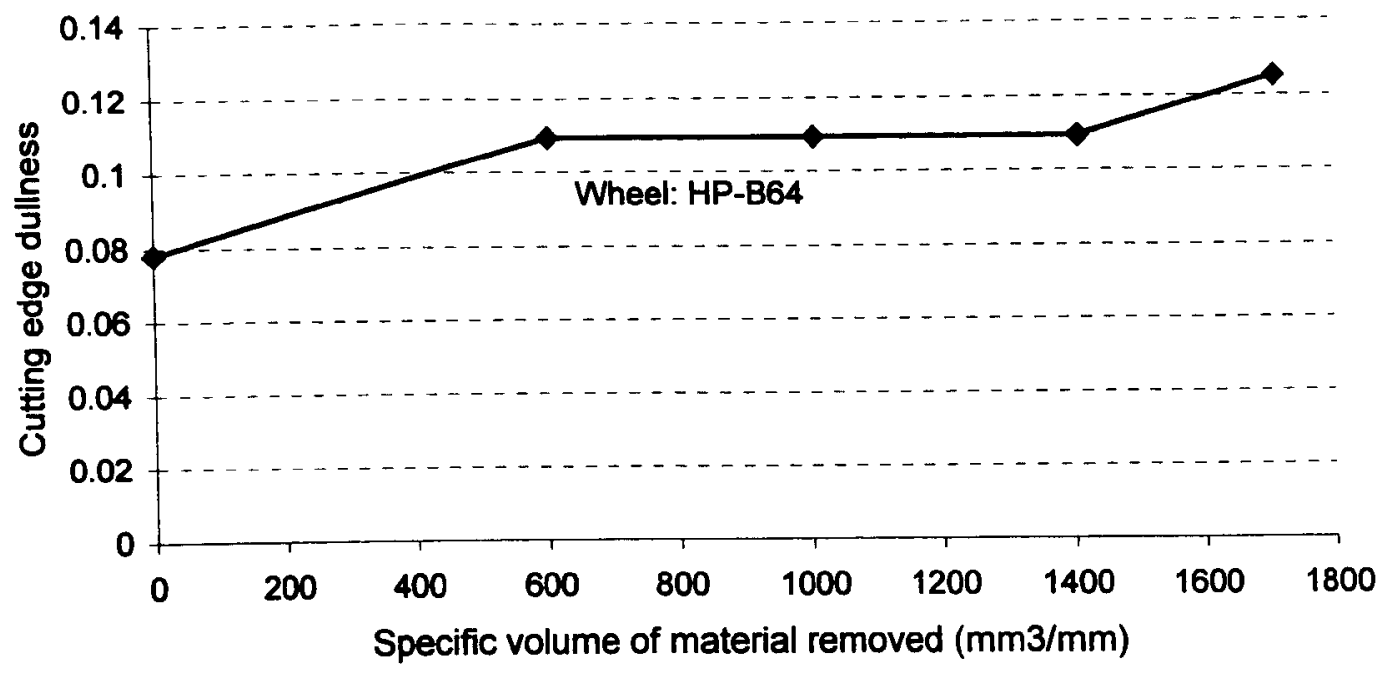

Fig. 6.15 (g) Cutting edge dullness when grinding M2 at a wheel speed of 110 $\mathrm{m} / \mathrm{s}$ 


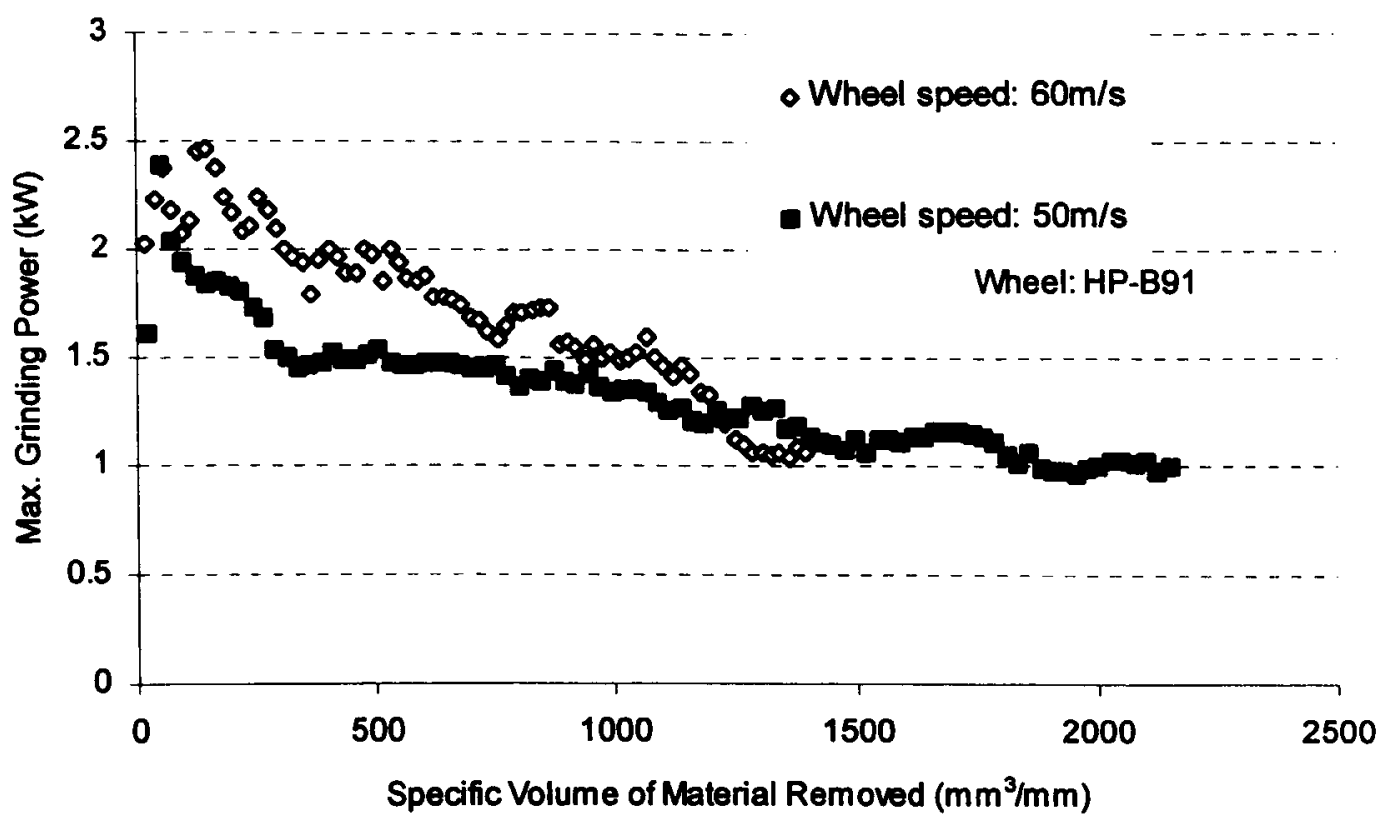

Fig. 6.16 (a) Maximum grinding power when grinding Inconel 718 at a wheel speed of $50-60 \mathrm{~m} / \mathrm{s}$

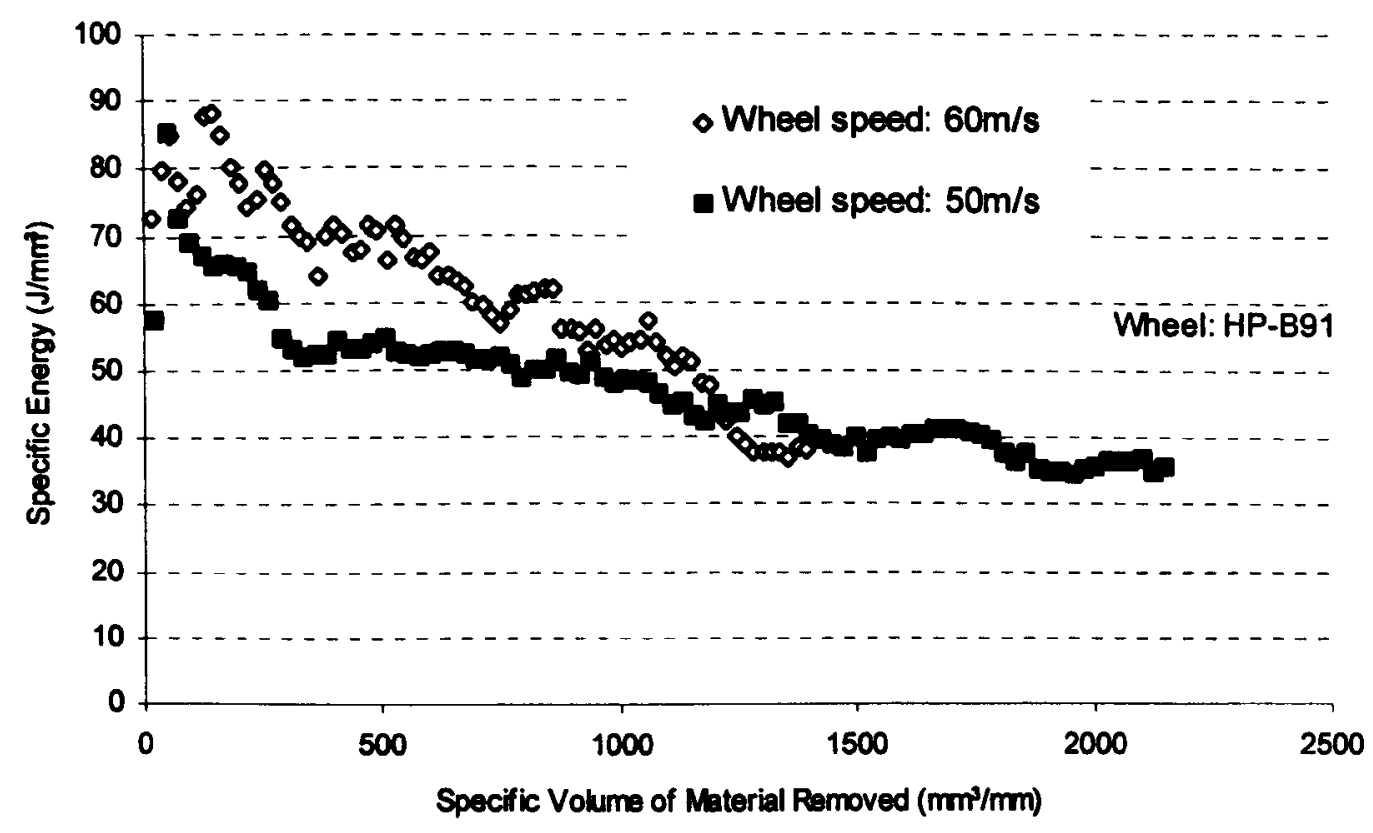

Fig. 6.16 (b) Specific energy when grinding Inconel 718 at a wheel speed of 50$60 \mathrm{~m} / \mathrm{s}$ 


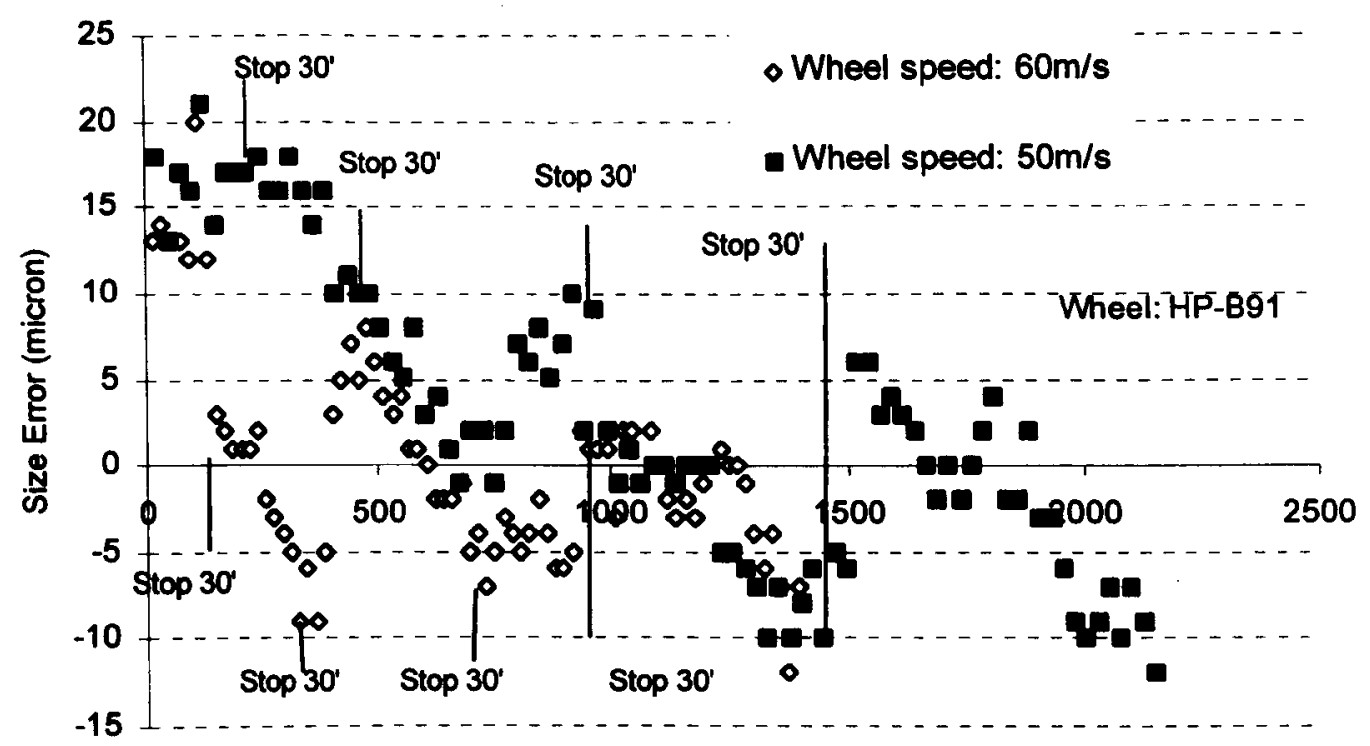

Specific Volume of Material Removed $\left(\mathrm{mm}^{3} / \mathrm{mm}\right)$

Fig. 6.16 (c) Workpiece size error when grinding Inconel 718 at a wheel speed of $50-60 \mathrm{~m} / \mathrm{s}$

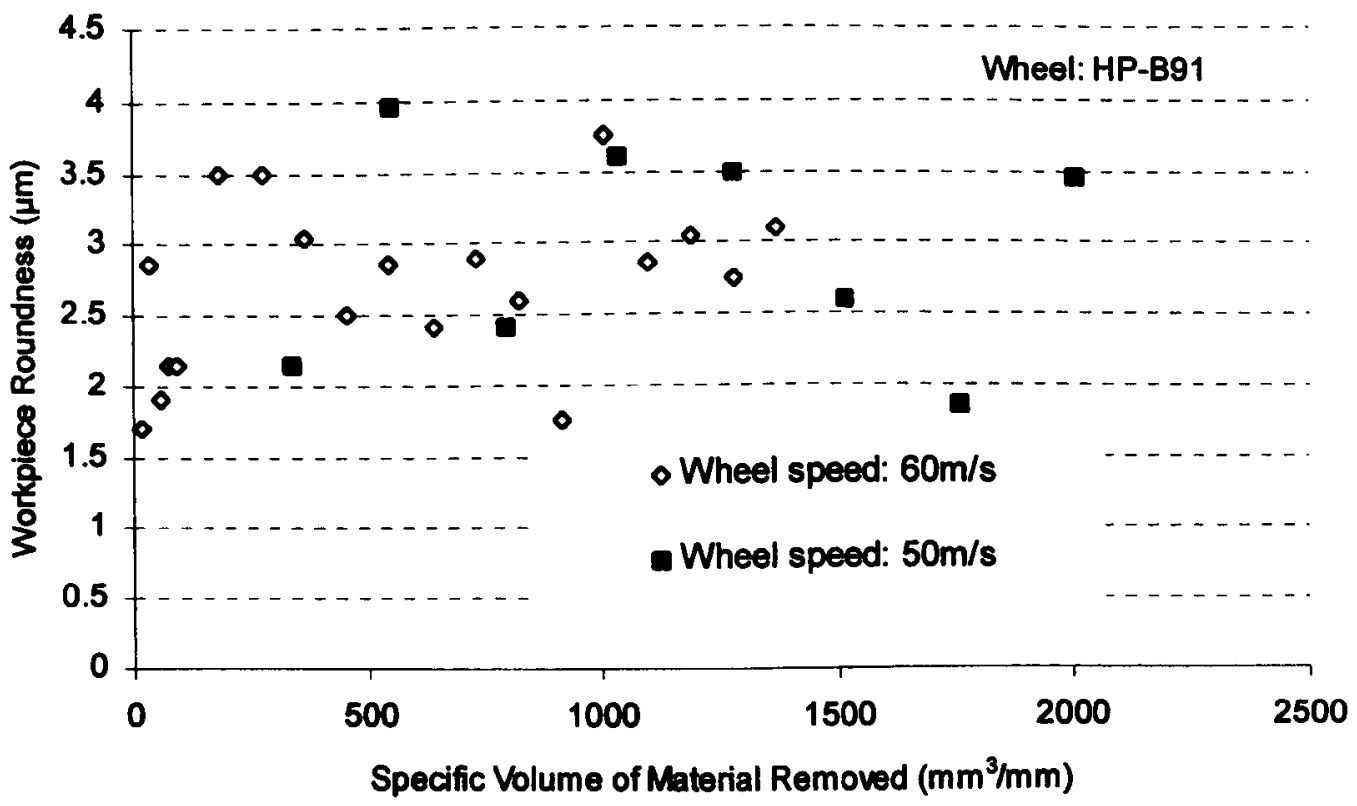

Fig. 6.16 (d) Workpiece roundness when grinding Inconel 718 at a wheel speed of $50-60 \mathrm{~m} / \mathrm{s}$ 


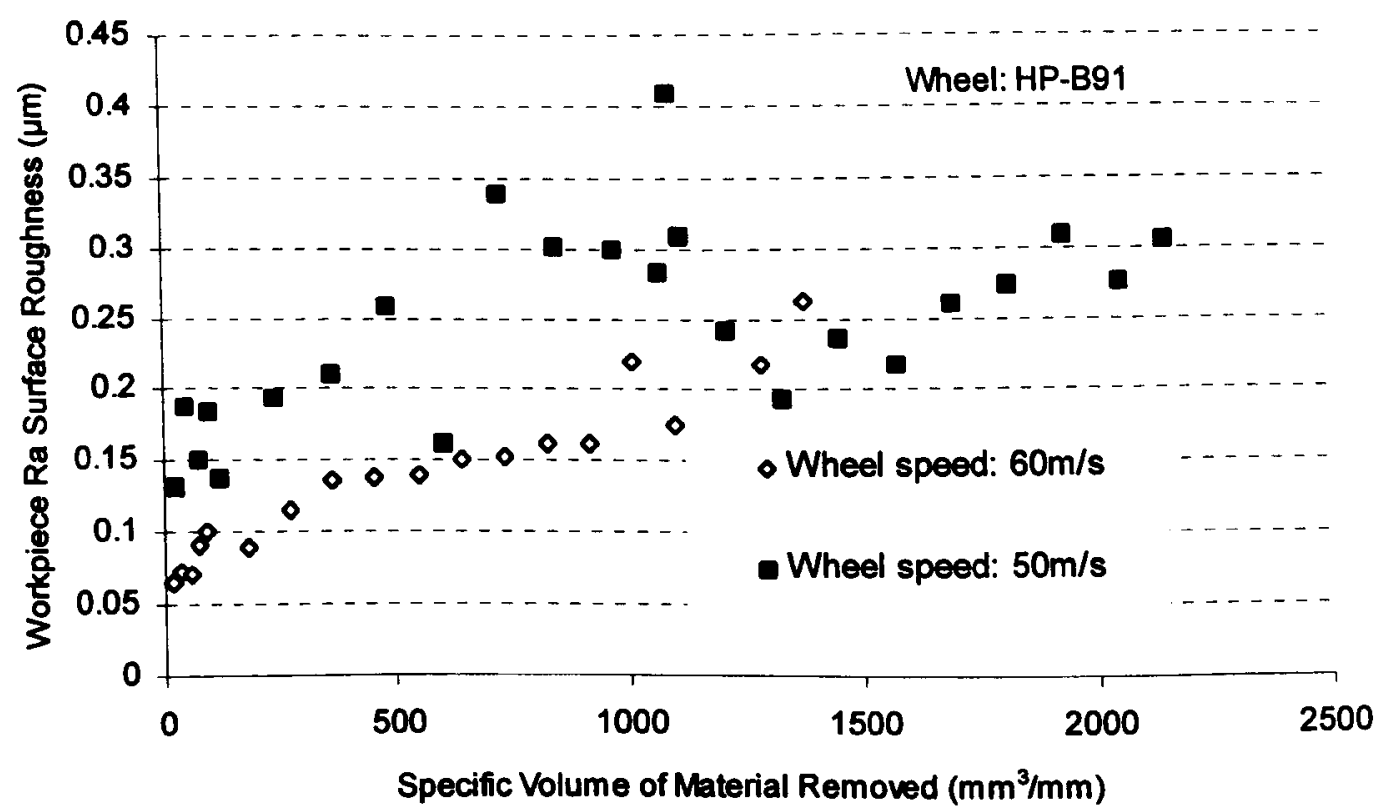

Fig. 6.16 (e) Workpiece Ra surface roughness when grinding Inconel 718 at a wheel speed of $50-60 \mathrm{~m} / \mathrm{s}$

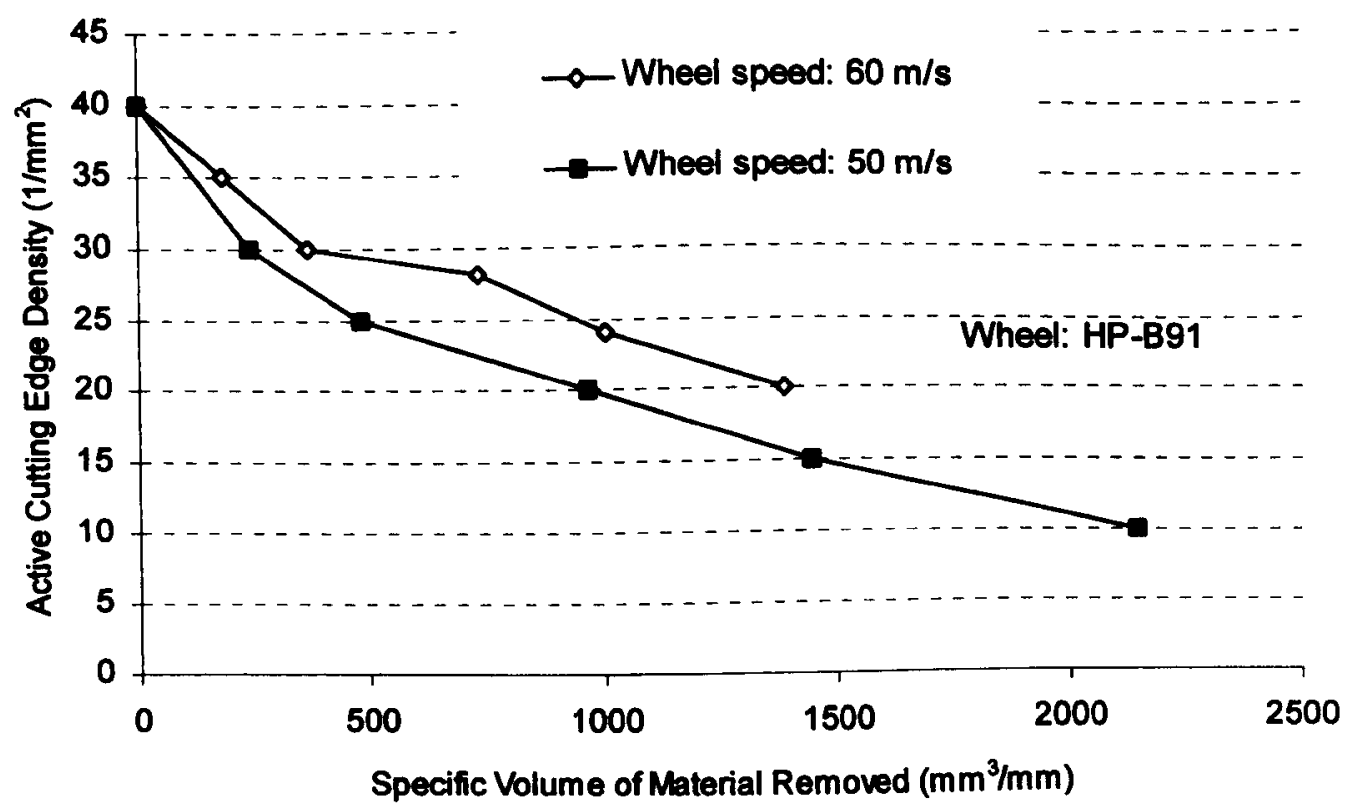

Fig. 6.16 (f) Active cutting edge density when grinding Inconel 718 at a wheel speed of $50-60 \mathrm{~m} / \mathrm{s}$ 


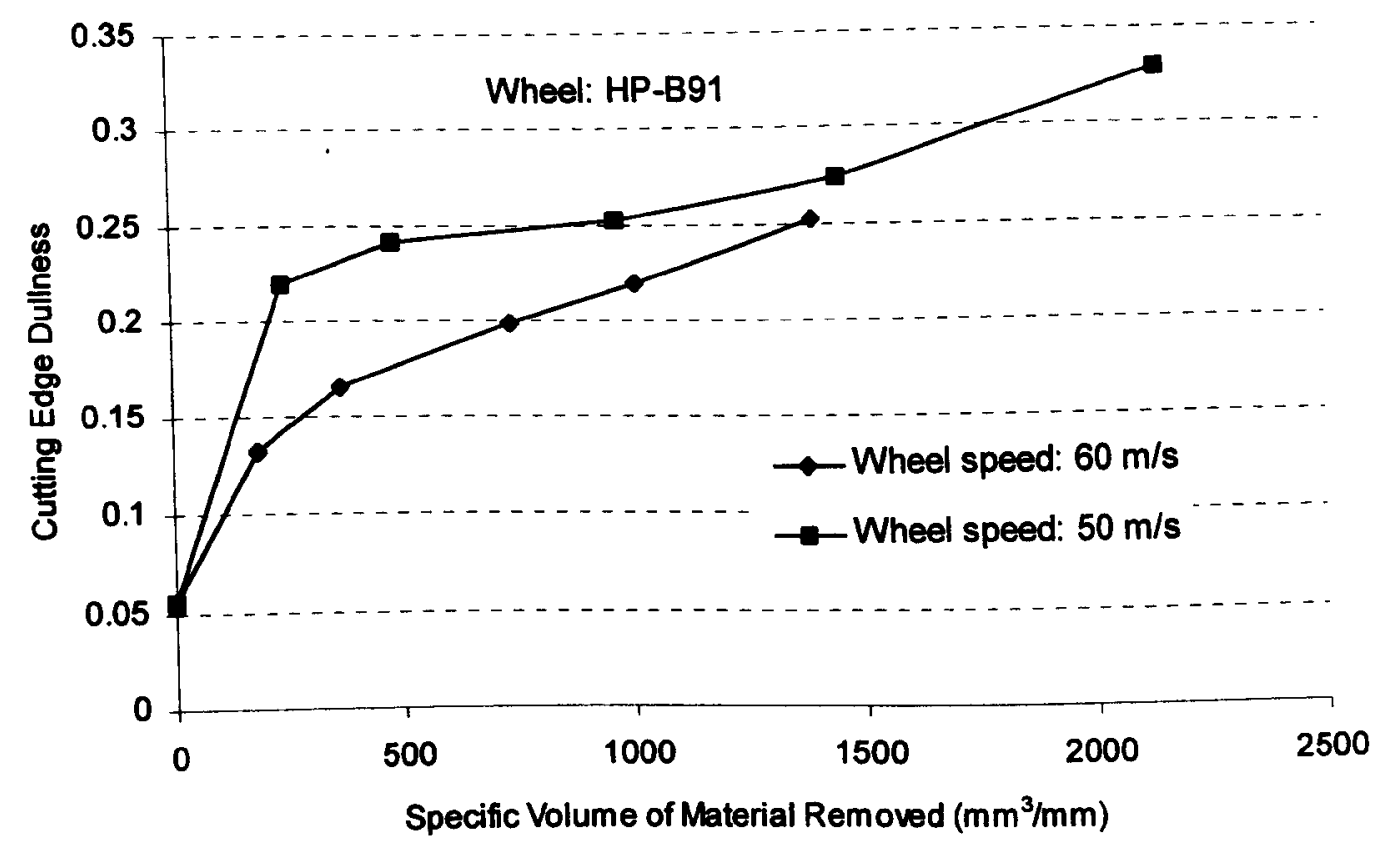

Fig. $6.16(\mathrm{~g})$ Cutting edge dullness when grinding Inconel 718 at a wheel speed of $50-60 \mathrm{~m} / \mathrm{s}$

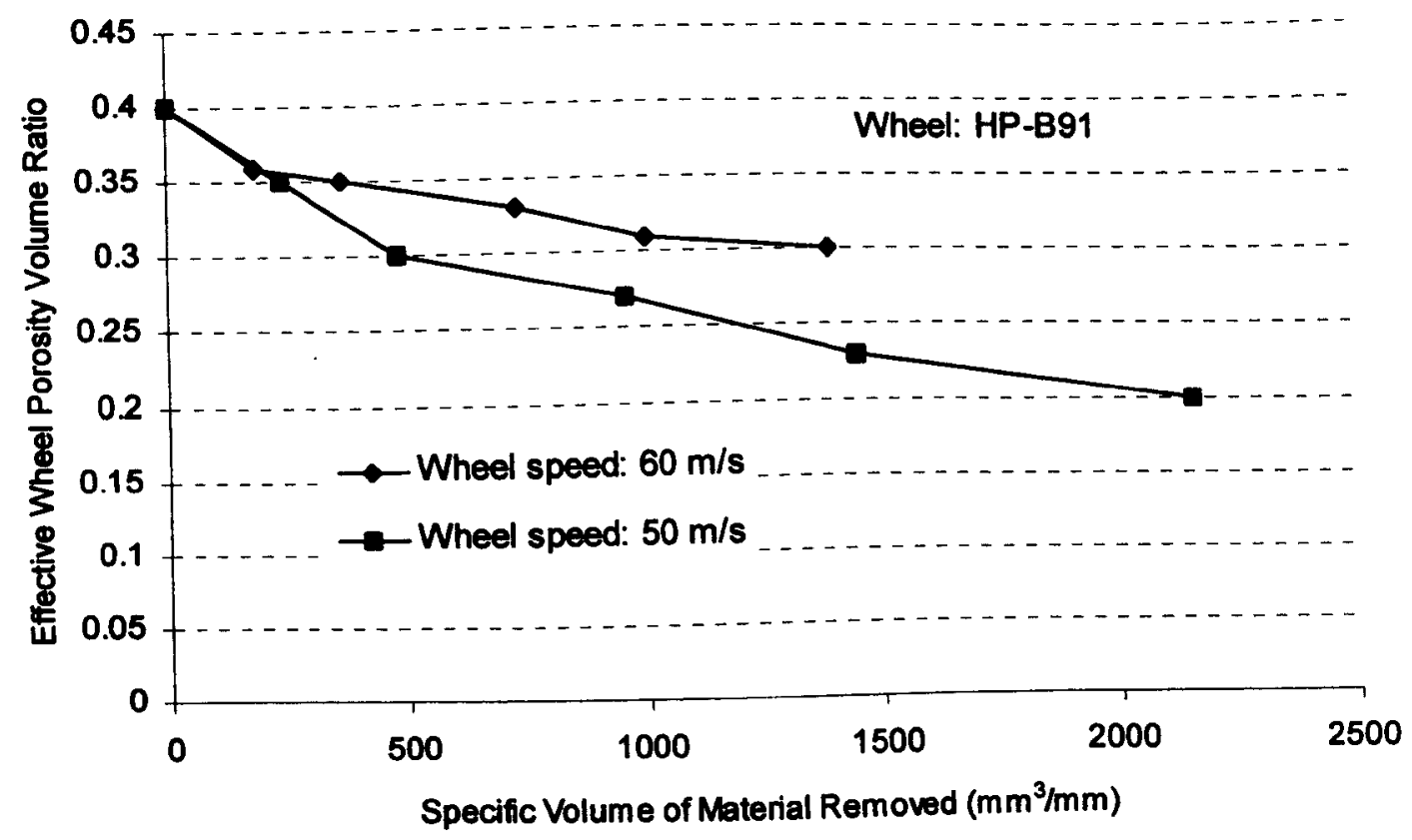

Fig. 6.16 (h) Effective wheel porosity volume ratio when grinding Inconel 718 at a wheel speed of $50-60 \mathrm{~m} / \mathrm{s}$ 
Grinding wheel: Medium-porosity CBN B91-150 VR

Equivalent diameter: $34 \mathrm{~mm}$

Coolant type: Hysol-X ( $10 \%$ emulsion)

Grinding machine: J\&S Suprema

Q' $10 \mathrm{~mm}^{3} / \mathrm{mm} . \mathrm{s} \quad\left(\mathrm{v}_{\mathrm{f}}=0.081 \mathrm{~mm} / \mathrm{s}\right)$

Coolant delivery: 36 litres/min ( 30 bar pump pressure)

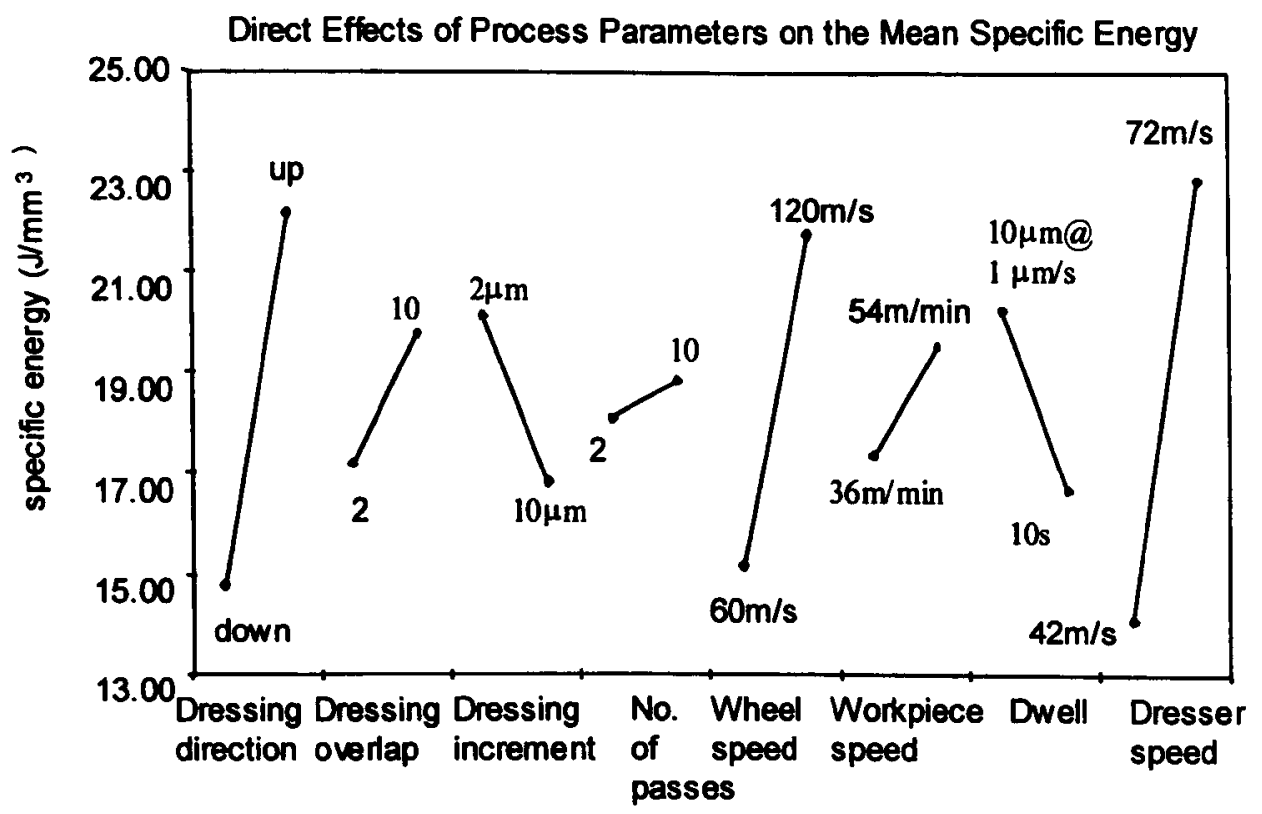

Fig. 6.17 (a) The direct effect of process parameters on the mean specific energy using a CBN wheel to grind AISI 52100

Direct Effects of Process Parameters on the Mean Surface Roughness

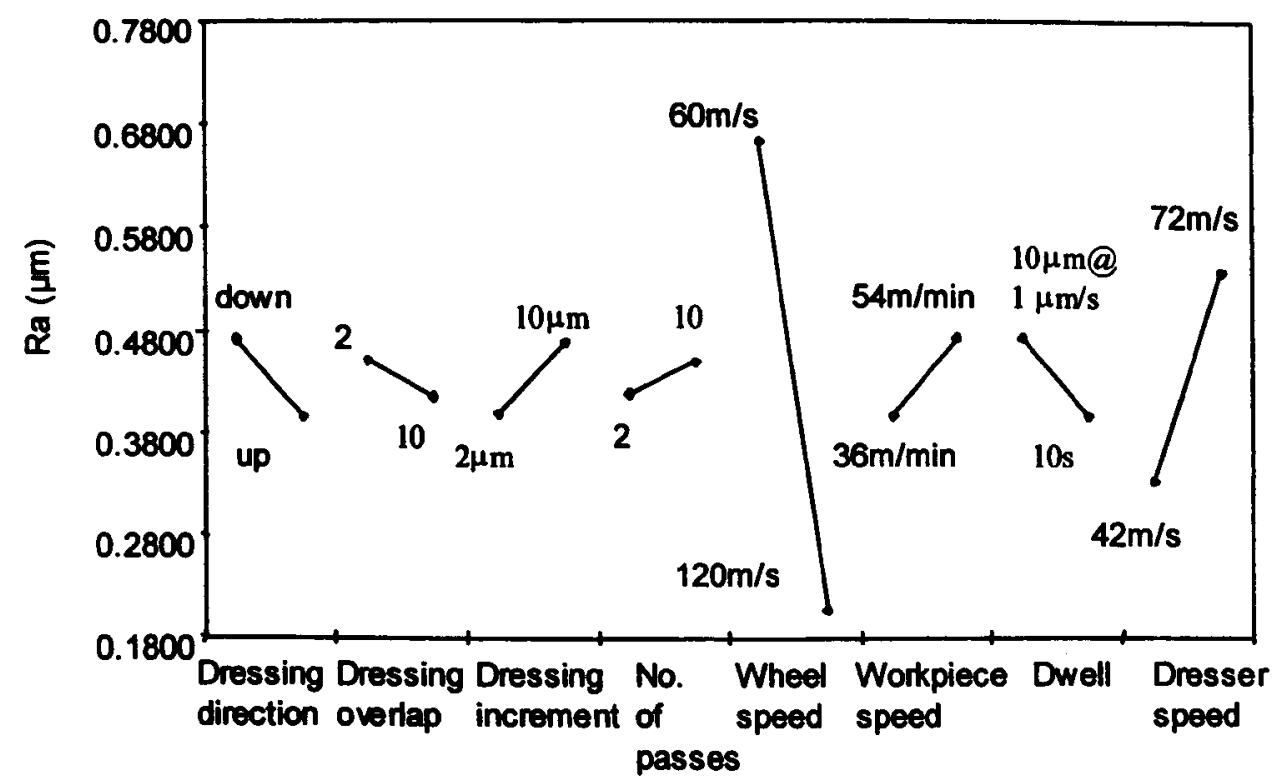

Fig. 6.17 (b) The direct effect of process parameters on the mean surface roughness using a CBN wheel to grind AISI 52100 
Direct Effect of Process Parameters on the Mean Roundness

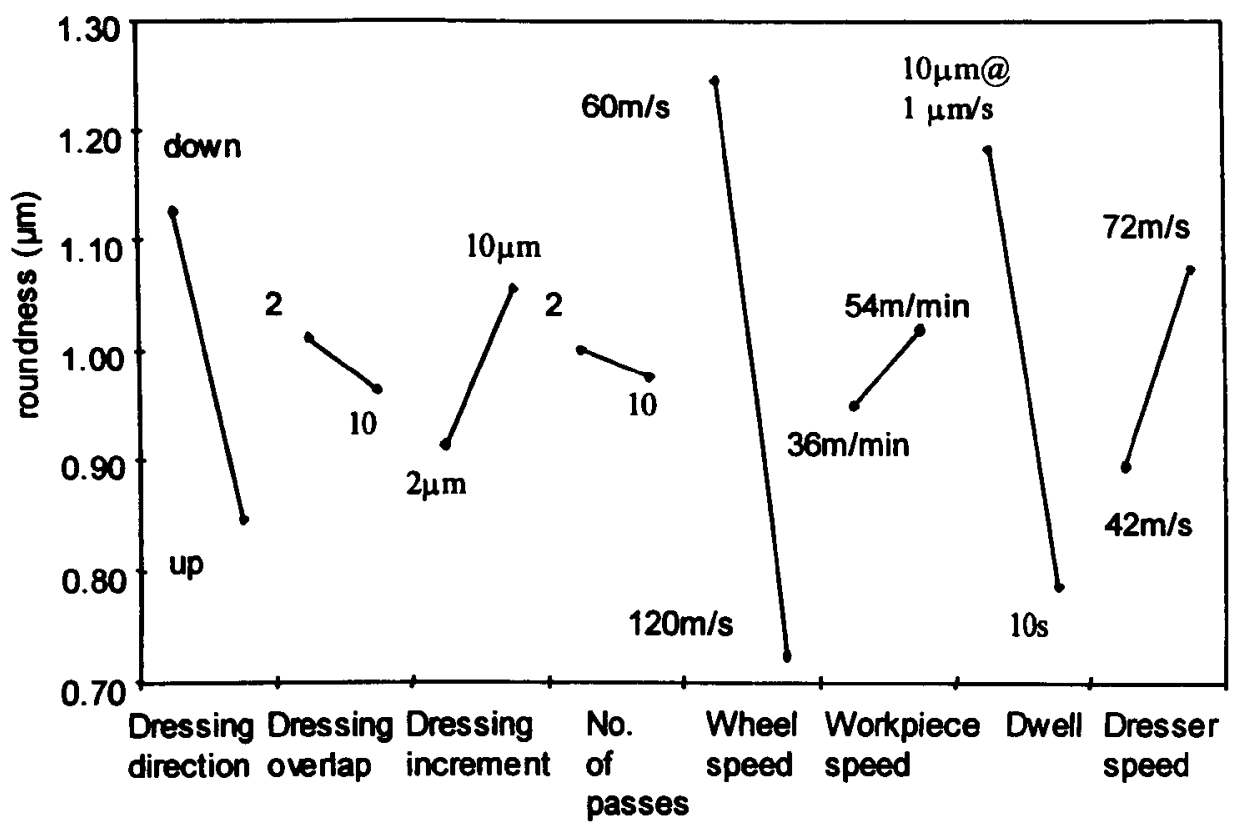

Fig. 6.17 (c) The direct effect of process parameters on the mean roundness using a CBN wheel to grind AISI 52100

Direct Effects of Process Parameters on the Mean Size Holding

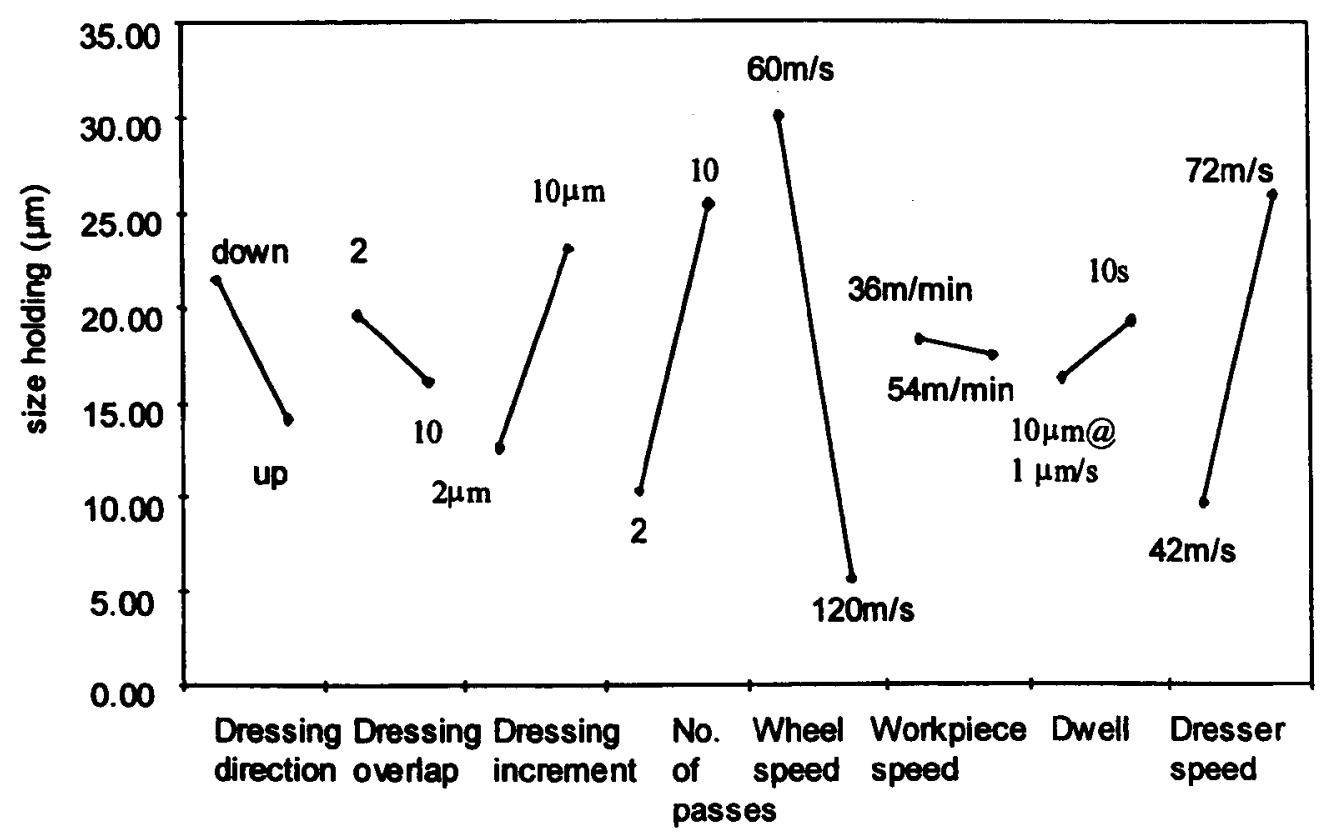

Fig. 6.17 (d) The direct effect of process parameters on the mean size holding using a CBN wheel to grind AISI 52100 
Direct Effects of Process Parameters on G-Ratio

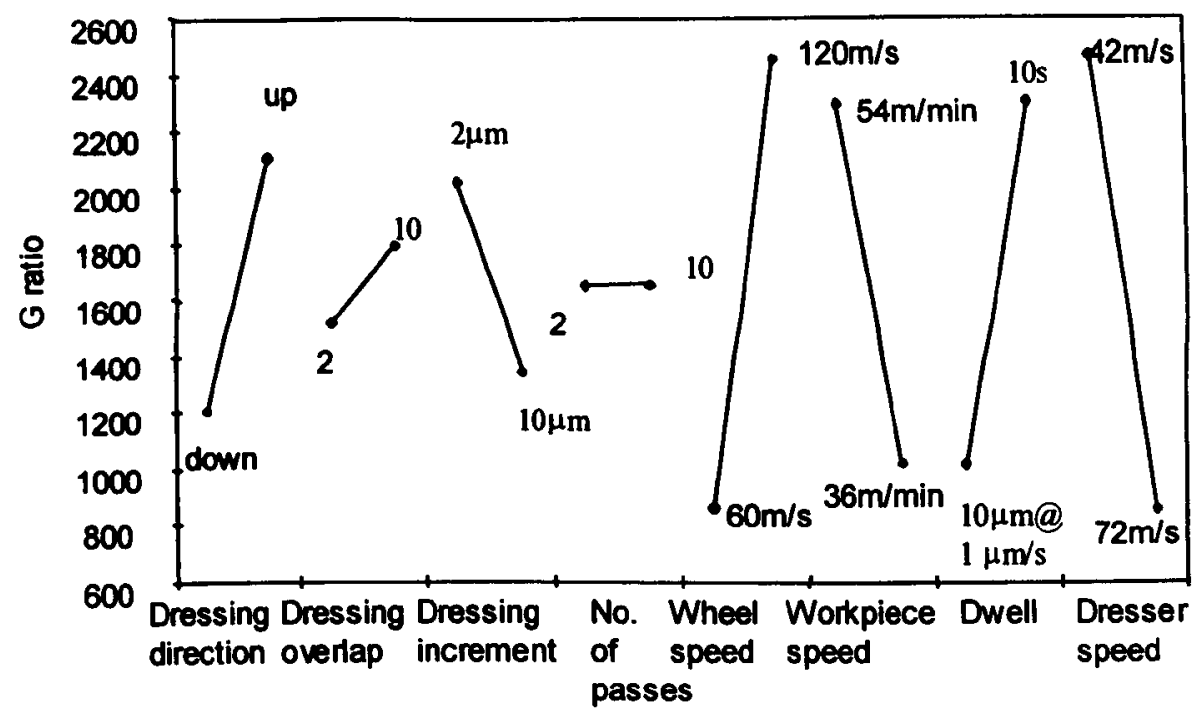

Fig. 6.17 (e) The direct effect of process parameters on G-ratio using a CBN wheel to grind AISI 52100 
Grinding wheel: Medium-porosity CBN B91-150 VR

Dresser rotational direction: Up

Dressing overlap: 10

Dressing increment: $2 \mu \mathrm{m}$

Dresser speed: $42 \mathrm{~m} / \mathrm{s}$

No. of dressing passes: 2

Wheel speed: $120 \mathrm{~m} / \mathrm{s}$

Workpiece speed: $36 \mathrm{~m} / \mathrm{min}$

Dwell: 10s

Q' ${ }_{\mathrm{w}}: 10 \mathrm{~mm}^{3} / \mathrm{mm} \mathrm{s}^{3}$

$\mathrm{V}_{\mathrm{w}}: 76,140 \mathrm{~mm}^{3}$

Coolant type: Hysol-X ( $10 \%$ emulsion)

Coolant delivery: 361/min @ 30bar pump pressure

Equivalent diameter: $34 \mathrm{~mm}$

Specific energy of consecutive diameters

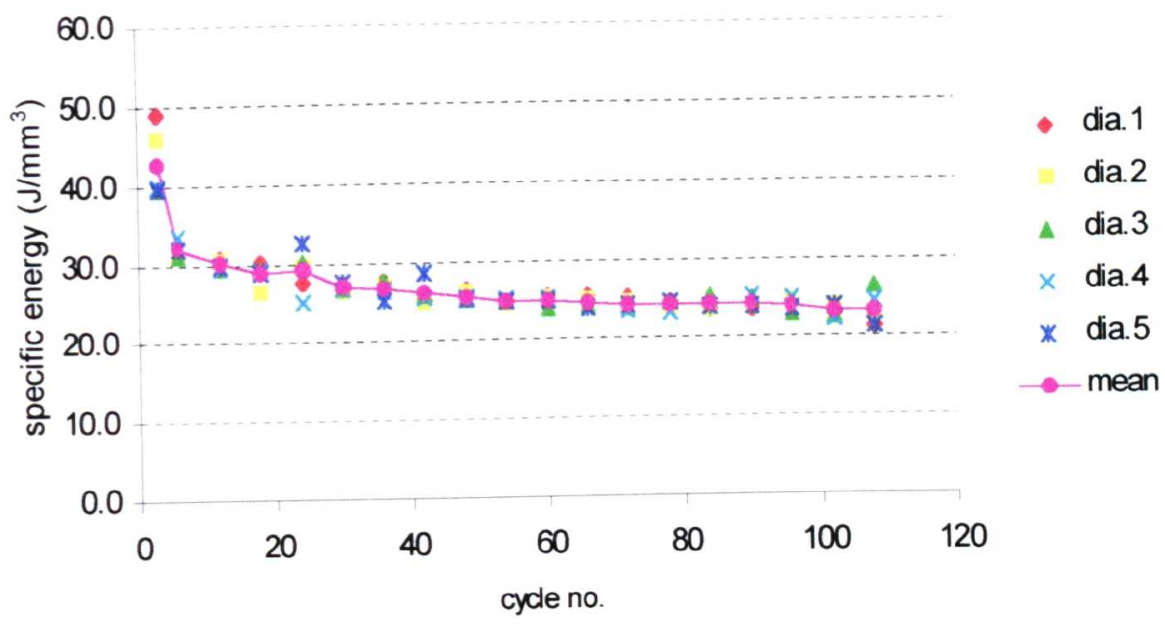

Fig. 6.18(a) Specific energy of Confirmation Trial 1 for improved workpiece quality grinding AISI 52100 on the J\&S Suprema (The G-Ratio for this trial was 5700)

$\mathrm{Ra}$ of consective diameters

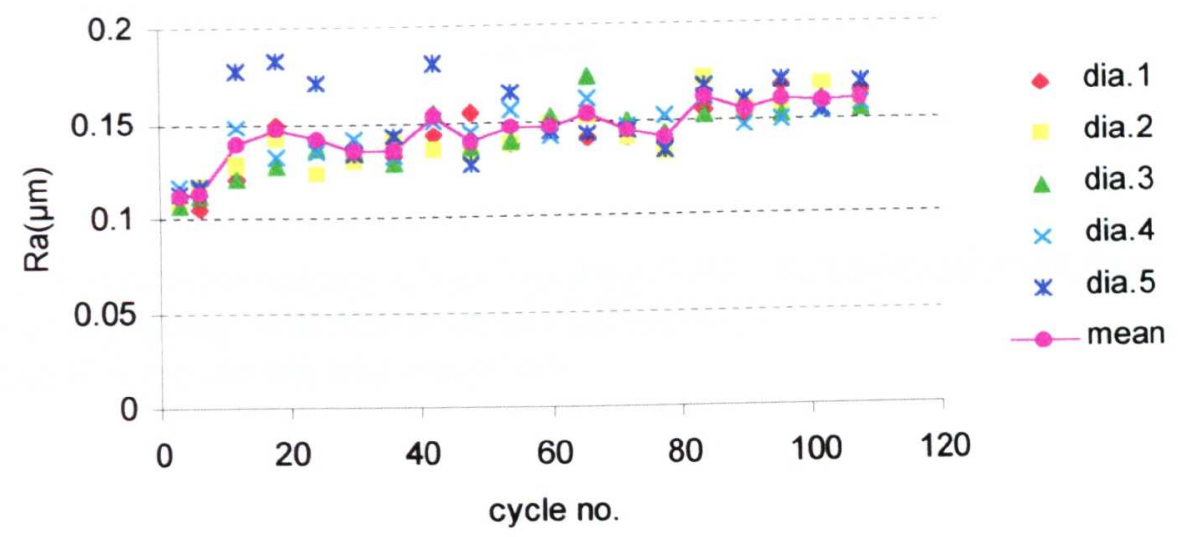

Fig. 6.18(b) Surface roughness of Confirmation Trial 1 for improved workpiece quality grinding AISI 52100 on the J\&S Suprema (The G-Ratio for this trial was 5700) 


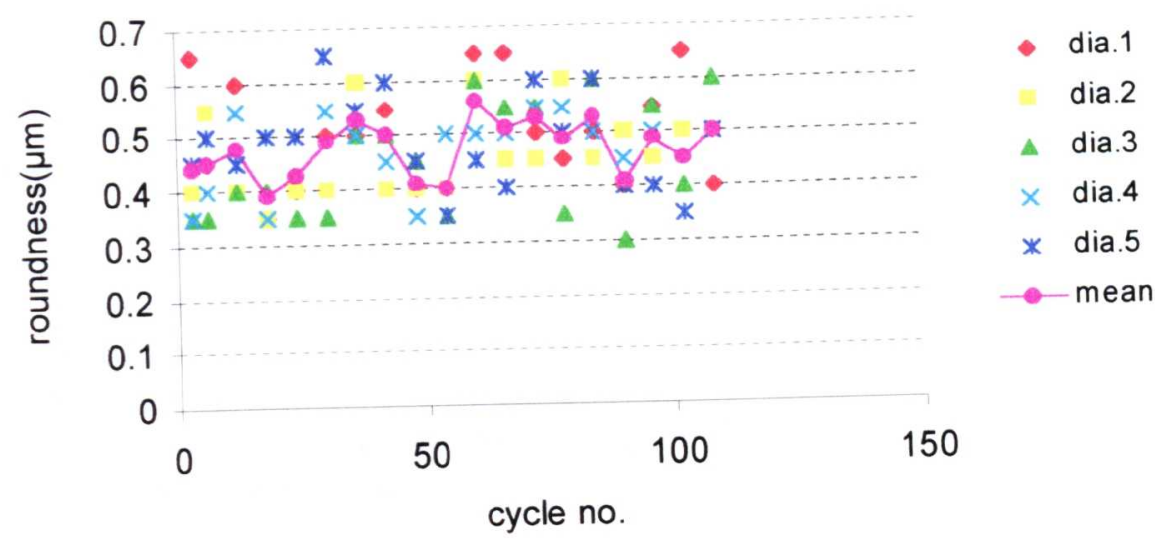

Fig. 6.18(c) Roundness of Confirmation Trial 1 for improved workpiece quality grinding AISI 52100 on the J\&S Suprema (The G-Ratio for this trial was 5700)

size holding of consecutive diameters

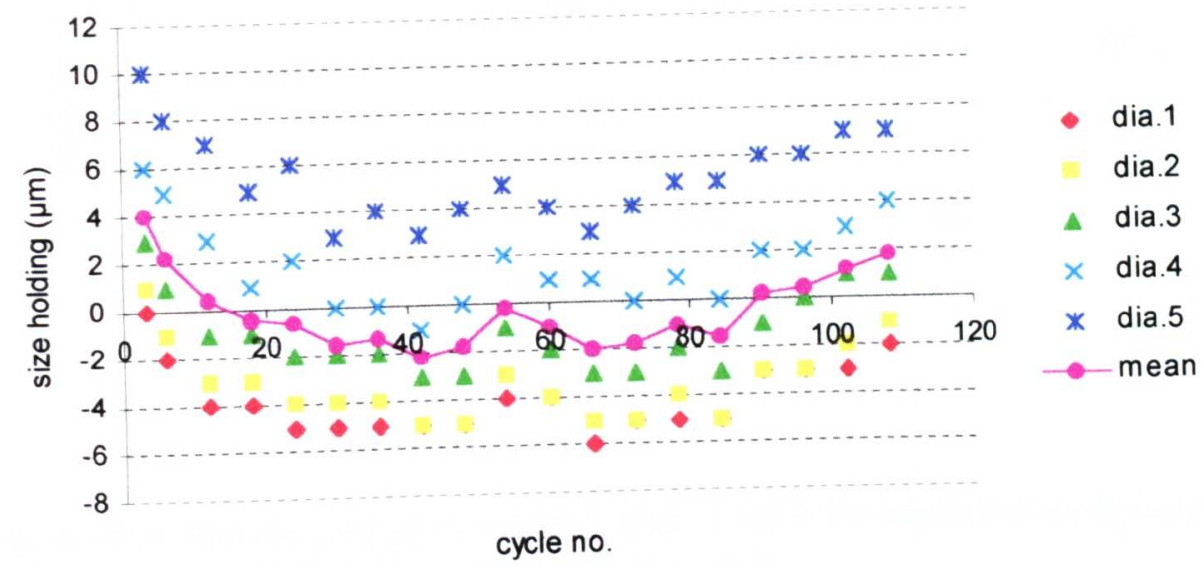

Fig. 6.18(d) Size holding of Confirmation Trial 1 for improved workpiece quality grinding AISI 52100 on the J\&S Suprema (The G-Ratio for this trial was 5700) 
Grinding wheel: Medium-porosity CBN B91-150 VR

Dresser rotational direction: Down

Dressing overlap: 2

Dressing increment: $10 \mu \mathrm{m}$

Dresser speed: $42 \mathrm{~m} / \mathrm{s}$

No. of dressing passes: 2

Wheel speed: $120 \mathrm{~m} / \mathrm{s}$

Workpiece speed: $36 \mathrm{~m} / \mathrm{min}$

Dwell: $2 \mathrm{~s}$

Q' ${ }_{w}: 20 \mathrm{~mm}^{3} / \mathrm{mm} \mathrm{s}^{3}$

$\mathrm{V}_{\mathrm{w}}: 76,140 \mathrm{~mm}^{3}$

Coolant type: Hysol-X ( $10 \%$ emulsion)

Coolant delivery: 361/min @ 30bar pump pressure

Equivalent diameter: $34 \mathrm{~mm}$

Specific energy of consecutive diameters

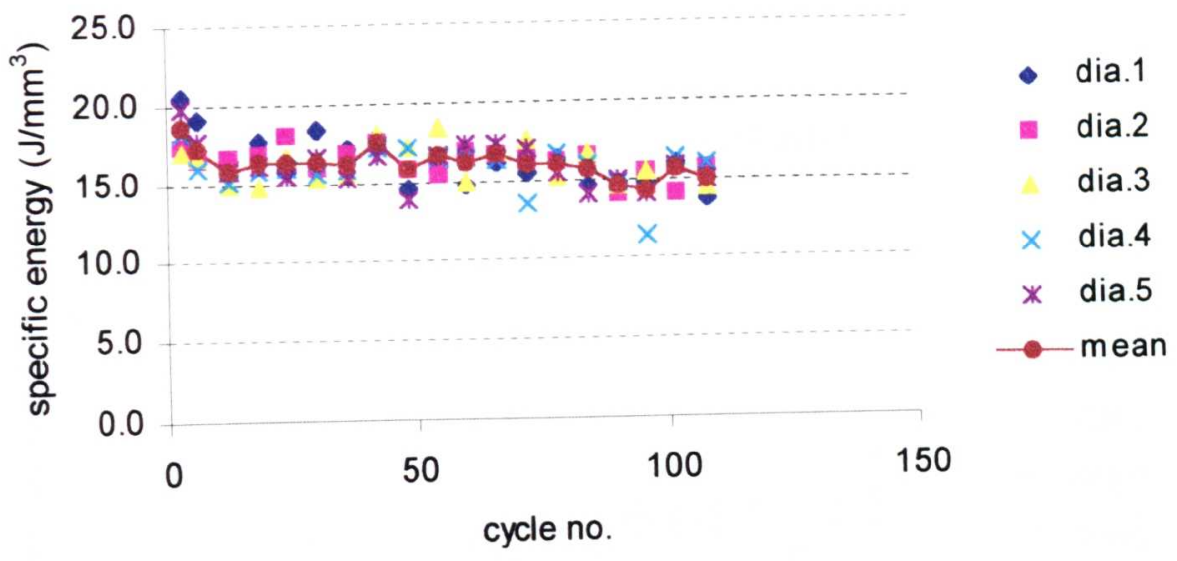

Fig. 6.19(a) Specific energy of Confirmation Trial 2 for improved workpiece quality grinding AISI 52100 on the J\&S Suprema (The G-Ratio for this trial was 720) 
$\mathrm{Ra}$ of consecutive diameters

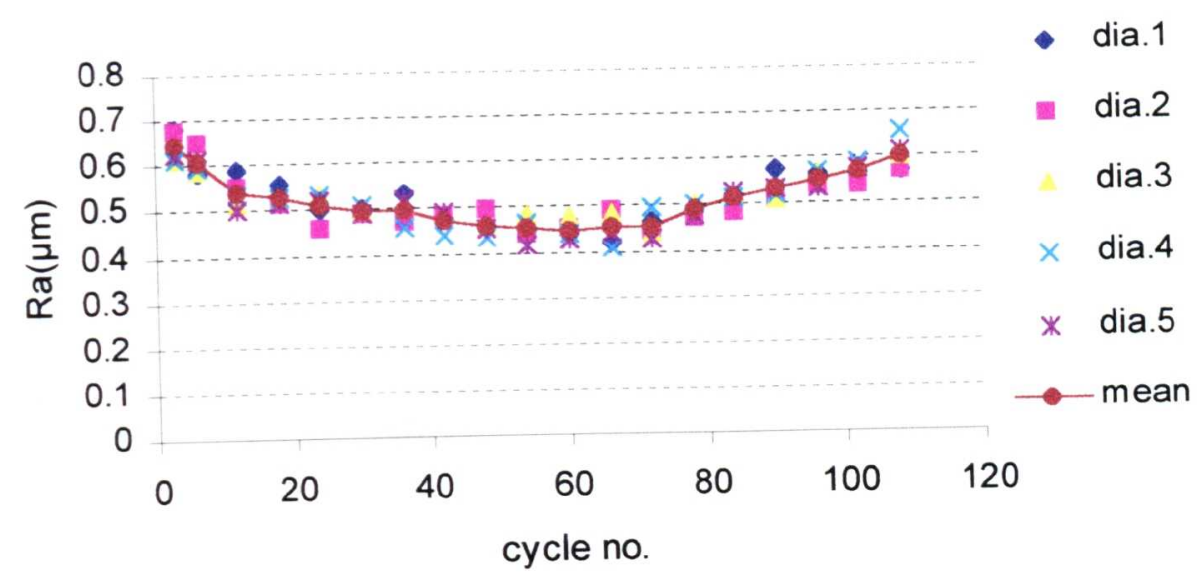

Fig. 6.19(b) Surface roughness of Confirmation Trial 2 for improved workpiece quality grinding AISI 52100 on the J\&S Suprema (The G-Ratio for this trial was 720)

Roundness of consecutive diameters

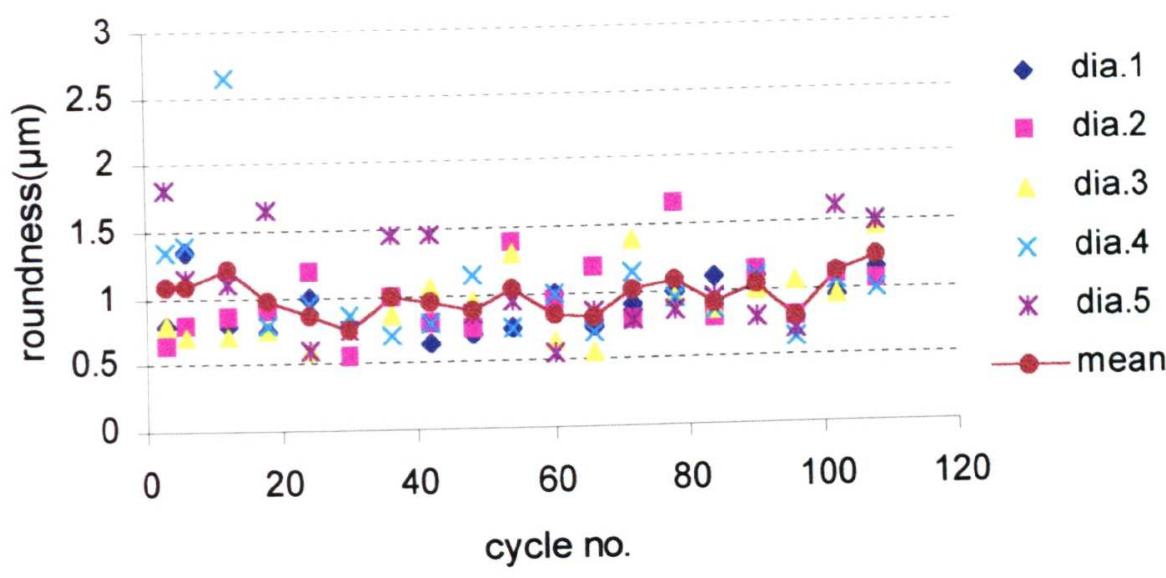

Fig. 6.19(c) Roundness of Confirmation Trial 2 for improved workpiece quality grinding AISI 52100 on the J\&S Suprema (The G-Ratio for this trial was 720) 
Size holding of consecutive diameters

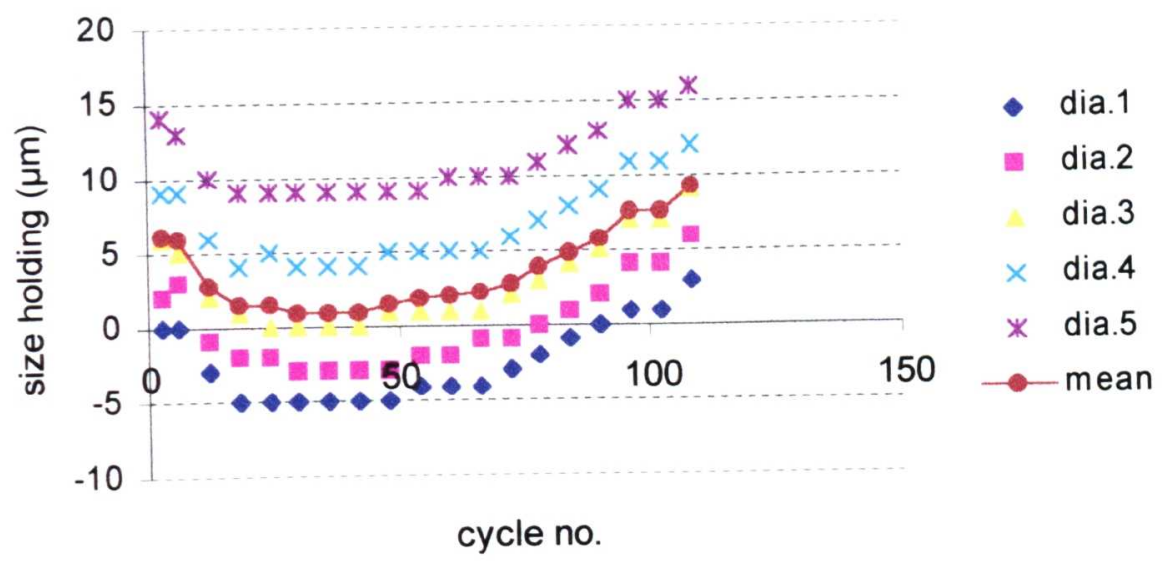

Fig. 6.19(d) Size holding of Confirmation Trial 2 for improved workpiece quality grinding AISI 52100 on the J\&S Suprema

(The G-Ratio for this trial was 720) 
Grinding wheel: High-porosity CBN B151-150 VR

Grinding Machine: J\&S Suprema

Coolant type: Hysol X (10\% emulsion)

Coolant delivery: 361/min @30bar pump pressure

Equivalent diameter: $36.6 \mathrm{~mm}$

$Q_{\text {w }}^{\prime}: 2 \mathrm{~mm}^{3} / \mathrm{mm} . \mathrm{s}$

Direct Effects of Process Parameters on the Mean Specific Energy

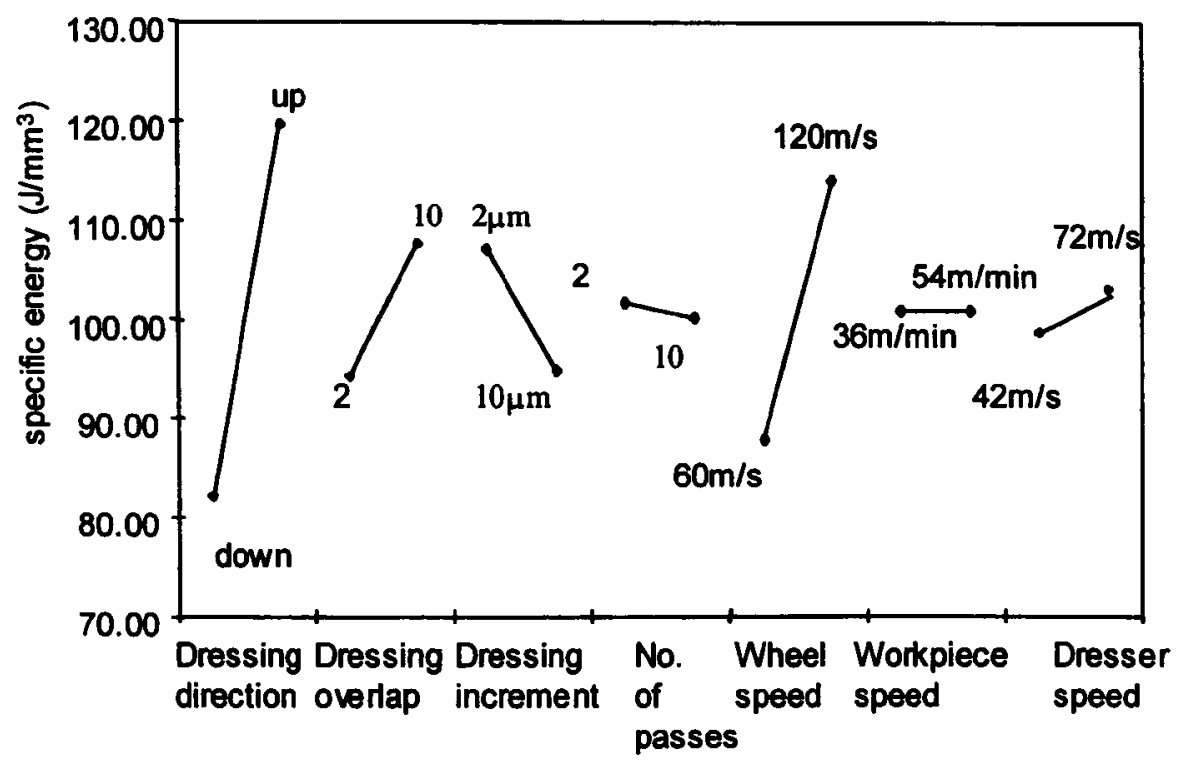

Fig. 6.20 (a) Direct effects charts of process parameters on the mean specific energy for Inconel 718 on Suprema 


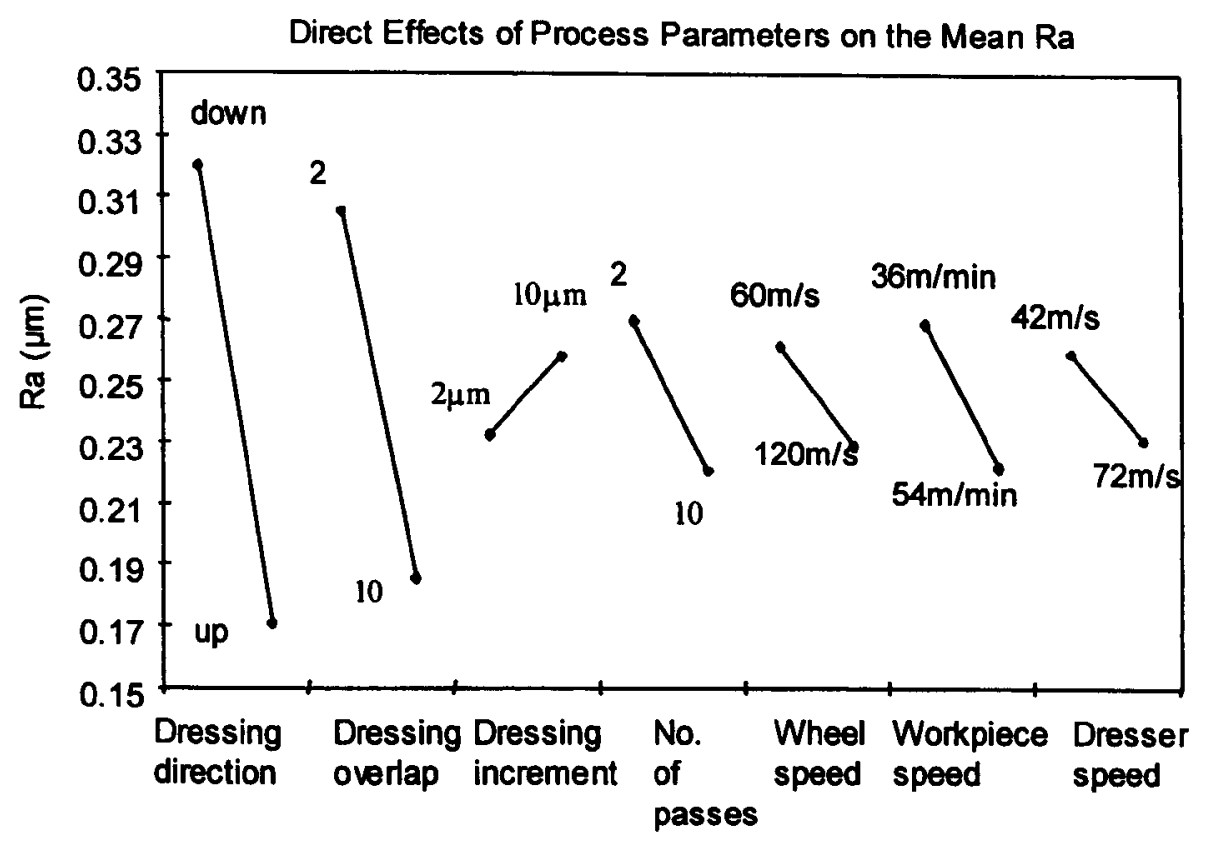

Fig. 6.20 (b) Direct effects charts of process parameters on the mean surface roughness for Inconel 718 on Suprema

Direct Effects of Process Parameters on the Mean Roundness

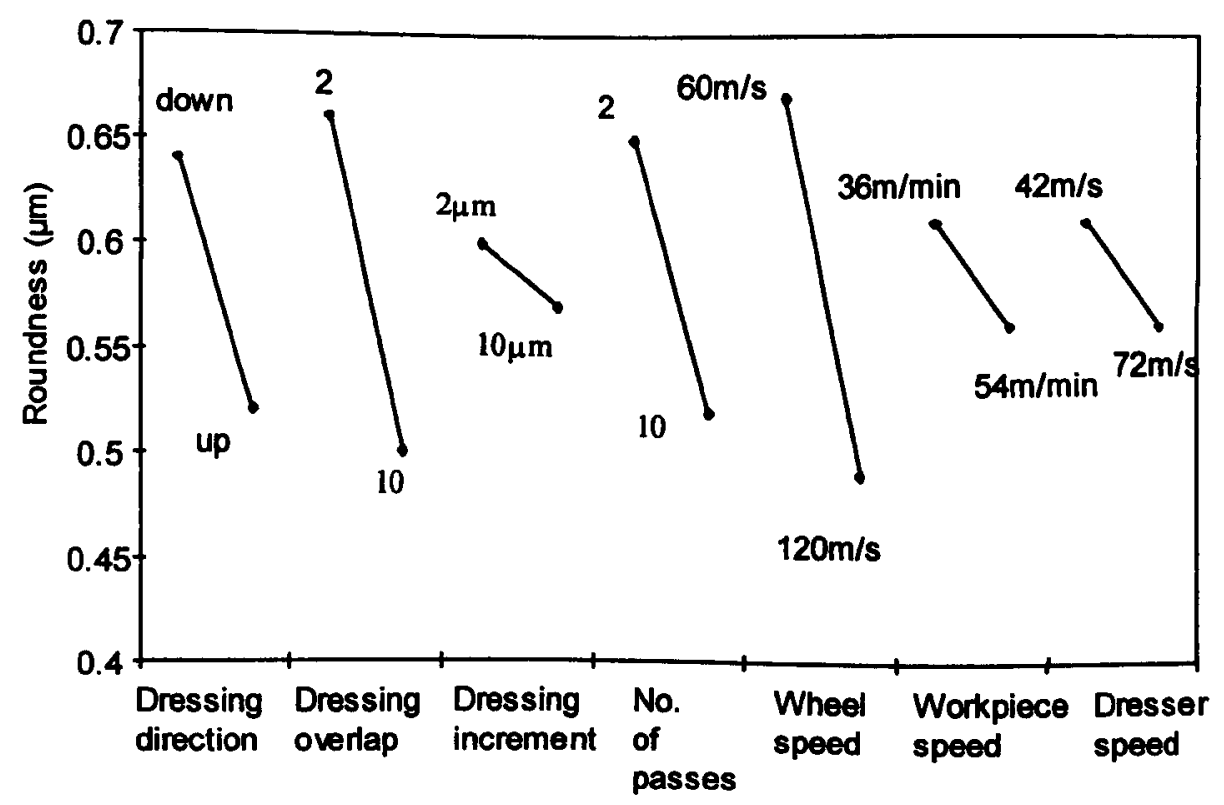

Fig. 6.20 (c) Direct effects charts of process parameters on the mean roundness for Inconel 718 on Suprema 
Direct Effects of Process Parameters on the Mean Size Holding

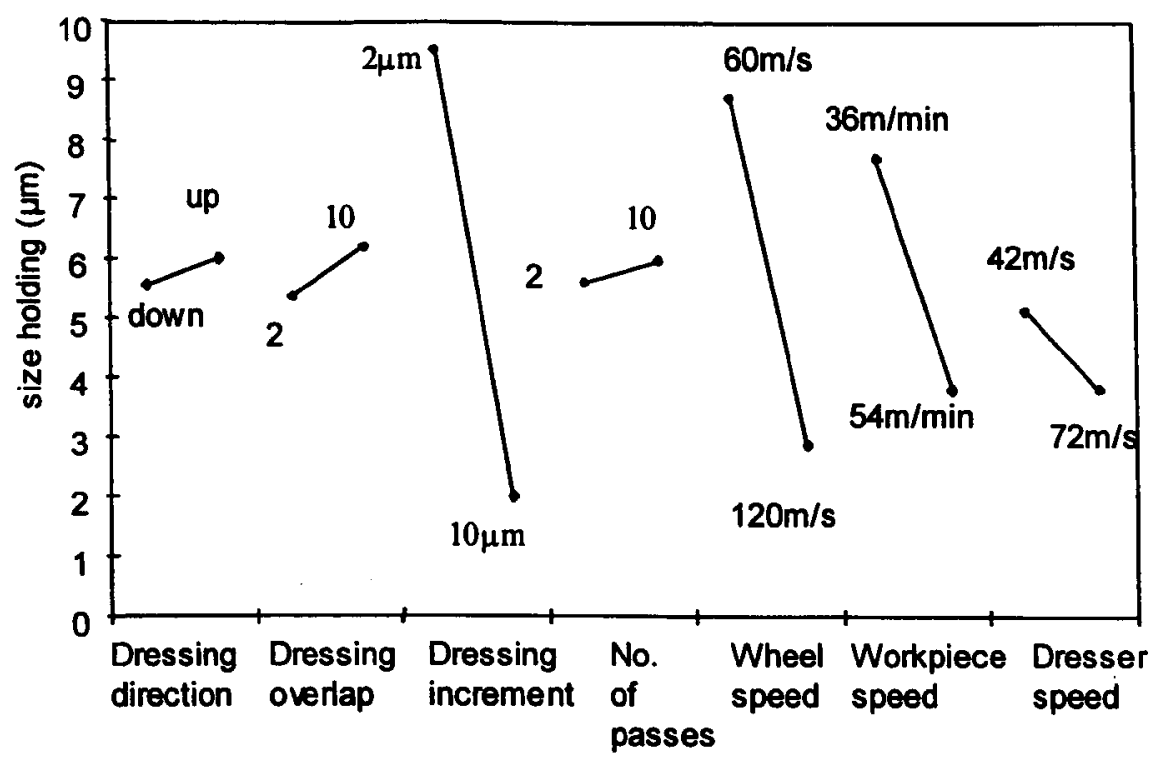

Fig. 6.20 (d) Direct effects charts of process parameters on the mean size holding for Inconel 718 on Suprema

Direct Effects of Process Parameters on G-Ratio

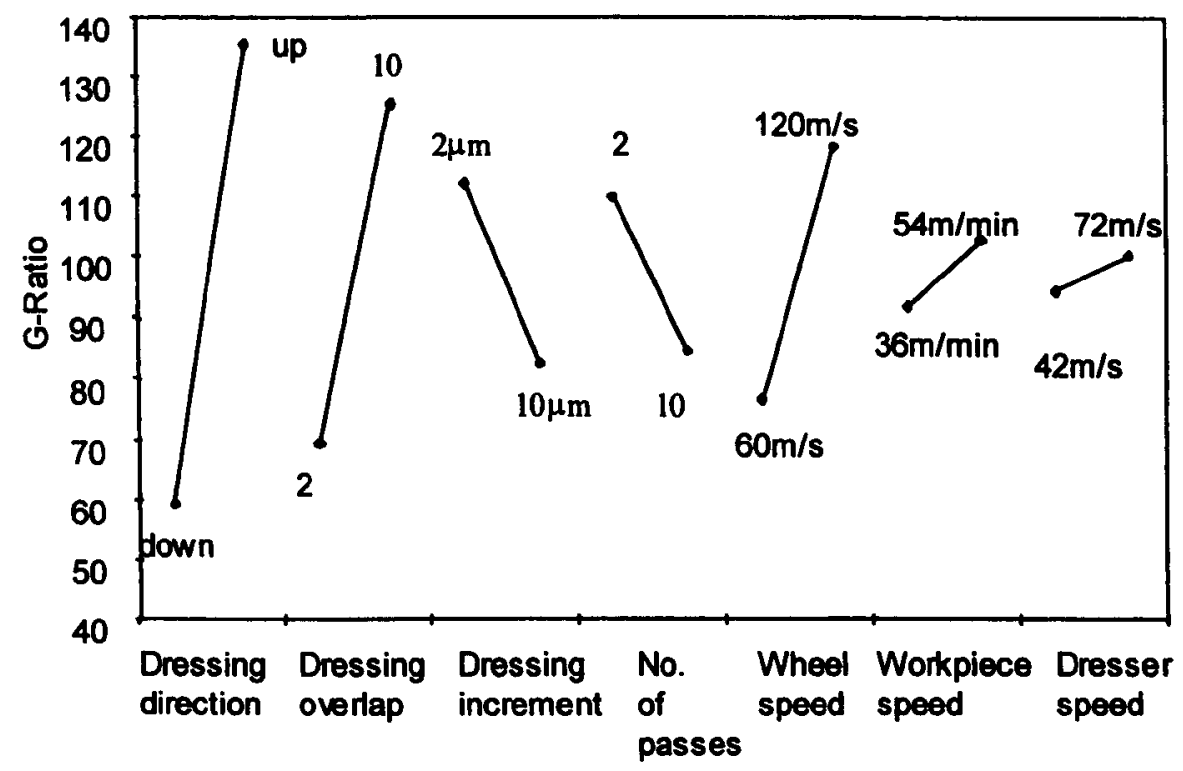

Fig. 6.20 (e) Direct effects charts of process parameters on the mean G-ratio for Inconel 718 on Suprema 
Grinding wheel: High-porosity CBN B151-150 VR

Equivalent diameter: $40 \mathrm{~mm}$

Grinding machine: J\&S Suprema

Dresser rotation direction: up

Dressing overlap: 2

Dressing increment: $2 \mu \mathrm{m}$

Dresser speed: $42 \mathrm{~m} / \mathrm{s}$

No. of dressing passes: 10

Wheel speed: $120 \mathrm{~m} / \mathrm{s}$

Workpiece speed: $42 \mathrm{~m} / \mathrm{min}$

Dwell: $2 \mathrm{~s}$

$Q_{w}^{\prime}: 2 \mathrm{~mm}^{3} / \mathrm{mms}^{3}$

$\mathrm{V}_{\mathrm{w}}: 10,530 \mathrm{~mm}^{3}$

Coolant type: Hysol-X (10\% emulsion)

Coolant delivery: 36 litres/min (30 bar pump pressure)

Specific Energy for CBN Wheel Life Trial

Grinding Inconel 718 at $120 \mathrm{~m} / \mathrm{s}$ (Suprema)

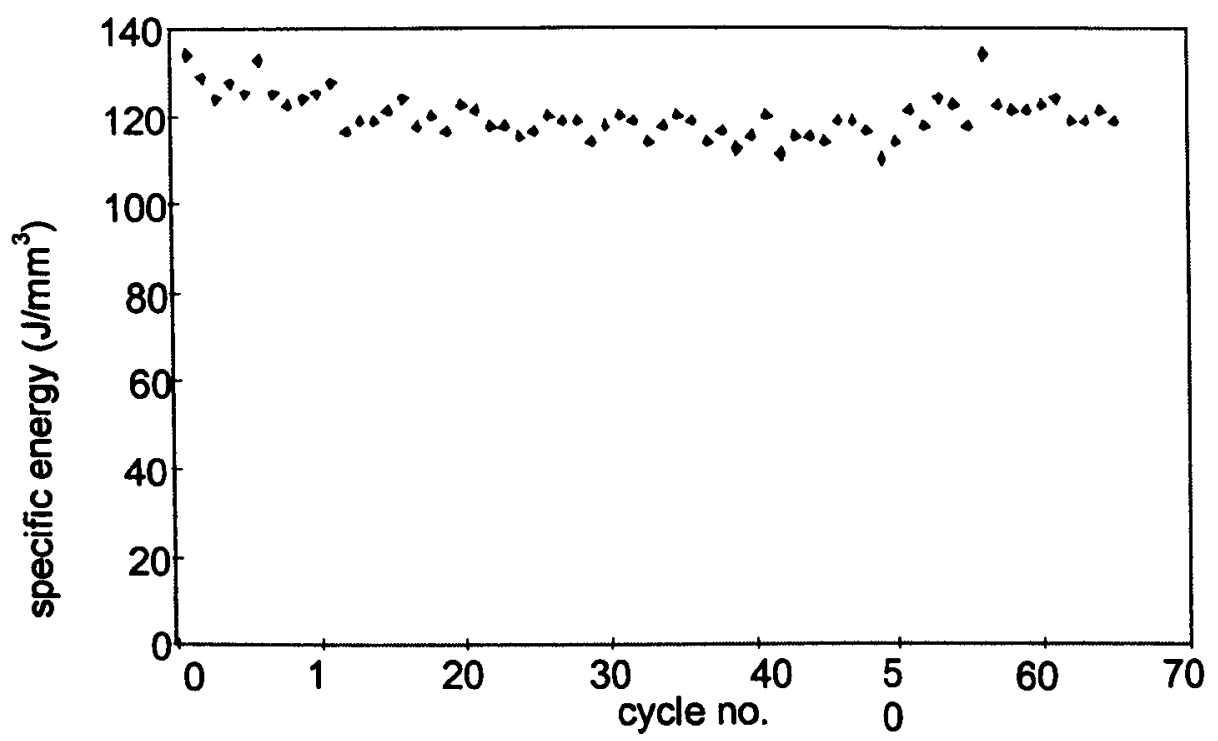

Fig. 6.21(a) Specific energy of wheel life trial for grinding Inconel 718 on the J\&S Suprema 
Surface Roughness for CBN Wheel Life Trial Grinding Inconel 718 at $120 \mathrm{~m} / \mathrm{s}$ (Suprema)

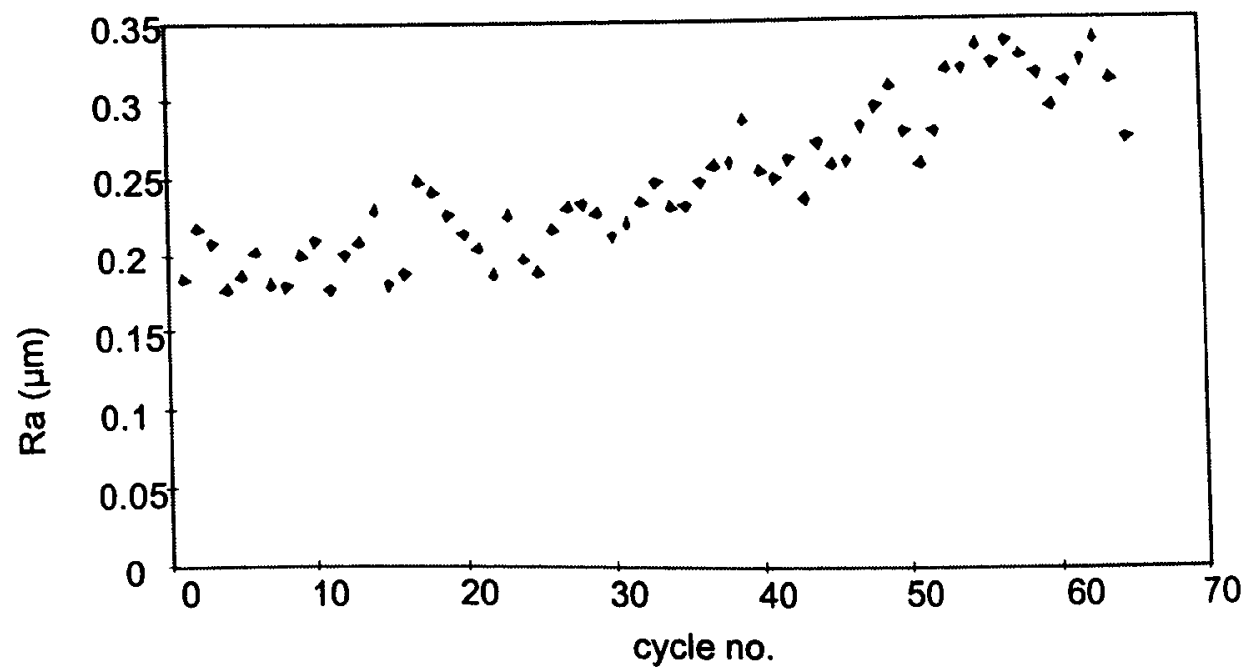

Fig. 6.21(b) Surface roughness wheel life trial for grinding Inconel 718 on the J\&S Suprema

Roundness for CBN Wheel Life Trial Grinding Inconel 718 at $120 \mathrm{~m} / \mathrm{s}$ (Suprema)

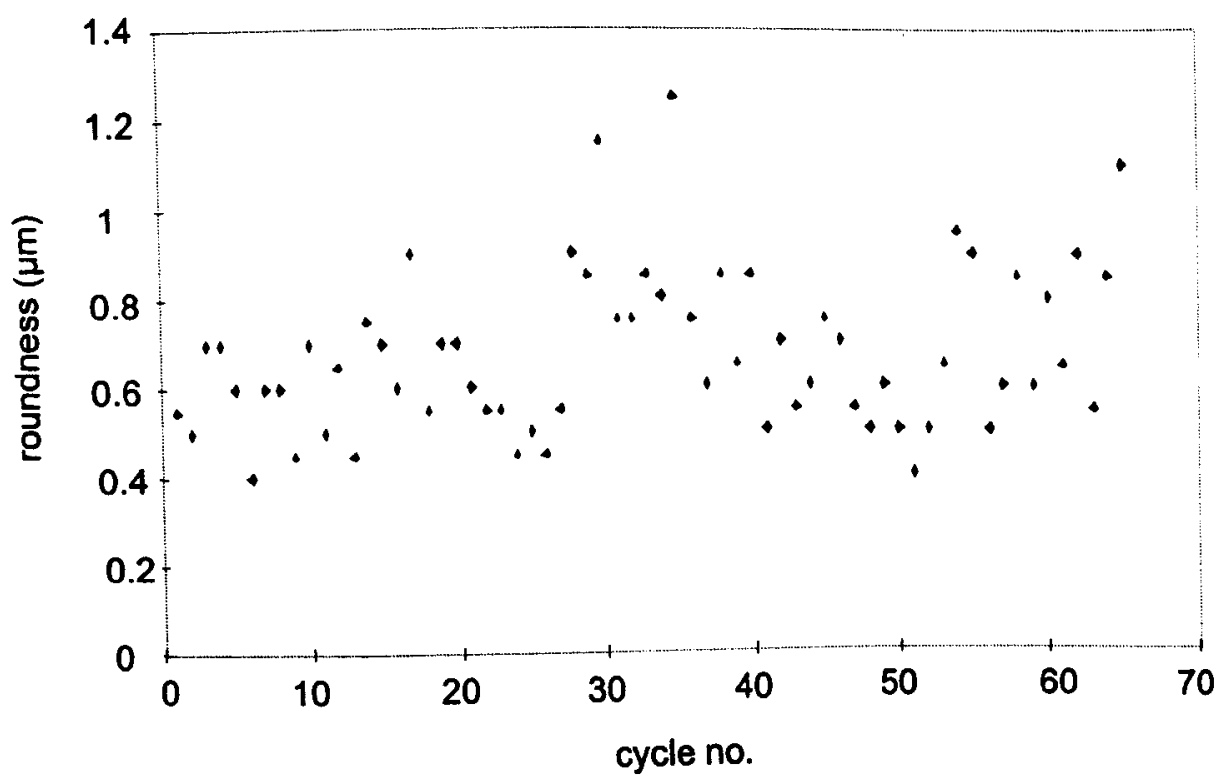

Fig. 6.21(c) Roundness of wheel life trial for grinding Inconel 718 on the J\&S Suprema 
Size Holding for CBN Wheel Life Trial Grinding Inconel 718 at $120 \mathrm{~m} / \mathrm{s}$ (Suprema)

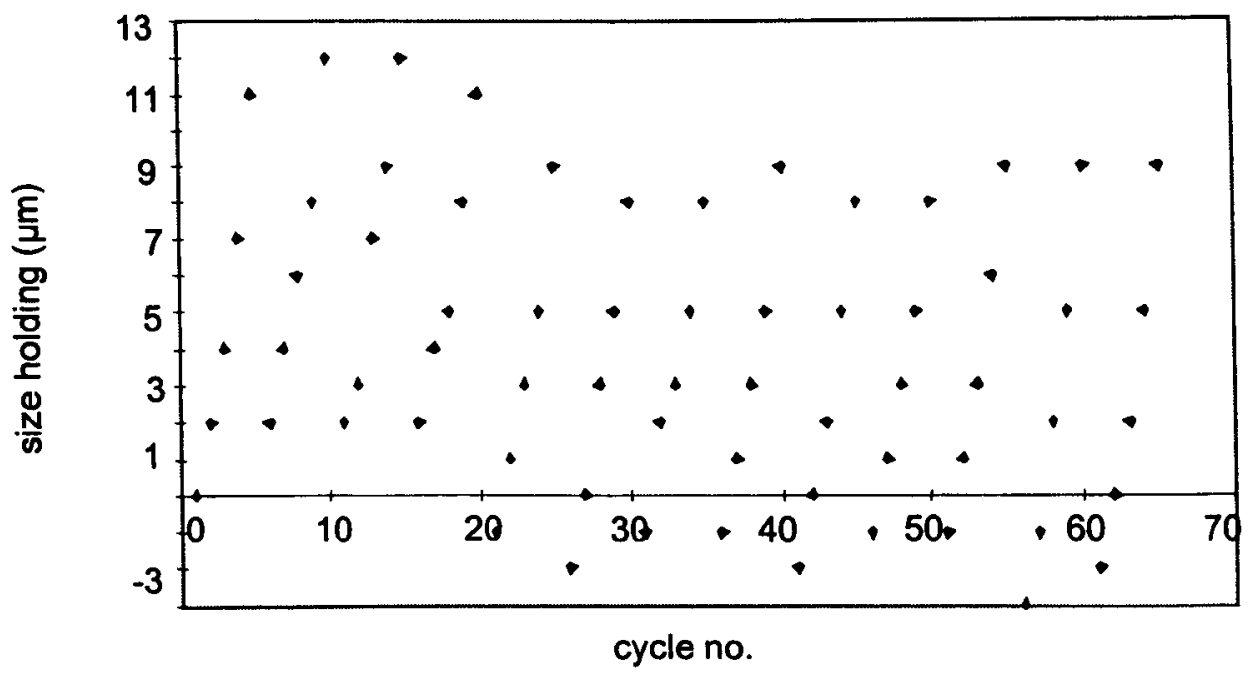

Fig. 6.21(d) Size holding of wheel life trial for grinding Inconel 718 on the J\&S Suprema 


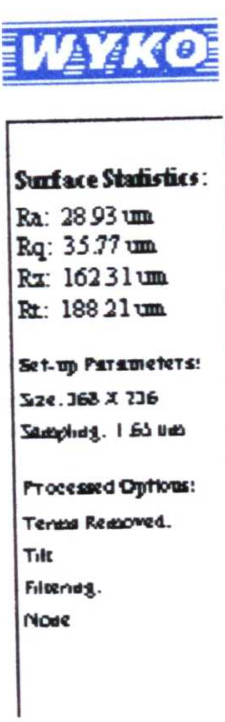

Title:

Note:

Fig. 6.22 (a) Adhesive loaded grains when grinding Inconel 718
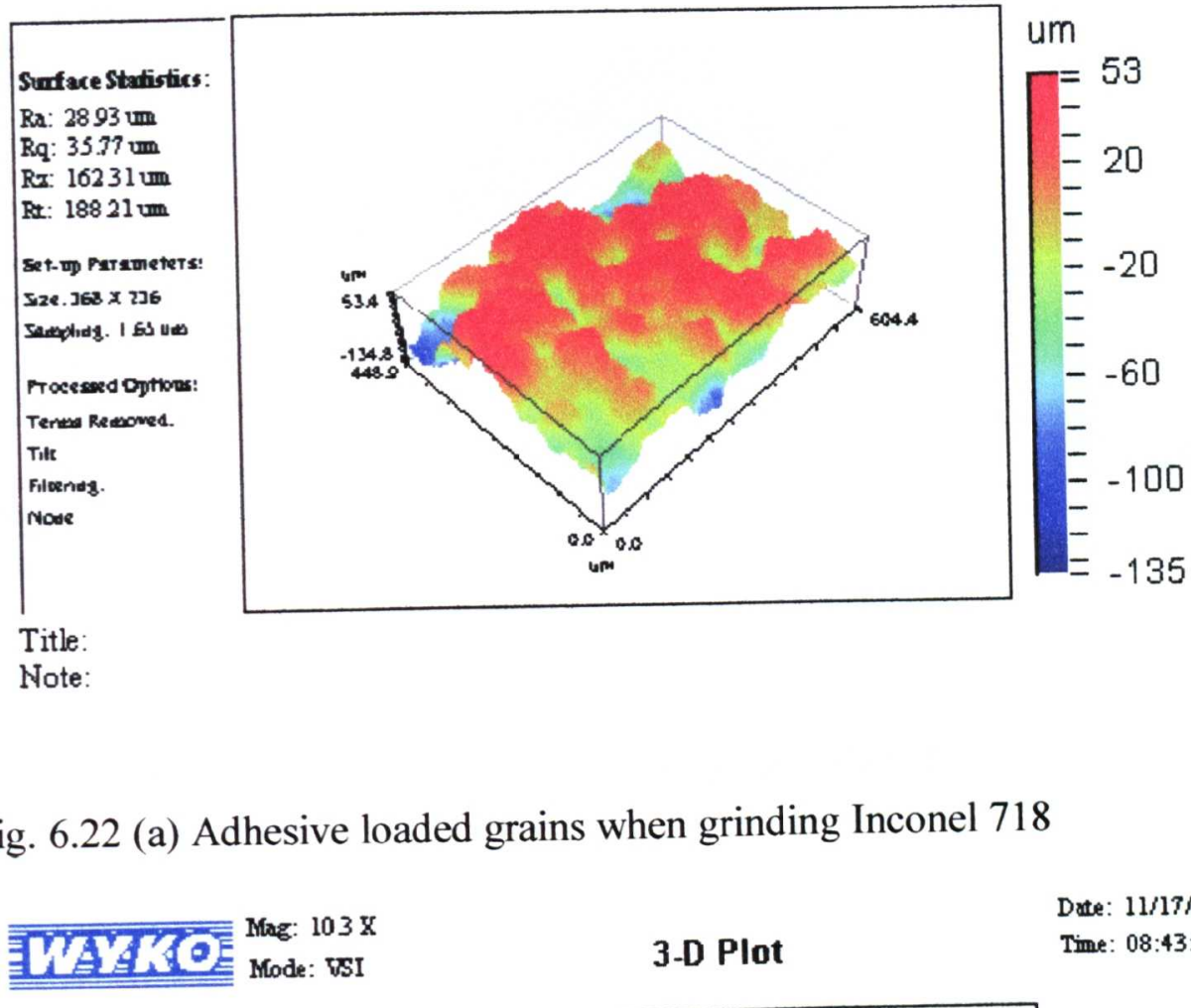

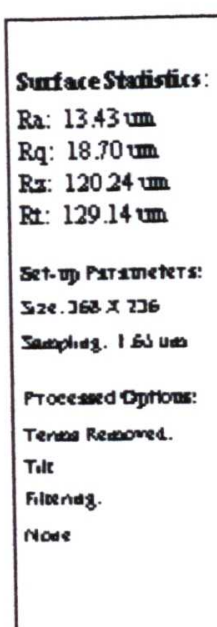

Title:

Note:
Date: $11 / 17 / 00$

Time: 08:43:38

um

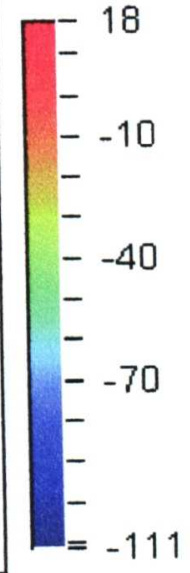

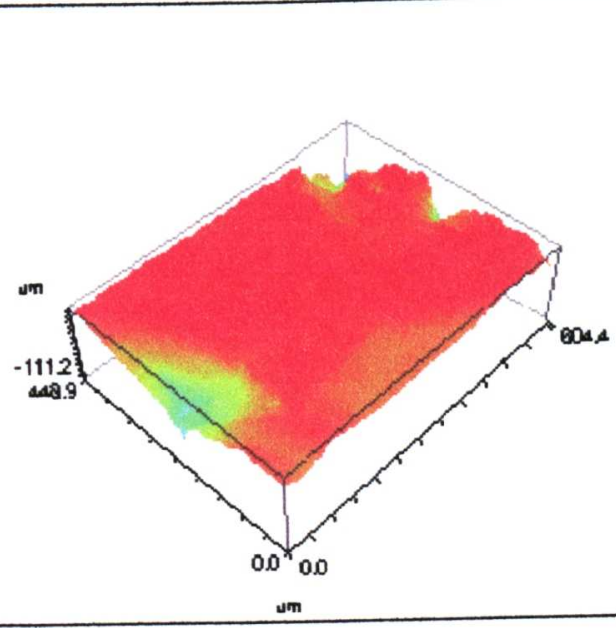

3-D Plot

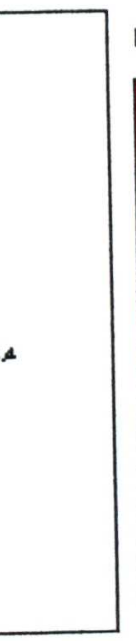

Fig. 6.22 (b) Compact mass loading when grinding Inconel 718 


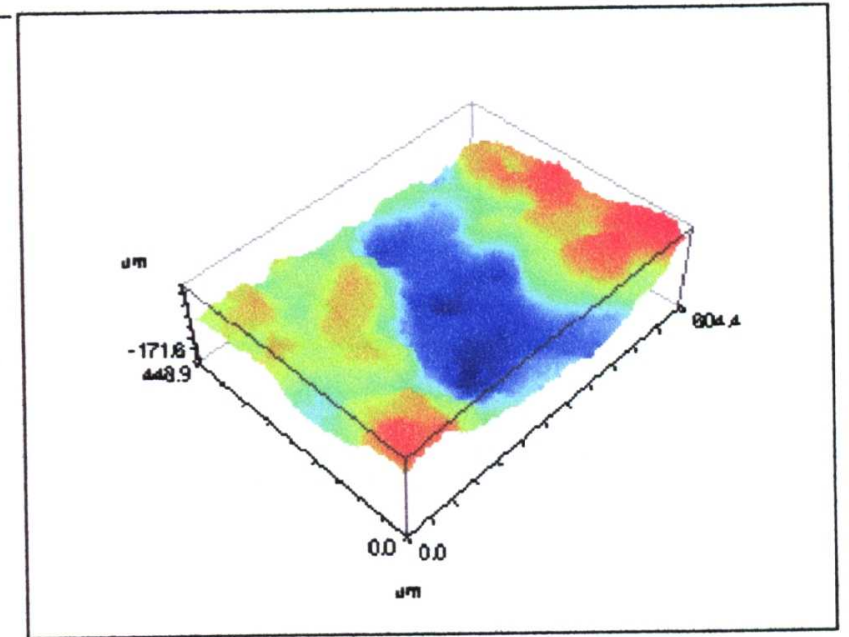

Time: 08:28:41

Title:

Note:

Fig. 6.22 (c) Grains pulled off when grinding Inconel 718 


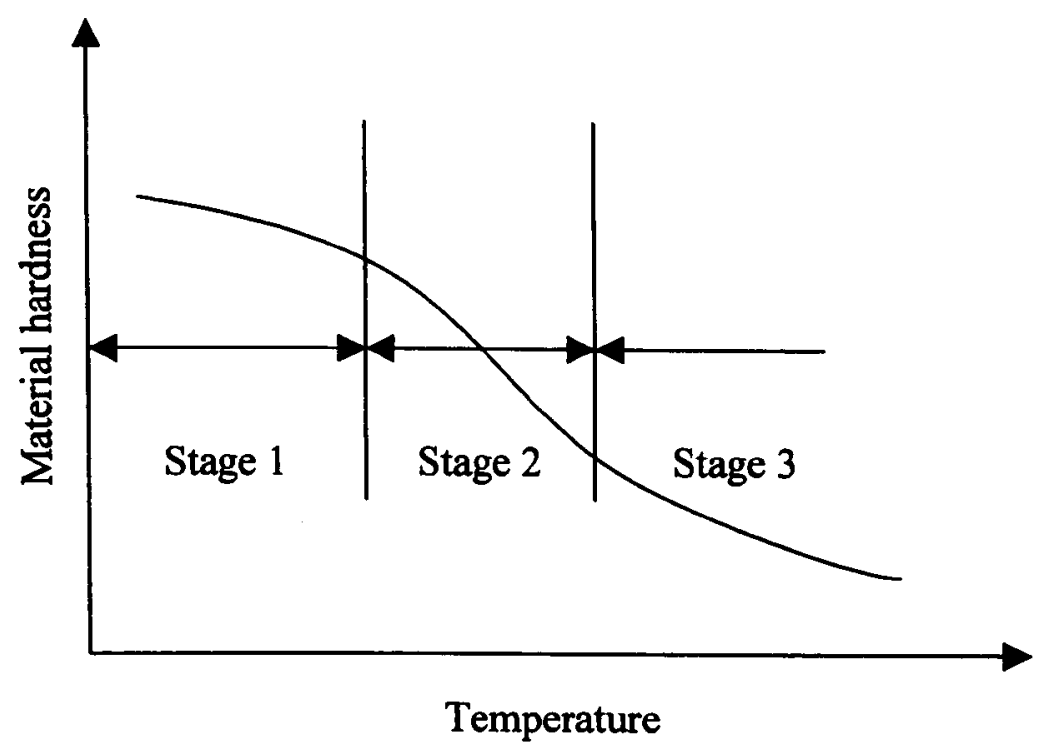

Fig. 6.23 Changes in material hardness with temperature

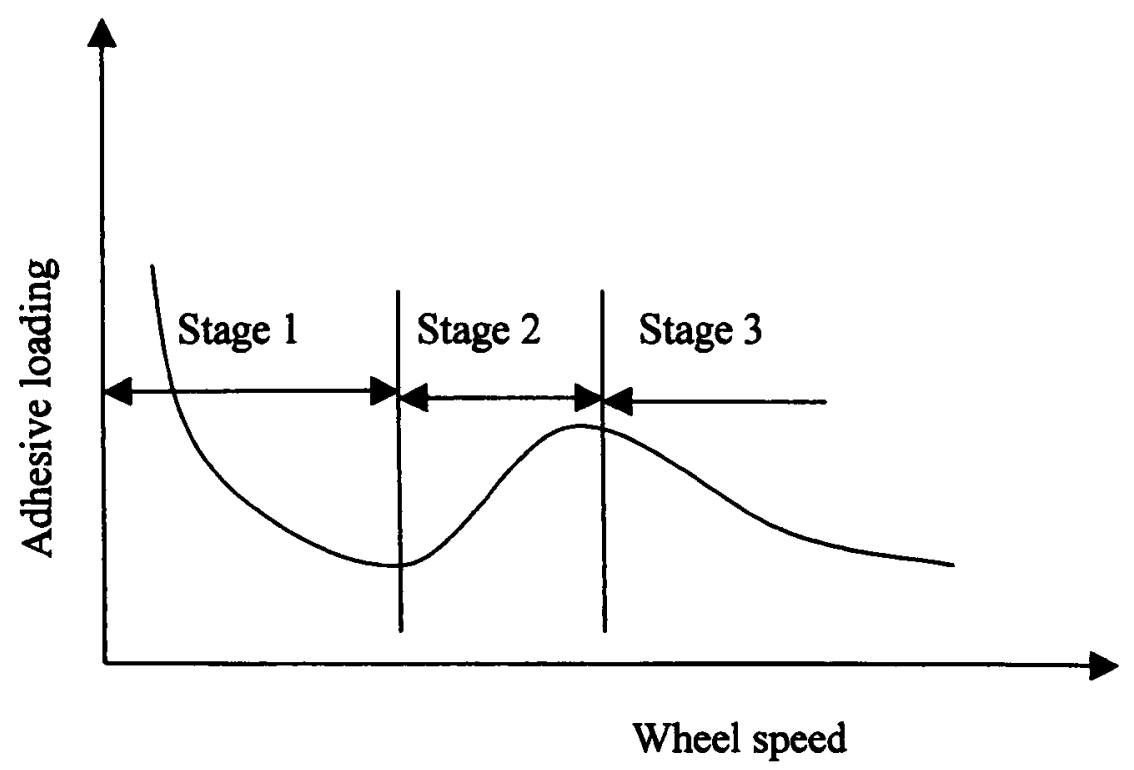

Fig. 6.24 Proposed adhesive loading with increase of wheel speed for a particular grinding situation and a particular grinding distance 


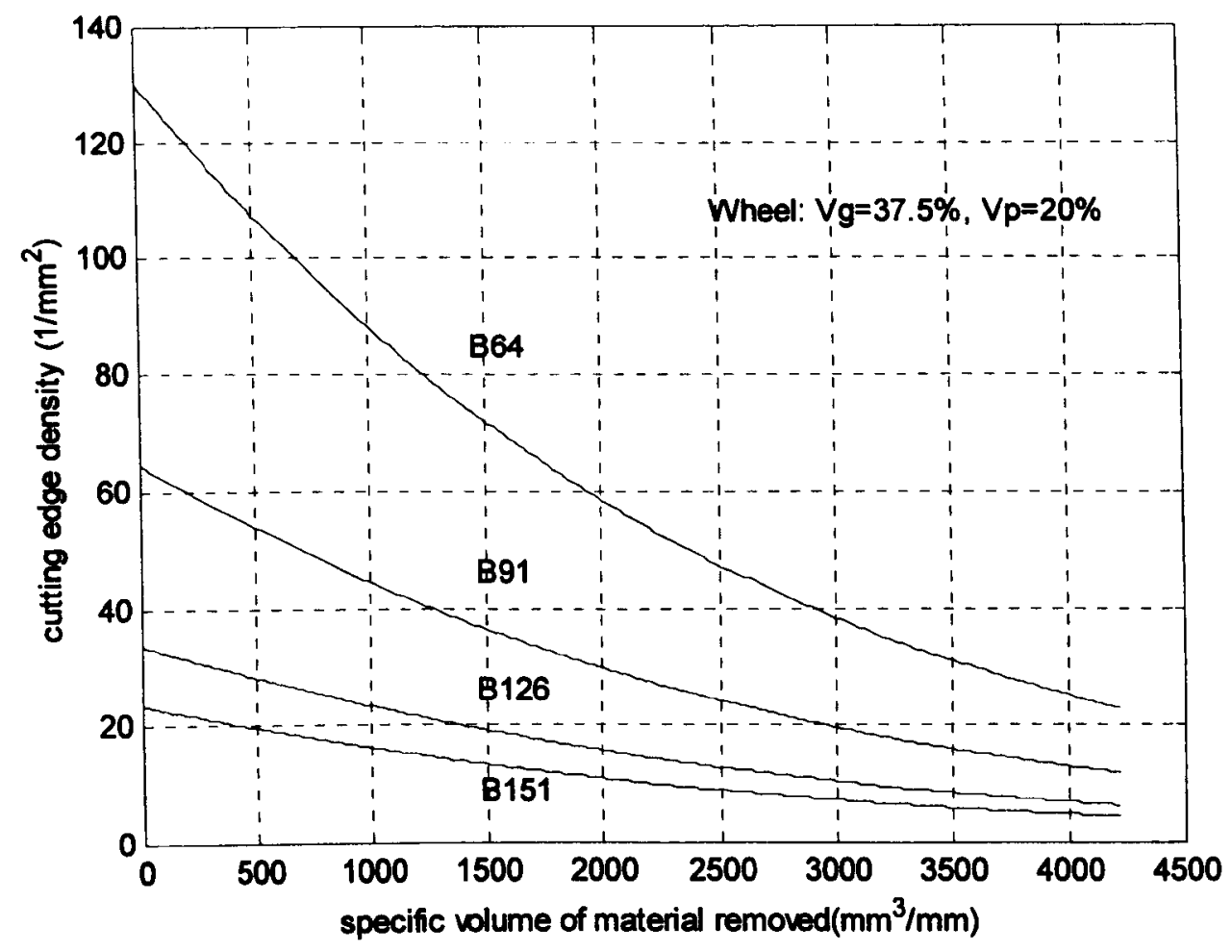

Fig. 7.1(a) The effect of grain size on cutting edge density in peridiction of wheel wear

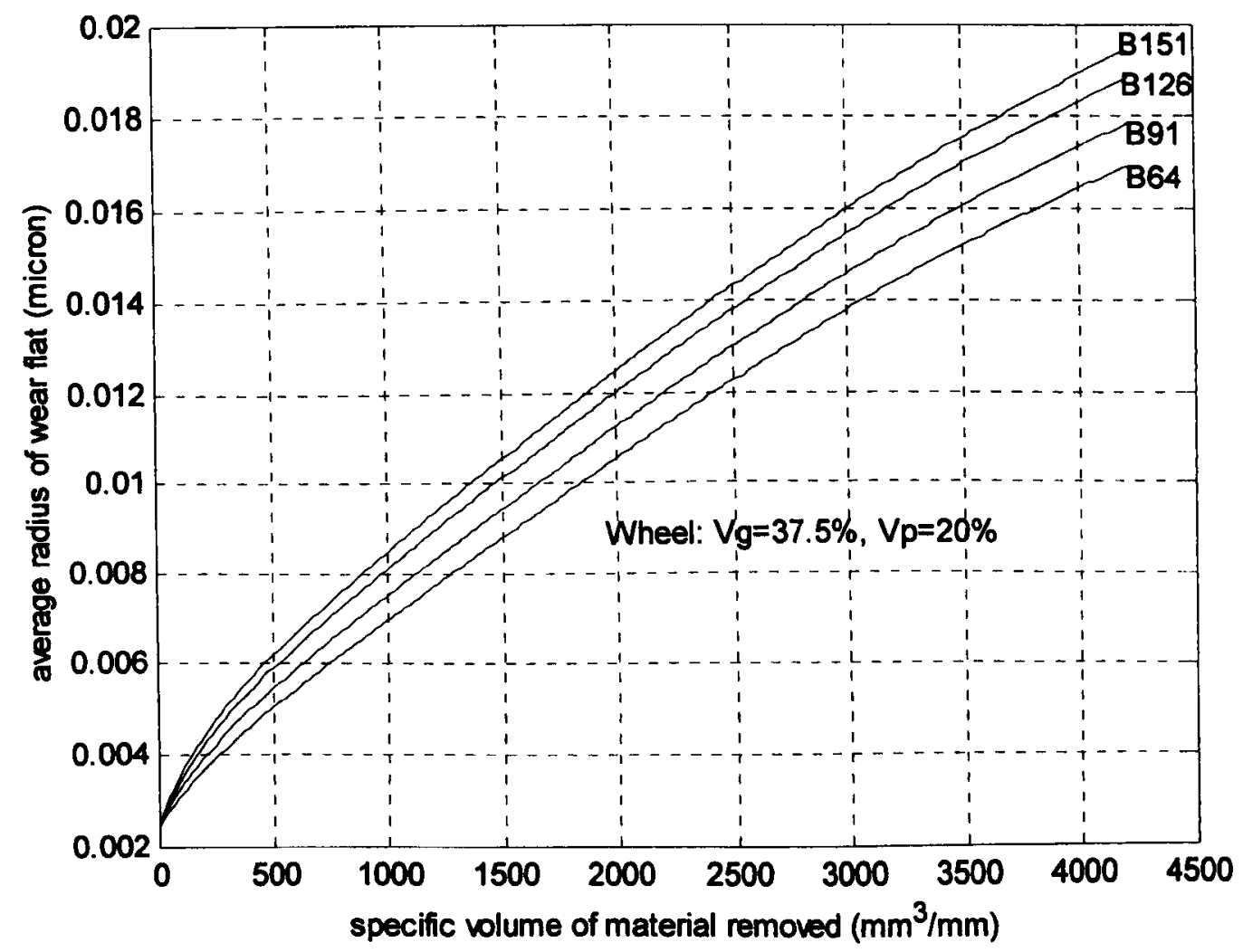

Fig. 7.1(b) The effect of grain size on wear flat radius in peridiction of wheel wear 


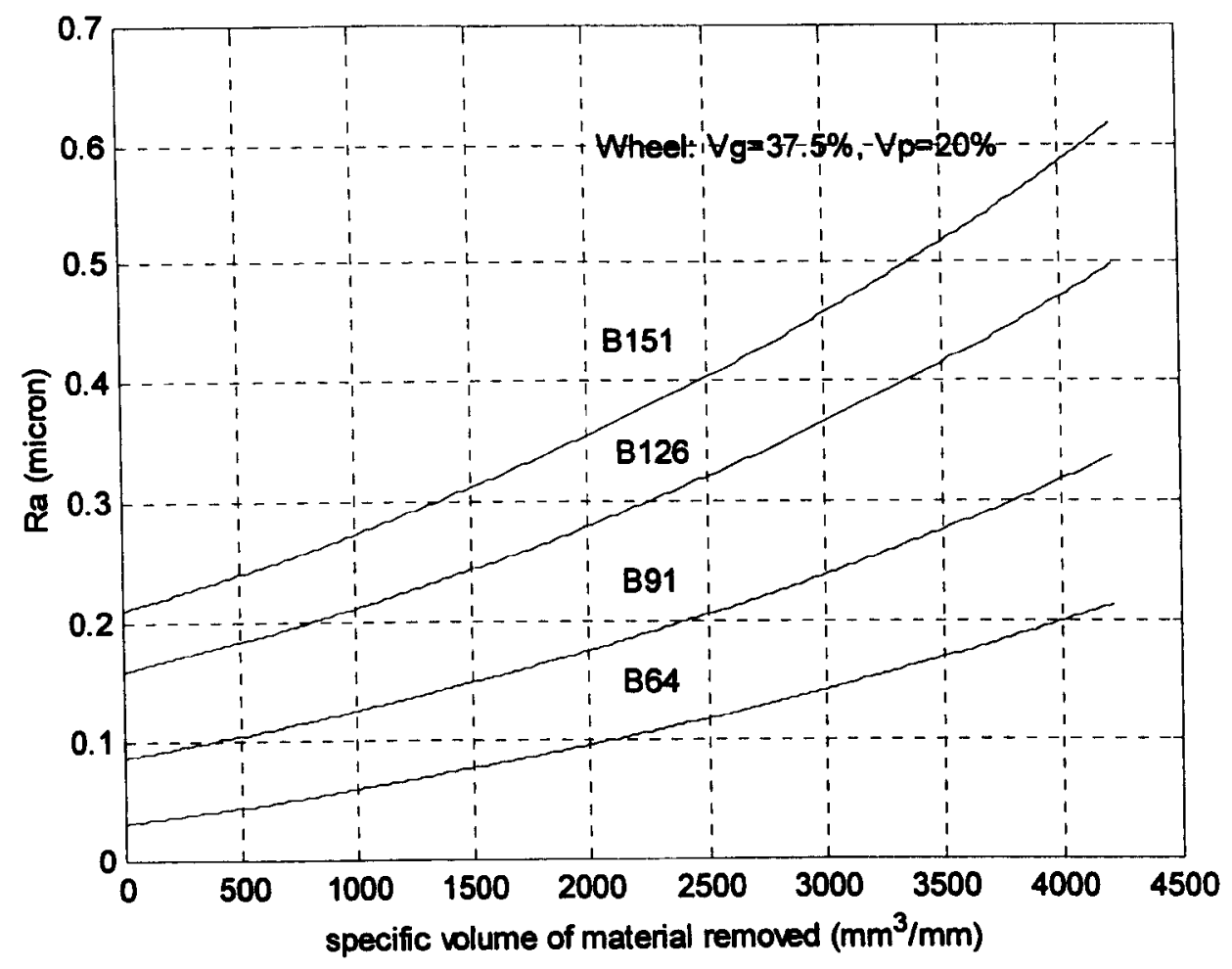

Fig. 7.1(c) The effect of grain size on workpiece Ra surface roughness in peridiction of wheel wear 


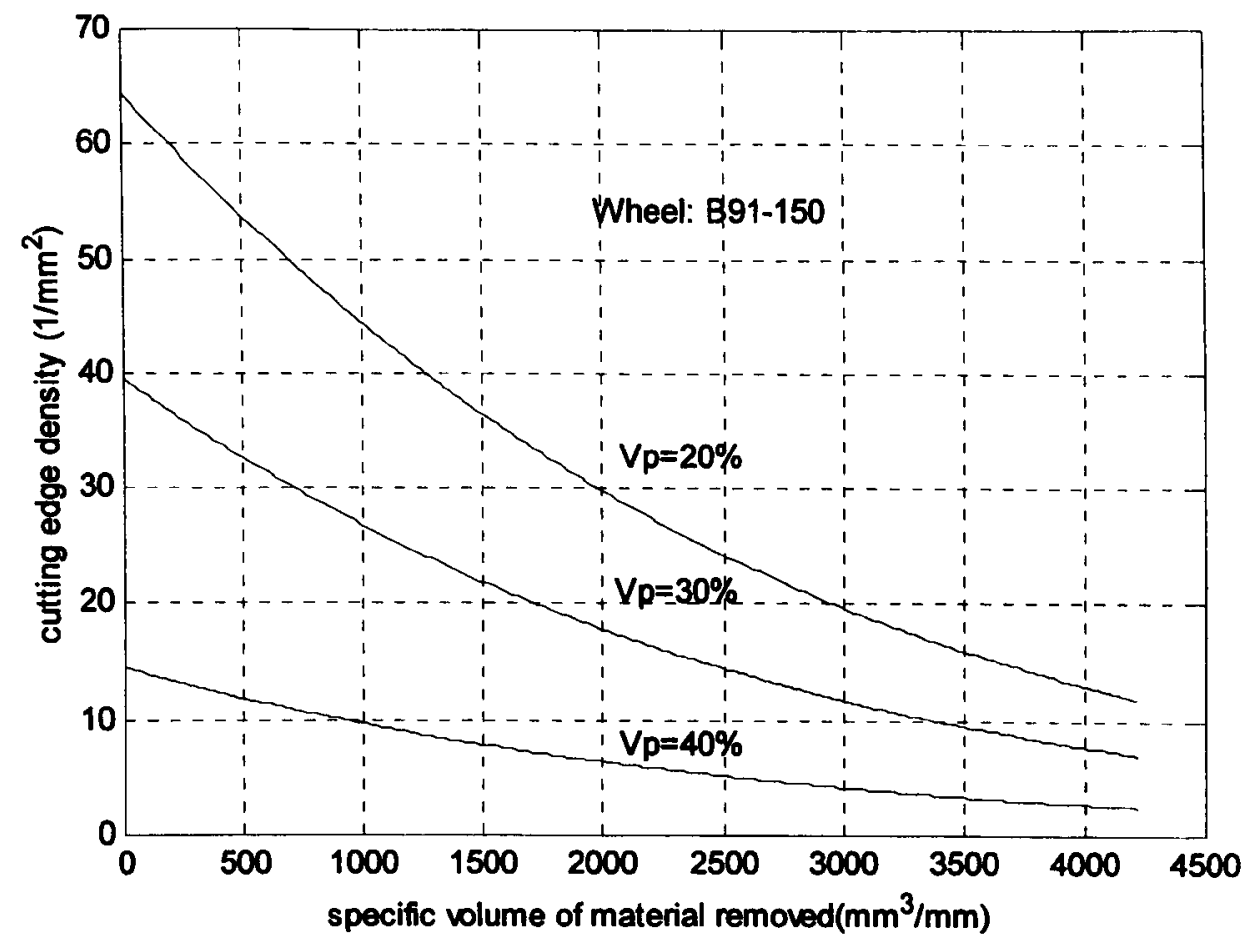

Fig. 7.2(a) The effect of percentage of pore volume on cutting edge density in prediction of wheel wear

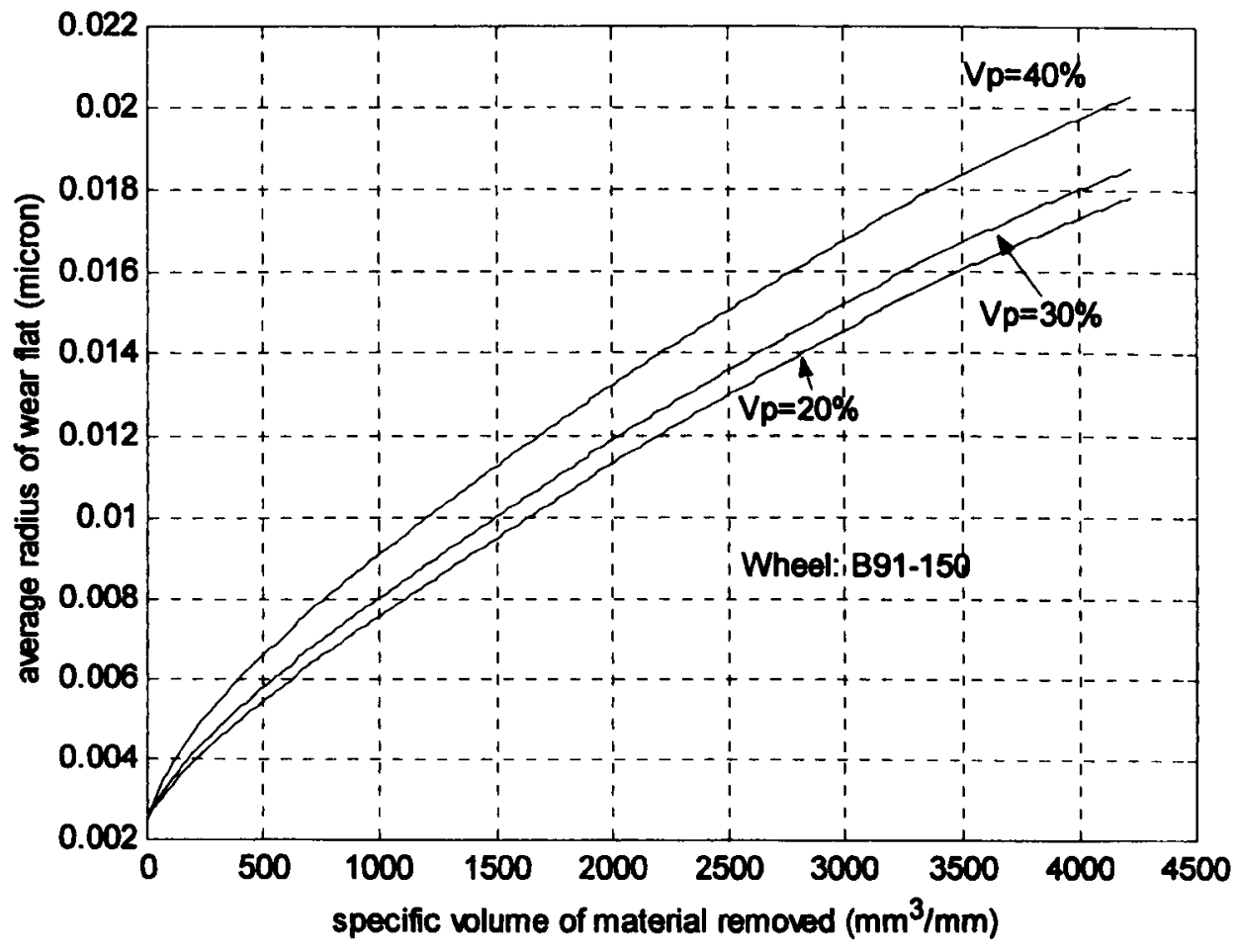

Fig. 7.2(b) The effect of percentage of pore volume on wear flat radius in prediction of wheel wear 


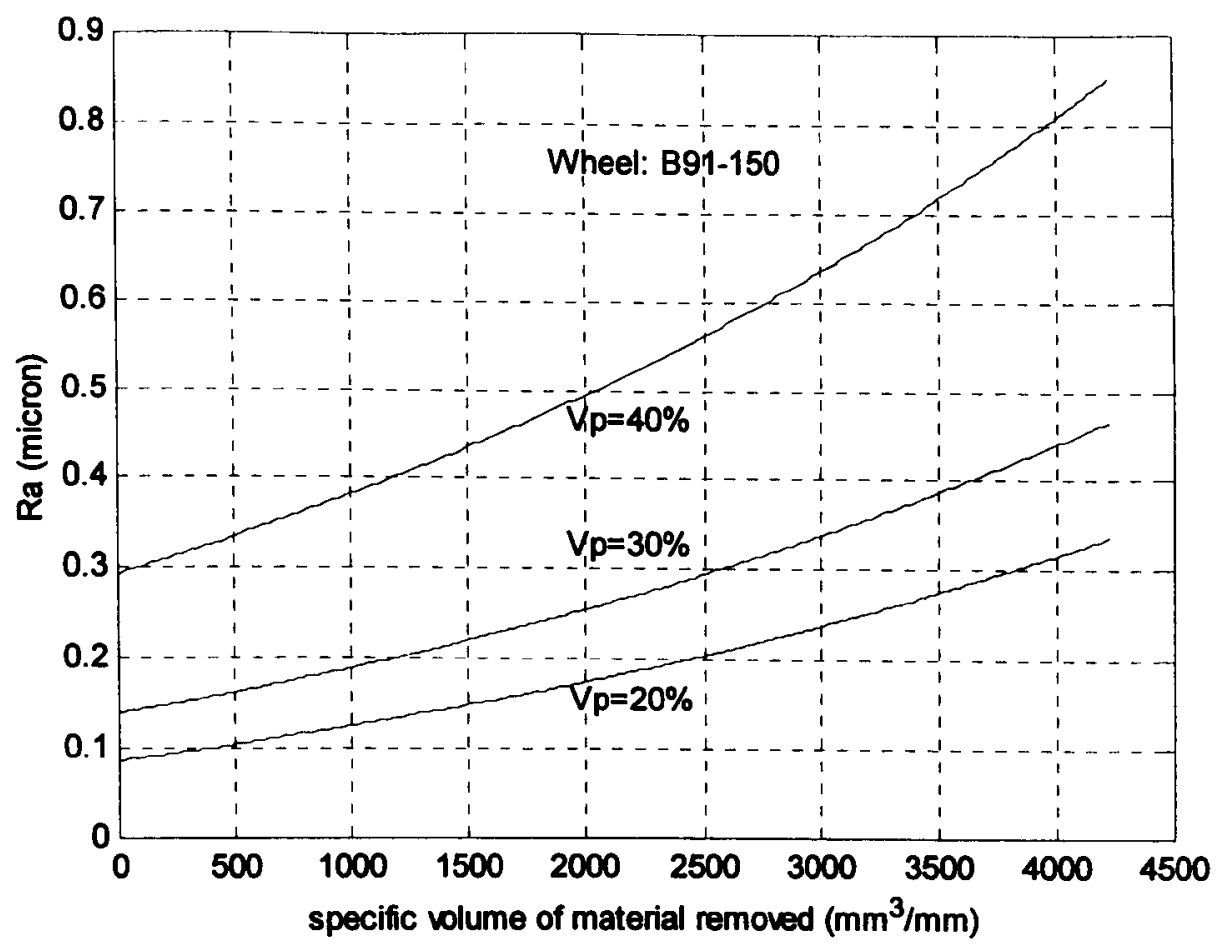

Fig. 7.2(c) The effect of percentage of pore volume on workpiece Ra surface roughness in prediction of wheel wear 


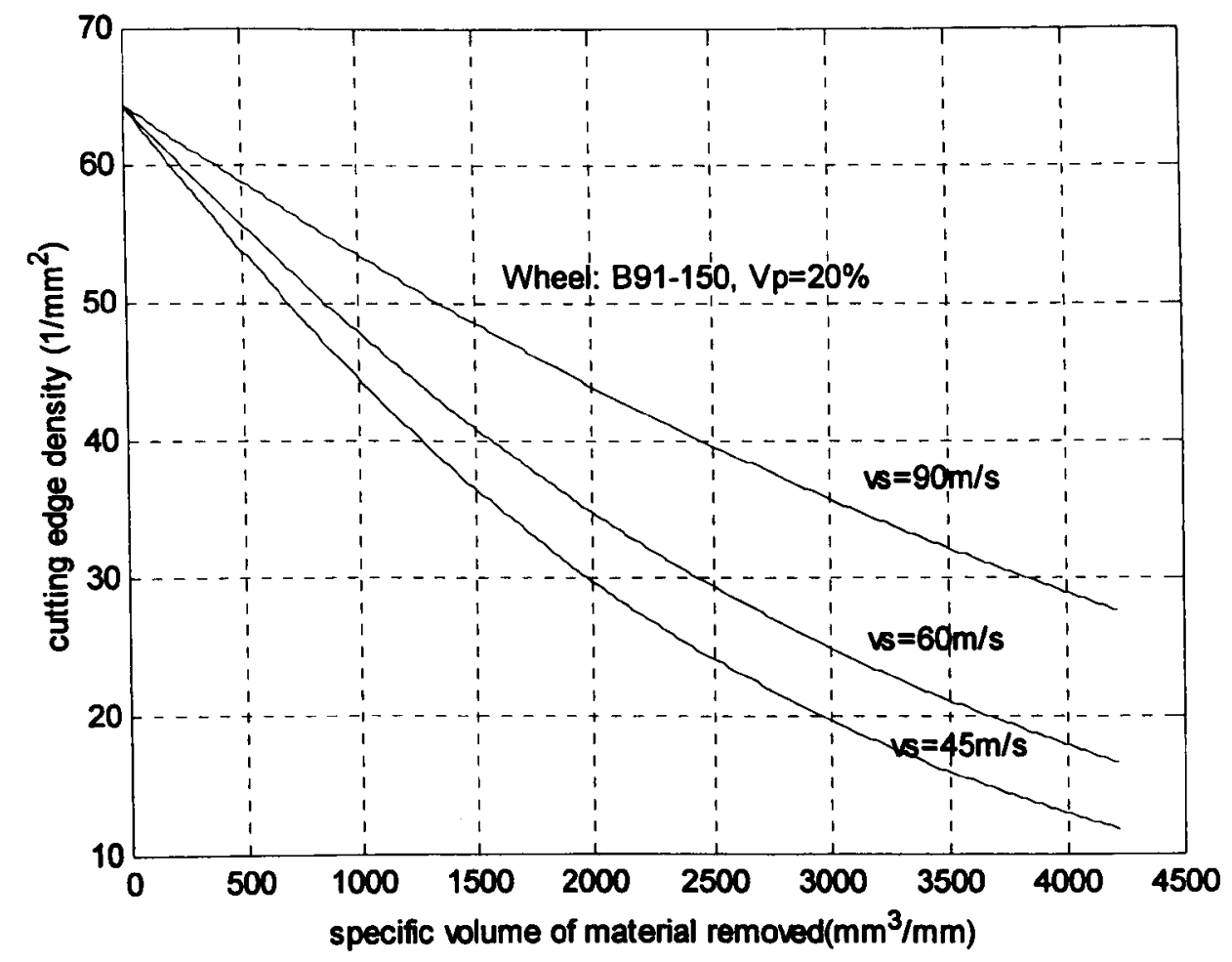

Fig. 7.3(a) The effect of wheel speed on cutting edge density in prediction of wheel wear

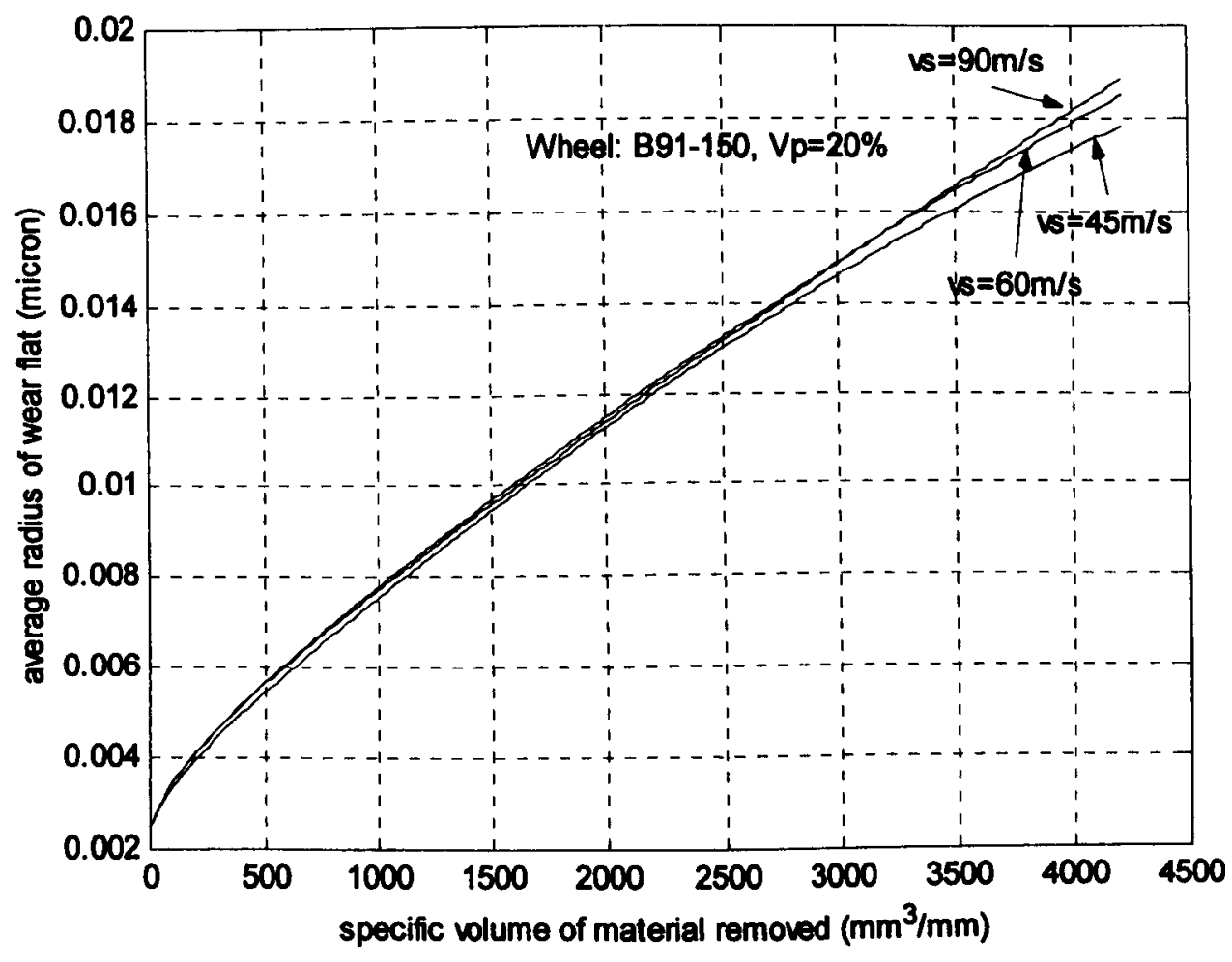

Fig. 7.3(b) The effect of wheel speed on wear flat radius in prediction of wheel wear 


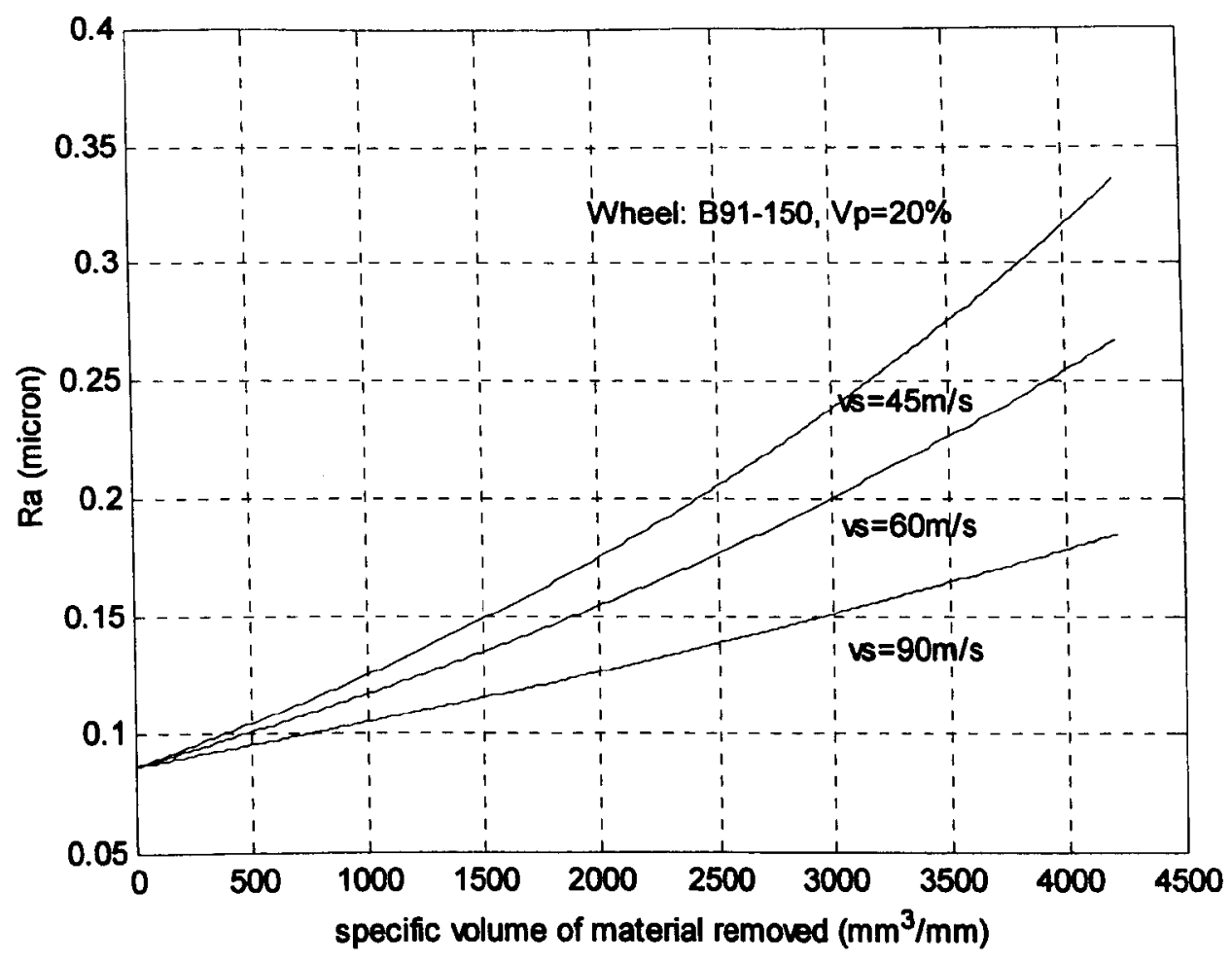

Fig. 7.3(c) The effect wheel speed on workpiece Ra surface roughness in prediction of wheel wear 


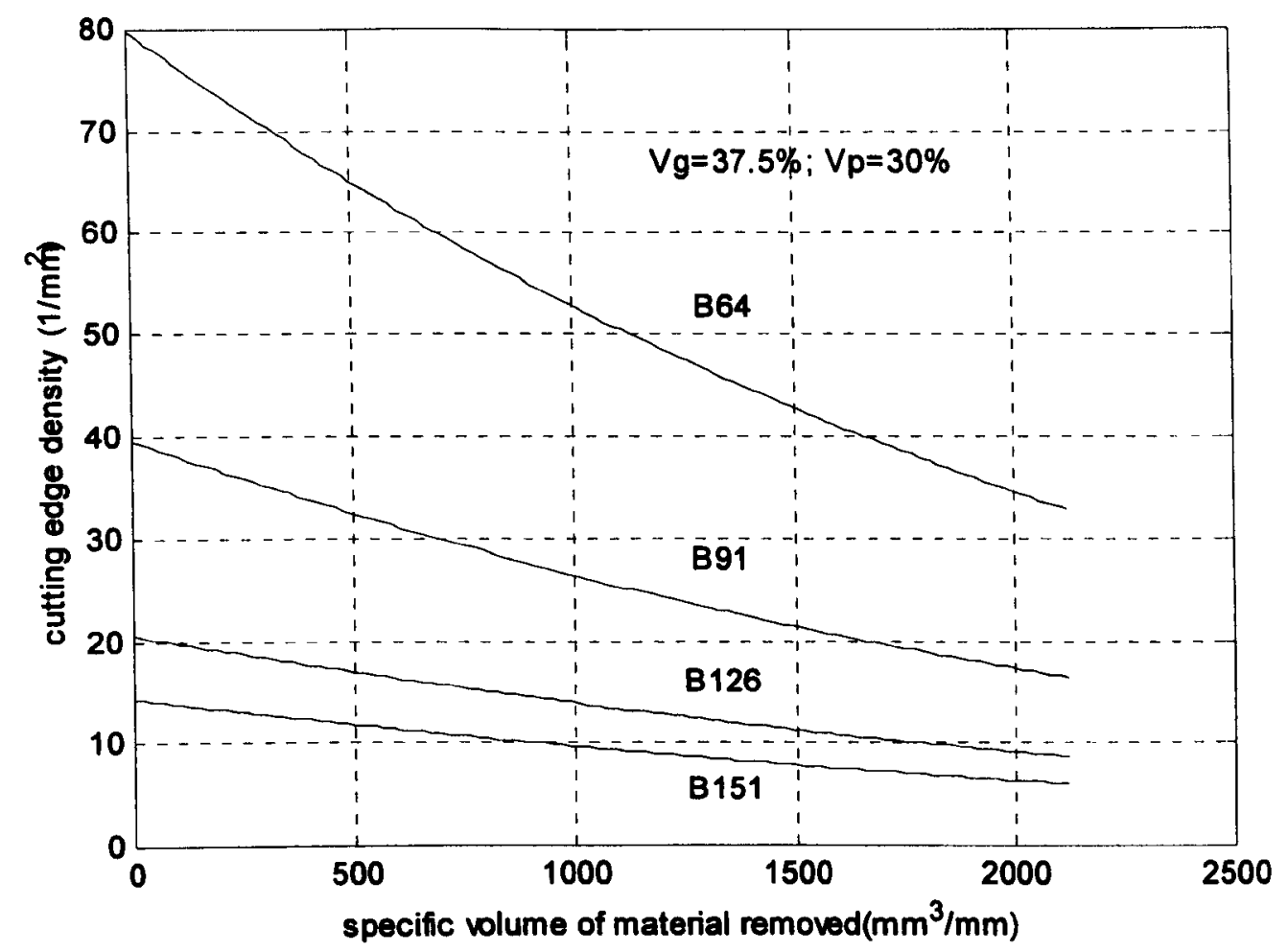

Fig. 7.4(a) The effect of grain size on cutting edge density in prediction of wheel loading

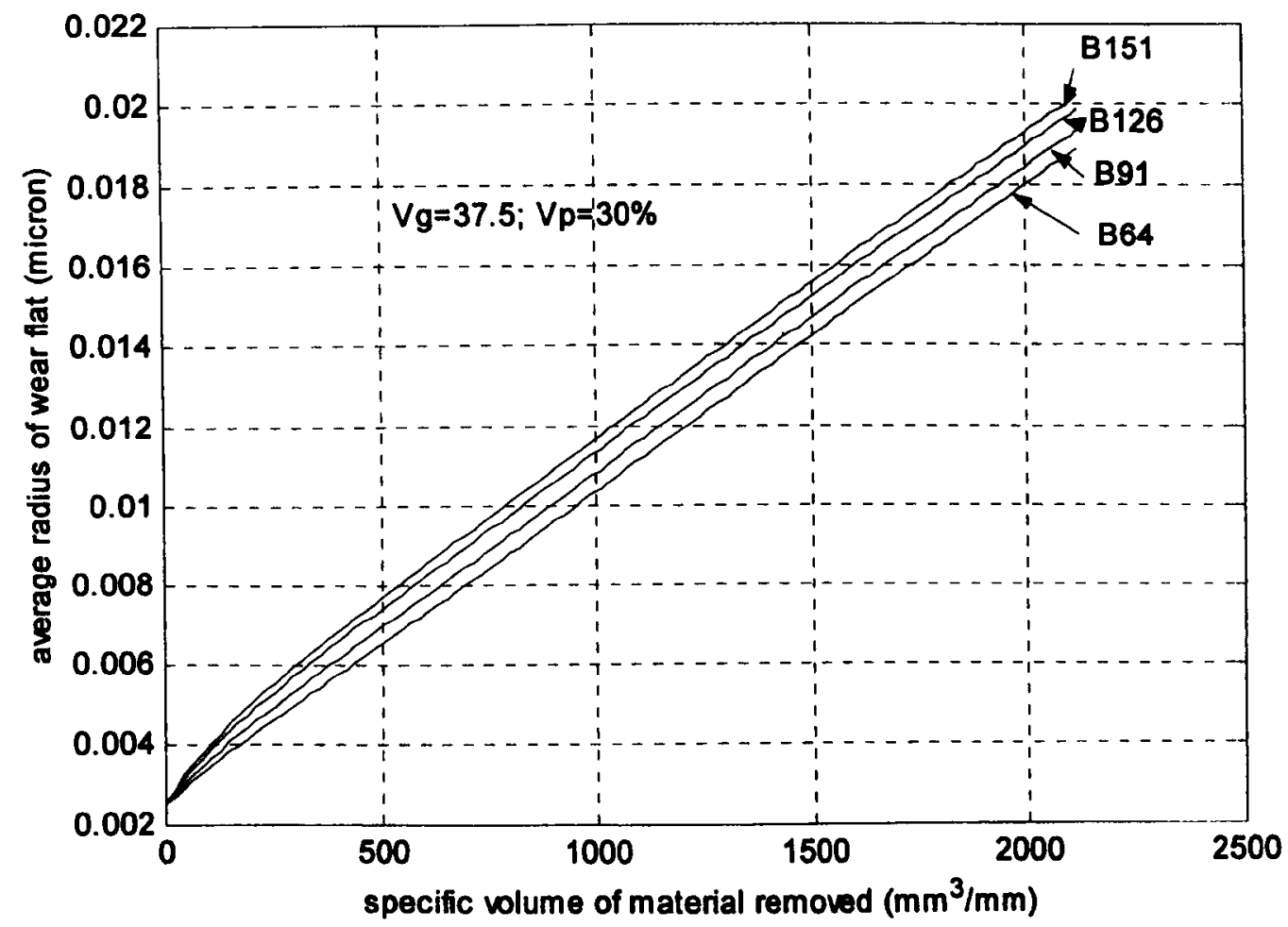

Fig. 7.4(b) The effect of grain size on wear flat radius in prediction of wheel loading 


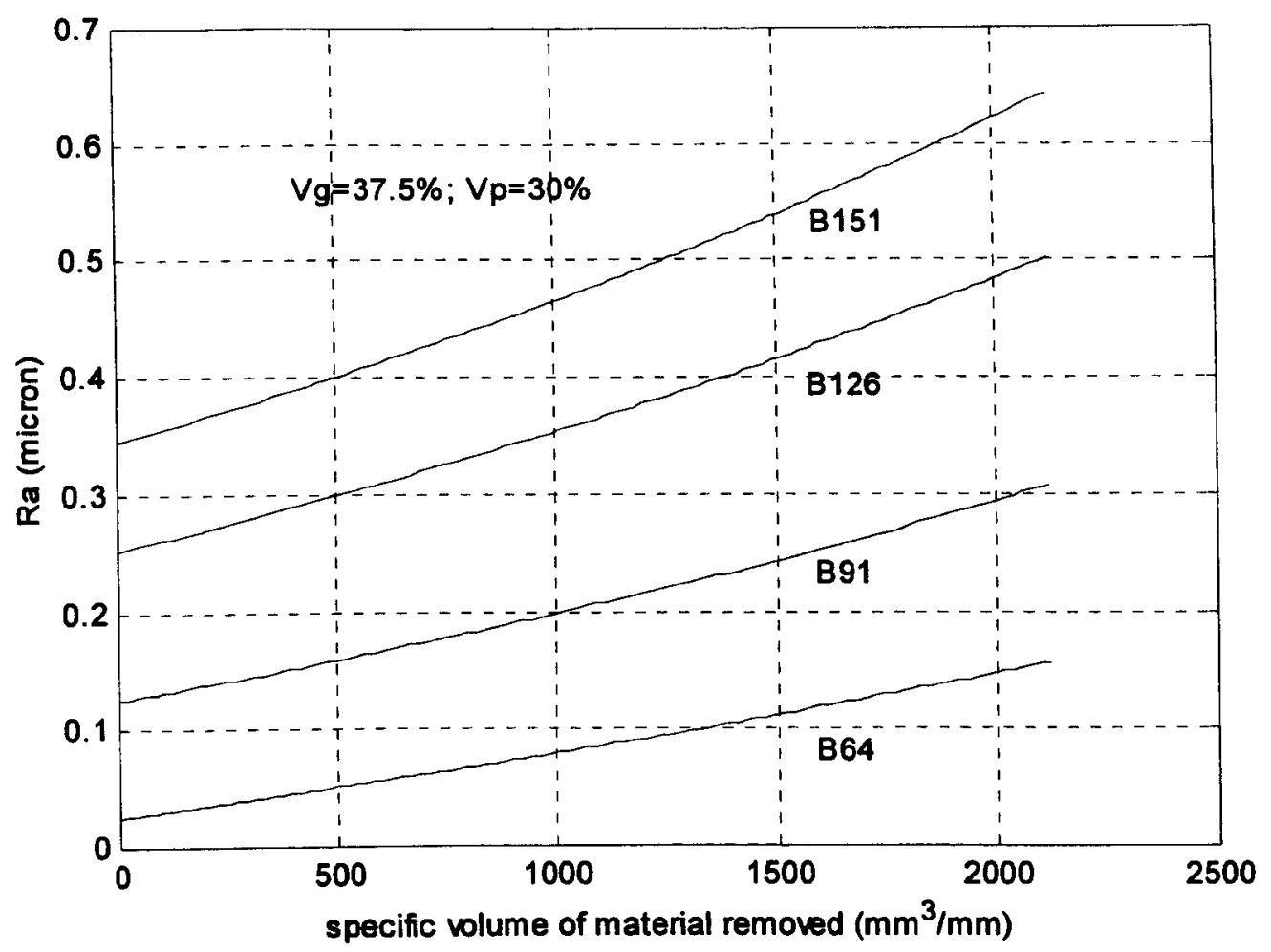

Fig. 7.4(c) The effect of grain size on workpiece Ra surface roughness in prediction of wheel loading

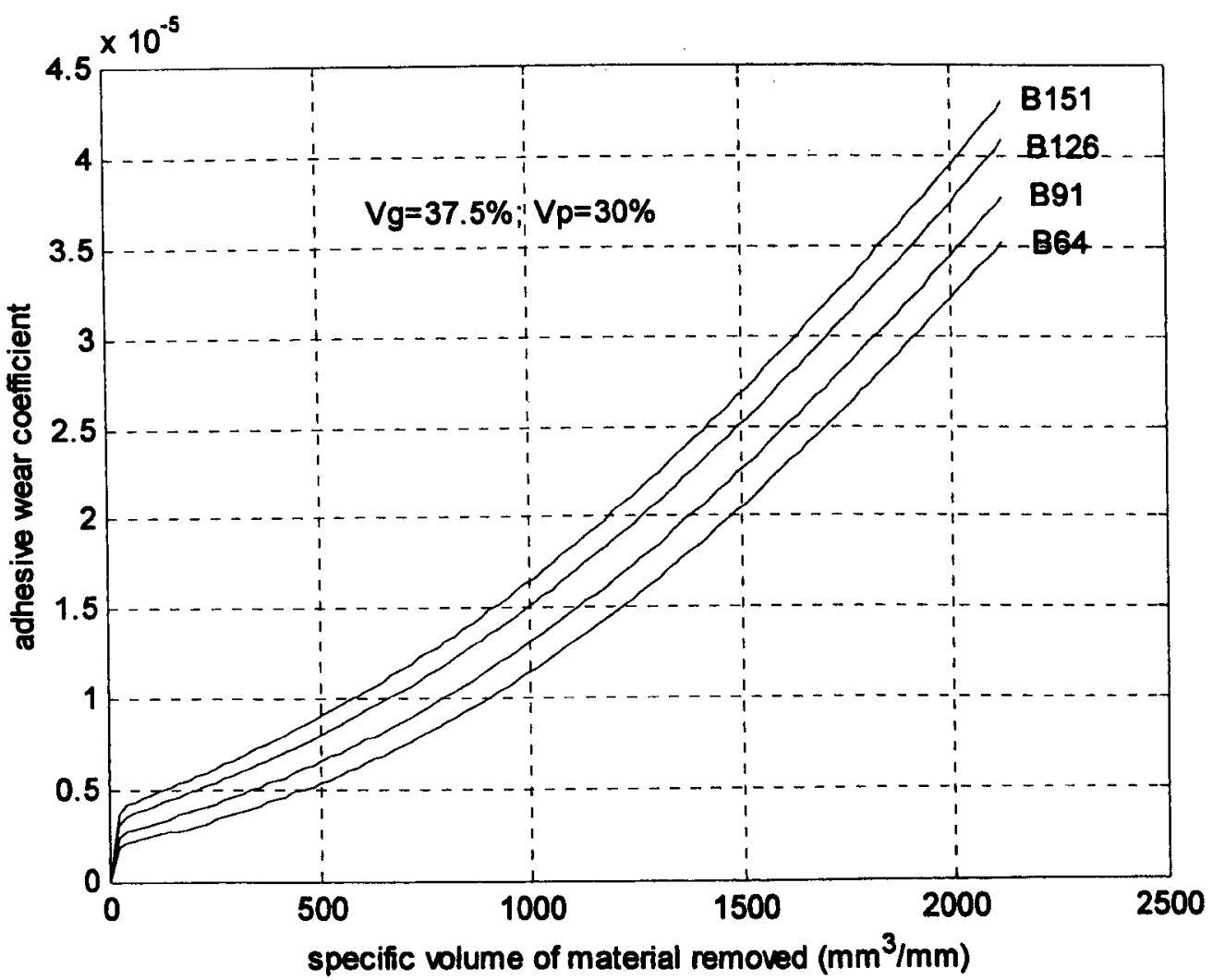

Fig. 7.4(d) The effect of grain size on adhesive wear coefficient in prediction of wheel loading 


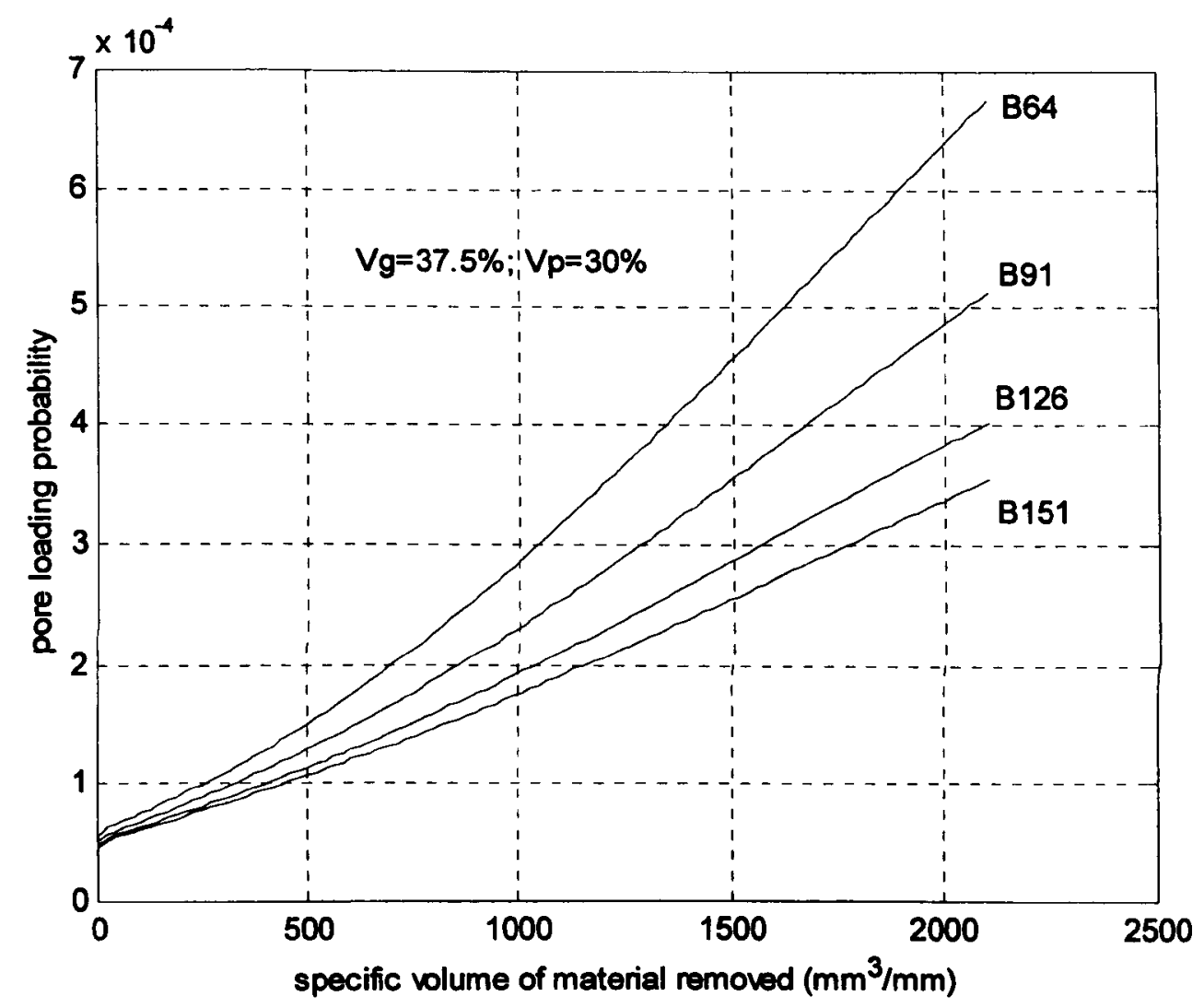

Fig. 7.4(e) The effect of grain size on pore loading probability in prediction of wheel loading 


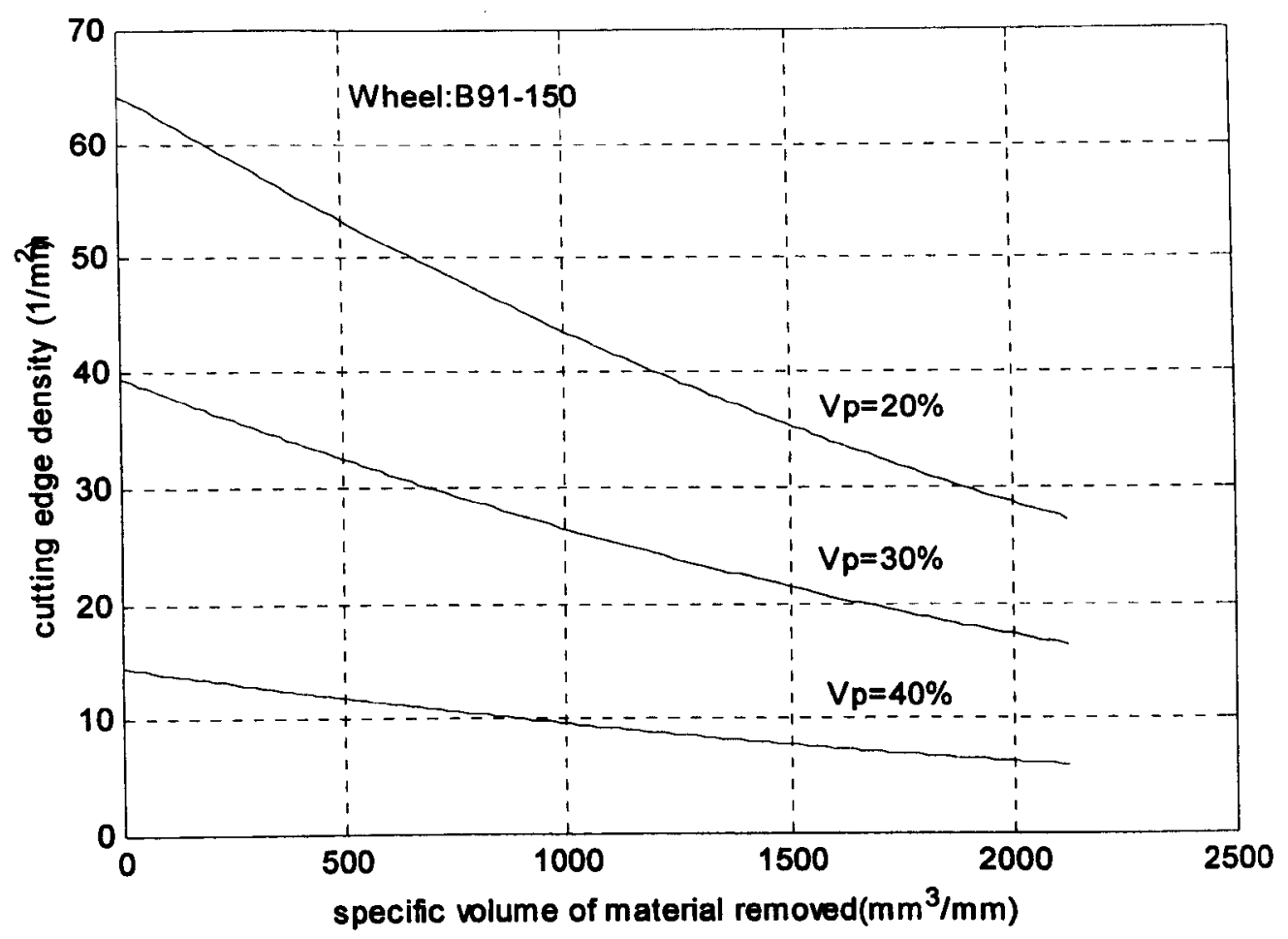

Fig. 7.5(a) The effect of percentage of pore volume on cutting edge density in prediction of wheel loading

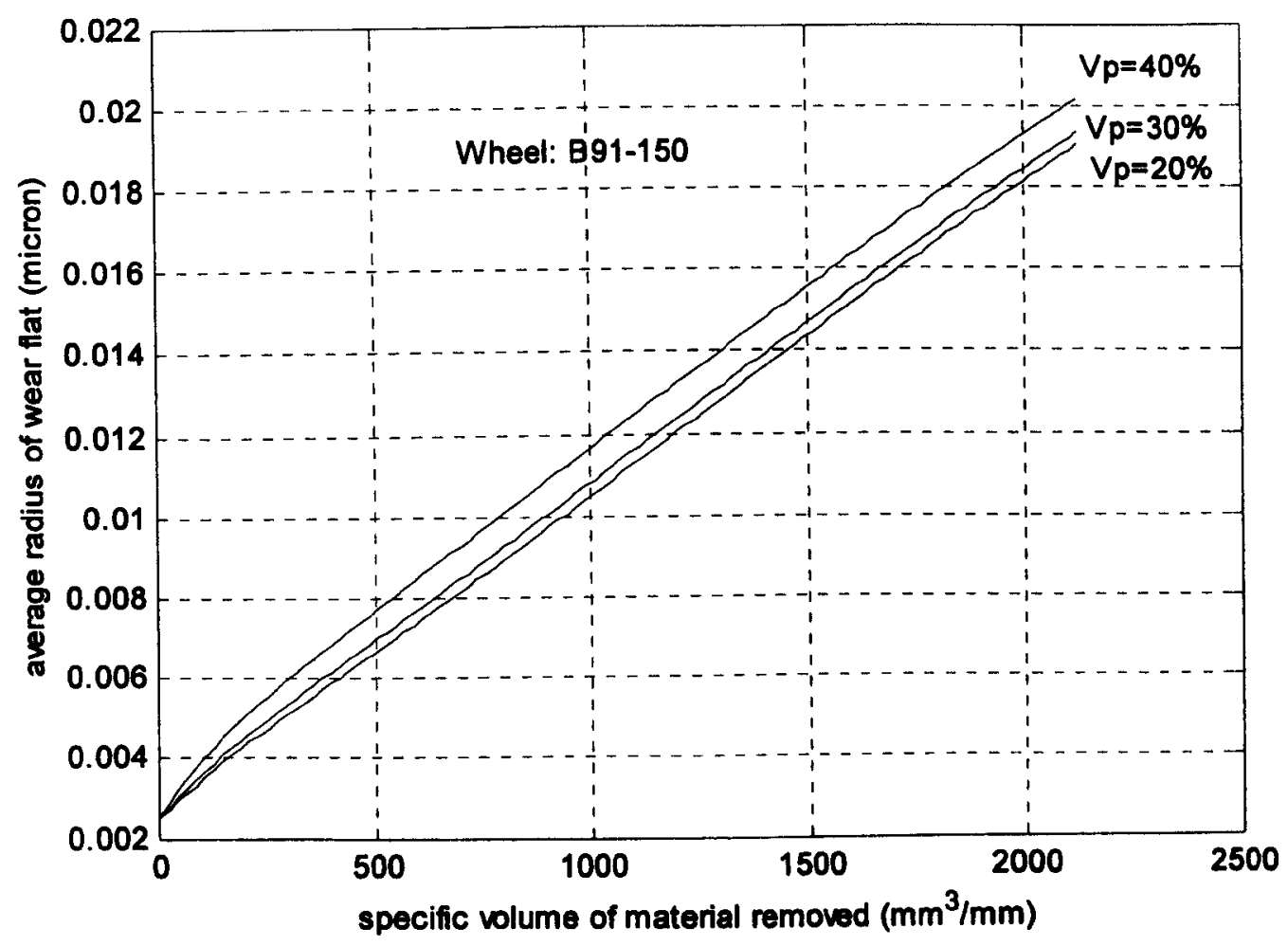

Fig. 7.5(b) The effect of percentage of pore volume on wear flat radius in prediction of wheel loading 


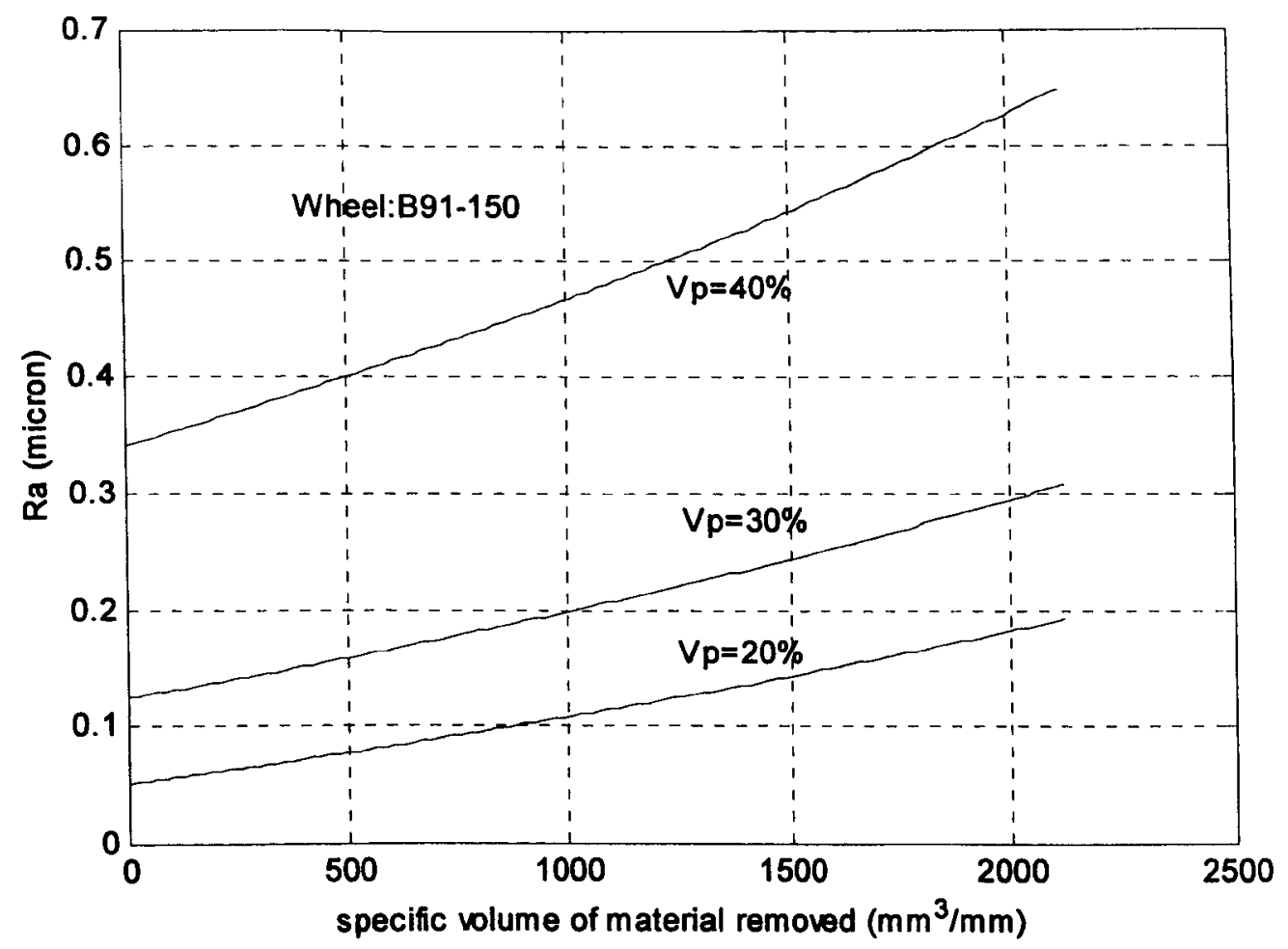

Fig. 7.5(c) The effect of percentage of pore volume on workpiece Ra surface roughness in prediction of wheel loading

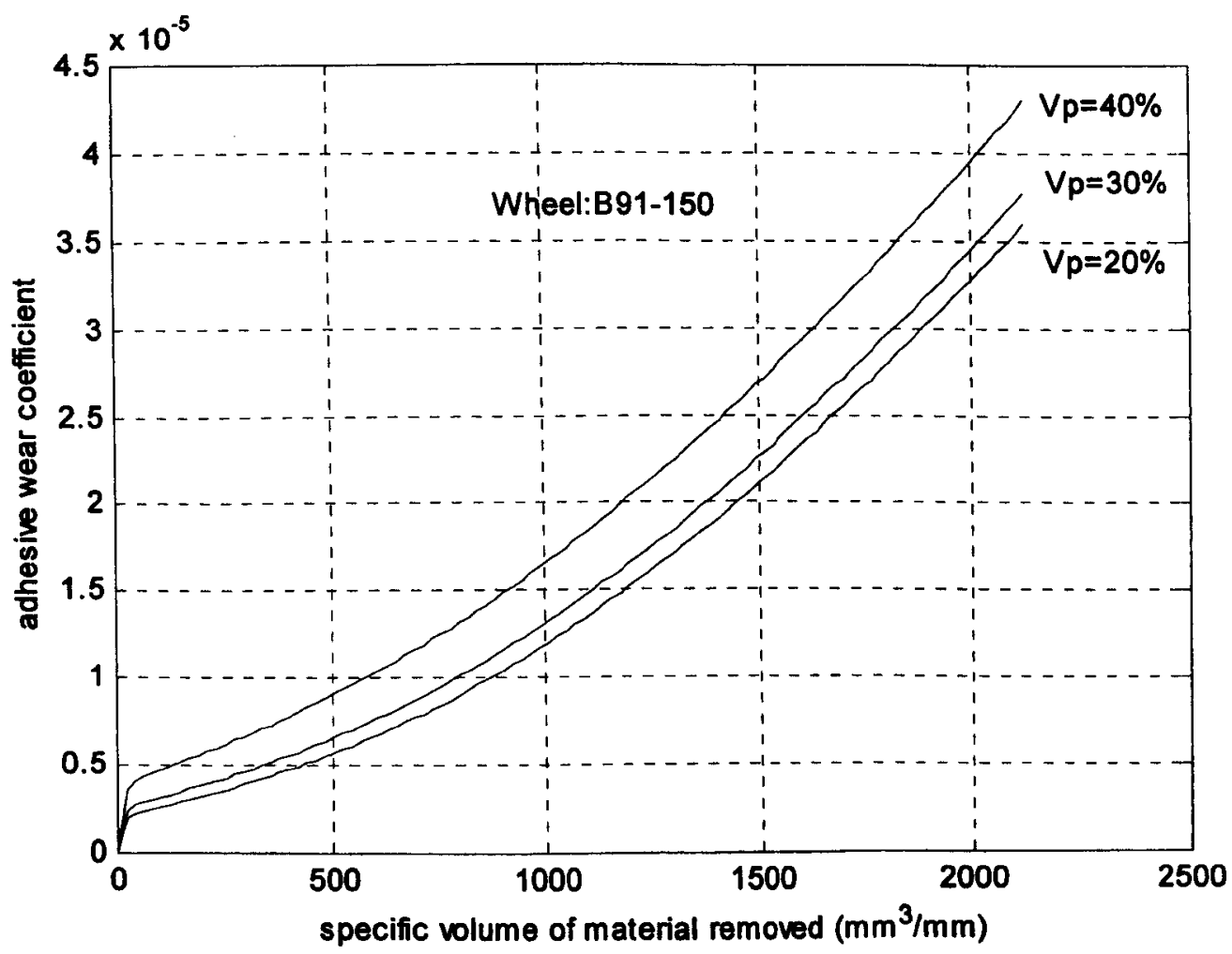

Fig. 7.5(d) The effect of percentage of pore volume on adhesive wear coefficient in prediction of wheel loading 


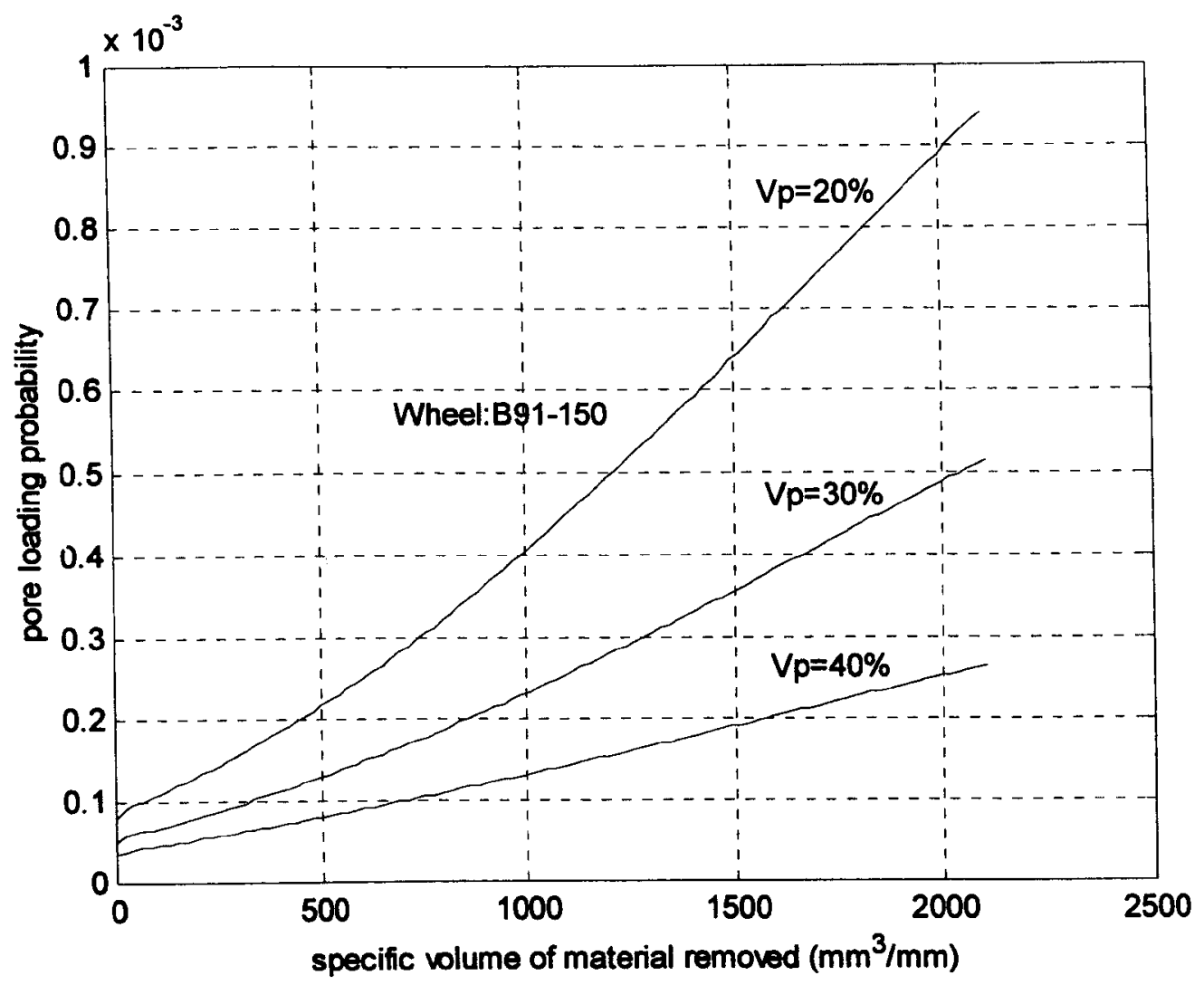

Fig. 7.5(e) The effect of percentage of pore volume on pore loading probability in prediction of wheel loading 


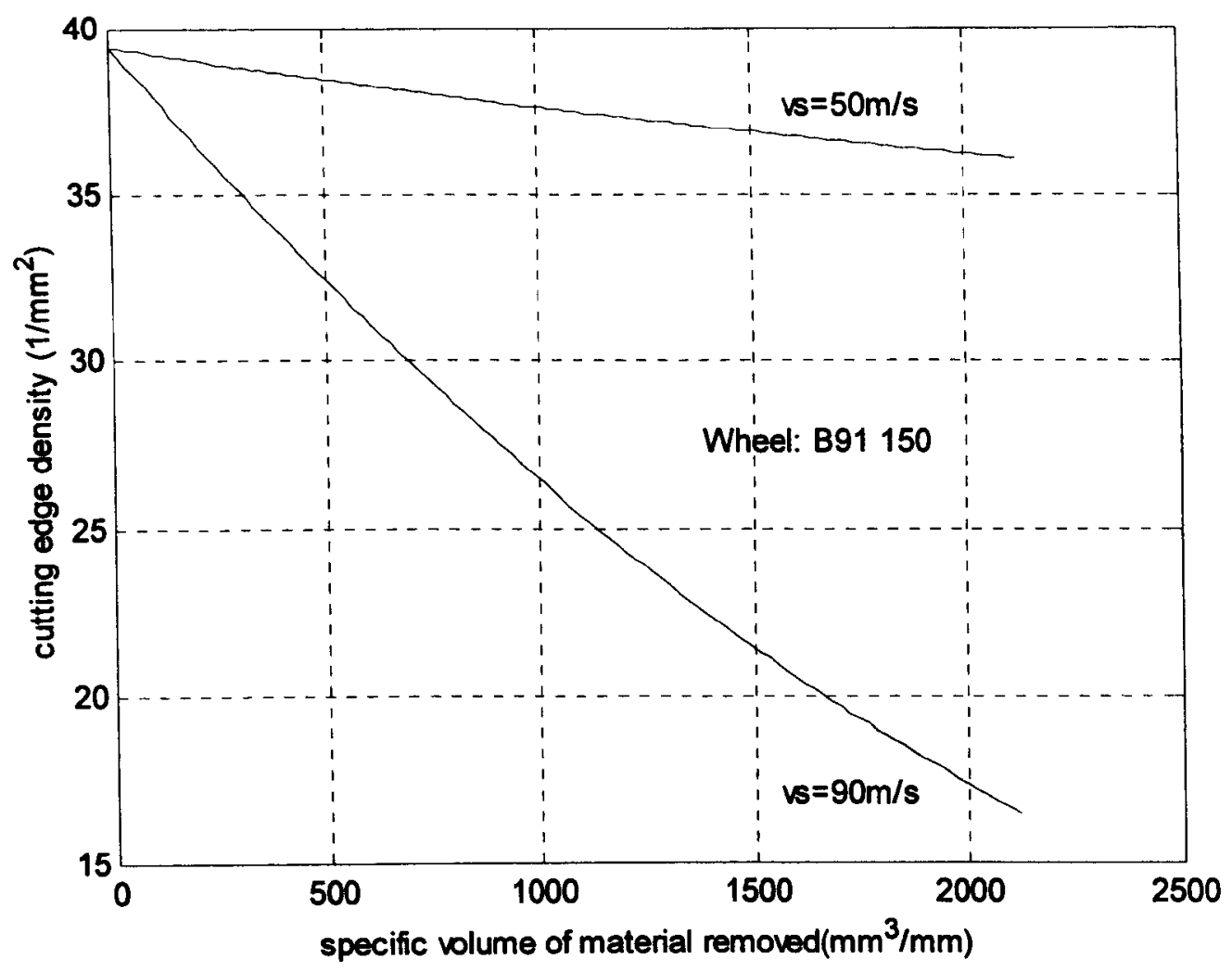

Fig. 7.6(a) The effect of wheel speed on cutting edge density in prediction of wheel loading

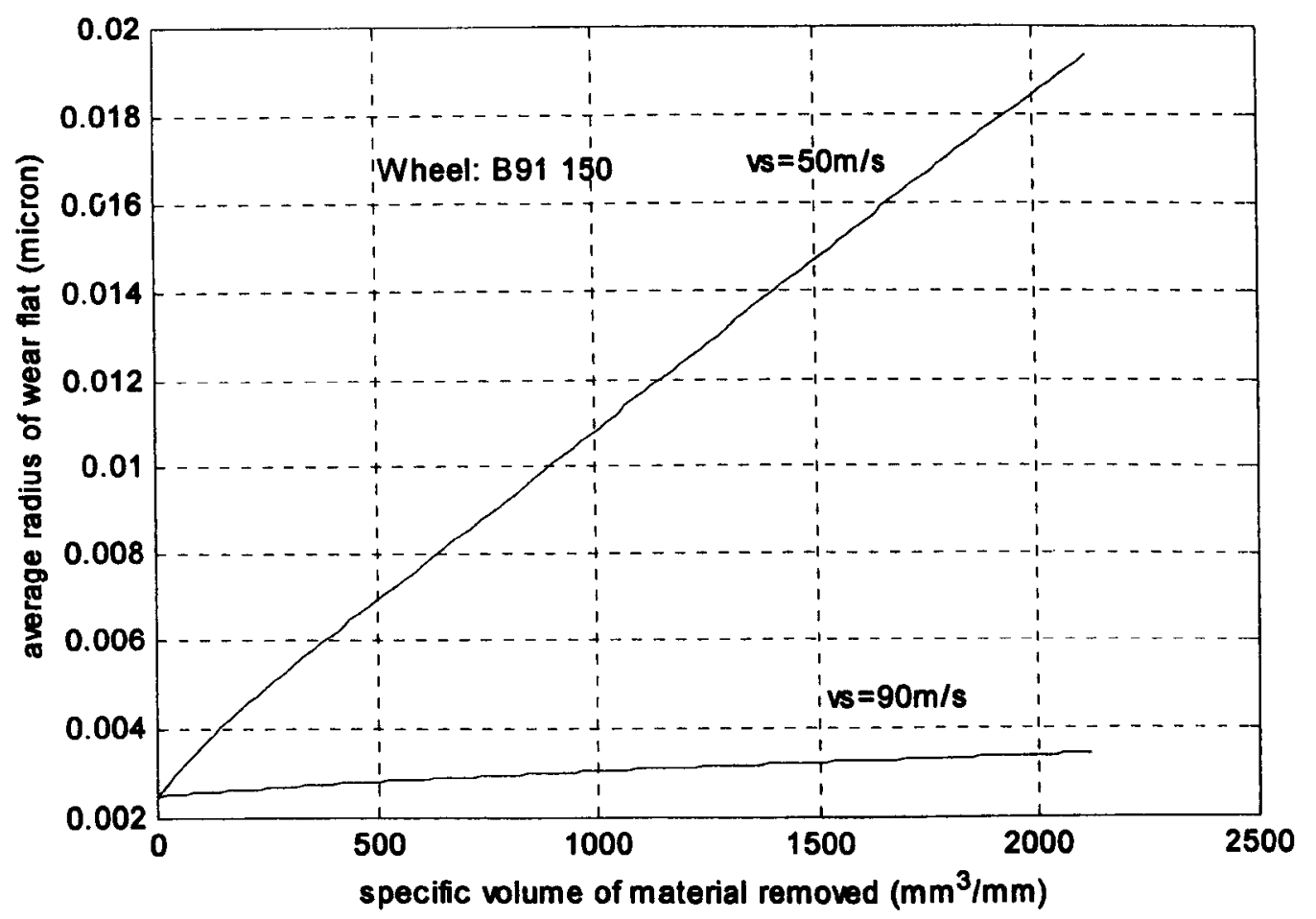

Fig. 7.6(b) The effect of wheel speed on wear flat radius in prediction of wheel loading 


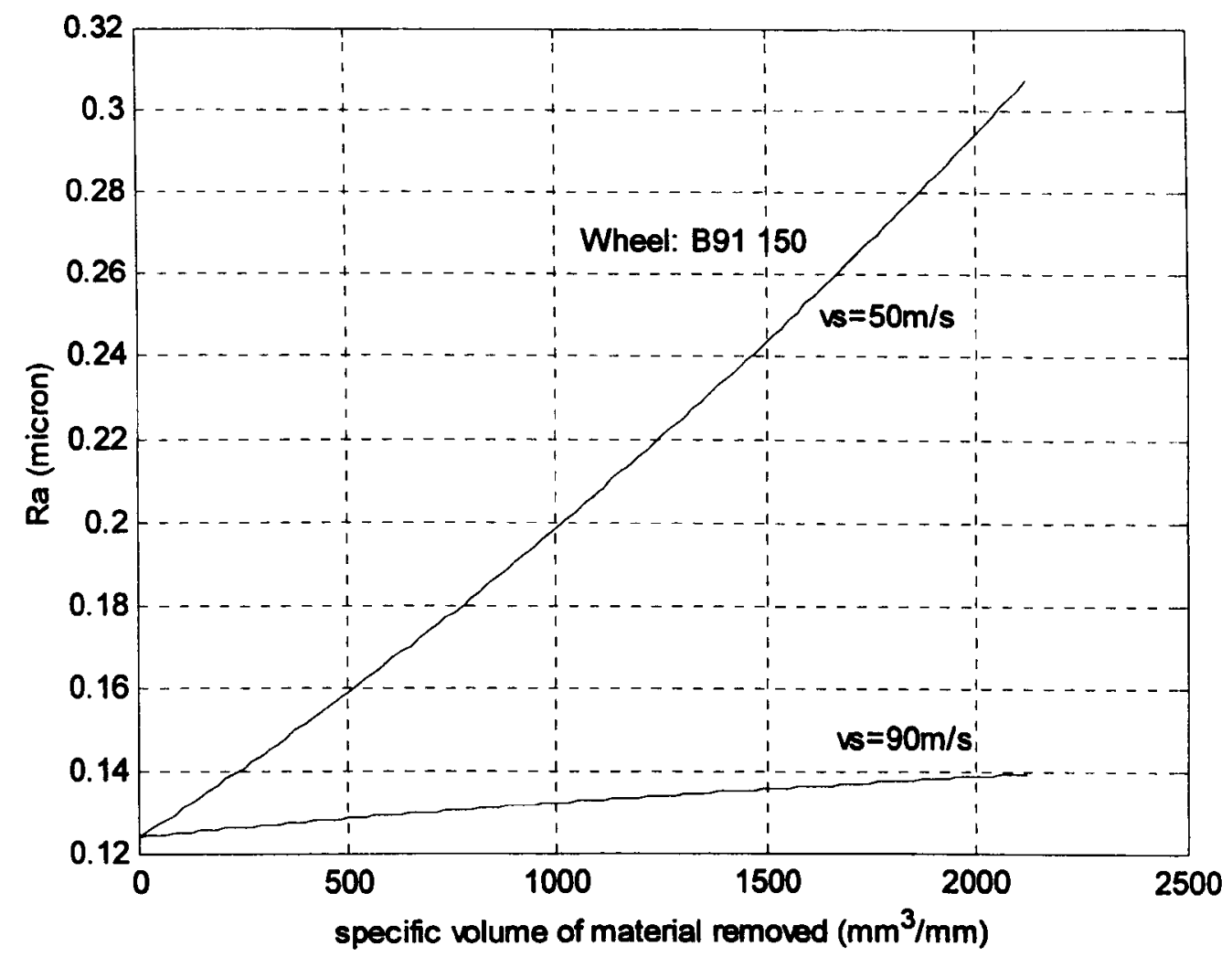

Fig. 7.6(c) The effect of wheel speed on workpiece Ra surface roughness in prediction of wheel loading

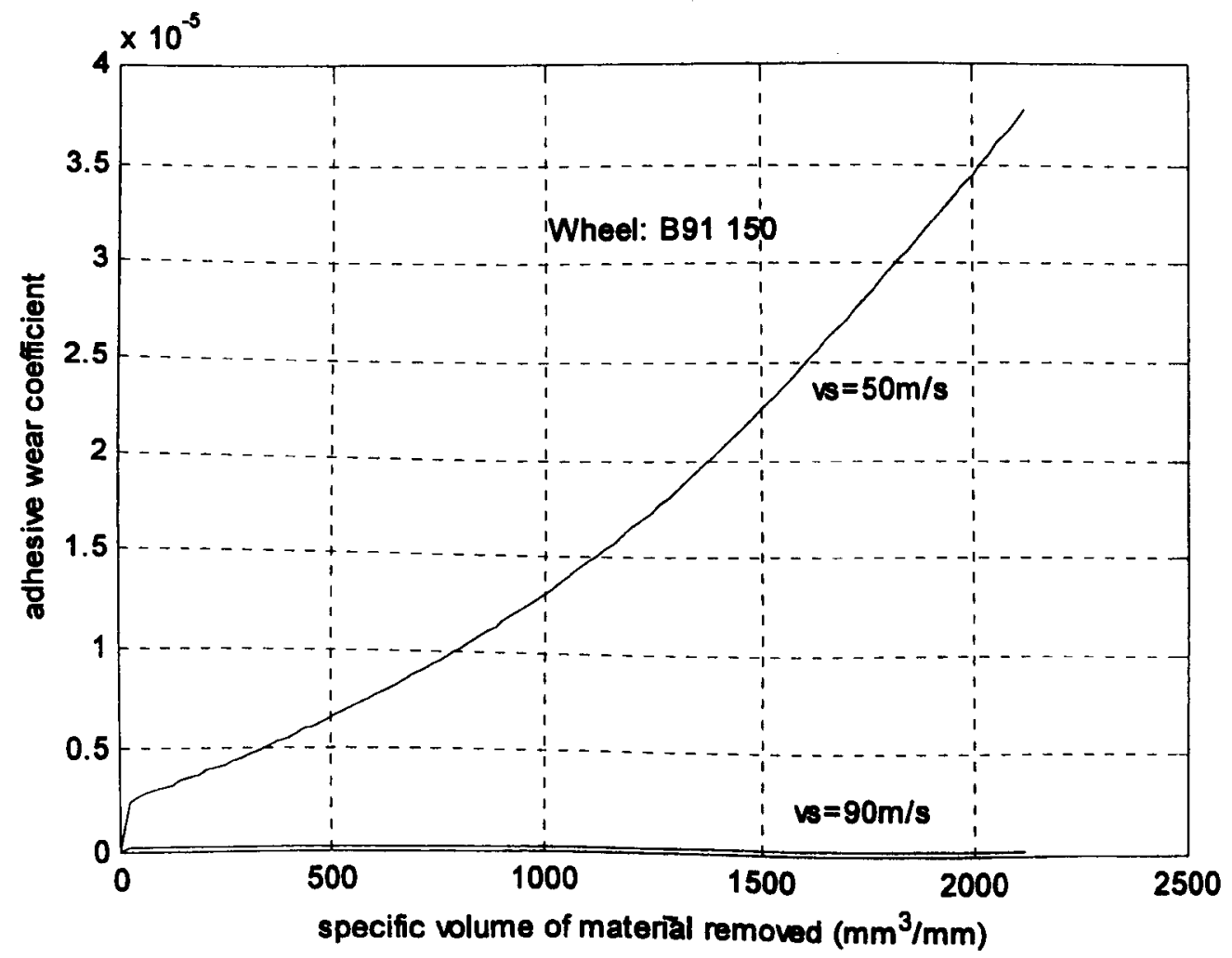

Fig. 7.6(d) The effect of wheel speed on adhesive wear coefficient in prediction of wheel loading 


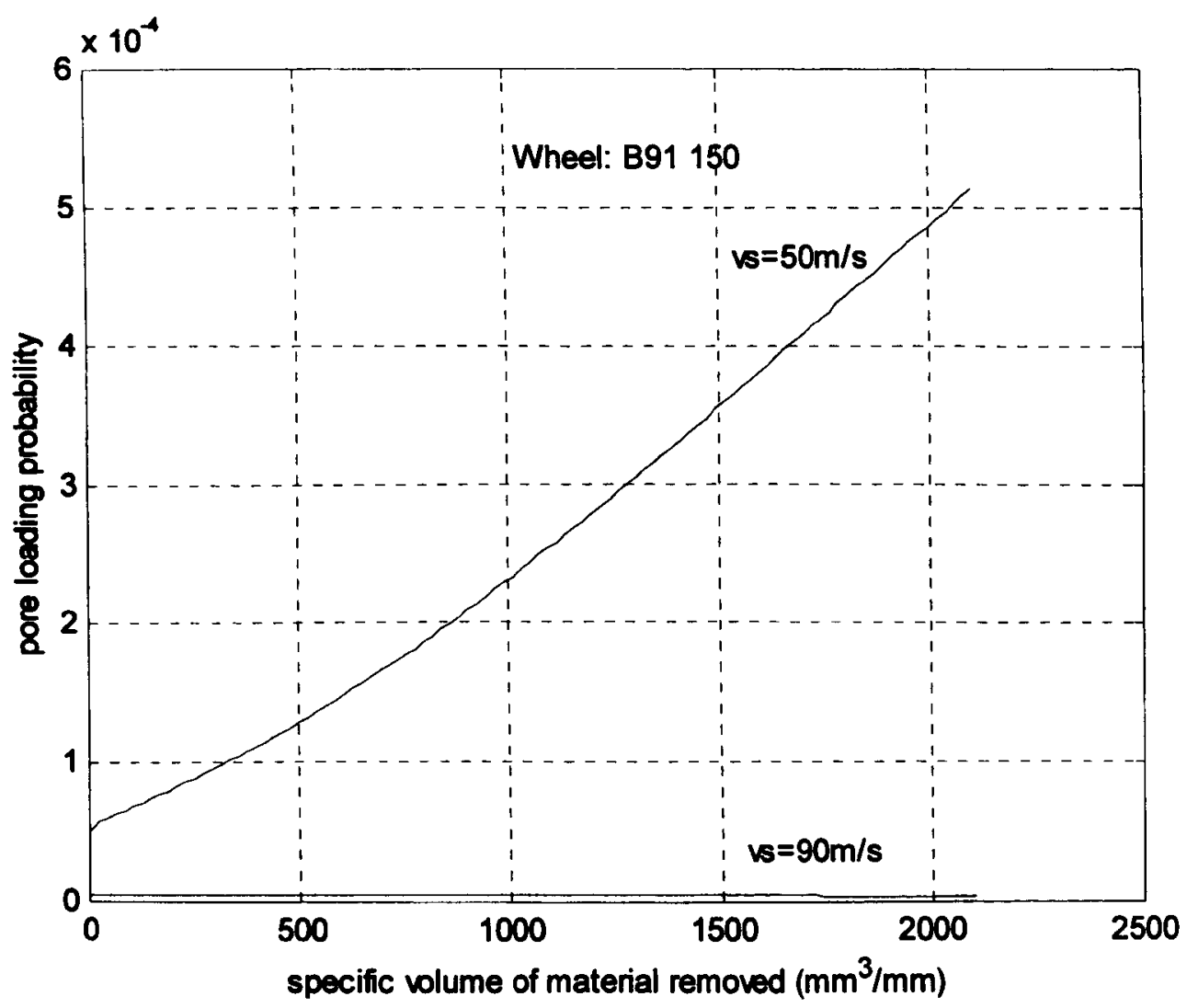

Fig. 7.6(e) The effect of wheel speed on pore loading probability in prediction of wheel loading

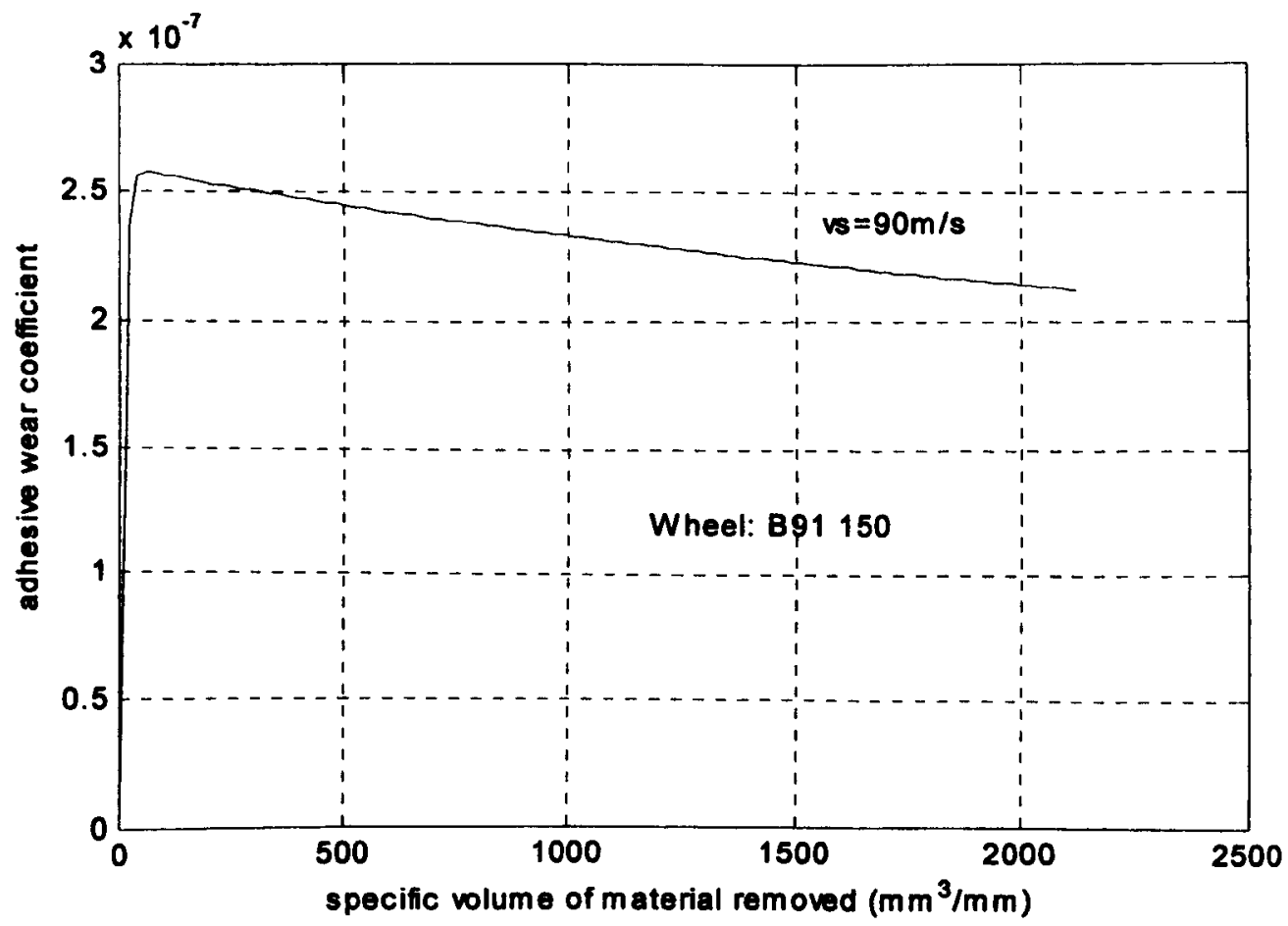

Fig. 7.6(f) The adhesive wear coefficient at wheel speed of $90 \mathrm{~m} / \mathrm{s}$ in prediction of wheel loading 


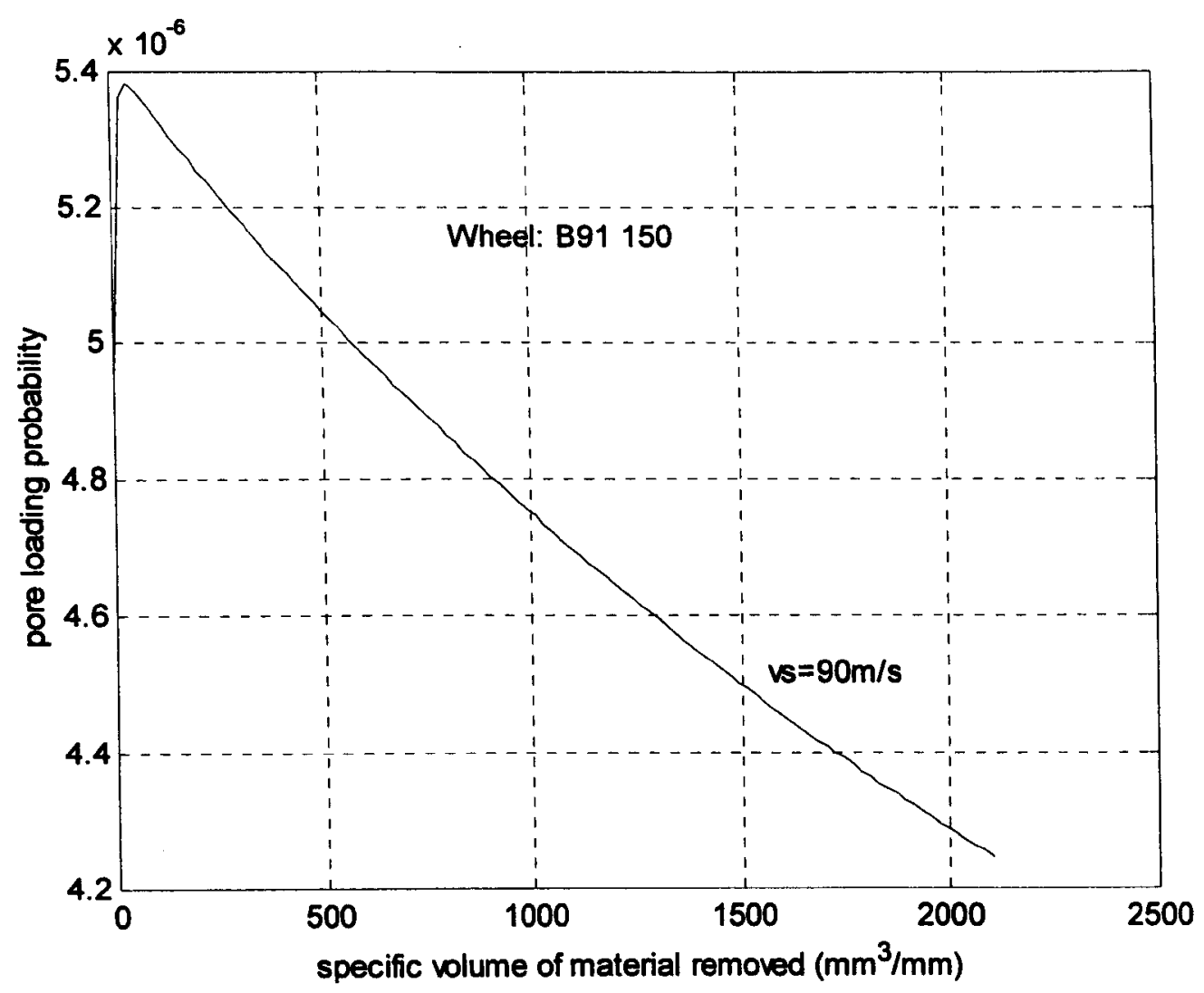

Fig. 7.6(g) The pore loading probability at wheel speed of $90 \mathrm{~m} / \mathrm{s}$ in prediction of wheel loading 